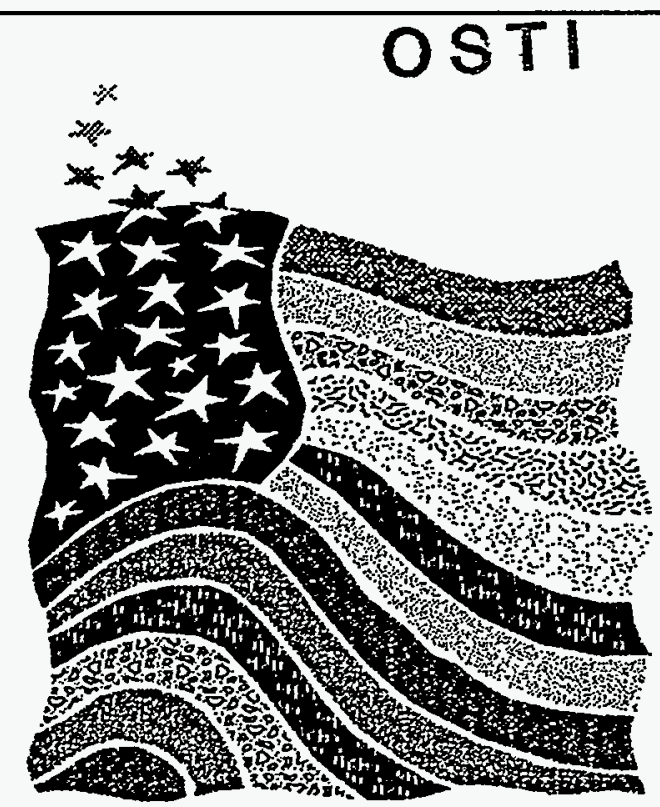

\title{
Ames Expedited Site Characterization Demonstration at the Former Manufactured Gas Plant Site Marshalltown, lowa
}

\author{
A. J. Bevolo, B. H. KJartanson \\ and J. D. Wonder
}

Report Date: March 1996

Ames Laboratory*

lowa State University

Ames, lowa 50011-3020

*Operated by lowa State University for the U. S. Department of Enorgy undor Contract No. W-7405-ENG-82 


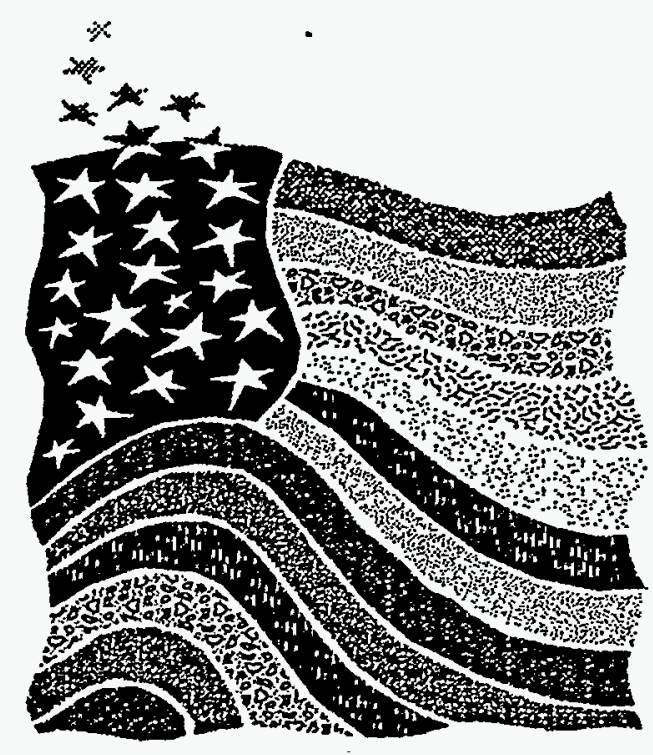

\title{
Ames Expedited Site Characterization Demonstration at the Former Manufactured Gas Plant Site Marshalltown, lowa
}

\author{
A. J. Bevolo, B. H. Kjartanson \\ and J. D. Wonder
}

Report Date: March 1996

Ames Laboratory* lowa State University Ames, lowa 50011-3020

*Operated by lowa State University for the U. S. Department of Energy under Contract No. W-7405-ENG-82 


\section{DISCLAIMER}

Portions of this document may be illegible in electronic image products. Images are produced from the best available original document. 


\section{This report was prepared as an account of work sponsored by an agency of the
United States Government. Neither the United States Government nor any
agency thereof, nor any of their employees, makes any warranty, express or
implied, or assumes any legal liability or responsibility for the accuracy,
completeness or usefulness of any information, apparatus, product, or process
disclosed, or represents that its use would not infringe privately owned rights.
Reference herein to any specific commercial product, process, or service by trade
name, trademark, manufacturer, or otherwise, does not necessarily constitute or
imply its endorsement, recommendation, or favoring by the United States
Government or any agency thereof. The views and opinions of authors expressed -
herein do not necessarily state or reflect those of the United States Government
or any agency thereof.}

This report has been reproduced directly from the best available copy.

AVAILABILITY:

To DOE and DOE contractors: Office of Scientific and Technical Information

P.O. Box 62

Oak Ridge, TN 37831

prices available from: (615) $576-8401$

FTS: 626-8401

To the public:

National Technical Information Service

U.S. Department of Commerce

5285 Port Royal Road

Springfield, VA 22161 
Ames Expedited Site Characterization - Marshalltown FMGP Site

FINAL Site Report

March 25, 1996

\section{TABLE OF CONTENTS}

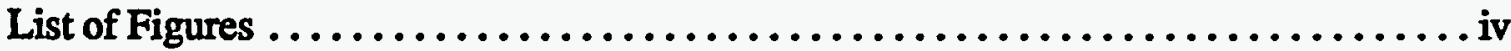

List of Tables..$\ldots \ldots \ldots \ldots \ldots \ldots \ldots \ldots \ldots \ldots \ldots \ldots \ldots \ldots \ldots \ldots \ldots$

EXECUTTVE SUMMARY $\ldots \ldots \ldots \ldots \ldots \ldots \ldots \ldots \ldots \ldots \ldots \ldots \ldots \ldots \ldots$

1.0 INTRODUCTION $\ldots \ldots \ldots \ldots \ldots \ldots \ldots \ldots \ldots \ldots \ldots \ldots \ldots \ldots \ldots \ldots \ldots \ldots \ldots$

1.1 Project Goals............................................

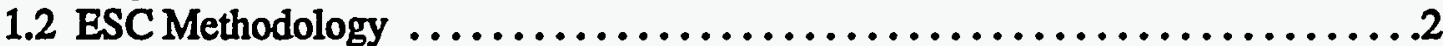

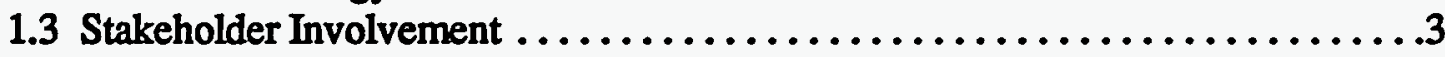

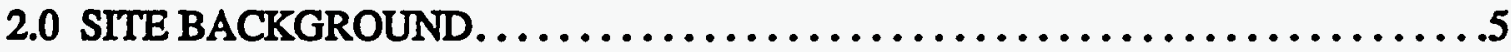

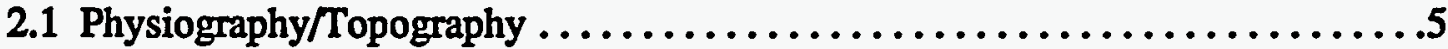

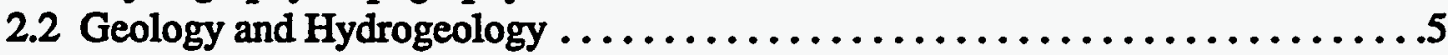

2.3 Marshalltown FMGP Site History $\ldots \ldots \ldots \ldots \ldots \ldots \ldots \ldots \ldots \ldots \ldots . \ldots \ldots$

2.4 Previous Investigations/Existing Data $\ldots \ldots \ldots \ldots \ldots \ldots \ldots \ldots \ldots \ldots, \ldots, \ldots$

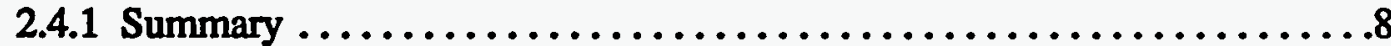

2.4.2 Remedial Investigation Data Analysis $\ldots \ldots \ldots \ldots \ldots \ldots \ldots \ldots \ldots . . .10$

2.4.3 Remedial Investigation Results .......................11

3.0 CHARACTERIZATION STRATEGY $\ldots \ldots \ldots \ldots \ldots \ldots \ldots \ldots \ldots \ldots \ldots \ldots \ldots$

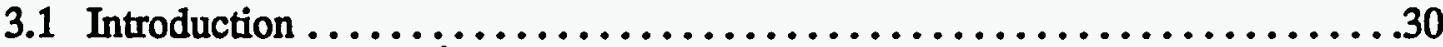

3.2 Geophysical Technologies . . . . . . . . . . . . . . . . . . . . . . . . . 30

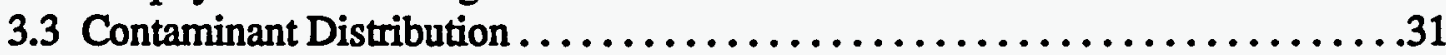

3.3.1 Screening Phase......................................

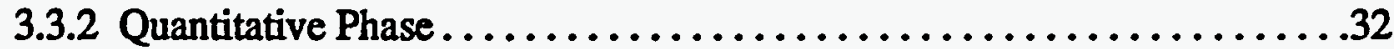

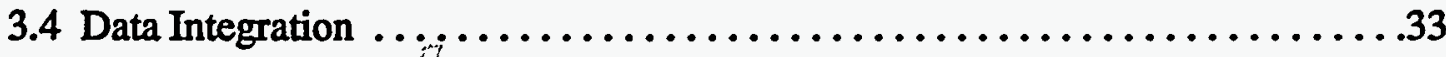

4.0 RESULTS AND EVALUATIONS $\ldots \ldots \ldots \ldots \ldots \ldots \ldots \ldots \ldots \ldots \ldots . \ldots . \ldots . \ldots . \ldots$

4.1 Non-Intrusive Methods for Geological Characterization - Geophysical

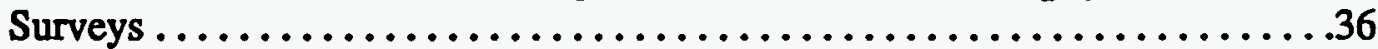

4.1.1 Ground Penetrating Radar $\ldots . \ldots \ldots \ldots \ldots \ldots \ldots \ldots \ldots \ldots \ldots . ., \ldots \ldots$

4.1.2 Seismic Reflection...............................41

4.1.3 Seismic Refraction..............................44

4.1.4 Borehole Geophysical Logging. ........................47

4.1.5 Electromagnetic Offset Logging (EOL) ..................50

4.1.6 Summary - General Comments on Geophysical Surveying Results . . . . .52

4.2 Phase I Contaminant Screening Technologies $\ldots \ldots \ldots \ldots \ldots \ldots \ldots \ldots .53$ 
Ames Expedited Site Characterization - Marshalltown FMGP Site

FINAL Site Report

March 25, 1996

4.2.1 Immunoassay $\ldots \ldots \ldots \ldots \ldots \ldots \ldots \ldots \ldots \ldots \ldots \ldots \ldots \ldots . . . \ldots \ldots$

4.2.2 Chemiluminescence................................56

4.2.3 Passive Soil Gas. ..................................57

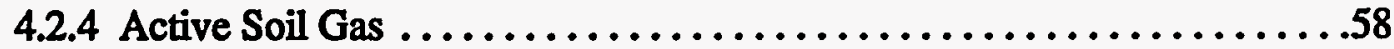

4.2.5 Summary - Phase I Contaminant Screening Technologies ..........59

4.3 Phase II Minimally Intrusive Methods for Geological Characterization and

Contaminant Screening. . . . . . . . . . . . . . . . . . . . . . . . . . . 59

4.3.1 Cone Penetration Testing. ..............................61

4.3.2 Soil Conductivity Probe..............................67

4.3.3 Interpretation from Minimally Intrusive Methods $\ldots \ldots \ldots \ldots \ldots \ldots . \ldots 71$

4.4 Quantitative Chemical Analyses of Soil Samples ...................79

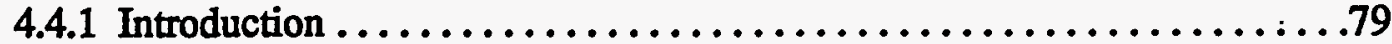

4.4.2 Comparison of Extraction Methods......................82

4.4.3 Site Characterization Implications. .....................89

4.5 Analysis of Groundwater Samples $\ldots \ldots \ldots \ldots \ldots \ldots \ldots \ldots \ldots \ldots \ldots . .92$

4.6 Stakeholder Activities $\ldots \ldots \ldots \ldots \ldots \ldots \ldots \ldots \ldots \ldots \ldots \ldots \ldots \ldots \ldots . . .63$

4.6.1 Communications Strategy $\ldots \ldots \ldots \ldots \ldots \ldots \ldots \ldots \ldots \ldots \ldots . . .63$

4.6.2 Objectives and Audience...........................93

4.6.3 Identification of Stakeholders and Planned Activities ............95

4.6.4 Evaluation of Objectives $\ldots \ldots \ldots \ldots \ldots \ldots \ldots \ldots \ldots \ldots \ldots \ldots . .96$

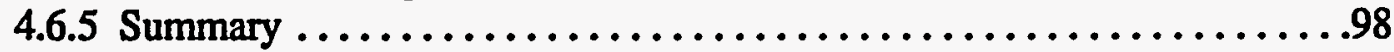

5.0 SUMMARY AND CONCLUSIONS $\ldots \ldots \ldots \ldots \ldots \ldots \ldots \ldots \ldots \ldots . \ldots \ldots 2$

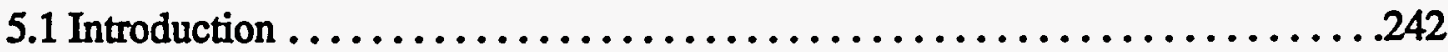

5.2 Geological/Stratigraphic Characterization $\ldots \ldots \ldots \ldots \ldots \ldots \ldots \ldots \ldots . .243$

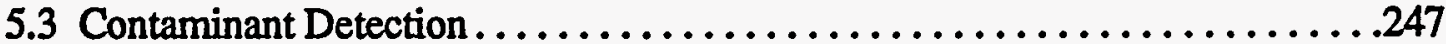

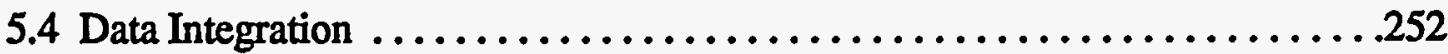

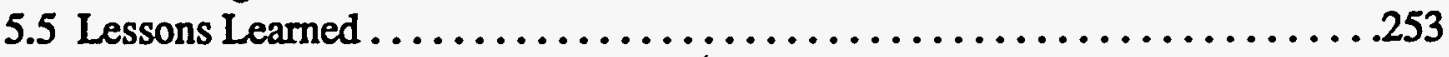

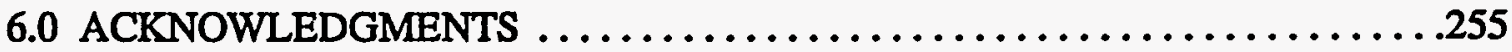

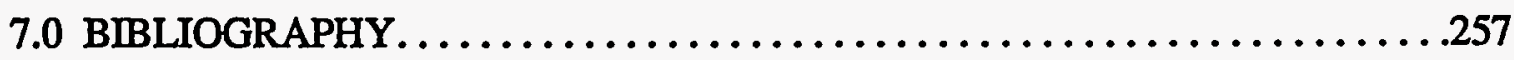

8.0 APPENDICES

Appendix A: Stratigraphic Logs for Boreholes HSA-1 and HSA-2

Appendix B: Panel Plots for CPT Pushes

Appendix C: Conductivity Profiles - Conductivity Versus Depth Logs

Appendix D: Analytical Results of each Sample for the 16 PAHs

Appendix E: Contractors and Consultants List 
Ames Expedited Site Characterization - Marshalltown FMGP Site FINAL Site Report

March 25, 1996

Appendix F: Stakeholder List

Appendix G: Attendee Lists for Conference Days

Appendix H: Abbreviations and Acronyms List

\subsection{DISTRIBUTION LIST}


Ames Expedited Site Characterization - Marshalltown FMGP Site

FINAL Site Report

March 25, 1996

\section{List of Figures}

Figure 2.1 Marshalltown FMGP Site Location Map (from BVWST, 1992) . . . . . 14

Figure 2.2 Marshalltown FMGP Site Map (from BVWST, 1992) ...........15

Figure 2.3 Auger Borehole Locations............................

Figure 2.4 Typical Site Stratigraphic Units and Stratigraphic Zones ...........17

Figure 2.5 BVWST Naphthalene and Phenanthrene

Concentrations - Stratigraphic Zone $1 \ldots \ldots \ldots \ldots \ldots \ldots \ldots$. 18

Figure 2.6 BVWST Naphthalene and Phenanthrene

Concentrations - Stratigraphic Zone 2 ..................19

Figure 2.7 BVWST Naphthalene and Phenanthrene

Concentrations - Stratigraphic Zone 3 ................20

Figure 2.8 BVWST Naphthalene and Phenanthrene

Concentrations - Stratigraphic Zone 4 .................21

Figure 2.9 BVWST - Observed Level of PAH Contamination in

Soils - Stratigraphic Zone $1 . \ldots \ldots \ldots \ldots \ldots \ldots \ldots \ldots . .22$

Figure 2.10 BVWST - Observed Level of PAH Contamination in

Soils - Stratigraphic Zone 2......................23

Figure 2.11 BVWST - Observed Level of PAH Contamination in

Soils - Stratigraphic Zone $3 . \ldots \ldots \ldots \ldots \ldots \ldots \ldots \ldots .24$

Figure 2.12 BVWST - Observed Level of PAH Contamination in

Soils - Stratigraphic Zone 4.......................25

Figure 2.13 BVWST - Observed Level of PAH Contamination in

Soils - Stratigraphic Zone $5 . \ldots \ldots \ldots \ldots \ldots \ldots \ldots \ldots \ldots .26$

Figure 2.14 BVWST - Observed Level of PAH Contamination in

Soils - Stratigraphic Zone $6 \ldots \ldots \ldots \ldots \ldots \ldots \ldots \ldots \ldots . \ldots \ldots \ldots$

Figure $3.1 \quad$ ESC Phase I Timeline..$\ldots \ldots \ldots \ldots \ldots \ldots \ldots \ldots \ldots \ldots \ldots \ldots \ldots \ldots$

Figure 3.2 ESC Phase II Timeline. $\ldots \ldots \ldots \ldots \ldots \ldots \ldots \ldots \ldots \ldots \ldots \ldots . . . \ldots \ldots$

Figure 4.1 Location of GPR Profiles (from D'Appolonia, 1994) ............99

Figure 4.2 GPR 300 MHZ Survey - Profile \#13 (from D'Appolonia, 1994) ..... 100

Figure 4.3 GPR 300 MHZ Survey - 13 ns Time Slice (from D'Appolonia, 1994) .101

Figure 4.4 GPR 100 MHZ Survey - CMP Profile (from D'Appolonia, 1994).... 102

Figure 4.5 Location of Seismic Reflection Lines

(from Resolution Resources, 1994)..................103

Figure 4.6 Interpreted Seismic Reflection Profiles

(from Resolution Resources, 1994)..................104 
Ames Expedited Site Characterization - Marshalltown FMGP Site

FINAL Site Report

March 25, 1996

Figure 4.7 Seismic Reflection - Check Shot Survey

(from Resolution Resources, 1994)..................105

Figure 4.8 Location of Seismic Refraction Lines (copy to Figure 4.8) ........106

Figure 4.9 Seismic Refraction Survey - Line 3 (from CEES - Blackhawk

Geosciences Division, 1994).....................107

Figure 4.10 Seismic Refraction Survey - Line 4 (from CEES - Blackhawk

Geosciences Division, 1994)......................108

Figure 4.11 Seismic Refraction Survey - Line 5 (from CEES - Blackhawk

Geosciences Division, 1994)......................109

Figure 4.12 Seismic Refraction Survey - Line 7 (from CEES - Blackhawk

Geosciences Division, 1994)......................110

Figure 4.13 Seismic Refraction Survey - Elevation of Bedrock from BVWST

Boreholes (from CEES - Blackhawk Geosciences Division, 1994) .111

Figure 4.14 Seismic Refraction Survey - Elevation of Bedrock from BVWST

Boreholes and Seismic Refraction Survey Results (from

CEES - Blackhawk Geosciences Division, 1994)...........112

Figure 4.15

Figure 4.16

Figure 4.17

Figure 4.18

Figure 4.19

Figure 4.20

Figure 4.21

Figure 4.22

Borehole Geophysical Logs - MW1 (from Technos, 1994) ..........113

Borehole Geophysical Logs - MW2B (from Technos, 1994) ..........114

Borehole Geophysical Logs - MW3B (from Technos, 1994) . . . . . . . 115

Borehole Geophysical Logs - HSA-2 (from Technos, 1994) . . . . . . . 116

Borehole Geophysical Logs - MW5 (from Technos, 1994) ..........117

Borehole Geophysical Logs - HSA-1 (from Technos, 1994) .........118

Electromagnetic Offset Logging - Source and Receiver Map

Locations (from GEHM, 1994) .....................119

Electromagnetic Offset Logging - 3-D Image of Resistivity $>55$

Ohm-meters (from GEHM, 1994)...................120

Figure 4.23 Seismic Refraction Survey - Depth to Bedrock from BVWST

Boreholes and Seismic Refraction Survey Results (from

CEES - Blackhawk Geosciences Division, 1994)............121

Figure 4.24

ESC Phase I Contaminant Screening Locations . . . . . . . . . . . 122

Figure 4.25

Figure 4.26

IMA Shallow Zone Screening - ENSYS ................... 123

IMA Shallow Zone Screening - Millipore. . . . . . . . . . . . . . 124

Figure 4.27

IMA Shallow Zone Screening - Quantix .................. 125

Figure 4.28

Figure 4.29

Figure 4.30

Figure 4.31

Figure 4.32

Figure 4.33

Figure 4.34

IMA Deep Zone Screening - ENSYS . . . . . . . . . . . . . . . 126

IMA Deep Zone Screening - Millipore ..................... 127

IMA Deep Zone Screening - Quantix .....................128

Chemiluminescence Shallow Zone Screening ................129

Chemiluminescence Deep Zone Screening ..................130

Passive Soil Vapor Shallow Zone Screening - Naphthalene.........131

Passive Soil Vapor Deep Zone Screening - Naphthalene .......... 132 
Ames Expedited Site Characterization - Marshalltown FMGP Site

FINAL Site Report

March 25, 1996

Figure 4.35 Passive Soil Vapor Shallow Zone Screening -

Anthracene/Phenanthrene .......................133

Figure 4.36 Passive Soil Vapor Deep Zone Screening - Anthracene/Phenanthrene .134

Figure 4.37 Passive Soil Vapor Shallow Zone Screening - Fluoranthene/Pyrene ...135

Figure 4.38 Passive Soil Vapor Deep Zone Screening - Fluoranthene/Pyrene ....136

Figure 4.39 Active Soil Vapor Shallow Zone Screening - Naphthalene .........137

Figure 4.40 Active Soil Vapor Deep Zone Screening - Naphthalene...........138

Figure 4.41 CPT Panel Plot for ML-109............................139

Figure 4.42 CPT Push Locations .............................140

Figure 4.43 Comparison of CPT ML-28 Stratigraphic Log with

BVWST MW-3 Log ..........................141

Figure 4.44 Comparison of CPT ML-32 Stratigraphic Log with

BVWST MW-4 Log ..........................142

Figure 4.45 CPT Stratigraphic Repeatability Comparison - ML-32..........143

Figure 4.46 CPT LIF Fluorescence Intensity - Stratigraphic Zone $1 \ldots \ldots \ldots \ldots \ldots 14$

Figure 4.47 CPT LIF Fluorescence Intensity - Stratigraphic Zone 2 . . . . . . . . . 145

Figure 4.48 CPT LIF Fluorescence Intensity - Stratigraphic Zone 3 . .........146

Figure 4.49 CPT LIF Fluorescence Intensity - Stratigraphic Zone $4 \ldots \ldots \ldots \ldots . .147$

Figure 4.50 CPT LIF Fluorescence Intensity - Stratigraphic Zone $5 \ldots \ldots \ldots \ldots 148$

Figure 4.51 CPT LIF Fluorescence Intensity - Stratigraphic Zone $6 \ldots \ldots \ldots \ldots . .149$

Figure 4.52 Geoprobe Soil Conductivity Push Locations.................150

Figure 4.53 Soil Stratigraphic Comparison - ML-28 Soil Conductivity vs

BVWST MW-3A vs CPT ML-28 ...................151

Figure 4.54 Soil Stratigraphic Comparison - Soil Conductivity ML-45 vs

BVWST B-8 vs CPT ML-60......................152

Figure 4.55 Coal Tar Residue Contamination Comparison - Soil Conductivity

ML-45 vs BVWST B-8 vs CPT LIF ML-60.............153

Figure 4.56 Coal Tar Residue Contamination Comparison - CPT LIF

ML-109 vs Soil Conductivity ML-54 ..................154

Figure 4.57 Location of Data Clusters Used for Stratigraphic and

Contaminant Level Comparisons ....................155

Figure 4.58 Locations of Stratigraphic Cross Sections Developed with

Push Technology Data ...........................156

Figure 4.59 Stratigraphic Cross Section AA' $\ldots \ldots \ldots \ldots \ldots \ldots \ldots \ldots \ldots \ldots \ldots \ldots \ldots$

Figure 4.60 Stratigraphic Cross Section BB' ..........................

Figure 4.61 Earth Vision Stratigraphic Model - East-West Cut at $500 \mathrm{~N}$.........159

Figure 4.62 EarthVision Stratigraphic Model - North-South Cut at 850 E ......160

Figure 4.63 EarthVision Stratigraphic Model - North-South Cut at 825 E .......161

Figure 4.64 EarthVision Stratigraphic Model - North-South Cut at 750 E .......162

Figure 4.65 Granular Unit/Lower Cohesive Unit Contact - Day $1 \ldots \ldots \ldots \ldots \ldots 163$ 
Ames Expedited Site Characterization - Marshalltown FMGP Site

FINAL Site Report

March 25, 1996

Figure 4.66 Granular Unit/Lower Cohesive Unit Contact - Day 2 $\ldots \ldots \ldots \ldots \ldots 164$

Figure 4.67 Granular Unit/Lower Cohesive Unit Contact - Day 4............165

Figure 4.68 Granular Unit/Lower Cohesive Unit contact - Day $10 \ldots \ldots \ldots \ldots \ldots 6$

Figure 4.69 Lower Cohesive Unit Surface Elevation Based on Kriged

Median Polish Residuals..........................167

Figure 4.70 Lower Cohesive Unit Surface Elevation Prediction Standard

Deviation Based on Kriged Median Polish Residuals ...........168

Figure 4.71 Seismic Refraction Survey Line \#5 - Comparison

with Direct Push Data ...............................

Figure 4.72 Seismic Refraction Survey Line \#7 - Comparison

with Direct Push Data ............................170

Figure 4.73 Seismic Refraction Survey Line \#9 - Comparison

with Direct Push Data ...........................171

Figure 4.74

Figure 4.75

Figure 4.76

Seismic Reflection Survey - West Line Profile...............172

Earth Vision Stratigraphic Model - North-South cut at 450 E ........173

McLaren/Hart Samples - Observed Level of PAH

Contamination in Soils - Stratigraphic Zone 1 ..............174

Figure 4.77 McLaren/Hart Samples - Observed Level of PAH

Contamination in Soils - Stratigraphic Zone 2 .............175

Figure 4.78 McLaren/Hart Samples - Observed Level of PAH

Contamination in Soils - Stratigraphic Zone 3 ............176

Figure 4.79 McLaren/Hart Samples - Observed Level of PAH

Contamination in Soils - Stratigraphic Zone 4 .............177

Figure 4.80 McLaren/Hart Samples - Observed Level of PAH

Contamination in Soils - Stratigraphic Zone 5 .............178

Figure 4.81 McLaren/Hart Samples - Observed Level of PAH

Contamination in Soils - Stratigraphic Zone 6 ..............179

Figure 4.82 Stratigraphic Cross Section AA' showing Zones of High

Coal Tar Residue Contamination ....................180

Figure 4.83

Figure 4.84

Figure 4.85

Figure 4.86

Three Dimensional EarthVision Visualization - LIF Intensity ........181

Three Dimensional EarthVision Visualization - Regions

with LIF Intensity $>100$ Counts . . . . . . . . . . . . . . . . . 182

McLaren/Hart Soil and Groundwater Sampling Locations...........183

Extraction Method Analytical Results - Means of Combined

Sandy and Clayey Soil Samples Plotted by Analyte............184

Figure 4.87 Extraction Method Analytical Results - Clayey Soil Sample

Means Plotted by Analyte - Data Corrected for Percent Recovery .185

Figure 4.88 Extraction Method Analytical Results - Sandy Soil Sample

Means Plotted by Analyte - Data Corrected for Percent Recovery .186 
Ames Expedited Site Characterization - Marshalltown FMGP Site

FINAL Site Report

March 25, 1996

Figure 4.89 McLaren/Hart GC/MS Soil Sample Analysis

Results - Naphthalene - Stratigraphic Zone 1 .............187

Figure 4.90 McLaren/Hart GC/MS Soil Sample Analysis

Results -Phenanthrene -Stratigraphic Zone 1 .............188

Figure 4.91 McLaren/Hart GC/MS Soil Sample Analysis

Results -Naphthalene - Stratigraphic Zone 2 ..............189

Figure 4.92 McLaren/Hart GC/MS Soil Sample Analysis

Results -Phenanthrene - Stratigraphic Zone 2 . . . . . . . . . . 190

Figure 4.93 McLaren/Hart GC/MS Soil Sample Analysis

Results-Naphthalene - Stratigraphic Zone 3 . . . . . . . . . . . 191

Figure 4.94 McLaren/Hart GC/MS Soil Sample Analysis

Results -Phenanthrene - Stratigraphic Zone 3.............. 192

Figure 4.95 McLaren/Hart GC/MS Soil Sample Analysis

Results -Naphthalene - Stratigraphic Zone 4 ..............193

Figure 4.96 McLaren/Hart GC/MS Soil Sample Analysis

Results -Phenanthrene - Stratigraphic Zone 4.............. 194

Figure 4.97 McLaren/Hart GC/MS Soil Sample Analysis

Results -Naphthalene - Stratigraphic Zone 5 ...............195

Figure 4.98 McLaren/Hart GC/MS Soil Sample Analysis

Results -Phenanthrene - Stratigraphic Zone 5.............196

Figure 4.99 McLaren/Hart GC/MS Soil Sample Analysis

Results -Naphthalene - Stratigraphic Zone 6 ............... 197

Figure 4.100 McLaren/Hart GC/MS Soil Sample Analysis

Results -Phenanthrene - Stratigraphic Zone 6..............198

Figure 4.101 McLaren/Hart GC/MS Soil Sample Analysis

Results -Total PAH in Stratigraphic Zone 1 ................199

Figure 4.102 McLaren/Hart GC/MS Soil Sample Analysis

Results -Total PAH in Stratigraphic Zone 2 ..............200

Figure 4.103 McLaren/Hart GC/MS Soil Sample Analysis

Results -Total PAH in Stratigraphic Zone 3 ..............201

Figure 4.104 McLaren/Hart GC/MS Soil Sample Analysis

Results -Total PAH in Stratigraphic Zone 4 .............. 202

Figure 4.105 McLaren/Hart GC/MS Soil Sample Analysis

Results -Total PAH in Stratigraphic Zone 5 .............203

Figure 4.106 McLaren/Hart GC/MS Soil Sample Analysis

Results -Total PAH in Stratigraphic Zone 6 ..............204

Figure 4.107 McLaren/Hart GC/MS Groundwater Sample Analysis -

Naphthalene and Phenanthrene in Shallow Samples ..........205

Figure 4.108 McLaren/Hart GC/MS Groundwater Sample Analysis -

Naphthalene and Phenanathrene in Deep Samples ...........206

viii 
Ames Expedited Site Characterization - Marshalltown FMGP Site

FINAL Site Report

March 25, 1996

List of Tables

Table 2.1 BVWST RI - Naphthalene and Phenanthrene Concentration in Soil (mg/kg) by Stratigraphic Zone $\ldots \ldots \ldots \ldots \ldots \ldots \ldots .28$

Table 2.2 BVWST RI - Observed Level of PAH Contamination by

Stratigraphic Zone ............................29

Table 4.1 GPR Velocity vs Depth - Derived from Moveout

Measured at CMP Test .........................207

Table 4.2 Approximate Electrical Conductivities, Relative Dielectric Constants

and Travel Times for Various Earth Materials .............207

Table 4.3 Phase I Contaminant Screening - HPLC Analytical

(EPA Method 8130) and Immunoassay Results ...........208

Table 4.4 Phase I Contaminant Screening - HPLC Analytical

and Chemiluminescence Results....................211

Table 4.5 Phase I Contaminant Screening - Passive Soil Gas Results ........212

Table 4.6 Phase I Contaminant Screening - Active Soil Gas Results ........213

Table 4.7 CPT LIF Data for Soil Samples Held Against Sapphire

Window vs Split Sample Laboratory TRPH ..............215

Table 4.8 Panel Plot CPT LIF Intensity vs Laboratory Analysis of

Adjacent Soil Samples ..........................216

Table 4.9 CPT LIF Intensities with Stratigraphic Zones...............217

Table 4.10 Comparison of Geoprobe Conductivity Dips with McLaren/Hart

and CPT Logs ...................................

Table 4.11 Soil Classification Comparisons by Selected Sample

Location Clusters..............................220

Table 4.12 Depths of Stratigraphic Contacts from Direct Push Technologies . . . .223

Table 4.13 Comparison of Kriged LCU Surface Elevations with LCU

Surface Elevations from BVWST Borehole Logs ..........226

Table 4.14 Soil PAH Contamination Comparison by Selected Sample

Location Clusters...........................227

Table 4.15 McLaren/Hart Soil Samples - Observed Level of PAH

Contamination by Stratigraphic Zone $\ldots \ldots \ldots \ldots \ldots \ldots \ldots . .230$

Table 4.16 McLaren/Hart Soil Sample Attributes...................231

Table 4.17 McLaren/Hart Soil Samples Used for Extraction Method

Comparisons .............................234

Table 4.18 McLaren/Hart Soil Sample Analysis - Average Percent

Recovery by Extraction Method and Analyte .............235 
Ames Expedited Site Characterization - Marshalltown FMGP Site

FINAL Site Report

March 25, 1996

Table 4.19 Summary Statistics over Soil Samples within Analyte/Method

Table 4.20

Subgroups ................................236

Table 4.21

Sandy vs Clayey Soil Sample Comparisons for all Data...........237

Summary of Method Detection Limit Data by Extraction

Method .....................................238

Table 4.22 Summary of Method Detection Limits Developed

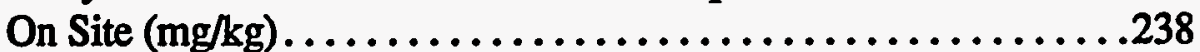

Table 4.23 Relative Error (\%) Calculated from Averages by Analyte for

Each Method ...............................239

Table 4.24 Summary of Method Bias and Precision Statistical Tests............239

Table 4.25

McLaren/Hart Groundwater Sample Results

.240 
Ames Expedited Site Characterization - Marshalltown FMGP Site

FINAL Site Report

March 25, 1996

\section{EXECUTIVE SUMMARY}

Ames Laboratory of the U.S. Department of Energy (DOE) undertook a project sponsored by the Office of Technology Development (OTD) in the Environmental Management (EM) program to promote the adoption of Expedited Site Characterization (ESC) and its associated technologies. The goals of the project were to identify and field site characterization technologies and approaches to solving DOE environmental problems, improve the basis for analyzing site characterization data, compare the various analytical technologies, and encourage stakeholder participation in the environmental arena. An ESC demonstration was carried out at a former manufactured gas plant site (FMGP) in Marshalltown, Iowa. The site was known to be impacted by polynuclear aromatic hydrocarbon (PAH) residues and was selected as the subject site because of its size, proximity, and favorable relations amongst Ames Laboratory, the Iowa Department of Natural Resources (IDNR), and the site owners. It should be noted that IDNR is the lead agency coordinating all the activities of the Marshalltown site and all data and reports must be validated and approved by IDNR before they can be used in any decision-making process.

The Marshalltown FMGP is owned by IES Utilities, Inc. and is located in an old industrial area adjacent to an active railroad switching yard and mainlines. The gas manufacturing processes, used during the operation of the site between the $1880 \mathrm{~s}$ and $1950 \mathrm{~s}$, resulted in a variety of potential environmental contaminants including "coal tar," petroleum products, condensates, and oxides. A portion of these materials were disposed on the site if they could not be resold on the market or used for other purposes. The primary contaminants of interest for this investigation were PAHs.

Previous remedial investigations were conducted at the site by Black\&Veatch (B\&V) Waste Science and Technology (BVWST). Historical and technical information, gathered in those previous investigations, were used to select technologies and develop scopes of work for the ESC demonstration. Available geologic data indicated a surface layer of fill overlying an upper cohesive unit which was overlying granular alluvial sediments that were overlying a lower cohesive unit and ultimately bedrock. All of these layers occurred at variable depths. Available contaminant data indicated the presence of dissolved, residual, and dense non-aqueous phase (DNAPL) liquid PAH's in soil and groundwater beneath the site. A suite of geophysical survey, geophysical/geotechnical screening-level chemical, and quantitative chemical analytical technologies were selected and applied at the site. The involvement of IDNR, IES and the Ames Laboratory with ESC should not be interpreted as an endorsement of any system or product used in the ESC investigation. 
Ames Expedited Site Characterization - Marshalltown FMGP Site

FINAL Site Report

March 25, 1996

Geophysical survey techniques including ground penetrating radar, seismic reflection and refraction, electromagnetic offset logging, and borehole logging were applied at the site primarily to define the surface of the bedrock and significant stratigraphic interfaces above the bedrock. Secondary objectives were to provide information regarding the distribution of PAH contamination. Geophysical survey techniques are considered to be the primary component in the early phase of the ESC process.

In addition to the geophysical survey techniques, two minimally intrusive geophysical/geotechnical techniques (cone penetrometer testing and soil electrical conductivity logging) were utilized to define the soil stratigraphy and, in particular, the surface topography of the lower cohesive unit (LCU).

Results of the geophysical surveying were calibrated against both the BVWST data and with the minimally intrusive data collected as a part of this investigation. Cultural interference, such as noise from the railroad tracks and from activities within the site, overhead power lines, buildings and surface metallic objects, near-surface fill material, and a weathered bedrock surface, contributed to difficulties encountered in making and interpreting geophysical measurements. The interpretation of the bedrock surface from the geophysical survey techniques was tenuous and found to be in error by as much as 10 to 15 feet when compared with data from direct intrusive technologies. The negative impact of the cultural interference and relatively difficult stratigraphic conditions make evaluation of the techniques very difficult. All the techniques, however, pointed to the fact that the bedrock surface is weathered, uneven and highly variable.

The Site Characterization and Analysis Penetrometer System (SCAPS) cone penetrometer unit and the Geoprobe soil conductivity probe provided very useful and reliable stratigraphic data. Side-by-side comparisons of the direct push technology logs with BVWST borehole logs indicated stratigraphic correspondence of the unit contacts generally within about one to two feet. It should be noted, however, that the BVWST data tended to produce a slightly deeper granular/lower cohesive unit contact than the direct push data. Usually the major unit stratigraphic contacts were easily picked off of both the cone penetration testing (CPT) and soil conductivity logs and were used to generate a database from which an EarthVision threedimensional site stratigraphic model was generated.

Based on the previous BVWST site characterization work, the lateral and vertical distribution of the dissolved PAHs and residual non-aqueous phase liquid contamination was estimated. Assessment of the nature and distribution of the PAH contaminants was carried out using three types of technologies: Phase I screening technologies [immunoassays (IMAs), passive and active soil gas, and chemiluminescence], Phase II screening technologies [laser-induced fluorescence probe, soil conductivity probe], and Phase II quantitative technologies [chemical 
Ames Expedited Site Characterization - Marshalltown FMGP Site

FINAL Site Report

March 25, 1996

analysis of soil samples with gas chromatography/mass spectrometry (GC/MS) instruments in field laboratories].

For the purpose of developing and assessing the site contamination model, the soil stratigraphy was subdivided into six zones:

- middle and bottom of upper cohesive unit ( zones 1 and 2 respectively)

- top, middle and bottom of granular unit ( zones 3,4 and 5 respectively)

- top of lower cohesive unit ( zone 6)

The Phase I suite of contaminant screening technologies were applied in an effort to evaluate their ability to identify the approximate boundaries of the organically contaminated area. At each surface grid node, Geoprobe sampling equipment was used to collect one shallow (4 to 5 feet deep) and one deep (10 to 15 feet deep) soil sample. Duplicates from soil core samples were analyzed by all three IMA techniques and the chemiluminescence system. In addition, a soil vapor sample was drawn at each sampling level and analyzed using a field gas chromatograph. Finally, a passive soil gas collector containing carbon adsorption elements in a resealable glass container was installed in the ground at the approximate depths of the soil samples.

When indicating the presence or absence of detectable PAHs, the data from each of the three IMA analyses correlated fairly well with each other. Furthermore, each of the three shallow and deep data sets agree very well with each other on the location and shape of the PAH

contamination distribution. Results from the chemiluminescence were reported as total PAH and did not correlate as well with other methods used.

Passive soil vapor samplers were analyzed off site by thermal desorption and direct mass spectrometer (MS) analysis. The shallow data agreed well with each other. Little of the heavier PAHs compounds were found in the deeper data set, and as expected, lighter molecular weight PAHs appears to correspond to higher volatility and concentration in soil gas. Active soil gas measurements for aromatic hydrocarbons and naphthalene showed good agreement with passive soil gas and IMA measurements. Overall, the results of the Phase I contaminant screening technologies generally compared well with the BVWST results for stratigraphic zones 1,2 and 3 . A significant finding of the Phase I contaminant screening study was that PAH contamination existed further to the west than it would appear from previous data.

Phase II contaminant screening was performed using the cone penetrometer laser-induced fluorescence (LIF) sensor system and the Geoprobe soil conductivity profiles. Chemical 
Ames Expedited Site Characterization - Marshalltown FMGP Site

FINAL Site Report

March 25, 1996

analysis of soil samples collected adjacent to LIF "hits" indicated that while the LIF sensor data could not be considered as quantitative, it could reliably detect regions of low, medium or high contamination in a qualitative sense. Average LIF intensities for each of the six stratigraphic zones were interpreted from the LIF panel plots. Contour plots for each zone gave an excellent indication of the distribution of contaminants at the site. The general trend of the contaminated region is still, however, from the northwest to the southeast through the central part of the site. Significant hits were detected in zone 6 (top of the LCU) at only two locations.

Many of the panel plots showed elevated fluorescent intensity values within the upper cohesive unit and within the upper and lower portions of the granular unit. Examination of the LIF panel plots revealed that elevated fluorescence intensity levels commonly occur in the two to four feet of sandy soil immediately overlying the top of the LCU indicating a pooling of DNAPLs on the LCU. When LIF measurements were taken in the top of the LCU, the fluorescent intensity reading typically dropped to near zero. This indicates that the LCU is tending to retard the downward migration of the DNAPL contamination at these locations. In terms of contaminant screening, the LIF may be considered the most direct qualitative methodology for indicating regions of PAH contamination.

Phase II quantitative plume delineation efforts were planned and implemented based on results of the BVWST Remedial Investigation (RI) report, contaminant screening data collected in Phase I and Phase II, and the updated site geologic model. The primary technology evaluation function of this part of Phase II was the comparison and assessment of five on-site extraction methods for PAHs in soil. Soil core samples for this effort were collected with minimal subsurface disturbance using a Geoprobe system. A total of 127 samples were run through one or more of the five extraction methods and analyzed by one of three identical GC/MS systems. The extraction methods used included three organic solvent-based methods (sonication, microscale, microwave-enhanced extraction) plus thermal desorption and supercritical fluid extraction (SFE).

Method bias comparisons indicated that the thermal extraction efficiency is less than the sonication extraction efficiency which is less than the microwave extraction efficiency. The microscale and SFE extraction methods generally gave intermediate results. There was no definitive indication that any extraction method was more precise overall, although there is a tendency for the method precision for the thermal and microscale extraction methods to be significantly less than the precision for the sonication method on the basis of all the available data. On the basis of all the available data, the clayey soil analytical results showed greater variation than the sandy soil results indicating greater precision with sandy samples for all but the thermal extraction method. The 38 subsets of this data, in which all methods gave a greater than non detect (ND) determination, indicate that only the microwave and SFE methods show 
Ames Expedited Site Characterization - Marshalltown FMGP Site

FINAL Site Report

March 25, 1996

greater variation with clayey than with sandy soil, while the other methods show no variation differences between soil types.

In summary, within the inherent limitations of these analyses, low thermal extraction results with high relative error and high microwave extraction results with low relative error were found, while the absolute value of the bias for both of these methods appears to decrease with increasing analyte molecular weight. A significant finding of the study was the potential for inconsistencies in procedures and results to arise, even within strict adherence to SW-846 methods.

The application, versatility, and high quality of data from direct push technologies was demonstrated at this site. The cone penetrometer demonstrated the reliability and efficiency of both the stratigraphic logging capabilities and LIF sensing capabilities in developing the site stratigraphic model and delineating areas of PAH contamination. The capabilities of the Geoprobe soil conductivity system with respect to maneuverability and operational efficiency were also demonstrated. With proper calibration, the Geoprobe system can be reliably used to fill in stratigraphic data between two locations with known stratigraphic profiles. In addition, again with proper calibration and verification, it can be used to enhance the site contamination model. Both direct push technologies have the capability to provide much more detailed stratigraphic information than conventional auger boring, which is important when considering contaminant fate and transport.

This study also indicated the potential for significant variation of chemical analysis results for PAHs in soils. The uncertainty and potential variability associated with soil matrix effects, sample selection, preparation and extraction procedures far outweigh inaccuracies in the chemical analysis methodologies themselves. The Phase I and Phase II screening results, including olfactory/visual data, gave a far better picture of the distribution and extent of contamination than the quantitative analysis results.

Ames Laboratory is operated for the U.S.-DOE by Iowa State University under Contract No. W7405-ENG-82 and is a member of ISU's Institute for Physical Research and Technology. This report was prepared as a deliverable on the project "Ames Laboratory Expedited Site Characterization Demonstrations," Technical Task Plan CH1-3-10-05, as part of the Characterization, Monitoring, and Sensor Technology Crosscutting Program in the OTD within the DOE's EM program. 
Ames Expedited Site Characterization - Marshalltown FMGP Site

FINAL Site Report

March 25, 1996

\subsection{INTRODUCTION}

\subsection{Project Goals}

The goal of the Ames Expedited Site Characterization (ESC) project is to evaluate and promote both innovative technologies (IT) and state-of-the-practice technologies (SOPT) for site characterization and monitoring. In April and May 1994, the ESC project conducted site characterization, technology comparison, and stakeholder demonstration activities at a former manufactured gas plant (FMGP) owned by Iowa Electric Services (IES) Utilities, Inc., in Marshalltown, Iowa. It should be noted that the Iowa Department of Natural Resources (IDNR) is the lead agency coordinating all the activities of the Marshalltown site and all data and reports must be validated and approved by IDNR before they can be used in any decision-making process.

Three areas of technology were fielded at the Marshalltown FMGP site: geophysical, analytical and data integration. The geophysical technologies are designed to assess the subsurface geological conditions so that the location, fate and transport of the target contaminants may be assessed and forecasted. The analytical technologies/methods are designed to detect and quantify the target contaminants. The data integration technology area consists of hardware and software systems designed to integrate all the site information compiled and collected into a conceptual site model on a daily basis at the site; this conceptual model then becomes the decision-support tool. Simultaneous fielding of different methods within each of the three areas of technology provided data for direct comparison of the technologies fielded, both SOPT and IT.

Ames Laboratory implemented the ESC model, first developed at Argonne National Laboratory (Burton,1993), by using some of the tools that are coming to be associated with ESC, such as a dynamic work plan and real time data analysis. Because of the Ames Laboratory's approach to stakeholder involvement, the Ames ESC project differs somewhat from past ESC practices. Because of their increasing role in the acceptance and ultimate commercialization of environmental technologies, early stakeholder involvement was sought; establishment and maintenance of close communications with regulators was viewed as particularly important. Significant efforts were made to invite participation from stakeholder communities including local residents, characterization and remediation technology providers, community organizations, and specialized and general state and local media. The demonstration activities of the ESC project provided opportunities not only for the Ames ESC project to communicate the ESC goals and activities to the stakeholders, but also provided opportunities for stakeholders to express their interest in and provide feedback about the technologies fielded as part of the project, and about ESC methodology and its future applications. 
Ames Expedited Site Characterization - Marshalltown FMGP Site

FINAL Site Report

March 25, 1996

All of these activities support the U. S. Department of Energy (DOE) environmental technology development goals. These goals, which include a directive to seek out and develop better, safer, faster, cheaper technologies and approaches to solving environmental problems, form the basis for analyzing the site characterization data, comparing the various analytical technologies, and encouraging stakeholder participation in the environmental arena.

This document reports the results of the site characterization, technology comparison, and ESC demonstration activities associated with the Marshalltown FMGP site. The involvement of IDNR, IES and the Ames Laboratory with ESC should not be interpreted as an endorsement of any system or product used in the ESC investigation.

The structure of the report basically follows the ESC process. The Marshalltown FMGP site background and the results of a previous remedial investigation (RI) are summarized in Chapter 2. This geological and contamination data was used to build the first conceptual model. Next, the overall ESC characterization strategy for this site is outlined in Chapter 3. The results and analyses of the Phase I and Phase II Ames ESC investigations are summarized in Chapter 4. The Phase I geophysical survey results are presented first and their contribution to the development of the site model assessed. Next, the Phase I contaminant screening results are presented. The results of the Phase I studies are both compared with each other and compared with the original RI data in terms of reliability of data and enhancement of the site conceptual model. Finally, the Phase II minimally intrusive data and the quantitative data are presented. Comparisons are again made with the already existing data and their contribution to the development of the site conceptual model assessed. The summary and conclusions of this investigation are presented in Chapter 5.

\subsection{ESC Methodology}

The ESC methodology incorporates on-site decision-support technologies that permit site characterizations to be completed in a consolidated package. The principal characteristics of ESC are:

- emphasis on geologic structure and hydrogeology as determinants of contaminant fate and transport,

- use of technologies by expert operators with flexible data quality objectives,

- on-site data processing using mobile laboratories, 
Ames Expedited Site Characterization - Marshallown FMGP Site

FINAL Site Report

March 25, 1996

- on-site decision making,

- preference for non-intrusive or minimally intrusive geophysical techniques,

- minimization of intrusive sampling techniques, and

- the same team that plans site work manages site work.

ESC has demonstrated that the characterization phase can be streamlined without compromising data quality. By using both on-site analytical and multiple hydrogeologic technologies, the need to send nearly all samples off site and the need to perform massive subsurface sampling in the absence of local hydrogeologic information is removed. By including on-site decision making, ESC can significantly reduce the probability of having to return to the site to fill data gaps. As a result, the current multiphase sequence of environmental data acquisition--consisting of sample, analyze, plan and sample, that typically takes years--becomes compressed into a single realtime phase, requiring only months to complete.

Because technology gaps exist, even within the context of ESC, the scope of this project involves the use of both SOPT as well as IT. By fielding ITs at actual sites, nontechnical as well. as technical barriers are simultaneously identified. Of prime importance is an opportunity to involve regulators in the evaluation and fielding of these technologies. Side-by-side comparison of IT with SOPT technologies, documented standard operating procedures, and confirmatory offsite analysis are some of the necessary features that encourage regulatory acceptance of ITs.

By integrating the innovative ESC technologies into an actual ESC project, it is expected that a more realistic evaluation of these technologies will be possible. Part of this benefit accrues from the active role of the regulator in the evaluation of these technologies. Additional benefit comes from exposure of these technologies to site-specific stakeholders, as well as those who cannot attend the demonstration but who will be reached by the information packages. This systematic approach, involving the demonstration of multiple ESC technologies to those who influence their adoption, is very cost effective.

\subsection{Stakeholder Involvement}

The Ames ESC team implemented the expedited site characterization model using many of the tools that are essential to ESC, such as a dynamic work plan and real-time data analysis, but Ames Laboratory's practice of ESC is distinctive in its. approach to stakeholder involvement. Because of the increasing role of stakeholders in the acceptance and ultimate commercialization 
of environmental technologies--and even more so in the case of a new methodology--early stakeholder involvement was sought.

Establishing and maintaining close communications with regulators was viewed as critically important; significant efforts were made to invite participation from stakeholder communities including local residents, community organizations, educators, students, association members, technology users and providers, trade press and local media. The public demonstration activities of the ESC project provided opportunities not only for the Ames Lab ESC team to communicate ESC goals and activities to the stakeholders, but also provided opportunities for stakeholders to express their interest and give feedback on the ESC methodology, its future applications and the technologies fielded as part of the project. 
Ames Expedited Site Characterization - Marshalltown FMGP Site

FINAL Site Report

March 25, 1996

\subsection{SITE BACKGROUND}

The Marshalltown FMGP is located south of East Nevada Street between 4th and 6th Avenue in the SE $1 / 4$ of the NE1/4 of Section 35, T.84N., R.18W (see Figure 2.1). The contiguous property presently owned by IES Utilities is also partially located in Section 36 . The site contains several buildings from the FMGP and former electric plant and is currently used as the service and materials distribution center for the IES gas and electric operations. It is located adjacent to an active railroad switching yard owned by the Chicago and Northwestern Railroad.

\subsection{Physiography/Topography}

The site is in an old industrial area of Marshalltown. Nearby industries include an inactive foundry, a scrap metal recycling business and a variety of manufacturers. The site is situated on the edge of the floodplain of a meandering stream (Linn Creek) within a glacial drift terrain. The ground surface is flat to gently sloping, with approximately 10 feet of relief across the site. Linn Creek is a tributary of the Iowa River and flows from west to east approximately 800 feet south of the FMGP site. Its confluence with the Iowa River is located approximately 2.5 miles northeast of the site.

Surface relief between the uplands and valleys is 50 to 150 feet. Maximum upland elevations range generally from 900 to 1000 feet above sea level (ASL) in the Marshalltown area. The Linn Creek and Iowa River floodplains vary in elevation from 860 to 880 feet ASL in the Marshalltown area.

\subsection{Geology and Hydrogeology}

The general stratigraphy of the glacial sediments in the Marshalltown area consists of loess over Kansan till. The terminal moraine of the younger Wisconsinian glaciation is located approximately 10 miles to the west of Marshalltown. The thickness of glacial deposition varies markedly around the Marshalltown vicinity, from 0 to 150 feet in the upland areas and from 25 to 250 feet in the valleys.

The glacial drift unconformably overlies Mississippian-age limestone and Pennsylvanian shale bedrock. The limestone units encountered immediately below the glacial/alluvial sediments at the site are identified in Remedial Investigation Report: Marshalltown, Iowa, Former Manufactured Gas Plant Site (BVWST 1992) as a part of the Mississippian Burlington and Gilmore City Formations and are part of the regional Mississippian aquifer. Outcrops are rare, but several are reported along railroad cuts and stream channels in the Marshalltown area. The 
Ames Expedited Site Characterization - Marshalltown FMGP Site

FINAL Site Report

March 25, 1996

former bedrock erosional surface is incised by multiple minor channels tributary to the preglacial channel of the Iowa River, referred to as the Poweshiek Channel. The Poweshiek Channel more or less follows the general trend of the present Iowa River floodplain and varies from one to two miles in width (Hansen, 1985). The City of Marshalltown is situated above a bedrock knob located south of the Poweshiek Channel. The FMGP site is situated over the northeast flank of the knob.

The near-surface stratigraphy of the site is reported in Remedial Investigation Report: Marshalltown, Iowa, Former Manufactured Gas Plant Site (BVWST 1992). According to drilling information reported by BVWST, the depth to bedrock varies from just over 20 feet in the northeast part of the site to about 35 to $\mathbf{4 0}$ (or more) feet in the western, central and southern parts of the site; a steep ridge in the bedrock surface with about 25 feet of relief, trends . northwest-southeast across the site. Near surface soils consist of a wide range of fill materials (clay, gravel, sand, cinder and other debris) of low plasticity and varying in thickness from 0.5 to 14 feet. This is underlain by fine-grained cohesive soils consisting of low plasticity silty clay with interbedded sandy and gravelly clays, ranging in thickness from 6 to 14 feet.

The cohesive silty clay unit grades into alluvial sands and gravels, varying significantly in silt and clay content, except at the farther northeast portion of the RI area where it is absent. The thickness of the alluvial sands and gravels varies from 0 to 26 feet, with the greatest thickness found near the center of the site, possibly indicating the present of a portion of a former stream channel.

A layer of low plasticity clayey lacustrine soil and low to high plasticity glacial till separates the alluvial soils from bedrock in most areas of the site. This unit was logged as absent in one boring near the east edge of the site. The upper surface of the unit slopes toward the south and generally mimics the bedrock surface.

The water table at the time of the BVWST investigation was approximately 18 to 20 feet below grade and within the granular alluvial soils. Hydraulic conductivity $(K)$ measurements obtained by falling head slug tests indicated $\mathrm{K}$ values in the range of 0.0029 to $0.00076 \mathrm{~cm} / \mathrm{sec}$ for the granular soils. According to the work done by BVWST (1992), groundwater flow in the alluvial sediments is to the south toward Linn Creek. Bedrock groundwater flow characteristics are not well established and appear to be strongly influenced by the activity of production wells in the area which tap the Mississippian aquifer. 
Ames Expedited Site Characterization - Marshalltown FMGP Site

FINAL Site Report

March 25, 1996

\subsection{Marshalltown FMGP Site History}

A comprehensive summary of the site history is included in Remedial Investigation Report: Marshalltown, Iowa, Former Manufactured Gas Plant Site (BVWST 1992). The BVWST report was a primary source for information contained in this section. Additional sources of historic information included a preliminary research report by McDonald (1986), Sanborn Company maps dated from 1884 to 1964, and the Marshall County Historical Museum Library.

When the plant first opened in the mid-1870's, gas manufacturing was accomplished by the coal carbonization process. At the time the plant opened under the name Marshalltown Gas Light Company, it occupied only Lot 5 of Barden's subdivision. In 1884, the site consisted of the gas plant building (at the approximate location of the present substation materials building), a coal house, and two gasometers (pressurized gas holders) located north of the plant building (see Figure 2.2). Barbed wire and furniture manufacturing facilities and a stock yard existed to the west of the plant on what is now IES Utilities property. The properties to the east were apparently vacant at this time.

Electric generation began at the site between 1888 and 1892. The electrical plant was built on a portion of the gas plant property and Lots 1,2 and 3 of Cunningham's Addition. Residential properties and warehouses were present to the east of the gas plant at this time, on what is now the IES property. In 1892, the Marshalltown Gas Company, the Marshalltown Electric Company, and the Marshalltown Street Railway Company consolidated into the Marshalltown Light, Power and Railway Company, bringing the electrical and gas operations under common ownership.

By 1901, the original, smaller gasometer had been removed, and the operation utilized a single 25,000 cubic foot gas holder located west of what is now the regulator building. Between 1901 and 1910, a new 200,000 cubic foot gas holder was built just east of the current regulator building (see Figure 2.2).

The gas manufacturing process was converted from coal carbonization to carbureted water gas between 1910 and 1921, and ownership transferred to the Iowa Railway and Light Corporation. During this period, the original purifier room operations were moved to a separate building (now the IES substation department building) and water gas generators were installed in place of the old purifiers. In addition, a 12,000-gallon tar well was installed east of the new water gas generator building, and an oil storage tank was installed at the north edge of the property west of the original gas holder to feed the carburetion process.

The original coal gasification facilities were demolished in the 1920s. Also during this period, a new 50,000 cubic foot gas holder was constructed immediately south of the 200,000 cubic foot gas holder, and the original 25,000 cubic foot holder was taken out of service but apparently left 
Ames Expedited Site Characterization - Marshalltown FMGP Site

FINAL Site Report

March 25, 1996

in place until the plant was shutdown completely in 1950. A second oil tank was also added in the 1920s north of the purifier building.

Although documentation was not available regarding the disposition of tar produced by the gas manufacturing processes, a substantial portion of the tar may have been disposed on site. This is evidenced by, among other things, a 1953 photograph in the McDonald (1986) report showing an excavation immediately east of the present electric substation filled with a substance described as tar. The pit was opened for a construction project, and the tar was pumped from the pit and disposed of at an unknown location.

At its maximum extent of operations, the FMGP occupied lots only in Barden's subdivision (lots 1-7) and Westlake's subdivision. The present IES Utilities property, located on and east of the vacated 6th Avenue, was not part of the gas plant and is, therefore, not expected to contain any source areas for gas plant wastes. The former electrical plant located primarily in Cunningham's addition to the west of the FMGP may contain source areas, since the two operations were under the same ownership. It has been documented that waste disposal, some of which appears to have been generated by the FMGP, did occur in the subfloor of the former electrical generation building (BVWST, 1992).

\subsection{Previous Investigations/Existing Data}

\subsubsection{Summary}

Documented investigation of waste disposal at this site began with a preliminary study by D. B. McDonald Research Associates in 1986. This investigation consisted of gathering regional hydrologic and geologic data and historical information about the operation of the plant, including interviews with former employees.

Later in 1986, a U. S. Environmental Protection Agency (U.S.-EPA) contractor, Ecology and Environment (E\&E), performed a reconnaissance investigation of the site. The E\&E investigation included similar historic data and added information regarding fate, transport, and exposure routes of the contaminants of interest. A followup site investigation by $E \& E$ in 1987 included surface geophysics and some shallow soil, groundwater, surface water and manhole sampling. The geophysics work consisted of ground penetrating radar in areas where subsurface structures were suspected. The soil samples indicated the presence of PAH compounds at levels substantially above background levels.

A report prepared by Tuthill, Inc., in 1988 contained a detailed synopsis of the site history, including the development and ownership changes of the site. Tuthill also prepared a report in 


\section{Ames Expedited Site Characterization - Marshalltown FMGP Site}

FINAL Site Report

March 25, 1996

1989 which provided a summation of the information contained in the previous reports discussed above. The 1989 report contained data from a public water quality assurance program which had been initiated locally. Also in 1989, Tuthill prepared a detailed report containing more information about the history of the site.

An underground storage tank (UST) was removed in November 1988 from an area near the west end of the site along the east wall of the former spray pond. Soil and groundwater samples were collected as a part of the UST closure operation. Petroleum hydrocarbons in excess of applicable action levels were detected.

BVWST began a detailed RI of the site in 1990. The investigation included a comprehensive program of soil and groundwater sampling and hydrogeologic characterization. The investigation also included sampling and characterization of material contained in the void space beneath the floor of the former electric generation building. The results are documented in a report submitted to the Iowa Department of Natural Resources (IDNR) (BVWST 1992).

As a result of the RI, the following potential contaminant source areas have been identified (refer to Figure 2.2):

1) The "tar pit" unearthed during a construction project in 1953, which is located immediately east of the existing electrical substation;

2) Tar separator indicated on the west side of former purifier building (Sanborn Company maps from 1921 and subsequent years). Based on references on maps dated after 1921, this tar separator may have been an above-grade construction;

3) Tar well located east of the original water gas generator building (Sanborn Company map from 1921) and now located off the northeast comer of the present substation material storage building; and

4) Tar separator located south of the former purifier building. This separator was unearthed and the liquid contents removed in 1992. Based on its location, this structure may have been the one identified in the 1921 map as a tar well.

Other subsurface areas potentially containing high concentrations of PAHs include the alleged coal storage area in the southeast corner of the FMGP operations and identified by EPA studies as containing high contaminant levels, and the perimeter of and below the subfloor of the former electrical generation plant, particularly the area west of the former electric plant. 


\subsubsection{Remedial Investigation Data Analysis}

The previous investigation performed by BVWST included their findings on the extent of the PAH contamination in soils. Seven monitoring wells and 16 borehole locations produced information of several types. Stratigraphic information was obtained from the cuttings and soil samples and is given in the borehole logs. The logs also contain observations on the appearance of the cuttings that were noted as either free product, oil sheen or discoloration or odor indications. Soil samples were taken and analyzed at an off-site laboratory. Seventy-eight samples were taken from locations within the property boundary, of which six were duplicates. Figure 2.3 shows the locations of the on-site boreholes, B-1 to B-16, and the three on-site monitoring wells MW-3, MW-4 and MW-5. No PAH chemical analyses were carried out on soil samples from the B-9, B-10, B-11, B-12 or MW-5 locations.

With respect to the PAH in soils, several trends were evident from the BVWST report. First, for nearly every sample in which PAHs were detected, either naphthalene or phenanthrene was the highest in concentration. [The same pattern was found in our 127 samples analyzed on and off site; see Section 4.4 for further discussion.) This observation permits a considerable simplification in the analysis of the PAH distribution. Instead of 16 PAH plumes we can focus on three types: naphthalene plume, phenanthrene plume and for some methods, as discussed below a modified total PAH plume. The latter type plume can be generated from any observation or measurement that integrates some or all of the PAH concentration distribution into a single value. For instance, visual and odor information from our work and BVWST are important observations because of the extremely low odor detection limit from some of the PAH contamination which we estimate to be in the few $\mathrm{mg} / \mathrm{kg}$ range. Also, some of the screening methods we used were biased against naphthalene, and, given the general dominance of phenanthrene, they very likely represent information that can be compared to the phenanthrene plume.

Second, the stratigraphic sequences at the site can be grouped into and analyzed in terms of five discrete units; from the ground surface down the units are fill, upper cohesive unit (UCU principally clay), granular unit (comprised mostly of various types of sand), lower cohesive unit (LCU - clay-like) and bedrock (often weathered). BVWST sampling focused on the upper two units and the top half of the third unit. Only one sample, at location B-4, was taken below the middle of the granular unit. However, visual and odor indications clearly implied that significant contamination was present in the bottom few feet of the granular unit. Based on these results, the PAHs were assumed to be principally located in the upper confining unit and throughout the granular unit with the latter's top and bottom deserving special focus. 
Ames Expedited Site Characterization - Marshalltown FMGP Site

FINAL Site Report

March 25, 1996

The design of our subsurface sampling program was based on these BVWST results and consisted of sampling the following zones:

- middle and bottom of UCU (termed zones 1 and 2 respectively);

- top, middle and bottom of granular unit (termed zones 3,4 and 5 respectively); and

- top of LCU (termed zone 6).

These zones are shown schematically in Figure 2.4. Instructions were given to all sampling crews to stop probing activities once they penetrated a few feet below the base of the granular unit to prevent introducing any new migration pathways.

Given these observations, we will present and discuss the BVWST and our PAH soil contamination data in the context of a simplified model that focuses on naphthalene, phenanthrene or modified total PAH plumes located in one of the six stratigraphic zones defined above.

\subsubsection{Remedial Investigation Results}

Table 2.1 lists the naphthalene and phenanthrene concentrations from the BVWST report that correspond to the six subsurface zones. With very few exceptions naphthalene and phenanthrene are co-located. Data from zones 1 through 4 are plotted in Figures 2.5 to 2.8. Because data are sparse and/or not available (NA), closed loop plume boundaries were not drawn. Areas of significant naphthalene and phenanthrene contamination generally lie along a NW-SE line from location B-1 to B-8 and B-13. The highest levels of naphthalene and phenanthrene contamination within the zones examined were found in boreholes B-2, B-4, B-5 and B-6. At the eastern plume edge, zone 4 has a non detect (ND) at B-7 while zone 1 has hits at B-7 and MW-3A indicating that the UCU is more contaminated in the eastern portion of the site than the granular unit. Along the southern site boundary from MW-4 to MW-3 differences occur between naphthalene and phenanthrene in that phenanthrene is present at a few of the borings while naphthalene is absent.

Important information resides in the visual and odor comments in the BVWST logs. To quantify this information, from both BVWST and our work, we employed a logarithmic type scale for these observations. Given that free product has a concentration of approximately $10^{6}$ 
Ames Expedited Site Characterization - Marshalltown FMGP Site

FINAL Site Report

March 25, 1996

$\mathrm{mg} / \mathrm{kg}$ and slight but definite odor corresponds to a concentration of about $1 \mathrm{mg} / \mathrm{kg}$, the following range was set up:

$\begin{array}{lc}\text { Observation } & \text { Scale } \\ \text { free product } & 6 \\ \text { product sheen } & 5 \\ \text { discoloration } & 4 \\ \text { strong odor } & 4 \\ \text { mild odor } & 3 \\ \text { slight odor } & 2 \\ \text { no odor } & 1 \\ \end{array}$

Table 2.2 contains information interpreted from the BVWST borehole logs. Most of the BVWST data are visual and therefore track the highest concentrations of the PAHs. From Table 2.1 it is clear that nearly all off-site samples were taken from zones 1 through 4 ; the visual/odor information contained in Table 2.2 adds considerable insight into the high concentration portions of the contaminant distribution. Plots of this visual/odor BVWST data for the six stratigraphic zones are shown in Figures 2.9 to 2.14. All BVWST boreholes penetrate to zone 6 except B-16 where the LCU is absent; therefore, no zone 5 and 6 can be defined. It was possible to draw closed loops for these odor and visually based plumes because there is odor and/or visual data at all locations. It must be kept in mind, however, that there is not a one-to-one correlation between Figures 2.5 to 2.8 and Figures 2.9 to 2.12. Even though BVWST sample analyses may indicate significant contamination, if direct visual or olfactory reference was not made at the appropriate location in the borehole $\log$, then the zone was ascribed a zero; i.e., it is assumed that the contamination was not recognized during the borehole logging or the appropriate depth is not referred to explicitly. Moreover, fuel odor was detected in zones 1 and 3 of B-1; this was, however, not included as a visual or olfactory sign of coal tar residue. Note that MW-4 has not been included in these contour plots as it would tend to draw the plumes across the generation plant building area where essentially no information was available.

All zones have plumes except for zone 6 where no contaminants were identified visually. Boreholes B-2, B-4, B-5, B-6 and B-8 show evidence of significant contamination in the upper 5 zones and establishes the core of the PAH plume. Hits at B-1 in zones 4 and 5 extend their plumes in the NW direction from the core plume. Hits at B-13 for zones 1 and 4 extend their plumes to the SW. Hits at B-14 for zones 1, 3 and 5 are interesting because in the case of zone 1 this merely extends its plume further to the $S W$ but for the other two plumes it indicates that local variations in stratigraphy over relatively small distances can significantly affect the distribution of contaminants in the subsurface. Hits at B-7 extend plumes in zones 2, 3 and 4 
further east from the core plume without the need to invoke a separate plume. Generally the plumes in the upper five zones are about equal in extent with perhaps the zone 3 plume being the smallest.

Given the preliminary plume outlines from the BVWST data and the thicker granular unit west of the B-7 location and its possible role as a migration pathway it was decided to focus the ESC effort on the eastern, southeastern and northwestern portions of the site. The grid laid out for screening activities described in section 4.2 was an attempt to overlap plume boundaries based on these BVWST results. 


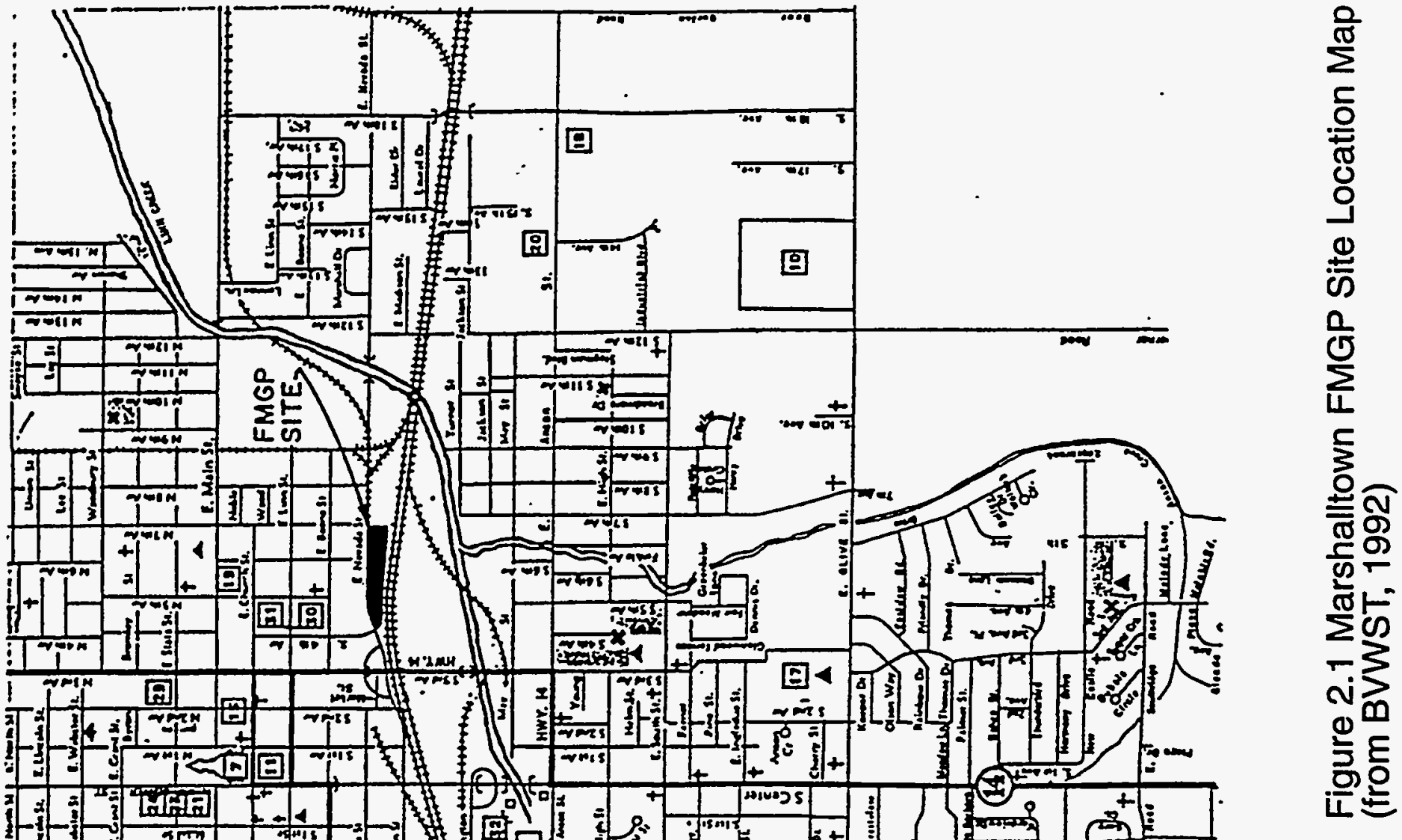

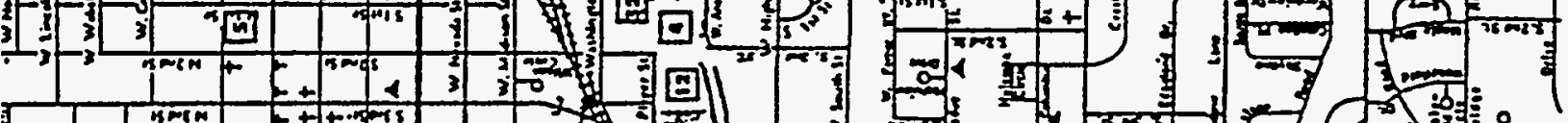

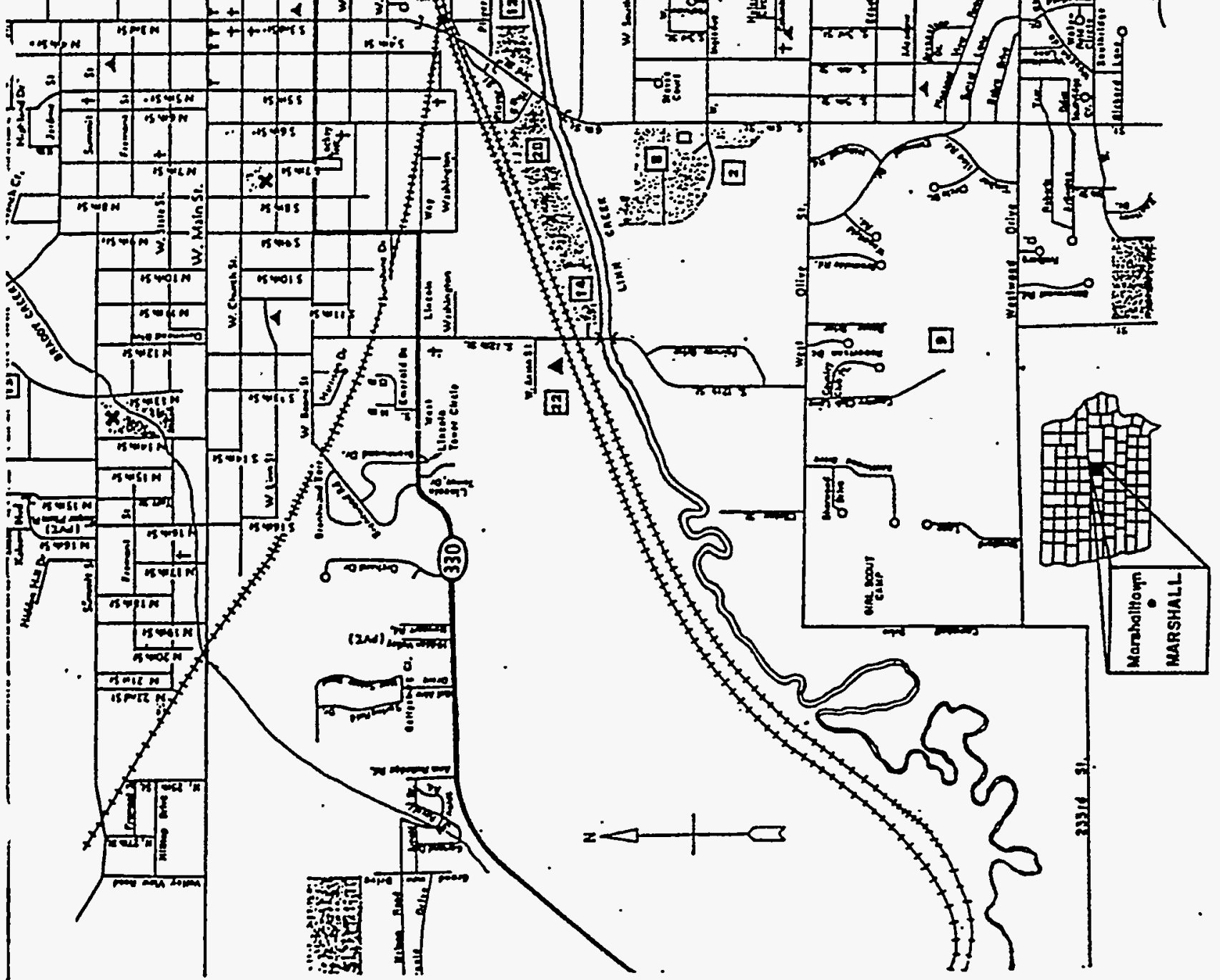



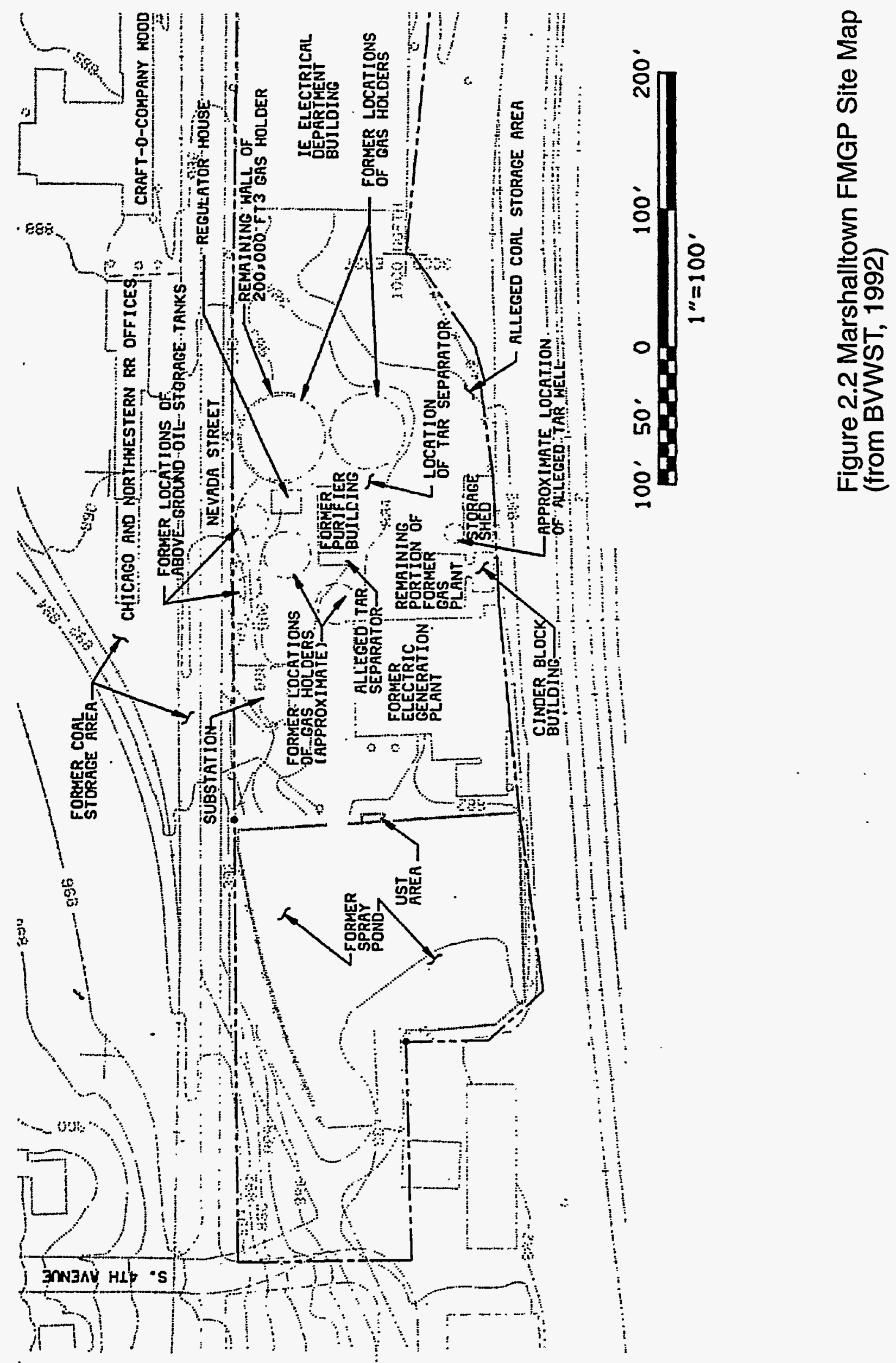


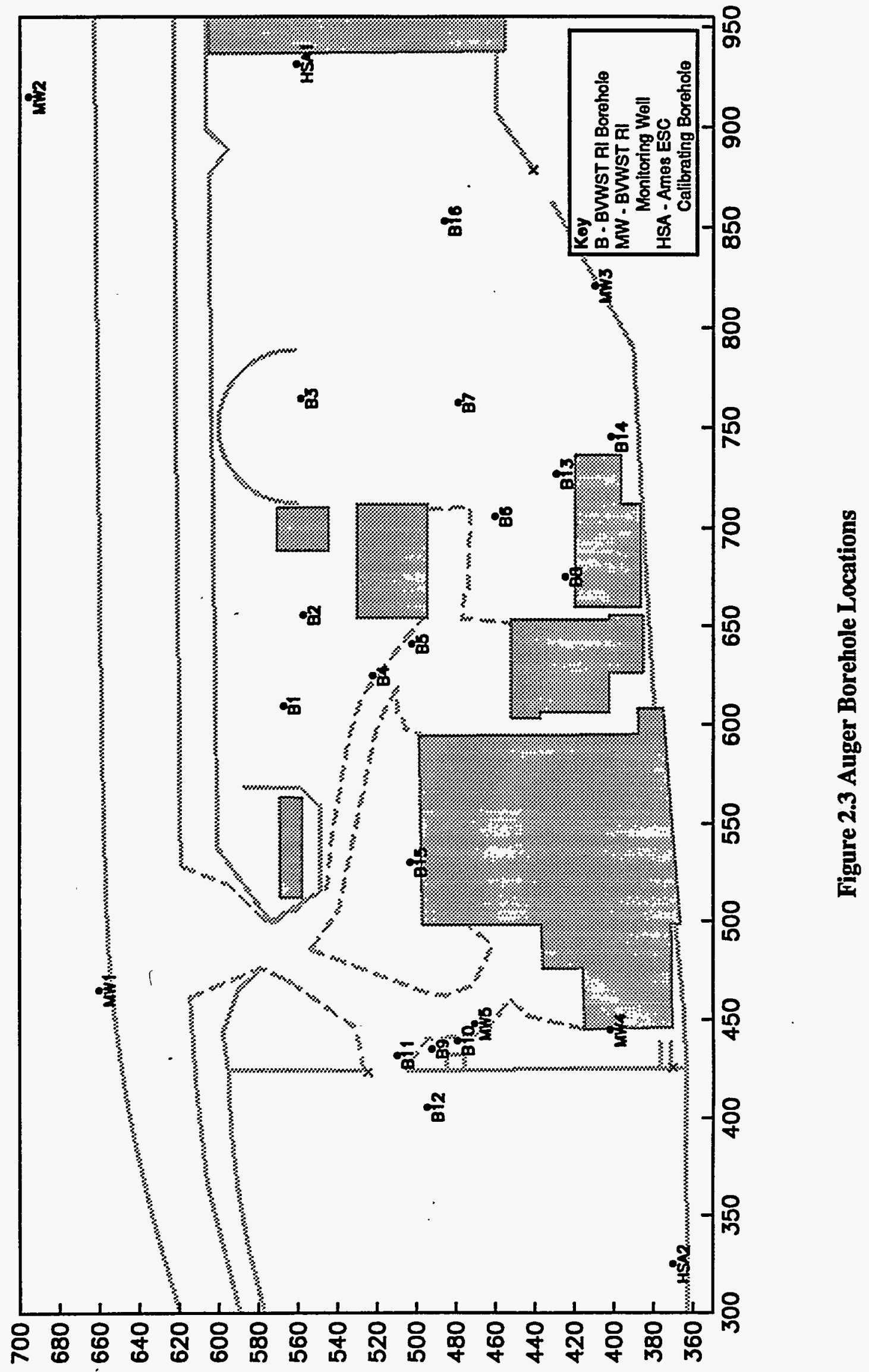


Figure 2.4 Typical Site Stratigraphic Units

and Stratigraphic Zones (after BVWST, 1992)

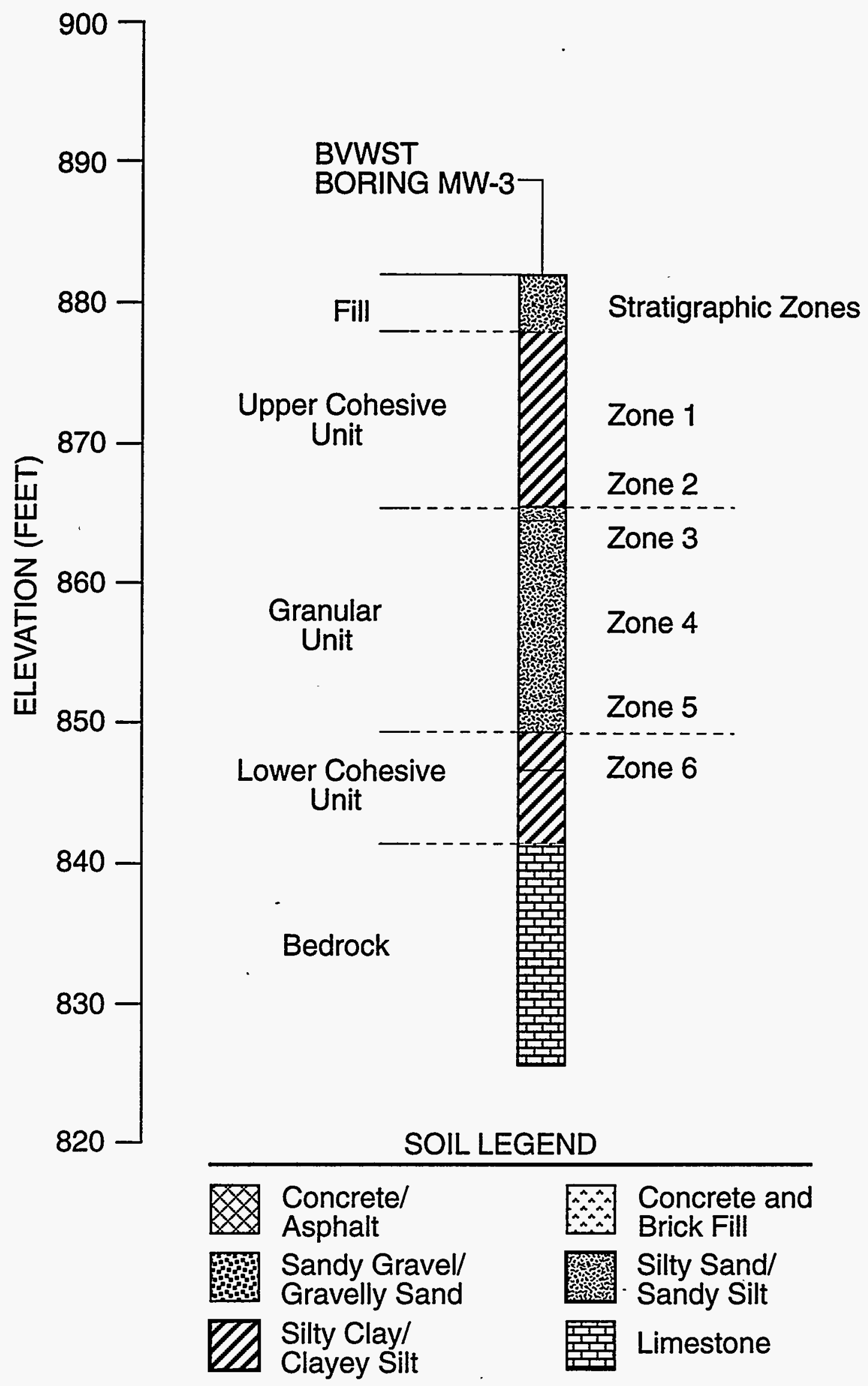




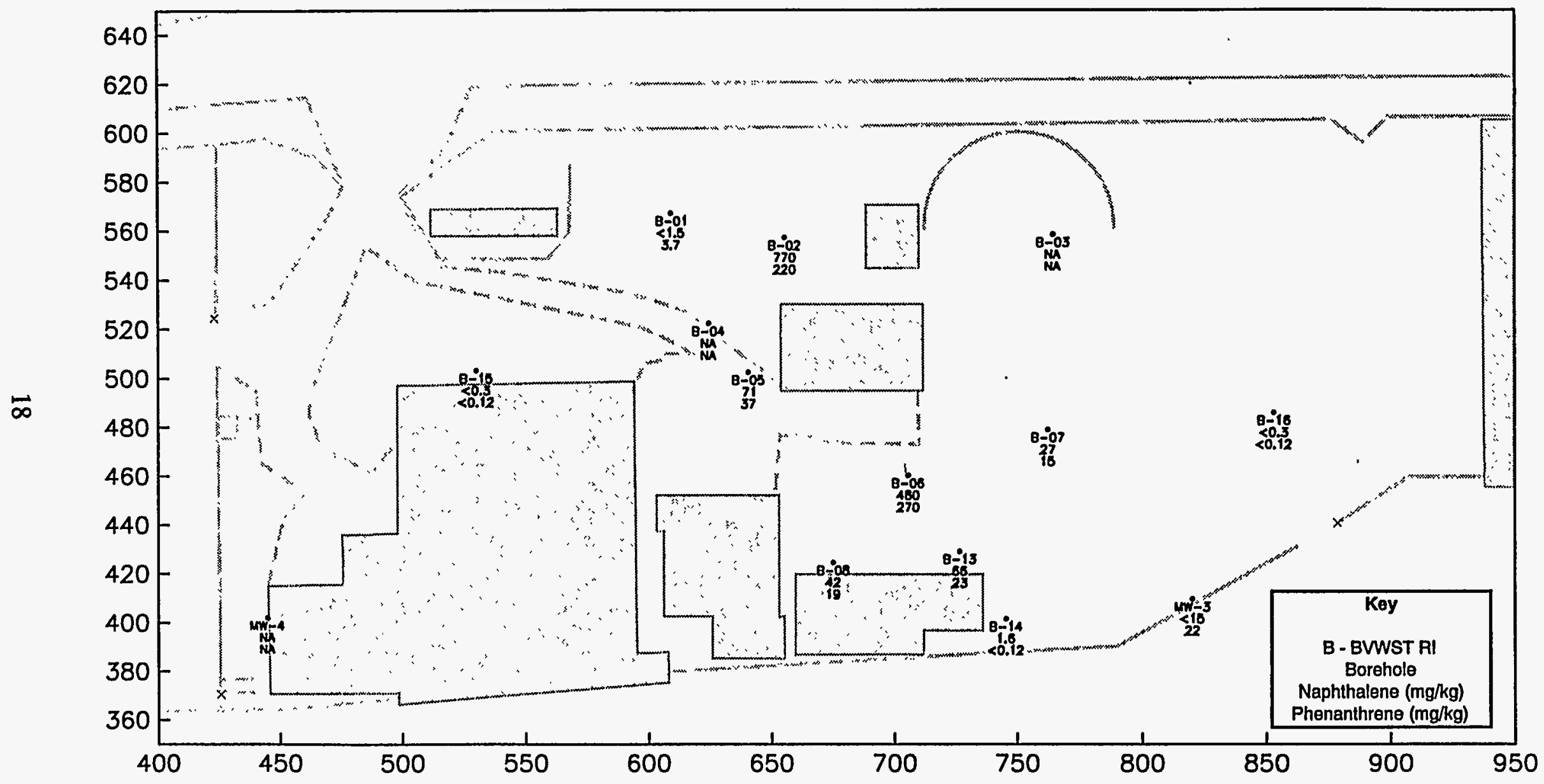

Figure 2.5 BVWST Naphthalene and Phenanthrene Concentrations - Stratigraphic Zone 1 


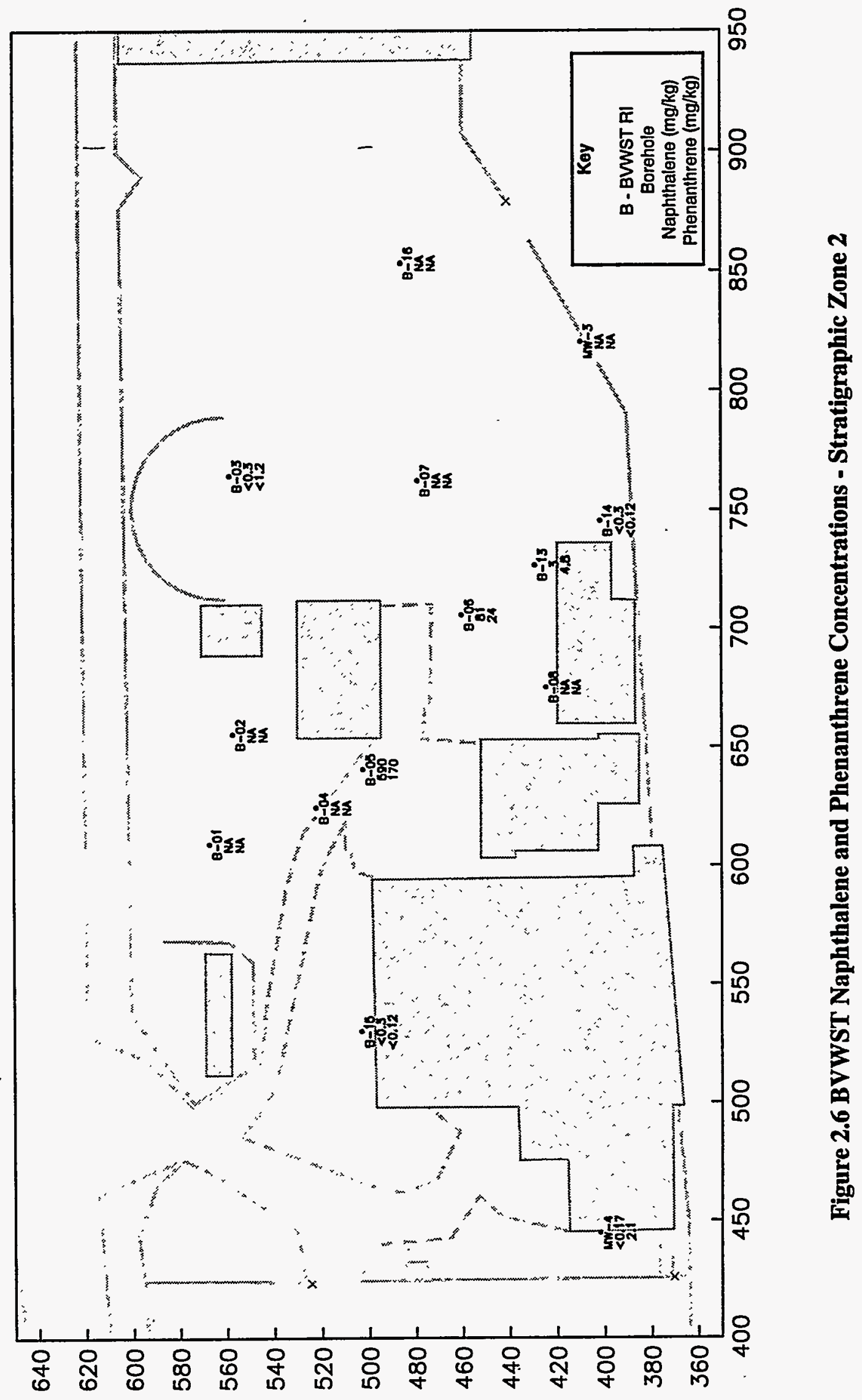




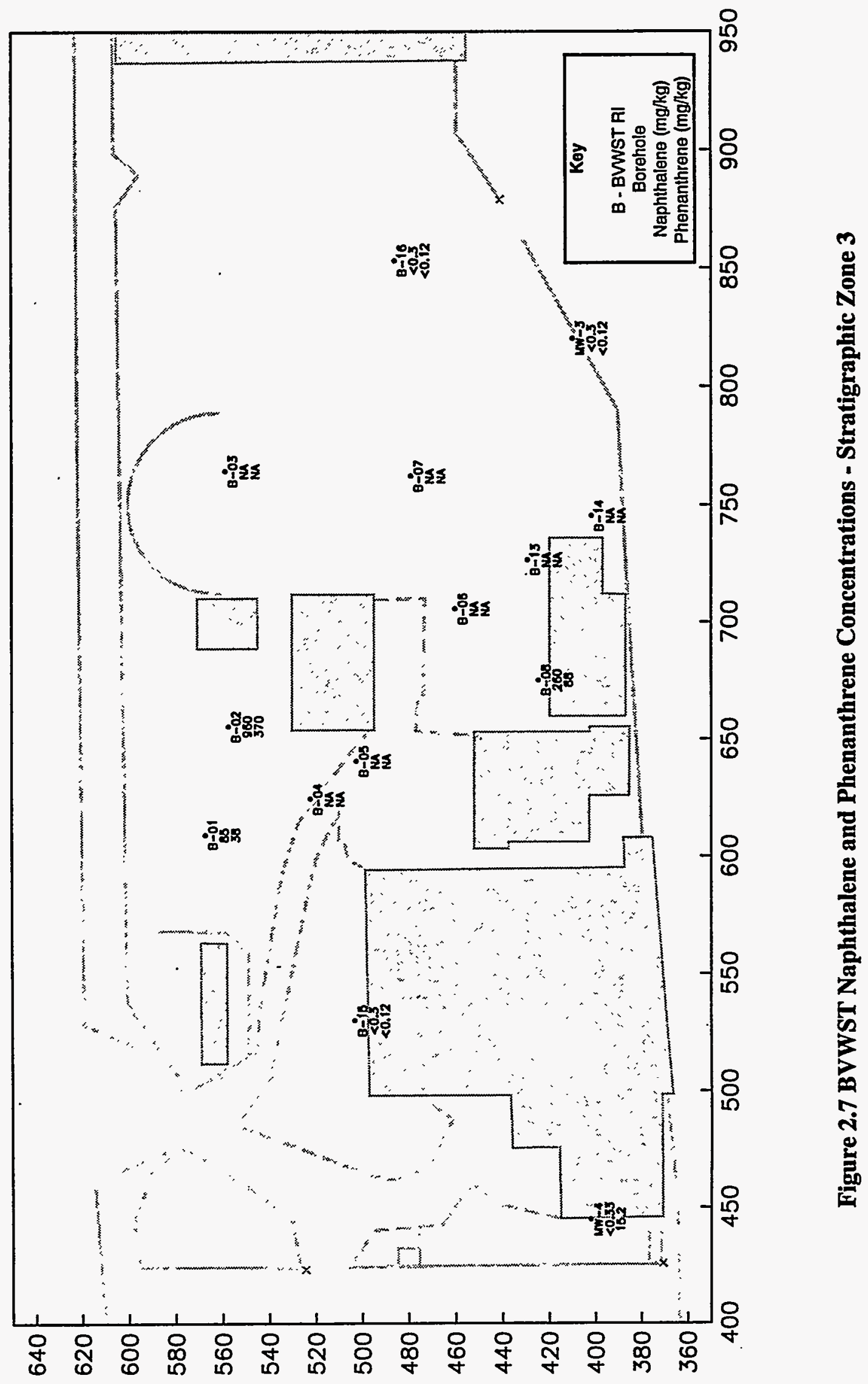




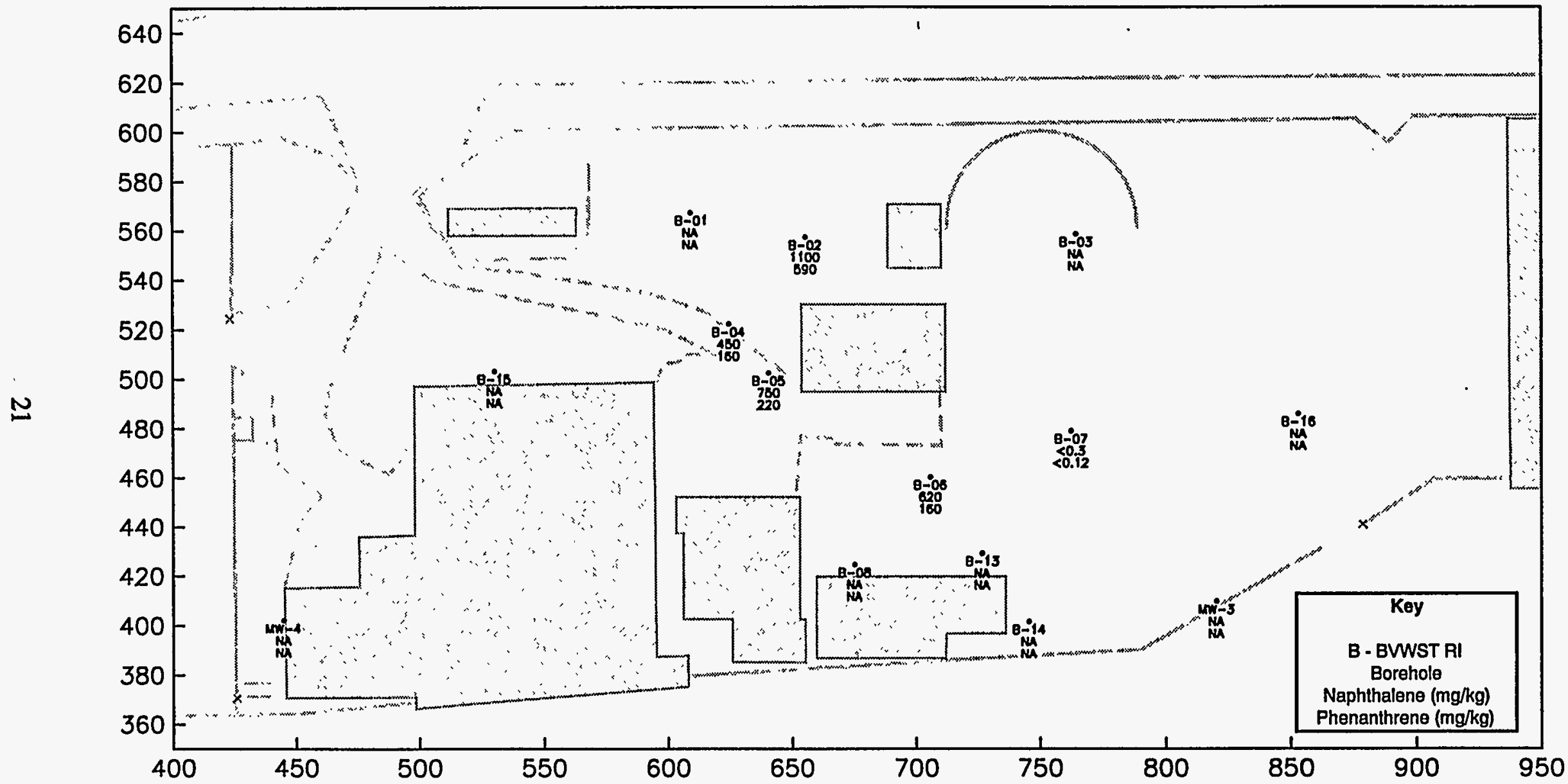

Figure 2.8 BVWST Naphthalene and Phenanthrene Concentrations - Stratigraphic Zone 4 


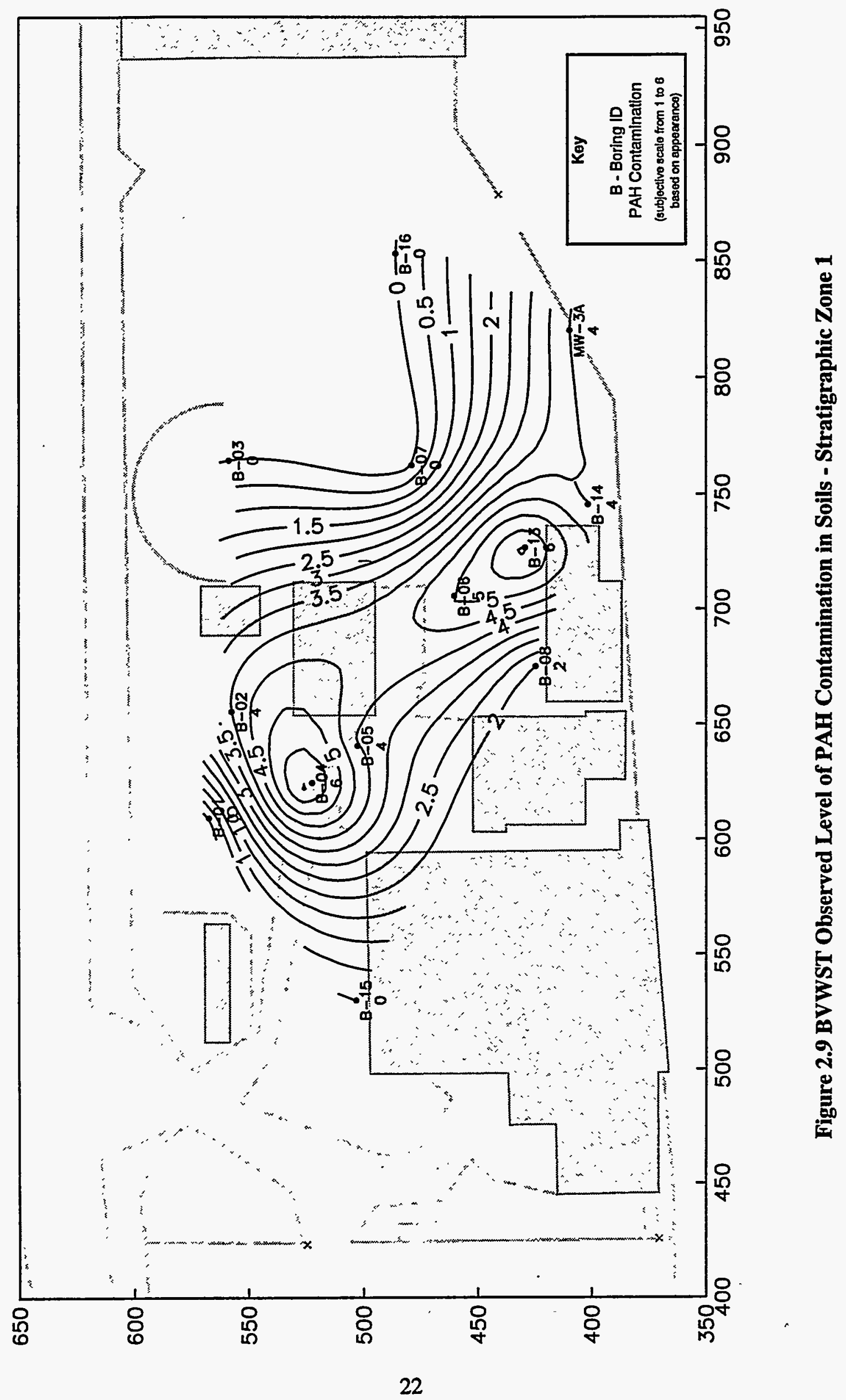




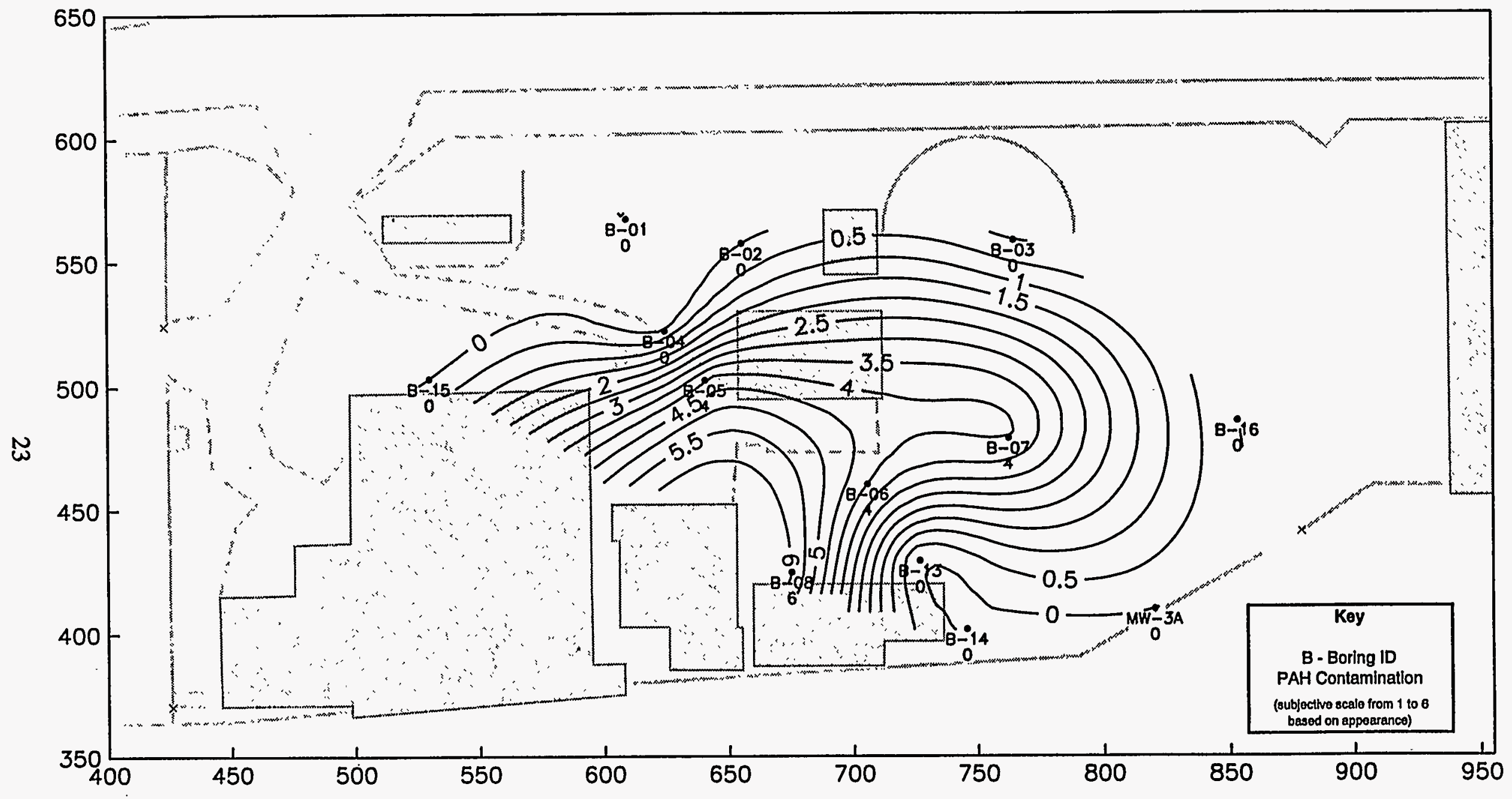

Figure 2.10 BVWST Observed Level of PAH Contamination in Soils - Stratigraphic Zone 2 


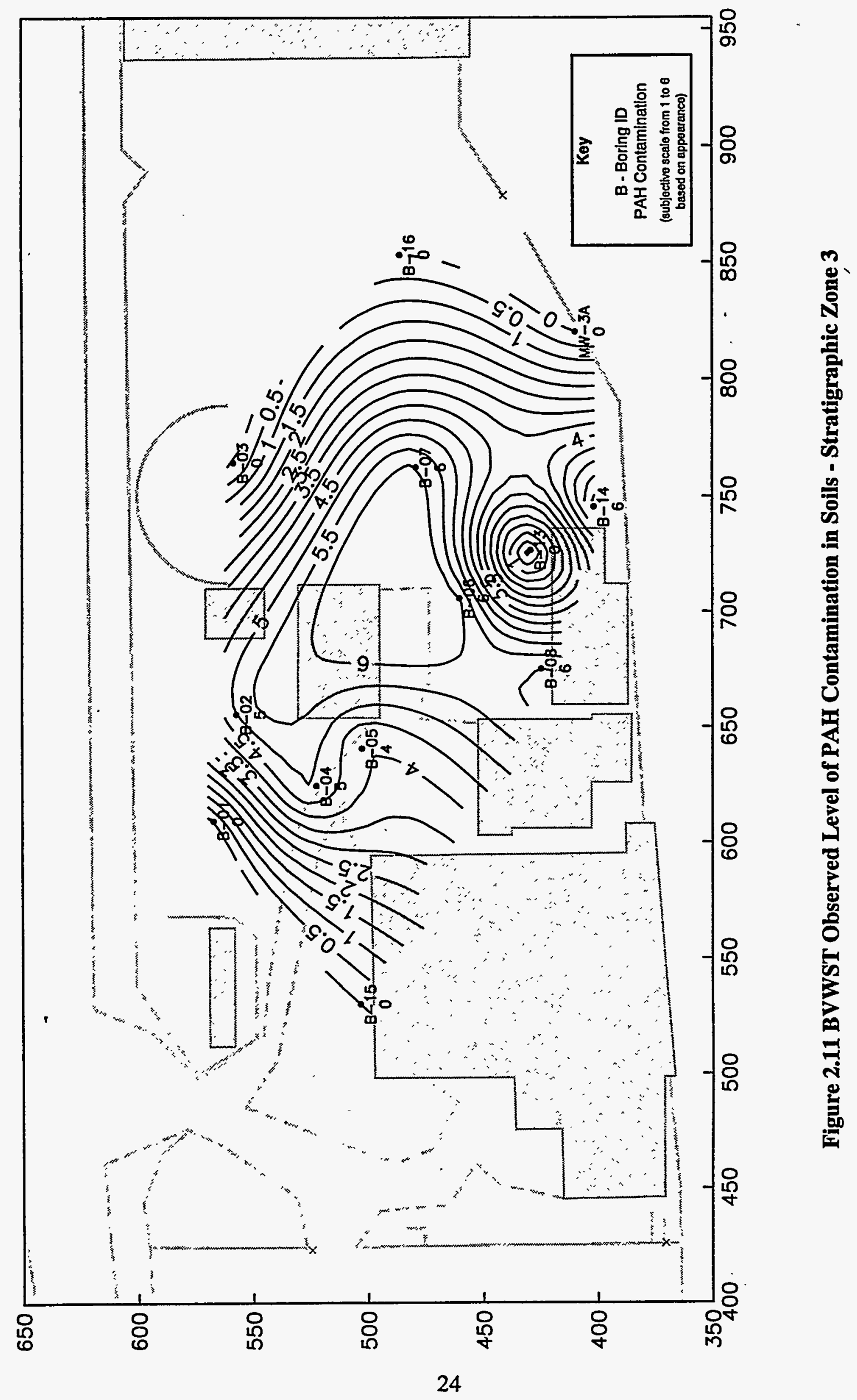




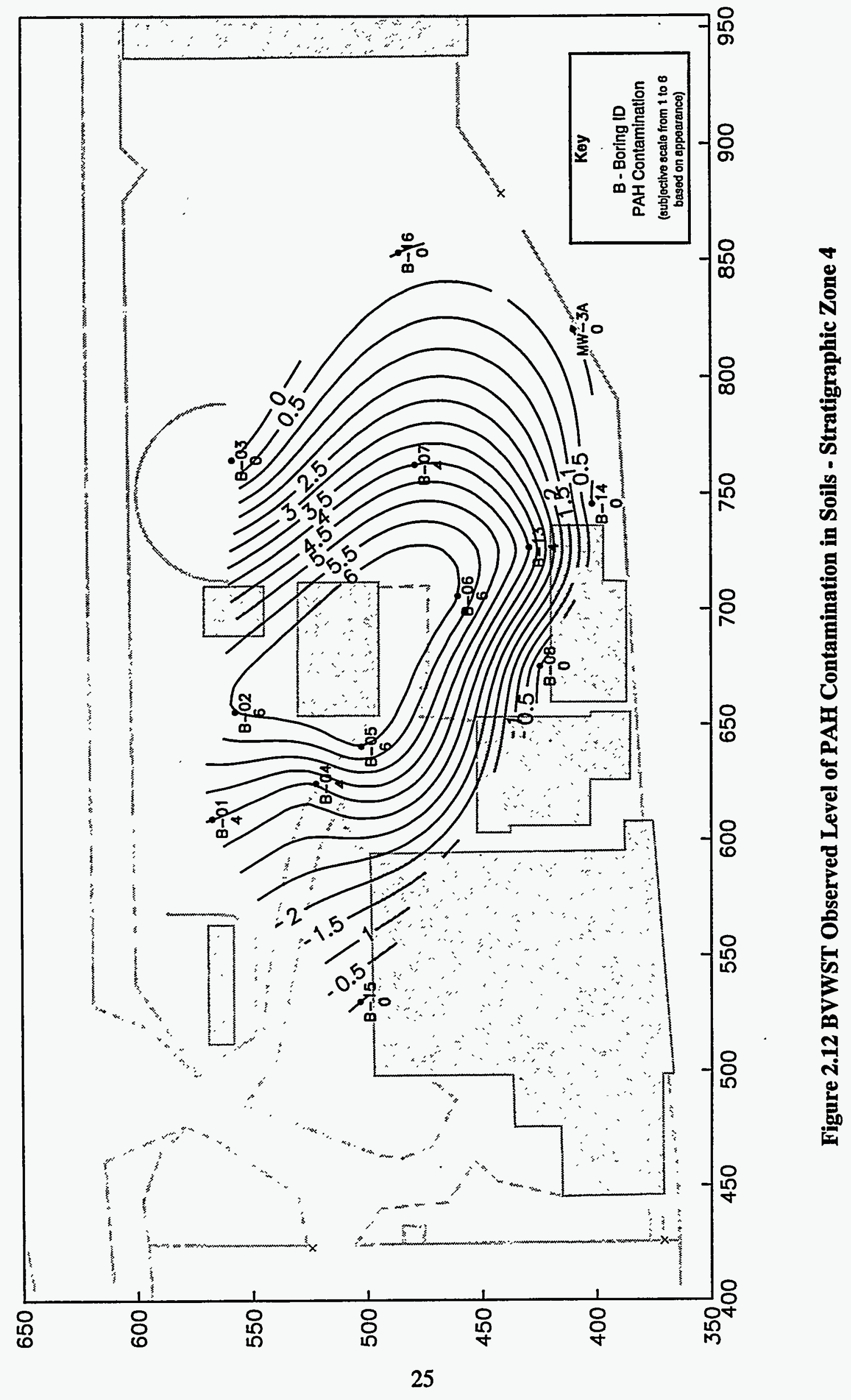




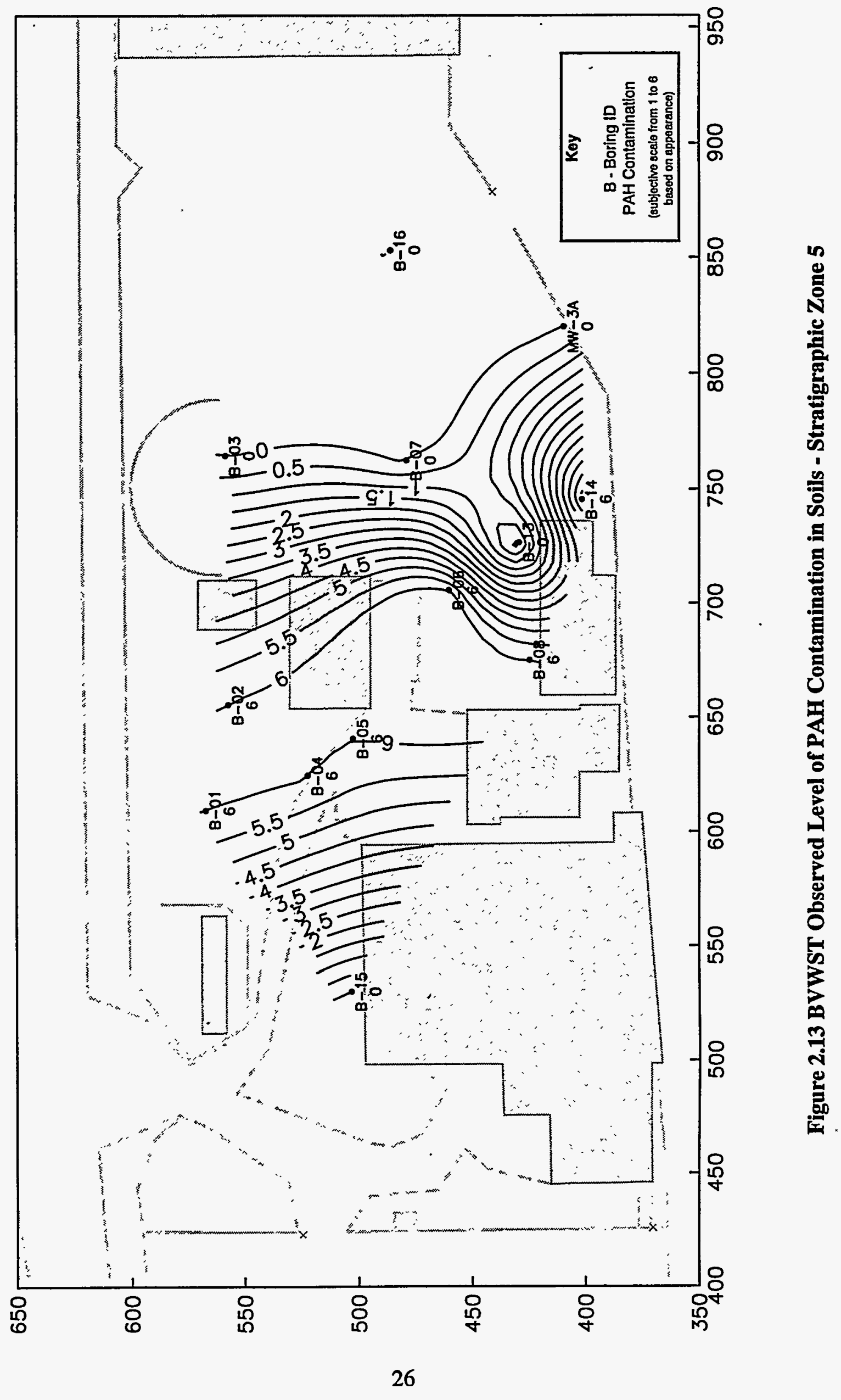




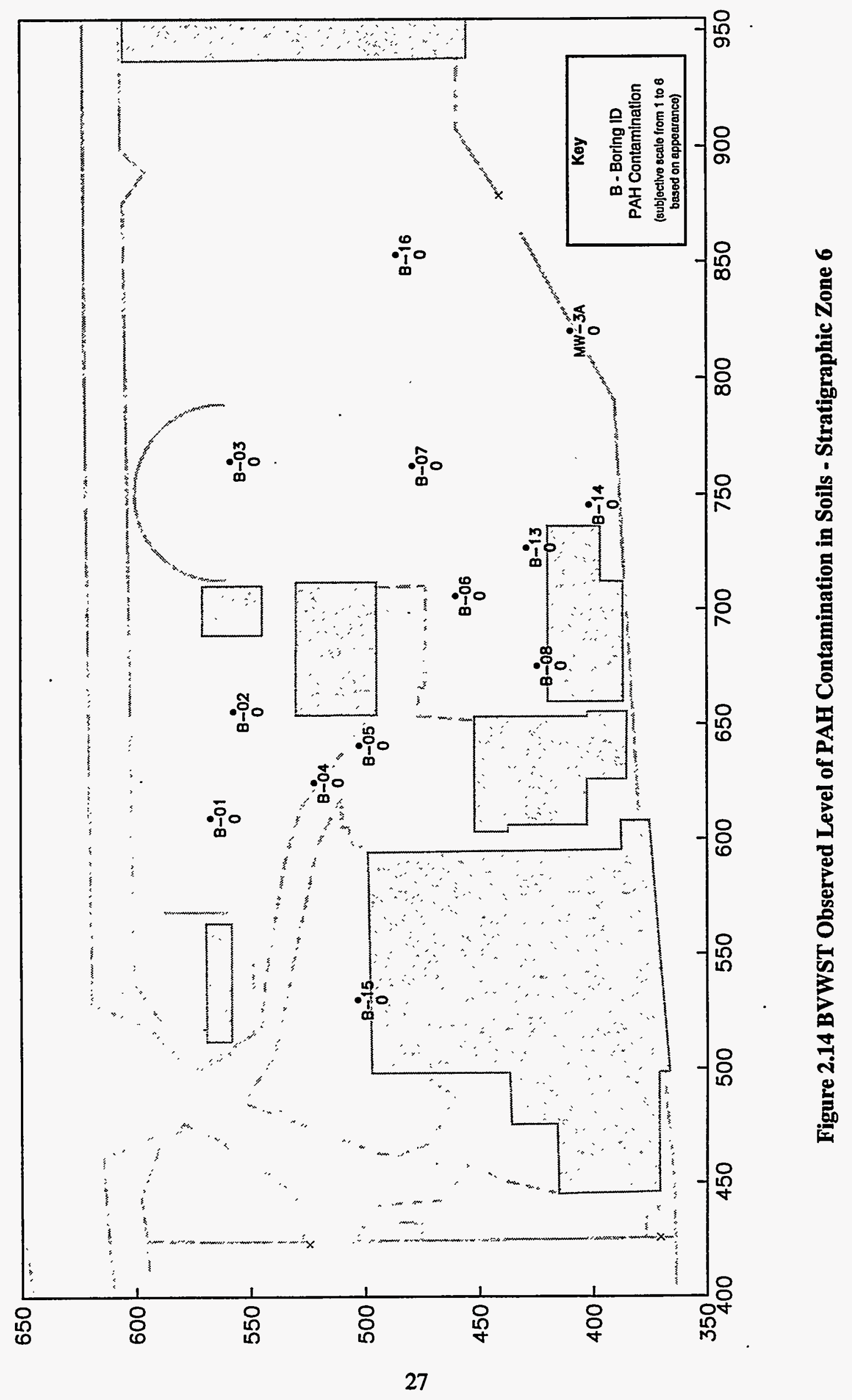


TABLE 2.1 BVWST RI NAPTHALENE AND PHENANTHRENE CONCENTRATIONS IN SOIL (mg/kg) BY STRATIGRAPHIC ZONE

\begin{tabular}{|c|c|c|c|c|c|c|}
\hline Borehole & & Zone 1 & Zone 2 & Zone 3 & Zone 4 & Zone 5 \\
\hline \multirow[t]{2}{*}{$\overline{B-1}$} & Naphthalene & $<1.5$ & NA & 85 & NA & NA \\
\hline & Phenanthrene & 3.7 & $\overline{N A}$ & 38 & NA & NA \\
\hline \multirow[t]{2}{*}{$\bar{B}-2$} & Naphthalene & 770 & $\overline{\mathrm{NA}}$ & 960 & 1100 & NA \\
\hline & Phenanthrene & 220 & NA & 370 & 590 & NA \\
\hline \multirow[t]{2}{*}{$\overline{B-3}$} & Naphthalene & $\overline{\mathrm{NA}}$ & $<0.3$ & NA & NA & NA \\
\hline & Phenanthrene & $\overline{\mathrm{NA}}$ & $<0.12$ & $\mathrm{NA}$ & NA & NA \\
\hline \multirow[t]{2}{*}{$\overline{B-4}$} & Naphthalene & $\overline{\mathrm{NA}}$ & NA & $\overline{N A}$ & 450 & 1800 \\
\hline & Phenanthrene & $\overline{\mathrm{NA}}$ & $\overline{N A}$ & $\mathrm{NA}$ & 160 & 430 \\
\hline \multirow[t]{2}{*}{$B-5$} & Naphthalene & 71 & 590 & $\mathrm{NA}$ & 750 & NA \\
\hline & Phenanthrene & 37 & 170 & $\mathrm{NA}$ & 220 & NA \\
\hline \multirow[t]{2}{*}{$\mathrm{B}-6$} & Naphthalene & 480 & $\overline{81}$ & NA & 620 & NA \\
\hline & Phenanthrene & 270 & 24 & $\overline{\mathrm{NA}}$ & 160 & NA \\
\hline \multirow{2}{*}{ B-7 } & Naphthalene & 27 & $\overline{N A}$ & $\overline{\mathrm{NA}}$ & $<0.3$ & $\overline{\mathrm{NA}}$ \\
\hline & Phenanthrene & 15 & $\mathrm{NA}$ & $\mathrm{NA}$ & $<0.12$ & NA \\
\hline \multirow[t]{2}{*}{$\overline{B-8}$} & Naphthalene & 42 & $\overline{\mathrm{NA}}$ & 260 & $\overline{N A}$ & NA \\
\hline & Phenanthrene & 19 & $\overline{\mathrm{NA}}$ & 88 & NA & NA \\
\hline \multirow[t]{2}{*}{$\overline{B-13}$} & Naphthalene & $\overline{66}$ & 3 & $\overline{N A}$ & $\overline{N A}$ & $\overline{\mathrm{NA}}$ \\
\hline & Phenanthrene & 23 & 4.8 & $\overline{\mathrm{NA}}$ & NA & NA \\
\hline \multirow[t]{2}{*}{ B-14 } & Naphthalene & $\overline{1.6}$ & $<0.3$ & $\overline{\mathrm{NA}}$ & NA & NA \\
\hline & Phenanthrene & $<0.12$ & $<0.12$ & $\overline{\mathrm{NA}}$ & NA & NA \\
\hline \multirow[t]{2}{*}{ B-15 } & Naphthalene & $<0.3$ & $<0.3$ & $<0.3$ & $\overline{\mathrm{NA}}$ & $\overline{\mathrm{NA}}$ \\
\hline & Phenanthrene & $<0.12$ & $<0.12$ & $<0.12$ & NA & NA \\
\hline \multirow[t]{2}{*}{ B-16 } & Naphthalene & $<0.3$ & $\overline{\mathrm{NA}}$ & $<0.3$ & $\overline{\mathrm{NA}}$ & $\overline{\mathrm{NA}}$ \\
\hline & Phenanthrene & $<0.12$ & NA & $<0.12$ & $\mathrm{NA}$ & NA \\
\hline \multirow[t]{2}{*}{ MW3 } & Naphthalene & $<15$ & $\mathrm{NA}$ & $<0.3$ & $\overline{N A}$ & $\overline{\mathrm{NA}}$ \\
\hline & Phenanthrene & 22 & $\overline{N A}$ & $<0.12$ & $\overline{N A}$ & $\overline{\mathrm{NA}}$ \\
\hline \multirow[t]{2}{*}{ MW4 } & Naphthalene & $\overline{N A}$ & $<0.17$ & $<0.33$ & $\overline{\mathrm{NA}}$ & $\overline{N A}$ \\
\hline & Phenanthrene & $\overline{N A}$ & 2.1 & 15.2 & NA & NA \\
\hline
\end{tabular}

Note: Entries with "<" symbol signify that the compound was not detected at a level equal to or greater than the value reported. 
TABLE 2.2 BVWST RI OBSERVED LEVEL OF PAH CONTAMINATION BY STRATIGRAPHIC ZONE

\begin{tabular}{|l|c|c|c|c|c|c|}
\hline Borehole & Zone 1 & Zone 2 & Zone 3 & Zone 4 & Zone 5 & Zone 6 \\
\hline B-1 & 0 & 0 & 0 & 4 & 6 & 0 \\
\hline B-2 & 4 & 0 & 5 & 6 & 6 & 0 \\
\hline B-3 & 0 & 0 & 0 & 0 & 0 & 0 \\
\hline B-4 & 6 & 0 & 5 & 4 & 6 & 0 \\
\hline B-5 & 4 & 4 & 4 & 6 & 6 & 0 \\
\hline B-6 & 5 & 4 & 6 & 6 & 6 & 0 \\
\hline B-7 & 0 & 4 & 6 & 4 & 0 & 0 \\
\hline B-8 & 2 & 6 & 6 & 0 & 6 & 0 \\
\hline B-13 & 6 & 0 & 0 & 4 & 0 & 0 \\
\hline B-14 & 4 & 0 & 6 & 0 & 6 & 0 \\
\hline B-15 & 0 & 0 & 0 & 0 & 0 & 0 \\
\hline B-16 & 0 & 0 & 0 & 0 & 0 & 0 \\
\hline MW3 & 4 & 0 & 0 & 0 & 0 & 0 \\
\hline
\end{tabular}

Note:

Observed Level Of PAH Contamination

Designation

Free Product

6

Product Sheen

Discoloration

5

Strong Odor

Mild Odor

Slight Odor

No Odor 
Ames Expedited Site Characterization - Marshalltown FMGP Site

FINAL Site Report

March 25, 1996

\subsection{CHARACTERIZATION STRATEGY}

\subsection{Introduction}

In keeping with the stated project goals (Section 1.1), the characterization strategy for the Marshalltown site focused on the evaluation, comparison and promotion of both SOPT and IT characterization and monitoring technologies. Geophysical, chemical analysis and data integration technologies were fielded in two phases. Phase I involved largely non-invasive, screening type measurements while Phase II involved more invasive geophysical and contaminant analysis measurements. The Phase I and Phase II timelines with the fielded technologies and providers are shown on Figures 3.1 and 3.2, respectively. The technologies fielded and approaches used in fielding the technologies are described in the following sections.

\subsection{Geophysical Technologies}

As geophysical survey technologies play an integral role in the ESC methodology, the evaluation of both IT and SOPT geophysical survey technologies formed a significant part of this project. It must be stressed, however, that the Marshalltown FMGP site conditions were far from ideal for conducting high quality geophysical surveys. Cultural interferences such as overhead power lines, buildings, fences and storage areas and the relatively high conductivity UCU impacted the resolution and depth of penetration, respectively, of the ground penetrating radar while noise from the nearby railroad yard and on the site itself and the relatively high velocity surface fill layer impacted the resolution and interpretation of seismic surveying. These factors need to be considered in the evaluation of the geophysical survey technologies.

ITs included a shallow reflective seismic technique using a high-frequency, vibratory energy source, a 3-D ground penetrating radar (GPR) data analysis technique and a vertical induction profiling technique termed electromagnetic offset logging. The SOPTs included seismic reflection and refraction, GPR data acquisition and borehole geophysics. Reasonable correlations with information from the invasive technologies were sought.

Mobilized invasive technologies included hollow stem auger equipment to drill boreholes for calibrating purposes and minimally intrusive technologies represented by Geoprobe $^{\circledR}$ and cone penetration test (CPT) direct push technologies. While the Geoprobe ${ }^{\triangleright}$ was used in Phase I as a part of the contaminant screening tests, the direct push technologies, following established ESC protocols, were largely used in Phase II to further develop the site model and confirm data from Phase I. Comparison was made between the capabilities of the CPT system and the Geoprobe ${ }^{\star}$, in terms of stratigraphic logging, sampling and contaminant screening capabilities. The 
Ames Expedited Site Characterization - Marshalltown FMGP Site

FINAL Site Report

March 25, 1996

Marshalltown site provided a nearly ideal setting to evaluate the respective merits of these two push technologies because the soil types are amenable, the depths of interest are 50 feet or less, and physical access issues that impact truck mobility were evaluated at this site. The heterogeneous upper fill layer and the uneven bedrock surface did, however, create difficulties for these direct push technologies as described later.

\subsection{Contaminant Distribution}

The contaminant distribution was investigated in two phases, first the screening phase, and second, the quantitative phase. The major goals of this part of the work were to assess the ease of use and reliability of the ITs fielded and to compare the results of the screening technologies with the quantitative and BVWST results.

\subsubsection{Screening Phase}

Nearly all of the techniques used in the screening phase, including the immunoassay kits (IMA), passive sorbent, and the chemiluminescence (CL) optical method can be considered innovative. The IMA methods are just being approved by U.S.-EPA. Our data, collected from kits from more than one IMA provider and taken from split samples under carefully controlled conditions, will add considerable weight to the effective adoption of IMA methods within and outside DOE.

Only a few passive sorbent methods are known for semivolatile PAHs and few potential users realize that they are applicable to saturated as well as unsaturated soils. This is in contrast to volatile organic compound (VOC) passive sorbent methods that are in widespread use but only in unsaturated soil conditions. Likewise, active soil gas methods have been rarely applied to PAHs because of the much reduced volatility of PAHs.

One of the target compound PAHs at Marshalltown is naphthalene, whose vapor pressure defines the boundary between semivolatile organic compounds (SVOCs) and VOCs. Thus naphthalene is the SVOC with the highest vapor pressure and likely would form the highest volume plume. Naphthalene is also an attractive target compound because it was one of the most common PAHs detected in the previous characterizations and it is one of the three most common compounds that make up coal tar. Typically naphthalene ranges from about one to ten percent by volume of typical coal tar waste. However, some of the methods, such as IMA, may not be very sensitive to naphthalene. The relative sensitivity of these screening methods to various PAHs were a focus of the technology evaluation.

Screening measurements with the active and passive soil gas equipment, IMA kits and the CL technique were carried out at the same sampling locations to facilitate direct comparison. A 
Ames Expedited Site Characterization - Marshalltown FMGP Site

FINAL Site Report

March 25, 1996

network of sampling locations, with a shallow and deep sample at every location was set up and carried out in Phase I.

The innovative CPT laser induced fluorescence (LIF) sensor, in its present state of development and validation, does not have the ability to assist in the quantitative delineation of the PAH plume near the action levels nor can it distinguish more than a subset of the 16 PAHs of interest. It can, however, detect the presence of petroleum, oil or lubricant contaminants down to the range of $100 \mathrm{mg} / \mathrm{kg}$ and also qualitatively distinguish areas of low, medium or high contamination. Moreover, a continuous profile of LIF response and stratigraphy with depth can be obtained from each push location. Given these capabilities, the role of the CPT unit was to confirm the degree and extent of contamination indicated by the BVWST data and the other screening technologies in Phase I and to provide guidance for selecting locations for soil samples for quantitative chemical analysis. In addition, once the detailed topography of the upper surface of the LCU has been defined, potential shallow depressions near the edges of the main body or bodies of the PAH plume(s) can be directly sampled for free phase, saturated soil, or less contaminated soil for the existence of isolated PAH plumes.

Although considered a stratigraphic logging tool, the Geoprobe ${ }^{\star}$ soil conductivity sensor provided an indirect indication of the presence of coal tar residue through a drop in conductivity. This technique of contaminant distribution screening was also investigated through comparison of the conductivity logs with nearby CPT LIF pushes and quantitative data from soil samples.

\subsubsection{Quantitative Phase}

The quantitative phase focused on comparing the performance and results of five field extraction methods: 1) sonic solvent (SOPT), 2) thermal desorption (IT), 3) supercritical fluid extraction (IT), 4) microwave extraction (IT), and 5) microextraction (IT) coupled with field gas chromatography/mass spectrometry (GC/MS) systems with the results of standard contract laboratory program (CLP) off-site chemical analyses. Samples for the field GC/MS systems were collected using a Geoprobe ${ }^{\oplus}$ truck. Although field duplicates were taken during the subsurface probing to address site soil inhomogeneity, it was necessary to collect a sufficient quantity of each sample so that, after homogenization, they could be split and analyzed by each of the extraction modules.

Little new information about the contaminant distribution was gained from these split samples. However, they were necessary for the comparative evaluation of the extraction methods. Although some of these extraction methods had been used before and may even have EPA approval, their evaluation under identical conditions will benefit their adoption and implementation by DOE and others. Variations in recovery percentages among the extraction 
methods that were due to soil type are suspected to be important in the selection of appropriate extraction methods for similar soil types. In order to isolate the extraction percentage from the variance among the GC/MS instruments, spiked aliquots were injected directly into the column. Moreover, spiked soil samples were used to evaluate the percent recovery and method detection limits for each of the extraction methods.

Although really a part of the screening phase, samples co-located with the contaminant screening network described above were taken and analyzed off site to provide a direct comparison for the screening technologies. In addition, soil samples were also collected within several feet of CPT LIF push locations to provide a direct comparison of the LIF results with an on-site total recoverable petroleum hydrocarbon (TRPH) method and off-site chemical analysis results.

\subsection{Data Integration}

EarthVision software from Dynamic Graphics, Inc., was used for data integration and visualization a daily basis to assess the status of the geologic and contaminate conceptual site models and to plan the next day's sampling locations. Data handling, analysis and visualization in Earth Vision could be improved with a more flexible database arrangement and also with the inclusion of geostatistical capabilities such as kriging and quantitative uncertainty analysis.

Data from the previous BVWST site investigation were processed with EarthVision prior to going to the site. An experienced operator from Dynamic Graphics, Inc. was on site to facilitate data integration and operation of the Earth Vision program during Phase II when daily upgrades of the site model were generated. In particular, 2-D contoured stratigraphic maps were produced which helped to identify gaps in the data; this information was extremely useful in planning the next sampling locations. A computer-compatible projection system facilitated the display of the site model and critical data. This system was used primarily during the stakeholder presentations. During Phase II geostatistical calculations were vital for making unbiased estimates of the subtle data gaps that remained. 


\section{April 1, 1994}

Drill two calibrating cored holes

Obtain site - specific soil banks

\section{April 4 to 9, 1994}

Analytical

Soil gas experiment

Active: Global Environmental - GC based

Passive: NERI - install sorbers

IMA analysisis

Ensys, Millipore and Quantix Chemiluminescence

Geologic

GPR - D'Appolonia

Seismic Reflection - Resolution Resources, Inc.

Borehole Logging - D'Appolonia

\section{April 18 to 22, 1994}

Collect seismic data - Coleman Black Hawk

Retrieve NERI sorbers for off-site analysis 


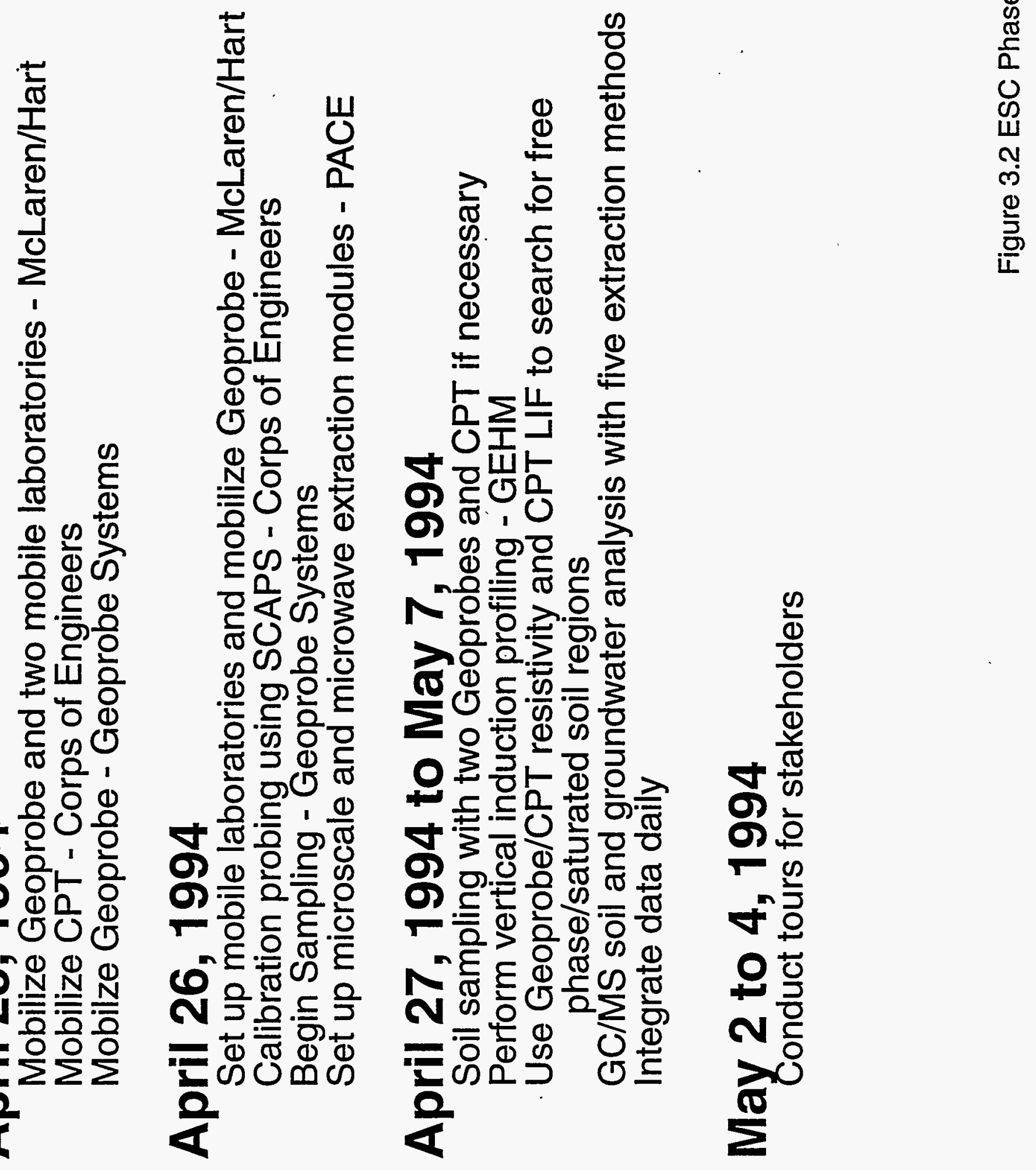


Ames Expedited Site Characterization - Marshalltown FMGP Site

FINAL Site Report

March 25, 1996

\subsection{RESULTS AND EVALUATIONS}

\subsection{Non-Intrusive Methods for Geological Characterization - Geophysical Surveys}

This section discusses the methods employed and the results obtained for non-intrusive geophysical survey technologies applied at the site. The technologies include ground penetrating radar (GPR), seismic refraction and reflection, electromagnetic offset logging (EOL) and borehole geophysical logging. The last two geophysical methods employed down-well tools but did not require any new intrusive activity to be accomplished. They are, therefore, included with the non-intrusive activities. A comprehensive evaluation of all geophysical applications was made by Technos, Inc. (1994).

\subsubsection{Ground-Penetrating Radar}

\section{Description}

The GPR survey, carried out by D'Appolonia Environmental Services, Inc., consisted of four phases: 1) common midpoint (CMP) testing to aid in the interpretation of signal velocity and depth of penetration; 2) a series of closely spaced, parallel profiles covering most accessible areas within the perimeter fence of the site; 3) processing of parallel profile data as a 3-D data set; and, 4) one common depth point (CDP) profile through the site using multiple transmitterreceiver offsets.

Common Midpoint Test. A CMP test was accomplished using a 100 megahertz (MHz) antenna in bistatic configuration and a Geophysical Survey Systems, Inc., (GSSI) SIR $\circledast$ System-10A. One north-south and one east-west trending line was run using a common central point (local coordinates 477N-845E; see Figure 4.1). Data were collected along each line beginning with minimum antenna separation and gradually increasing antenna offset to a maximum of 75 feet. A profile of average two-way velocities were determined from the data for the E-W line.

Parallel Profiles. A total of 36 radar profiles were obtained in parallel east-west orientations using a $300 \mathrm{MHz}$ antenna in monostatic mode and a GSSI SIR $\circledast$ System-10A (see Figure 4.1). A closely-spaced bistatic antenna configuration was originally planned but was abandoned due to instrumental interference. Profile lines were spaced 5 feet apart and set up in all areas sufficiently free of obstructions to allow data acquisition. Individual shots were taken at 6-inch intervals along each line. A total of 9851 shots were taken, with each shot consisting of 1024 time-amplitude measurements. Data were acquired by towing the antenna on a wheel-mounted sled and recorded on an Exabyte 8mm tape to a two-way travel time window of 200 nanoseconds 


\section{Ames Expedited Site Characterization - Marshalltown FMGP Site}

FINAL Site Report

March 25, 1996

(ns). Data for the profiles were processed and color depictions of the radar data were developed using the RADAN program.

3-D Data Set. Additional processing of the $300 \mathrm{MHz}$ data was performed by D'Appolonia Environmental Services for the purpose of compiling the data into a three-dimensional set. This was accomplished by first converting the RADAN data into. ASCII format using the RTOA16 provided by the GPR system manufacturer. The ASCII data represented the amplified and filtered trace amplitude profiles at each of the 9851 shot locations. Data was recorded to a range of about $180 \mathrm{~ns}$.

The number of time-amplitude measurements in each shot was then reduced to make data processing possible in a $\mathrm{PC}$ format. However, the reduction method was not consistent throughout the data set. The data set from the south half of the site was reduced to include only every other point in the top half and the bottom half was discarded, partly as a result of aerial interferences in the data. The remaining data set was reduced by inclusion of only every fourth measurement.

Map coordinates were then assigned to each shot measurement using Microsoft EXCEL. This was followed by a trace subtraction routine to eliminate background "noise." This was accomplished by subtracting from each individual shot trace the shot trace three measurements ahead (i.e., the fourth trace was subtracted from the first, the fifth was subtracted from the second, and so on). This routine was employed by D'Appolonia to effectively remove all but dipping reflections because these were the targets of interest for imaging as three-dimensional time slices. Following the trace subtraction routine, the individual line-specific files were compiled into master files. Due to the size of the master files and the memory limitations of the PC, the eastern half of the site had to be compiled into three separate master files and the western half of the site was compiled as a fourth master file.

The XYZ master files were imported into the GEOSOFT ${ }^{\mathrm{TM}}$ contouring program by segregating into smaller files with common $\mathrm{Z}$ coordinates (i.e., the same two-way travel time). These horizontal "time-slices" were then contoured as 2-D data sets by GEOSOFT ${ }^{\mathrm{TM}}$.

Common Depth Point Profile. Data were collected from the southeast edge to the northwest corner of the site along an uninterrupted 620-foot line. The location of the line is shown in Figure 4.1. Data were collected using $100 \mathrm{MHz}$ antennae in bistatic configuration with three different transmitter/receiver offsets. The purpose of this survey was to enable CMP processing of the data in a manner similar to seismic reflection data. The $100 \mathrm{MHz}$ antennae would presumably allow deeper penetration of the radar signal, but with less resolution than the 300 $\mathrm{MHz}$ antenna. 


\section{Ames Expedited Site Characterization - Marshalltown FMGP Site}

FINAL Site Report

March 25, 1996

A transmitter/receiver pair was first towed along the line in zero-offset configuration and measurements were made at 10-foot station spacings. Then two pairs of antennae at approximately 5-foot and 10-foot offsets were towed along the line in both directions. Measurements were taken with the transmitter antennae on the individual station marks. This system provided five measurements at each station (zero-offset, plus two non-zero offsets with receiver antennas on each side of the station). Individual measurements were recorded to a range of $900 \mathrm{~ns}$.

Data processing was conducted using PC-based software from the Lookout Geophysical Corporation of Golden, Colorado. Processing of the data was performed in the following steps:

1) Conversion of the raw RADAN data into SEG-Y format using software provided by GSSI.

2) Low and high pass filtering.

3) Derivation of average velocities.

4) Application of normal moveout correction.

5) Automatic statics correction.

6) CMP stacking of records (three-fold).

\section{Results}

Common Midpoint Test. Based on the CMP test and subsequent data acquisition and processing, the GPR contractor interpreted the maximum depth of resolvable reflections to exceed 75 feet (see Figure 4.4). Above ground reflectors, such as buildings and overhead wires, tended to interfere with some of the reflections from deeper than about 15-20 feet.

The interpreted velocity profile is provided in Table 4.1. For comparison and reference, a table of approximate electrical conductivities, relative dielectric constants and travel times for various earth materials is presented in Table 4.2. It is not clear why the two-way travel time in the depth range of 21 to 30 feet is so low; this depth would be below the water table and according to the value given for approximate travel time for a saturated sand material is low by about a factor of two.

ParallelProfiles. An example of a typical $300 \mathrm{MHz}$ profile collected from the site is reproduced in Figure 4.2. Based on velocity determinations made by the CMP test, the window of data to $180 \mathrm{~ns}$ is roughly equivalent to a depth profile to 30 feet. Equivalent interpreted depths have 
Ames Expedited Site Characterization - Marshalltown FMGP Site

FINAL Site Report

March 25, 1996

been superimposed along the left vertical axis of the figure. Complex reflections are demonstrated in the first $\sim 30 \mathrm{~ns}$, which corresponds to the top 6 to 7 feet in the subsurface. This may be attributable to fill material. Below this level, several strong horizontal reflections are interpreted by the contractor as instrument artifacts (e.g., reflection at $\sim 120 \mathrm{~ns}$ ). Reflections from overhead power lines are visible as broad parabolic reflections (see Figure 4.2 at $50 \mathrm{~ns}$ ). Reflections from buildings at the ends of profiles appear as steeply sloping multiple reflections at the ends of the lines, and buildings parallel to the lines appear as multiple strong, shallow reflections near the surface. Slabs of concrete are visible as bright "ringing" reflections near the surface.

In general, the data were less noisy on the eastern side of the property as a result of the relative openness of the site. On the western side, the former electric plant building on the south, the substation on the north, and the former purifier building on the east produced strong interfering reflections. No stratigraphic information could be obtained from this data set.

3-D Data Set. The processing performed on the 3-D data set (described above) was useful in reducing the intensity of or eliminating "noise" in the data, particularly in the first approximately $40 \mathrm{~ns}$ where ringing is strongest. Color contour images of the individual time slices were prepared and are included in D'Appolonia's report for various depths between approximately 2 feet and 12 feet. The time slice map for $13 \mathrm{~ns}$ (an interpreted depth of $\sim 3$ to 3.5 feet) is shown in Figure 4.3. Most of the visible anomalies display a strong north-south orientation as a result of the inhomogeneous distribution of data points (very closely spaced in the east-west direction (6 inches) as compared to the north-south direction (5 feet)).

The most visible anomalies in the time-slice maps are above-ground features, such as buildings and large metallic objects. The strong anomaly immediately to the east of the $500 \mathrm{~N}-800 \mathrm{E}$ intersection is surface interference from a parked trenching machine. Anomalies which are not as easily attributable to surface objects include a linear feature (interpreted as possibly a buried utility line) at the west end of the site trending NNE-SSW, a roughly square feature at the west end of the east half of the site (possibly an unusually strong reflection from the buildings), and numerous small anomalies dispersed across the site but particularly abundant in the southern portions. These small anomalies are notably more scarce to absent in certain areas, such as beneath the concrete pad of the former gas holder east of the former purifier building (see Figure 2.1 for reference). Several of these anomalies are interpreted by the contractor as buried metallic objects. In the deepest time slice ( $65 \mathrm{~ns}$ or about $12 \mathrm{feet}$ ), the interference from overhead power lines became the most salient features. No stratigraphic information could be obtained from this data set.

Common Depth Point Profile. Data collected by the $100 \mathrm{MHz}$ multi-offset, bistatic antenna configuration and processed by CDP methods are displayed in wiggle trace and trace amplitude 
Ames Expedited Site Characterization - Marshalltown FMGP Site

FINAL Site Report

March 25, 1996

format in Figure 4.4. Surface landmarks are indicated on the figure for reference. Strong coherent reflections are apparent between two-way travel times of about 60 and $130 \mathrm{~ns}$. This is followed by an absence of reflections down to about 240 ns. From 240 ns to about $700 \mathrm{ns,}$ reflections are intense and regular, with the exception of around $600 \mathrm{~ns}$; reflections beyond a two-way travel time of about $550 \mathrm{~ns}$ are interpreted by the contractor as "ringing" between horizons.

Based on the results of the CDP survey, a maximum depth to resolvable targets of 75 feet (approx. $550 \mathrm{ns)} \mathrm{was} \mathrm{interpreted} \mathrm{by} \mathrm{the} \mathrm{contractor} \mathrm{for} \mathrm{the} 100 \mathrm{MHz}$ antenna configurations, and reflections to a two-way travel time of $550 \mathrm{~ns}$ were interpreted to be from actual horizons in the ground. The reflection at about $100 \mathrm{~ns}$ is believed to represent the water table. The soil-bedrock interface was interpreted as the coherent reflections at about $240 \mathrm{~ns}$ or approximately 40 to 45 feet. An aberration in the data at shot points 18-20 (Figure 4.4) was interpreted as a possible bedrock pinnacle. However, the line passed within several feet of a metal gate and chain link fence at this location, which may provide a more likely explanation of the anomaly. In addition, the interpreted bedrock reflection contains gaps between shot points 13 and 19, shot points 23 and 26 , and shot points 49 and 52.

\section{Broductivity/Output}

The time needed to accomplish the scope of work described above was six field days, although half of this time was non-productive because of equipment problems. The actual collection of data required one day for the 3-D data set, less than one day for the bistatic multi-offset line, and several hours for the CMP test.

Post-processing was accomplished off site over a period of about four weeks. Delays in processing were encountered primarily as a result of difficulty in converting multi-offset radar data to SEG-Y format.

\section{Commentary}

Two significant indications suggest potentially poor radar performance at this site (Technos, Inc., 1994). It is generally accepted that penetration of radar signals is severely limited by geologic strata with relatively high values of electrical conductivity. Borehole geophysical logging and Geoprobe conductivity probing (discussed in Section 4.3.2) indicate that the conductivity of the UCU is on the order of 50 to $150 \mathrm{mS} / \mathrm{m}$. Empirical data suggests that the maximum depth of radar penetration is related to the electrical conductivity $(\sigma)$ by the expression:

Depth $(\max )<40 / \sigma$ to Depth $(\max )<80 / \sigma$. 
Ames Expedited Site Characterization - Marshalltown FMGP Site

FINAL Site Report

March 25, 1996

Using the lower range of $\sigma$ would thus limit the depth of penetration to about 0.8 to $1.6 \mathrm{~m}$ (2.6 to about 5 feet). Moreover, the heterogeneous nature of the upper fill would tend to disperse and attenuate the radar signal.

The second indication of poor radar performance is the abundance of air reflectors in the data. The $300 \mathrm{MHz}$ GSSI antennae are fully shielded and do not normally show any response from air reflectors (Technos, Inc., 1994). The fact that air reflectors are seen indicates a poor impedance match between the antennae and the ground (i.e., the signal is simply being reflected off the ground and into the air). In addition, the contractor refers to problems with the bi-static configuration of the $300 \mathrm{MHz}$ system, which was apparently unresolved, problems with a survey wheel and limitations with the PC based software being used to process the data. A significant part of the data set was not used because of the limitations of the software; this could have an adverse impact on processing and interpretation.

In short, the results of this GPR survey should be viewed with caution.

\subsubsection{Seismic Reflection}

Both seismic reflection and refraction surveys were completed at the site in an effort to provide detail of the bedrock surface beneath the alluvial soils an, if possible, to provide information regarding stratigraphic interfaces above the bedrock. The two surveys were conducted by different contractors, so the systems and operating parameters differ and will be discussed individually. The seismic reflection survey was carried out by Resolution Resources, Inc.

\section{Description}

Three seismic reflection profiles were collected on each of three sides of the site. Two eastwest trending lines were collected outside-the perimeter fence on the north and south, and one shorter line was collected along the west side within the site. These lines will hereafter be referred to as the "north," "south" and "west" lines, respectively. Figure 4.5 shows the locations of the lines. The line locations were chosen by the contractor to potentially identify geologic features which may influence shallow (i.e., above bedrock) and deep (i.e., below bedrock) groundwater flow and to verify the existence of lineaments in bedrock structure believed by the contractor to be visible in aerial photography. A total of 2,845 feet of 24 -fold seismic line data was collected.

A noise test was conducted at the west end of the south line on an unused portion of the railroad right-of-way. 
Ames Expedited Site Characterization - Marshalltown FMGP Site

FINAL Site Report

March 25, 1996

The following operating parameters were chosen by the contractor following the noise test:

$\begin{array}{ll}\text { Sample Rate } & 0.250 \mathrm{~ms} \\ \text { Channels } & 48 \\ \text { Record Length } & 250 \mathrm{~ms} \\ \text { Low Cut Acquisition Filter } & 70 \mathrm{~Hz} \\ \text { High Cut Acquisition Filter } & 500 \mathrm{~Hz} \\ \text { Geophone Spacing } & 5 \text { feet } \\ \text { Minimum Offset } & 0 \text { feet }\end{array}$

A 12-pound sledge hammer source with six pops per source location was used, and signals were received with Mark Products $40 \mathrm{Hertz}(\mathrm{Hz})$ geophones and recorded on a Geometrics Strataview seismograph.

Vertical seismic profiles (termed check shot surveys by Technos, Inc., 1994) were performed in wells $\mathrm{MW}-1 \mathrm{~B}$ and $\mathrm{MW}-3 \mathrm{~B}$ using a $30 \mathrm{~Hz}$ downhole receiver. The receiver was lowered into each of the wells and locked in place with an inflatable bladder. Measurements were taken at 2.5-foot intervals to a total depth of 55 and 57 feet, respectively, in the two wells. A 12-pound sledge was used at a distance 10 feet from the well at 10 pops per station.

Processing was performed using Seistrix 3 software developed by Interprex. The following data processing steps were performed following downloading of the data to a PC:

- Edit field data - remove noisy records or bad data traces;

- CDP sort -- assemble individual traces from different records into records having a common reflecting point;

- Digital filter -- eliminate noise in frequency domain;

- Velocity Analysis -- build model of velocity vs. depth, required to correct data for normal moveout and estimate depth from the time section, by applying various velocities to a select set of CDP records and choose the velocity which produced the most coherent section;

- Static correction - apply time corrections to compensate for elevations differences in geophones and sources;

- Mute -- remove refraction (first arrival) from reflection section; 
Ames Expedited Site Characterization - Marshalltown FMGP Site

FINAL Site Report

March 25, 1996

- Normal moveout correction -- remove the differences in travel time that result from differences in distance from source to near and far geophones;

- CDP stack -- summation of moveout-corrected traces in the CDP records; and

- Automatic gain compensation -- rescales amplitudes across the individual stacked traces, increasing the amplitude of late events.

The stacked and corrected traces were then plotted on hard copy for viewing. A static correction of $8.3 \mathrm{~ms}$ was added to traces in the south line to adjust for the difference in elevation between the north and south lines. No static correction appears to have been made along the individual lines.

\section{Results}

A copy of the interpreted seismic reflection profiles are contained in Figure 4.6. The vertical dimension is two-way travel time in milliseconds (ms) rather than depth in feet. The three key stratigraphic contacts between the UCU, granular unit, and LCU were interpreted from the profiles by the contractor. In addition, "disrupted" areas (incoherent reflections) in the bedrock structure were interpreted. These were interpreted by the contractor to be associated with the lineaments they had identified on the aerial photographs prior to commencement of work.

The check shot surveys carried out in MW-1 and MW-3 were used to calculate the reflection time to the stratigraphic contact of interest. The results are shown in Figure 4.7. An apparent low velocity zone between about 12 feet and 22 feet in well $\mathrm{MW}-1 \mathrm{~B}$ may be noted. In addition, the velocity below 25 feet differs greatly between the two wells. Correlation between the top of the LCU in the check shot survey and the interpreted cross section is good (see Figure 4.6a), but the bedrock contact appears to be somewhat deeper in the cross section than on the check shot survey (i.e., according to the formula presented in the contractor's report). The bedrock should be at about $43 \mathrm{~ms}$, whereas the cross section shows bedrock at a depth of about $50 \mathrm{~ms}$. Further comparisons of the seismic reflection interpretations with intrusive data are made in Section 4.3.3.

\section{Broductivity/Output}

Data was collected by a two-person crew over a period of five days, including time involved in reconnoitering the geology of the Marshalltown area. Data processing was accomplished on a daily basis, and updated plots of processed lines were viewed at the site as work progressed. All field work and data processing was completed by experienced personnel. 
Ames Expedited Site Characterization - Marshalltown FMGP Site

FINAL Site Report

March 25, 1996

\section{Commentary}

The air photo lineaments referred to above and illustrated in Figure 4.5 appear to be related to human activity (e.g., edges of rows of trees, dredging for drainage purposes, etc.) rather than geologic structure (Technos, Inc., 1994). In terms of acquisition of the data, it is believed that considering the depth to bedrock of about 40 to 50 feet, a geophone spacing of one to two feet would have been more appropriate than the 5 foot spacing that was used. The resulting long source-to-geophone offset (on the order of 240 feet) would cause serious phase distortions for all reflectors above and including those from the bedrock (Technos, Inc., 1994). It is also believed that the shallowest reflectors in the 20 to 30 ms range are too discontinuous to be considered as representing stratigraphic contacts.

As a final note on the efficacy of this work, a second contractor (Coleman Energy and Environmental Systems-Blackhawk Geosciences Division) was hired to conduct a seismic reflection survey using a variable frequency electromechanical source produced by OYO Corporation along side a manual hammer source. However, walkaway tests using both sources produced no identifiable reflecting events, as interpreted by the contractor. Despite this, the contractor collected 150 feet of data to be processed by reflection techniques, although no interpretations were derived from this data.

\subsubsection{Seismic Refraction}

\section{Description}

The seismic refraction survey carried out by Coleman Energy and Environmental SystemsBlackhawk Geosciences Division used both compressional (P-wave) and shear (S-wave) impact sources for the primary function of characterizing the bedrock surface. In general, the site exhibited some unfavorable features including a high velocity near-surface fill layer which attenuated the seismic signal (particularly high frequencies), and high ambient noise from the railyard and activity on and around the site.

Equipment used for the collection of refraction data included an OYO DAS-1 48-channel seismograph, OYO $28 \mathrm{~Hz}$ geophones, 24 channel CMP cable, Bison EWG-1 assisted weight drop, azimuthal traction plate, and an I/O 240M channel roll box.

Compressional Wave Survey Nine $\mathrm{P}$-wave refraction lines were collected at various locations around the site (see Figure 4.8); lines 1 and 2 were not interpreted due to significant cultural interferences. The lines were located primarily inside the perimeter fence and were constrained such that the effects of visible cultural refractors would be minimized. For six of the lines, a 24- 
Ames Expedited Site Characterization - Marshalltown FMGP Site

FINAL Site Report

March 25, 1996

geophone array was laid out at a spacing of 5 feet giving a total line length of 115 feet. A 48geophone array was used for lines 1,2 and 10, giving a total line length of 235 feet.

A mechanically assisted drop weight was used to generate the signal. Due to the low signal to noise ratio, up to 40 blows were used on long source-to-receiver offsets. Five shot locations were used for forward and reverse shot directions. Distant shot locations were restricted due to physical inaccessibility.

Refraction data processing consisted of the following steps:

1) Import data into OSIPAK software system for first break analysis;

2) Pick first breaks on individual traces;

3) Import first breaks into GREMIX program for refraction analysis;

4) Assign layers to arrivals;

5) Pick X-Y distances for Generalized Reciprocal Method (GRM) analysis; and

6) Compute depths and velocities of refracting layers.

Engineered fill at the near surface throughout the site created a near surface high speed $(11,000$ $\mathrm{ft} / \mathrm{s}$ ) refractor, which was the first seismic event to appear on the records. This event was ignored when picking first arrival from the records. The later events were picked based on consistency between adjacent records and their velocities.

As a result of the near-surface high velocity fill layer and possibly also a thin layer of saturated sediments, a condition called a "hidden layer" existed in which the layers below the high velocity layer cannot be recognized. This problem was resolved by applying the GRM analysis. GRM analysis does not allow mapping of the bottom of the hidden layer, but does allow mapping of layers below this hidden layer. A full treatment of the hidden layer case is given by Palmer (1980) and Lankston (1989). .

The velocity of a refractor is calculated from the slope of the time-distance relationship of the time of arrival picks. The velocity of the refractor interpreted to be the limestone bedrock at the site was in the range of 6,000 to $7,000 \mathrm{ft} / \mathrm{s}$, which is consistent with published velocities for weathered limestone. Some of the lines which were oriented N-S showed significantly higher velocities, although this fact may be the result of interpretive error. 


\section{Ames Expedited Site Characterization - Marshalltown FMGP Site}

FINAL Site Report

March 25, 1996

Shear Wave Survey Five S-wave refraction lines were collected at locations corresponding with $\mathrm{P}$-wave lines 1 to 5. S-waves were generated using an azimuthal traction plate to create waves of opposite polarity. The reversed polarity allowed the masking of compressional energy which may have been produced by the source, thus enhancing the ability to pick the first arrival of the shear wave. Internal geophone elements were modified from vertical orientation to horizontal with consistent polarity throughout the array.

First arrivals of shear waves were picked manually by superimposing corresponding traces and noting the location on each pair where simultaneous opposite polarity motion was observed. This process was made more difficult by ambient noise and a traction plate that provided lower energy signals than the EWG-1 wave generator.

\section{Results}

Compressional Wave Survey. Depth to bedrock profiles were interpreted by the contractor for eight of the $\mathrm{P}$-wave lines. The remaining two lines were not interpreted due to suspected interfering signals refracted from nearby buildings or other sources. Four of the interpreted profiles are reproduced in Figures 4.9 to 4.12. Note that depth to bedrock from nearby BVWST boreholes and seismic line intersection point depths to bedrock are also included on these profiles. Combining these results with the BVWST borehole data allowed the contractor to produce revised bedrock elevation contours based on combined borehole and seismic refraction data. Contours of bedrock elevation with the BVWST data alone are shown on Figure 4.13, and contours including the seismic refraction data are shown on Figure 4.14.

The following may be concluded regarding the seismic refraction profiling:

1) Overall, the depths to bedrock interpreted from the seismic survey correspond to the BVWST data. Comparisons with MW-3 and B-16 are within several feet. Moreover, the general trends observed with the contours correspond to general trends of the existing data; i.e., generally greater depth to bedrock in the vicinity of Lines 6 and 10 and shallower bedrock in the northern and eastern parts of the site. In particular, BVWST boreholes B-1, B-2, B-4 and B-5 all indicate depths to bedrock of over $\mathbf{4 0}$ feet. This tends to provide support for the results of line 6.

2) The seismic data also indicate a bedrock high or ridge which trends north-south in the region of $800 \mathrm{E}$ to $850 \mathrm{E}$ from the northern part of the site. A bedrock knob or pinnacle was also identified in the vicinity of $830 \mathrm{E}, 450 \mathrm{~N}$ (see Figure 4.14). 


\section{Ames Expedited Site Characterization - Marshalltown FMGP Site}

\section{FINAL Site Report}

\section{March 25, 1996}

3) Comparison of the depth to bedrock at points where the seismic lines intersect indicate correspondence within about two to three feet. A notable exception is at the intersection of lines 5 and 7 where the depths to bedrock deviate by more than 10 feet.

Shear Wave Survey The combined effects of high ambient noise and inherently low source energy from the traction plate resulted in indistinguishable first shear wave arrivals at far geophones for the S-wave surveys. As a result of the lack of far offset data, little information could be obtained from the S-wave effort, and this data was, therefore, not used to derive depth to bedrock information.

\section{Broductivity/Output}

Work was accomplished by a three-man crew over the course of seven work days, including walkaway tests for refraction and reflection. Raw field records were viewed in the field; however, processed refraction profiles were not available until after processing was completed off site.

\section{Commentary}

Despite the fact that a 1-foot geophone spacing was used, no reflections were observed from the shallow bedrock or overlying stratigraphic soil units using either the hammer or vibratory source. This result casts much of the seismic reflection survey reported in Section 4.1.2 in doubt.

Despite the cultural interferences at the site, the results of the seismic refraction survey correlate reasonably well (generally to within about two to three feet) with the BVWST existing data. It must be kept in mind, however, that most of the data is concentrated in the eastern portion of the site and the depth to bedrock shown in the westem portion of the site would have a higher level of uncertainty; note also that the most detail in the contour maps corresponds to the area of the most intensive surveying. Further comparisons with the intrusive data collected at the site will be made in later sections.

\subsubsection{Borehole Geophysical Logging}

\section{Description}

D'Appolonia Environmental Services, Inc., of Monroeville, PA, was contracted to apply a suite of borehole logging technologies to four existing wells and two new cased boreholes at the site. This information was then correlated with stratigraphic logs developed by BVWST and Ames 
Ames Expedited Site Characterization - Marshalltown FMGP Site

FINAL Site Report

March 25, 1996

Laboratory for the respective holes to evaluate the quality and quantity of stratigraphic data which can be obtained by these methods in similar settings.

Natural Gamma. Natural gamma logging is a passive-type log which measures naturally occurring gamma radiation in the soils or rock formation surrounding the well or borehole, primarily from isotopes of potassium, radium and thorium. These elements tend to be more abundant in clay-rich soil and shale. This tool usually provides qualitative information about the stratigraphy of the formation on a gross scale. Fine details of sedimentation can not be resolved by this method.

Gamma-Gamma. This logging tool uses a gamma ray source and gamma detectors to measure backscattering and attenuation by the materials surrounding the well or borehole to gain an understanding of bulk density. Only qualitative information can be obtain due to interference of well materials and annular media. The tool used for this project was a D'Appolonia Model 501DR Depthprobe with a $10 \mathrm{mCi}$ cesium-137 source and it had the capability of determining wet and dry density by using the moisture content output from the neutron log (discussed below).

Neutron. This method involves the use of a neutron source inside the logging tool which interacts with borehole and formation materials, resulting in backscattered neutrons and gamma ray production. Most of the interactions are with hydrogen atoms, primarily contained in water molecules. Therefore, neutron logging is used for determining moisture content and formation porosity in saturated conditions. The Model 501DR Depthprobe with a 50mCi americium$241 /$ beryllium source was used. The detectors on the tool sense either low energy (thermal) neutrons or gamma radiation from slow neutron absorption. The data generated was used in the calculation of wet and dry density values for the gamma-gamma logs.

Induction. The boreholes were also logged using electromagnetic induction. The tool used for this activity was a Geonics EM-39, with a transmitter-receiver coil spacing of $50 \mathrm{~cm}$. The transmitter coil induces electric currents in the formation media. The magnetic fields of these currents are then detected by the receiver coil. The coils are configured such that peak response is approximately $30 \mathrm{~cm}$ from the axis of the borehole, well outside of the well and annular sealing materials. The recorded data are computed as ground conductivity in units of milliSiemens per meter $(\mathrm{mS} / \mathrm{m})$.

Results

The density, natural gamma and induction logs for MW-1, MW-2, MW-3, HSA-2, MW-5 and HSA-1 have been plotted along with the BVWST borehole logs by Technos, Inc., in Figures 4.15 to 4.20 , respectively. The stratigraphic logs for boreholes HSA-1 and HSA-2 may be found in 
Ames Expedited Site Characterization - Marshalltown FMGP Site

FINAL Site Report

March 25, 1996

Appendix A. The natural gamma logs generated for MW-1, MW-2 and MW-3 displayed a relatively high degree of scatter with respect to stratigraphic variations. Subtle details in the soil units above bedrock could not be confidently identified. The transitions from the upper cohesive unit to the granular unit to the LCU were marked by a gradual decrease in the gamma count. However, the logs provided good information about the occurrence of unweathered bedrock, marked by a steady low gamma radiation level. The most abrupt change in the soil units in any of the gamma logs was in HSA-2 at a depth of 14 to 17 feet, where the gamma count dropped by a factor of 5.(see Figure 4.18). This depth corresponded to a transitional zone between firm clay above 14 feet and poorly graded sand below 17 feet.

The gamma-gamma logs showed scatter similar to the natural gamma logs, and the variations in density in the overburden soils were not sufficient to use this technology for detailed stratigraphic logging. However, the logs showed consistently high density readings at and below the bedrock surface (weathered and unweathered). A notable exception to these results was the $\log$ for HSA-2, which showed a dramatic rise in density between 23 and 24 feet that persisted to the bottom of the hole at 45 feet (although the density log data continues inexplicably to $>49$ feet; these two facts may indicate an error in the depth scale for this $\log$ ).

The induction (conductivity) data showed much less scatter than the nuclear-derived data, with the exception of some spurious readings in MW-2 at a depth range of 24 to 27 feet (Figure 4.16). While large differences exist between conductivity values in the cohesive units and the granular unit, the contacts are gradual in the EM-39 logs and cannot be pinpointed by this method alone. As with the natural gamma logs, HSA-2 displayed the sharpest boundary between the upper cohesive and the granular units.

Typical ranges in conductivity and resistivity for the stratigraphic units were as follows:

\begin{tabular}{lcc} 
Soil Unit & Conductivity (mS/m) & Resistivity (ohm-m) \\
\cline { 2 - 3 } UCU & $55-82$ & $12-18$ \\
Granular & $50-65$ & $15-20$ \\
Weathered Lmst. & $45-55$ & $18-22$ \\
Unweathered Lmst. & 45 & 22 \\
& & \\
ctivity/Output & &.
\end{tabular}

The suite of logs described above was accomplished on six wells with a total of approximately 250 feet of $\log$ collected. The work was accomplished in four (4) working days by a two-person crew. With the exception of an obstruction in well MW-4, preventing it from being logged, no significant logistical or equipment problems were encountered. 
Ames Expedited Site Characterization - Marshalltown FMGP Site

FINAL Site Report

March 25, 1996

\section{Commentary}

The advantage of using state-of-the-practice borehole logging techniques for geologic characterization of the site is that they provide qualitative records of subsurface physical properties useful in making further interpretations using other complimentary technologies. In particular, the borehole logging data was useful in making positive identification of unweathered bedrock horizons, which was not possible by any other means, including drilling logs which are typically highly subjective. A clear disadvantage of borehole logging is the requirement for new intrusive drilling activity or pre-existing wells and the concomitant generation of potentially contaminated waste material.

\subsubsection{Electromagnetic Offset Logging (EOL)}

\section{Description}

The electromagnetic offset logging technique used at this site is based on a geophysical technique originally used to prospect for metallic (conductive) orebodies and oil and gas reservoirs. The system used consisted of a surface source coil and a downhole receiver. The source coil is a 12-turn, 2-meter coil, operated with a very low frequency alternating current of 10 amperes, creating an effective electromagnetic moment of 500 ampere-meters squared (amp-meters ${ }^{2}$ ). The current induced in the downhole receiver coil by the primary and secondary magnetic fluxes created by the source coil is recorded as voltage data.

The EOL survey was performed by The GEHM Corporation and Entech Engineering, Inc. The source coil was placed on a 20-foot grid pattern. The survey area was approximately $200 \times 200$ feet (approximately 1 acre). A total of 126 offset logs were acquired, of which 29 were rejected during editing prior to modeling. The final offset log coverage and source locations are depicted in Figure 4.21, which shows that 97 offset logs were used for modeling. Data from each source coil location was logged from surface grade to 50 feet below grade. Vertical sampling in each log was performed on a 0.1 foot interval.

The signals from the receiver coil are passed through a High-Q, inverted-notch filter specific to the source coil frequency. This filter enhances signal-to-noise ratio. The filtered signal is then passed to an integrator which performs additional signal-to-noise enhancement by summing and averaging the signal over many tens of cycles.

At each source coil station location, the receiver travels the full depth of the selected well or borehole and records a "continuous" record of the induced current. Variations in the receiver coil signal should reflect variations in subsurface resistivity beneath the source coil. After a 
Ames Expedited Site Characterization - Marshalltown FMGP Site

FINAL Site Report

April 4, 1996

complete vertical offset log is recorded, the source coil is moved to another station and the process is repeated. A 3-D matrix of data is generated from this process.

The last steps of the field processing involve digital sampling of the integrator voltage output, plotting of field records of the output for quality control, and field evaluation of detected anomalies. The digital data passing quality control checks is stored on floppy disk for further processing off site. Details of the processing were not disclosed by the contractor but were identified as involving the following steps:

1) Automated editing and removal of extreme noise as unusable offset logs.

2) Automated amplitude static corrections to eliminate variations in the individual logs caused by changes in source strength in and around noise features.

3) Automated signal-to-noise enhancement using 0.1-foot samples to generate resolution for the final 0.5-foot sampled offset logs input to the model process.

4) Generation of one-dimensional log models.

5) Design of two-dimensional and three-dimensional model weights.

6) Three-dimensional surface-integral modeling.

The final steps are development of 3-D images, maps and cross-sections using EarthVision software and annotation using Silicon Graphics' Showcase software.

Results

A simple resistivity spectrum involving six resistivity ranges (colors) was chosen to model and present the data. The resistivity values in the spectrum reflect the following geologic strata:

\begin{tabular}{|c|c|c|c|}
\hline Soil Type & Resistivity $(\Omega-m)$ & Resistivity $(\Omega$-m)* & Conductivity $(\mathrm{mS} / \mathrm{m})+$ \\
\hline clay & $<45$ & $45-55$ & $>22$ \\
\hline silty clay & $45-55$ & $55-80$ & $18.2-22$ \\
\hline silty sand & $55-80$ & $80-120$ & $12.5-18.2$ \\
\hline clean sand & $80-120$ & $120-300$ & $8.3-12.5$ \\
\hline
\end{tabular}

and gravel

- - same soils containing hydrocarbons

+ - corresponding conductivity of water saturated soil types indicated in the first column 
Ames Expedited Site Characterization - Marshalltown FMGP Site

FINAL Site Report

March 25, 1996

A 3-D resistivity image produced from the data is shown as Figure 4.22. This figure illustrates two significant regions with resistivity $>55 \mathrm{ohm}$-meters (conductivity $<18.2 \mathrm{mS} / \mathrm{m}$ ). The eastern region, which comes very close to the surface near borehole B-7, contains a significant core of material with $>120 \mathrm{ohm}$-meters resistivity. Having ruled out the possibilities of an enormous free-product plume (based on intrusive sampling) or a large bedrock knob, the contractor attributes this to "a dichotomy condition of electrical resistivity resulting from the presence of hydrocarbon containing vertical fractures." It is not clear in the contractor's report where these fractures are believed to be located. The presence or absence of such fractures could not be verified within the scope of this investigation.

\section{Productivity/Output}

Data acquisition began on April 27, 1994 and was completed April 29, 1994. Processing began the following day with the first model being created on May 1, 1994. Ames Laboratory personnel were briefed on the preliminary findings on May 2, 1994.

\section{Commentary}

The contractor has not alluded to the potential for significant cultural interferences to affect the EOL results at this site. In fact, the transmitter loop was deployed within a few feet of vehicles, chain link fences, stacks of steel piping and even within buildings (including a steel shed). Adding to this the fact that only one receiver well was used, a limitation which the contractor did recognize, it may be concluded that the data were acquired under less than ideal conditions. There presently is no physical evidence to support the contractor's hypothesis of vertical fractures containing hydrocarbons in this region. Furthermore, the proprietary algorithm for processing raw logs could not be evaluated. Without a clear understanding of how the data was handled, it is not possible to comment on the integrity of the method used.

The resistivity values measured in this survey are significantly higher than those measured with the EM-39 logging tool (i.e., the highest resistivity values measured with the EM-39 are on the order of $22 \mathrm{ohm}-\mathrm{m}$ for the unweathered limestone (corresponding conductivity of $45 \mathrm{mS} / \mathrm{m}$ )). Since the EM-39 borehole logging technique is a well established SOPT, these results may be considered as more representative of the site soil and rock resistivity characteristics.

Comparisons will also be made with the Geoprobe conductivity logging in a later section.

\subsubsection{Summary - General Comments on Geophysical Surveying Results}

Of the geophysical techniques used at the site, only the seismic refraction and borehole logging appear to provide confident data. The results of the seismic refraction compared reasonably well 
(usually within about two to three feet) with the existing BVWST data in terms of defining the bedrock surface. The borehole logging results were also verified by the BVWST borehole logs. The borehole logging results added no new information to the characterization effort beyond the interpreted interface between weathered and unweathered bedrock; these results, however, were useful for calibrating and verifying some of the other intrusive techniques discussed in later sections of this report.

The GPR data added little useful information to the characterization effort. The $300 \mathrm{MHz}$ data appeared to locate shallow foundations and services, but this aspect of GPR logging was not an important part of this project. For the $100 \mathrm{MHz}$ survey, it is unlikely that the depth of penetration of the radar signal was as deep as the contractor claims. There is also significant potential error in trying to determine the depth to bedrock. The bedrock high or pinnacle noted by the contractor at stations 18 to 20 (see Figure 4.4) tends to correspond to a bedrock high determined by the refraction survey. The GPR $100 \mathrm{MHz}$ line is superimposed on the refraction depth to bedrock map on Figure 4.23. Significant uncertainty in the two-way travel times, however, make interpreting the depths to any better than within 5 to 10 feet impossible.

As there are no depth indications on the seismic reflection "interpreted" profiles and no clear indication in the contractor's report as to how to calculate the depths, quantitative evaluations and comparisons with other data are not possible. Qualitative comparisons of stratigraphic profiles produced by the seismic reflection interpretations with those produced from intrusive data showed poor matches. The EOL survey data does not appear to correlate with any observed features at the site and the contractor's explanation of the observed anomalies appears questionable.

It appears that the seismic reflection and EOL contractors may have come to the site with preconceived notions of the conditions at the site. The seismic reflection contractor found lineaments in the bedrock from the same aerial photos that other experts who viewed them could not confirm, and the EOL contractor seemed to be focused on finding large hydrocarbon plumes. The level of confidence in the results obtained by these two methods is not high.

\subsection{Phase I Contaminant Screening Technologies}

Based on a previous site characterization performed by BVWST, the lateral and depth distribution of the PAH contamination could be qualitatively estimated (see Chapter 2). This site was considered an important opportunity to evaluate a variety of screening technologies for SVOCs. In addition, the results could be used to confirm the previous plume estimates and to assist with the planning of the Phase II contaminant screening and sampling/quantitative analysis activities. 


\section{Ames Expedited Site Characterization - Marshalltown FMGP Site}

FINAL Site Report

March 25, 1996

MLs 3 to 27 were laid out to bound the expected edges of the PAH plumes (see Figure 4.24). The sampling locations were set up in triangles with about a 50 foot separation between the locations; due regard was given to the interfering structures on the site. ML-1 and ML-2 were assigned to the calibrating hollow stem auger boreholes which were placed on the extreme east and west edges of the site (HSA-1 and HSA-2, respectively), well away from suspected contamination zones. The sampling network covered most of the site except its extreme western edge.

The suite of Phase I screening technologies fielded included kits from all three of the available IMA vendors that could detect PAHs at the time of the demonstration (Ensys, Millipore and Quantix), an innovative CL system, and active and passive soil gas measurements.

A Geoprobe system mobilized on site by the active soil gas measurement contractor, Global Environmental, Inc., was used to actively sample for soil gas, install the passive gas instruments and take soil core samples to provide duplicate samples for the IMA and CL techniques at each of the $24 \mathrm{MLs}$. Two depths were investigated at each ML; one shallow, typically at a depth of about 5 feet, and the other deeper, at a depth of 10 to 15 feet. As a result, there were a total of 48 sampling locations for the screening program. The shallow depth samples were recommended by Northeast Research Institute (NERI), the passive soil gas vendor. The deeper measurements were an attempt to sample below the relatively impermeable upper cohesive unit and improve the detectability of the active soil gas measurements for the deeper lying PAHs. According to BVWST, the water table was typically in the middle of the granular unit in 1992. However, historic heavy rains in the summer of 1993 raised the water table into the lower levels of the upper cohesive unit on the western half of the site. Since active soil gas measurements require unsaturated soils, the deeper set of samples on the west portion of the site had to be taken in the upper cohesive unit rather than in the upper reaches of the more permeable granular unit.

Because the results of the IMA and CL tests are basically only qualitative, it was decided that duplicate soil samples would be used rather than splits. These soil samples were taken as close as possible to the locations where the soil gas measurements were made. In addition, six duplicate soil samples were taken and analyzed off site for moisture content and PAH concentration by high pressure liquid chromatography (HPLC).

\subsubsection{Immunoassay}

\section{Description}

IMA is a technology that was developed by the medical testing community and is based on the very high selectivity of enzyme reactivity that permits sensitive analysis of organics in the presence of many potentially interfering compounds. The IMA kits, which require little training 
Ames Expedited Site Characterization - Marshalltown FMGP Site

FINAL Site Report

March 25, 1996

or expertise to use, give results on site in a matter of minutes using color-coded liquid extractants. However, they detect a weighted average of the PAHs of interest and can only be used in a screening mode. Ensys and Millipore are gravimetric measurements while Quantix is volumetric. Each company was contracted for 75 kits and associated training for three employees, which was done at the Ames Laboratory prior to site measurements. The three Ames Laboratory employees who were trained by the IMA vendors made all of the on-site IMA measurements. The employees had no formal training in the use of the IMA test kits prior to this project. Evidence of operator dependence was not apparent in the IMA results, confirming the ease of use of this method and minimal requirements for the analyst.

\section{Results}

The results from the three IMA analyses are reported in units of parts per million (ppm) by weight for Ensys and Millipore and ppm by volume for Quantix in Table 4.3. In some cases values are given as ranges. A row with n.a. means that the sample was not analyzed or that a sample suitable for IMA analysis could not be obtained.

From Table 4.3 it can be seen that the results from the three different IMA test kits for each sample correlate very well in terms of indicating the presence or absence of detectable PAHs. In order to examine the spatial variation of the level of PAH contamination indicated by the IMA results, the results of Table 4.3 have been plotted at their respective MLs and contoured. Considering that the Ensys and Millipore data are reported in terms of concentration ranges, $10 \mathrm{~g}$ scale-type contours were used; the Quantix results were contoured directly. The contour plots are presented in Figures 4.25 to 4.27 for the shallow depths and in Figures 4.28 to 4.30 for the deeper measurements.

Several trends are evident from these figures. Careful inspection of the six figures shows that a single curve can be drawn that separates the ND results from those with some detected level of PAH and that these curves are nearly coincident for each of the two measurement depths. Moreover, the three shallow and three deep contour plots agree very well with each other on the location and shape of the PAH contamination distribution. The deep measurements tend to place the centroid of the plume further west than the shallow data; this result was not anticipated based on the BVWST measurements (e.g., see Figures 2.9 to 2.11). Indeed, the sampling network chosen for this data set was based on the BVWST data set and was expected to overlap both the eastern and western edges of the PAH plume. This was the first sign that contamination in the 10 to 15 foot depth range extended further west than expected.

The efficacy of the IMA test results may be evaluated by comparison with the HPLC analytical results contained in Table 4.3. Since the IMA technique is relatively insensitive to naphthalene, the total PAH concentration less the concentration of naphthalene is used for comparison. Of 
Ames Expedited Site Characterization - Marshalltown FMGP Site

FINAL Site Report

March 25, 1996

six possible agreements, Ensys had four agreements and two false positives; Millipore had three agreements, two false positives and one false negative; and Quantix had two agreements, three false negatives and one false positive. In assessing these results, it is important to note that:

- as IMA kits are biased to false positives, the relative percentage of false negatives is a better indicator of performance,

- duplicate rather than split samples were used, and,

- Ensys and Millipore are gravimetric, while Quantix is volumetric.

\subsubsection{Chemiluminescence}

\section{Description}

The CL technique, developed and manufactured by Tauw Milieu of The Netherlands, was discovered by Golder Associates of Albuquerque, New Mexico through a DOE EM-50 OTD contract to seek out foreign-based environmental technologies. Golder Associates arranged for Tauw Milieu to field and demonstrate their CL technique at the Marshalltown site. The technology is being commercially marketed in Europe. It was selected because of its simplicity and potential for quantitative analysis. Because we used duplicate and not split samples, however, the quantitative analysis capability could not be reliably evaluated.

After proprietary liquid phase extraction from a soil sample, a chemical is added to the extract and the luminescence is monitored by a simple solar cell-based detector that converts the emitted light signal into an electrical current. The enhanced luminescence is easily visible to the naked eye in the shade of a sunlit day. Calibration factors, using the results of HPLC analyses on representative site samples, are required to convert the measured intensity of the light to concentration. Results are reported in terms of total PAH concentration (not including naphthalene) in units of ppm.

\section{Results}

The results of the CL analysis are given in Table 4.4 and log contour plots of the CL results for the two depths are given in Figures 4.31 and 4.32. The shallow and deep plume configurations defined by the $\mathrm{CL}$ results generally correspond with those defined by the IMA results. Some western movement of the deeper plume, as indicated by the deep IMA results, is also evident in the $\mathrm{CL}$ results. The presence of a single plume as evidenced by the capability to draw one curve 
Ames Expedited Site Characterization - Marshalltown FMGP Site

FINAL Site Report

March 25, 1996

separating ND measurements from detects is also confirmed for the CL data and correlates well with the IMA results.

When comparing the CL measured concentrations with the HPLC results (less naphthalene), within a factor of two, three agreements, two false negatives and one false positive may be observed. The measured results also agree in a general sense with the IMA results.

\subsubsection{Passive Soil Gas}

\section{Description}

The passive soil gas measurements were made using the NERI Petrex system, which has been widely used for VOC detection but only rarely for SVOC detection. Unlike active gas measurements, this system has the advantage of being able to operate within the saturated zone. (Attempts to field a similar system from Gore Technologies were not successful because of procurement difficulties.)

Each Petrex soil gas sampler consists of two or three adsorption elements or collectors (ferromagnetic wire coated with activated charcoal) housed in a resealable glass container in an inert atmosphere. The Petrex system requires placement of an unsealed sampler into a covered hole for a period of 5 to 10 days. Volatile gases are adsorbed through vapor diffusion from the soil over the exposure period in the soil. After the samplers are retrieved from the holes, resealed and returned to the vendor, one collector from each sampler is analyzed by Thermal Desorption/Mass Spectrometry (TD-MS). Selected second collectors were analyzed by Thermal Desorption-Gas Chromatography/Mass Spectrometry (TD-GC/MS) for compound confirmation. GC/MS measurements are only attempted if the MS spectra are too complex in the direct mode and some degree of separation is required before MS analysis can be reliably made. Total time from insertion of the collectors to receipt of the analytical results was 20 days for the Ames Laboratory's samples. The results for naphthalene, anthracene/phenanthrene (A/P) and fluoranthene/pyrene (F/P) are reported in ion counts in Table 4.5. Anthracene/phenanthrene and fluoranthene/pyrene are grouped together because they have the same atomic masses and their spectral peaks are indistinguishable.

\section{Results}

Figures 4.33 and 4.34 display contour plots of the ion count results for the shallow and deep naphthalene measurements. Comparing shallow to deep data for naphthalene clearly shows the previously noted western trend of this contaminant plume for the deep measurements. The shallow and deep results for A/P are plotted in Figures 4.35 and 4.36, respectively, while the shallow and deep data for F/P are plotted in Figures 4.37 and 4.38, respectively. The plots for the 
Ames Expedited Site Characterization - Marshalltown FMGP Site

FINAL Site Report

March 25, 1996

deep A/P and F/P measurements continue to show hits in the western portion of the site which the shallow plots do not indicate. In addition, it may be noted that the relative size of the plumes shrinks from naphthalene to A/P to F/P. Little detectable F/P or A/P was found in the deep data set. In fact only ML-12 and ML-18 have detectable concentrations of $A / P$ in the shallow zone while ML-12, ML-18 and ML-25 have detectable concentrations in the deep zone. Clearly the higher number of naphthalene hits compared to the other measured PAHs is due to its relatively higher volatility, which is crucial for this type of adsorptive technique which relies on vapor transport through the soil; the higher the volatility, the larger the volume of soil which will have vapors accessible to the detector. The relative distribution and concentration of naphthalene and phenanthrene, derived from the quantitative information and discussed later, strongly suggests that the reduced counts for the phenanthrene relative to naphthalene is not due to reduced concentration of phenanthrene.

\subsubsection{Active Soil Gas}

\section{Description}

The active soil gas measurements were performed by Global Environmental, Inc., of South Dakota' using their Geoprobe system to extract soil gas from the shallow and deep zones as defined previously. The active soil gas technique basically requires unsaturated soils; if saturated soil was encountered and soil vapor could not be collected, the space in the Geoprobe core sleeve was sampled before capping. The concentrations of BTEX and naphthalene gases were measured directly in the field with both a gas chromatography/flame ionization detector (GC/FID) and gas chromatography/photoionization detector (GC/PID) instrument. (Although the contract called for measurements of naphthalene as the most volatile PAH, the vendor also provided quantitative results for BTEX gases as well.) The HP5890-Series II gas chromatography (GC) instrument was calibrated on site using reference gas standards. The results for both the GC/FID and GC/PID measurements for both the shallow and deep zones are given in Table 4.6; the BTEX results, although included in Table 4.6, are not discussed in this report. The results are reported in units of $\mu \mathrm{g} / \mathrm{L}$ of vapor.

\section{Results}

The data in Table 4.6 shows that the PID was more sensitive to naphthalene than the FID. In cases where naphthalene was detected by the FID the agreement with the corresponding PID measured concentration was very good. Log contour plots of the shallow and deep FID naphthalene data are shown in Figures 4.39 and 4.40. A single plume is evident at the lateral resolution of the grid at both depths. Some data are not available due to difficulties with inserting the Geoprobe rod to the proper sampling depth. The centroid of the plume at the shallow depth is to the southeast of the centroid of the deeper plume and, as seen with the some 
Ames Expedited Site Characterization - Marshalltown FMGP Site

FINAL Site Report

March 25, 1996

of the other screening technologies, the deeper plume extends further to the west than the shallow plume. The active soil gas plumes generally agree with the plumes deduced from the IMA and the CL results. The naphthalene passive gas contour plots (see Figures 4.33 and 4.34) tend to indicate larger plumes than the active soil gas results in both the shallow and deep zones. Note in particular that significant hits were detected at ML-11, ML-12, ML-13 and ML-18 in the passive gas measurements while the active gas measurements had non detects at these locations. The passive gas measurements thus seem to be more sensitive to detecting naphthalene than the active gas measurements.

This use of GCs to search for SVOC plumes is not typically attempted because of the lowered volatility of the SVOCs compared to the VOCs. Clearly, where naphthalene is suspected, active soil gas measurements with unmodified VOC GC columns can be used with some expectation of success.

\subsubsection{Summary - Phase I Contaminant Screening Technologies}

The results of the Phase I contaminant screening technologies generally compare well with the BVWST results for stratigraphic zones 1,2 and 3 presented in Chapter 2. The plumes are generally centered around the former purifier building (see Figure 2.2) and extend down toward the storage shed at the southern end of the site. Analytical results for BVWST boreholes B-02, B-04, B-05 and B-06 generally indicated the highest levels of PAH contamination. All of the Phase I screening results tend to yield plumes centered near the former purifier building. Analytical results for boreholes B-08, B-13 and B-14 indicated that the top two stratigraphic zones in the southcentral part of the site also contained PAH contaminants. The Ensys and Millipore IMA results and the passive gas results for the deep screening zone for Ml-12 and ML18 have indicated significant PAH contamination in this region; the other screening techniques have partially detected it (CL) or completely missed it (active gas). A significant finding of the Phase I contaminant screening study was that PAH contamination existed further to the west than it would appear from the BVWST data. In particular, the BVWST analytical data for B-15 from zone 2, corresponding to the deep screening tests, indicate no PAH contamination (the analytical results for samples from this depth range gave non detects). The deep zone IMA results and passive gas results, in particular, exhibit significant levels of PAH contamination at ML-24 and ML-25, indicating that the plume could in fact extend farther to the west.

\subsection{Phase II Minimally Intrusive Geological Characterization and Contaminant Screening}

Two minimally intrusive, direct push characterization technologies were fielded during the Phase II investigations at Marshalltown: an innovative cone penetrometer testing (CPT) system 
Ames Expedited Site Characterization - Marshalltown FMGP Site

FINAL Site Report

March 25, 1996

(the Site Characterization and Analysis Penetrometer System (SCAPS)) and an innovative Geoprobe direct sensing soil conductivity sensor. The SCAPS was contracted through the Kansas City District, Corps of Engineers while the soil conductivity sensor unit was contracted through Geoprobe Systems of Salina, Kansas. The objectives of this phase of the investigation were to confirm and further refine the site geologic and contamination conceptual models as defined through the Phase I activities (following the ESC approach) and to compare and evaluate the CPT and Geoprobe systems. A key objective for the direct push technologies was to further define the topography of the LCU. While an integral part of the stratigraphy, this is especially important considering the transport characteristics of the denser-than-water non-aqueous phase liquid (DNAPL) coal tar residue and potential remediation schemes, such as free product pumping. These technologies, however, are limited in providing direct data to the define the surface of the bedrock.

Besides providing continuous stratigraphic profiles, the SCAPS was equipped with a LIF sensor system which could detect petroleum, oil and lubricant contaminants in the subsurface. A continuous log of LIF response with depth could be provided at a push location. The Geoprobe conductivity sensor provided a continuous $\log$ of conductivity with depth at a push location. As indicated in Section 4.1.5, the resistivity of a soil increases (conductivity decrease) with increasing hydrocarbon contamination; thus, in addition to providing stratigraphic information, the Geoprobe conductivity logs could also be used as a contaminant screening tool.

Another valuable source of data for comparison with the CPT and Geoprobe conductivity data and for further development of the site geologic and contamination models was the visual description logs associated with each of the soil samples collected for quantitative chemical analysis (which is described in Section 4.4). The soil samples for this effort were collected by McLaren/Hart Environmental Engineering Corporation personnel operating a Geoprobe Model 8-MU sampling system. Core samples two feet long by one inch in diameter were collected from the six subsurface zones at specified depths. A detailed descriptive log of each of the 127 samples collected was kept and data on core recovery, date, time, sample number, depth, soil description, water content and visual/odor evidence of contamination were recorded.

The following sections describe the SCAPS CPT and the Geoprobe systems and geological characterization results using these technologies. This is followed by contaminant screening results using these technologies. Comparisons with other technologies and results were made and the site geologic and contamination models were updated and revised in the light of these results. 
Ames Expedited Site Characterization - Marshalltown FMGP Site

FINAL Site Report

March 25, 1996

\subsubsection{Cone Penetration Testing}

\section{Description}

The SCAPS CPT system was developed by the U.S. Army Engineer Waterways Experiment Station (WES) of Vicksburg, Mississippi under the sponsorship of the U.S. Army Environmental Center. The objective of SCAPS is to provide a cost-effective and rapid method to determine in situ soil conditions and detect and delineate petroleum, oil, and lubricant (POL) contaminants for site screening and characterization. The SCAPS is comprised of a twenty ton truck equipped with hydraulic rams used to push instrumented sensor probes into the ground. Data acquisition, processing, and storage are handled via electronic signal processing and an onboard computer system. The SCAPS utilizes tip and sleeve resistance measurements for continuous soil stratigraphic profiling while simultaneously using either resistivity or LIF to obtain further subsurface information such as pore fluid characteristics and the presence of POL contaminants. The SCAPS graphic output, or "panel plot," displays the soil classification data, and either fluorescence intensity and peak wavelength or resistivity data plotted against penetration depth (in feet) below ground surface. An example panel plot is shown in Figure 4.41. The panel plot information may be viewed in real time on an onboard computer screen; this real-time information is especially useful in determining when to terminate the push. In addition, soil and groundwater samples may be obtained from selected depths. A Hogentogler sampler was used at this site to collect one inch diameter by eight inch long soil samples. The SCAPS has the ability to grout through the cone tip on retraction of the push rods.

Soil Profiling Capabilities. Strain gages in the cone tip are used to measure the resistance against the cone tip (end bearing stress) and sleeve friction as the cone is advanced into the ground. Both measurements are recorded in tons per square foot. Empirical relationships have been developed to classify the soil type based on the cone tip and sleeve friction measurements (e.g., Olsen, 1988; Olsen and Farr, 1986; and Chiang, Loos and Klopp, 1992). This technology has been applied in geotechnical engineering site investigations for several decades.

Panel plot outputs from the CPT instrumentation show the tip and friction sleeve resistance as well as the soil classification based on the friction ratio versus depth (see Figure 4.41). Environmental factors such as grain size and gradation, angularity of particles, overconsolidation ratio, soil density, and coefficient of lateral pressure influence the tip and friction sleeve resistance (Douglas and Olsen, 1981). CPT soil classification may be ambiguous in mixed soils. Therefore, a continuously logged soil borehole from the site should be used to calibrate and verify the CPT soil classification log.

Laser Induced Eluorescence (LIF) Sensing. The SCAPS LIF system sends $337 \mathrm{~nm}$ excitation pulses from a nitrogen laser down a fiber optic cable in the cone rods through a sidewall 
Ames Expedited Site Characterization - Marshalltown FMGP Site

FINAL Site Report

March 25, 1996

mounted sapphire window to induce fluorescence of the POL fluorophores. The sapphire window is about two feet above the cone tip and provides a rugged and relatively optically clear . interface between the soil and the ends of the fiber optic cable. The induced fluorescence is collected on a separate fiber optic cable and returned to the surface where it is spectrally analyzed using a linear photodiode array in a continuous window mode.

The fluorescence spectrum consists of photon counts measured at a rate of 1024 points over the wavelength range from 300 to $800 \mathrm{~nm}$ for every $2 \mathrm{~cm}$ depth interval. The panel plots showing the fluorescence results are produced by plotting the maximum fluorescence intensity over the 350 to $600 \mathrm{~nm}$ range, and the corresponding wavelength at which the maximum intensity occurred (see Figure 4.41). Since different fluorescing compounds have different characteristic fluorescent spectra, a change in wavelength associated with the maximum intensity is indicative of a different compound or set of compounds giving rise to the fluorescence.

Laser excitation of many (three or more) ring PAH compounds will cause fluorescence, however, excitation wavelengths lower than $337 \mathrm{~nm}$ are required to cause fluorescence of lighter aromatic hydrocarbons such as BTEX compounds. Interferences which can affect LIF response include fluorescing minerals, fluorescent dyes used as tracers or in antifreeze fluids, optical brighteners used in laundry detergents and septic system effluents, and sunlight penetrating the optical window in the top few inches of soil. Accordingly, a high intensity fluorescence does not always signify that a contaminant is present; however, experience with LIF technology has shown that the technique is generally reliable as an indicator of PAH contamination (Stenback et al. 1994).

The efficiency of the laser signal depends on several factors including abrasion of the sapphire window and pitting and misalignment of the optic fiber. To recognize and correct for a signal degradation, the energy transmission of the LIF sensor is evaluated before and after each LIF penetrometer push. Prior to each LIF push, the LIF probe is suspended from the chuck and a cuvette of rhodamine solution is placed against the sapphire window. The laser is fired on the cuvette. The process is repeated after each push. Any degradation of the LIF response to the rhodamine solution is assumed to be linear and a linear correction is applied to the fluorescence data obtained during that particular push.

LIF intensity data is commonly presented as the response or counts above background, where the background LIF response is obtained from an uncontaminated, representative sample of soil from the site. The soil sample is pressed against the sapphire window and the laser pulsed to obtain ten emission spectra. This background distribution would then be subtracted from subsequent LIF measurements to obtain the LIF response above background. 
Ames Expedited Site Characterization - Marshalltown FMGP Site

FINAL Site Report

March 25, 1996

\section{Results}

The CPT push locations are shown on Figure 4.42. A total of 43 stratigraphic logs were acquired at 34 push locations. The panel plots for all of the pushes are in Appendix B. To avoid opening a conduit for potential contaminant migration into lower stratigraphic units, penetration tests were ended, in most cases, as soon as fluorescence data were collected down to the top of the LCU. Because of problems with probe breakage and apparent coarse gravel and cobbles in the 16 to 20 foot depth range early in the program, an uninstrumented "dummy probe" was used to pre-push holes at many of the locations. In fact, an LIF and a resistivity probe were broken at ML-33 and another resistivity probe was broken at ML-115. Due to the dummy rod pushes, however, many of the CPT logs have no soil stratigraphic data in the upper 20 feet. Of the several resistivity pushes which were accomplished at this site, review of the data indicates significant fluctuation of resistivity values from one location to another, in much the same material. Hardware difficulties are suspected; the data were not used.

Soil Stratigraphy. To calibrate the stratigraphic information generated by the CPT, two pushes were made in close proximity to wells MW-3 and MW-4 installed and logged by BVWST. Side-by-side comparisons are made in Figures 4.43 and 4.44. A high degree of correlation can be seen between the adjacent logs. The CPT push location ML-28 was about 19 feet northeast of MW-3; the surface elevations are within several inches of each other. There is less than a one foot difference in the depth to the upper cohesive unit/granular unit contact when the logs are compared, and there appears to be a gradational contact between the base of the granular unit and the LCU demonstrated in both logs. However, the LCU is about three feet deeper at MW-3 than at the CPT push location.

The CPT push at ML-32 was located about 9 feet north of MW-4. The depth to the upper cohesive unit/granular unit contact is within one foot. In both the CPT log and the BVWST borehole $\log$, the UCU grades over about a 2-foot depth from clay to a sand-gravel mix (top of the sand-gravel at 19.5 to 20 feet). In both logs, the bottom portion of the granular unit or top portion of the LCU contain interbedded sandy and silty clay strata, although the CPT logs seem to portray slightly thinner layers; a gradational contact between the granular unit and the LCU is again apparent. It should be noted that the CPT stratigraphic logs yield significantly more detail on fine stratification than the hollow stem auger boreholes. This can be extremely important when considering the fate and transport of DNAPLs, which would be influenced by finer details of the stratigraphic layering. The CPT is, however, susceptible to unfavorable subsurface conditions such as rubbly fill, coarse gravels and cobbles.

The repeatability of the CPT stratigraphic logging capability was evaluated by comparing CPT logs ML032-F and ML032-R (LIF and resistivity pushes, respectively), which were pushed near well MW-4 and.separated by about 5 feet (see Figure 4.45). The most significant differences 
Ames Expedited Site Characterization - Marshalltown FMGP Site

FINAL Site Report

March 25, 1996

visually between the CPT logs occurs in the top 10 feet, presumably in fill material. Otherwise the logs show virtually the same stratigraphic profile. A clear conclusion of the comparison between the borehole logs and CPT data is the increased level of stratigraphic resolution which can be obtained with the CPT.

Contacts between the stratigraphic units at the site were interpreted from the CPT panel plots and used to develop the site geological model. This is discussed in more detail in Section 4.3.3.

Contaminant Screening. In order to assess the efficacy of the LIF sensor response, nineteen soil samples were collected by the SCAPS truck at locations where LIF pushes indicated the presence of significant subsurface contamination. The soil samples were collected within several feet laterally of LIF hits of interest. The samples were analyzed by WES personnel for TRPH, (mg/kg) by EPA Method 418.1 using a field-portable equipment. The samples were homogenized and a portion of each sample was held against the LIF probe sapphire window while a fluorescence spectra was obtained for comparison with the TRPH analyses. These data are listed in Table 4.7. The first number in the Sample ID represents the map location. LIF intensity data from the adjacent CPT pushes were available for direct comparison with the TRPH analytical results. These data are listed in Table 4.8. Generally, the data from Tables 4.7 and 4.8 indicate that fluorescence intensities greater than about 200 counts are associated with moderate to high TRPH levels (high defined as $>500 \mathrm{mg} / \mathrm{kg}$ and low defined as $<100 \mathrm{mg} / \mathrm{kg}$ ), fluorescence intensities less than 100 counts are associated with low TRPH levels, and fluorescence intensities between 100 and 200 counts are associated with TRPH levels ranging from low to high. However, four samples with TRPH reported as $<2 \mathrm{mg} / \mathrm{kg}$ show fluorescence intensities near 150 counts (see Table 4.7) and sample 81-1 shows high TRPH contamination with a fluorescence intensity (collected following a dummy probe pre-push) less than 100 counts (see Table 4.8). These exceptions aside, the LIF intensity shows qualitatively where contaminated soil exists.

Also noted in Table 4.8 are the locations where LIF readings were taken in dummy probe prepush areas. Both large diameter $(1.75$ inch) and small diameter $(1.44$ inch) dummy probe rods were used in pre-pushing. The diameter of the LIF probe is 1.44 inches. Three factors could potentially affect LIF readings taken in a pre-push hole: the sapphire window may not be in proper contact with the soil; the hole may cave slightly on removal of the pre-push rods; or, contaminants from lower zones may be smeared on the pre-push hole wall as the rods are removed. Table 4.8, however, indicates reasonable correspondence between the LIF readings and TRPH results from adjacent soil samples, even in push ML-60 where the larger pre-push rods were used.

The panel plots for all of the LIF pushes carried out were examined and average LIF intensities were determined for each of the six stratigraphic zones. These data are tabulated in Table 4.9. 


\section{Ames Expedited Site Characterization - Marshalltown FMGP Site}

FINAL Site Report

March 25, 1996

Details on pre-pushes are also included in Table 4.9. Zones 1,2 and 3 would be most affected by the pre-pushes. In this regard, while data for the top three zones are presented, and the adjacent samples lend support to the efficacy of the data in the pre-pushed region of the hole, LIF data in the top three zones should be viewed as having a higher uncertainty than the data in the lower zones.

Logarithmic scale contour plots of the average LIF intensity for the six stratigraphic zones are shown in Figures 4.46 through 4.51. In the upper four zones the most significant feature is the heavily contaminated zone in the northwest portion of the site, just to the east of the substation. The general trend of the contaminated region is still, however, from the northwest to the southeast through the central part of the site. Relatively high LIF readings were also noted in the region of ML-97 in the upper four zones. The main plume for zone 5 (base of the granular unit) extends from just west of the former purifier building to the southern portion of the site; elevated LIF readings near the southern site boundary indicate that contamination has most likely moved off the site in this zone. Significant hits were only detected in zone 6 (top of the LCU) at ML76 and ML-95 (see Figure 4.51).

Examination of the LIF panel plots revealed that elevated fluorescence intensity levels commonly occur in the two to four feet of sandy soil immediately overlying the top of the lower cohesive unit indicating a pooling of DNAPL on the lower cohesive unit. When LIF measurements were taken in the top of the lower cohesive unit, the fluorescence intensity reading typically dropped to near zero (for example, see Figure 4.41). This indicates that the lower cohesive unit is tending to retard the downward migration of the DNAPL contamination at these locations.

Many of the panel plots show elevated fluorescence intensity values within the upper cohesive unit and within the upper and lower portions of the granular unit (see Appendix B for plots). Elevated fluorescence intensities are occasionally found at several locations in sandy soils overlying fine grained soils suggesting the presence of lenses acting as localized barriers to vertical migration of contaminants causing contamination to pool on top of the lens (e.g., see Figure 4.41). Elevated fluorescence intensities also occur in the granular unit with no apparent stratigraphic barrier to limit contaminant transport. These occurrences appear to be associated with light non aqueous phase liquids which tend to float on the groundwater table at about 20 to 22 feet below ground surface (see Figure 4.41). An interpretation suggesting varying compounds with differing transport behavior is consistent with the distinct fluorescence spectra observed from the samples analyzed by WES as mentioned above (Stenback and Kjartanson, 1994 and 1995).

Although the BVWST borehole B-1 indicates some fairly significant contamination in zone 3 (see Figure 2.7) in the northwest part of the site, the CPT LIF data suggest that this area of the 
Ames Expedited Site Characterization - Marshalltown FMGP Site

FINAL Site Report

March 25, 1996

site is more heavily contaminated than is indicated by the BVWST data alone. Moreover, the CPT LIF results in this region tend to confirm the hits in the deep zone by the IMA (see Figures 4.28 to 4.30) and the passive gas naphthalene tests (see Figure 4.34) - the deep zone for these tests approximately corresponds to zone 2 . The CPT LIF responses in the southem part of the site agree both with the BVWST data and the IMA and passive soil gas screening data for the deep zone. In addition, the lobe centered at ML-97 in the LIF data is captured very nicely by the deep IMA screening data, which has this lobe centered on ML-9 some 4 feet to the south of ML97. No evidence of this lobe appears in the shallow IMA data. The passive soil gas data for the shallow and deep zones, especially for naphthalene, shows this feature as well. Only shallow GC/PID naphthalene shows this feature while no evidence for it appears in any of the CL data. The relatively low but persistent LIF hits at ML-33 on the eastern side of the site are not confirmed by any other measurements of the PAH concentration in this region.

\section{Productivity/Output}

A total of 43 stratigraphic logs at 34 locations were generated by the CPT system over the course of 10 working days. The system averaged approximately 208 pushed feet per day. Encounters early on with coarse gravel and cobbles that resulted in broken probes, led to a decision to push an uninstrumented "dummy" probe to a depth of 15 to 20 feet at many of the locations before advancing the instrumented probes. For this reason, stratigraphic information was not generated in the upper 15 to 20 feet of many probe holes. Moreover, this reduced the stratigraphic profiling productivity to an average of about 90 feet per day.

Several factors limited the use of the SCAPS in some parts of the site. The unit is designed for use on level to gently sloping terrain with clayey to sandy soils with small gravel sizes. The air-space above the truck must be free of obstructions such as power lines, and the large size of the truck requires ample open space to maneuver. The CPT probes will not penetrate boulders, cobbles, rubble, highly compacted soils, or sound bedrock.

Analog printouts of each log were produced within an hour of completion of the hole. Digital data (as text files) were not available until near the end of the field work because of formatting problems.

The CPT instrumentation was unique in its efficient production of reproducible stratigraphic information which was of high vertical resolution and accuracy, coupled with minimum ground disturbance. A clear disadvantage was its high susceptibility to unfavorable subsurface conditions (coarse gravels, buried debris, etc.) and to above ground features (tight work spaces, surface pavements, etc.). 
Ames Expedited Site Characterization -Marshalltown FMGP Site

FINAL Site Report

March 25, 1996

\subsubsection{Soil Conductivity Probe}

\section{Description}

A percussion soil probe system designed for direct sensing of soil conductivity was developed recently by Geoprobe Systems, Inc., (Christy et al., 1994). The system was field tested at Marshalltown as a comparable technology to the stratigraphic logging capabilities of the CPT system.

The probe is approximately 1-1/8 inches in diameter and about 8 inches long with four electrically isolated contact rings. The inner rings are 1 inch apart while the outer rings are separated by a little over 2 inches. The probe is advanced into the ground using a hydraulically powered percussion probing machine. Percussion is applied to the top of the probe rods at a rate of about $30 \mathrm{~Hz}$ and may result in instantaneous forces greater than 12,000 pounds transmitted through the probe rods (Christy et al., 1994). A signal cable attached to the probe is run through the inside of the rods to a laptop computer-based data recording system. Depth measurements are obtained from a stringpot system configured to measure the distance from the driving mechanism to the ground surface. Data derived from this system are used to determine both the depth of the probe and the probing speed. Conductivity measurements are displayed with respect to depth in near-real-time on the computer screen. Data were recorded in spreadsheet format and downloaded at the end of each field day.

Soil bulk electrical conductivity (termed simply 'conductivity') varies with soil type, water content, pore fluid chemistry and type and level of contamination. High conductivities are associated with clayey soils, low conductivities with sandy and gravelly soils, and sand/silt/clay mixtures have conductivities that lie somewhere in between. Because the conductivity varies somewhat for a given soil type between sample locations, comparing the conductivity log with a soil boring $\log$ or CPT soil classification $\log$ is essential to properly interpret the conductivity log. The true value in this technique is being able to extend stratigraphic information laterally from a known vertical profile or to fill in areas between known vertical profiles. Changes in conductivity are caused by changes in soil type, water content, and soil or groundwater contamination. Accordingly, when used alone, the conductivity log leaves some doubt regarding an accurate soil classification. It is quite effective, however, at delineating changes in the subsurface properties.

Research by Geoprobe Systems, Inc., has shown the probe to produce reproducible logs and to provide a high degree of vertical resolution for the discrimination of soil/sediment units (Christy, et al., 1994). The probe electrodes can be operated in either a Schlumberger array (using all four electrodes) or dipole array (using only the upper pair of electrodes). The dipole array has the potential of providing a higher degree of vertical resolution due to the closer electrode spacing. 
Ames Expedited Site Characterization - Marshalltown FMGP Site

FINAL Site Report

March 25, 1996

Results

A total of 27 conductivity profiles were collected. Their locations are shown on Figure 4.52. The conductivity versus depth logs may be found in Appendix C. Each push was terminated on a confident identification of having encountered the LCU (typically indicated by a rapid rise in soil conductivity) or, in locations where the LCU was absent, on probe refusal.

Soil Stratigraphic Logging Capabilities. As with the CPT technique, the first soil conductivity push was made adjacent to BVWST MW-3; this location is also called ML-28. The ML-28 locations were within 19 feet of MW-3; surface obstructions disallowed a closer approach. Figure 4.53 shows the three profiles arranged with correlating vertical scales: soil conductivity on the left, the BVWST borehole log in the middle, and the CPT stratigraphic log on the right. The conductivity log is plotted in reverse (i.e., increasing conductivity toward the left) so that an inflection to one direction or the other in conductivity is analogous to a similar inflection on the CPT stratigraphic $\log$ (e.g., an inflection to the left in either log would typically be indicative of a higher content of fine grained soils). With the exception of approximately the top six feet, where the conductivity data is erratic, the correlation with the borehole log for MW-3 is good. The top three to five feet of most conductivity logs demonstrated this same erratic conductivity behavior. Possible explanations for this behavior include nonuniform infiltration of highly saline solutions from winter road salting operations, poor ground-to-probe contact at shallow depths and/or the erratic nature of the surficial fill.

Although, the soil conductivity log does not give a soil type interpretation directly, the UCU/granular contact could be inferred by the distinct drop in soil conductivity between about 15 and 17 feet. It should be noted, however, that drops or dips in conductivity may also be associated with coal tar residue contamination (discussed in more detail in the following section) or gravelly layers.

Figure 4.53 shows that the depth to the gradational UCU-granular contact is within one foot on all three of the logs. In terms of identifying the granular unit/LCU contact, however, both the CPT and conductivity log indicate that the contact is about two to three feet higher in the stratigraphic profile than the BVWST log indicates. The granular unit/LCU contact is inferred by a distinct rise in the conductivity values. Both of these contacts could be easily identified in most of the soil conductivity logs. The largest variation among the logs is the significant amount of silt and clay between about 21 and 25 feet indicated by the CPT log. Neither the conductivity $\log$ nor the BVWST log gave an indication of this. The soil conductivity logs can give the same level of stratigraphic detail as the CPT logs when properly calibrated and verified. 


\section{Ames Expedited Site Characterization - Marshalltown FMGP Site \\ FINAL Site Report}

March 25, 1996

Figure 4.54 shows another comparison of CPT and soil conductivity pushes close to a BVWST borehole. The CPT push at ML-60 and the soil conductivity push at ML-45 are within 13 feet of B-8. The three logs gave good stratigraphic correlation. The soil conductivity log gives the UCU/granular unit contact within one to two feet of the depth indicated on the BVWST log. Similarly, the granular unit/LCU contact is at a depth within one to two feet on all three logs. .The CPT log tends to show it about two feet higher than the BVWST log.

Conductivity levels change somewhat from one location to the next; some trends are apparent, however. The clayey soils at the site tend to have conductivities in the range of 60 to $140 \mathrm{mS} / \mathrm{m}$ (resistivity from 7 to $17 \mathrm{ohm}-\mathrm{m}$ ) and the sands about 30 to $40 \mathrm{mS} / \mathrm{m}$ (25 to $33 \mathrm{ohm}-\mathrm{m})$, with gravels generally showing a conductivity dip of about 5 to 10 units below the level for a sandy soil. These values are similar to those reported in Christy et al., 1994 for an alluvial site in central Kansas containing interbedded sands, silts and clays. When the soil conductivity log ML-28 is compared with the borehole induction log for MW-3 (Figure 4.17), it may be noted that the borehole induction log is flatter both in terms of the ranges in magnitude of the values (i.e., the soil conductivity log shows higher clay conductivities and lower sand conductivities) and definition of stratigraphic contacts (i.e., the borehole induction log shows a much smoother or "rounder" transition from one unit to another). The soil conductivity probe is in direct contact with the soil and has a rather small measuring volume due to the relatively close spacing of the electrodes; the induction borehole logging, on the other hand, needs to extend farther out into the soil to avoid measuring simply the conductivity of the monitoring well materials. This may have resulted in a loss of resolution.

Contaminant Screening Capabilities. During the implementation of the Geoprobe soil conductivity probe system for the purpose of defining stratigraphic variations, a marked downward inflection or dip in conductivity was recorded in several logs at the bottom of the granular unit. This was underlain directly by the LCU, as interpreted from the logs. It was surmised that the dip in the conductivity log may be the result of the presence of free phase coal tar residue containing PAHs in that zone, rather than a natural condition arising out of stratigraphic changes. As noted previously, the bulk soil conductivity is significantly influenced by the pore fluid conductivity; soils contaminated with PAH will have a lower conductivity and will show a dip in their conductivity log. An example of this is illustrated in Figure 4.55. This is the same group of BVWST boring, soil conductivity and CPT logs presented in Figure 4.54, except that the CPT LIF log is given in this figure rather than the stratigraphic log. A subtle dip in the conductivity log from about 33 to 35 feet corresponds to a strong LIF hit from about 32 to 34 feet. Moreover, dips in the conductivity $\log$ at about 22 to 23 feet tend to correspond to an LIF hit at about 20 to 22 feet. Both the soil conductivity and LIF hits are confirmed by the visual descriptions in the BVWST log (see Figure 4.55). A comparison between a soil conductivity push (ML-54) and a nearby CPT push (ML-109) is shown in Figure 4.56. Conductivity dips corresponding to LIF hits may be noted from 5 to 7 feet, 20 to 21 feet and 34 


\section{Ames Expedited Site Characterization - Marshalltown FMGP Site}

FINAL Site Report

March 25, 1996

to 36 feet. Following these observations, an analysis was conducted to evaluate potential correlations of soil conductivity dips with the presence of coal tar residue.

By comparing the Geoprobe conductivity logs with the CPT logs (soil classification and LIF) and/or the physical soil sample descriptions from the daily logbook, we gained some understanding regarding the extent to which such dips in the conductivity correlate with PAH contamination and/or the presence of gravel (or rock fragments as they are often referred to in the field logbook) which will also cause a lower conductivity. The conductivity logs contain many such dips, some obvious, others more subtle, as well as portions of conductivity trace that are quite erratic showing irregular peaks and troughs. Most of these dips do not have either a CPT log or physical soil sample to compare with to assess the cause of the conductivity change; however, a few do and these are listed in Table 4.10. Table 4.10 shows the location identification where a particular conductivity sounding was performed, the depths at which dips in the trace occurred which can be correlated with the CPT or McLaren/Hart (MLH) data, the suspected cause of the conductivity dip based on the CPT or sample description logs, the source of information (CPT or MLH and location), and the approximate horizontal distance between the conductivity push and the information source. In some cases the information source and conductivity were taken at the same location and show identical $(X, Y)$ coordinates, however, such cases were actually separated by one or two feet and are listed as being two feet apart in Table 4.10.

Based on the data presented in Table 4.10, we find that dips in the conductivity profile occur adjacent to uncontaminated gravelly soil about 25 percent of the time, and adjacent to contaminated soil free of gravel about 60 percent of the time. The remainder of the dips for which a correlation was possible occurred near gravelly soil which showed evidence of $\mathrm{PAH}$ contamination; this is not surprising since the PAH contaminants tend to migrate through the coarse grained soil matrix (as well as within tension cracks, root holes and fractures within clayey soils). It seems clear that, although the bulk of conductivity dips correspond to PAH contaminated soil, occasionally we see that dips in the conductivity trace are due to the presence of gravel (or cobbles) with little or no PAH contamination.

\section{Broductivity/Output}

The conductivity logging system produced 1022 feet of $\log$ in 32 holes over a period of six working days. The system was operated by a two-person crew. Operation by a one-person crew is possible, although productivity would be significantly lower. The data required minimal postprocessing (deletion of negative or repeat values). Digital conductivity and probing speed data and field printouts were provided at the end of each work day for integration into the existing site model. 
Ames Expedited Site Characterization - Marshalltown FMGP Site

FINAL Site Report

March 25, 1996

Occasional problems encountered during the completion of this work included electrode failure, probe point mechanical failure, intermittent negative values in conductivity data and erratic or extremely high conductivity values in the upper few feet of most profiles. Another short-coming of the system was its inability to easily identify weathered carbonate bedrock, which was encountered in some locations where the LCU was absent. Weathered bedrock displayed erratic conductivity values which were not easily distinguished from inhomogeneity in the overlying units. The probing speed output might be useful in this regard. In any case, positive identification of the material could only be made subsequently with core samples.

The advantage of this system is its ability to provide a large number of profiles in a relatively short time. Moreover, if required, it can be operated by a single person. It is also rather versatile in that it can maneuver into small spaces and can penetrate most soil subsurface materials.

\subsubsection{Interpretation from Minimally Intrusive Methods}

In this section the data collected by the minimally intrusive methods are compared at a number of locations where CPT, soil conductivity and soil sampling pushes and BVWST boreholes were advanced within several feet of one another, these data collections are referred to as "data clusters." The data are compared both in terms of stratigraphy and level of contamination. In addition, the minimally intrusive data are compiled and analyzed on a site wide basis and assessed in terms of their contribution to the development and refinement of the site geologic and contamination models. Specific comparisons are made with both geophysical survey and BVWST data. Data resolution, sensitivity and reliability issues are addressed. Moreover, the evolution of the site geologic model, on a day-by-day basis, according to the ESC approach, is presented. The usefulness of 3-D visualization and geostatistical techniques, such as kriging, is also assessed.

\section{Stratigraphic Interpretation}

Twelve clusters of CPT stratigraphic, MLH soil core sample, Geoprobe soil conductivity and BVWST borehole logs were identified; the locations of these data clusters are shown in Figure 4.57. The selected clusters and stratigraphic details are listed in Table 4.11.

The SCAPS CPT panel plots give a soil classification according to clay, silt mixtures, sand mixtures, sands, and sands and gravels. The BVWST boreholes provide a stratigraphic log. The MLH soil classification is a visual description recorded in the daily logbook as each soil sample was collected. The Geoprobe soil conductivity log (Geoprobe in the table) provides a more subjective soil classification interpretation; when calibrated against known soil types, however, the main soil unit contacts and stratigraphy become relatively easy to interpret from the logs. As shown in Table 4.11, the soil classification schemes agree quite well, with only a few exceptions, 
Ames Expedited Site Characterization - Marshalltown FMGP Site

FINAL Site Report

March 25, 1996

and these can generally be explained by local soil heterogeneities which may exist both vertically and horizontally over a distance of several feet at this site.

Depths to contacts between the upper cohesive unit and the granular unit, the granular unit and the LCU and the overlying soil (either LCU or granular unit) and bedrock were interpreted from the CPT stratigraphic and soil conductivity logs and input into a database for EarthVision. This database is included as Table 4.12. The stratigraphic correlations between the push technologies and the BVWST borehole log data in the two previous sections demonstrated that the contacts between soil units can generally be interpreted from the CPT and soil conductivity logs with confidence; interpretation of the bedrock surface from the CPT and soil conductivity logs is somewhat more subjective. In most cases the advance of the CPT or soil conductivity probe was terminated after having penetrated a few feet of the LCU; the surface of the bedrock in the threedimensional EarthVision model may be considered only approximate, therefore. In the eastern part of the site, however, both the CPT and the soil conductivity probes are believed to have come to refusal in bedrock at a number of MLs (e.g., broken resistivity and LIF CPT probes at ML-33 at depths of 16 feet and 26.5 feet, respectively). Moreover, a continuously sampled Geoprobe push was carried out directly adjacent to the soil conductivity push at ML-34 (see Figure 4.52) to provide quantitative stratigraphic data to calibrate the soil conductivity sensor in the eastern portion of the site where the bedrock is relatively shallow. The surface of the weathered bedrock is not readily apparent even in this calibrated soil conductivity push, however. Defining the surface of the weathered bedrock with the push technologies, therefore, may be considered quite uncertain.

Two stratigraphic cross sections were prepared using the push technology stratigraphic data alone. The locations of the cross sections are shown on Figure 4.58. Section AA (Figure 4.59) was prepared using soil conductivity data as the primary stratigraphic information; the soil conductivity logs are shown superimposed on the figure. As noted previously, the contacts between the soil stratigraphic units are generally well defined, as illustrated on the western side of the cross section between ML-45 and ML-59. Stratigraphic contacts from BVWST borehole and CPT push logs close to the cross section were added at the appropriate locations along the cross section; it may be noted that the UCU/granular unit contact given by the three technologies' logs corresponds to within about two feet while the granular unit/LCU contact corresponds to within about one foot. This may be considered excellent stratigraphic correspondence between the technologies. The surface of the bedrock rises sharply in the region of ML-37 to ML-36; sections of weathered bedrock several feet thick overlying unweathered bedrock have been interpreted from the soil conductivity logs in the eastern part of the section. It may be noted that the contacts between stratigraphic units are not nearly so clear in the eastern part of the cross section as in the western part. 
Ames Expedited Site Characterization - Marshalltown FMGP Site

FINAL Site Report

March 25, 1996

The second cross section $\mathrm{BB}^{\circ}$ runs from the northwestern part of the site to the extreme eastern part of the site (see Figure 4.58). This section, shown in Figure 4.60, was drawn using both soil conductivity and CPT stratigraphic data. This cross section illustrates that significantly more stratigraphic detail may be interpreted from the push technology logs than from borehole logs. This cross section also shows the same general pattern of a relatively sharply rising bedrock surface in the eastern part of the site. Comparisons with BVWST boreholes B-4 and B-6 are good (see Figure 4.60). BVWST borehole B-5 shows the LCU surface about three feet lower than in the cross section. This could be indicative of a localized low spot in the LCU surface; note that the LCU surface drawn from the push technology data also dips down, but not as strongly, in the same general area. This could also be a result of differing interpretations of the depth to the frequently gradational lower contact.

A three-dimensional stratigraphic model of the subsurface was created using EarthVision based on the interpreted stratigraphic contacts from the push technologies as noted above and listed in Table 4.12. Several sections or vertical "cuts" were made through the model to allow comparison with the BVWST borehole data. An east-west cut was made through the model at $500 \mathrm{~N}$ (see Figure 4.61). Logs from nearby BVWST boreholes were overlaid on the cross section at the appropriate locations. Correlation is generally quite good, except in the region of B-7 where the depth to the LCU is about 12 feet deeper. This cross section has the same general stratigraphic patterns as section BB (see Figure 4.60), including the dip in the LCU in the vicinity of $600 \mathrm{E}$. A major difference is, however, the rise in the LCU surface near $800 \mathrm{E}$.

Further comparisons between BVWST data and the three-dimensional model were made using several north-south cuts through the three-dimensional model. The north-south cut at $850 \mathrm{E}$ is shown as Figure 4.62. The base of the granular unit is indicated to be one foot higher at the location of BVWST borehole B-16 which is superimposed on the cut. Note that the LCU was not detected at B-16. A second north-south cut at 825E is included as Figure 4.63. The log for MW-3 is superimposed on the cut. Agreement of the model soil stratigraphic contacts with those shown on MW-3 is within about one foot. A third north-south cut at 750E is included as Figure 4.64. The BVWST logs for boreholes B-3, B-7 and B-14 are included for comparison. The surface of the LCU is within about one foot of the modeled surface at BVWST boreholes B3 and B-14. The surface of the LCU is indicated as being three feet deeper than the model at the location of borehole B-7. Correlation between the UCU/granular unit contact is within one foot at the locations of boreholes B-7 and B-3. Borehole B-14 shows this contact about six feet deeper than the model. These cross sections indicate generally good correspondence with the BVWST logs. 
Ames Expedited Site Characterization -Marshalltown FMGP Site

FINAL Site Report

March 25, 1996

Key aspects of the ESC process are:

- data integration

- evaluation

- updating of the site geologic and contamination conceptual models on a daily basis, and

- flexible decision making in the field.

To demonstrate this aspect of the process, the evolution of an important geologic site characteristic, the surface elevation of the LCU (or base of the granular unit where the LCU is absent), was examined as the Phase II investigation progressed. The LCU surface elevation plan was generated using soil conductivity, soil sampling and CPT stratigraphic data. The surface elevation plans for Day 1, Day 2, Day 4 and Day 10 are shown as Figures 4.65 to 4.68, respectively. It may be noted that the most significant changes in the LCU elevation plan occurred in the first 4 days; the Day 4 and Day 10 plans are not significantly different. Figure 4.68 illustrates that the LCU generally dips towards the southwest; a relatively steep ridge with a rise of about 10 feet trends from the northwest to the southeast across the site.

As noted above, EarthVision uses a spline function to develop the contours and no estimate of uncertainty is made. Stenback (1995) has used a geostatistical approach to characterize the elevation of the LCU surface and to estimate the degree of uncertainty in the contours themselves. This approach uses a median polish algorithm (see Cressie, 1991) to account for trend in the surface elevations. The residuals from the median polish were analyzed using a geostatistical approach whereby a variogram was generated and kriging equations were used to model the correlation structure in the residuals. A measurement error variance of $0.25 \mathrm{feet}^{2}$ was included in the calculations. The values generated by the kriging algorithm were added to the median polish surface. These values were in turn input into the contouring program Surfer, the contour plot shown in Figure 4.69 was generated. Note that this surface is in general similar to that shown in Figure 4.68. A contour plot of the prediction standard deviation corresponding to the surface elevation contours shown in Figure 4.69 is shown as Figure 4.70. Note that the standard deviations are lowest in the areas where there is the most data. A further evaluation of the veracity of the kriged surface was carried out by comparing the LCU surface elevation given by kriging to that given by the BVWST boreholes at the BVWST borehole locations. The results are summarized in Table 4.13. It may be noted that the BVWST data suggest a lower LCU surface for 12 of the 17 comparisons. In addition, the most significant deviations are at BVWST boreholes B-2, B-7 and B-13. These differences are also apparent by direct comparison of the nearest direct push log to the BVWST borehole location. The differences are probably due to some local variation and heterogeneity in the stratigraphy as well as some interpretation error. This is not surprising given the gradational contacts in some locations. 


\section{Ames Expedited Site Characterization - Marshalltown FMGP Site \\ FINAL Site Report}

March 25, 1996

the conductivity trace was generally irregular and a clear signal was not apparent; a "no PAH signal" entry indicates that the conductivity trace was relatively level with no apparent dips which would indicate PAH contamination. The MLH sample logs generally indicate whether any product was noticed visually, such as sheen, or free phase product, together with an olfactory determination, such as no odor, mild odor, or strong odor. The CPT data is reported as an average LIF intensity over the region of comparison. Finally, the BVWST logs generally give a visual indication of contamination, such as free product, tarry substance or oil sheen.

The data in Table 4.14 indicate generally good agreement between the olfactory, visual and CPT classifications. Discrepancies can generally be accounted for by comparisons within several feet vertically. For example, in Cluster 5 both McLaren/Hart and BVWST show free product from depths of $\mathbf{3 0}$ to 32 feet, but the CPT LIF intensity is only about 50 counts indicating relatively low PAH levels within this depth range. However, the CPT LIF intensity is about 300 counts or more over the depth range from 32 to 34 feet, indicating high PAH levels over that interval. This example provides further evidence of local heterogeneities in the soil stratigraphy.

The Geoprobe conductivity often shows good agreement with the other methods, but occasionally shows ambiguous results. The ambiguity arises for several reasons. First, because factors other than PAH contamination, such as soil type or pore fluid chemistry, can contribute to a low conductivity reading. Second, the conductivity trace is often quite irregular due to thinly interbedded seams of silts, sands, clays and gravels; accordingly, abrupt swings in the conductivity trace make interpreting the dips very difficult. An example of a strong correlation between soil conductivity(ML-54), CPT LIF (ML-109) and soil sample descriptions (ML-118) is data cluster 4. Recall that excellent contamination correlation was observed between ML-54 and ML-109 in Figure 4.56. The MLH soil sample descriptions support the soil conductivity and CPT LIF interpretations.

Because the CPT soil classification is based on a cone tip and friction sleeve resistance, which depends on the shear strength of the soil, among other things, one might expect that the soil classification could be affected by the PAH contamination. However, examination of data showing levels of PAH contamination based on LIF measurements, no evidence is seen to suggest that the CPT soil classification is affected by the presence of PAH contamination. In particular, compare clusters 4,5,6 and 7 in Tables 4.11 and 4.14. In addition, examination of Table 4.14 shows no evidence that any significant PAH contamination is drawn down with the CPT probe thereby producing falsely elevated LIF response in a relatively clean soil underlying a contaminated region.

As discussed and illustrated in Chapter 2 for the BVWST RI data, important information resides in the visual and odor comments in the MLH logs. The same logarithmic type scale used in Chapter 2 is used again here. Given that free product corresponds to a concentration of about $10^{6}$ 
Ames Expedited Site Characterization - Marshalltown FMGP Site

FINAL Site Report

March 25, 1996

$\mathrm{mg} / \mathrm{kg}$ and slight but definite odor corresponds to a concentration of about $1 \mathrm{mg} / \mathrm{kg}$, the following range is again appropriate:

$\begin{array}{lclc}\text { Observations } & \text { Scale } & \text { Observations } & \frac{\text { Scale }}{2} \\ \text { free product } & 6 & \text { mild odor } & 2 \\ \text { product sheen } & 5 & \text { slight odor } & 1 \\ \text { discoloration } & 4 & \text { no odor } & 0 \\ \text { strong odor } & 3 & & \end{array}$

Table 4.15 contains visual/odor information interpreted from the MLH sample logs using the above scale. Contour plots of this MLH data for the six stratigraphic zones are shown in Figures 4.76 to 4.81. In general, these results compare closely with what was interpreted from the CPT LIF pushes (see Figures 4.46 to 4.51 ). The most significantly contaminated regions are to the north and east of the storage shed and to the south and east of the substation. The eastern limits of the most significantly contaminated areas identified by the MLH visual/odor data closely correspond to those indicated by the CPT LIF contour plots. When comparing the CPT LIF and visual/odor plots, it should be noted that different map (data) locations are being used to generate the plots and thus the shapes of the plumes will be somewhat different. The density of MLH sampling locations is heavily biased to the eastern part of the site; the rationale and background for this bias is discussed in more detail in the next section. Examination of the visual/odor contours for zone 5 (Figure 4.80) indicates that the most significantly contaminated regions are confined to the depressed area (i.e., below the NW-SE trending ridge) of the surface of the LCU; this trend corresponds to that observed in the CPT LIF zone 5 contour plot (see Figure 4.50). Visual levels of contamination identified in the soil samples at ML-94, ML-96 and ML-57 confirm the significant contamination detected by the LIF sensor in the northwestern part of the site near the substation. Moreover, major hits at ML-86 and ML-88 indicate that the contamination extends to the extreme northwest corner of the site. Therefore, as was first indicated by the Phase I screening data, and confirmed by the CPT LIF data, these data again confirm regions of significant contamination in the northwestern part of the site.

Figure 4.82 shows the same cross section produced in Figure $4.59^{\circ}$ (AA ${ }^{\circ}$ in Figure 4.58) with zones of heavy coal tar residue contamination added as indicated from the BVWST logs and interpreted from the soil conductivity and CPT LIF logs. Only regions with LIF intensity greater than about 200 counts were included. The most heavily contaminated regions are at the base of the fill, within the UCU, at the top of the granular unit and at the base of the granular unit. Continuity of contamination from one location to another is evident. The apparent levels of contamination drop off sharply between ML-59 and ML-37; this is the same region where the bedrock and LCU rise sharply. 
Ames Expedited Site Characterization - Marshalltown FMGP Site

FINAL Site Report

March 25, 1996

In terms of contaminant screening, the CPT LIF is the most direct qualitative methodology for indicating regions of PAH contamination. The LIF responses have been corroborated by sideby-side sampling with chemical analyses, by correspondence with nearby BVWST logs and indirectly by the soil conductivity logs. The CPT LIF contaminant screening program has indicated the extent of the heavily contaminated zone in the northwestem part of the site near the substation and also highlighted the extent of coal tar residue contamination which is sitting on the LCU. An attempt to capture a three-dimensional view of the LIF signal intensity is given in Figure 4.83. These are visualizations based on the continuous nature of the LIF data with depth and the extensive probings near the edges of the PAH plume. Figure 4.84 is a three-dimensional EarthVision plot showing areas of significant contamination (LIF intensity $>100$ ) sitting on top of the LCU shown as a continuous pink layer. It may be noted that the region of coal tar residue contamination resting on the LCU generally follows the surface topography of this unit. There is a good chance, therefore, that the DNAPL is moving off the site to the south, flowing down the gradient of the LCU.

\subsection{Quantitative Chemical Analysis of Soil Samples}

\subsubsection{Introduction}

As noted in the introduction to Section 4.3, soil samples for quantitative chemical analysis were collected by McLaren/Hart Environmental Engineering Corporation of Warren, NJ using a twoperson crew operating a Geoprobe Model 8-MU sampling system. Cores two feet long and one inch in diameter were collected from the six subsurface zones at specified depths. In general, soil samples were only taken at locations and depths where information content was expected to be maximum based on all prior evidence, including BVWST information, Geoprobe conductivity logging, CPT LIF and CPT soil stratigraphic data. Occasionally a two-foot section was requested from a depth with the expectation that two contiguous zones would contribute equally to the two-foot core. In these cases the prior stratigraphic evidence was of such quality that nearly all of these types of cores met our stringent expectations and two distinct samples could be generated from one core. A detailed descriptive log of the 123 core samples collected from 29 MLs (see Figure 4.85 for locations) was kept and data on recovery, date, time, sample number, depth, soil description, water content and visual/odor evidence of contamination were recorded. A total of 127 soil samples were selected from the core samples and analyzed on site for the 16 target PAH compounds. Twenty-one additional samples were so contaminated that they were simply archived and qualitative GC/MS fingerprinting of the free product phase was done using the sonication extraction method.

A major effort at Marshalltown was the fielding of five separate on-site GC/MS-based extraction schemes for semi-volatile PAHs in soils. The extraction methods used were sonication, 


\section{Ames Expedited Site Characterization - Marshalltown FMGP Site}

FINAL Site Report

March 25, 1996

supercritical fluid extraction (SFE), thermal desorption, microscale and microwave extraction. These five extraction schemes were coupled to three GC/MS instruments. Three subcontractors were involved in this unusual activity; successful implementation of this activity required their complete cooperation in the field. McLaren/Hart provided all three GC/MSs in two mobile laboratories and three of the extraction modules and their associated expert operators. Their sonic solvent system consisted of a Tekmar Sonic Disrupter coupled to an HP Series II 5890 GC with an HP 5970B MS detector, while their SFE system included an HP Model 7680T SFE Module coupled to an HP Series II GC with an HP 5972 MS detector. Their thermal extraction unit consisted of a Thermex extractor from Ruska Instruments with the same GC/MS unit as was used for the SFE. They provided space in their mobile laboratories to accommodate the microscale extraction module and a qualified chemist who was fielded by PACE of Minneapolis, MN. A CEM Corporation Model 1000 Microwave Extractor was used by the same PACE chemist inside the McLaren/Hart mobile laboratory. Very little additional training was required to operate the CEM unit and it was provided by CEM at the site. Eighteen samples were also sent off site and analyzed by MBT Environmental Laboratories, a division of McLaren/Hart, using Method 8270B for SVOCs. Only the 16 targeted PAHs were measured by MBT. Autosamplers were used to permit overnight analysis. The sonication and microscale extraction analyses were done on one GC/MS instrument, the supercritical fluid and microwave extraction analyses were done on a second GC/MS instrument, and the thermal extraction was done on a third GC/MS instrument.

A critical issue in the evaluation of the extraction methods was how the samples were to be homogenized before being split and sent to the six extraction methods (five on site and one off site). Unless the split samples could be considered homogeneous, any deviation of the reported PAH concentration could not be assigned confidently to the extraction method rather than the sample concentration variance. A minimum of six inches of core length was required to satisfy the soil quantity requirements for all six methods. In consultation with local and national experts, the homogenization process decided on was to select the core to be homogenized, remove it, cut it into small chunks with a knife and mix it thoroughly using a gloved hand. The chopping and kneading process was repeated until the resulting specimen was visually and tactually uniform. The cycle was then repeated once more. The sample was then separated into seven pieces and distributed to the various measurement systems, with one sample held in reserve.

In terms of the site characterization goal of this project, samples were selected starting from outside the expected plume area followed by moving toward the suspected plume with the intent of using the low quantitation limits of the GC/MSs to map the action level limits of the plume. This plan conflicted with the technology evaluation goal of the project because it led to a large number of ND samples that could not be used for the technology comparison task. Nevertheless, this site sampling plan was followed for the first several days of soil sampling. In addition, 
estimates of the sample analysis throughput were lower than expected and if this approach were continued very few contaminated samples would be available for a systematic comparison of the five extraction methods. As a result, after the first few days the sampling and analysis plan was modified so that only samples judged to be contaminated were automatically sent to all five extraction modules. Other samples were first analyzed using the sonication method. These samples were then sent to the other extraction systems only if the sonication results confirmed that PAH contamination was present. Even with this revised system implemented, however, a significant number of the samples analyzed returned ND results.

A fortunate circumstance was realized after a few days that made the criteria for selecting the contaminated samples for further analysis relatively easy. It was noticed that soil samples that were characterized as having a slight but definite odor turned out to have PAH concentrations in the 1 to $10 \mathrm{mg} / \mathrm{kg}$ range. This allowed better estimates as to which samples to send for analysis without incurring any idle GC/MS instruments.

Table 4.16 lists the attributes of the samples that were analyzed by one or more of the five onsite extraction methods, including sample number, map location (ML), depth, soil type, stratigraphic zone from which the sample was taken, recovery, extraction methods used and an indication of whether the sample contains PAHs above the detection limits. The analytical results of each sample for the 16 PAHs, organized by on-site extraction type, are given in Appendix D. A uniform method detection limit of $1 \mathrm{mg} / \mathrm{kg}$ was applied by McLaren/Hart for each extraction method and for each analyte for the data reported in Appendix D. Some of the samples were diluted five, ten, or even 100 fold prior to testing; if such a diluted sample returned a non-detect result (ND, below the method detection limit), then it was recorded as $<5,<10$, or $<100 \mathrm{mg} / \mathrm{kg}$, respectively (depending on the level of dilution) on the original MLH data summary (McLaren/Hart, 1994). Due to the inherent significant uncertainty, these results were not used in any of the analyses reported here and were excluded from the data set. Moreover, the undiluted sample non detect values ( $<1 \mathrm{mg} / \mathrm{kg}$ ) were assigned values of $0.5 \mathrm{mg} / \mathrm{kg}$ for analysis purposes. This assumption is relatively conservative (as opposed to assigning them a value of zero) and minimizes potential error. Data reported as being outside the linear working range of the GC/MS instrument were simply used as reported.

The analytical results reported in Appendix $D$ will be used both for comparing and evaluating the on-site extraction methods in Section 4.4.2 and for characterizing the PAH plume in Section 4.4.3. 
Ames Expedited Site Characterization - Marshalltown FMGP Site

FINAL Site Report

March 25, 1996

\subsubsection{Comparison of Extraction Methods}

\section{Extraction Method Data}

Because a substantial portion of the results of the 127 soil samples tested on site by McLaren/Hart were below the method detection limit, 15 samples taken from 10 MLs with a relatively high proportion of results above the detection limit were chosen for comparison purposes and extraction method analysis. Moreover, most of these samples contain data from three or more of the five extraction methods that we wish to compare. The sample (map) locations, MLH sample identification, site coordinates, sample depth, and logged soil type from which the data were taken are listed in Table 4.17. For the data. in this analysis, some samples were not tested by all extraction methods resulting in a 12.4 percent loss of potential data; of the remaining data, 33.4 percent are recorded as below the method detection limit. Out of 240 possible data subgroups (15 samples times 16 analytes), only 38 subgroups contain a measured value above the method detection for all five extraction methods. Because of this difficulty, all ND data were excluded from our analyses, unless stated otherwise.

\section{DataAnalysis}

A series of percent recovery tests were carried out at the onset of the field program to examine the relative extraction efficiency of the different extraction methods. The percent recovery data were generated, in most cases, by testing five or six soil samples in duplicate, spiked with $\mathbf{5 0}$ $\mathrm{mg} / \mathrm{kg}$ of each of the PAH compounds, for each method. One exception to this is the microwave percent recovery, which is based on only two samples run in duplicate. The data from the percent recovery tests are summarized in Table 4.18.

A preliminary analysis of the extraction method comparison data was carried out by Stenback and Kjartanson, 1994 (samples MT-2-G017.2 and MT-2-G111.1 were not included in this analysis). This preliminary analysis indicated that the microscale and microwave extraction techniques were the most efficient techniques while the off site and the thermal techniques were generally the least efficient. The differences in concentration given the techniques for the same sample were significant, up to an order of magnitude or more. Possible explanations for this behavior were explored. During the microscale and microwave extraction procedures the extract was exposed to the atmosphere for a short time, potentially allowing some evaporation of extract solvent. This was not accounted for in subsequent calculations and could be responsible for the positive bias observed in the associated PAH concentration measurements. The off-site, fixed base laboratory method called for concentration of the soil sample extract to a greater extent, in accordance with the EPA 3550 method, than was done with the on-site extractions. Because the PAH analytes tested are semivolatile, it is possible that some analyte could be lost along with solvent during the concentration step thereby resulting in a bias downward. The off-site samples 


\section{Ames Expedited Site Characterization - Marshalltown FMGP Site}

FINAL Site Report

March 25, 1996

were capped and cooled during shipping to avoid losses due to evaporation during sample shipping and handling. Shipping and handling losses are known to be potentially significant with VOCs, but were not thought to be significant with SVOCs. The significant differences and deviations observed in the preliminary analysis triggered a more in depth analysis of the results; this more in depth analysis is reported in Stenback and Kjartanson, 1995.

A major difference in this analysis from the preliminary analysis is that each datum was divided by the average percent recovery values from Table 4.18, expressed as a decimal, for that method and analyte. Because no percent recovery data for the thermal extraction procedure are available, no adjustment for this method was made in the following data analysis. (As an aside, the percent recovery data for the remaining four extraction methods are in agreement directionally with the biases noted in the Stenback and Kjartanson, 1994 report.\} The off-site analysis results were also excluded from this analysis. Additionally, nonparametric statistical significance tests were performed because some of the data being compared do not appear to come from normally distributed populations. Other minor differences are discussed as they arise below. There is no accepted method, or known true values, against which to compare these data; accordingly, method comparisons here are made relative to the other methods in this study.

Method Bias and Precision. To detect method bias, examine method precision, compare sandy versus clayey soil sample results and look for differences between methods as a function of analyte, the data were grouped by soil sample and analyte ( 15 soil samples by 16 analytes $=240$ categories). Depending on which extraction methods were used to obtain a result for that particular sample and analyte category, up to five measurements may exist within each category. The arithmetic average within each category was determined, i.e., averaging over extraction methods, and each individual value was then normalized by dividing it by its category mean. A derivation of some properties of this normalized variable, and reasons for its use, was given in Stenback and Kjartanson, 1994 and is not elaborated on here. The means, standard deviations and number of observations (soil samples) for the normalized analyte/method subgroups are listed in Table 4.19. These give some idea of method bias and relative precision for each analyte and method.

Due to the data normalization, bias is measured relative to one (1.0), i.e., means significantly less than one suggest a bias downward while means significantly greater than one suggest a bias upward. Method precision is measured by the standard deviations under the assumption that the method standard deviation is proportional to the mean analyte concentration. In other words, the standard deviations give an estimate of the proportionality constant, $K$, between the method standard deviation $(\sigma)$ and the mean analyte concentration $(\mu)$, that is, $\sigma=\mathrm{K} \mu$.

Means of the normalized data by extraction method, i.e., averages taken over samples and analytes, were compared to detect relative biases between methods using the nonparametric 


\section{Ames Expedited Site Characterization - Marshalltown FMGP Site}

\section{FINAL Site Report}

March 25, 1996

Kruskal-Wallis procedure using a 0.01 level of statistical significance. The results of the bias comparisons are displayed below.

\section{Kruskal-Wallis Analysis}

\begin{tabular}{lccccrr} 
Method & Mean & Standard Deviation & $\mathbf{n}$ & \multicolumn{2}{c}{ Mean Comparisons } \\
Thermal & 0.71 & 0.55 & 112 & $*$ & & \\
Sonication & 0.84 & 0.36 & 128 & & $*$ & $*$ \\
SFE & 1.01 & 0.38 & 166 & & $*$ & $*$ \\
Microscale & 1.13 & 0.46 & 178 & & & \\
Microwave & 1.28 & 0.43 & 100 & & &
\end{tabular}

Comparisons with no overlapping asterisk are significantly different at the 0.01 level, based on the Kruskal-Wallis test at an overall significance level of 0.01 . $n$ is the number of observations above ND.

On the basis of the results presented in the table above, several of the extraction methods appear to provide significantly different results. The thermal and sonication methods show a bias downward. The microwave method shows a bias upward. The microscale and SFE methods show no bias relative to each other. With the exception of the SFE and microscale methods which do not show a statistically significant difference, this indicates that the extraction efficiency increases in the order in which the extraction method means increase, as listed in the Kruskal-Wallis analysis table above.

Soil samples were treated as replicates and differences between method precision were evaluated using a nonparametric procedure. The variances, $\sigma^{2}$, provide an estimate of the proportionality constant, $\mathrm{K}$, between the method standard deviation, $\sigma$, and the mean analyte concentration $(\mu)$, that is, $\sigma=\mathrm{K} \mu$. Two variance estimates are given here. The first comes from a combination of all the data for a given method and calculation of the variance by standard formulae. This may be biased upward due to differences between analyte means because the variance estimated this way includes variation between samples and variation, if any exists, due to differences between analyte means. The second calculation gives a pooled estimate of the variance by calculating a variance for each analyte (for each method) and pooling these together using their degrees of freedom according to standard variance pooling formulae. This method accounts for differences between analyte means, if any exists. Comparison of the two estimated variances (or standard deviations) will indicate whether differences between analyte means are significant or not. The table below shows the results of the two estimates of the standard deviation. A nonparametric test for variance differences between extraction methods (Conover, 1980, page 241) using an overall significance level of 0.01 was performed. The comparison results are displayed in the table on the next page. 
Ames Expedited Site Characterization - Marshalltown FMGP Site

FINAL Site Report

March 25, 1996

Nonparametric Variance Analysis

\begin{tabular}{lcrrrrr} 
Methed & $\boldsymbol{\sigma}$ & df & Pooled $\sigma$ & df & \multicolumn{2}{c}{ Comparison } \\
Sonication & 0.36 & 127 & 0.37 & 113 & $*$ & \\
SFE & 0.38 & 165 & 0.39 & 150 & $*$ & $*$ \\
Microwave & 0.43 & 99 & 0.44 & 85 & $*$ & $*$ \\
Microscale & 0.46 & 177 & 0.46 & 163 & & $*$ \\
Thermal & 0.55 & 111 & 0.52 & 96 & & $*$
\end{tabular}

Comparisons with no overlapping asterisk are significantly different at the 0.01 level of significance.

$\sigma=$ standard deviation, $\mathrm{df}=$ degrees of freedom.

Comparison of the two standard deviation estimates displayed in the table above show that there is not an appreciable difference between the two. The implication of this is that the majority of observed variation exists between samples, and only a relatively small amount of variation exists between analytes. One exception to this is seen in the thermal extraction data for the analyte benzo(a)pyrene which is discussed further in the Clayey Versus Sandy Soil Samples section below. Additionally, the Extraction Method Bias by Analyte section below does indicate some effect on some measured analyte levels with respect to the molecular weight of the analyte.

On the basis of the comparisons in the table above, the sonication method shows significantly less variation, or greater precision, than the microscale and thermal extraction methods. The microscale, microwave, thermal and SFE methods do not show any clear significant differences in method precision from one another. Due to the nature of our data transformation (division of each data value by its analyte/sample subgroup mean), the presence of a negative bias in the method mean will tend to result in an underestimation of the method standard deviation while a positive bias will tend to result in an overestimation of the method standard deviation.

Accordingly, the low standard deviation for the sonication method may be due to the fact that the sonication mean also appears to be biased downward. However, this does not explain the large standard deviation observed with the thermal extraction data, hence, it seems clear that the thermal extraction data are quite variable, i.e., this analysis suggests that the thermal extraction method has poor analytical precision (the method detection limit data discussed below are in agreement with this conclusion).

Clayey Versus Sandy Soil Samples. To detect differences in method extraction between sandy versus clayey soil samples, data as described above and used to generate the Kruskal-Wallis and nonparametric variance analysis tables above were split into clayey and sandy groups. The nonparametric Mann-Whitney test was used to test for differences between clayey versus sandy sample means by extraction method. A squared ranks test described by Conover (1980, page 
Ames Expedited Site Characterization - Marshalltown FMGP Site

FINAL Site Report

March 25, 1996

239) was used to compare method precisions. The results of these comparisons are displayed in Table 4.20.

The results shown in Table 4.20 indicate that the extraction efficiency for clayey samples is significantly less (lower mean) than for the sandy samples for supercritical fluid extraction. Otherwise, the other extraction methods show no significant differences (mean test p-values $>0.01$ ) in extraction efficiency between sandy and clayey soil samples. All extraction methods except the thermal extraction method indicate that the variation with clayey samples is significantly greater (less precision) than the variation with sandy samples at the 0.01 level of statistical significance.

It is worth noting that an analysis of the sandy versus clayey sample subgroups, as listed in Table 4.20 , using pooled estimates of the standard deviations and classical Student's t-tests yields the same conclusions as the nonparametric mean tests listed in Table 4.20. Comparison of the variances using pooled standard deviations and classical F-tests give the same conclusions as reached in Table 4.20 with the one exception that the variance for sandy samples is significantly less than the variance for clayey samples for the thermal extraction method. This occurs because the pooled standard deviation estimates are nearly identical to those given in Table 4.20 with the one exception that the standard deviation estimate for the sandy soils with the thermal extraction data is $s$ (pooled) $=0.30$ (rather than $s=0.42$, as in Table 4.20). This is due to significant differences between analytes for the thermal method, in particular, the extremely low results for benzo(a)pyrene. This results in a statistically significant difference between sandy and clayey samples with $p$-value $<0.001$ using an F-test.

Extraction Method Bias by Analyte The means, sample sizes, and molecular weights for the extraction methods are listed in Table 4.19 and these means are plotted by analyte in Figure 4.86. Figures 4.87 and 4.88 show the means for clayey and sandy samples plotted by analyte, respectively. The analytes are listed in order of increasing molecular weight (MW), with the slight exception of the last two, dibenzo( $\mathrm{a}, \mathrm{h})$ anthracene $(\mathrm{MW}=278)$ and benzo $(\mathrm{g}, \mathrm{h}, \mathrm{i})$ perylene ( $M W=276)$, in Figures 4.86, 4.87 and 4.88. Bars indicating a significant difference (two-sigma, or $95 \%$ confidence level) between two means are shown on each figure. These figures indicate the trends in the extraction methods noted previously; i.e., thermal extraction generally least efficient and microwave and microscale generally the most efficient. The figures also indicate a general decrease in absolute bias with increasing analyte $\mathrm{MW}$ for microscale, microwave and thermal extraction.

Method Detection Limit Data A soil sample selected from the site was spiked at $10 \mathrm{mg} / \mathrm{kg}$ with each contaminant, homogenized and split into 35 subsamples (five methods times seven individual analytical tests) for a method detection limit (MDL) study that took place during the first few days that the McLaren/Hart mobile laboratory was on site. Analyses for each of the 16 
Ames Expedited Site Characterization - Marshalltown FMGP Site

FINAL Site Report

March 25, 1996

analytes of interest were run seven times (six for SFE) using each of the five extraction methods. The standard deviations of these analyses were used to obtain a method detection limit that is equal to a Student's t quantile for $99 \%$ confidence (upper-tail) times the standard deviation for each method and analyte. The averages and standard deviations for the MDL data are summarized in Table 4.21 .

Using the spiked value of $10 \mathrm{mg} / \mathrm{kg}$ as a basis for comparisons, the column averages and their standard errors (Std. Error of the Ave.) listed in the last two rows of Table 4.21 indicate that the sonication method shows a slight overall negative bias, microscale shows an overall positive bias and SFE shows no significant overall bias. These results are consistent with previous analyses. The microwave method shows an overall negative bias and the thermal method shows a large overall positive bias by a factor of about two. These results are inconsistent with previous analyses, as indicated in the Kruskal-Wallis analysis. It is not clear why the thermal method results are so high and variable and why the microwave method results are so low; however, these data were collected early in the program before laboratory procedures were well established.

The method detection limits for each analyte and method are listed in Table 4.22. With the exception of the microwave method MDL data, these data suggest that the detection limit of 1.0 $\mathrm{mg} / \mathrm{kg}$ that was used in reporting analytical results was too low. All methods other than microwave show MDLs consistently greater than $1.0 \mathrm{mg} / \mathrm{kg}$. The thermal extraction method MDL values range from 8.2 to $58.4 \mathrm{mg} / \mathrm{kg}$ over all analytes. The SFE extraction method MDL values range from 6.4 to $10.8 \mathrm{mg} / \mathrm{kg}$ over all analytes.

For comparison, the overall relative errors based on the MDL study (Table 4.21) and from data presented previously are listed in Table 4.23. The relative error is the standard deviation divided by the mean, and is expressed here as a percentage. For the clayey, sandy, and combined relative errors, the means listed in Table 4.20 were used along with pooled estimates of the standard deviations. The pooled standard deviation estimates are essentially identical (within about 10 percent) to the standard deviations listed in Table 4.20, with the exception of the sandy/thermal extraction data (as mentioned above) which shows nearly a 30 percent decrease with pooling.

Examination of Table 4.23 indicates that, with the exception of the sandy soil SFE data, the relative errors for the field measurements are considerably greater than indicated by the data collected for the MDL study. This is not too surprising for several reasons. It is not unreasonable to expect field data to be somewhat more variable than data collected under controlled laboratory conditions. Due to the ND data and the fact that not all extraction methods were used with each sample, under-representation of data within some analyte/sample subgroups may have introduced some bias and increased variance in our analysis of this data. 


\section{Ames Expedited Site Characterization - Marshalltown FMGP Site}

FINAL Site Report

March 25, 1996

The relative error calculations shown in Table 4.23 clearly indicate that the microscale and microwave methods have low relative error, sonication has intermediate relative error, and the thermal and SFE methods have high relative error, based on the MDL study data. For all five extraction methods, the relative error with clayey soils is greater than the relative error with sandy soils. For the field clayey and sandy samples combined, the microwave extraction shows the lowest relative error and the thermal method shows the greatest relative error, in agreement with the MDL study data. The combined sample field data indicate that the sonication, microscale, and SFE methods show only slightly greater relative error than the microwave method, and are not significantly different from one another.

\section{Summary of Extraction Method Analysis}

Method bias and precision conclusions are summarized in Table 4.24 and are discussed below. The method bias comparisons clearly indicate that the thermal extraction efficiency is less than (lower mean, negative bias) the sonication extraction efficiency which is less than the microwave extraction efficiency (higher mean, positive bias). The microscale and SFE extraction methods generally give intermediate results.

There is no definitive indication that any extraction method is more precise overall (see Table 4.24). On the basis of all the available data, the method precision for the thermal and microscale extraction methods is significantly less than the precision for the sonication method. As indicated in Table 4.24, a second parallel statistical analysis was carried out using 38 cases from the data set which gave analytical determinations for all five extraction methods above the method detection limit; this would eliminate this potential source of bias. These results indicate that there are no significant differences in precision between any of the extraction methods (see Table 4.24).

On the basis of all the available data, the clayey soil analytical results show greater variation than the sandy soil results indicating greater precision with sandy samples for all but the thermal extraction method. The 38 subsets of this data whereby all methods gave a greater than ND determination indicate that only the microwave and SFE methods show greater variation with clayey than with sandy soils, while the other methods show no variation differences between soil types.

The means for the microwave extraction data tend to decrease with analyte molecular weight (see Table 4.24). The means for the thermal extraction data tend to increase with analyte molecular weight. This indicates that the absolute value of the microwave and thermal method biases decrease for the higher molecular weight analytes. The SFE means tend to decrease with 


\section{Ames Expedited Site Characterization - Marshalltown FMGP Site}

FINAL Site Report

March 25, 1996

increasing analyte molecular weight with the sandy soil samples, but this effect was not observed with the clayey samples.

The MDL study performed on site indicates that the MDL is greater than $1.0 \mathrm{mg} / \mathrm{kg}$ for all extraction methods except the microscale method. The relative errors based on the field data generally indicate poorer analytical precision than is indicated by the MDL data. The MDL study data sets are small and were collected over a relatively short time frame during which the on-site procedures were being established. The MDL data indicate that the microwave method shows a negative bias while the thermal method shows a large positive bias; these results conflict with biases indicated by the field data for both the thermal and microwave methods.

Because of the large portion of potential data for which no analysis was performed and because of the high proportion of ND data, the data in this analysis do not always have equal representation between soil types, analytes, or extraction methods, i.e., the data are unbalanced. Accordingly, we have attempted to draw conclusions with some scrutiny and caution. Nevertheless, the above mentioned differences between these extraction methods seem apparent on the basis of this analysis. In particular, we find low thermal extraction results with high relative error and high microwave extraction results with low relative error, while the absolute value of the bias for both of these methods appears to decrease with increasing analyte molecular weight.

As is often the case with studies of this nature, similar field studies performed by other workers may yield somewhat different, possibly even conflicting, results. Even our analysis does not give clear results in some cases, for example, compare columns two and three in Table 4.24 above. In light of this, and because of the need to gain regulatory acceptance of field laboratory data for a full realization of the expedited site characterization process, at the very least, these data clearly point out the need for enhanced field quality assurance and quality control (QA/QC) and further field- and field-versus-fixedlaboratory method comparisons and/or method validations.

\subsubsection{Site Characterization Implications}

To enable comparisons with the BVWST data and the contaminant screening data presented previously, the quantitative data are plotted at the relevant MLs in terms of naphthalene concentration, phenanthrene concentration, and total PAH concentration per zone. As discussed in Section 4.4.1, five on-site extraction techniques and three GC/MS instruments were used to generate the quantitative data. Due to its strong negative bias, the off-site laboratory data is not included in this analysis. Moreover, because the thermal extraction results are generally low, have high relative error and suspicious method detection limit data (see Table 4.21), the thermal extraction data were also excluded from the analysis. Because there was no clear indication as to 
Ames Expedited Site Characterization - Marshalltown FMGP Site

FINAL Site Report

March 25, 1996

which of the remaining methods produced the "best" overall data, in terms of extraction efficiency, precision and accuracy, a representative concentration for each PAH compound for each of the samples listed in Appendix D was calculated by taking an average over the extraction method concentration results available (without the thermal extraction results) for each sample. These results are presented in Appendix D. Note that ND data were treated as discussed previously; i.e., ND data from diluted samples were deleted and the standard ND data were assigned a concentration of $0.5 \mathrm{mg} / \mathrm{kg}$.

\section{Naphthalene and Phenanthrene Distribution}

The naphthalene and phenanthrene concentration distributions by zone are given in Figures 4.89 to 4.100. In these figures the label NA means that no average was calculated for that location while no label below the ML designator mean that no data was taken at that location. It is instructive to first compare these results with the BVWST RI results presented in Chapter 2. As noted previously, the heavy concentration of MLH measurement locations on the eastern part of the site has defined the eastern edge of the contaminated zone well, but makes direct comparison with other areas of the site difficult. Comparisons which can be made are as follows.

For zone 1, comparing Figures 4.89 and 4.90 with Figure 2.5, it may be noted that the concentrations measured at ML-112 and ML-118 are generally in the same order of magnitude as those measured at B-6, which is situated between the two MLs. In addition, the concentrations measured at ML-116 closely correspond with those measured at the nearby B-7. Significant differences between these same BVWST and MLH locations, however, in zones 2, 3 and 4 indicate that quantitative levels of contamination can vary significantly over relatively small distances, due largely to the heterogeneous movement of the DNAPL coal tar residue through the subsurface and also subsampling techniques; i.e., the measured concentration could be very much a function of how the analytical samples are actually selected from the core sample. In this regard, as noted previously, some of the most heavily contaminated MLH samples were fingerprinted for PAH compounds rather than analyzed for concentrations of individual PAHs. The areas of contamination outlined by the BVWST and MLH data, taking into account the different sampling locations, are, however, generally the same.

It is also instructive to compare the MLH naphthalene data from the upper two zones with the deep Phase I passive and active screening naphthalene results. Comparing Figures 4.34, 4.40, 4.89 and 4.91 indicates that the plume defined by the passive gas system (Figure 3.34) more closely corresponds to the MLH data than the active gas plume (Figure 4.40), particularly on the eastern edge. The plumes are, however, generally similar in extent. This further supports, in particular, the passive gas screening technique in defining the general areas of contamination. 
Ames Expedited Site Characterization - Marshalltown FMGP Site

FINAL Site Report

March 25, 1996

\section{TotalPAH Distribution}

Contour plots of total PAH concentration from the MLH data are given in Figures 4.101 to 4.106. In these figures the label NA means that no average was calculated for that location while no label below the ML designator mean that no data was taken at that location. These plots are included as they would be used in regulatory decision making. As with the naphthalene and phenanthrene contour plots discussed in the last section, the data are biased to the eastern part of the site, thus allowing the eastern edge of the contaminated zone to be well defined. In addition, some of the more heavily contaminated samples, particularly in the northwestern part of the site near the substation have not been analyzed for PAH compound concentrations. In this regard, the visual/odor plots presented in Figures 4.76 to 4.81 , although only qualitative, are probably a better representation of the distribution of contaminants on the site than the quantitative data presented here. As in the previous section, this data are compared with Phase I screening data. Moreover, comparisons with Phase II screening data are made.

Comparison of zones 1 and 2 (Figures 4.101 and 4.102) with the deep zone IMA results (Figures 4.28 to 4.30 ) indicate general similarity of the plumes defined except in the area near the substation in the northwestem part of the site, for the reasons discussed previously. The eastem edges of the plumes defined by the IMA results conform to the McLaren/Hart results reasonably well.

Comparison of the MLH data with the CPT LIF data (contoured in Figures 4.46 to 4.51) gives the eastern edge of the plume in approximately the same locations for the stratigraphic zones. As with the MLH visual/odor data, however, much more CPT LIF data is available to better define the extent of contamination within the zones than the MLH quantitative data.

\section{Summary of Site Characterization Implications}

Due to the poor distribution of the sampling locations and the fact that heavily contaminated samples were systematically excluded from quantitative PAH analysis, this data adds little to further the development of the site contamination model. It does, however, confirm the eastern edge of the contaminated area as defined by the screening techniques and also correlates well, in places, with the quantitative BVWST data. A key issue is that while the screening tools have been able to define the areas of contamination much more clearly, they do so only in a qualitative sense. Quantitative data like the MLH data presented in this section is required for regulatory decision making. 
Ames Expedited Site Characterization - Marshalltown FMGP Site

FINAL Site Report

March 25, 1996

\subsection{Analysis of Groundwater Samples}

Groundwater samples were collected from ML98, ML90, ML104, ML80 and ML106 using a Geoprobe mill-slotted well point and Watera-type (tubing with bottom check valve) sampling tools. Samples were collected from two depths at each map location; the shallow samples were collected from the upper region of the granular unit while the deep samples were collected from the lower part of the granular unit. Three $40 \mathrm{~mL}$ volatile organic analysis (VOA) samples with no headspace and a one liter PAH sample were collected at each location. The samples were analyzed on site using the ultrasonic extraction method. The chemical analysis results of the samples for the 16 target PAHs are given in Table 4.25. Also given in Table 4.25 are the sample MLs, depths acquired and, for reference, the solubility of each of the PAH compounds in water at $25^{\circ} \mathrm{C}$. All of the groundwater samples were unfiltered except for a second GW005 sample which was passed through a $0.45 \mu \mathrm{m}$ filter. Sample GW006 at ML98 contained significant free product and was deemed too contaminated for analysis. Note that the IDNR standard for individual PAHs in groundwater is $0.2 \mu \mathrm{g} / \mathrm{L}$, except for naphthalene which is $20 \mu \mathrm{g} / \mathrm{L}$.

Table 4.25 indicates that the solubilities of the PAH compounds in water generally decrease as the molecular weight of the compound increases. The measured concentrations of the PAH compounds generally follow the trend of their solubilities; as with the results of the soil samples, naphthalene and phenanthrene are generally the two PAH compounds with the highest concentrations. Filtering, based on the analysis of the one unfiltered sample, appears to reduce the concentration of PAH contaminants in groundwater by about $50 \%$. The distribution of naphthalene and phenanthrene in groundwater for the shallow depths are shown in Figure 4.107 while the distribution of naphthalene and phenanthrene in groundwater for the deep region are shown in Figure 4.108. The concentrations are the highest in the region north of the storage shed, at ML98 and ML90, and decrease from ML80 to ML106 toward the eastern portion of the site. This pattern corresponds to the distribution of contaminants and the plumes defined by the Phase I screening and Phase II screening and quantitative technologies results.

It may be noted that PAH compounds were detected above the IDNR standards in every one of the groundwater samples analyzed. Due to the distance of the sampling locations from BVWST monitoring wells and the different groundwater sampling collection procedures, direct comparison of these results with the BVWST results is not possible. It is apparent, however, that the concentrations given in the central part of the plume are significantly higher than any concentrations recorded by BVWST. 


\subsection{Stakeholder Activities}

\subsubsection{Communications Strategy}

Ames Laboratory's application of ESC is distinctive in its approach to stakeholder involvement. Because of the increasing role of stakeholders in the acceptance and ultimate commercialization of environmental technologies (DOE, 1994)--or in this case, an environmental methodology--early stakeholder involvement was sought. Early communication with stakeholders is important because it enables ESC project managers to apply the approach so that ESC meets the technical, regulatory and public interest needs at a specific site.

In our ESC communication efforts with Marshalltown stakeholder groups, we employed a two-way symmetric communication model (Grunig, 1984) that aims for a balanced exchange of information between the organization (or program) and stakeholders, so that each group can learn from the other. The model seeks mutual understanding as its goal and is characterized by formative and evaluative research on audience/stakeholder interests and attitudes.

\subsubsection{Objectives and Audience}

Our objectives were to 1) increase the awareness of the ESC methodology among stakeholder groups, and 2) encourage a two-way exchange of information. We began by identifying stakeholders using a framework of organizational linkages as shown below.

Sample framework of organizational linkages used to identify stakeholders.

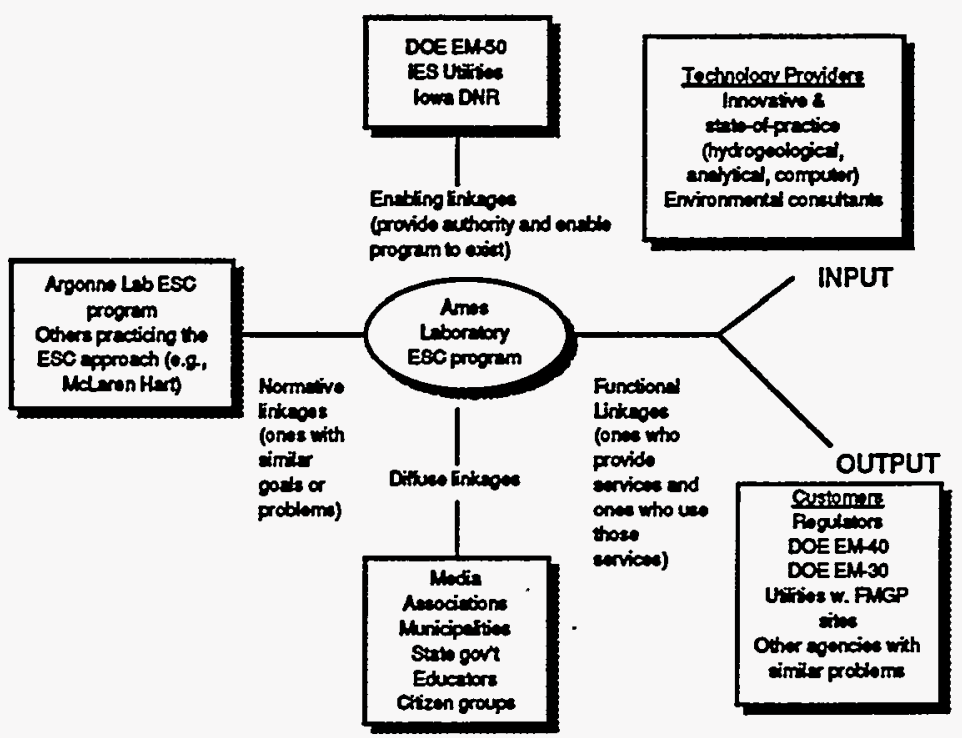


Ames Expedited Site Characterization - Marshalltown FMGP Site

FINAL Site Report

March 25, 1996

IES Utilities, Inc., the site owner, was an enabling "public" or stakeholder, critically important to the success of our communication efforts. Shortly after the company had agreed to let their site be used for the demonstration, and several months before the field work was to begin, the Ames ESC technical and communications team members met with both IES environmental managers and public relations representatives to discuss the public events desired for ESC stakeholder involvement.

The early scheduling of this meeting was critical for effectively introducing the Ames ESC project not merely as a technical effort but also as a stakeholder involvement effort. We presented the idea of sponsoring an open session giving interested publics (community members, regulators, DOE officials, educators, students, etc.) an opportunity to observe the approach, discuss its use, express concerns, tour the site and see the technologies used for ESC at work.

These activities met with IES approval and were in fact consistent with the company's philosophy of openness and proactive communications which they had used on several previous environmental projects, and had seen the resulting benefit.

The stakeholders or "publics" that were of primary concern to IES Utilities were:

- their own employees: that they understand the ESC work that was to take place and have the first chance to ask questions about it;

- the regulatory community: being under the oversight of the Iowa Department of Natural Resources (IDNR), IES Utilities was concerned that stakeholder/public involvement efforts be responsive to the opinions and suggestions of the overseeing regulators;

- the general public: that members of IES' large customer base would recognize that the company's support of this project demonstrated their commitment to economic incentives and customer focus; and

- environmental communicators: that Ames ESC efforts include a focus on reporters with an environmental focus.

In response to these communication concerns, the Ames ESC team agreed to:

- present information sessions to explain ESC to IES employees before the work began and allow for open dialogue;

- meet with overseeing regulators to discuss stakeholder involvement, and to include these regulators in the planning and approval of such things as press releases, announcements, etc.; 


\section{Ames Expedited Site Characterization - Marshalltown FMGP Site}

FINAL Site Report

March 25, 1996

- prepare a publicity plan for inviting specific groups, organizations and individuals in Iowa, the region and the nation to the ESC public conference/tour and share this plan with IES; and

- maintain regular communication with IES and regulators to keep them informed and get their input on specific stakeholder involvement plans as they were being developed.

In subsequent meetings, the overseeing regulator, Dr. Johanshir Golchin, environmental engineer and project coordinator with the IDNR, encouraged broad public participation and suggested names of organizations and individuals to invite to the planned conference.

\subsubsection{Identification of Stakeholders and Planned Activities}

Using the framework of organizational linkages, Ames developed a detailed list of stakeholder groups to invite (see Appendix F), and distributed this list to IES Utilities and IDNR for comments. We collected names of members of these stakeholder groups, totalling approximately 900 individuals. In addition, we sent announcements to newsletters, newspapers and electronic bulletin board services to publicize our event to an even wider group of stakeholders.

Personal phone calls were made to association presidents, newsletter editors, regulatory branch heads, DOE funding officials and other influential individuals to draw their attention to our written material and to solicit their help and support in promoting our event to their constituents. As we did this, we received suggestions for tailoring the content of the conference to the needs of particular stakeholders. For example, an environmental consultant in South Dakota suggested that by offering CEU credits for the event, regulators in that region could more easily arrange to attend. Personal contact with the public information bureau chief at the Iowa Utilities Board helped us assess the level of technical understanding of our potential attendees.

Because our objective was to increase the awareness and understanding of ESC (and its relationship to cleanup needs) and to encourage a two-way exchange of information, we designed the public conference to include a non-technical overview of the cleanup process, a presentation on ESC concepts and how it was applied to the Marshalltown FMGP site, tours of the various technologies at the site and a question and answer wrap-up. We mailed the conference brochure to over 900 people approximately one month before the event. Participants were only be charged for the luncheon meal. We offered an identical agenda on three consecutive days, May 11, 12,13,1994, to give stakeholders more flexibility in attending. The final agenda included the following sessions:

Overview of Environmental Cleanup. Designed to provide a context for understanding the importance of characterization, this session offered, in lay terms, background information on a number of aspects of environmental remediation: steps in the remediation process, the regulations involved, interpreting EPA numbers, assessing risk and defining technical terms. 
Ames Expedited Site Characterization - Marshalltown FMGP Site

FINAL Site Report

March 25, 1996

ESC at Marshalltown. IA. This session described ESC within the context of environmental management elements: risk, economics, stakeholder involvement, cleanup problems and cleanup technologies. We then described details of the approach, as applied to the characterization of coal tar wastes at the Marshalltown FMGP site, and the technologies used.

Tours of the site. Participants visited the Former Manufactured Gas Plant Site, and viewed more than 12 SOPT and ITs in action in the field. Contractors were present to explain their technologies and answer questions.

Question and Answer Wrap-UD. This session allowed DOE Headquarters program managers, IDNR regulators, Ames ESC team members, technology providers and other participants to openly exchange ideas, questions and comments on the ESC approach.

\subsubsection{Evaluation of Objectives}

Our first objective was to increase awareness. Conference attendance totalled 177 registrants, most of whom were not familiar with the ESC methodology or the technologies beforehand. The following stakeholder groups were represented:

$\begin{array}{lr}\text { Interested citizens and activists } & 3 \% \\ \text { Educators } & 5 \% \\ \text { International visitors (Bolivia) } & 8 \% \\ \text { City government } & 2 \% \\ \text { State government } & 4 \% \\ \text { Regulators (state level) } & 22 \% \\ \text { Site owners (utility industry primarily) } & 10 \% \\ \text { Technology providers and users } & \\ \quad \text { Industry (consultants \& contractors) } & 34 \% \\ \text { DOE } & 2 \% \\ \text { Other federal agencies } & 2 \% \\ \text { Ames Laboratory } & 8 \%\end{array}$

Comments received on the participant evaluation forms helped us assess, in a more qualitative way, whether the ESC event had increased awareness. The tour of the FMGP site, and of the technologies being used to characterize the site, seemed particularly effective in increasing an awareness and understanding of what is involved in ESC. Comments from the Visitors' Day included:

- "[Attendance] gave me a better grasp of what is involved and the associated time and expense. As a regulator, we only get to see dollars, with no idea of the work involved." 
Ames Expedited Site Characterization - Marshalltown FMGP Site

FINAL Site Report

March 25, 1996

- "Very worthwhile to attend. We see the clean up and costs associated with FMGP sites when utilities file rate cases. This was a good opportunity to see how those monies might be used more effectively and to see what is involved in cleanup activities."

- "This type of information is very difficult to get via other methods of information sharing (magazines, books, conferences, etc.). Very helpful seeing the data collected."

- "It is very helpful to see the various technologies in action."

- "I think it is very important to invite members of the public and locally concerned people to learn about cleanup efforts."

- "I'd like to be able to bring a high school class to a session like this. They could see the technologies being used. I appreciate so much the opportunity to attend."

On the day of the event, we met the Des Moines Register reporter and gave him direct access to the scientists and field managers involved with the work. The objectives and technical aspects of the project were accurately reported in the Des Moines Register, a state-wide distributed newspaper. Our work was also publicized in The Probing Times, Geoprobe System's in-house newsletter that is distributed to consultants, contractors and the regulated community nationwide. An article appeared in the Iowa Groundwater Association Newsletter, and resulted in an invitation to speak at the Iowa chapter meeting of that association. We also received an invitation to submit an article to the Journal of Soil and Water Conservation. In addition, our ESC Marshalltown project was mentioned in numerous DOE reports and presentations, furthering our objective of increasing awareness.

With the collection of video footage and photos that we had shot during the ESC demonstration, we were able to create hypertext documents and post multimedia descriptions of the ESC work on the Internet's World Wide Web (WWW). Using the suggestions of the IDNR regulator Dr. Johanshir Golchin, and the Iowa Citizens Action Network environmental specialist Julianna Johnston, we also began developing an on-line forum on the WWW to promote a discussion of ESC. A New Jersey video production company shot footage that was used in a promotional video for McLaren/Hart Environmental Engineering. The company shared this footage with the Ames Lab for our use in producing a video about ESC.

Our second objective was to encourage a two-way exchange of information. Evaluation comments indicated that a two-way exchange of information did take place but that more would have been desirable. The causes for the lack of interaction were partly logistical--e.g., running behind schedule which cut into the question and answer time, and not allowing enough time and structure for the tours. 
Ames Expedited Site Characterization - Marshalltown FMGP Site

FINAL Site Report

March 25, 1996

Some of the feedback we received included:

- "The on-site tour was very interesting. Each of the speakers took the time to fully answer our questions. The best part was that the speakers were the ones doing the work."

- "I would have enjoyed more discussion of the data fusion process...both days I attended there was no question and answer portion of the presentation."

- "I felt rushed and missed one of the vendors due to time. Very good information at the ones I did attend though."

- "Need more time for demonstrations. Allow more time per station [on tour]. More Ames staff could have been available at the tent for general questions."

But even with these logistical problems, two-way communication and an exchange of ideas did take place.

These and other comments on the evaluation form provided a very useful mechanism for listening to participants' concerns. Other feedback was obtained through phone calls to attendees after the event. Many of the comments and suggestions have been incorporated into subsequent $\mathrm{ESC}$ demonstrations.

\subsubsection{Summary}

Several factors contributed to the success of the communications effort. One was that IES Utilities' offered their support and cooperation with the public relations/stakeholder involvement effort early on in the project which allowed time for planning. A second, perhaps more important factor was that this support came from IES technical project managers who had a good understanding of, and appreciation for, two-way public relations activities. And a third was that the company had a history of openness with the public and good relationships established with regulators and the media. These factors provided a foundation on which to develop ESC stakeholder activities.

Of those activities, the tours appeared to be one of the most effective means of involving a diverse group of stakeholders and increasing their awareness of the ESC methodology. Allowing participants to go on to the site, to talk directly with the contractors using the technology and discuss the findings demonstrated our commitment to openness and a two-way exchange of ideas. 


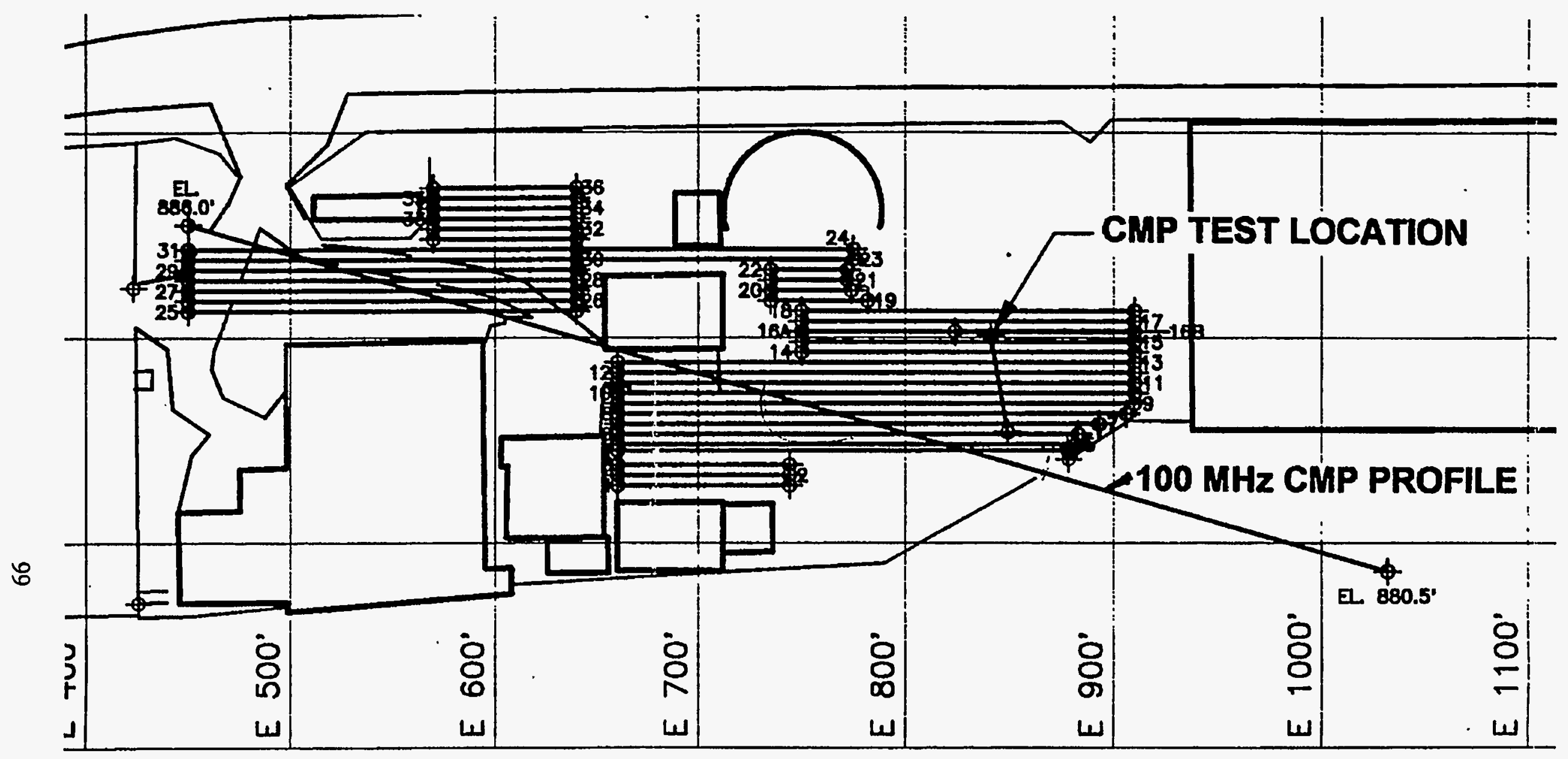

Figure 4.1 Location of GPR Profiles (from D'Appolonia, 1994) 


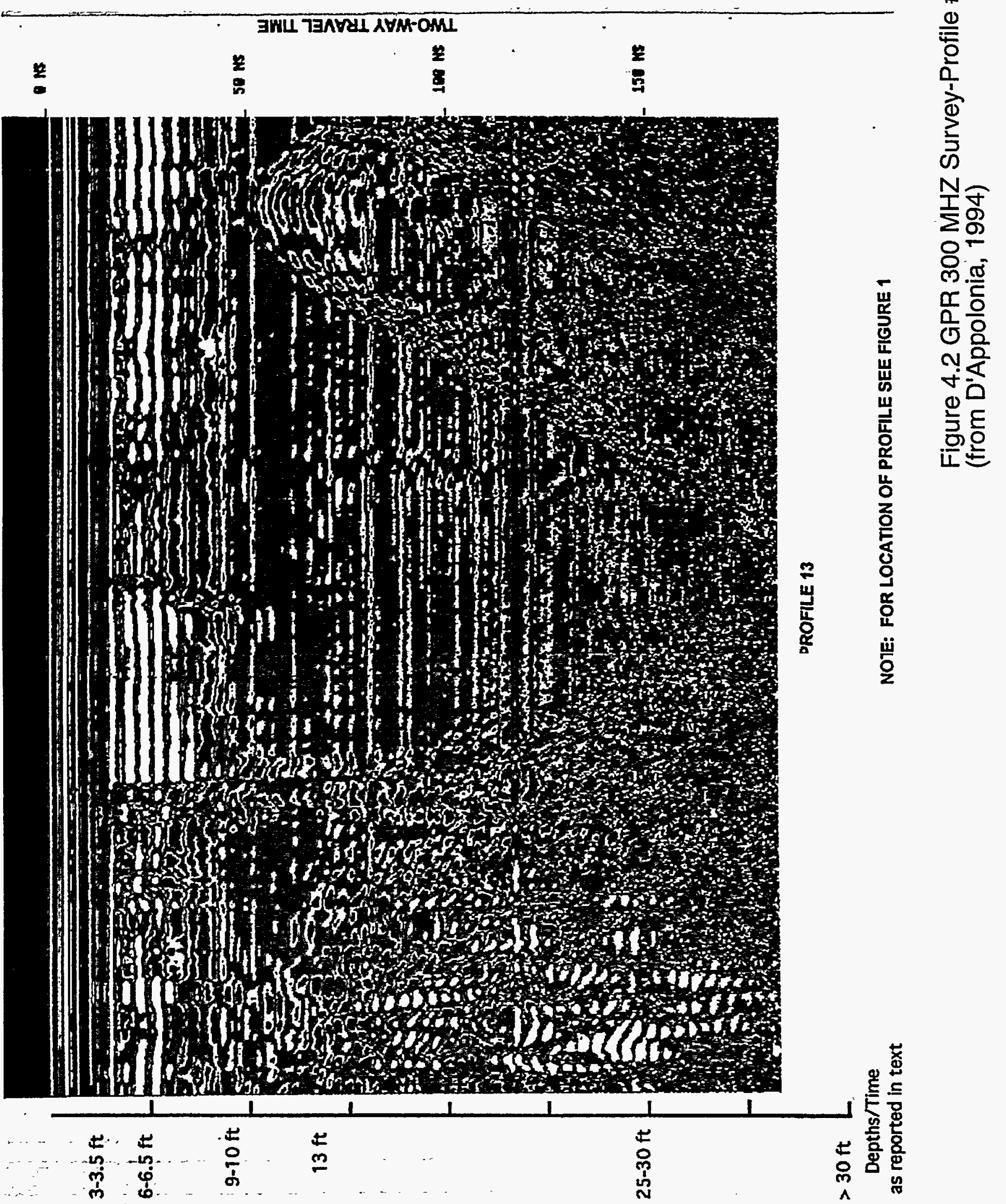




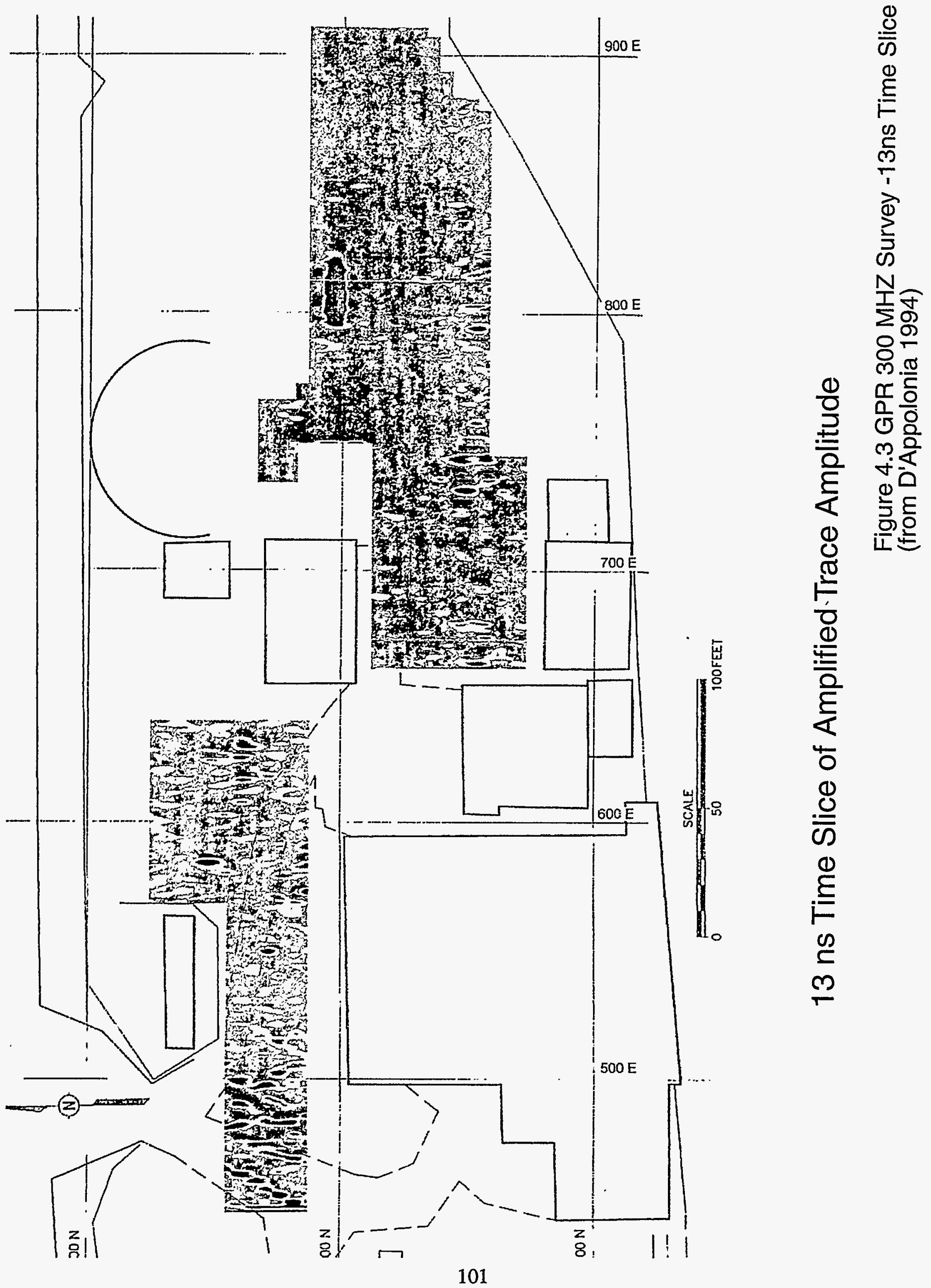




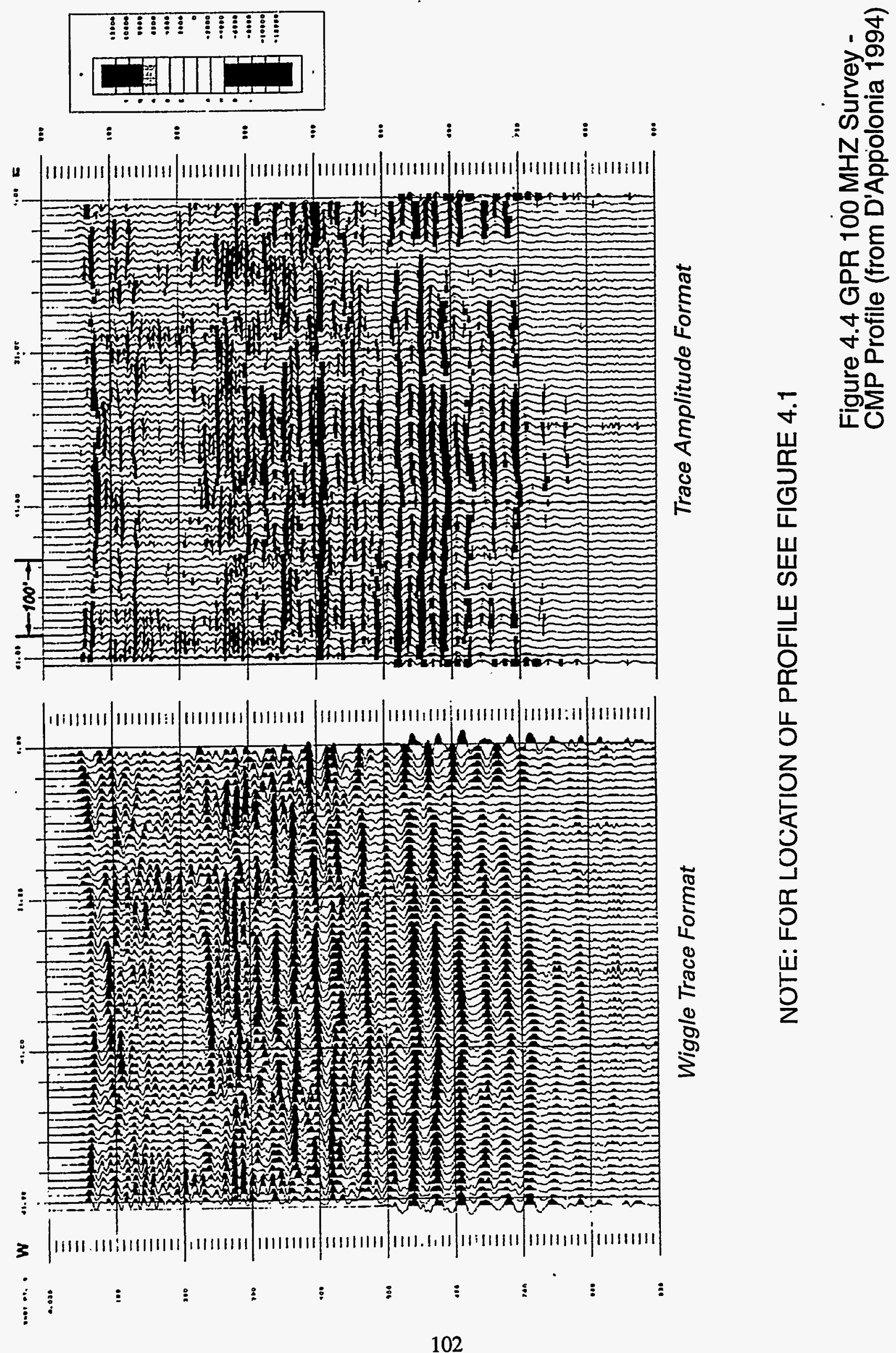




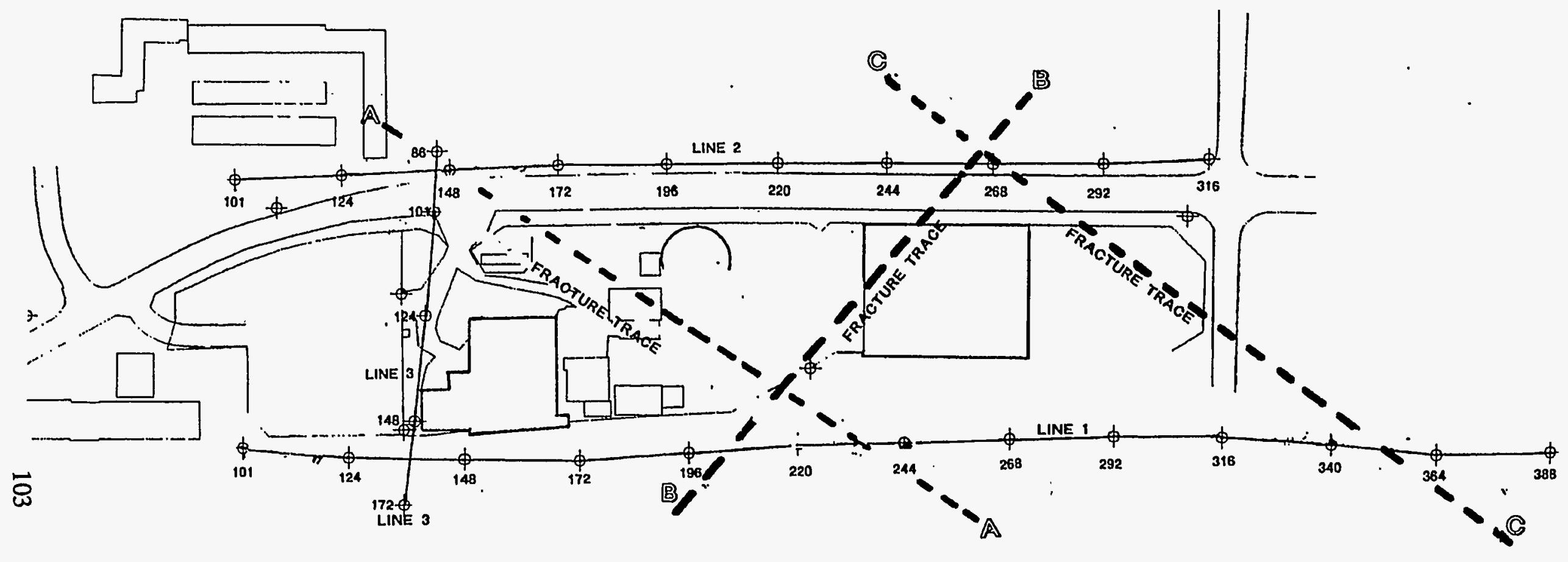

Figure 4.5 Location of Seismic Reflection Lines (from Resolution Resources, 1994) 


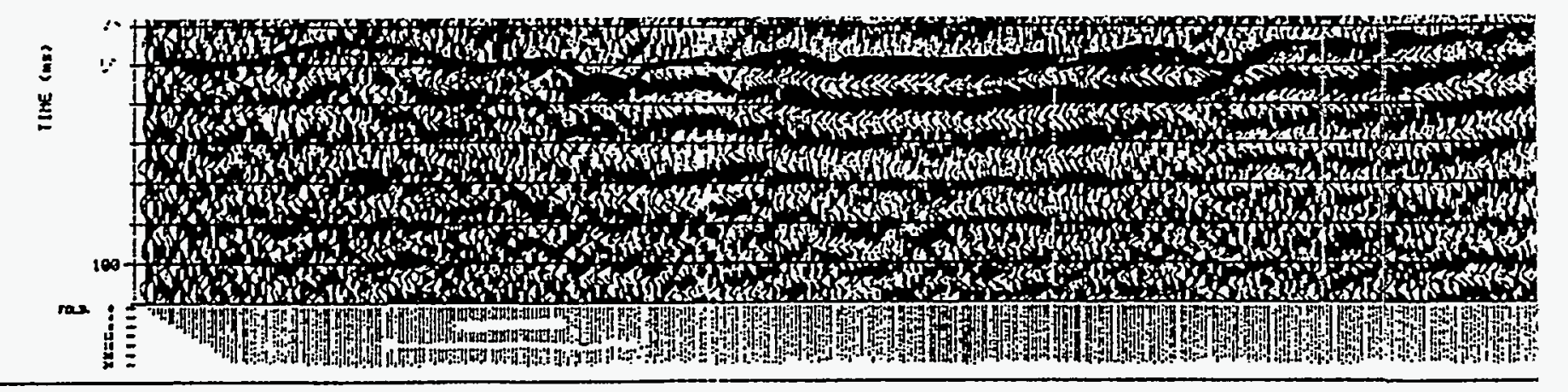

Figure $4.6 a$

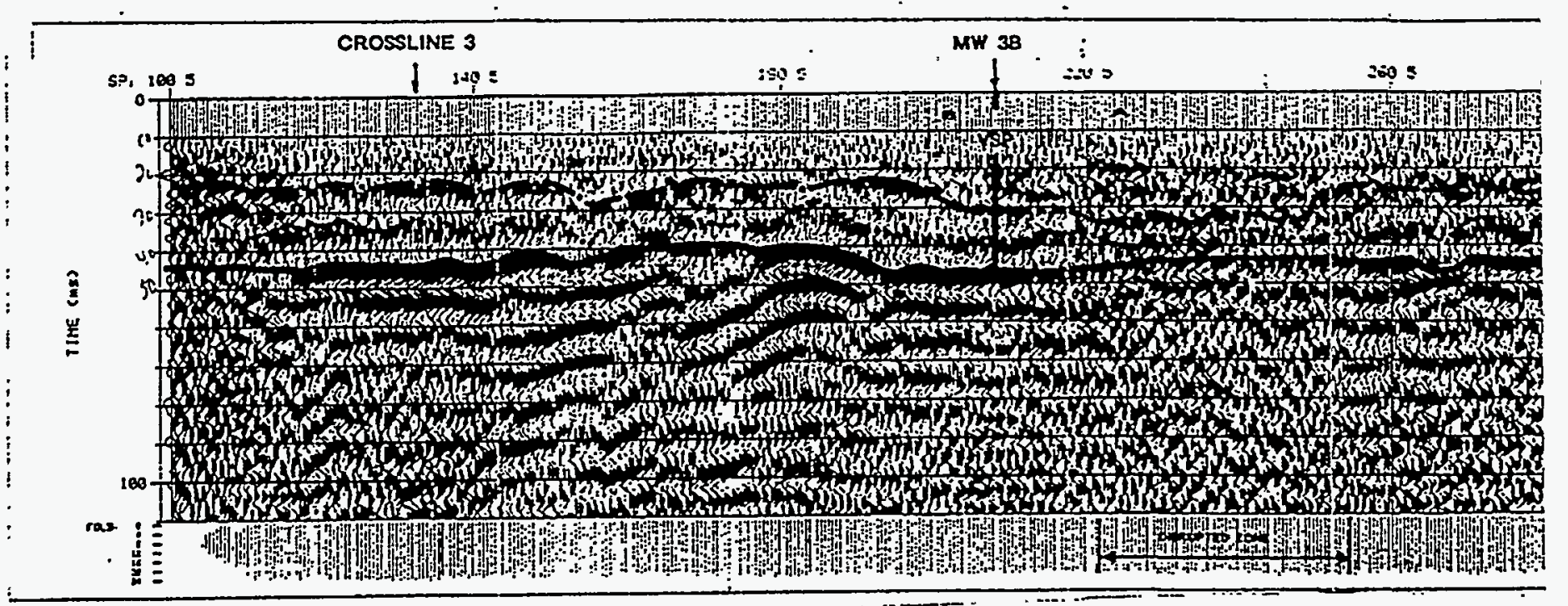

Figure $4.6 b$
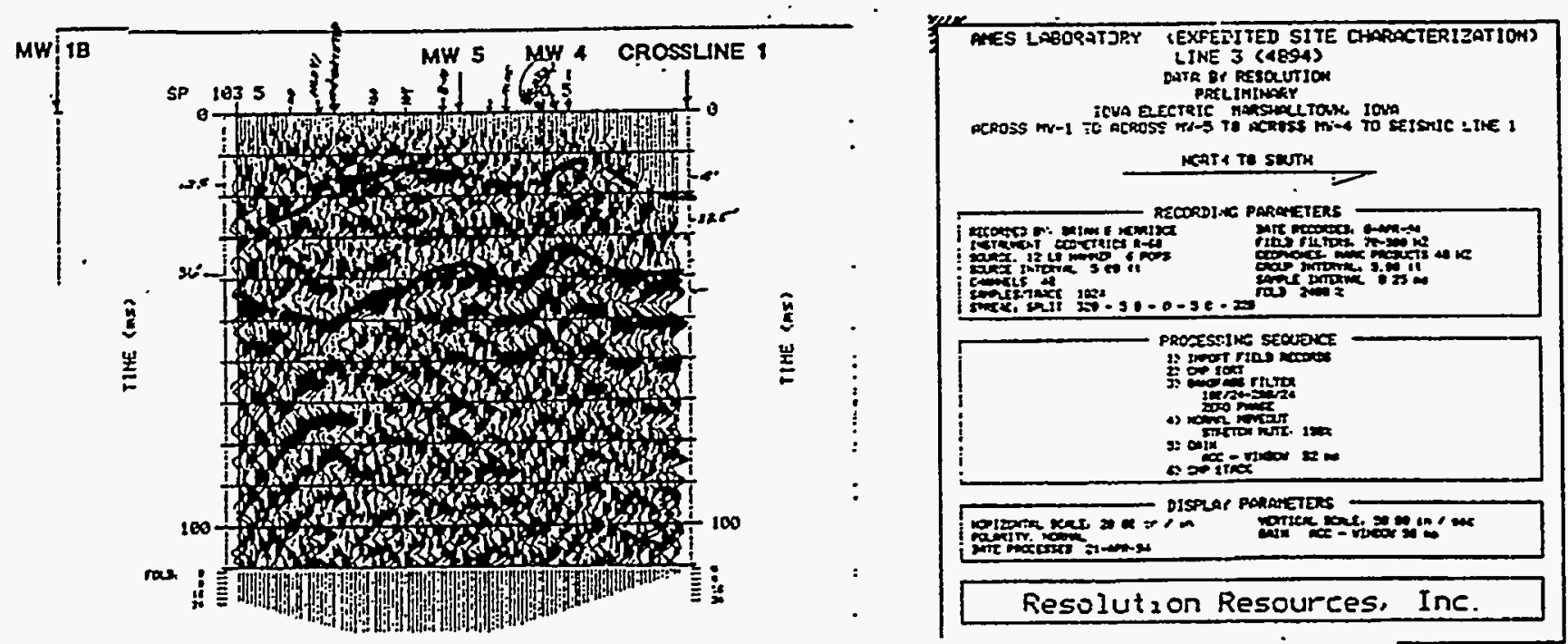

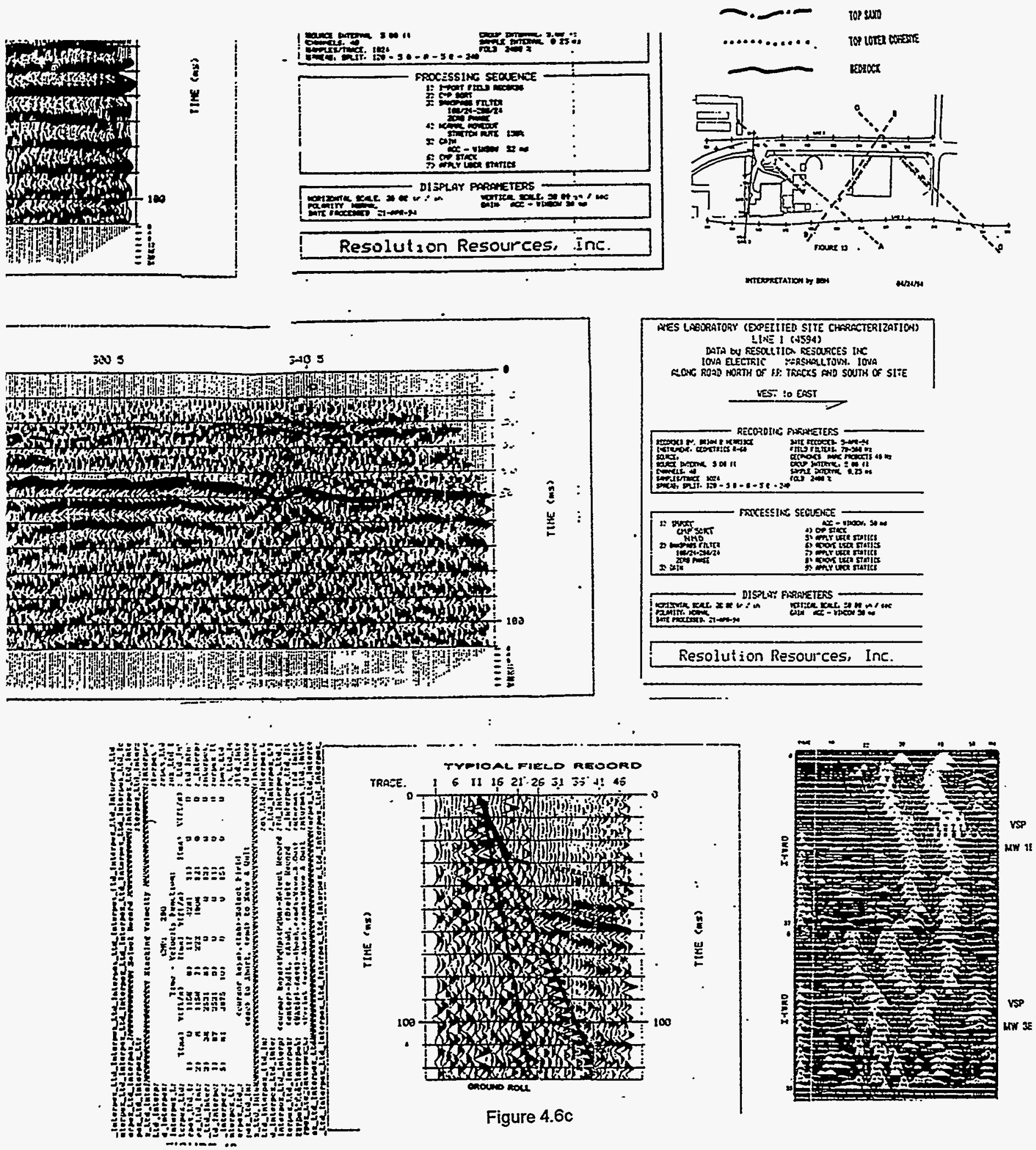

Figure 4.6c

Figure 4.6 Interpreted Seismic Reflection P (from Resolution Resources, 1994) 


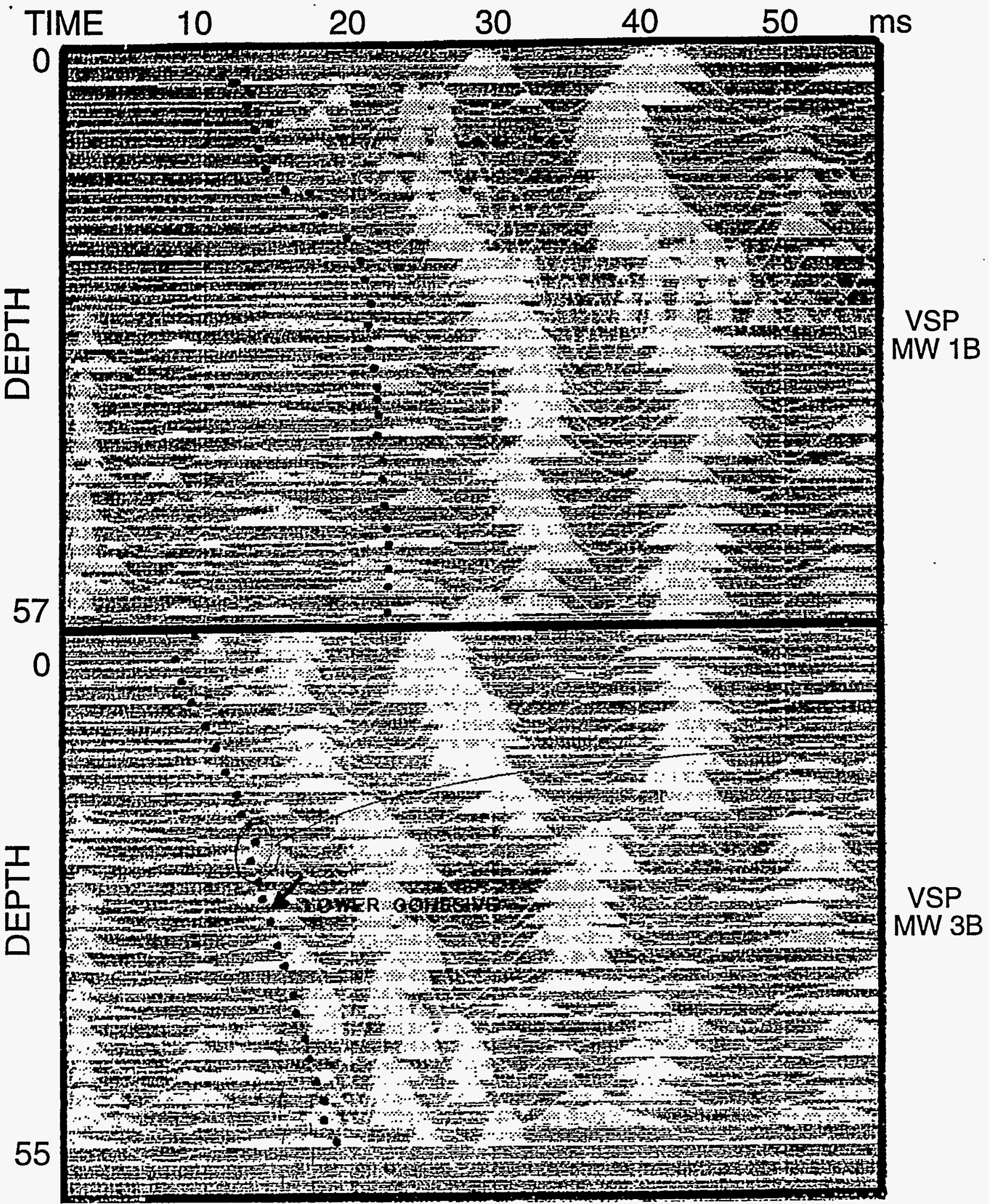

Figure 4.7 Seismic Reflection - Check Shot Survey (from Resolution Resources, 1994) 


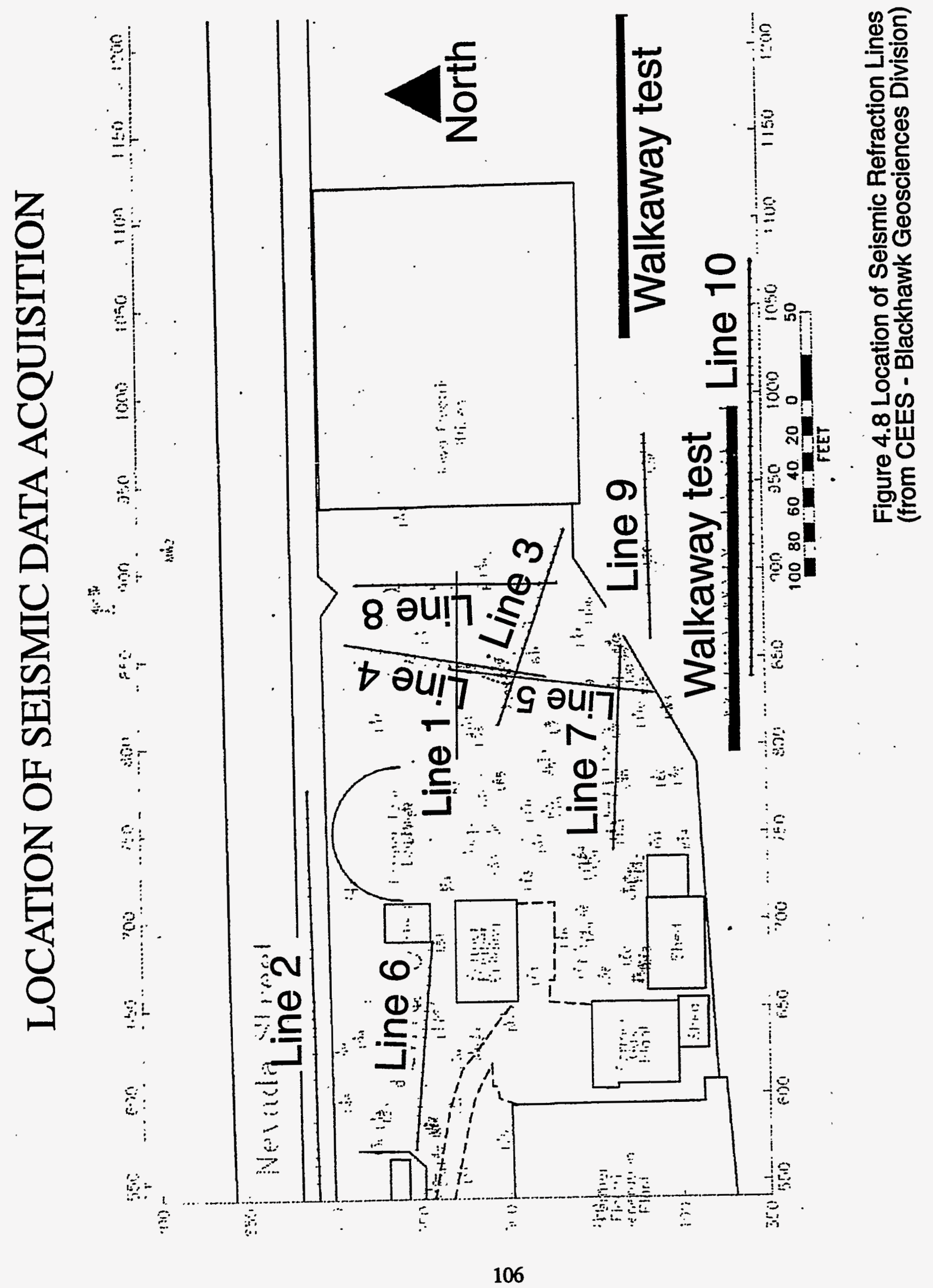




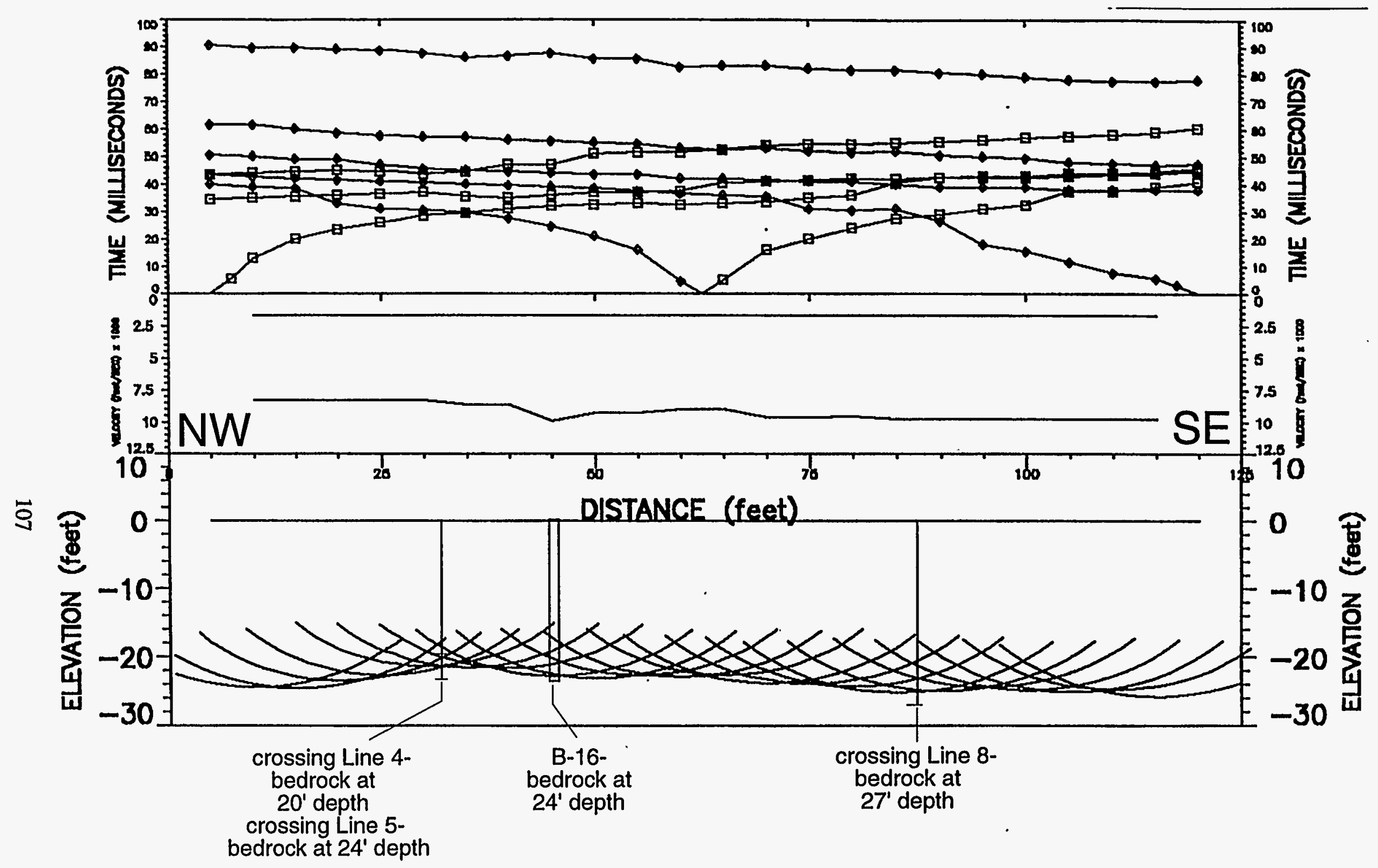

Figure 4.9 Seismic Refraction Survey Line 3 (after CEES - Blackhawk Geosciences Division) 


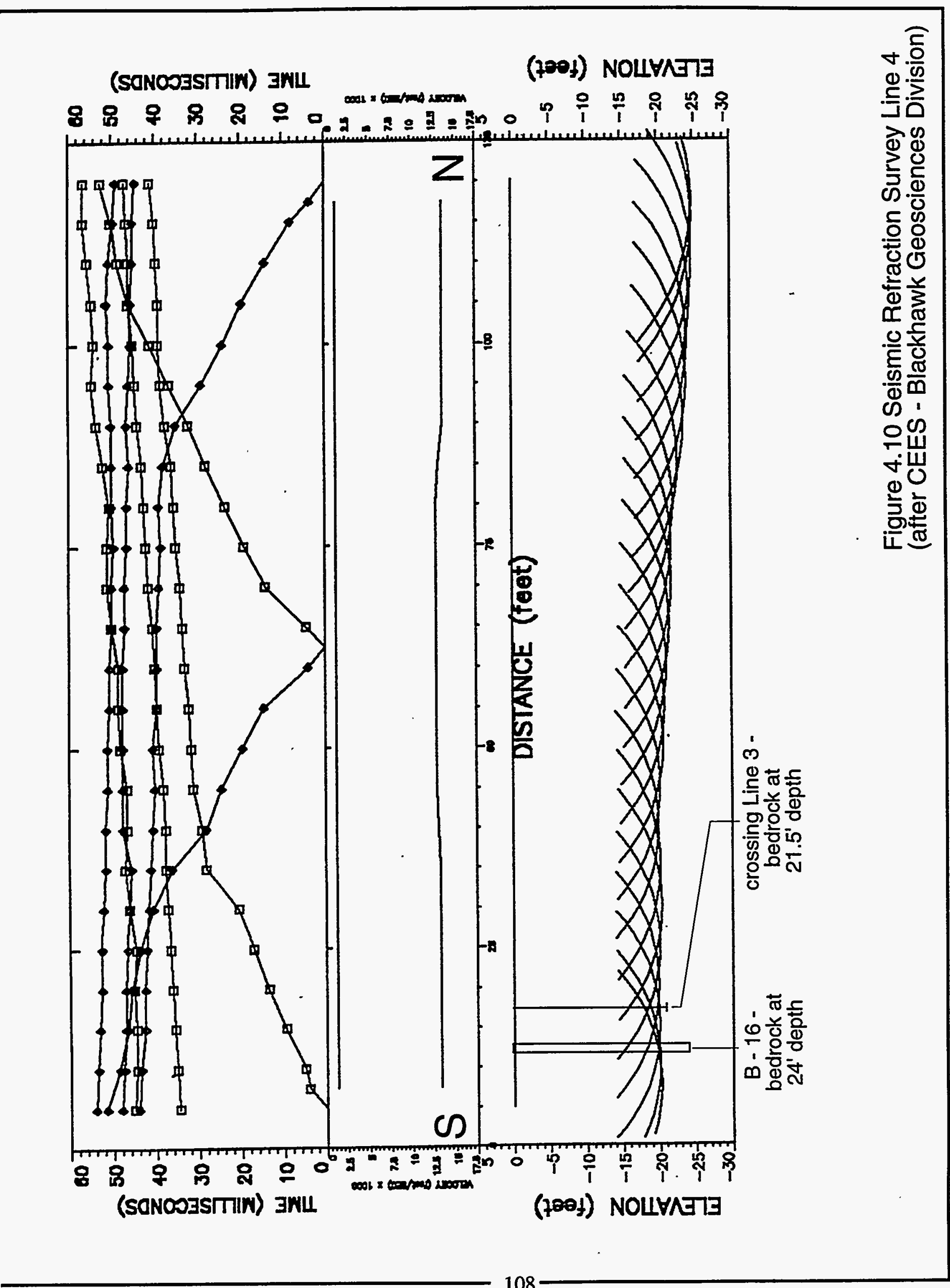




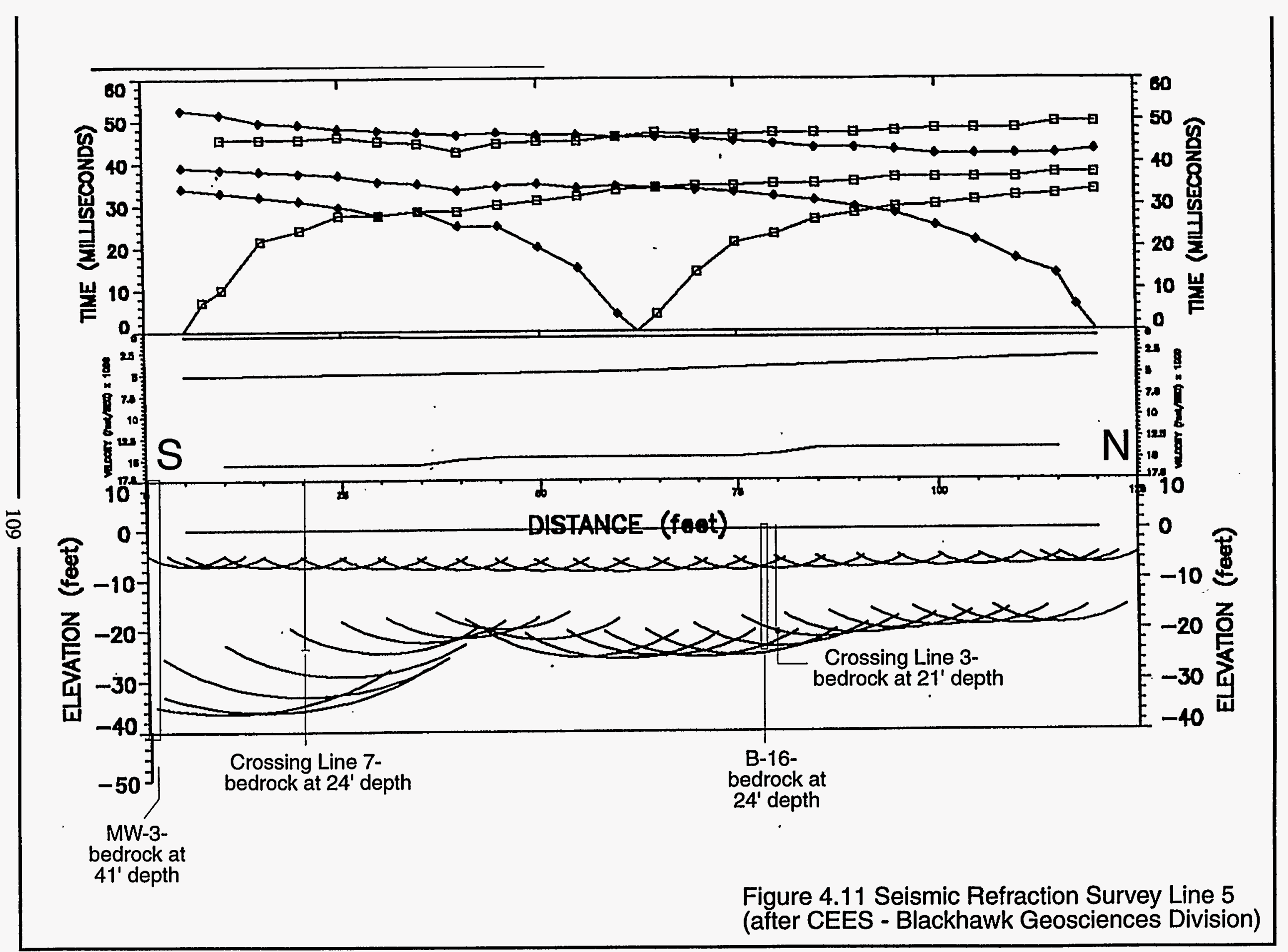




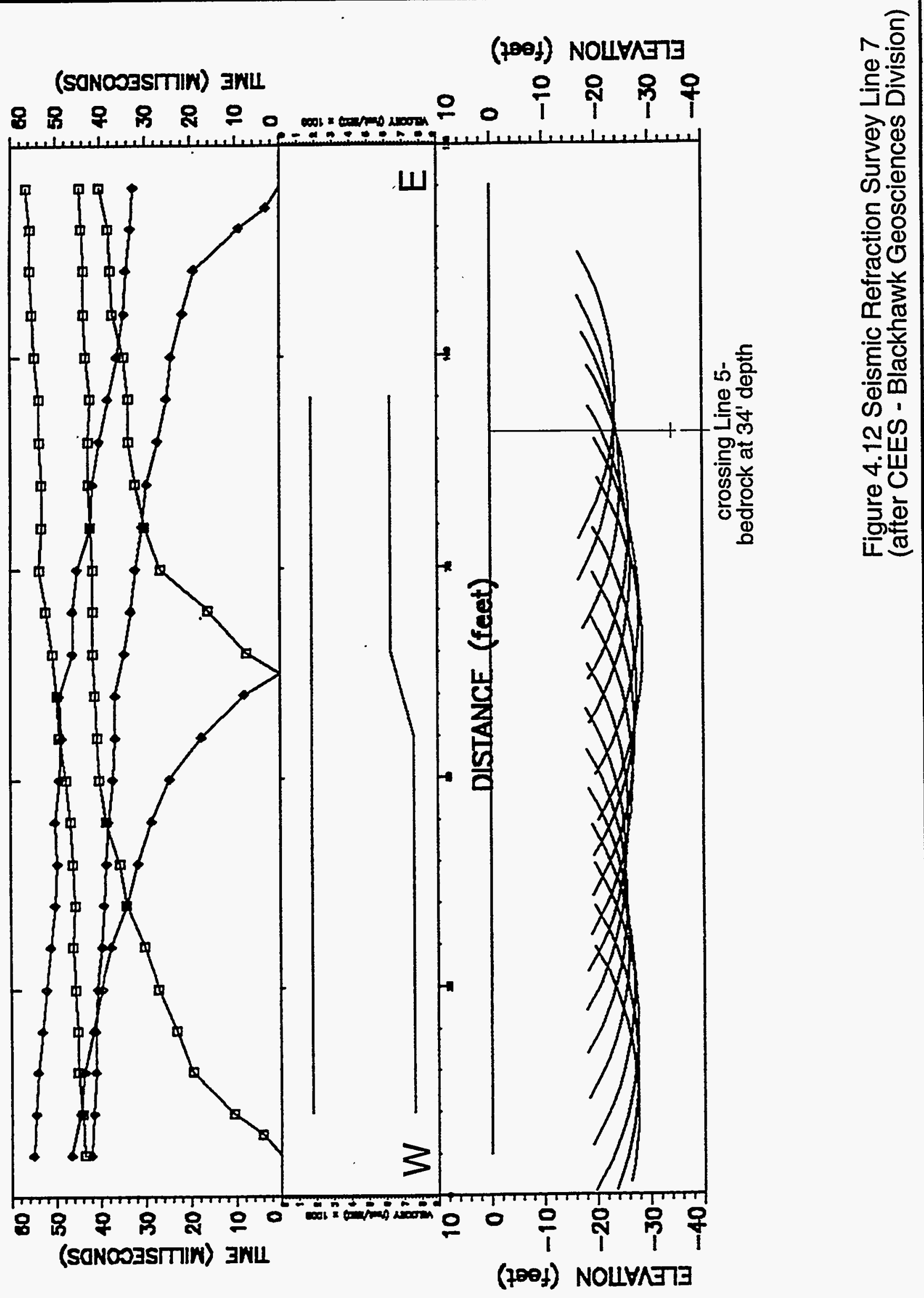




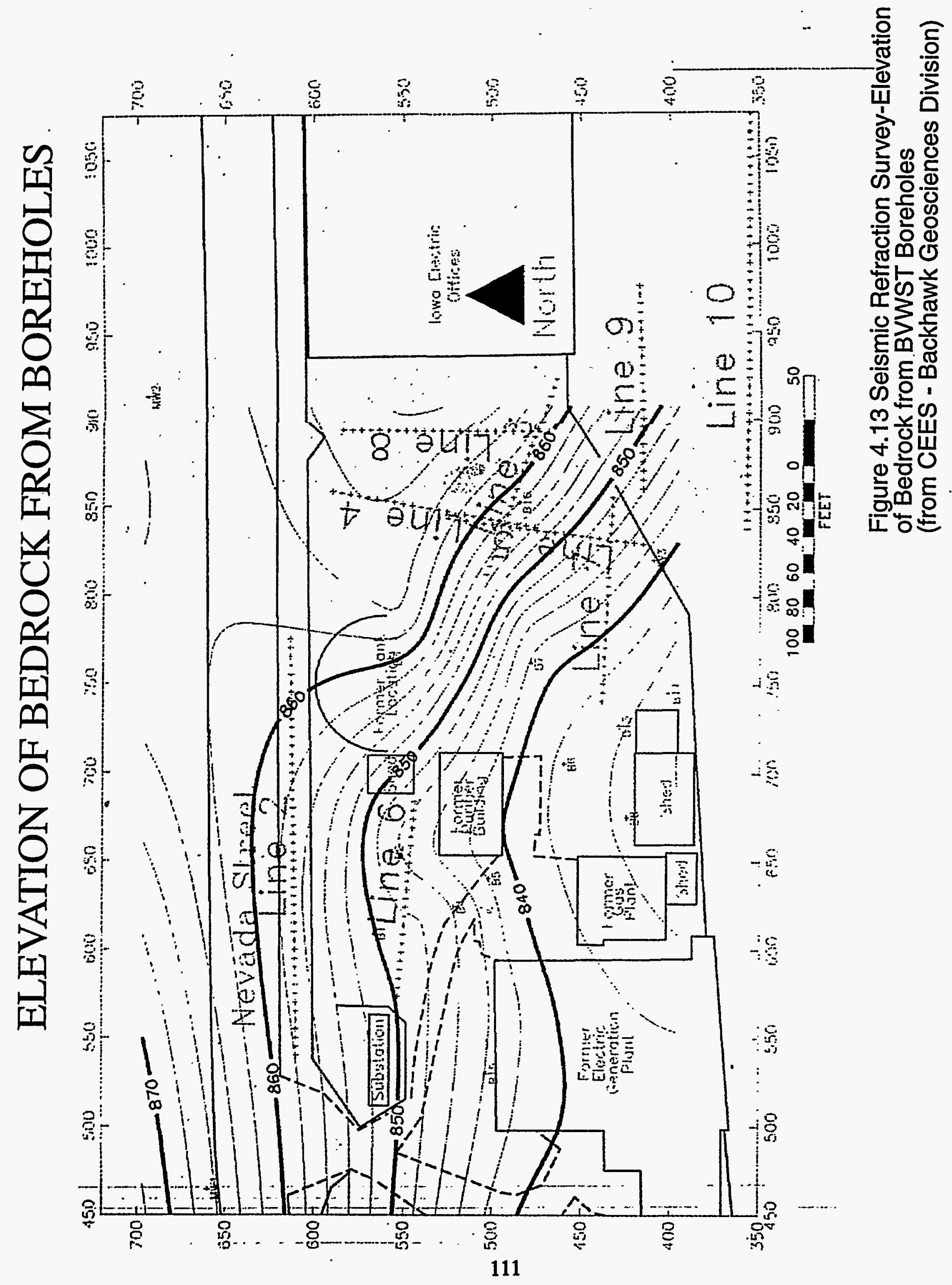




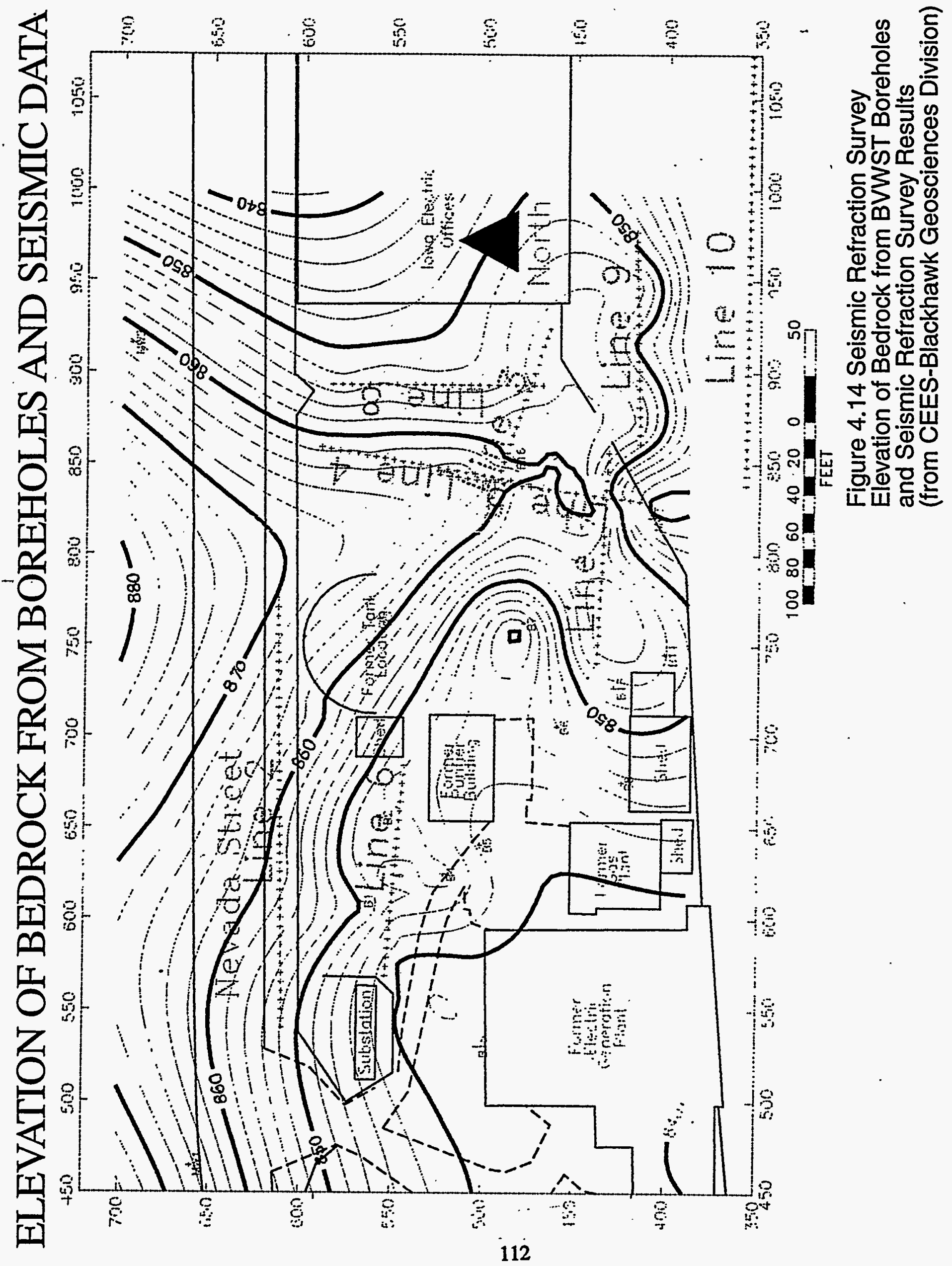




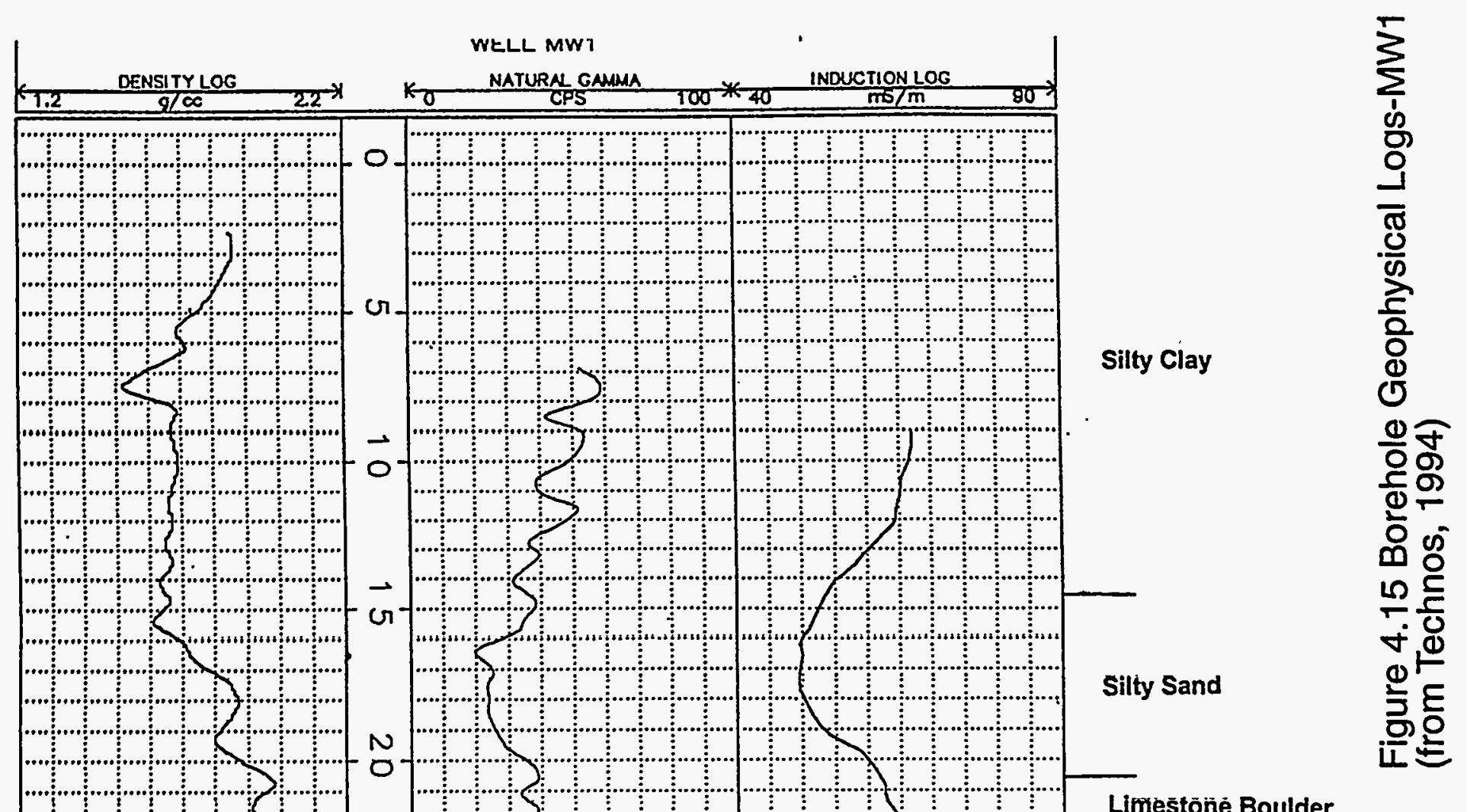

Limeštōnèe Boulder

Silty Clay

Limestone

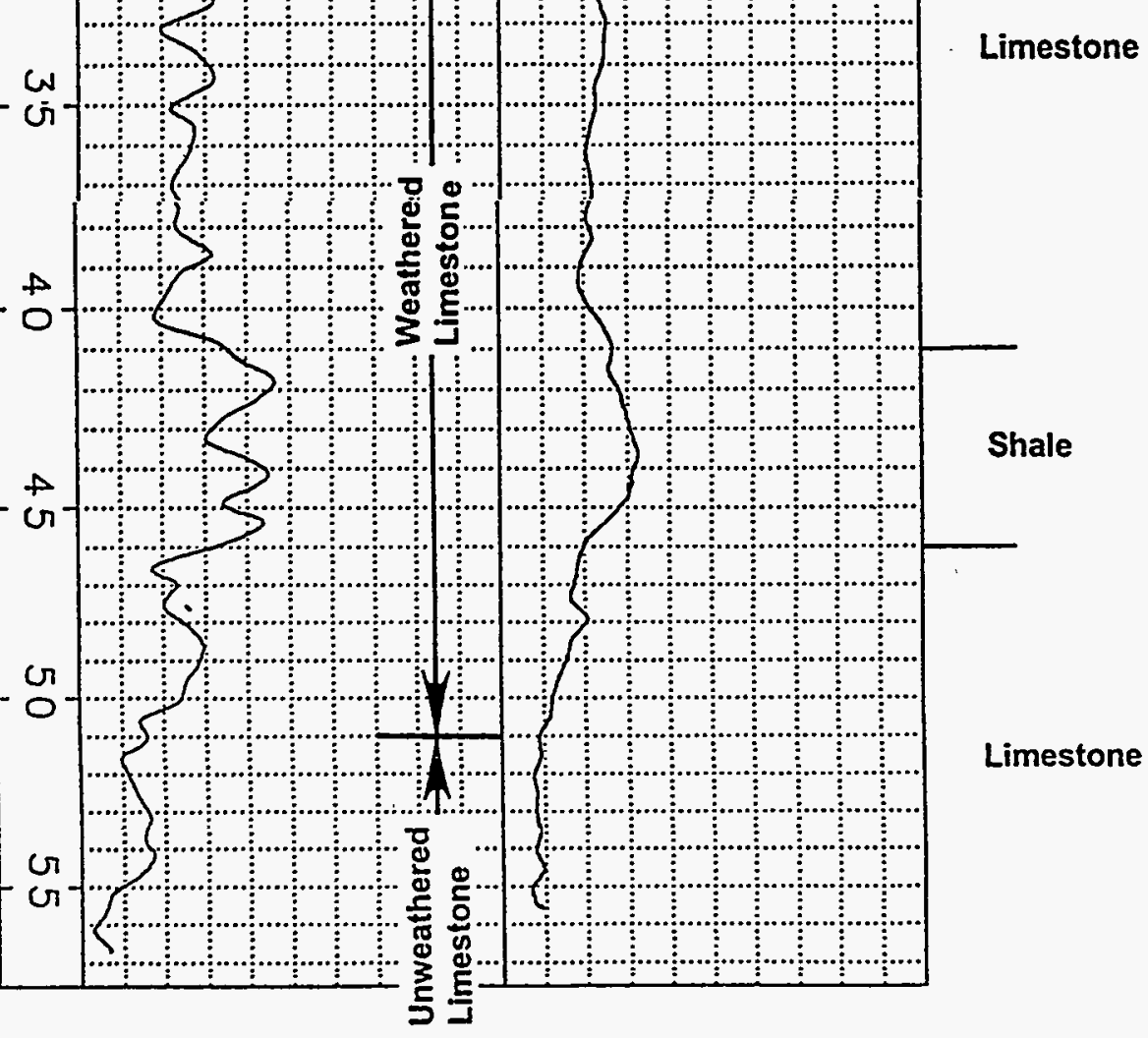




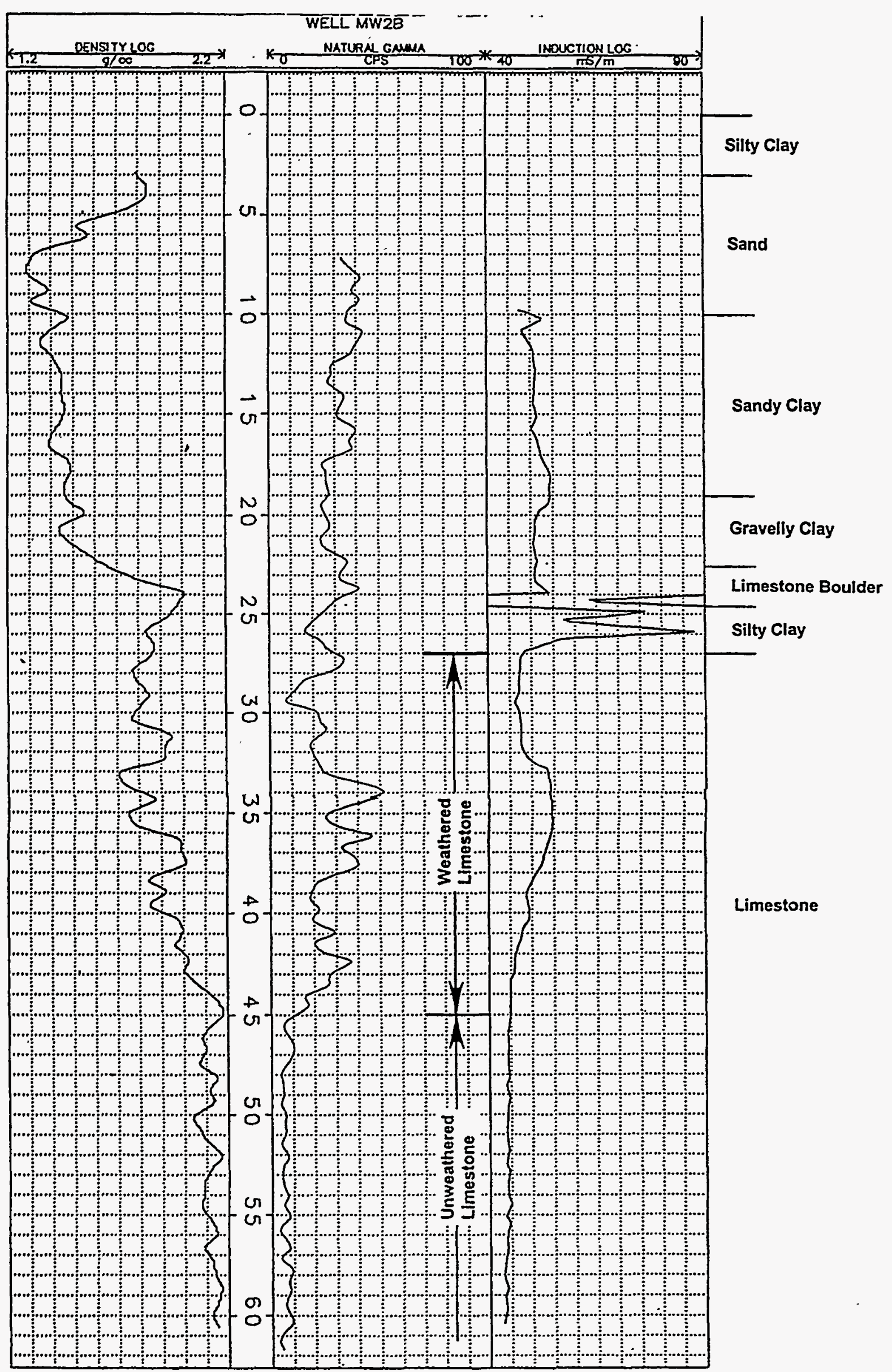




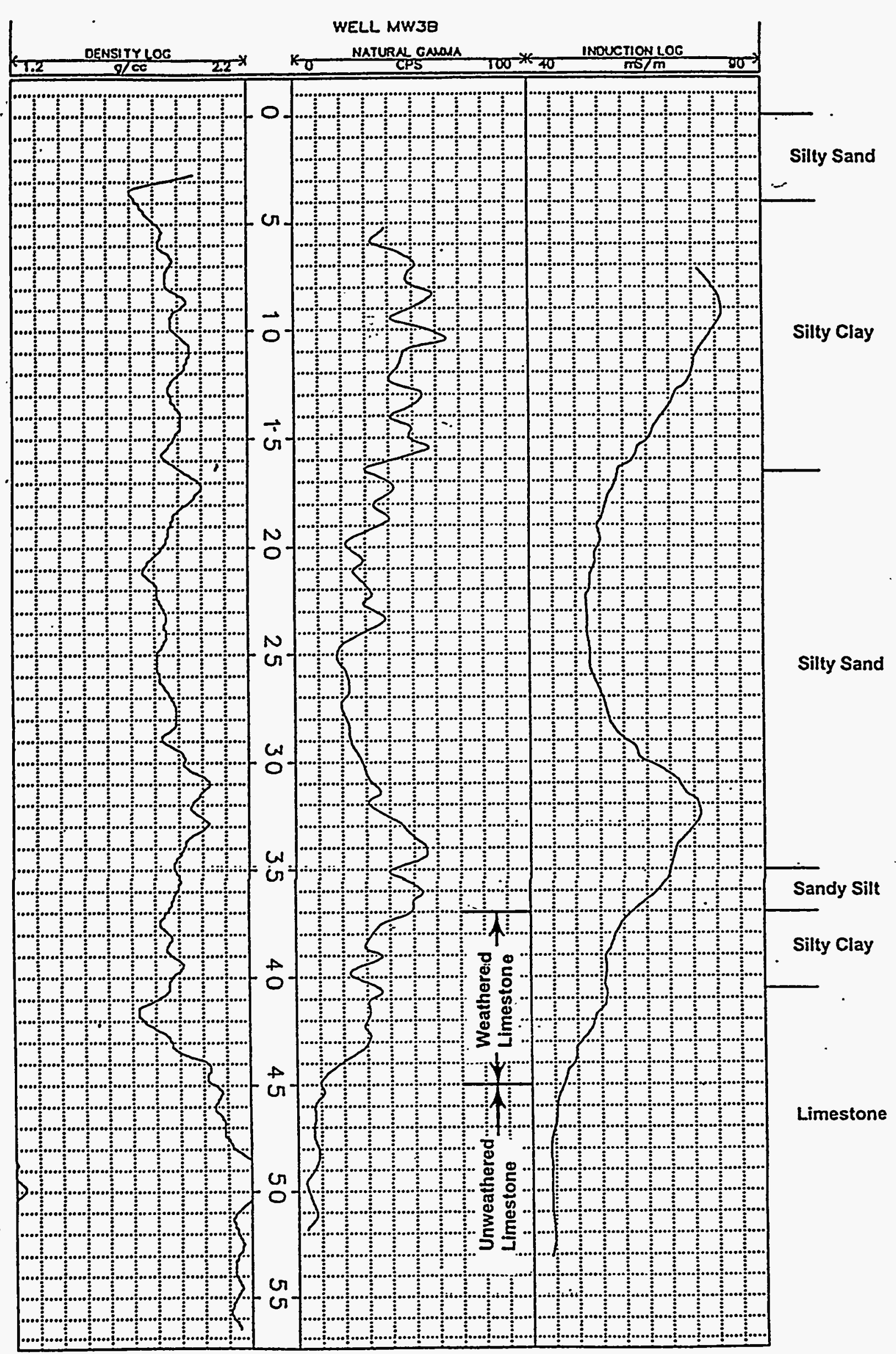




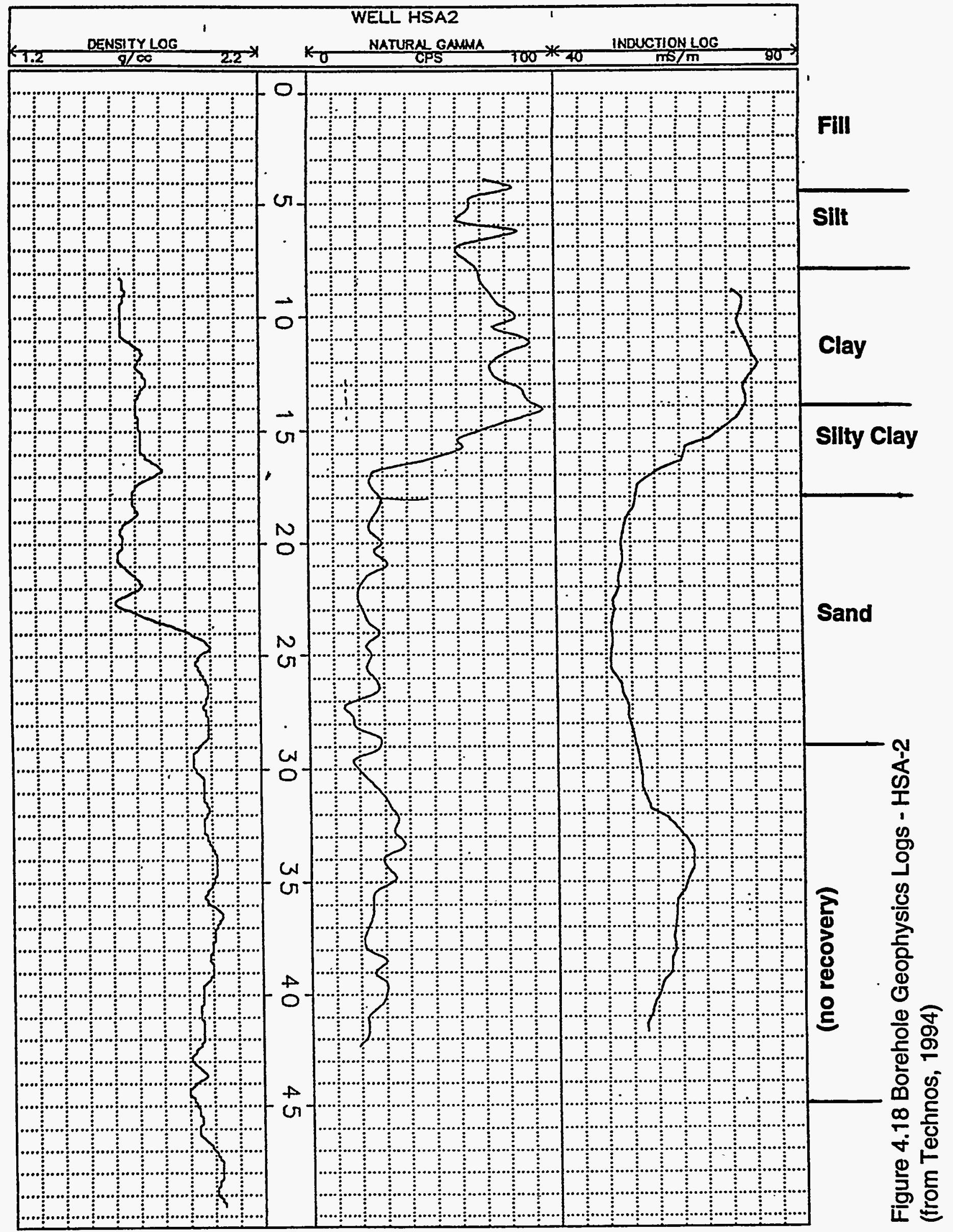




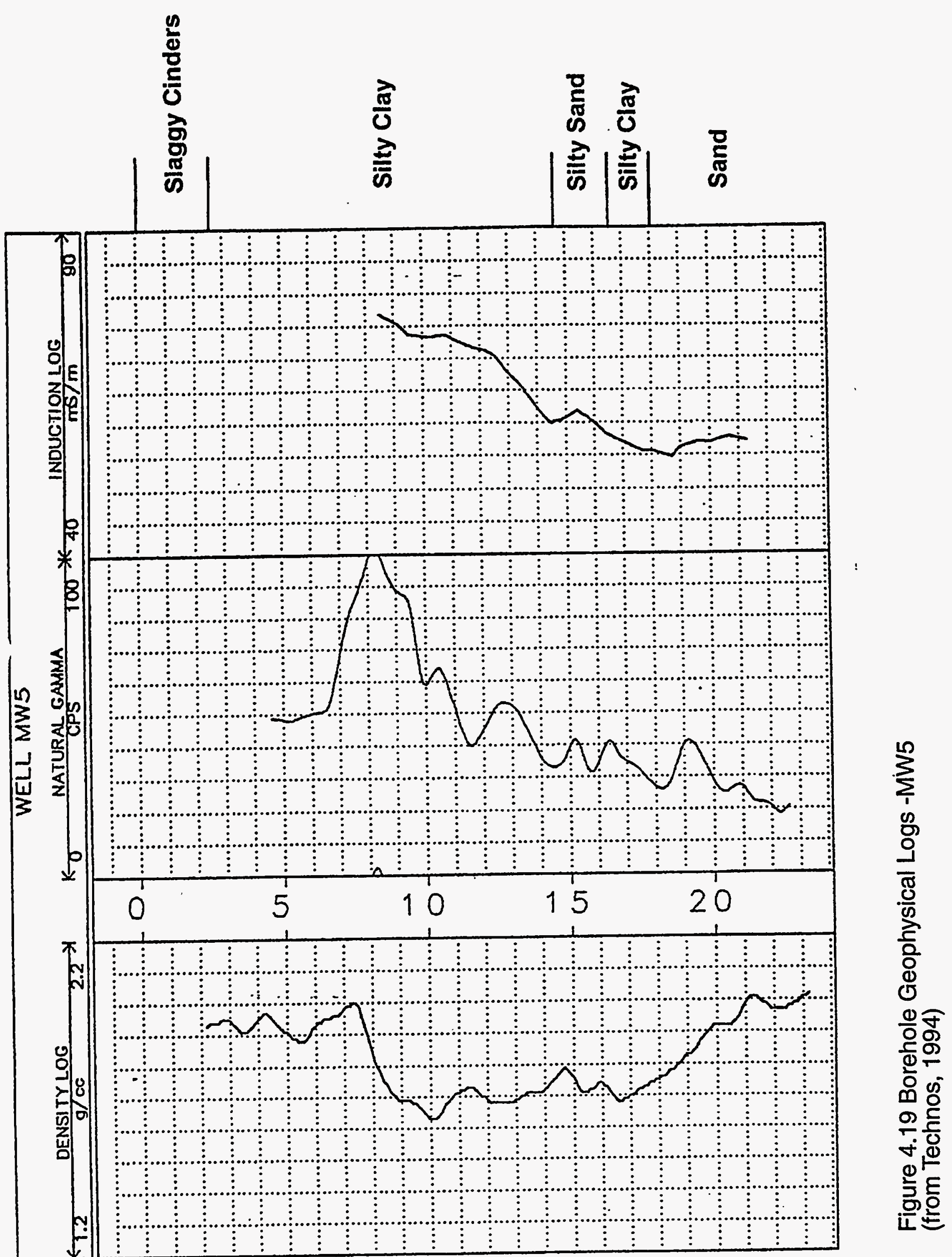




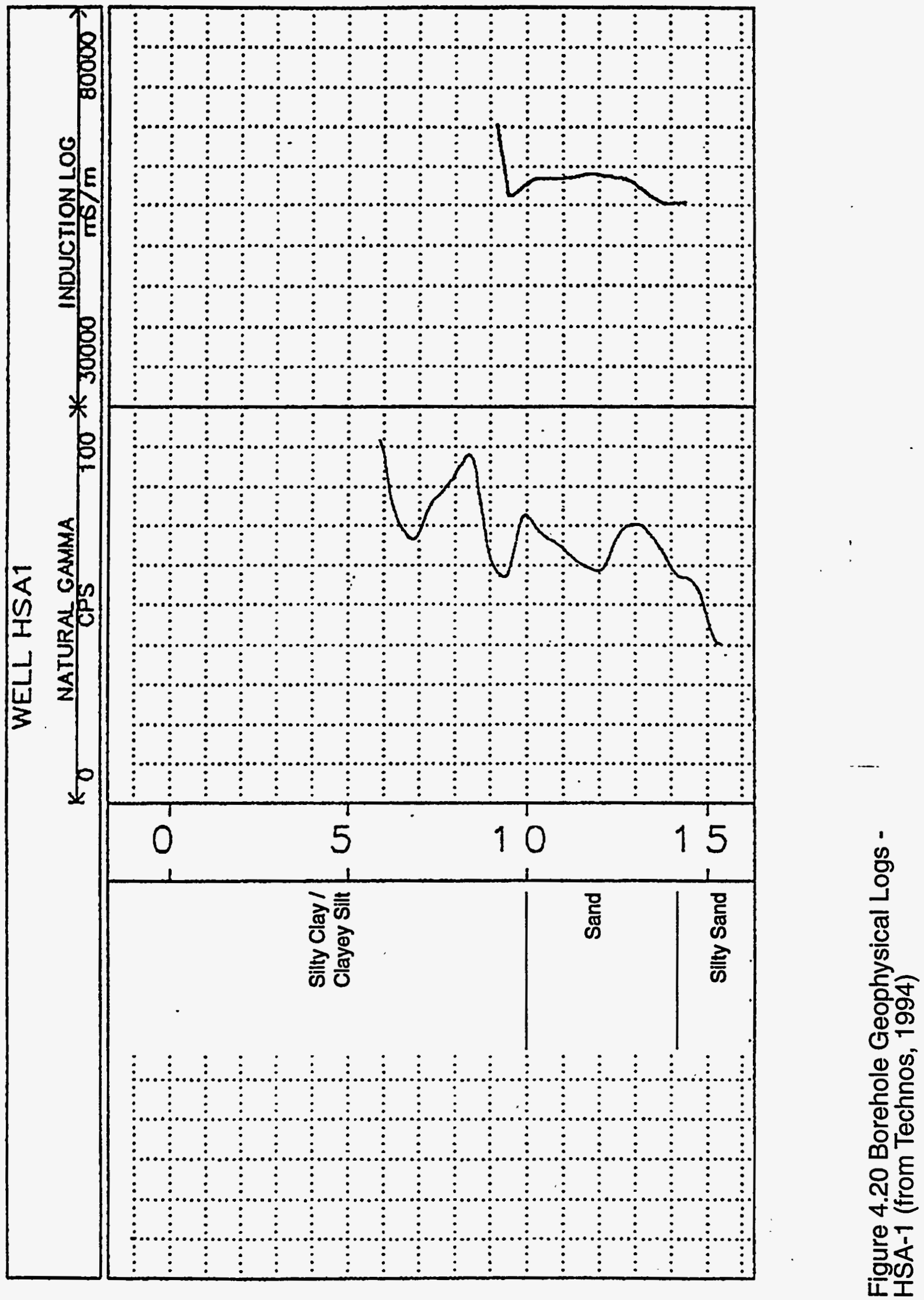




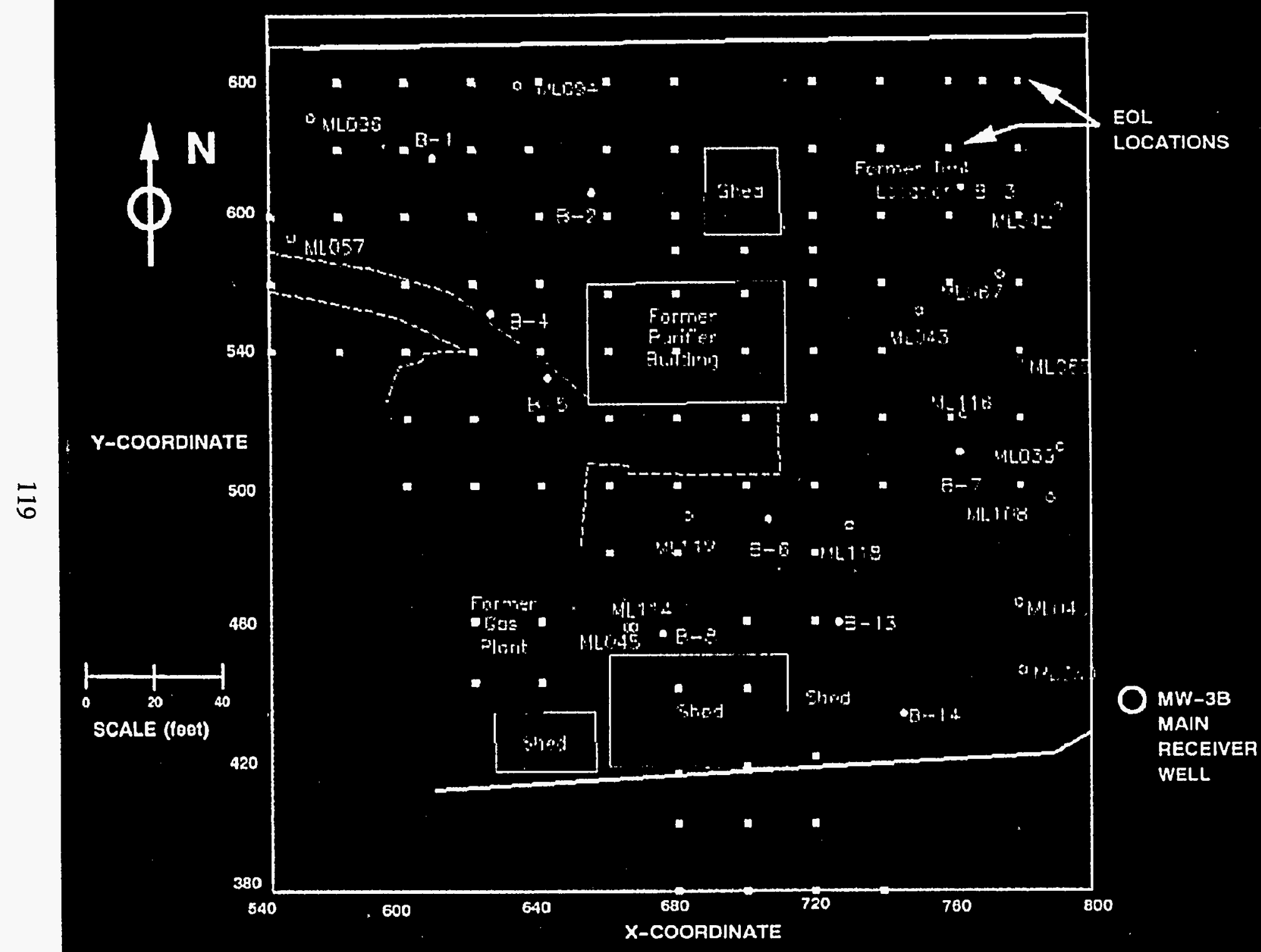

Figure 4.21

Electromagnetic Offset Logging - Source and Receiver map Locations (from GEHM, 1994)

LEGEND:

a

EM Source Coil (EOL) Locations

O MW-3B EM Aeceiver Well

O MW-3B Monitor Well

B-14 Soll Borings

- ML-80 Cone Penetrometer

\begin{tabular}{|c|}
\hline 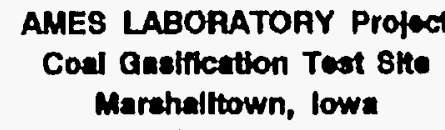 \\
\hline 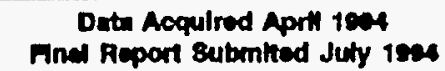 \\
\hline 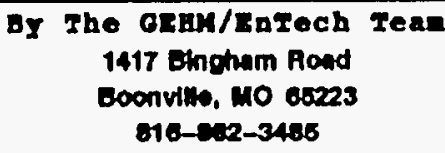 \\
\hline
\end{tabular}




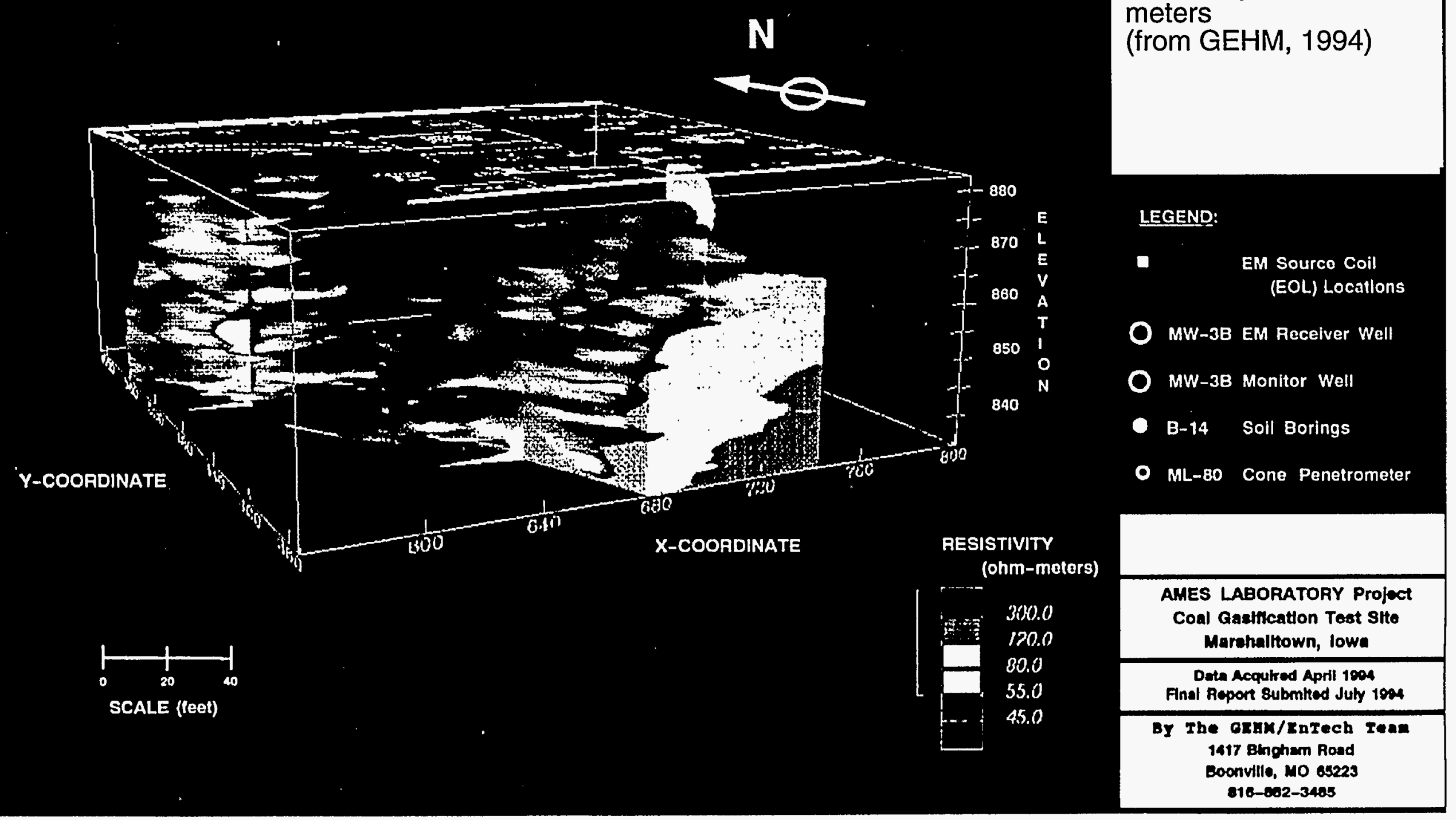

Figure 4.22

Electromagnetic Offset Logging - 3-D Image of Resistivity $>55$ Ohmmeters

(from GEHM, 1994) 


\section{DEPTH TO BEDROCK FROM BOREHOLES AND SEISMIC DATA}

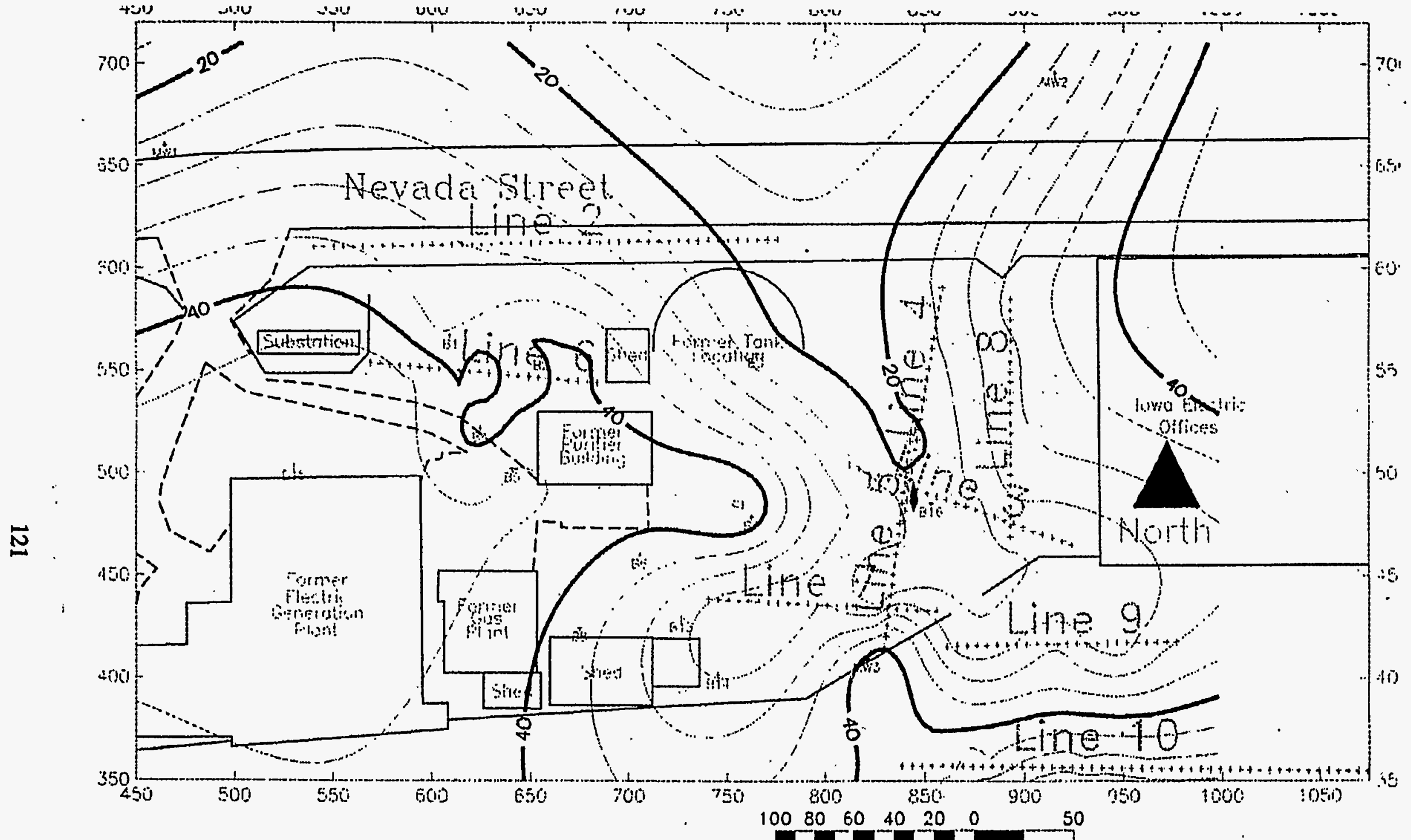

Figure 4.23 Seismic Refraction Survey -

Depth to Bedrock from BVWST Boreholes and Seismic Refraction Survey Results

(from CEES-Blackhawk Geosciences Division) 


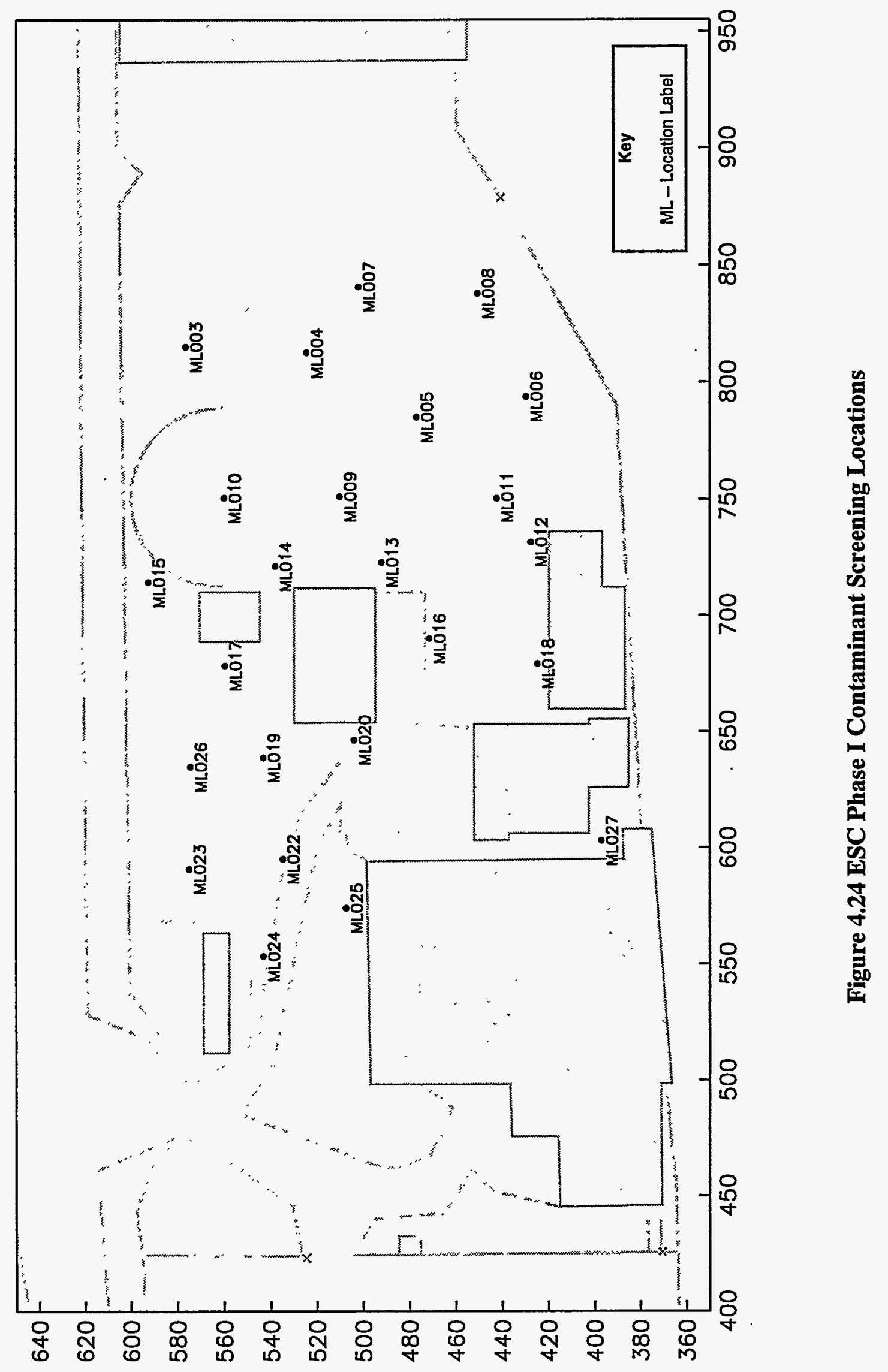




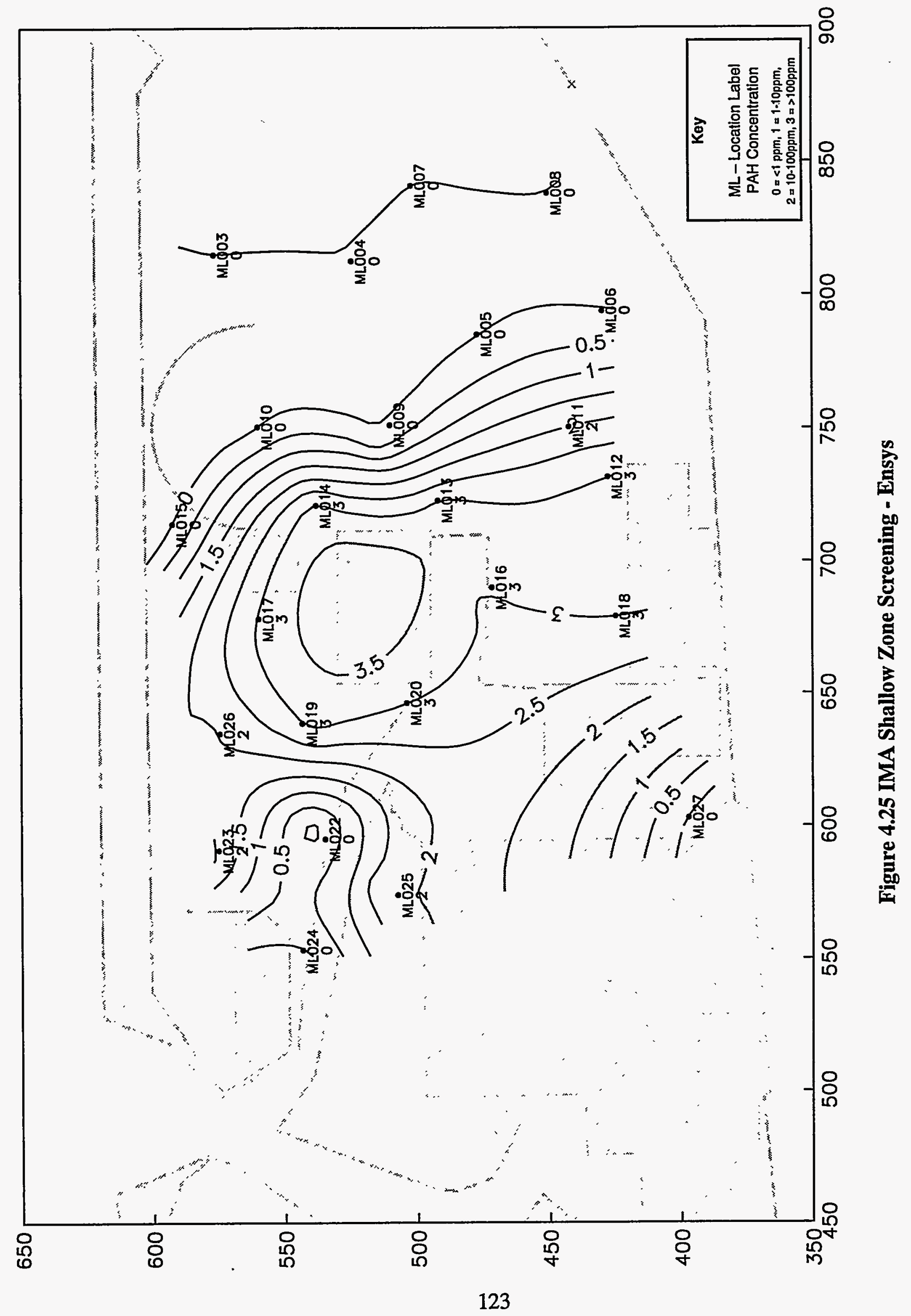




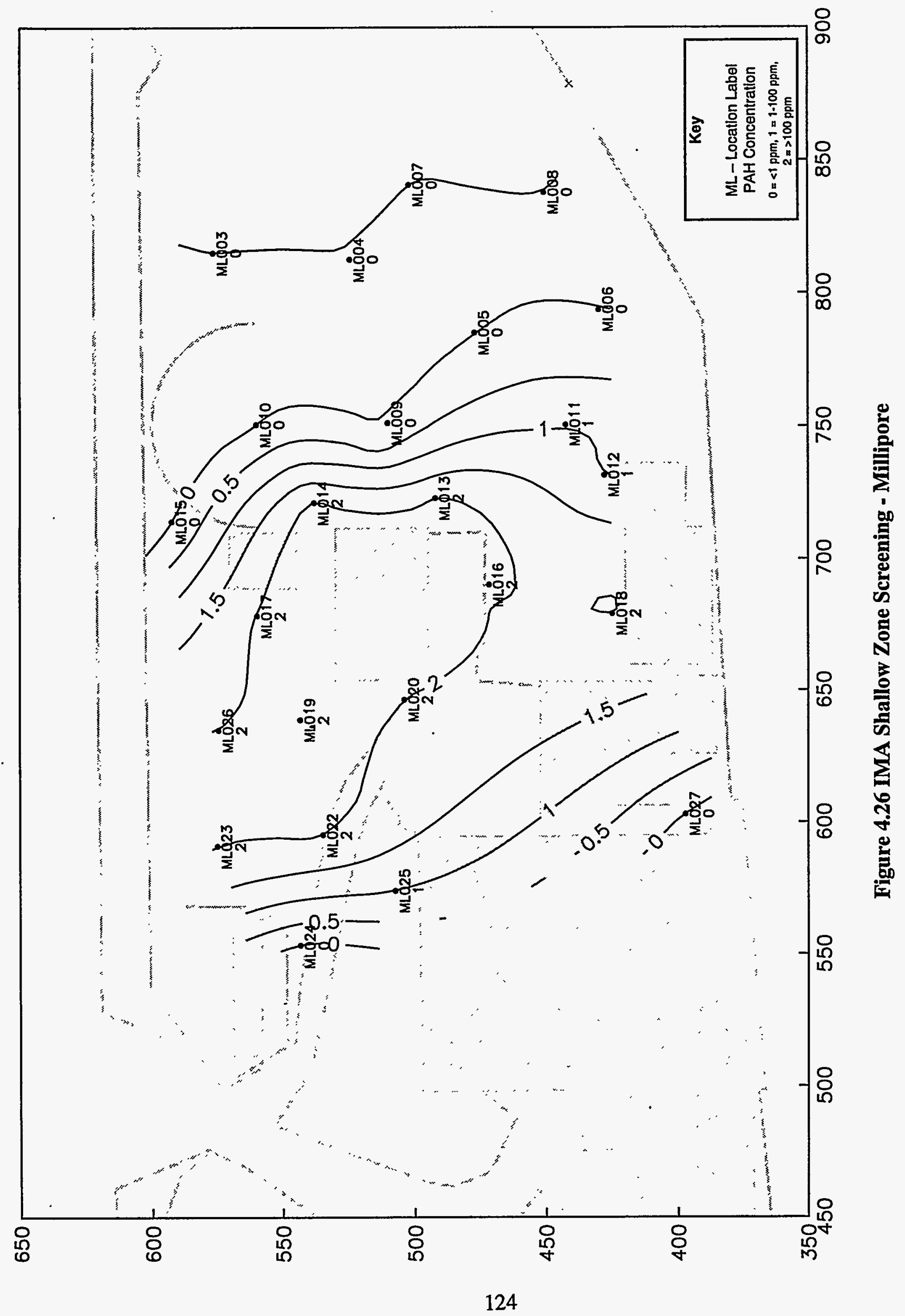




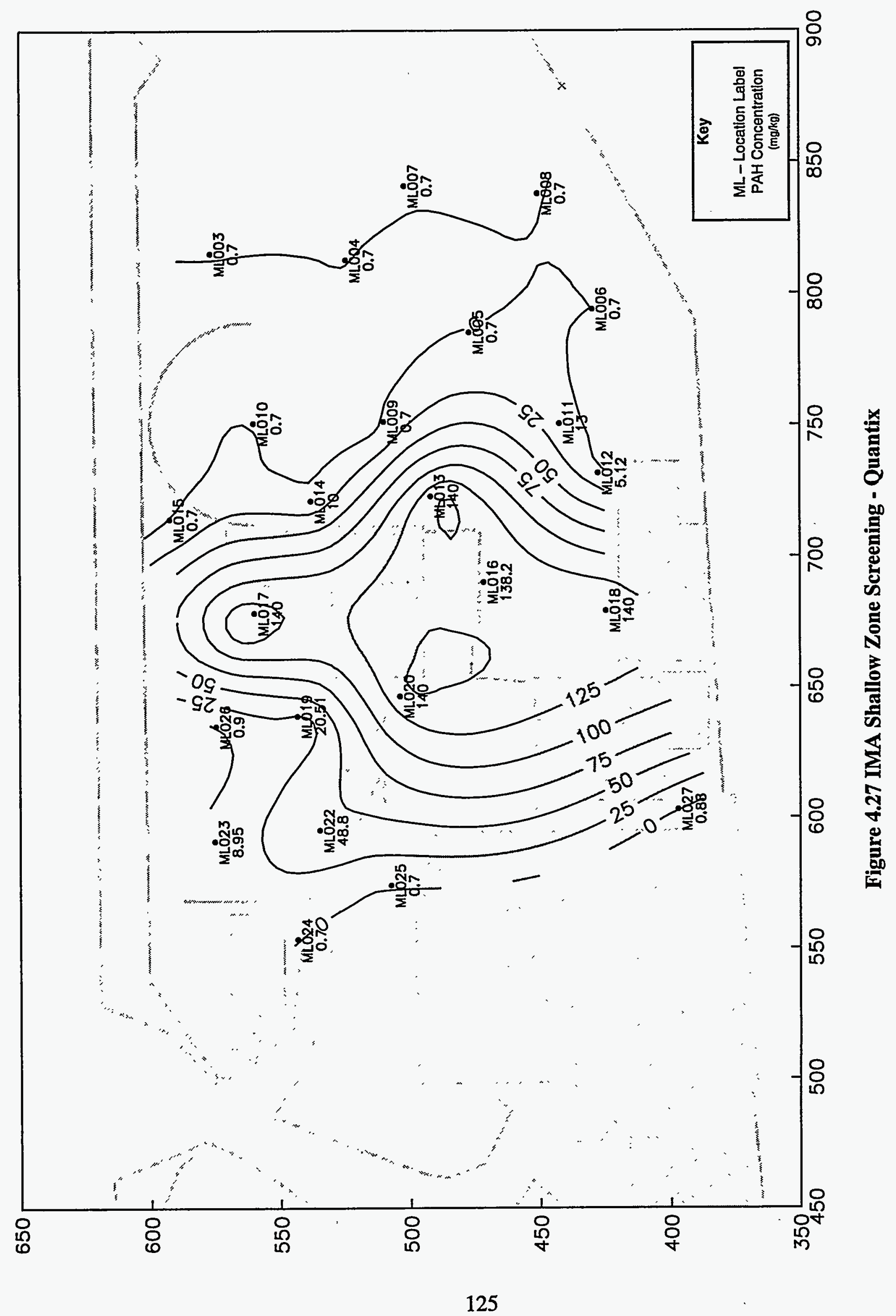




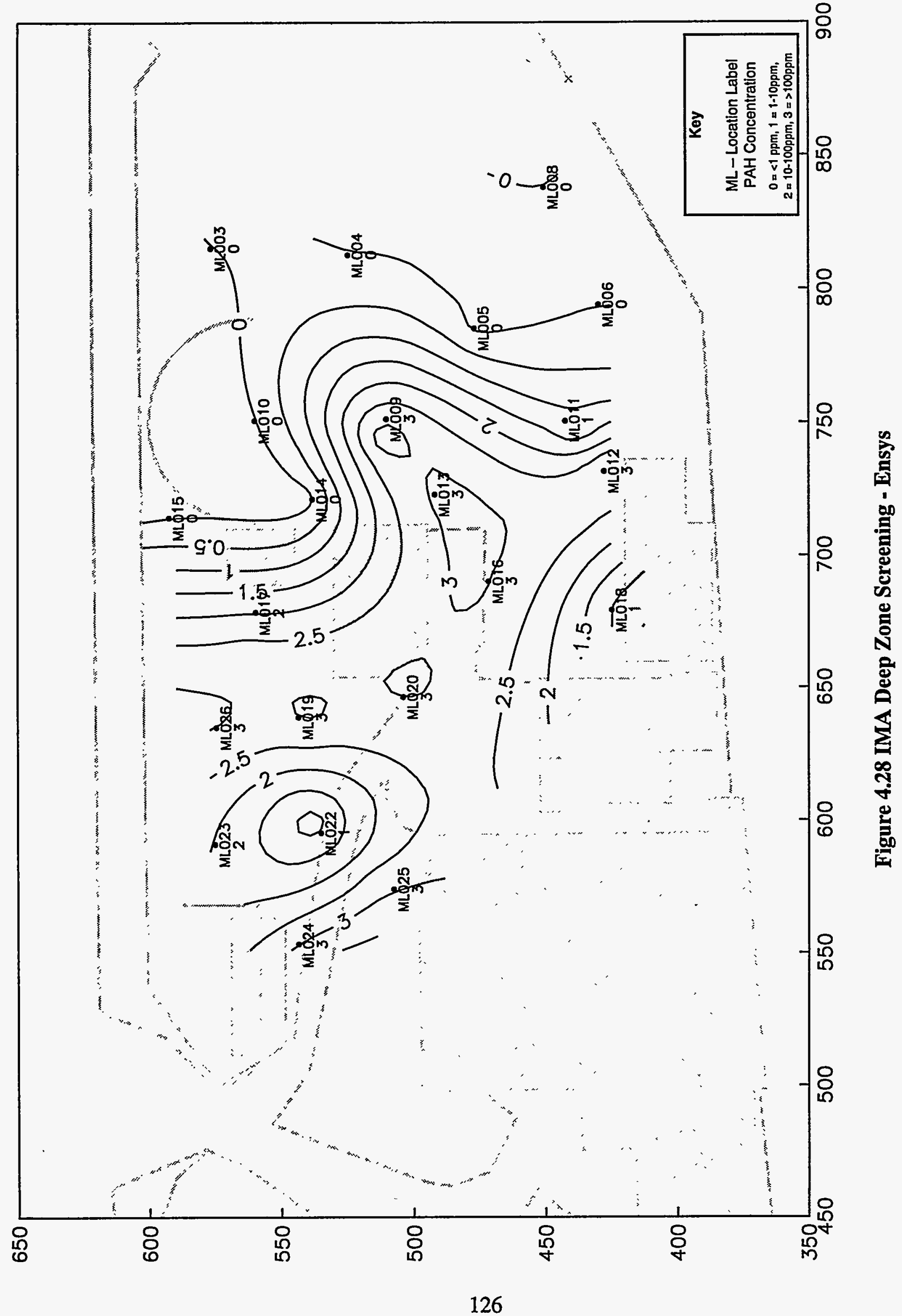




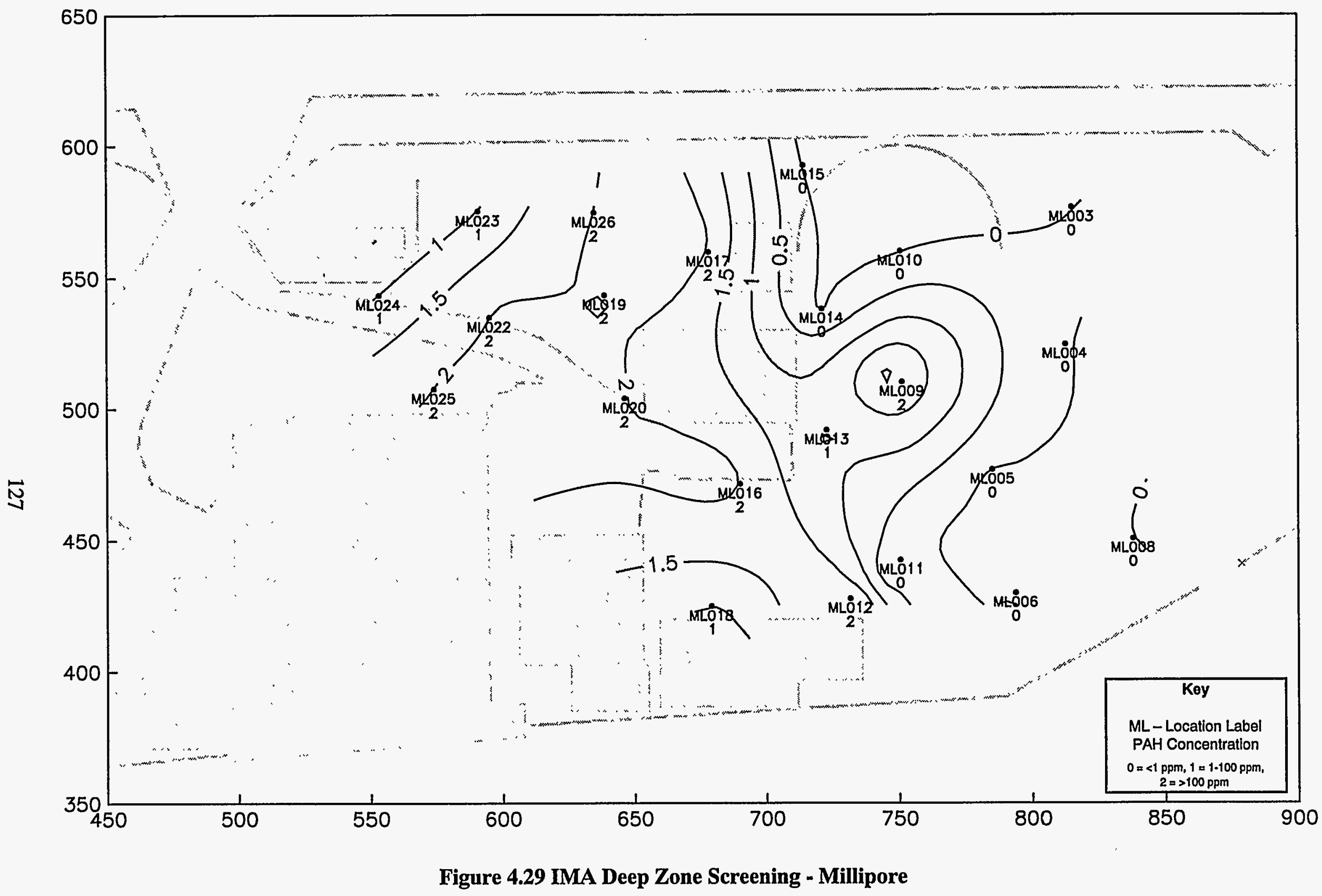




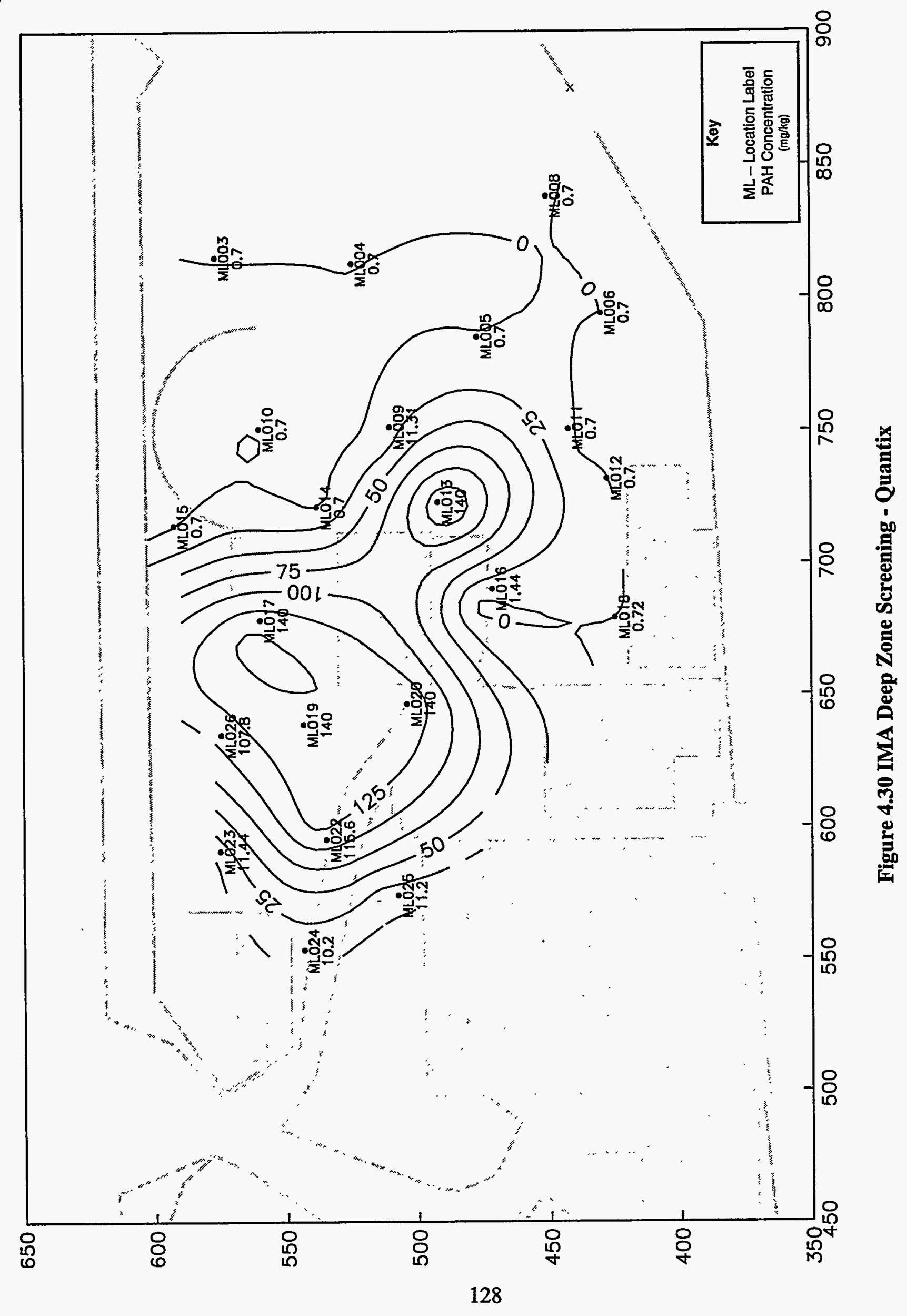




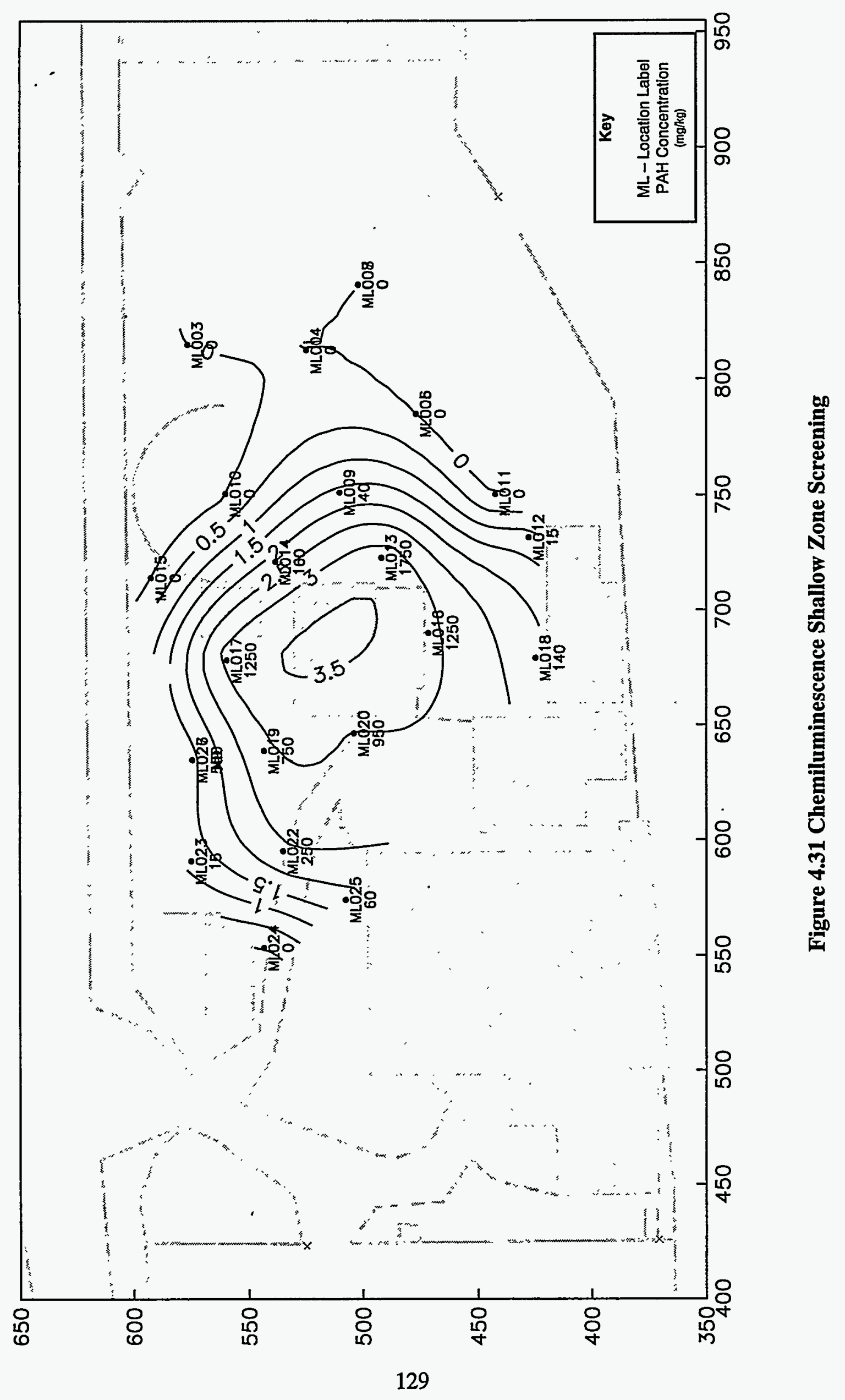




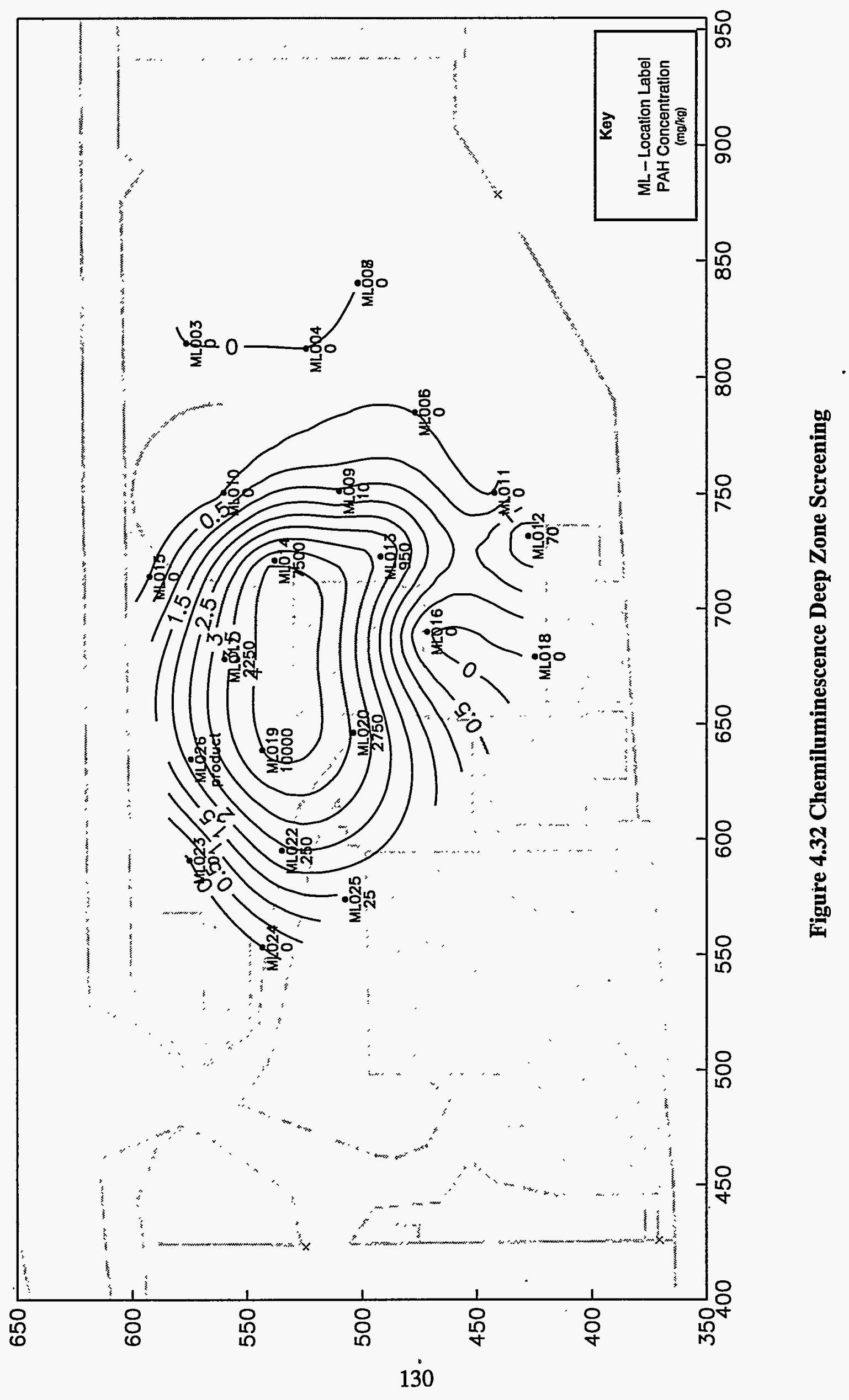




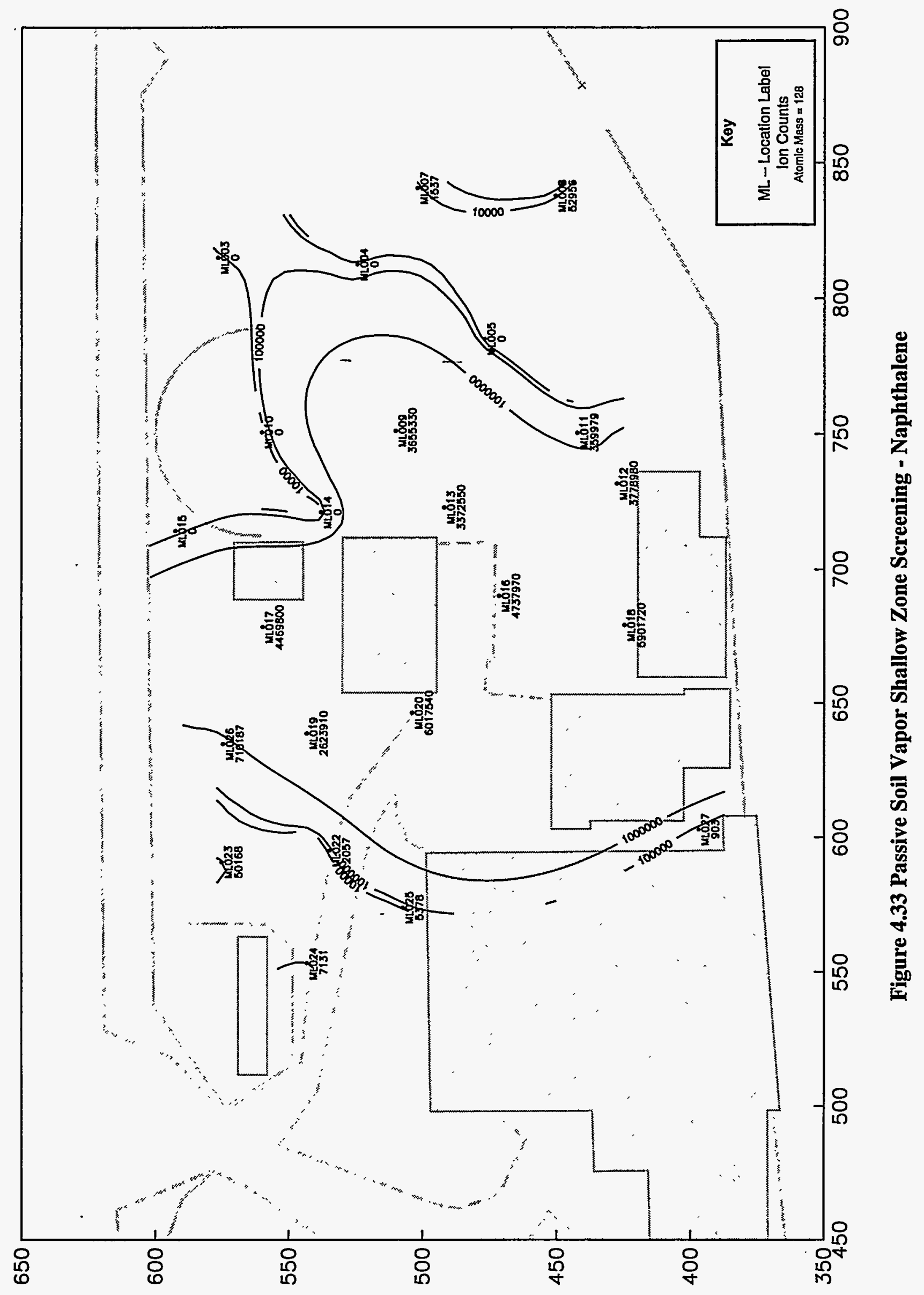




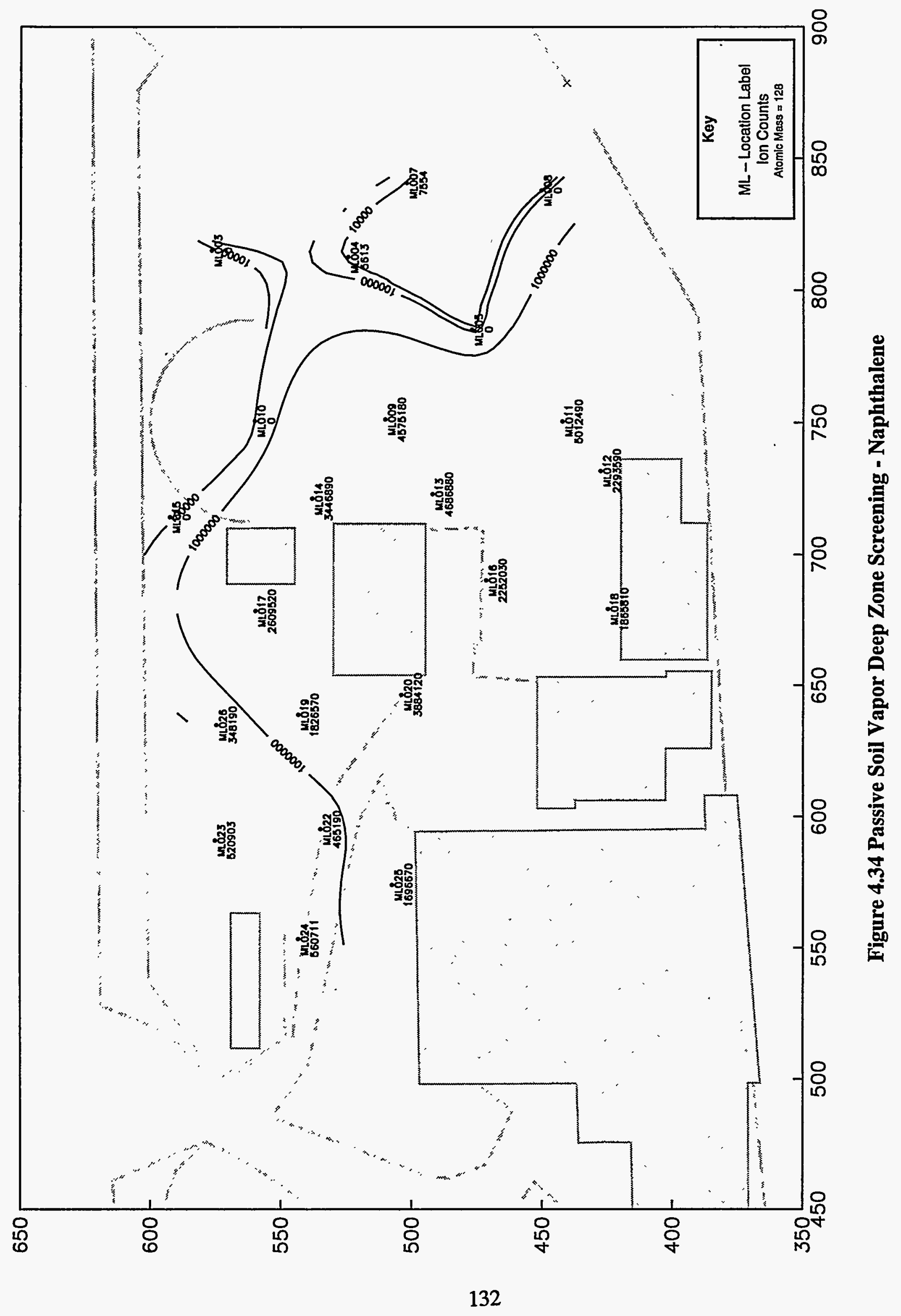




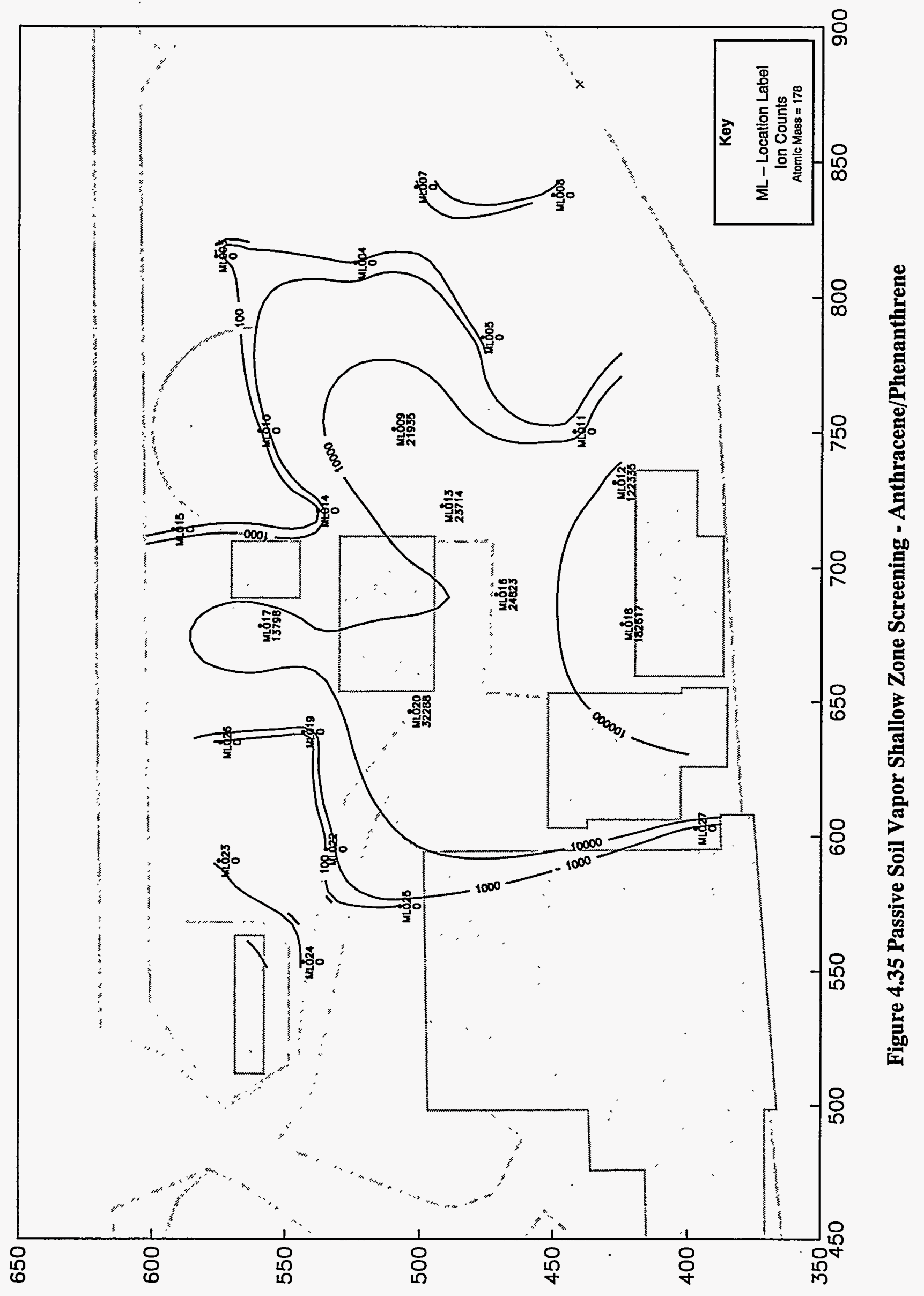




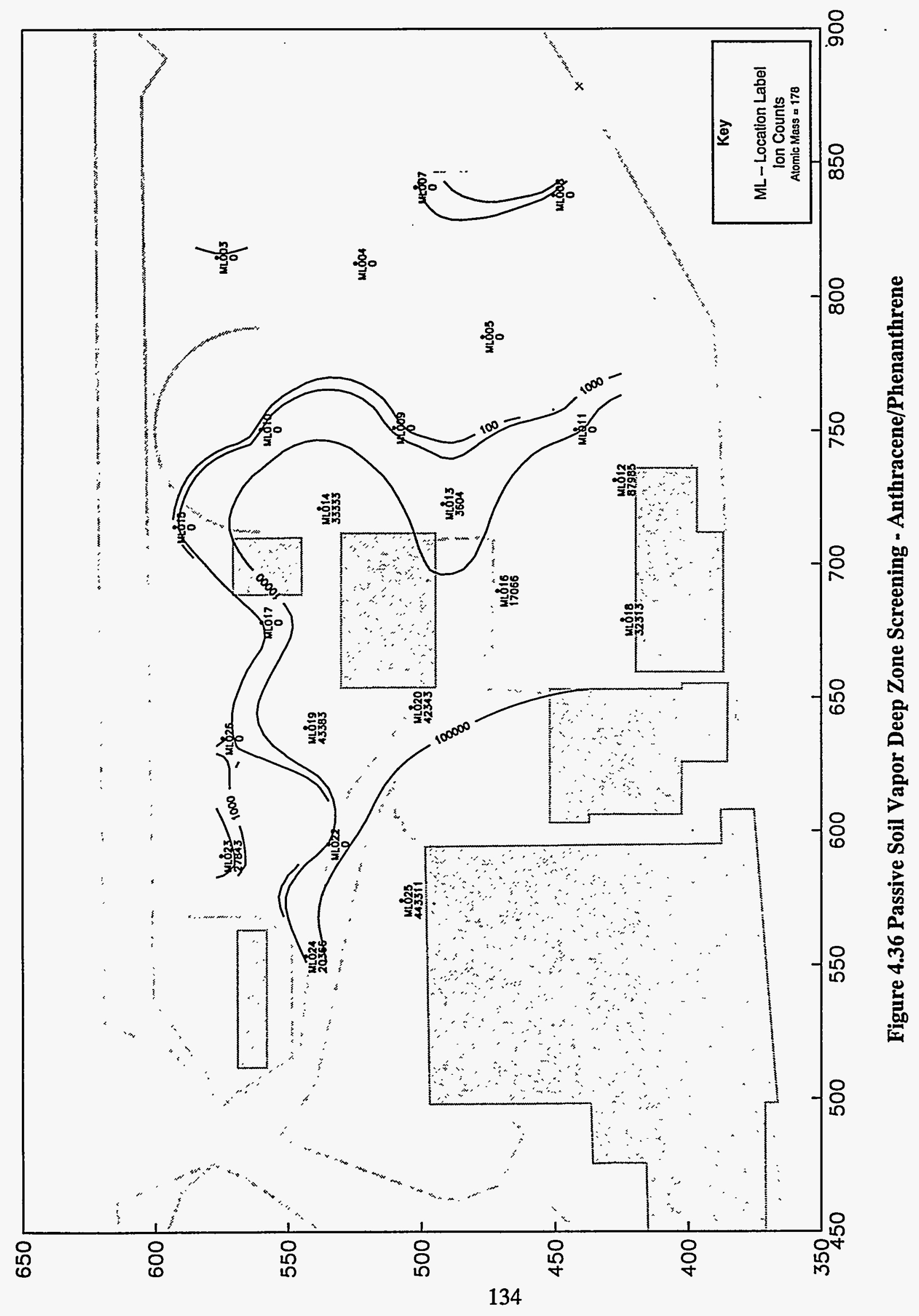




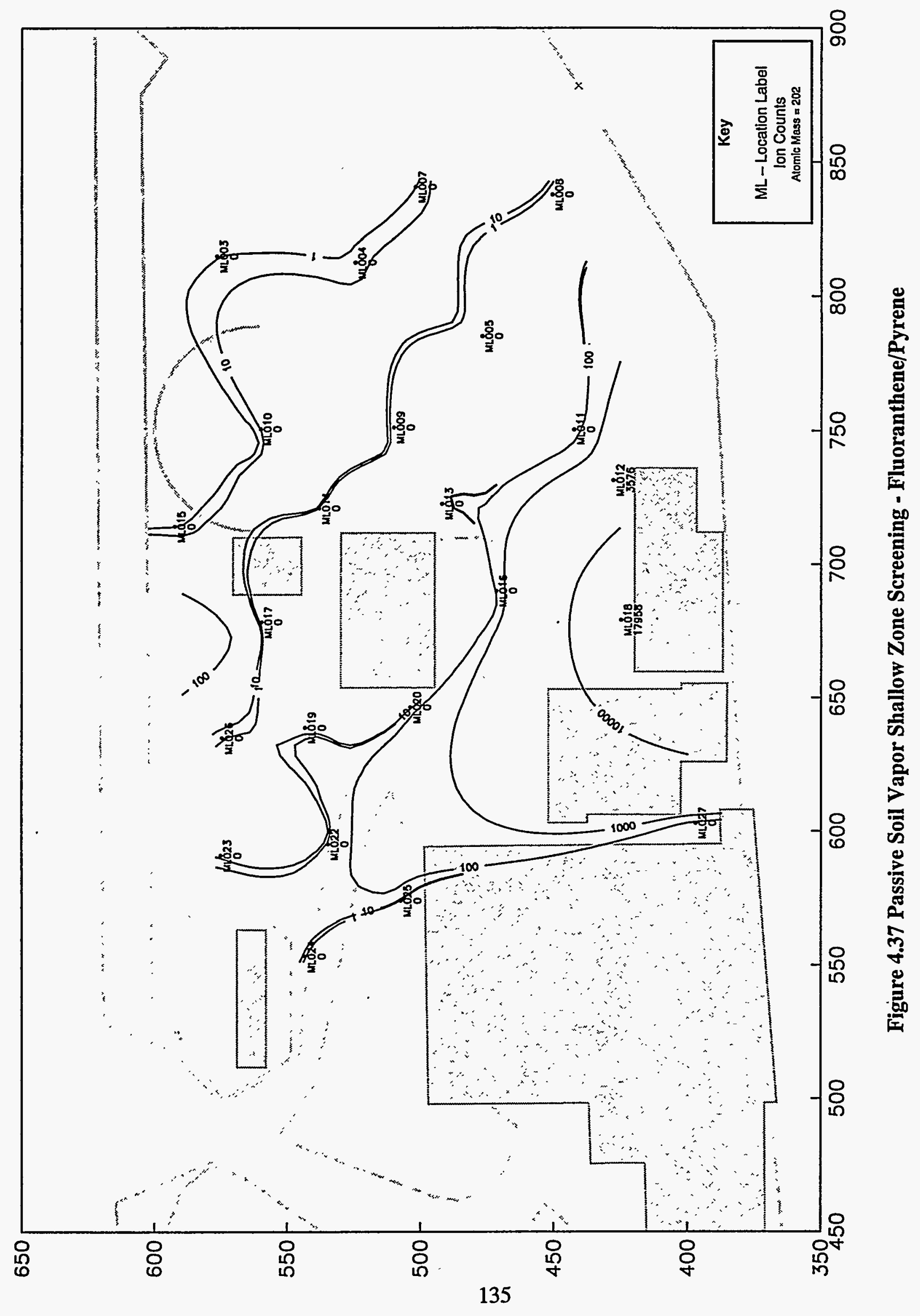




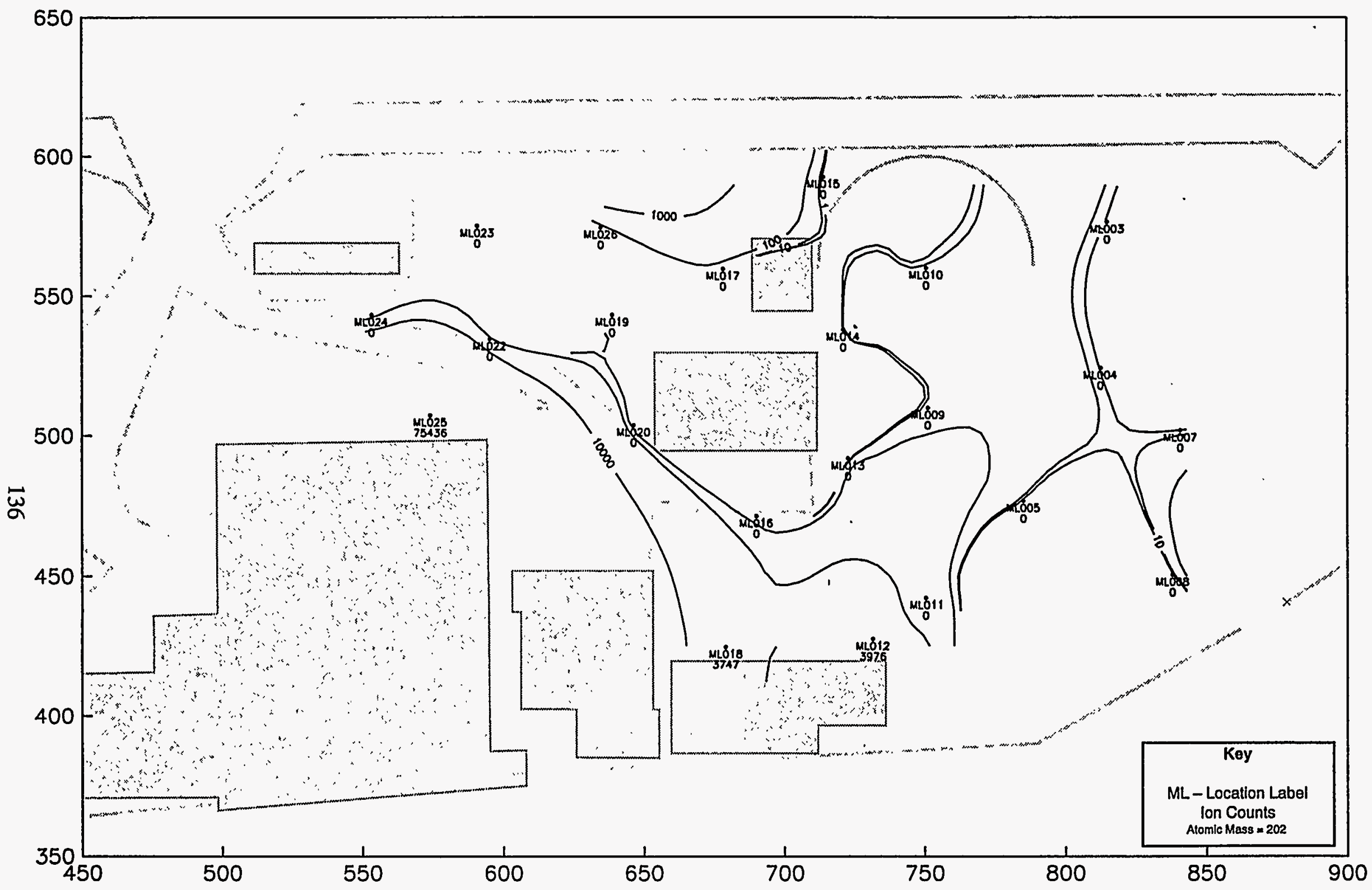

Figure 4.38 Passive Soil Vapor Deep Zone Screening - Fluoranthene/Pyrene 


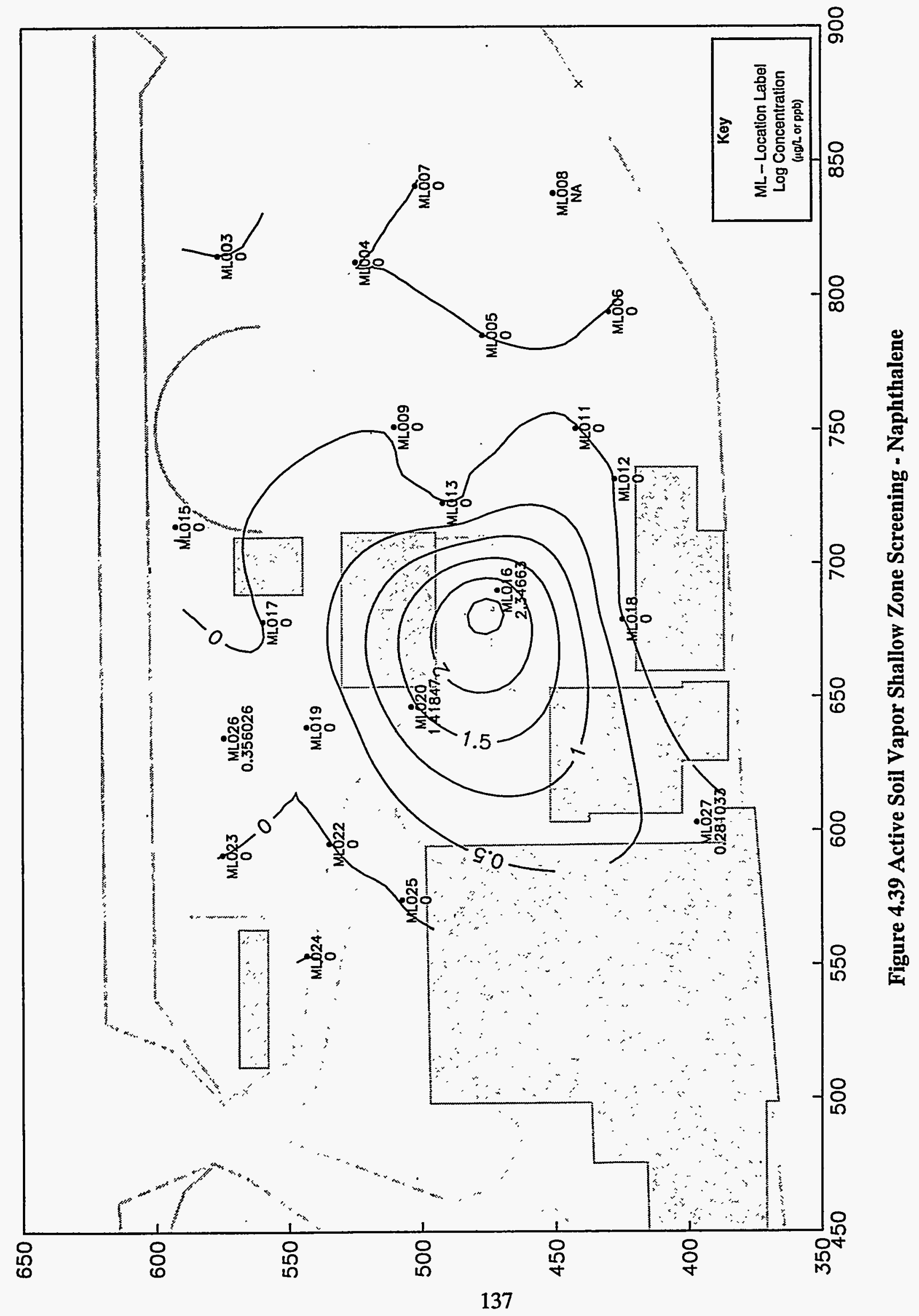




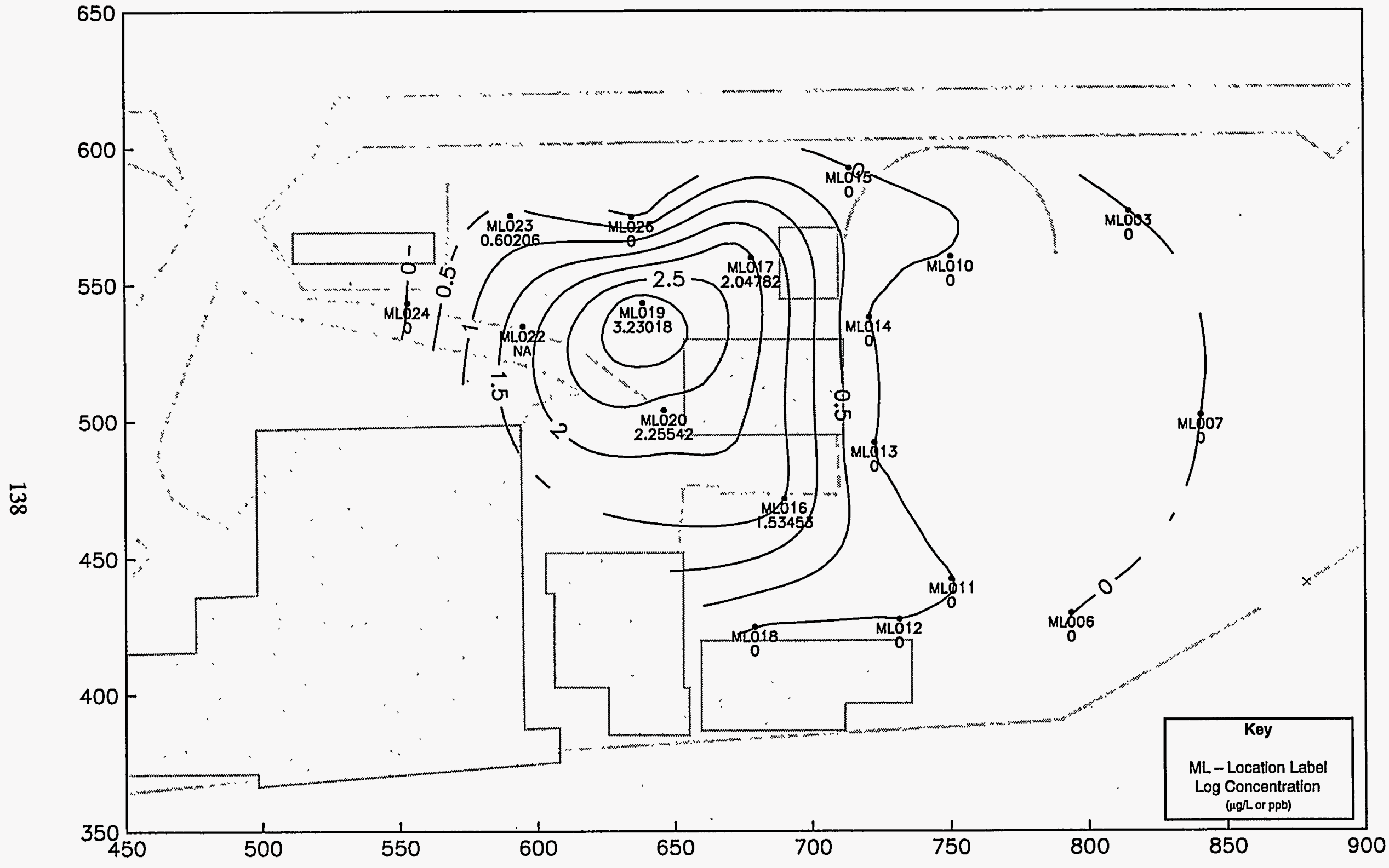

Figure 4.40 Active Soil Vapor Deep Zone Screening - Naphthalene 
(7әа\$) 47da0
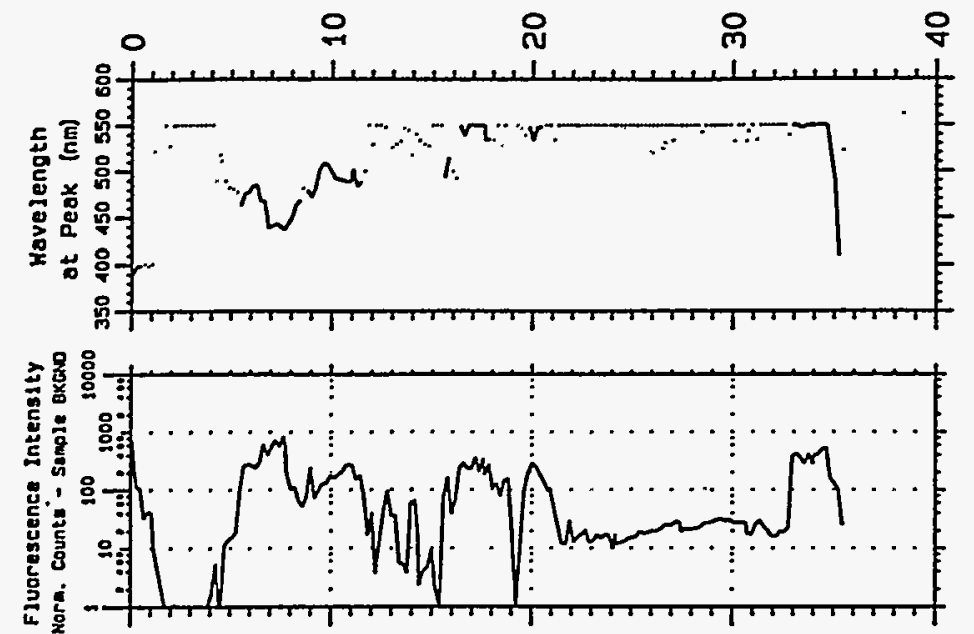

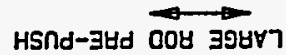
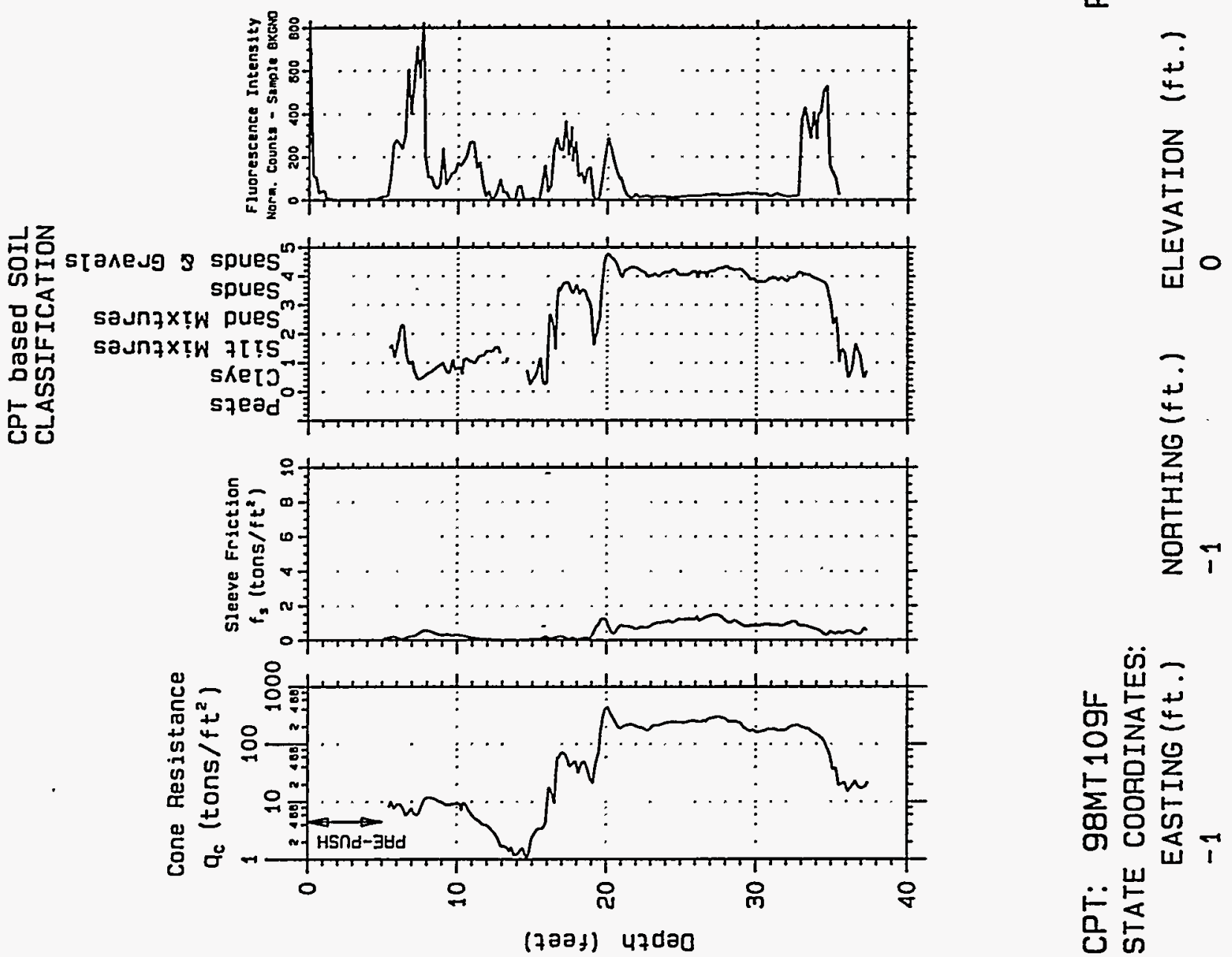


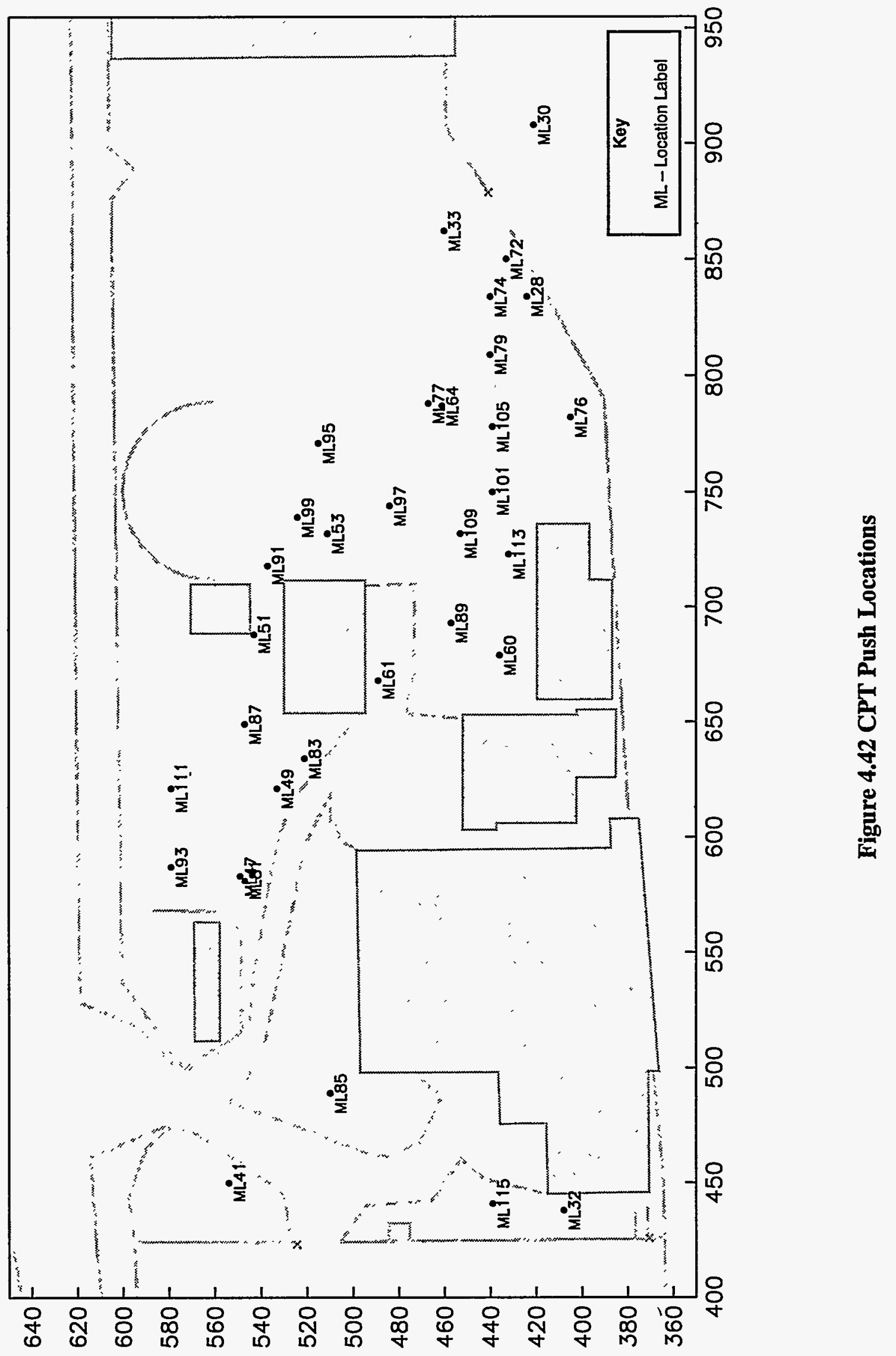


Figure 4.43 Comparison of CPT ML-28 Stratigraphic Log with BWWST
MW-3 Log

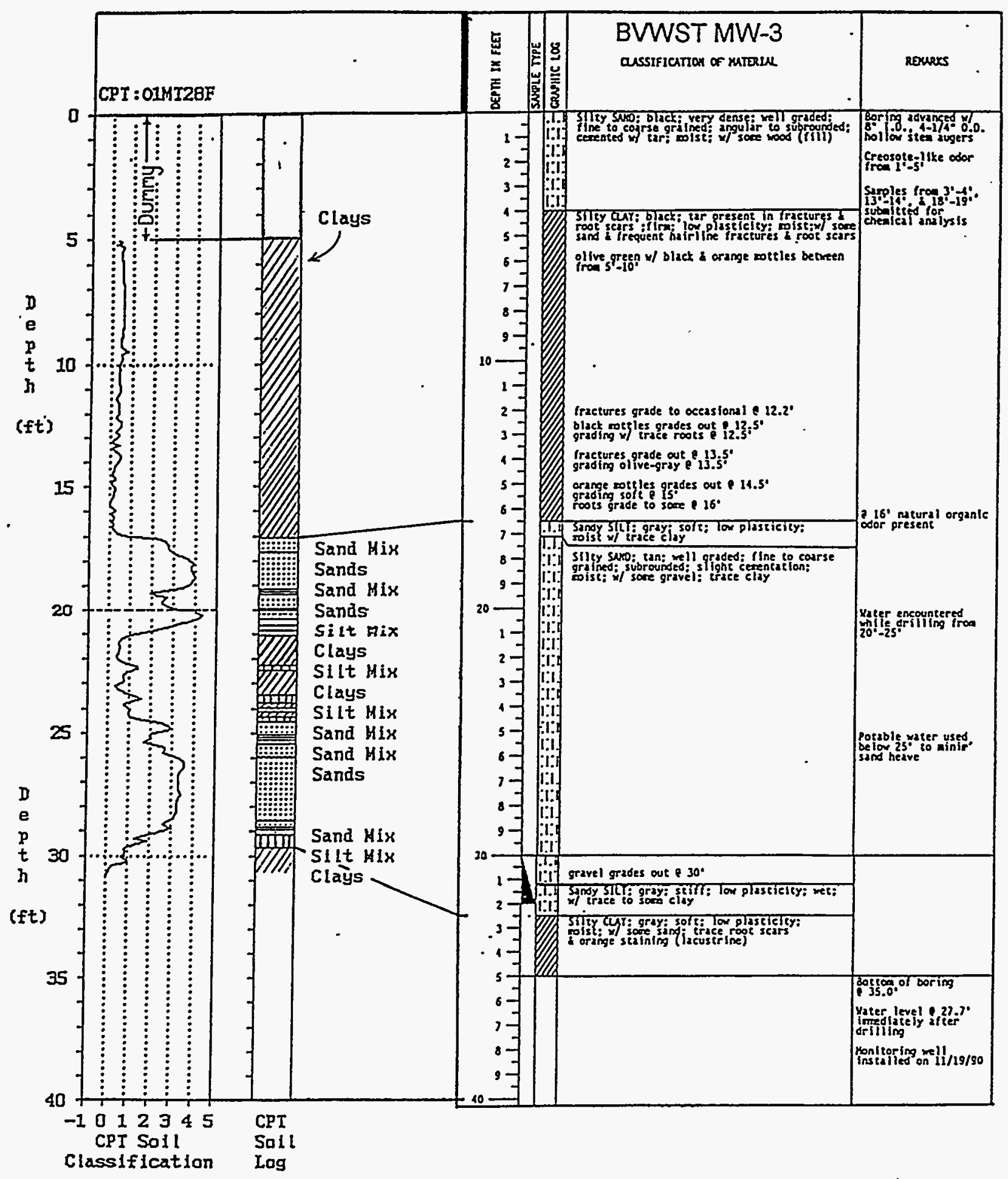

- 0-1=Clays, 1-2=Silt Mixtures, 2-3=Sand Mixtures, 3-4=Sands, 4-5 =Sands and gravels. 
Figure 4.44 Comparison of CPT ML-32 Stratigraphic Log with BVWST
MW-4 Log

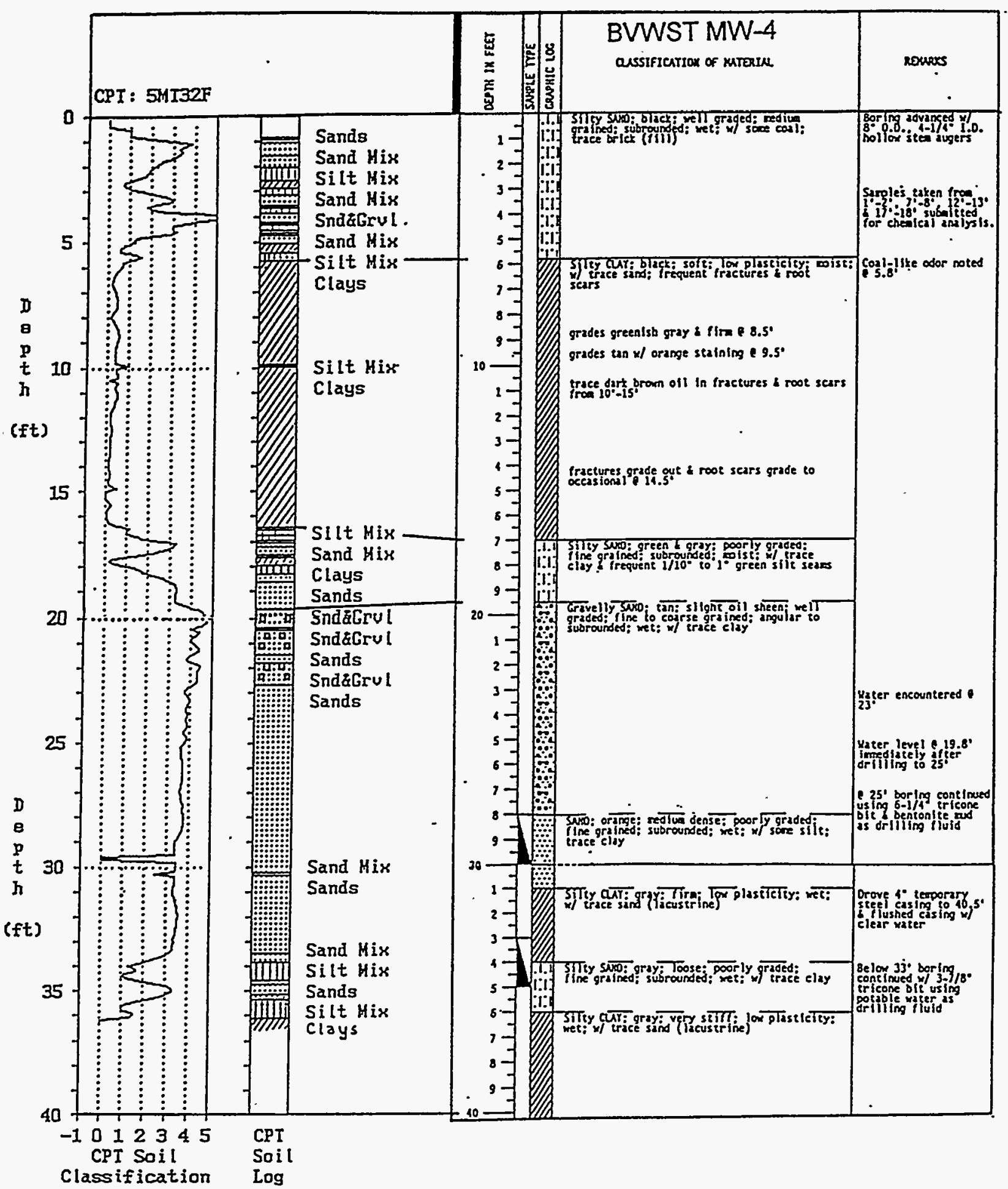

- $0-1=$ Clays, $1-2=$ Silt Mixtures, 2-3=Sand Mixtures, 3-4=Sands, 4-5=Sands and gravels. 


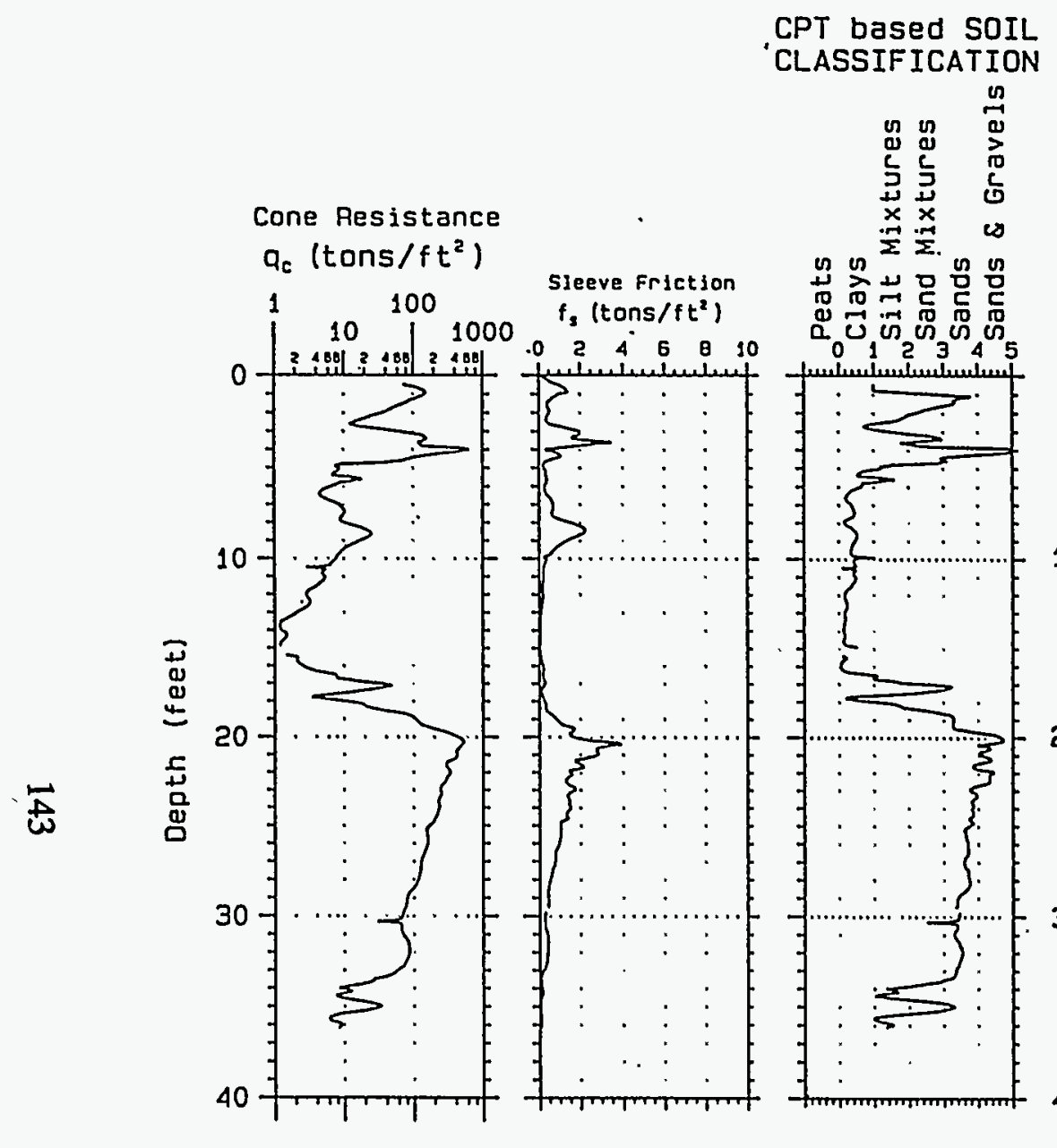

MI-32 - LIF

STATE COORDINATES: EASTING (ft.)
CPT based SOIL

CLASSIF ICATION

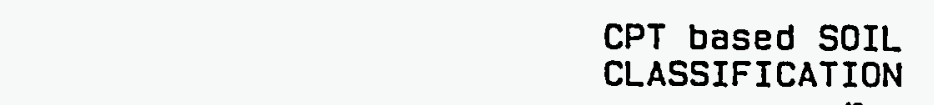

Cone Resistance

$\mathrm{q}_{\mathrm{c}}$ (tons $/ \mathrm{ft}^{2}$ )

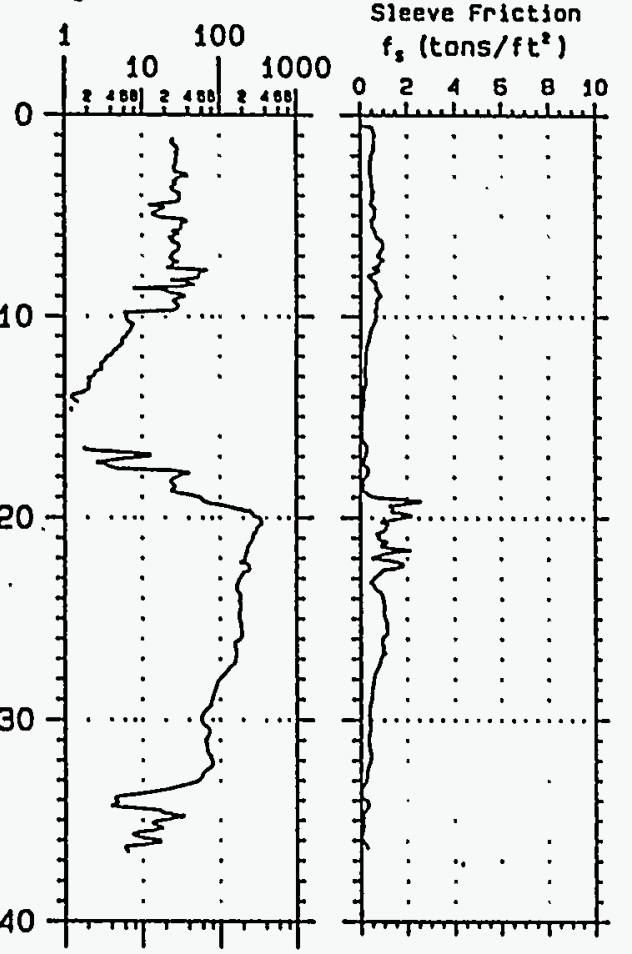

Sleeve Friction

$f,\left(\right.$ tons $\left./ \mathrm{ft}^{2}\right)$

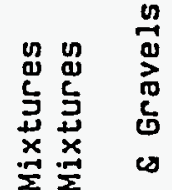

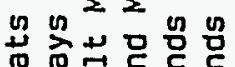

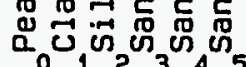

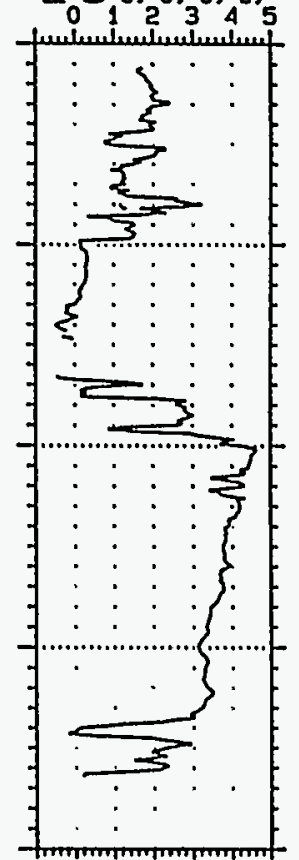

\section{ML-32 - Resistivity}

Project: Marshalltown

NORTHING ( $f t$.)

$-1$
ELEVATION ( $\mathrm{ft}$.)

0
Probe date

04-26-1994

Figure 4.45 CPT Stratigraphic Repeatability Comparison - ML-32 


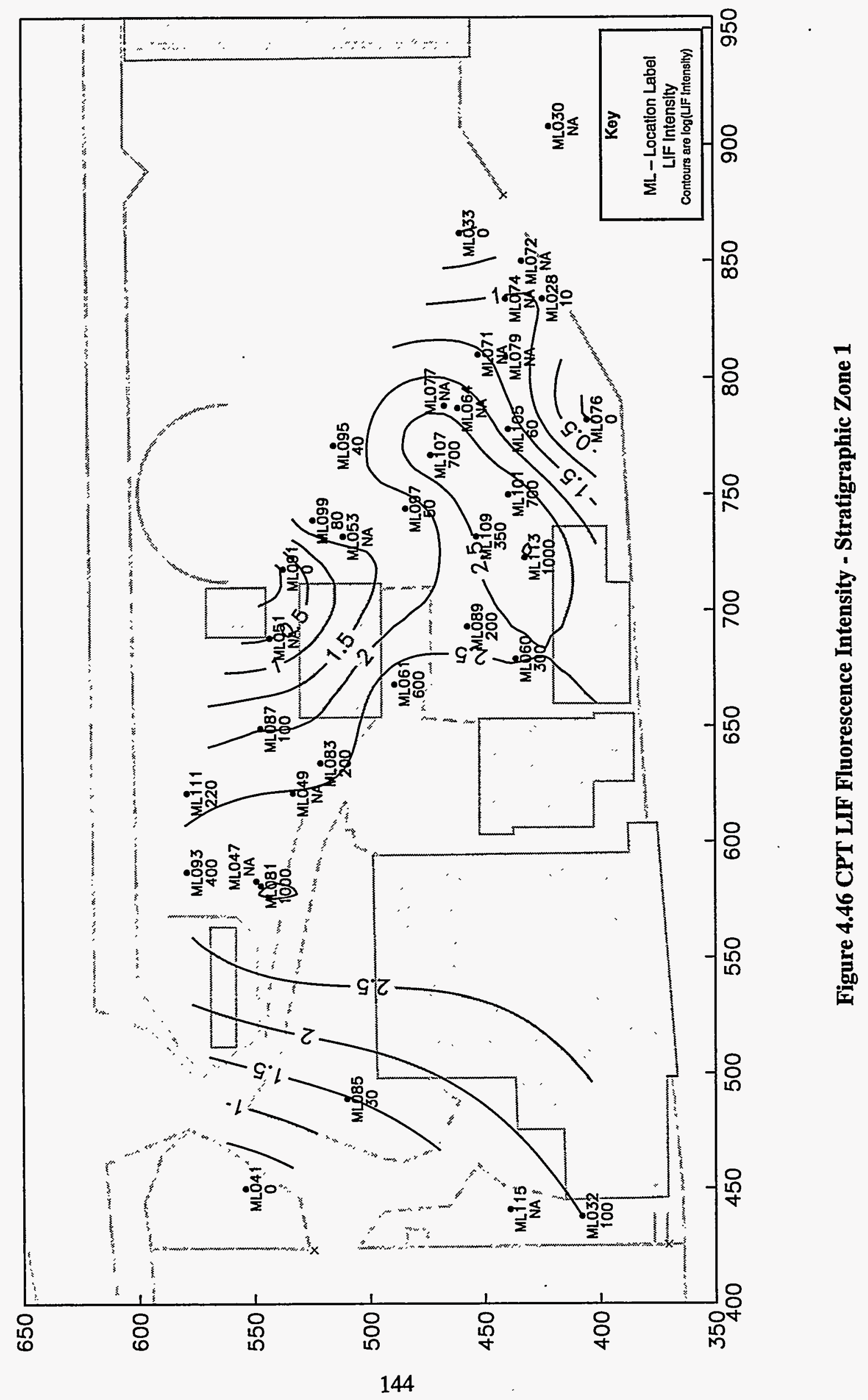




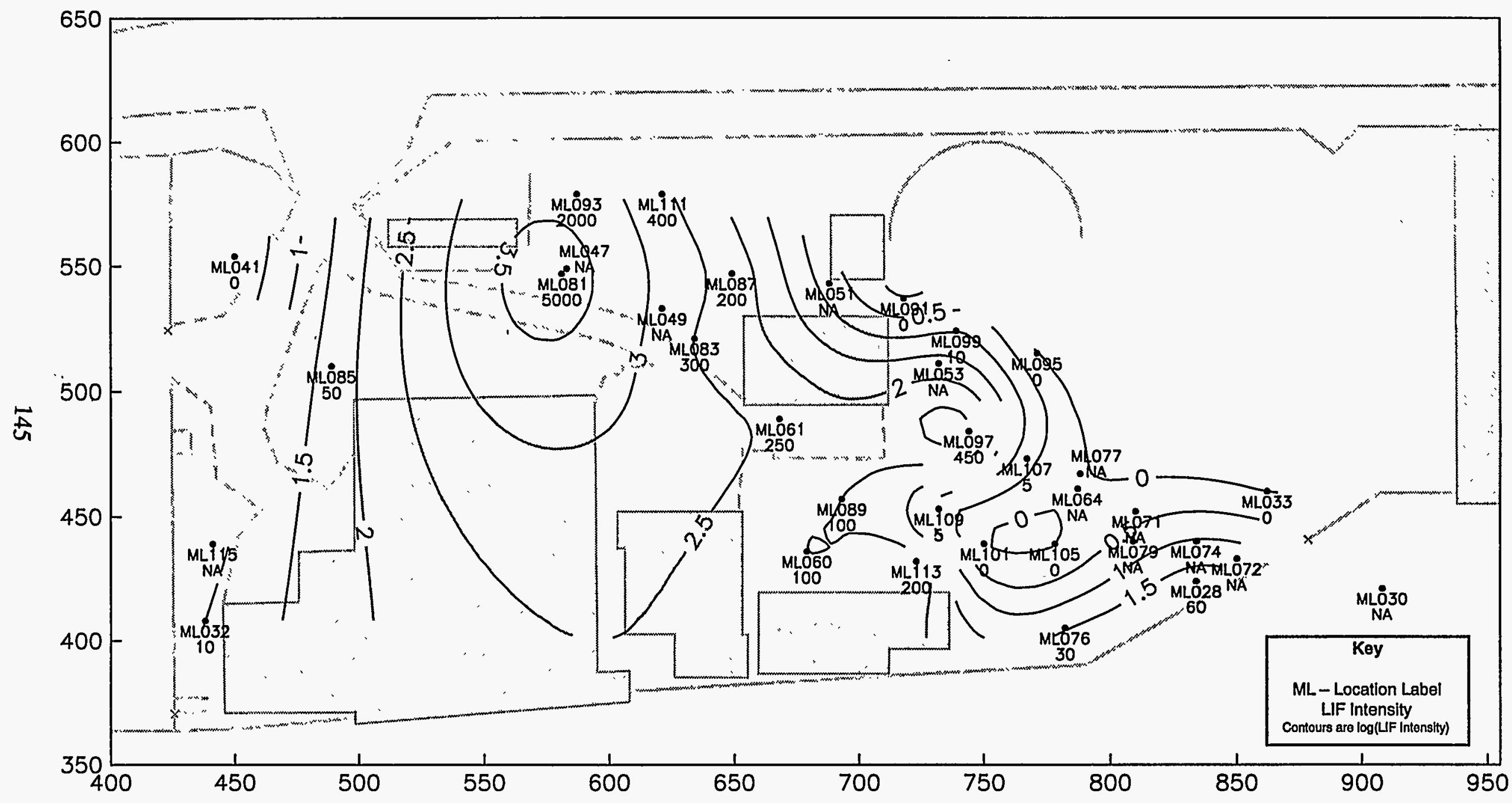

Figure 4.47 CPT LIF Fluorescence Intensity - Stratigraphic Zone 2 


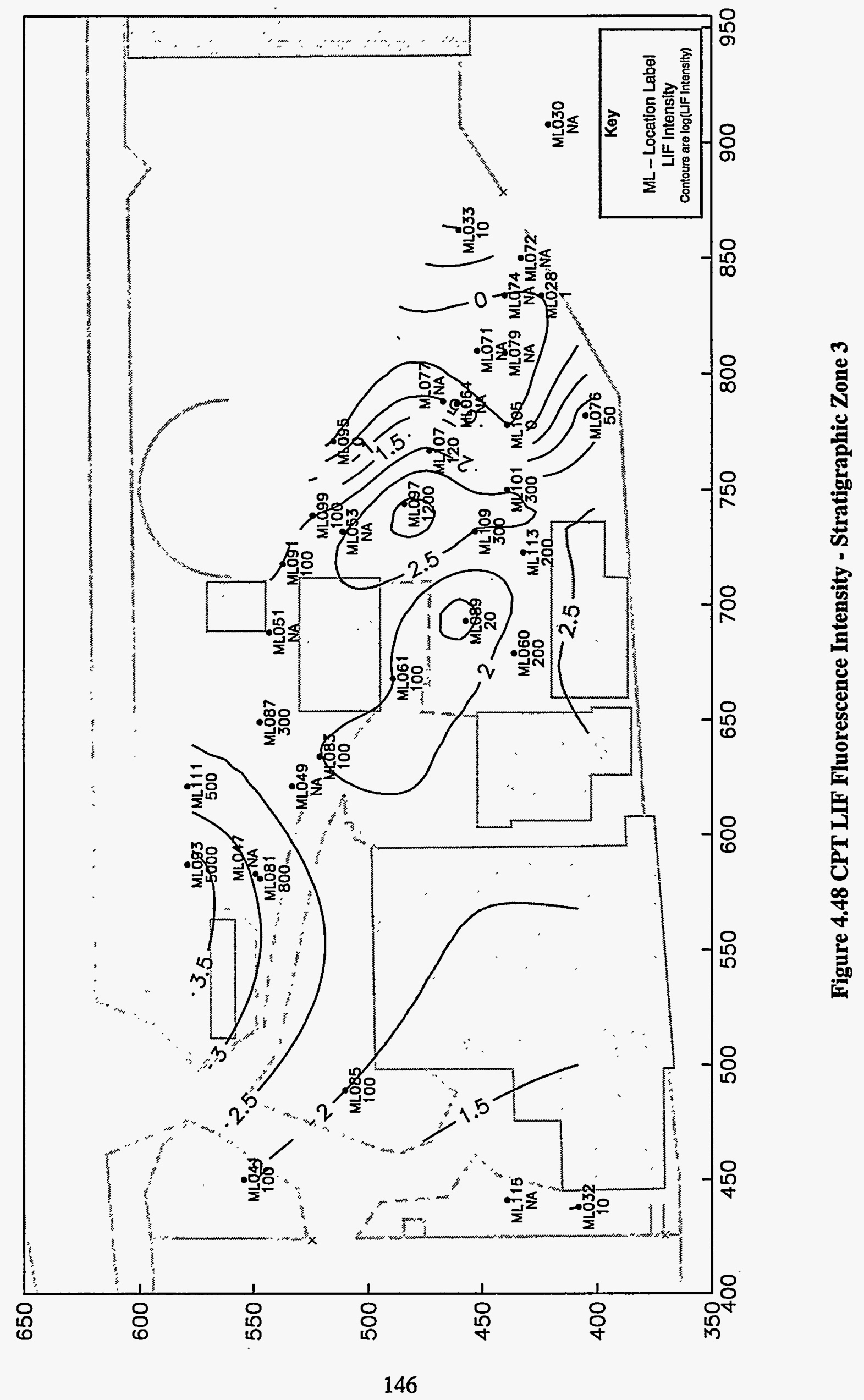




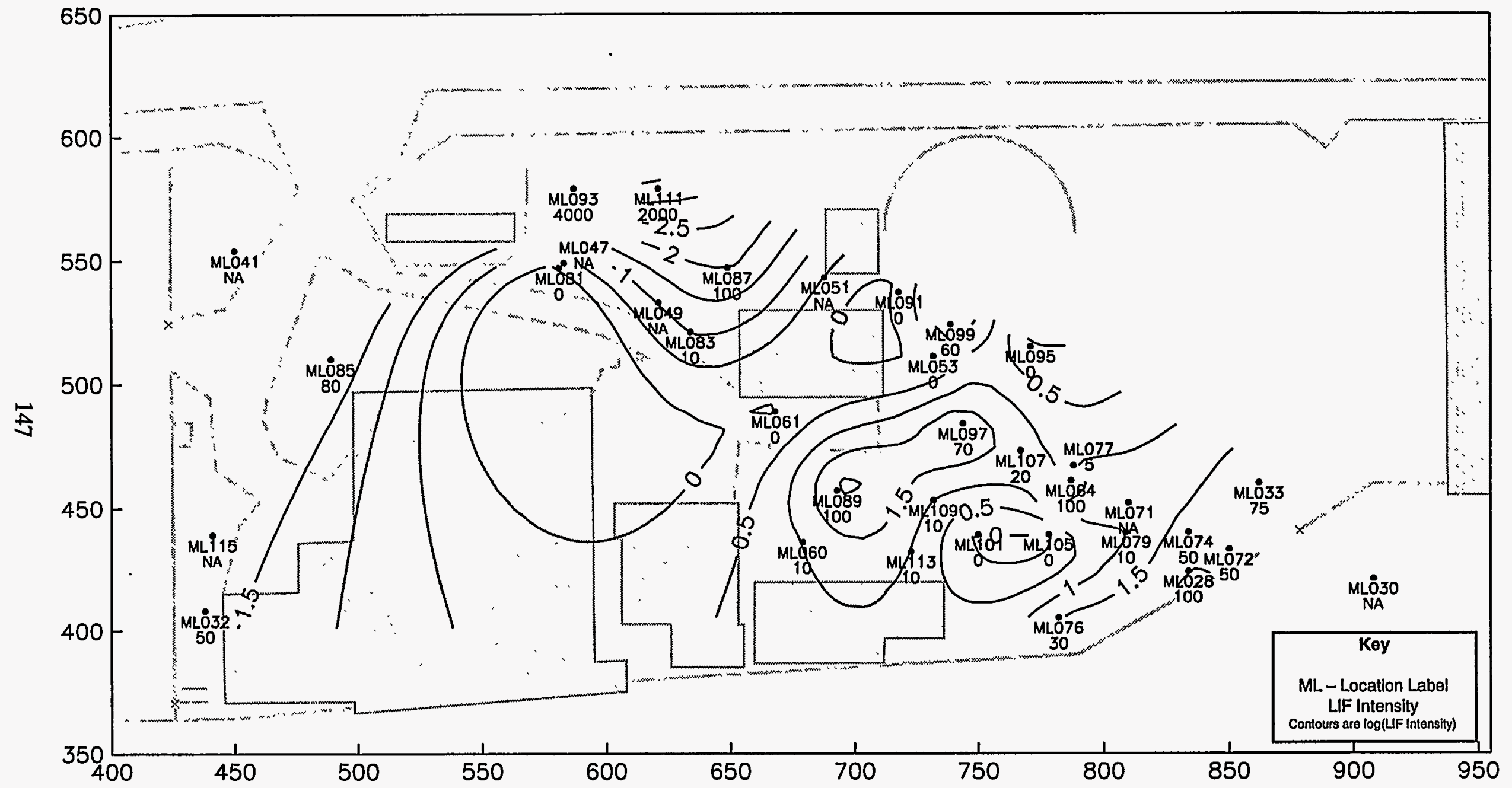

Figure 4.49 CPT LIF Fluorescence Intensity - Stratigraphic Zone 4 


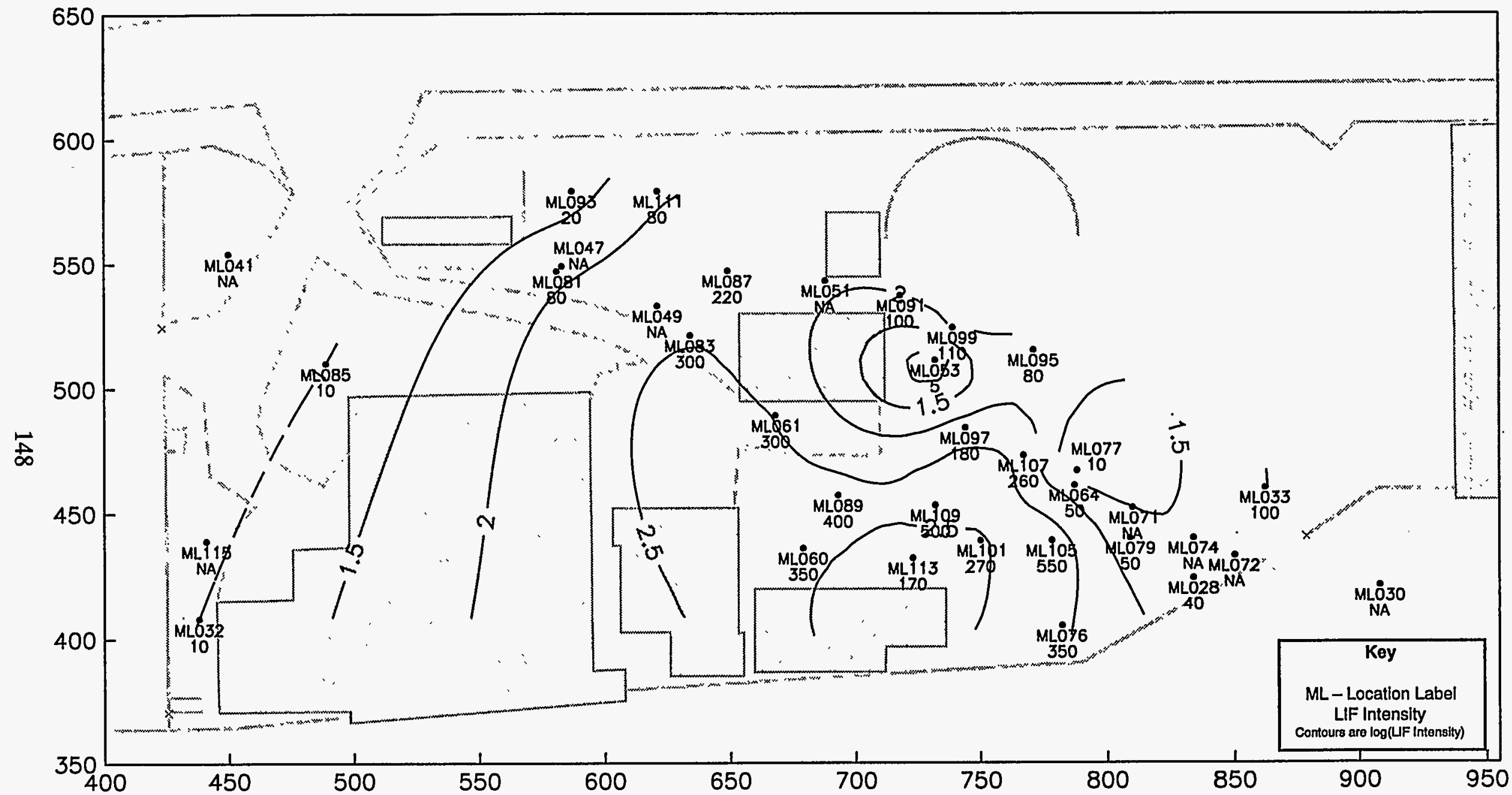

Figure 4.50 CPT LIF Fluorescence Intensity - Stratigraphic Zone 5 


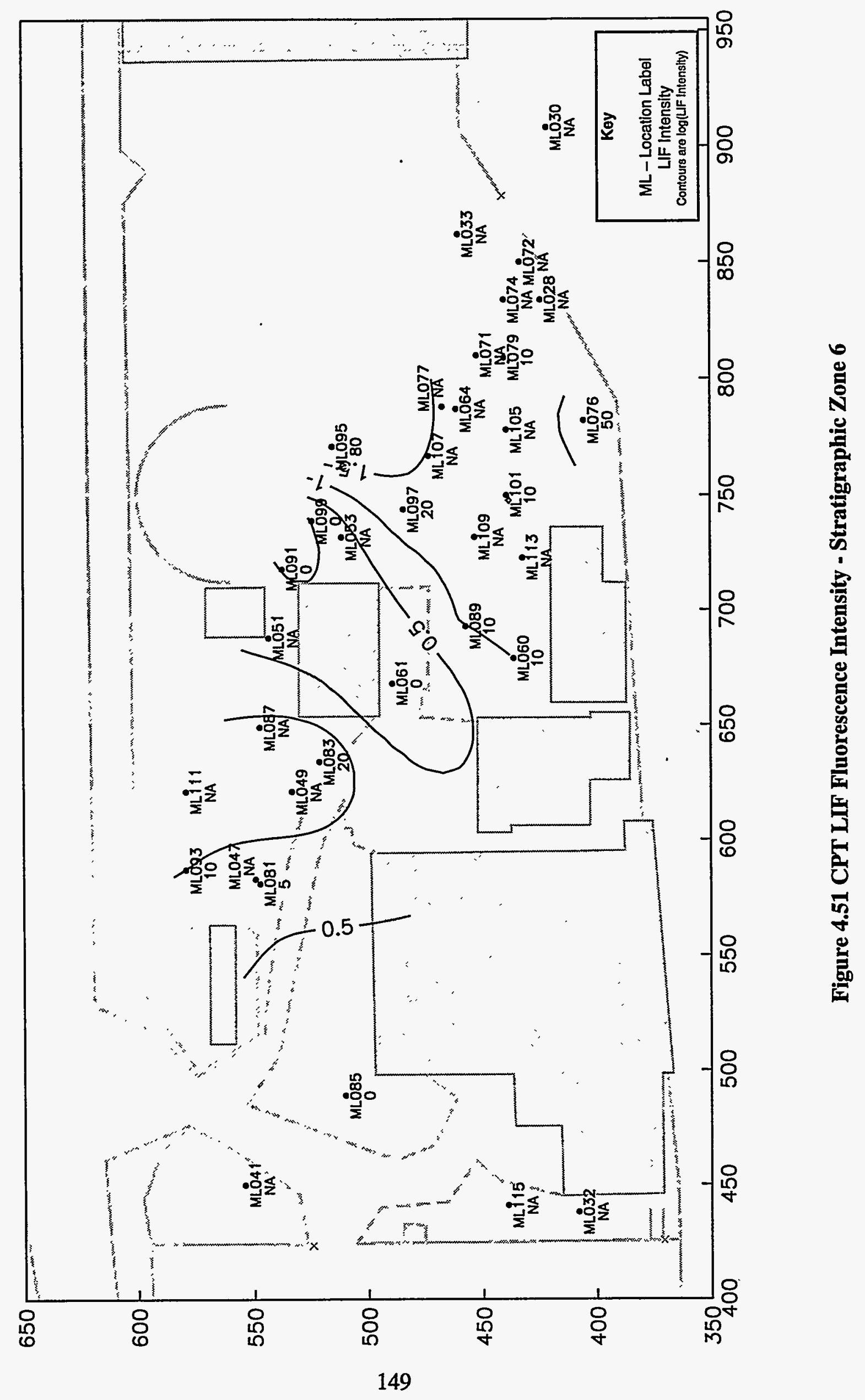



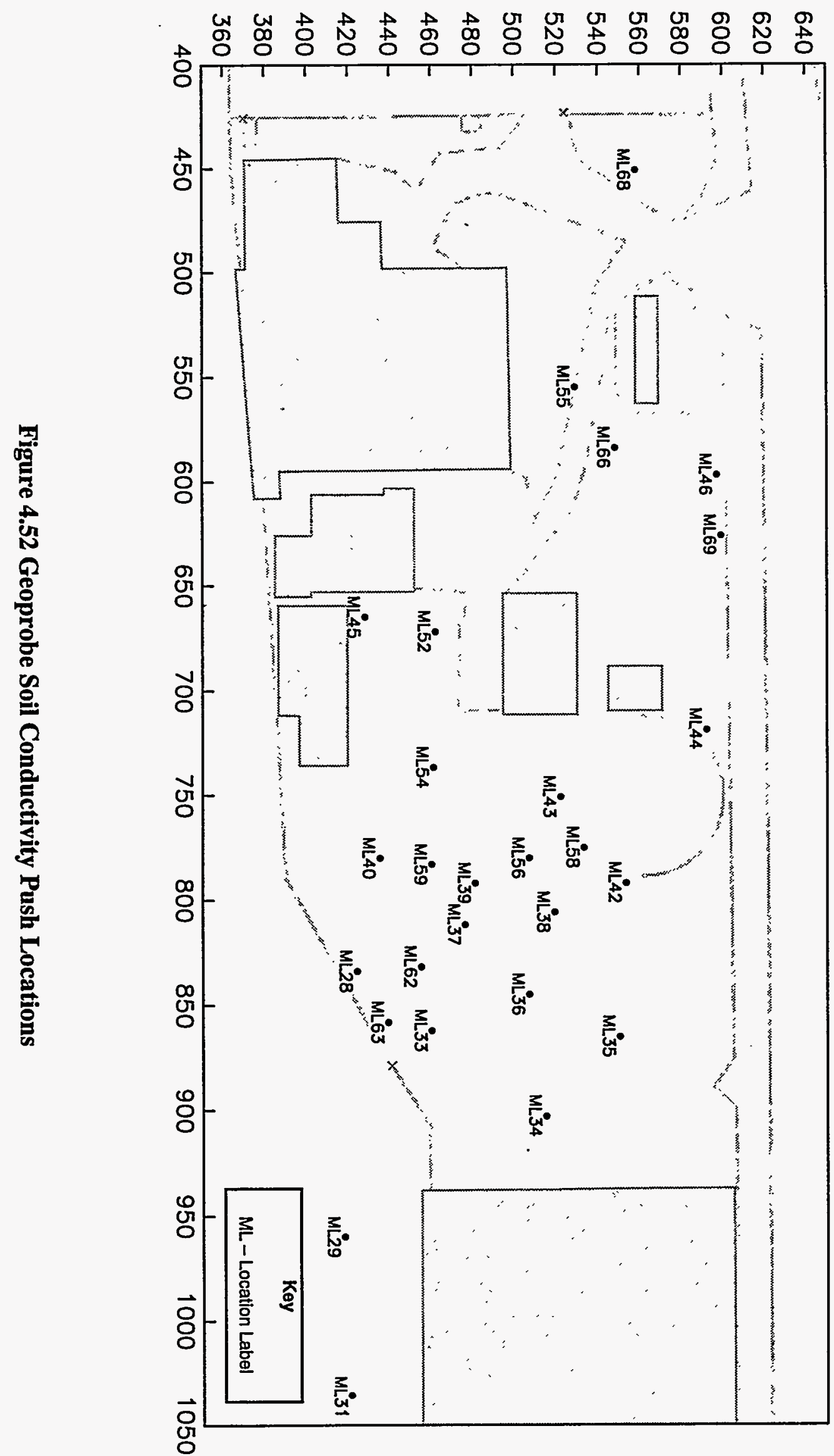


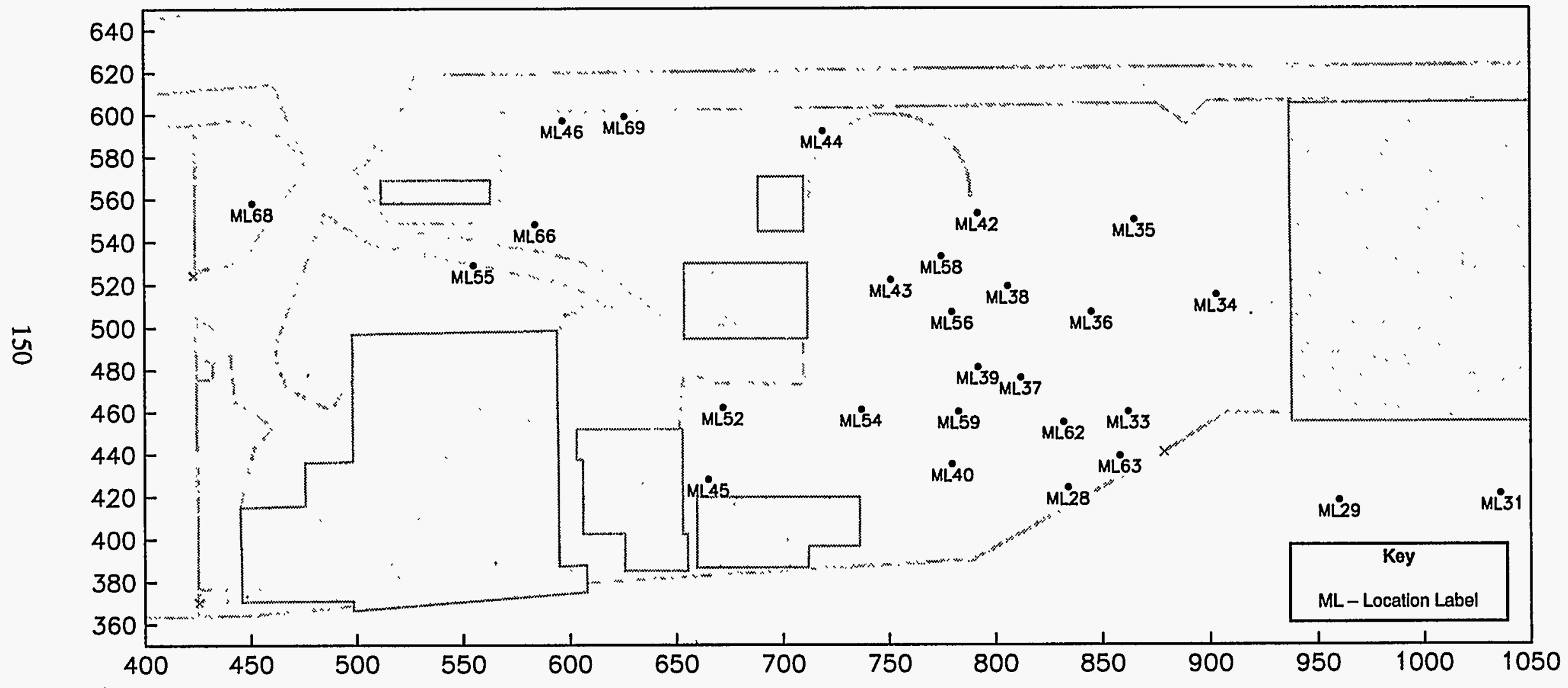

Figure 4.52 Geoprobe Soil Conductivity Push Locations 


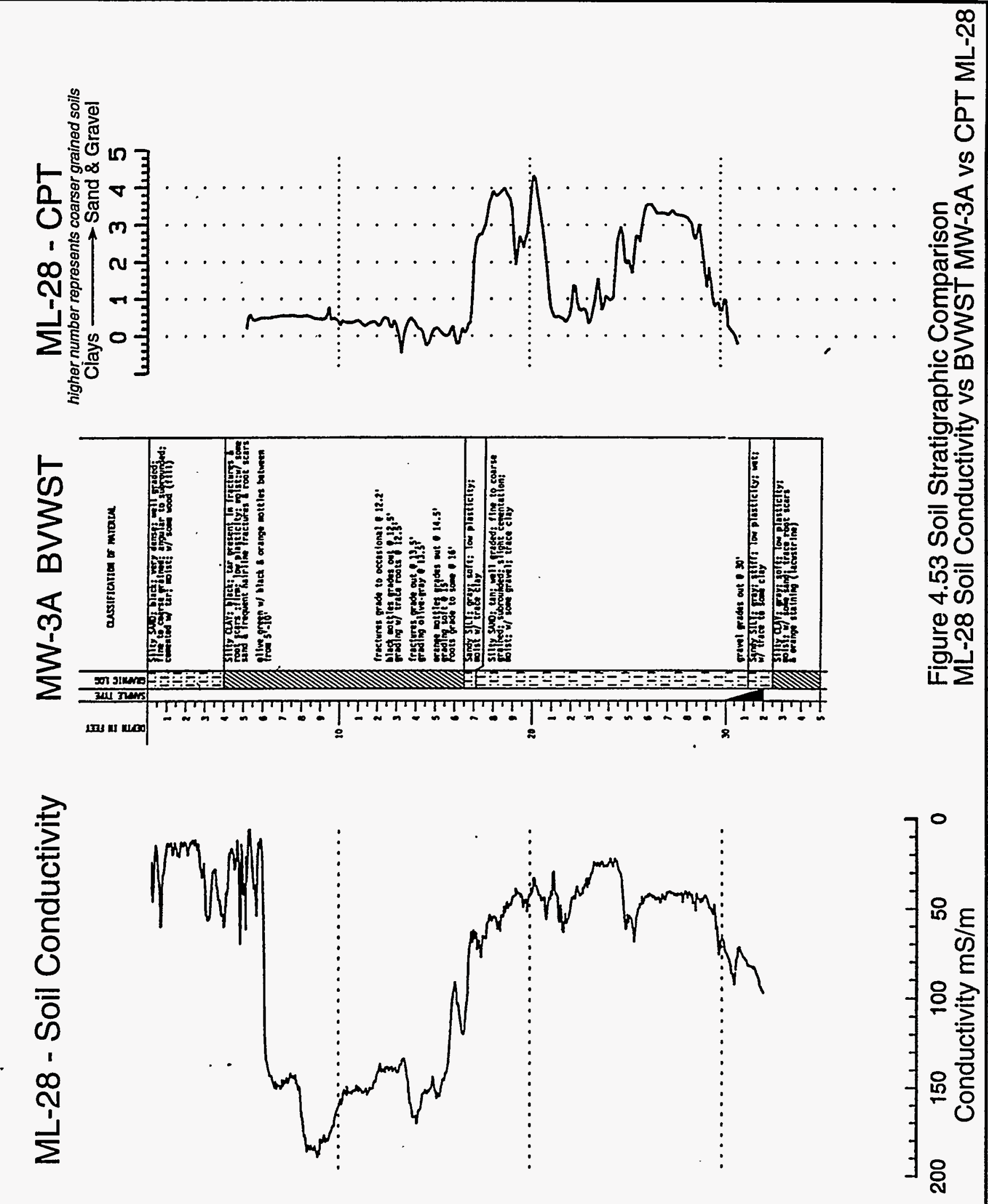



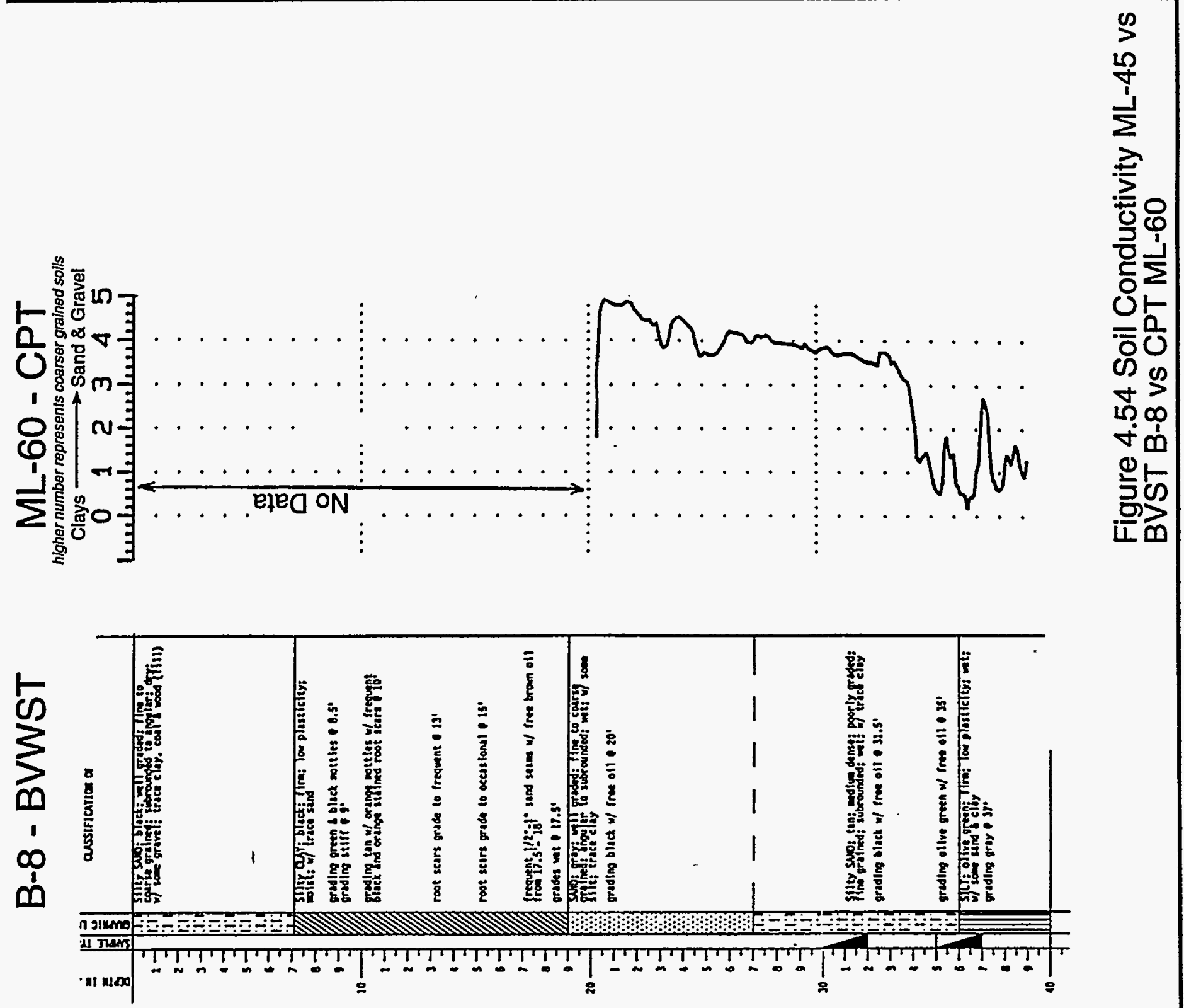

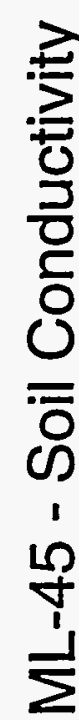

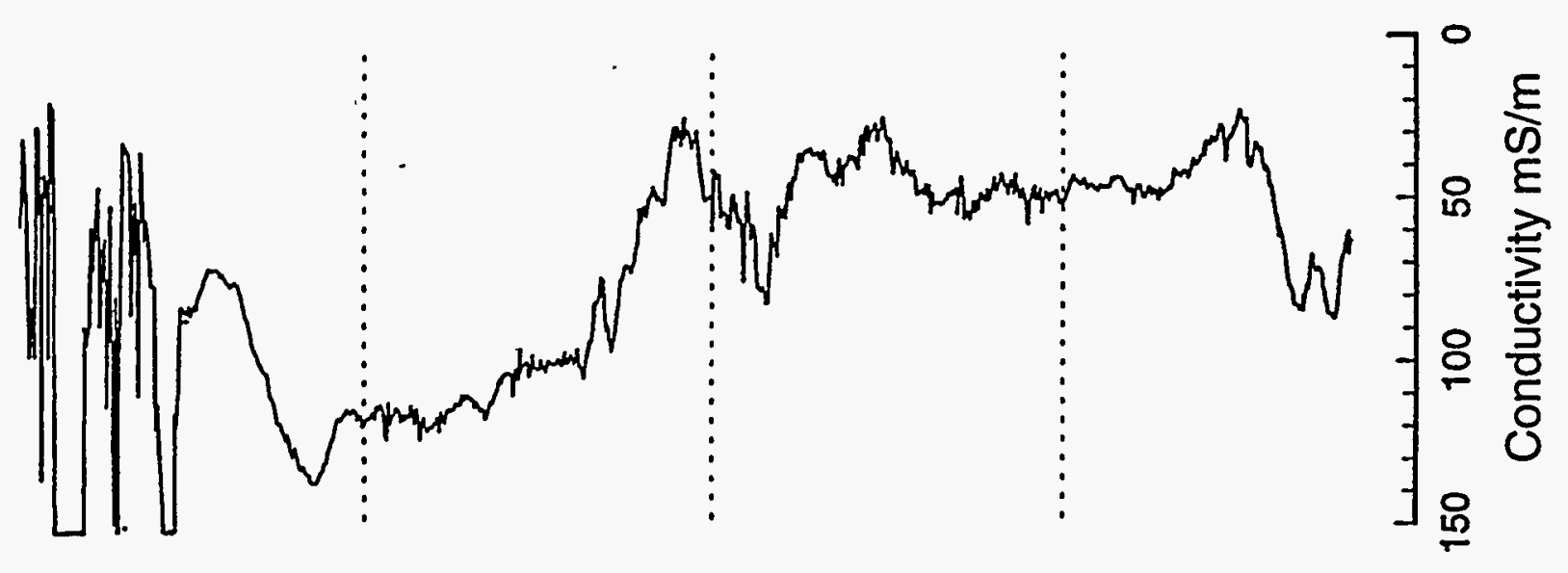


ML-45 - Soil Conductivity

$\vec{w}$

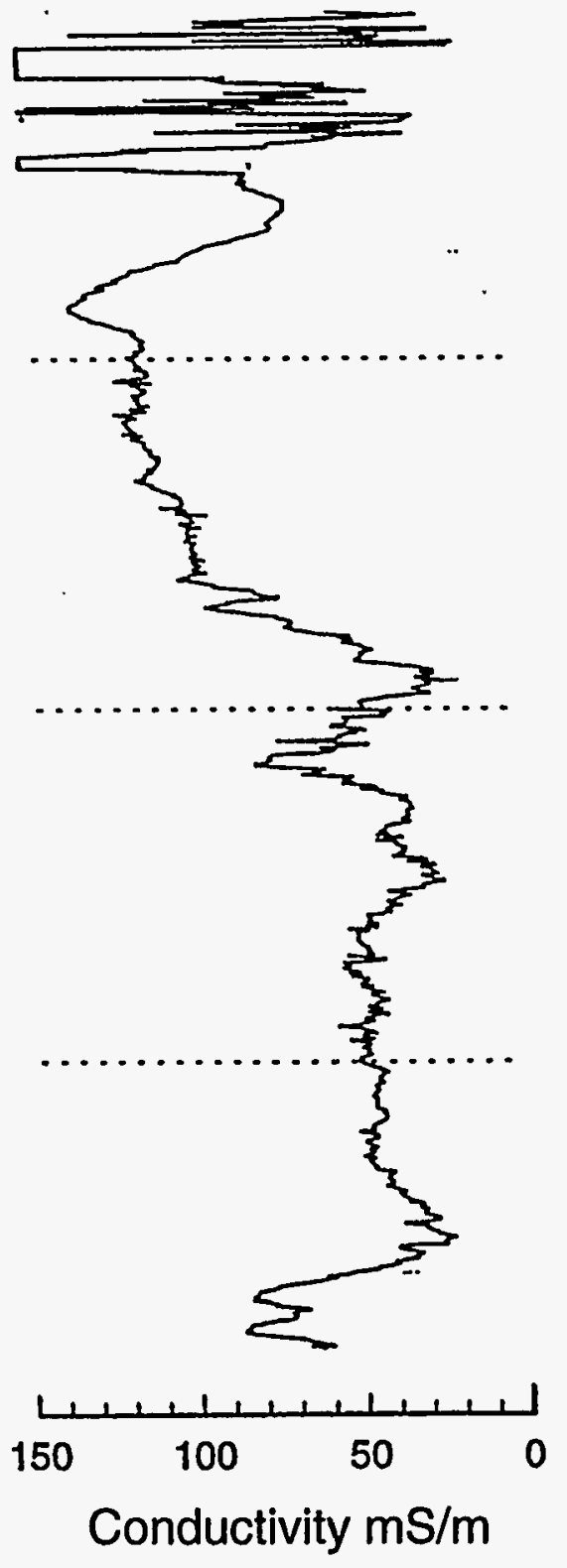

\section{B-8 BVWST}

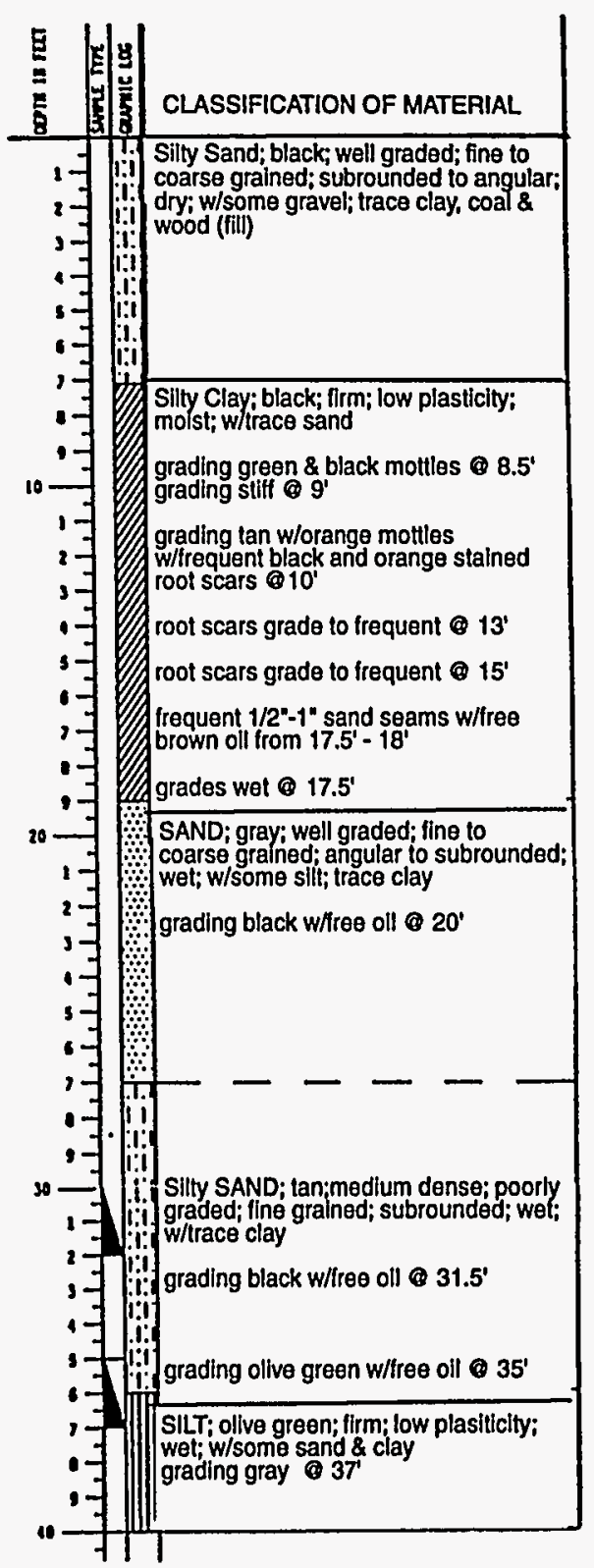

ML-60 - CPT

Fluorescence Intensity

Norn. Counts - Samol: BXGNO

- $200 \quad 400 \quad 500 \quad 800$

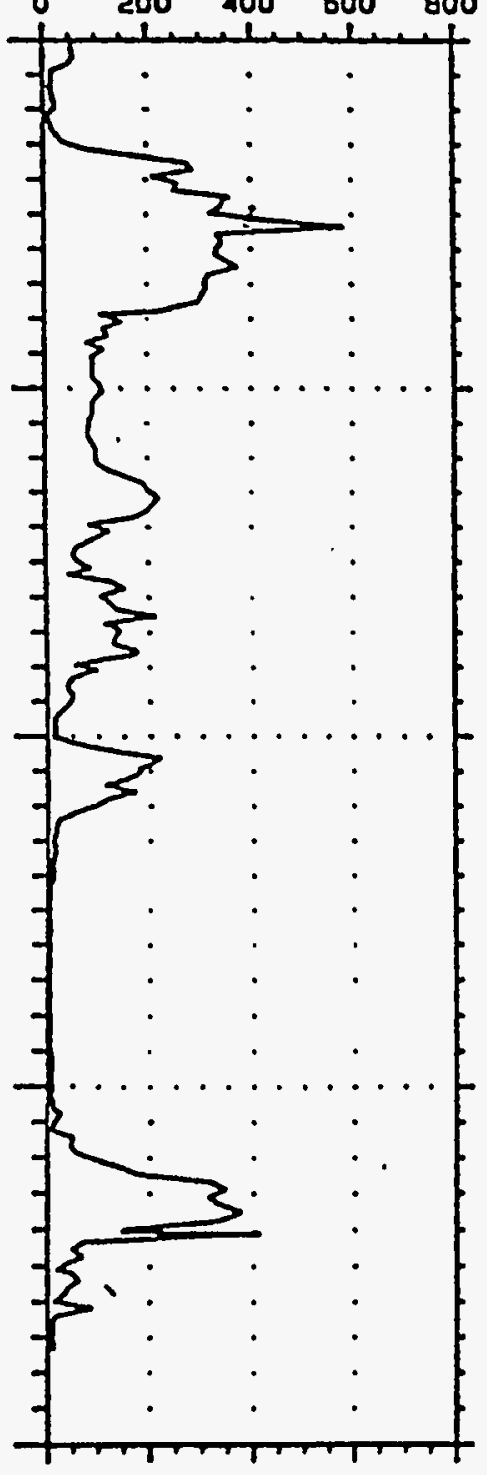

Figure 4.55 Coal Tar Residue Contamination Comparison Soil conductivity ML-45 vs BVWST B-8 vs CPT LIF ML-60 


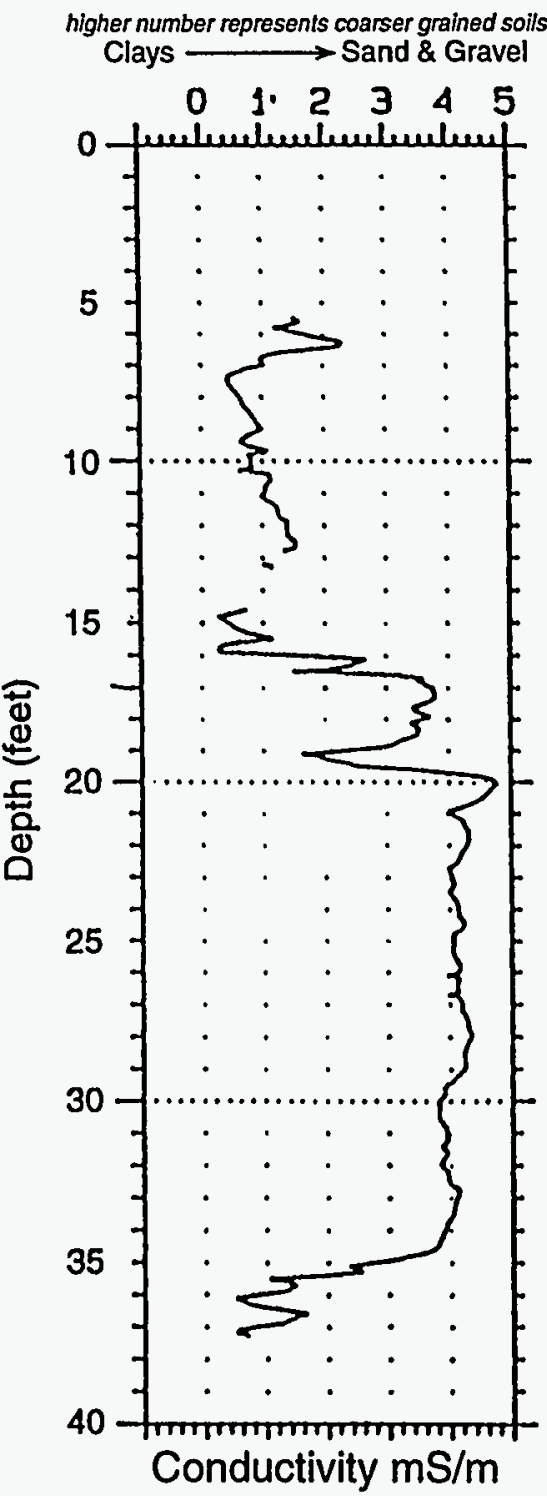

Fluorescence Intensity Norm. Counts - SAmple BKGNO

O $200 \quad 100 \quad 600 \quad 800$ ML-54- Soil Conductivity
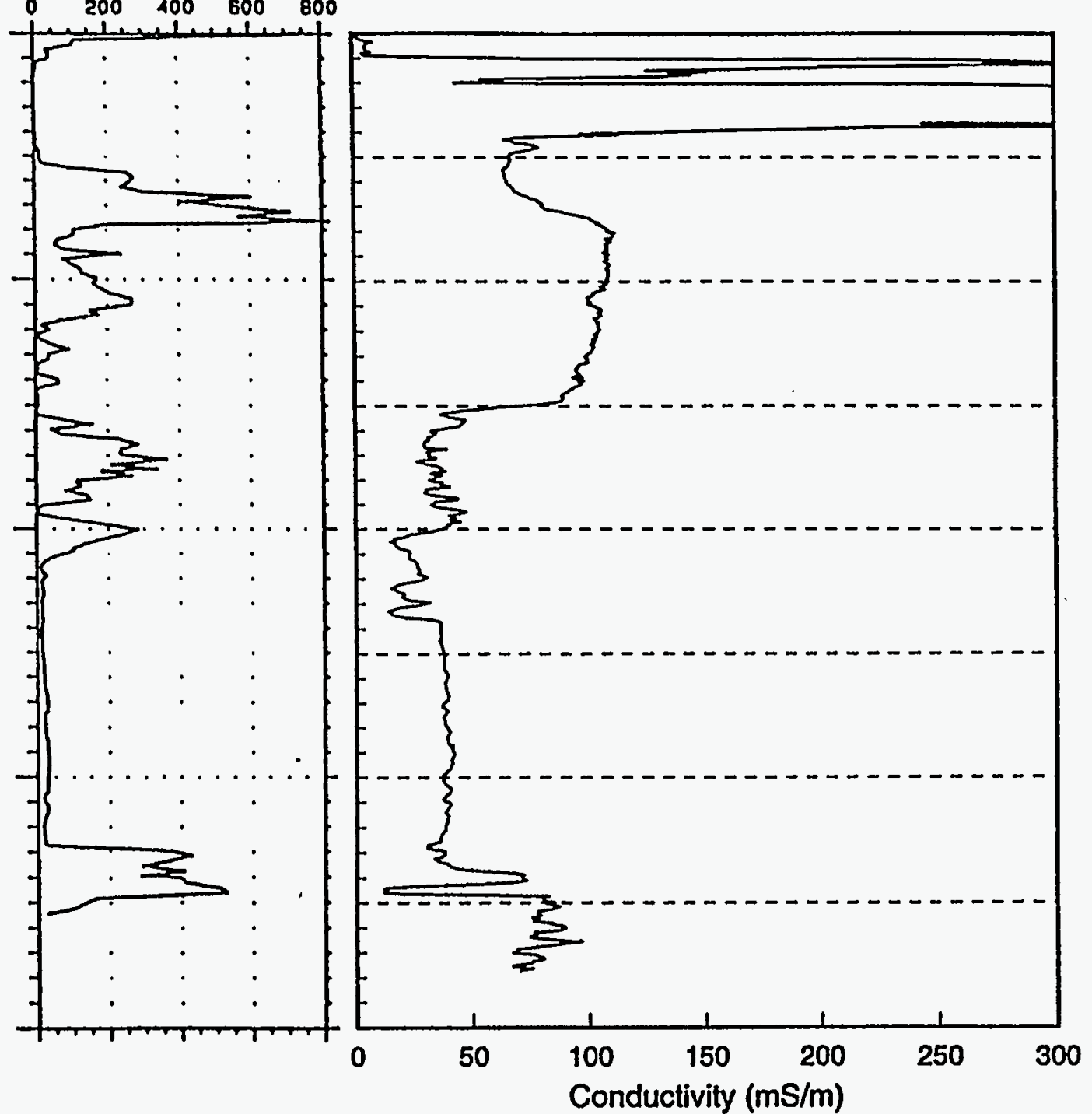

Figure 4.56 Coal Tar Residue Contamination Comparison CPT LIF ML-109 vs Soil conductivity ML-54 


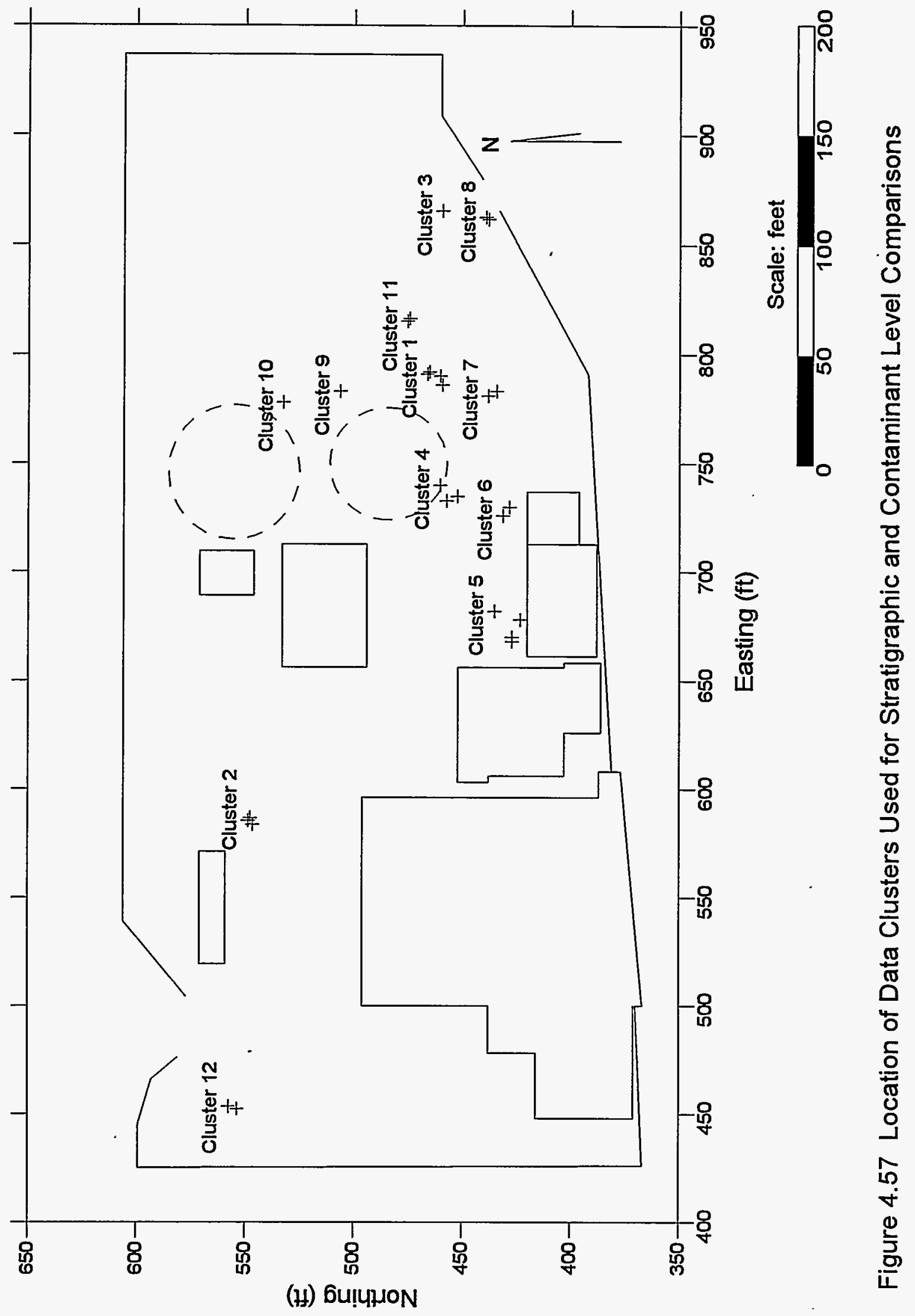




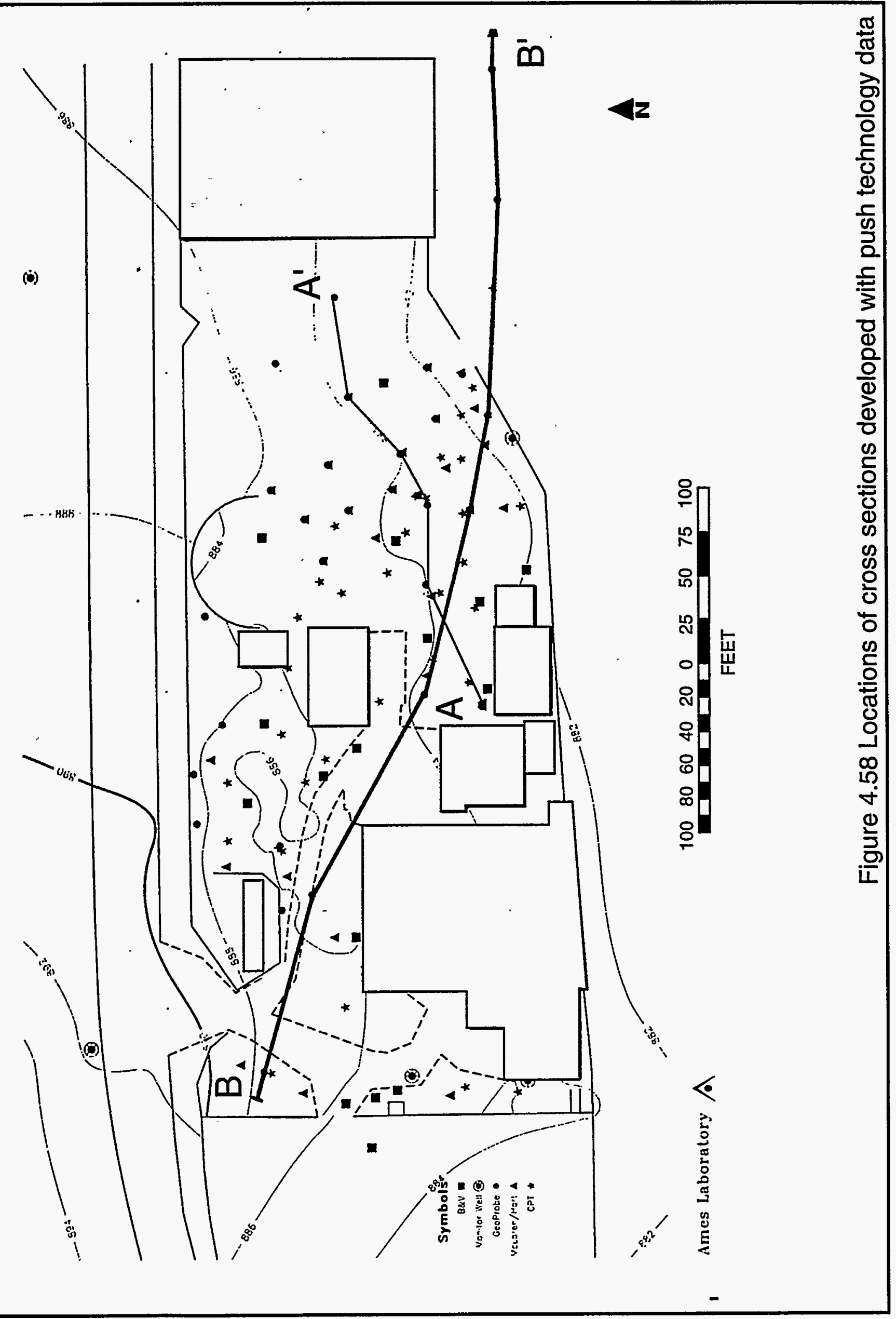




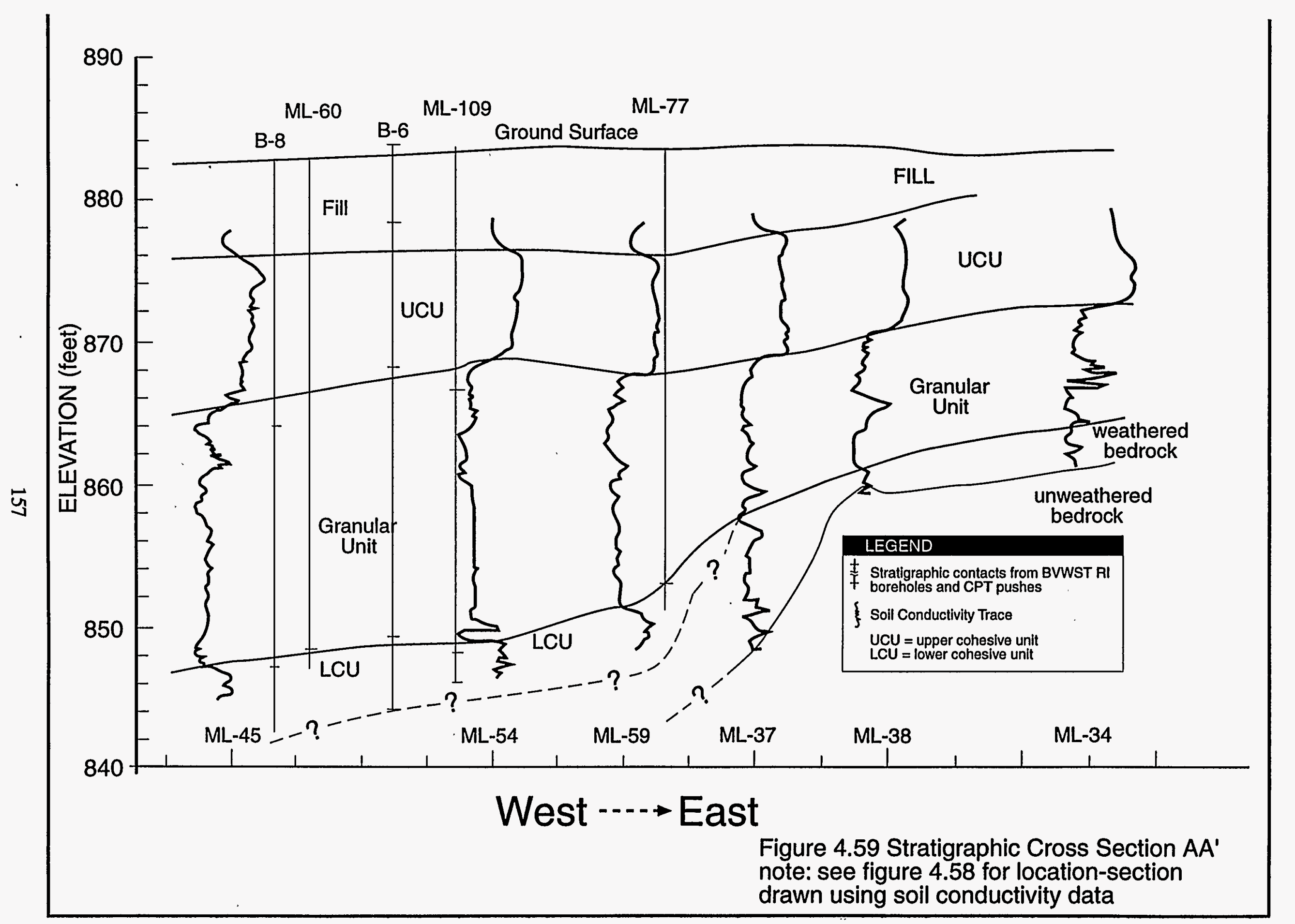




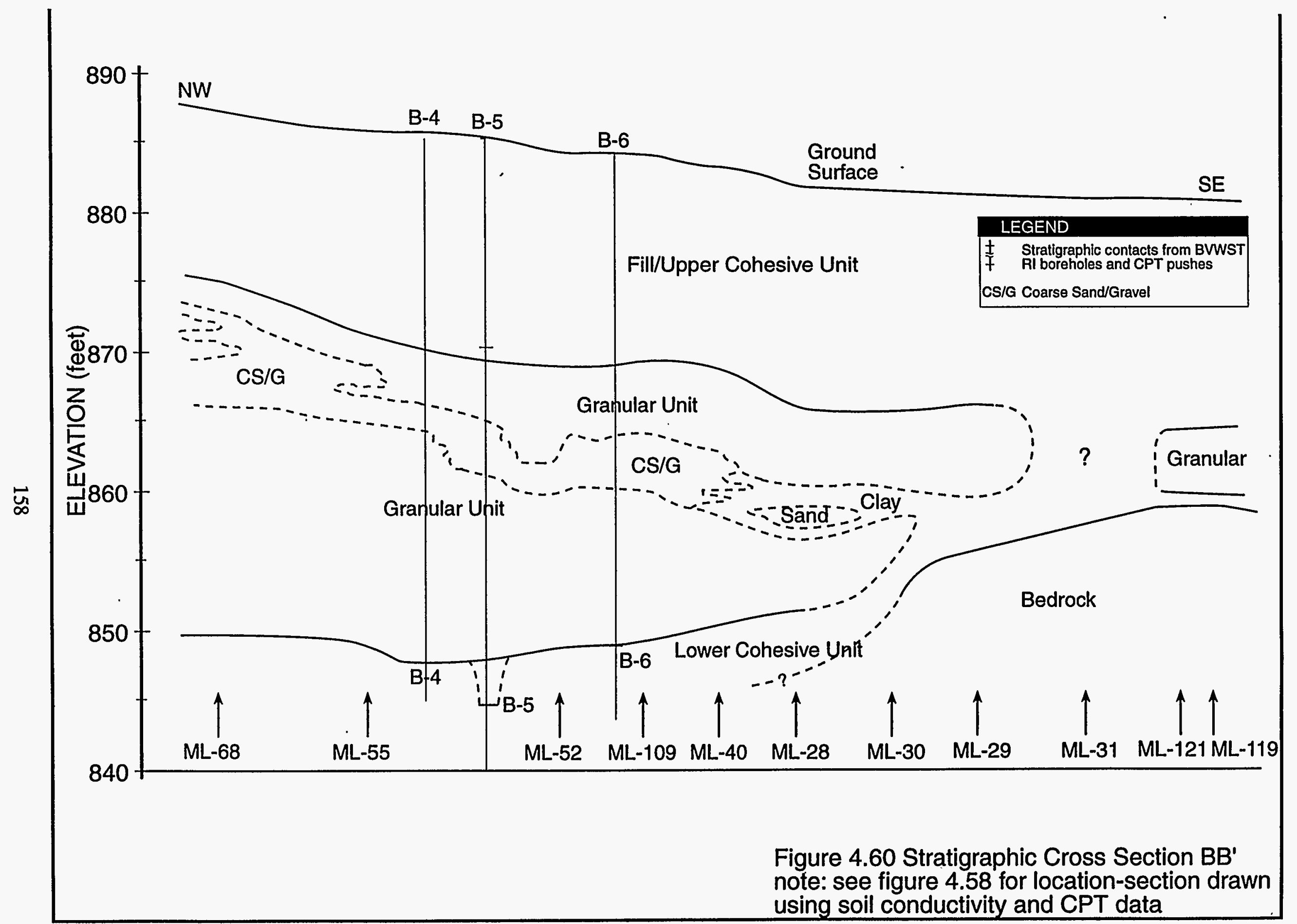



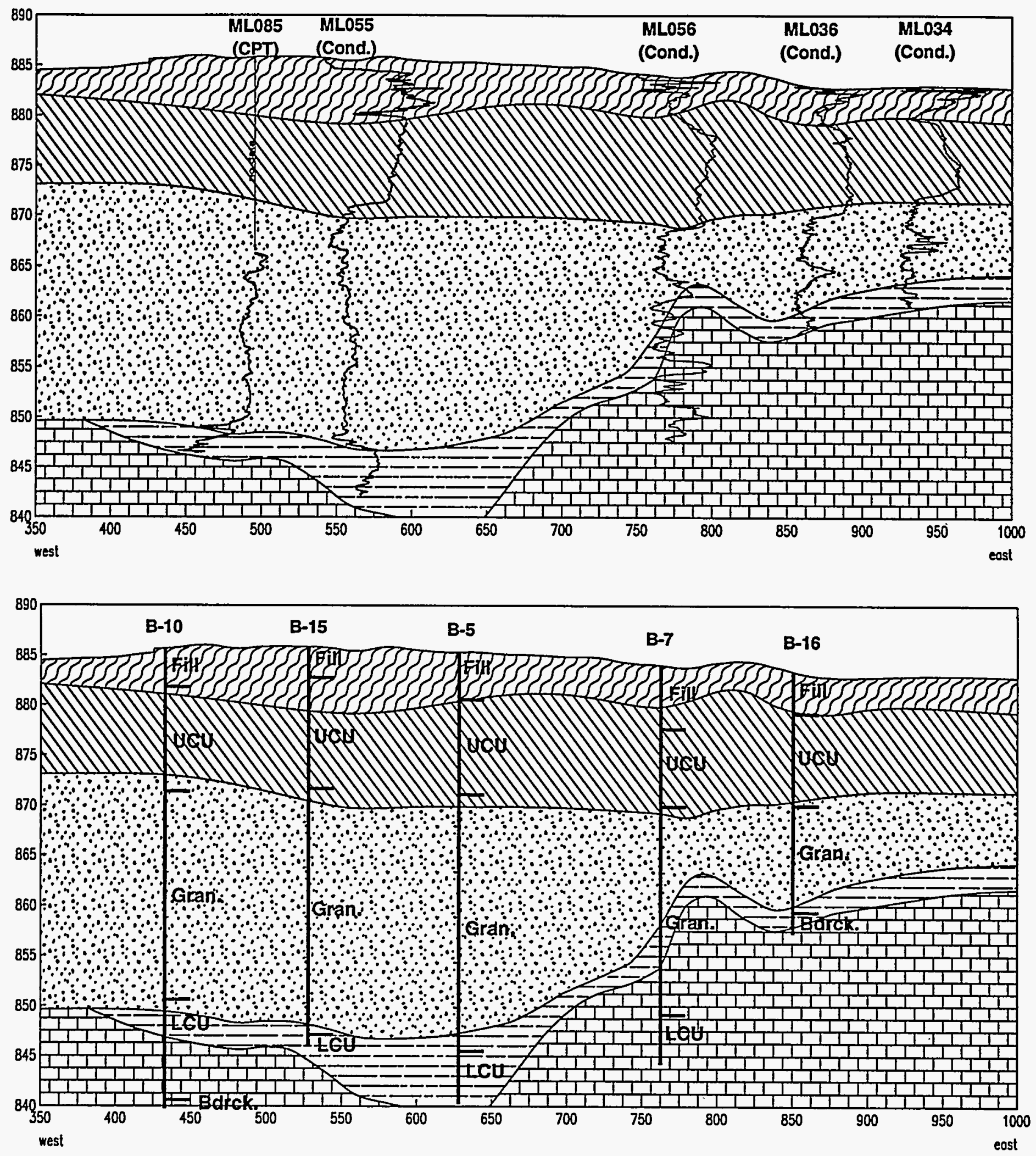

Figure 4.61 EarthVision Stratigraphic Model - East-West Cut at $500 \mathrm{~N}$

(Top - Projected ESC data overlay;

Bottom - Projected BVWST data overlay) 

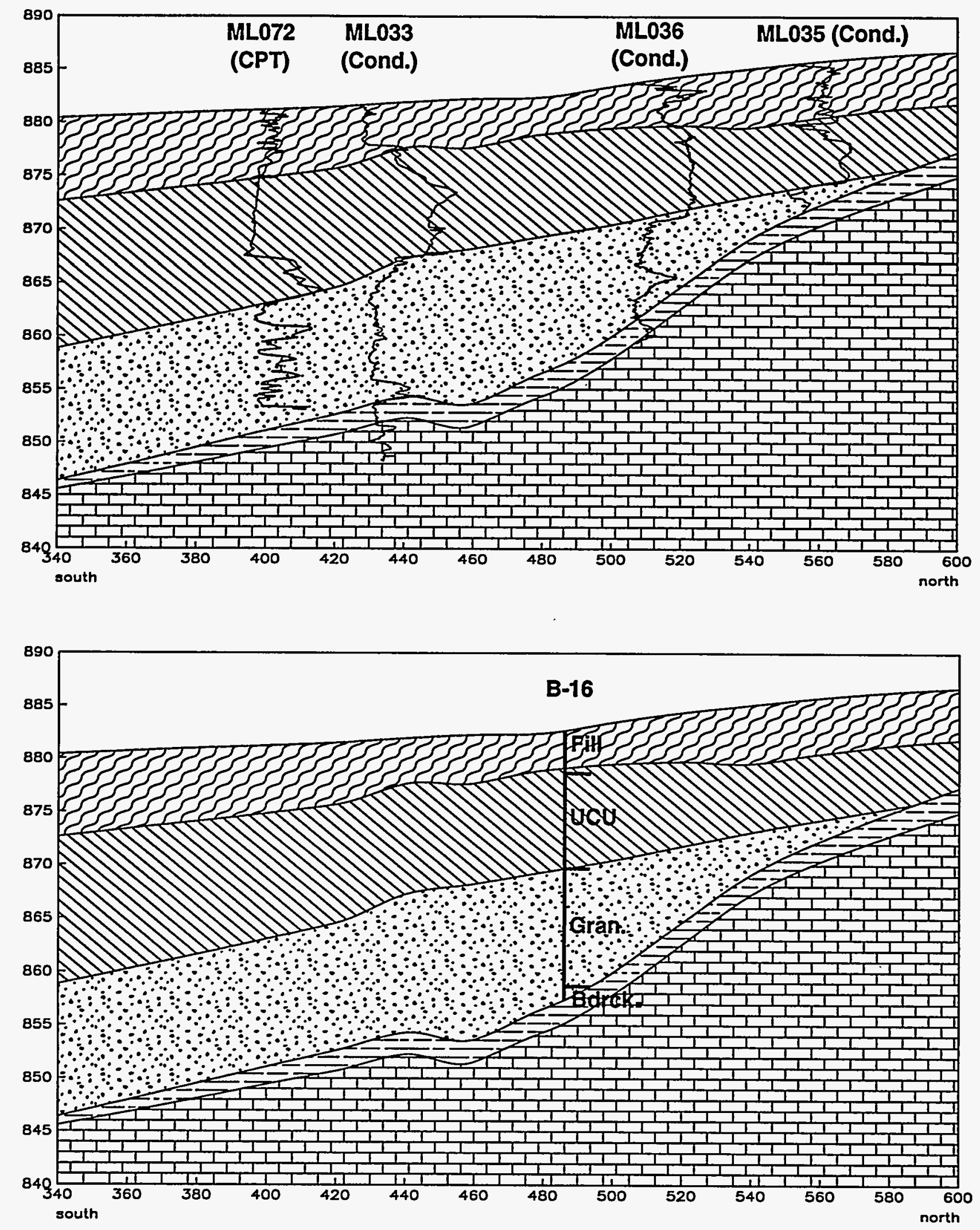

Figure 4.62 EarthVision Stratigraphic Model - North-South Cut at 850 E (Top - Projected ESC data overlay;

Bottom - Projected BVWST data overlay) 

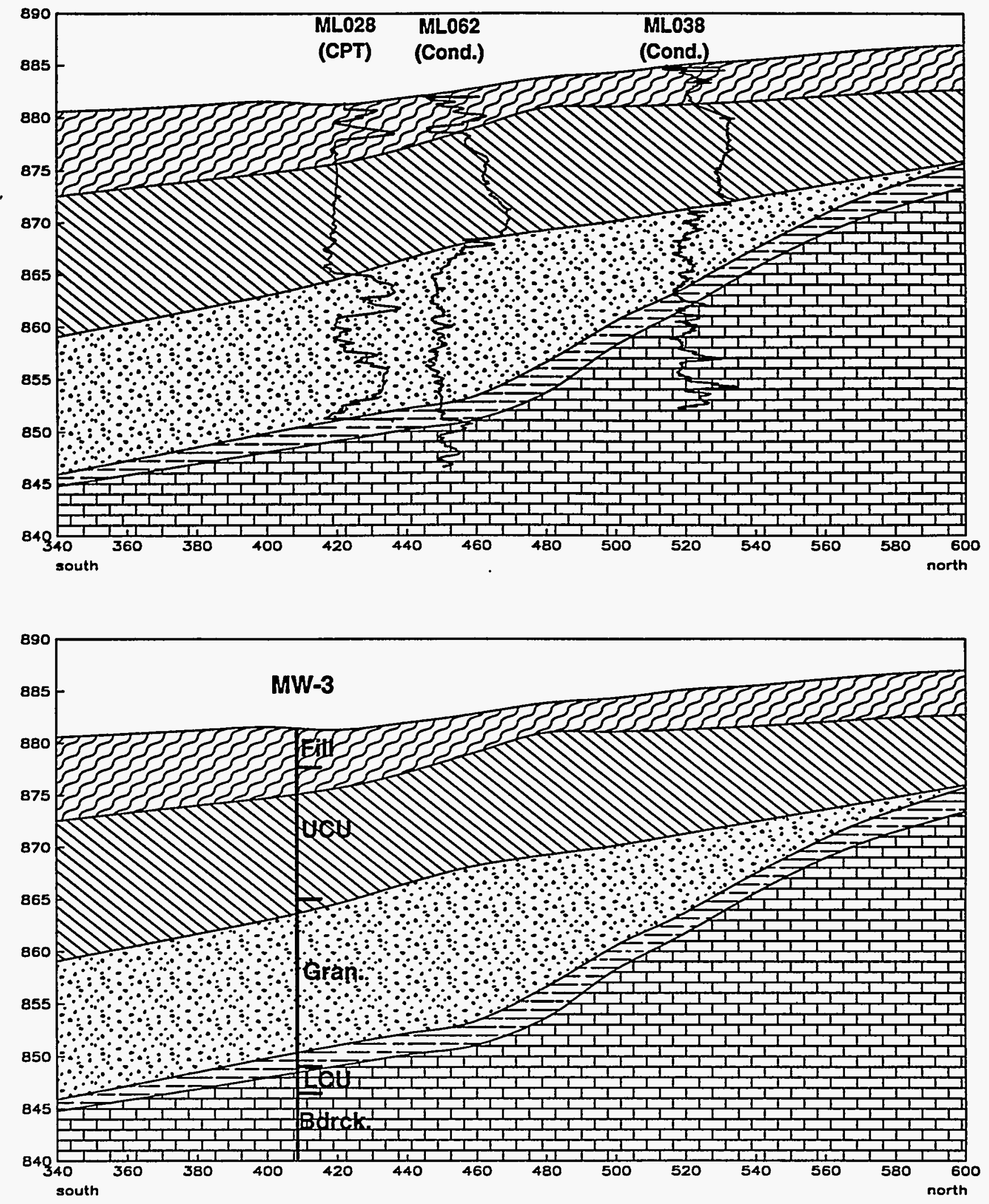

Figure 4.63 EarthVision Stratigraphic Model - North-South Cut at 825 E (Top - Projected ESC data overlay;

Bottom - Projected BVWST data overlay) 

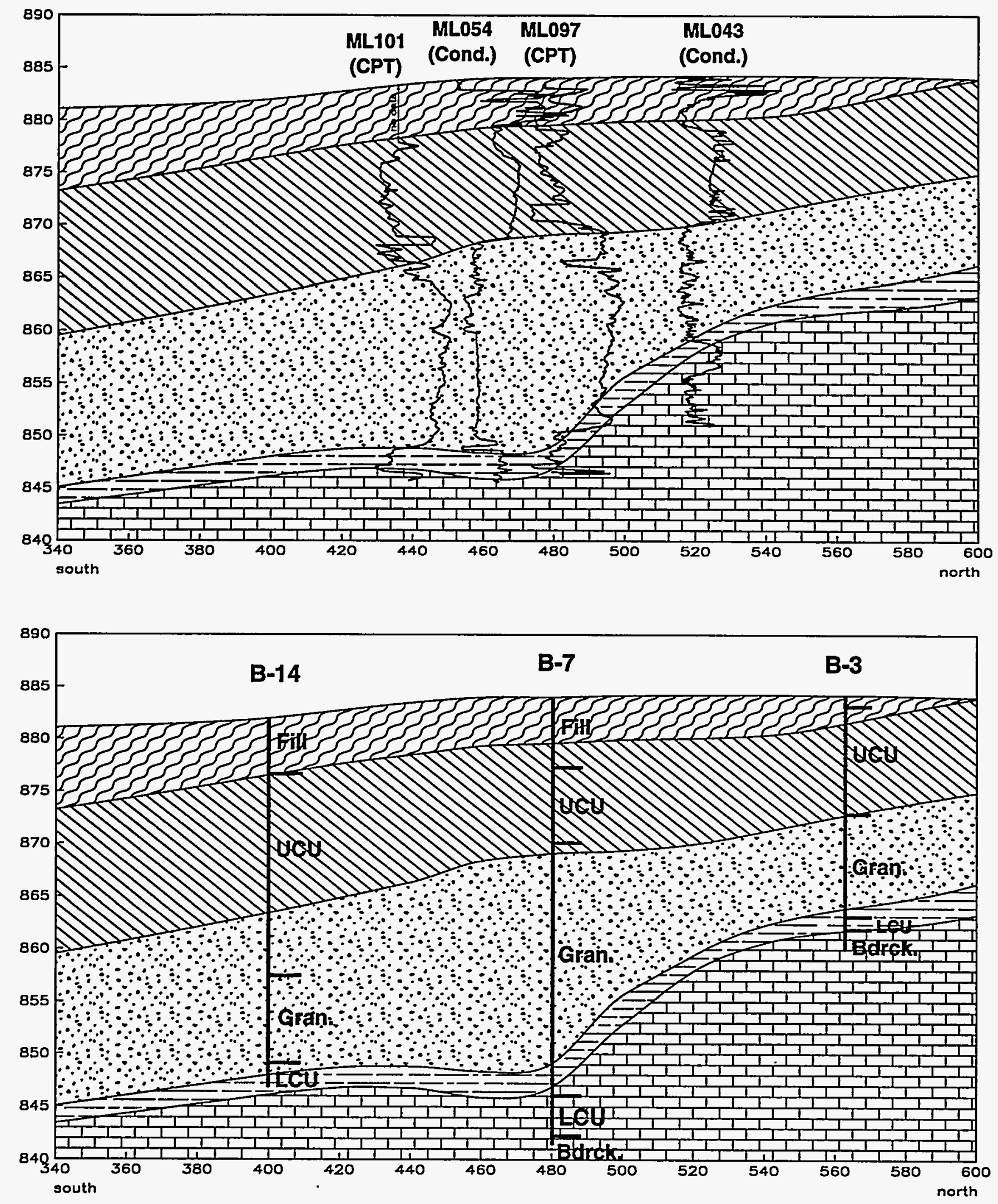

Figure 4.64 EarthVision Stratigraphic Model - North-South Cut at 750 E

(Top - Projected ESC data overlay;

Bottom - Projected BVWST data overlay) 


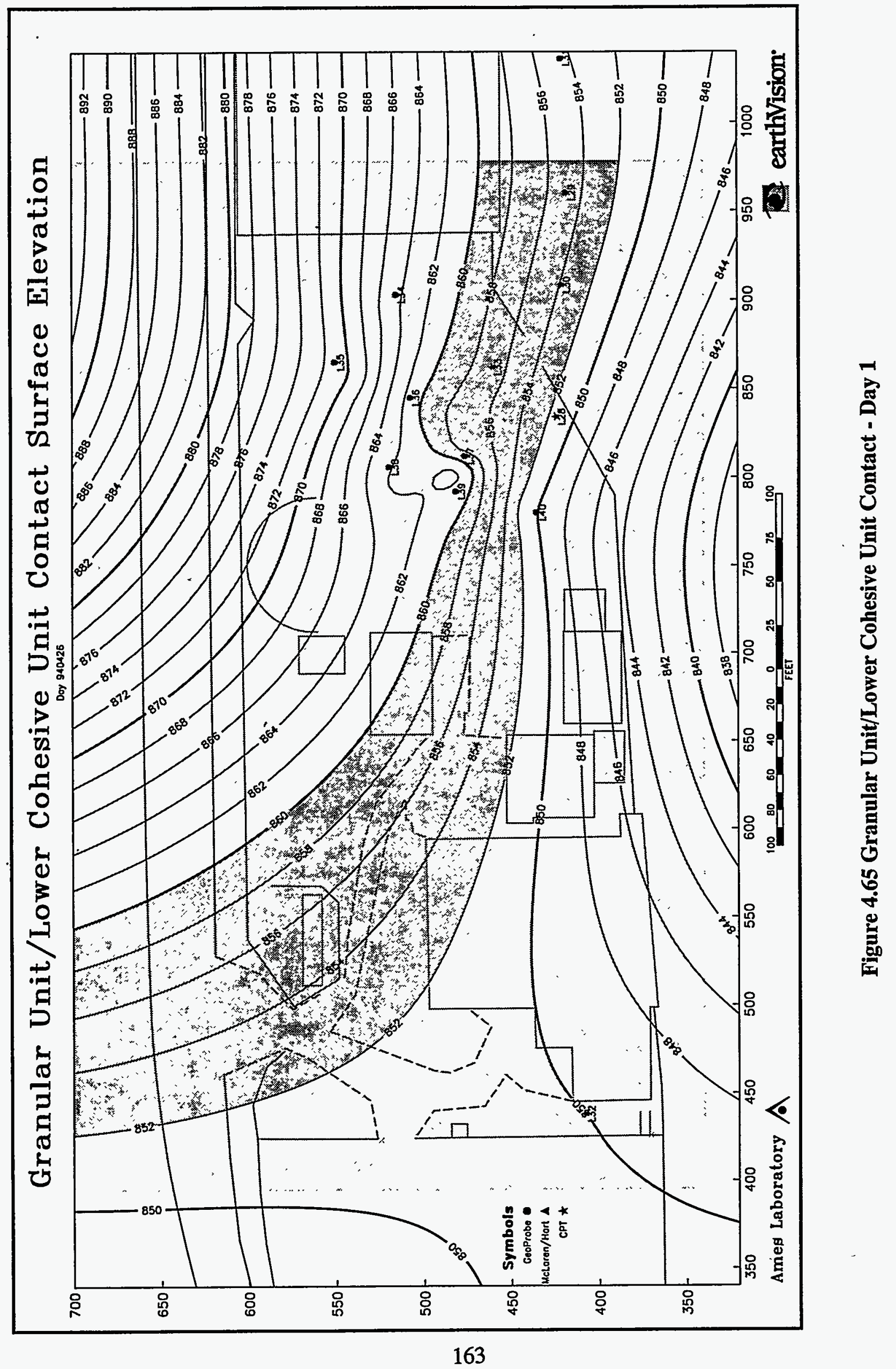




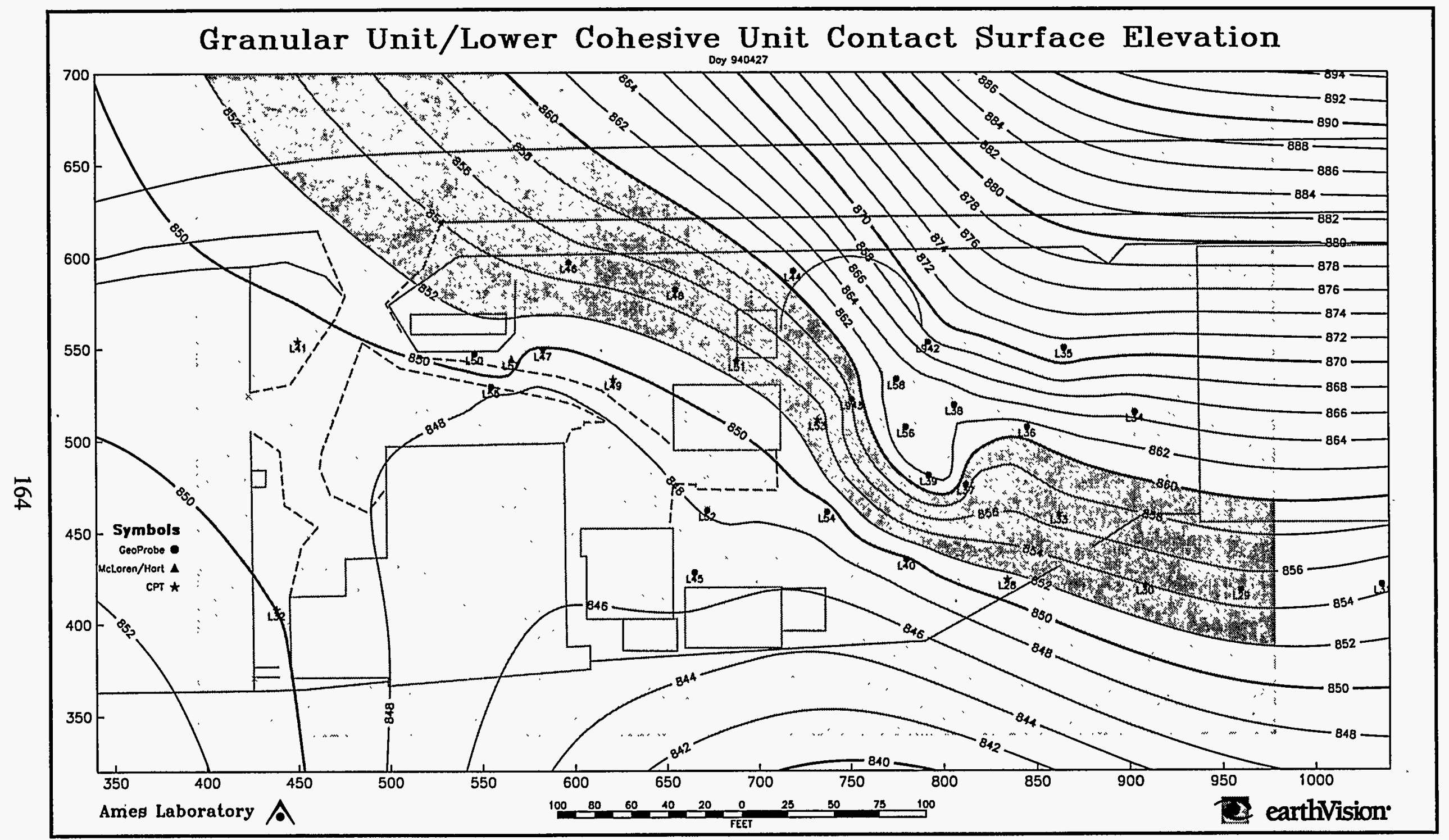

Figure 4.66 Granular Unit/Lower Cohesive Unit Contact - Day 2 


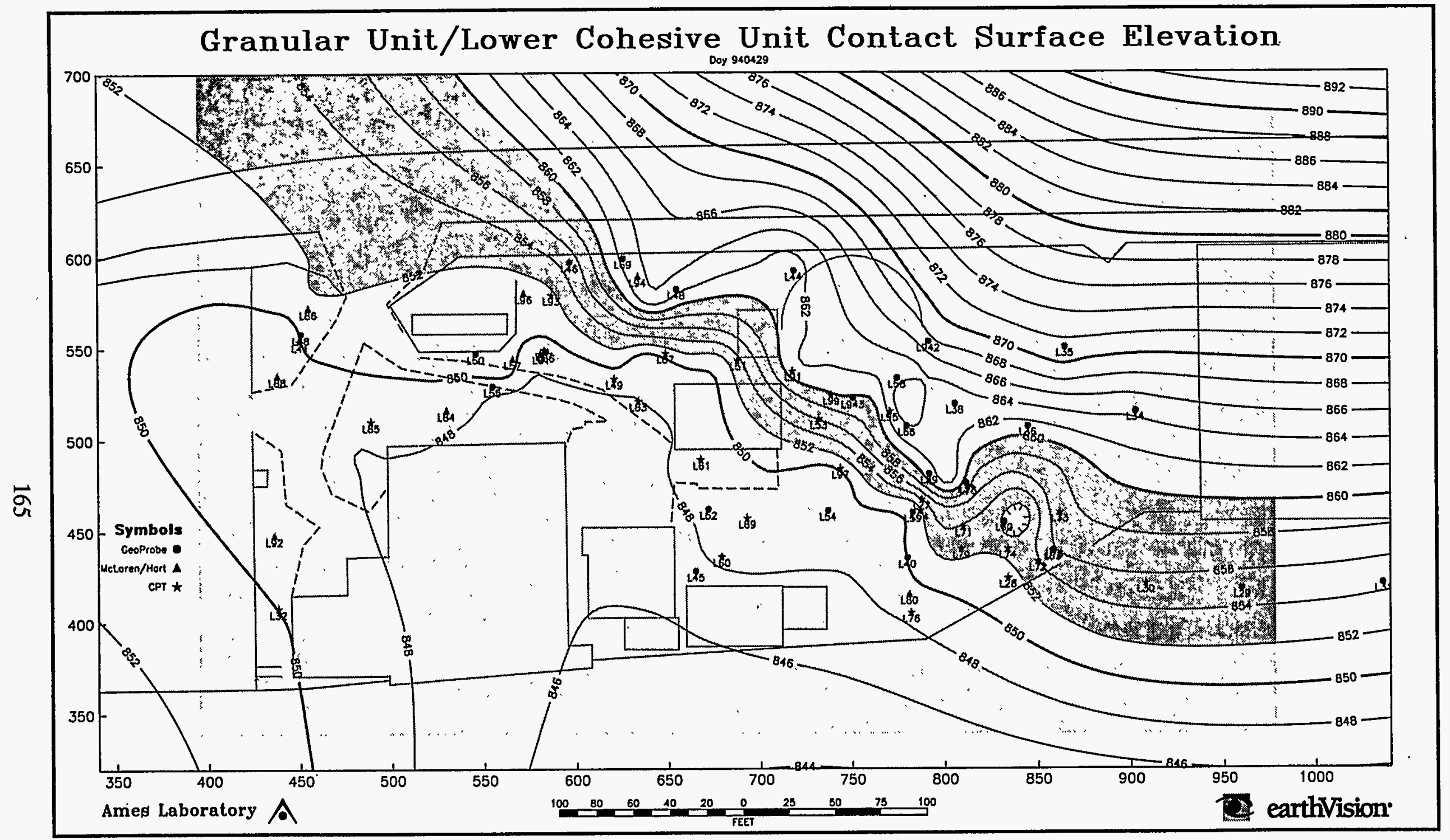

Figure 4.67 Granular Unit/Lower Cohesive Unit Contact - Day 4 


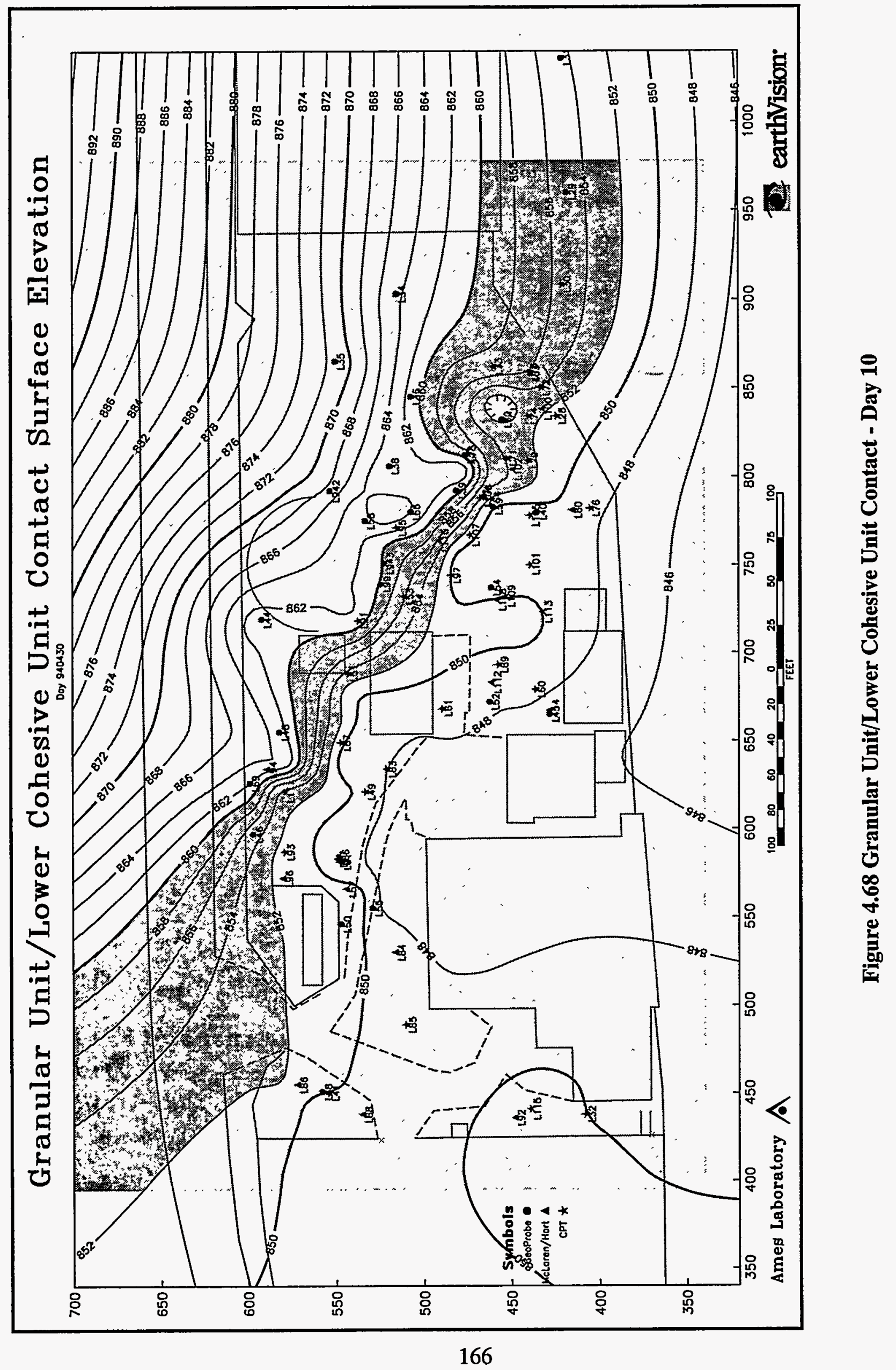




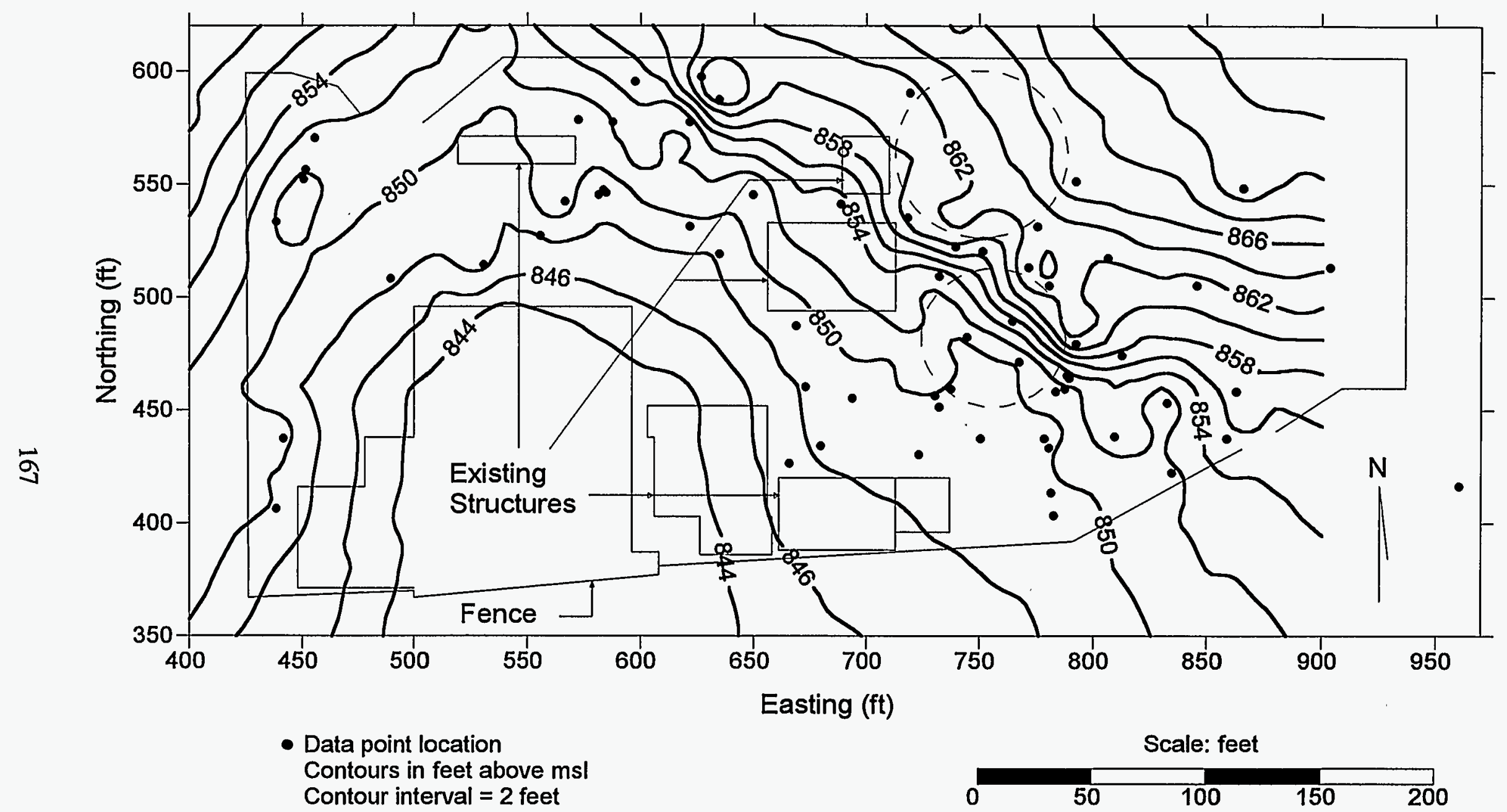

Figure 4.69 Lower Cohesive Unit Surface Elevation Based on Kriged Median Polish Residuals 


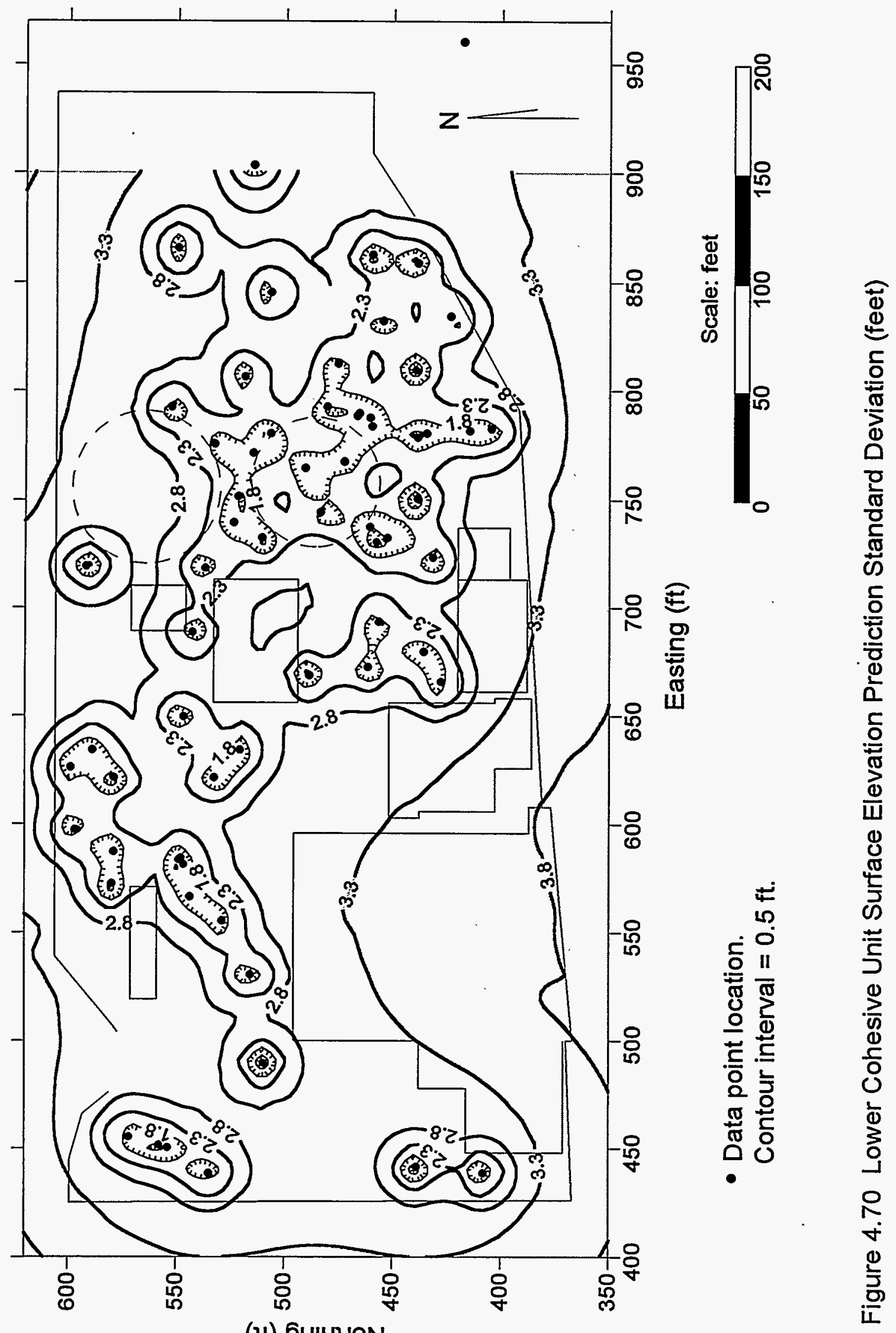




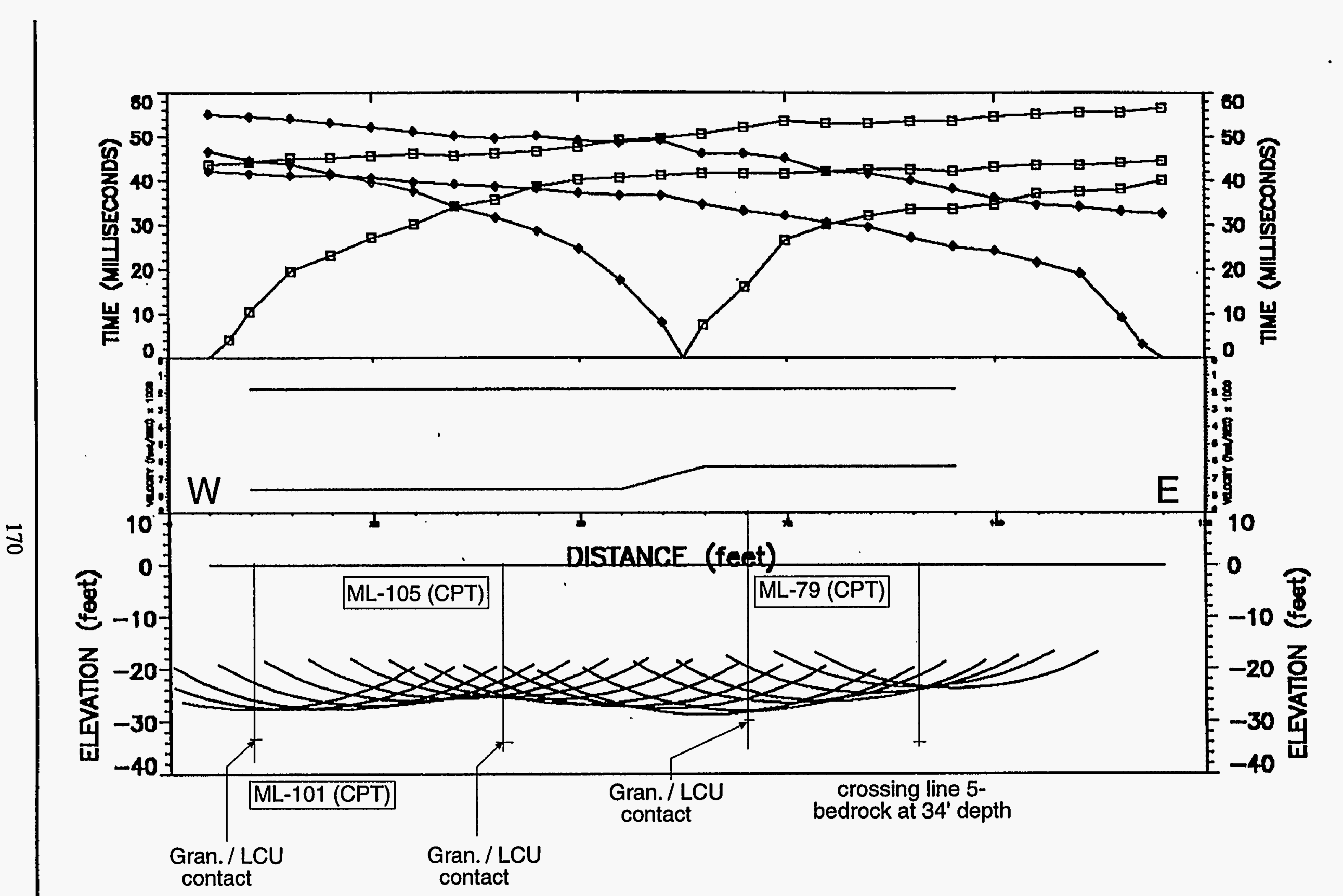

Figure 4.72 Seismic Refraction Survey Line 7 Comparison with Direct Push Data (after CEES - Blackhawk Geosciences Division) 


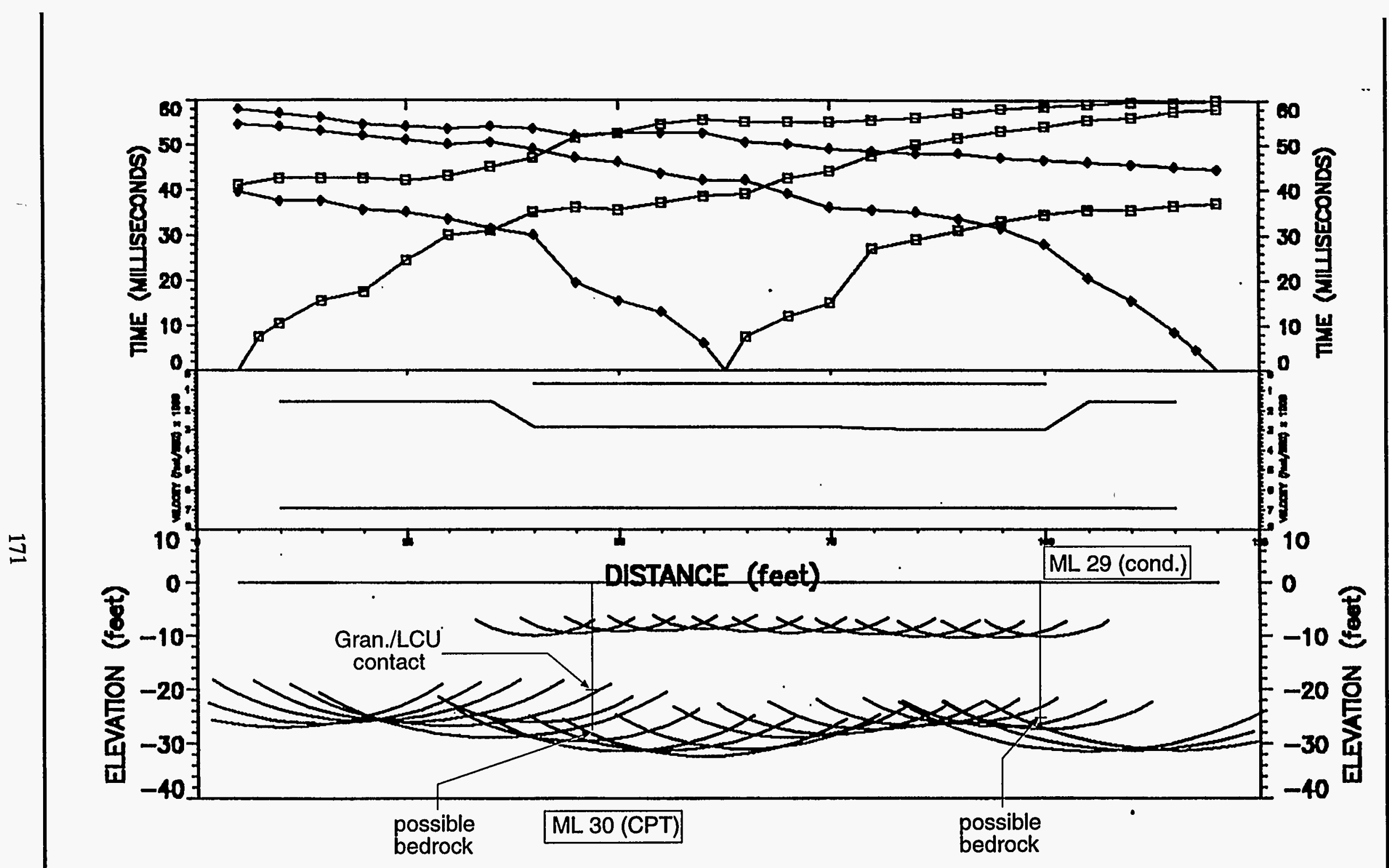

Figure 4.73 Seismic Refraction Survey Line 9 Comparison with Direct Push Data (after CEES - Blackhawk Geosciences Division) 


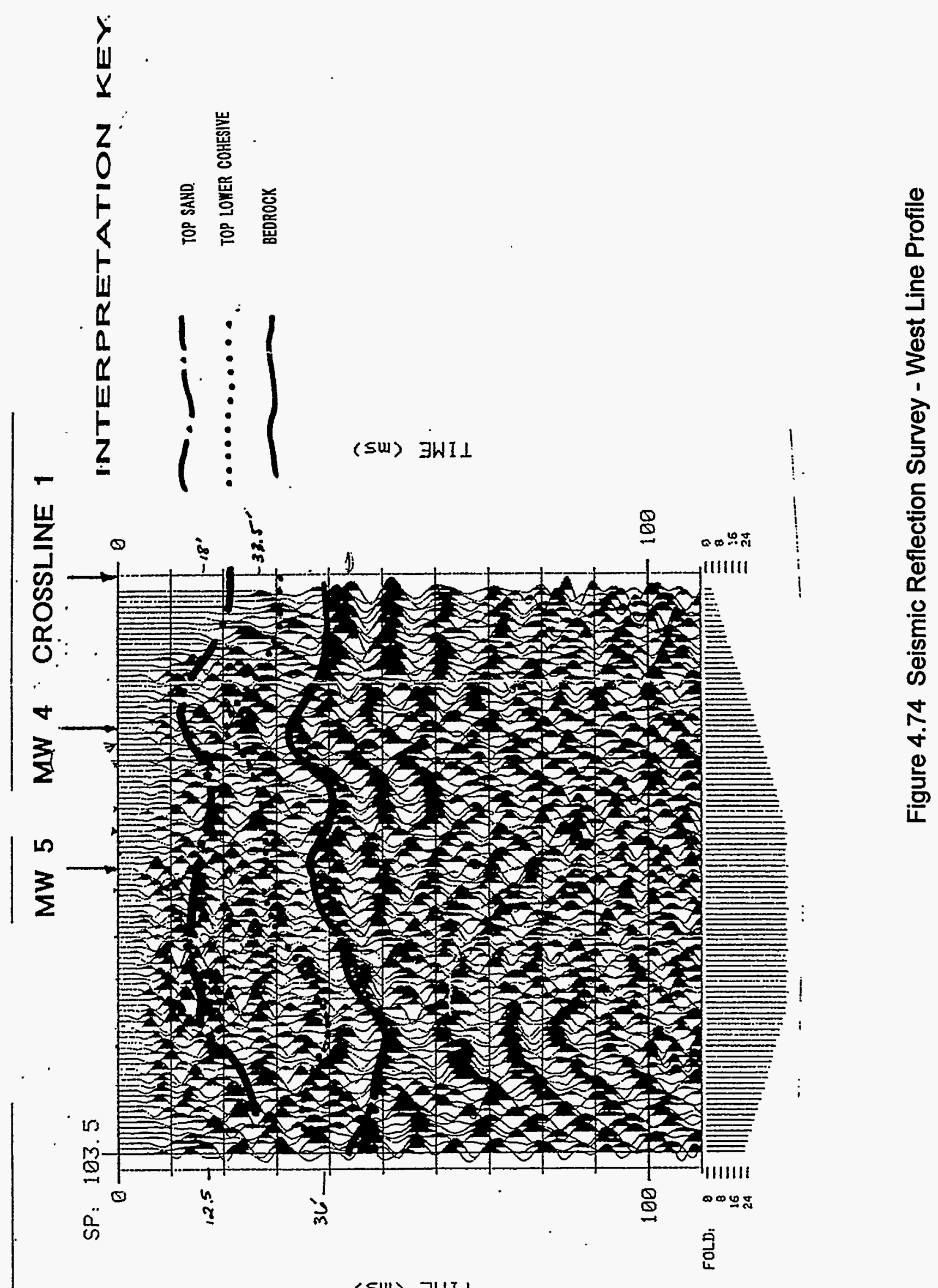

(SW) $\exists W I \perp$ 


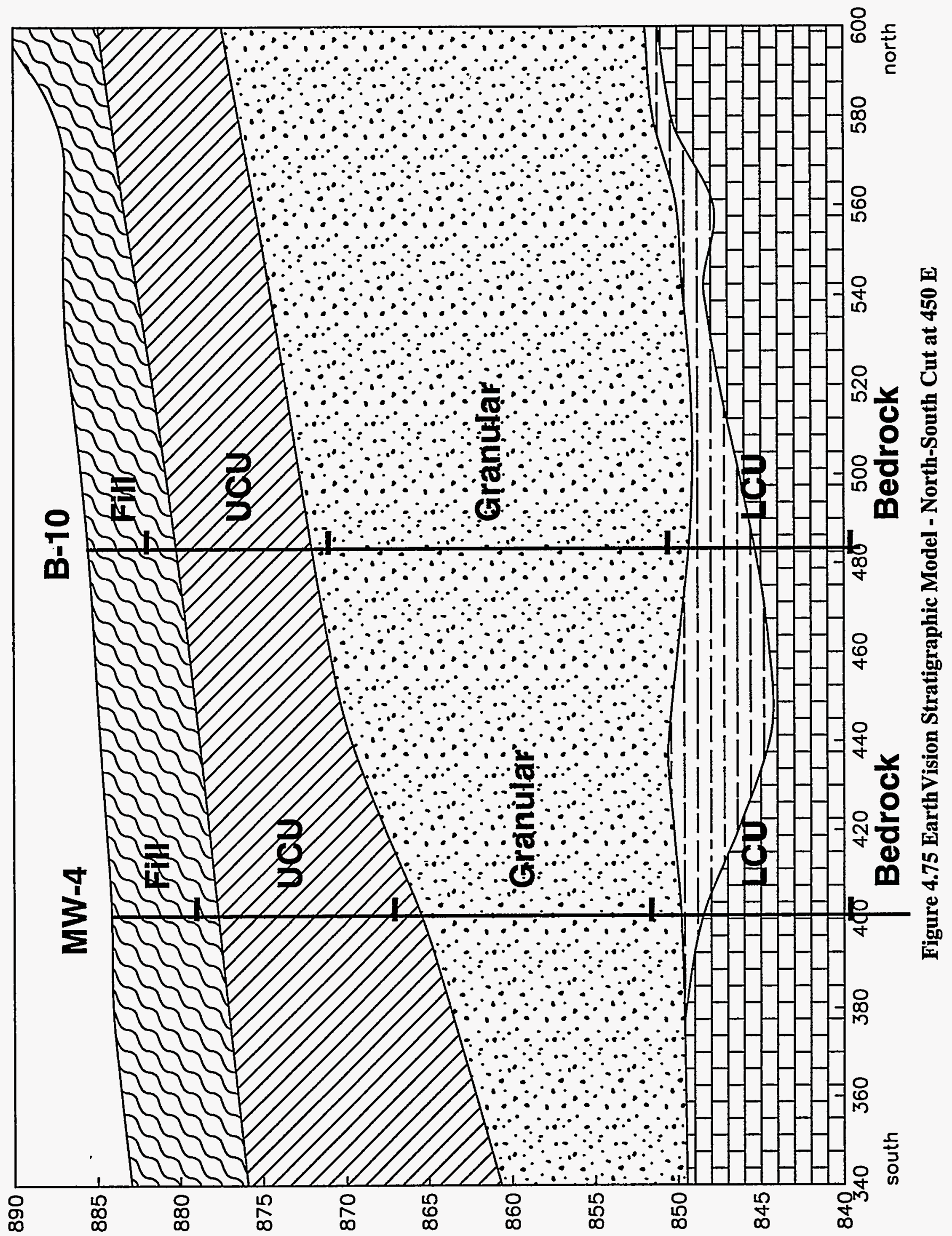




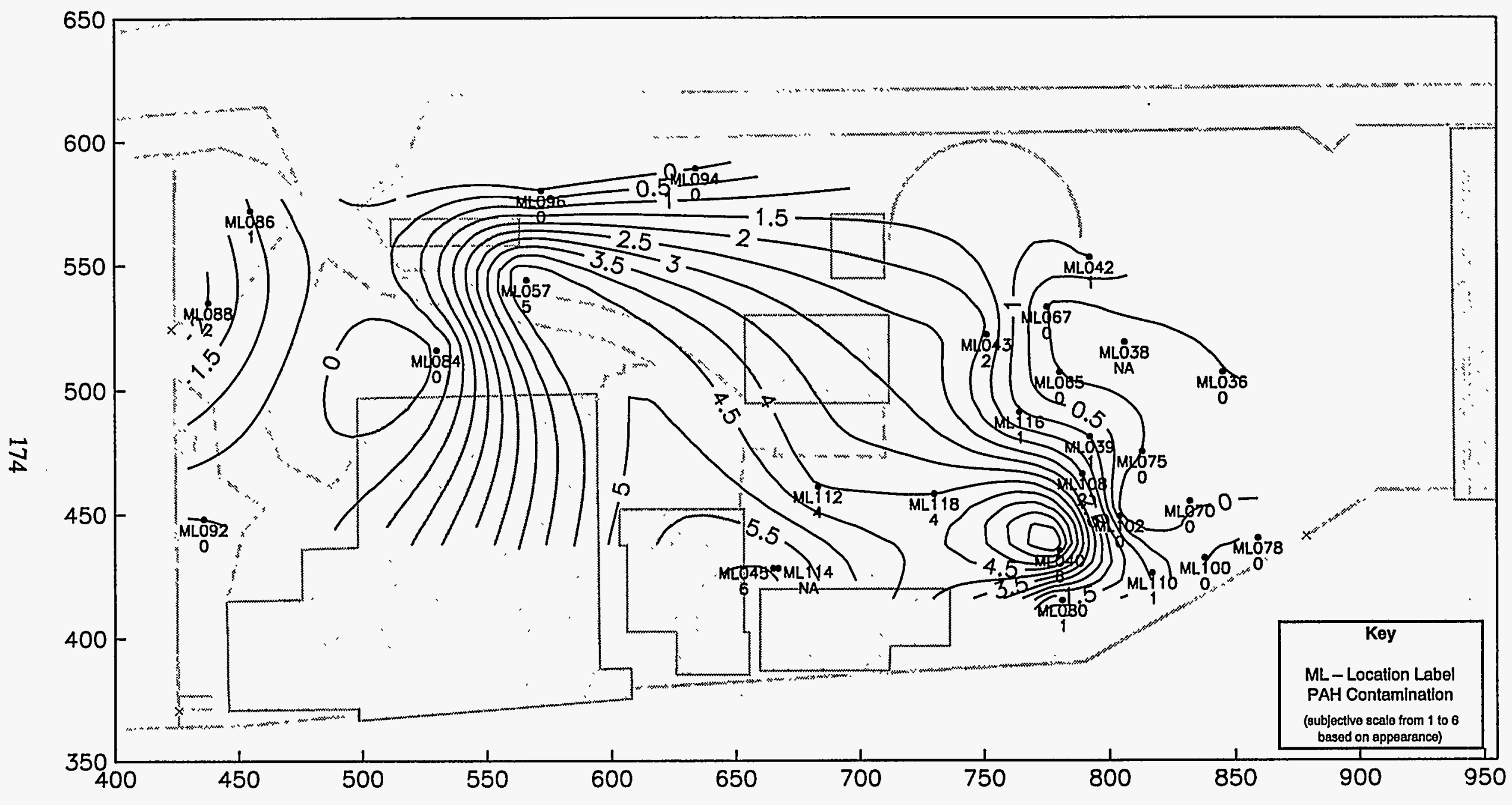

Figure 4.76 McLaren/Hart Samples - Observed Level of PAH Contamination in Soils - Stratigraphic Zone 1 


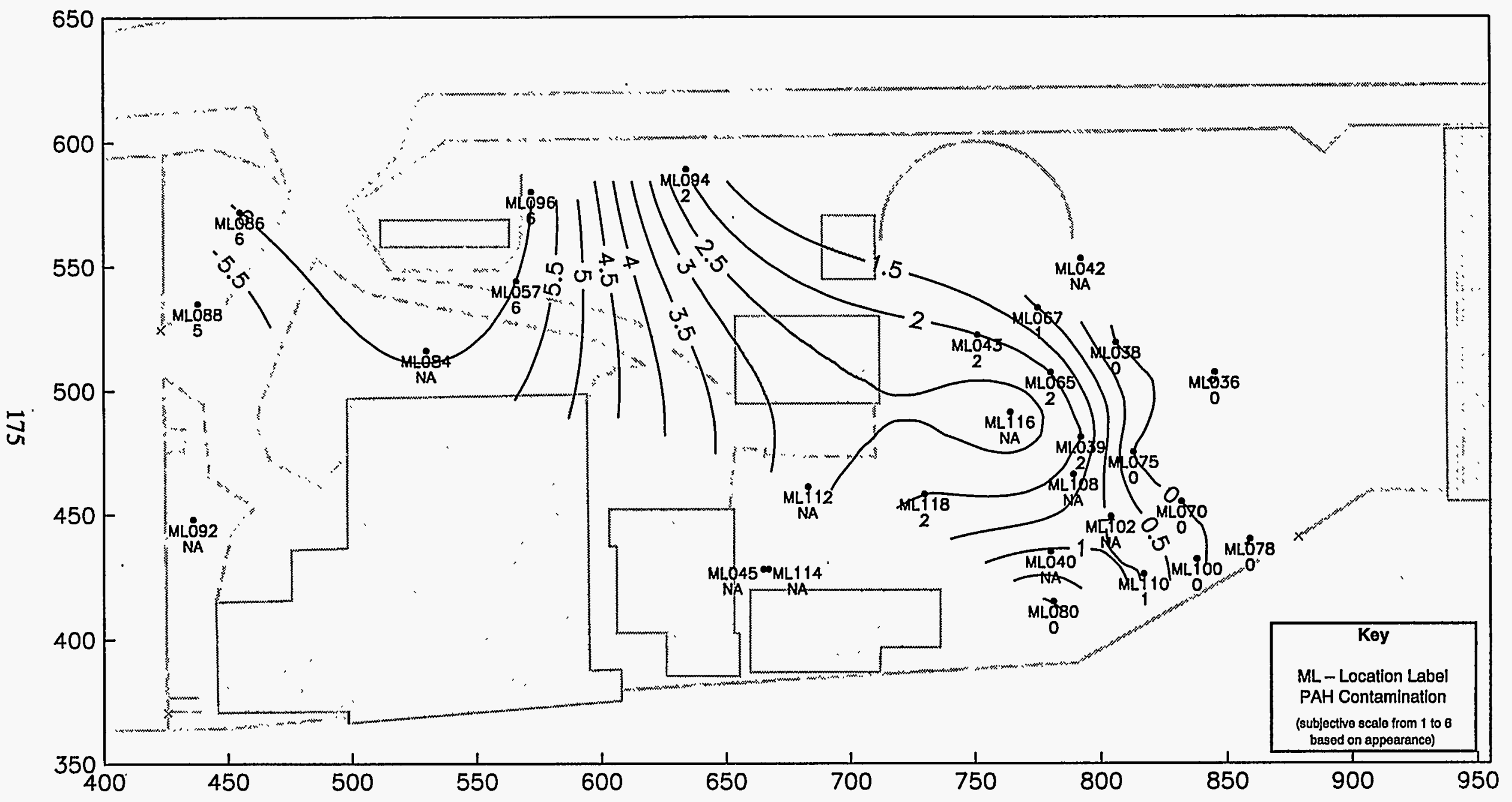

Figure 4.77 McLaren/Hart Samples - Observed Level of PAH Contamination in Soils - Stratigraphic Zone 2 


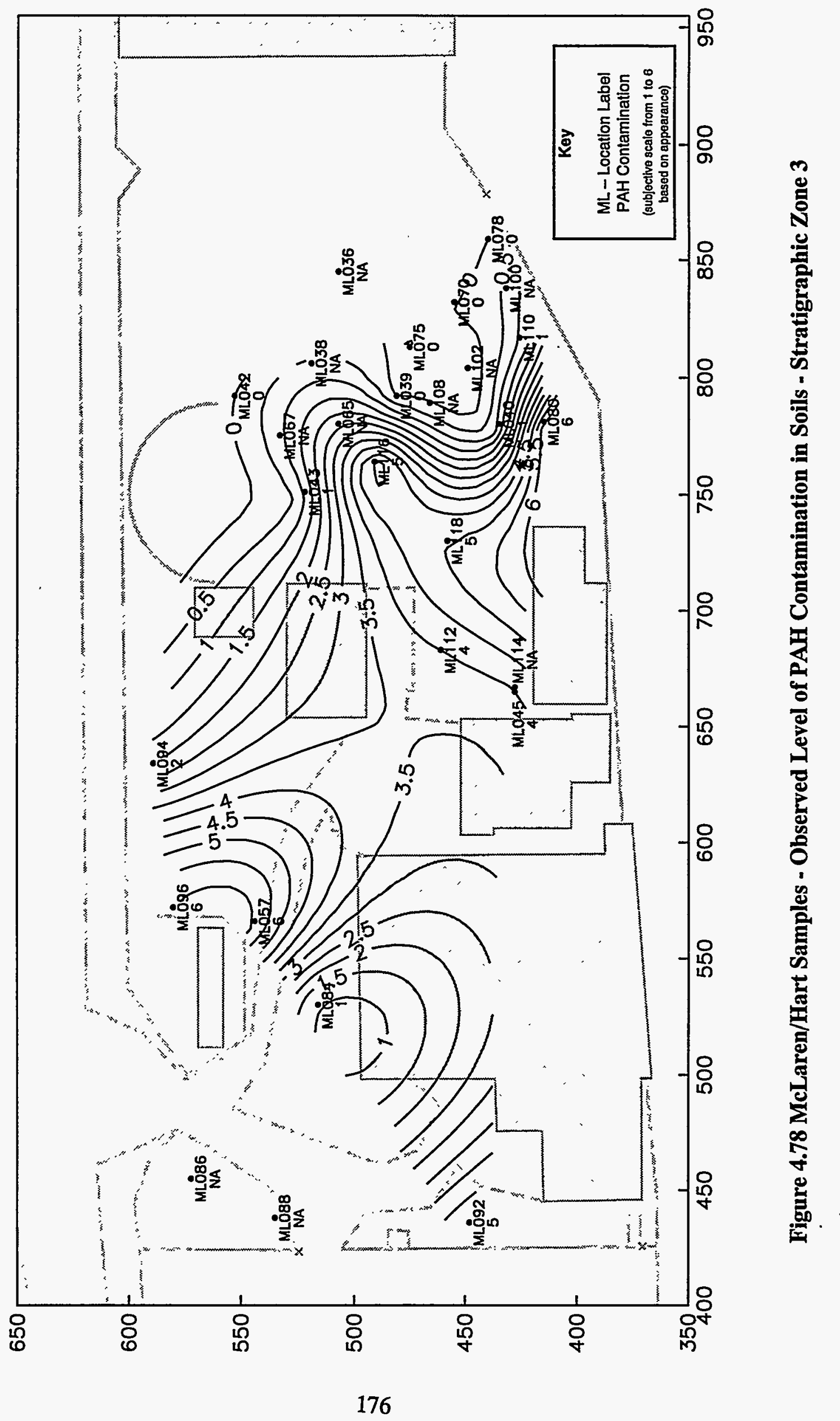




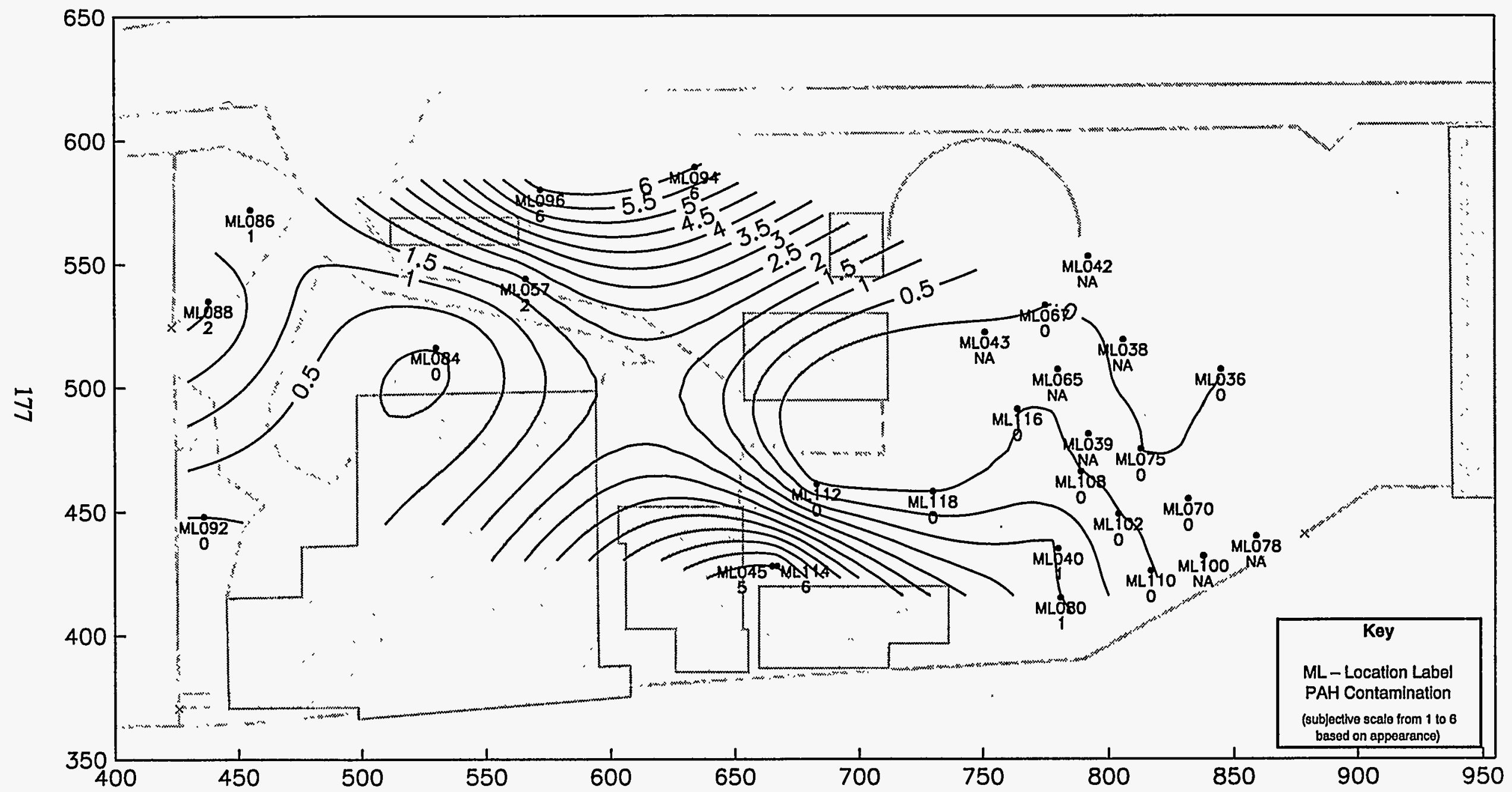

Figure 4.79 McLaren/Hart Samples - Observed Level of PAH Contamination in Soils - Stratigraphic Zone 4 


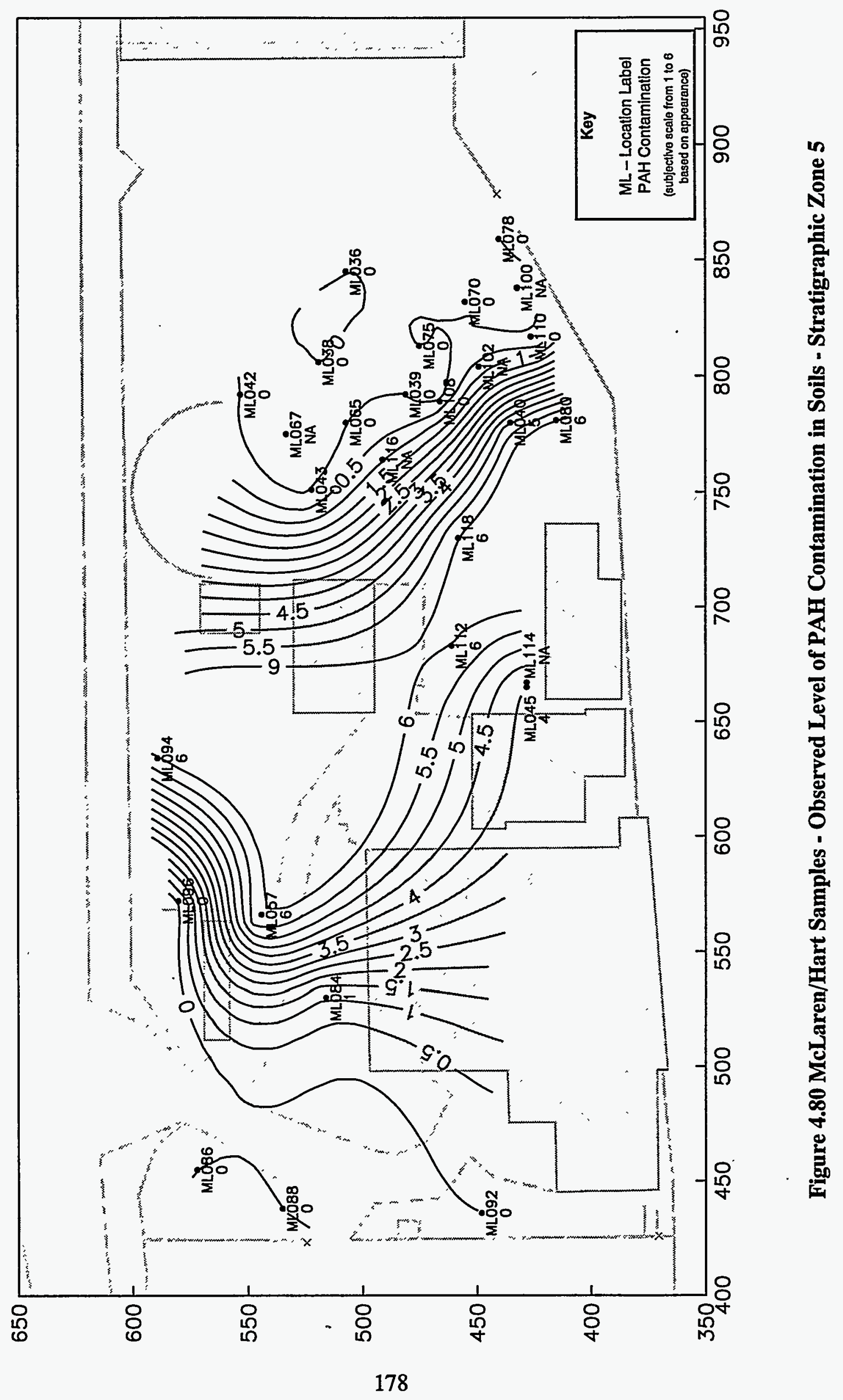




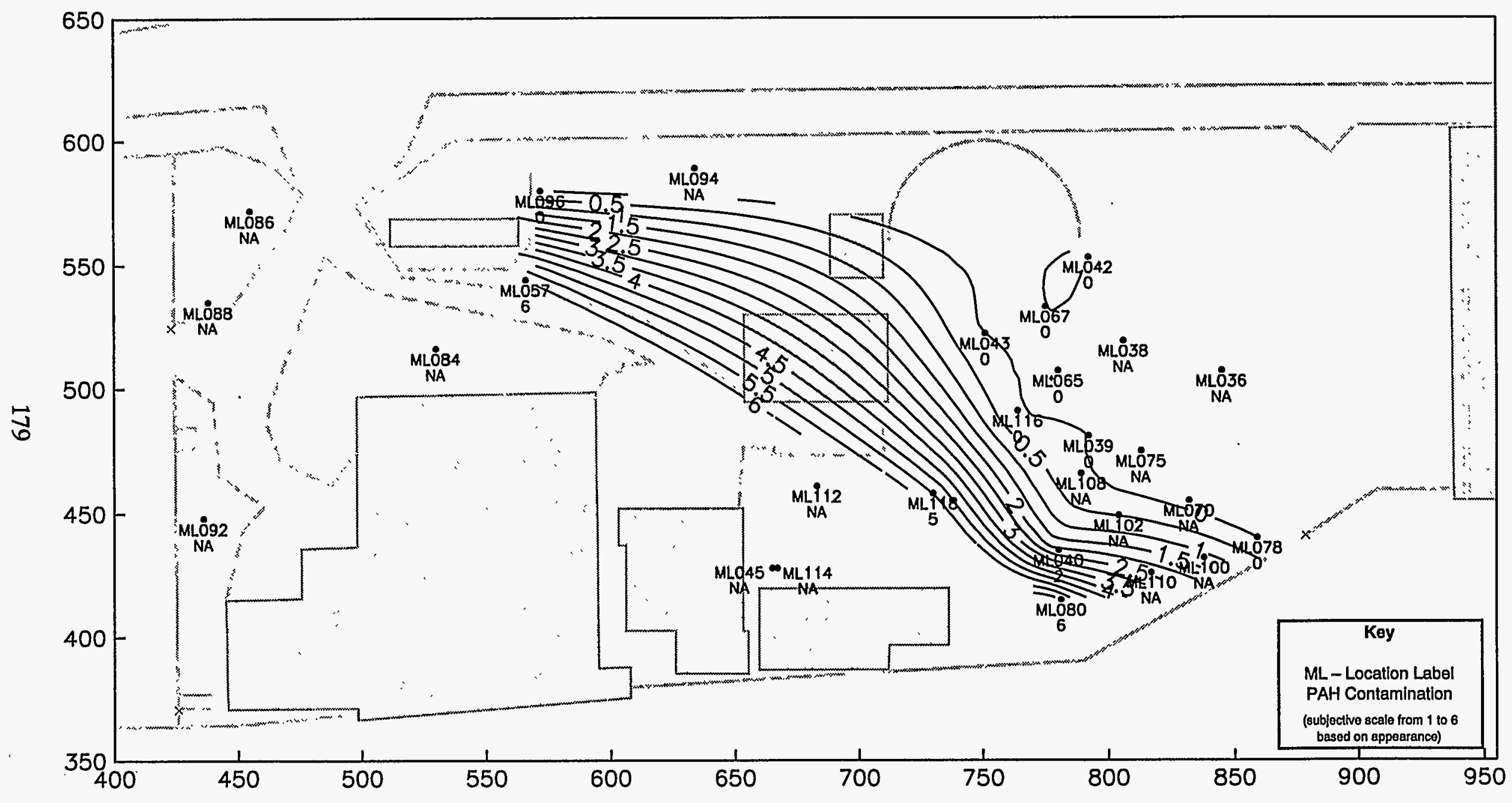

Figure 4.81 McLaren/Hart Samples - Observed Level of PAH Contamination in Soils - Stratigraphic Zone 6 


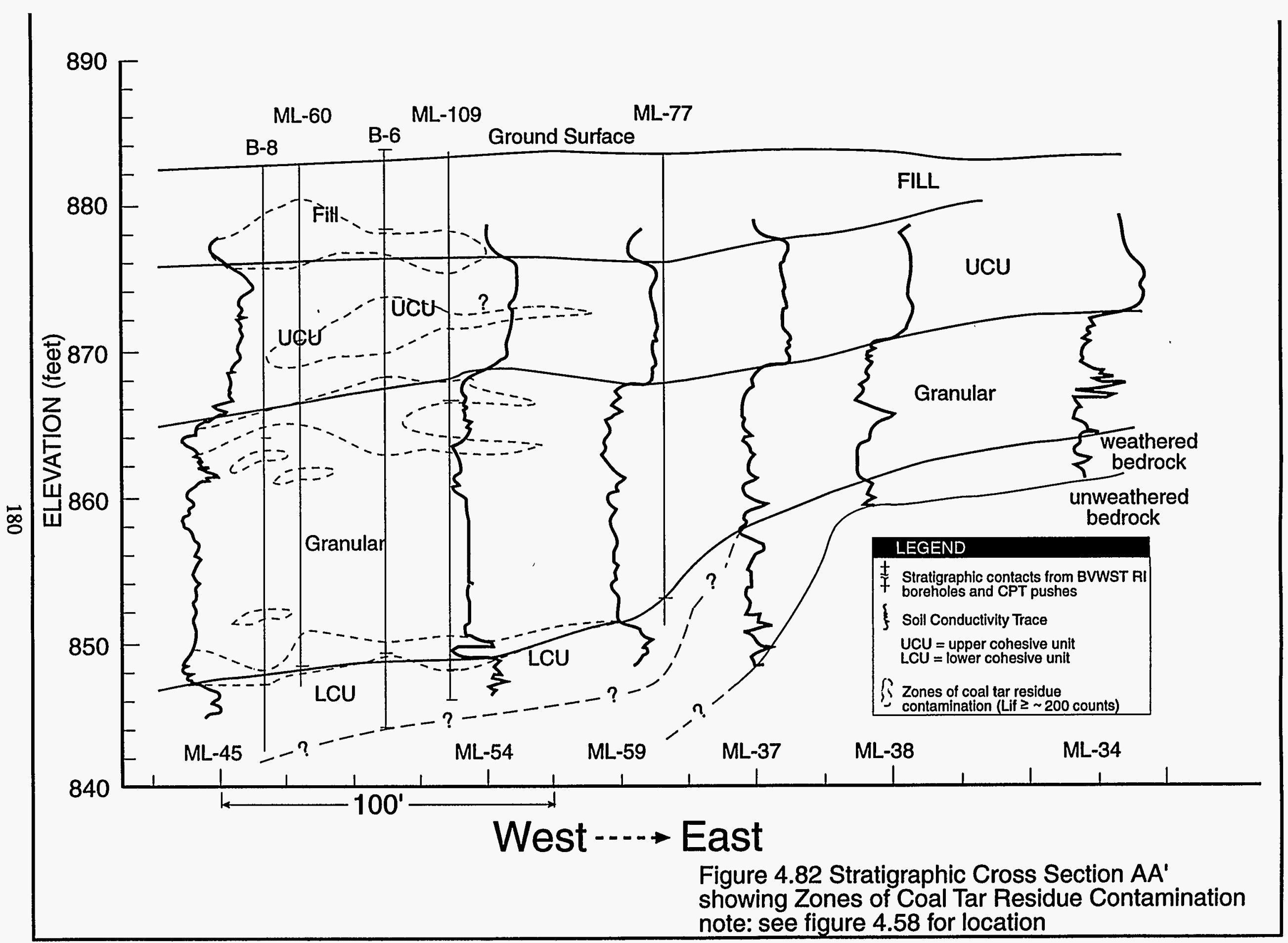




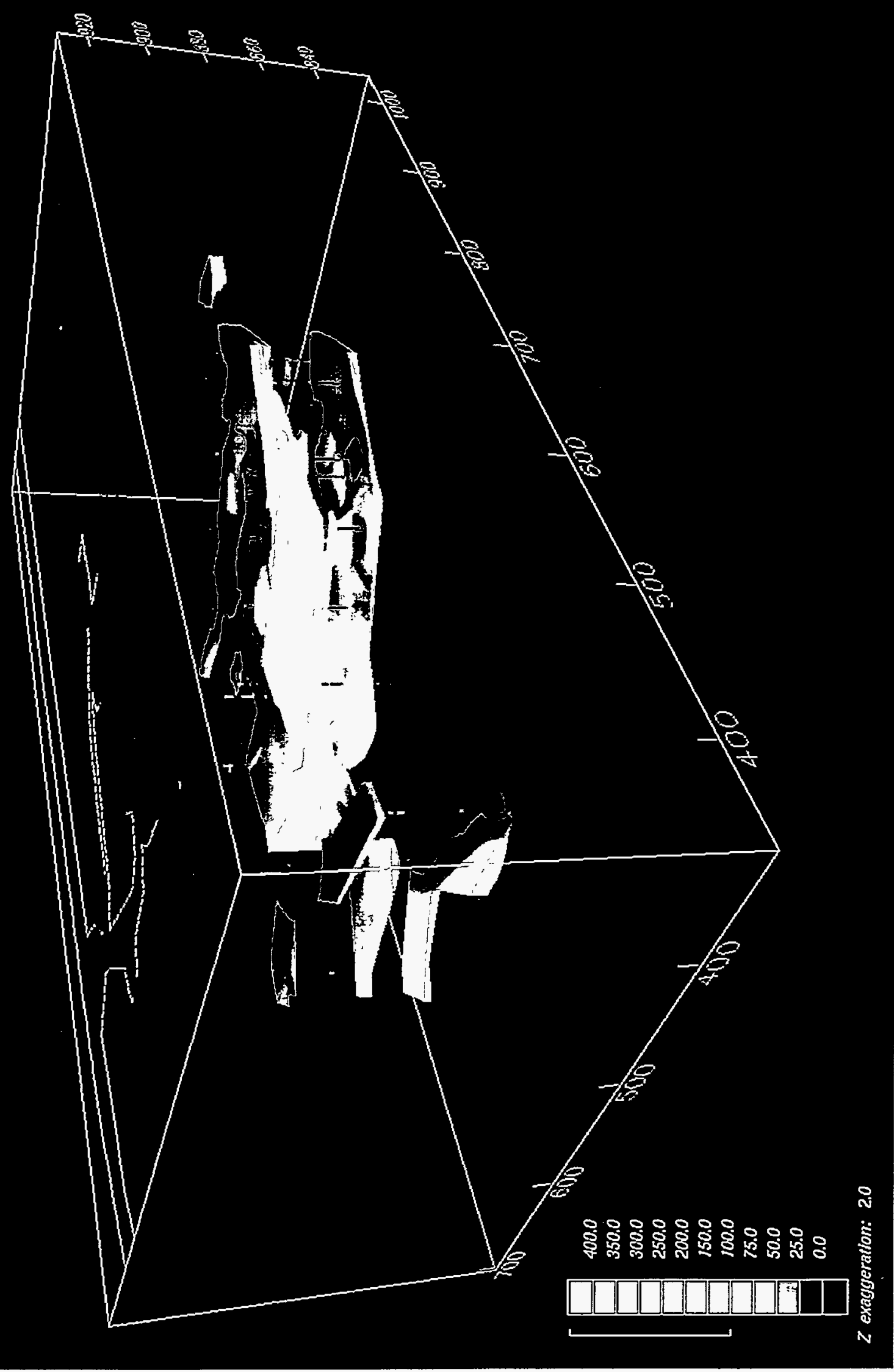

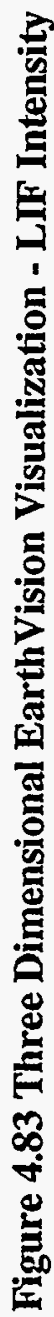




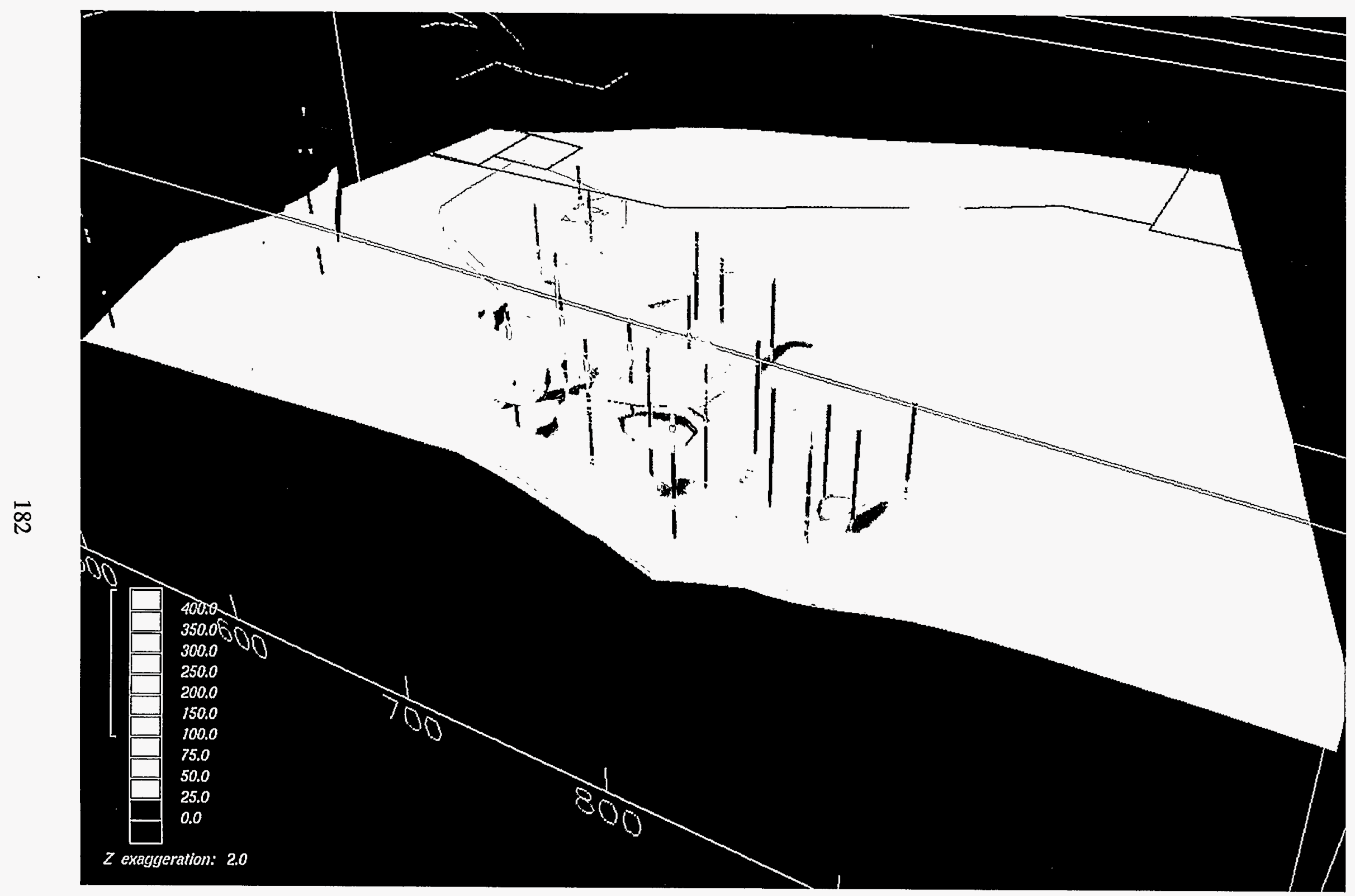

Figure 4.84 Three Dimensional Earth Vision Visualization - Regions with LIF Intensity > 100 Counts 


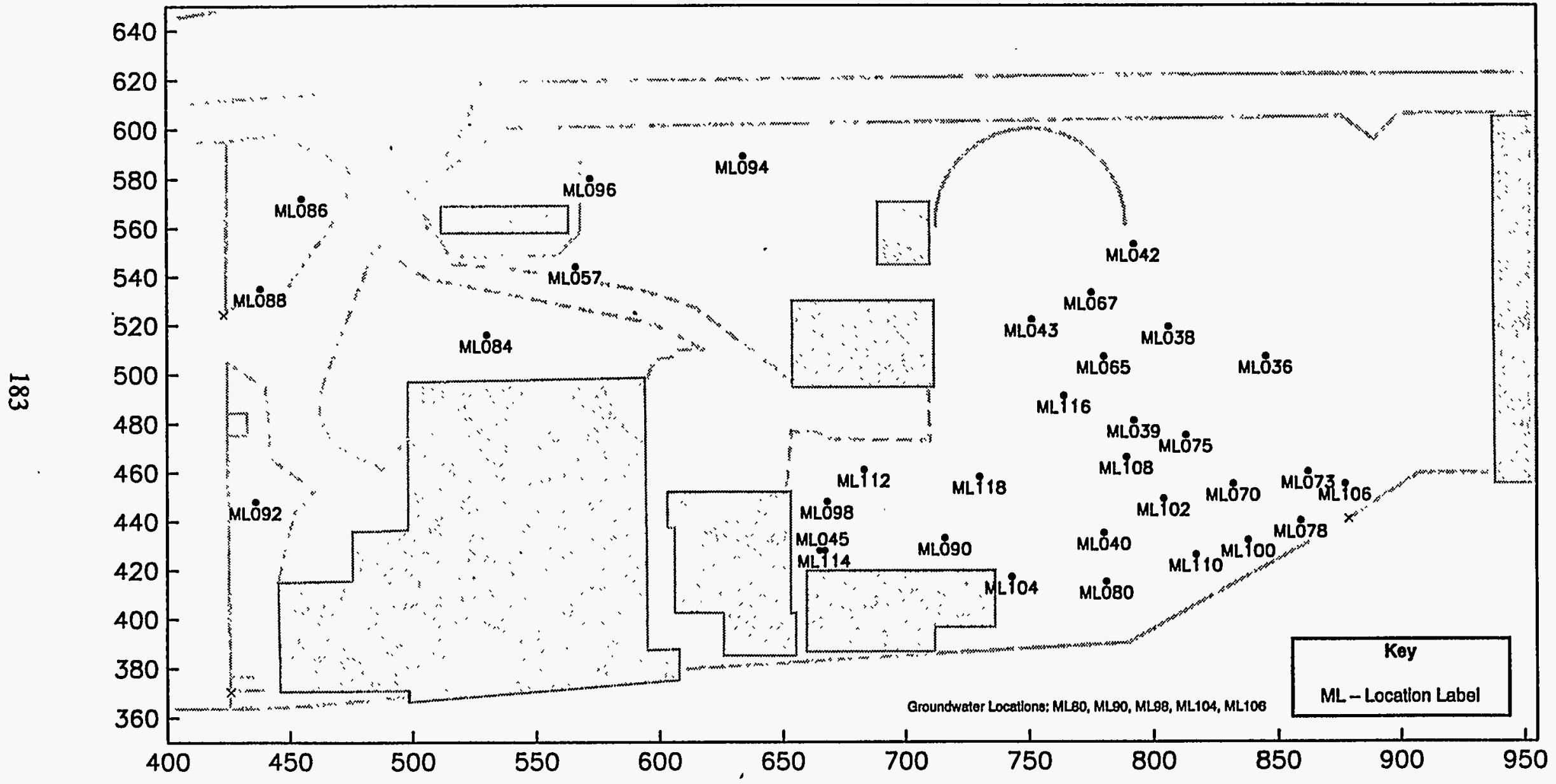

Figure 4.85 McLaren/Hart Soil and Groundwater Sampling Locations 


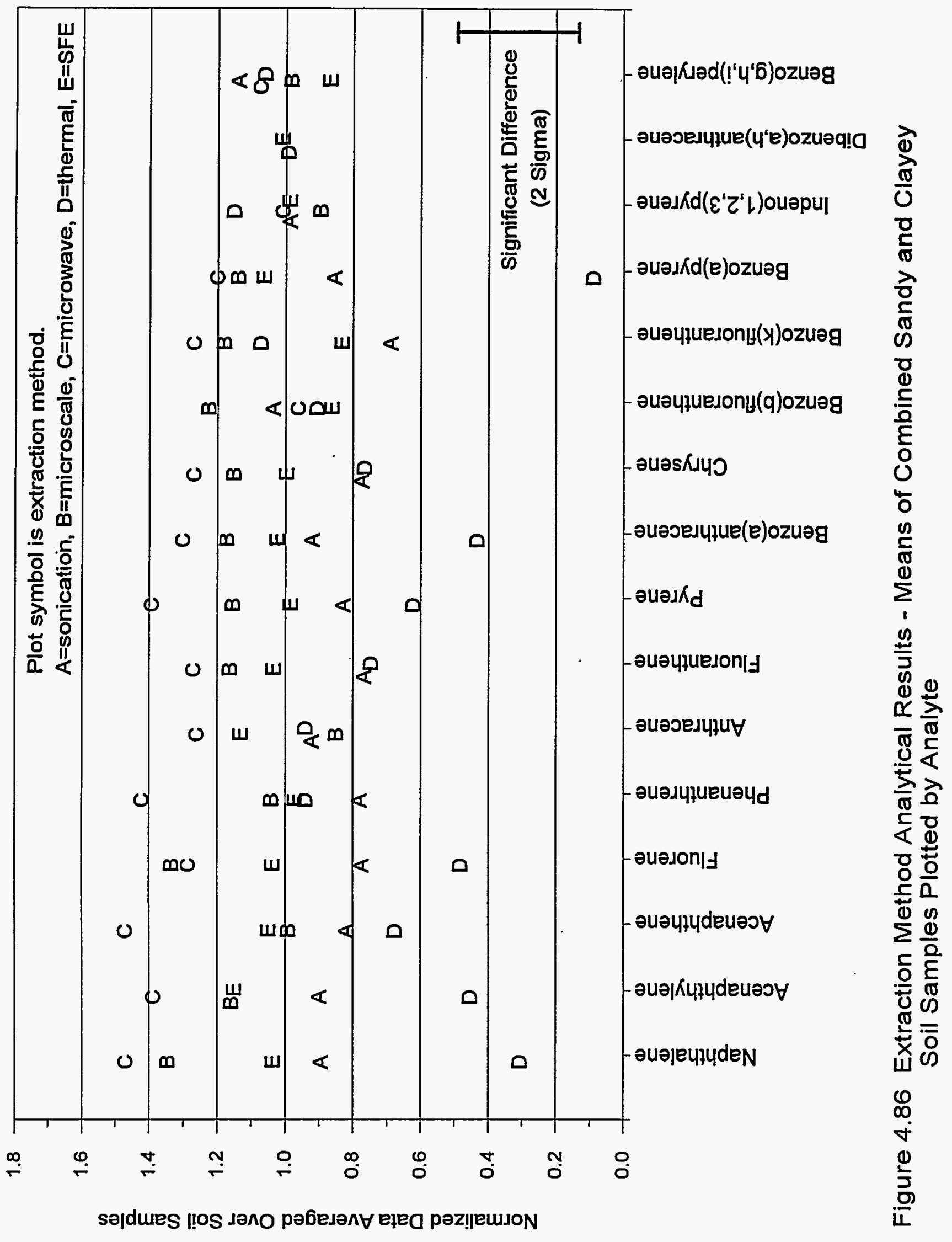




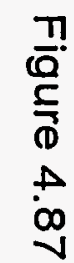

밀

ญ

○ 을

은

궁 3

O욤

ช

品

○

赵事

กิ

党

分

员

齐

$\Omega$

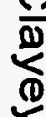

() Benzo(a)anthracene-

으.

os

$\frac{0}{3}$

$\frac{\bar{D}}{3}$ Benzo(b)fluoranthene-

文

$\overbrace{0}^{0}$

0
0
0
0
0
0
2
2
0
0

Phenanthrene

Anthracene

Fluoranthene

Pyrene

Normalized Data Averaged Over Soil Samples

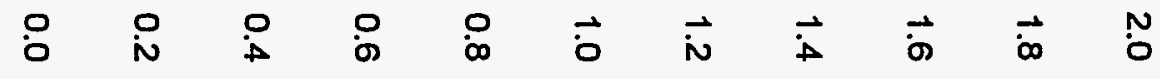

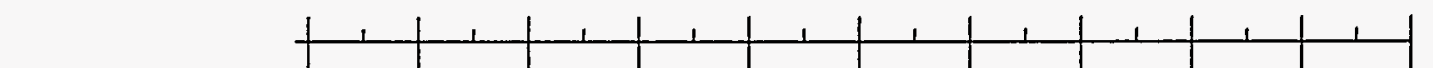




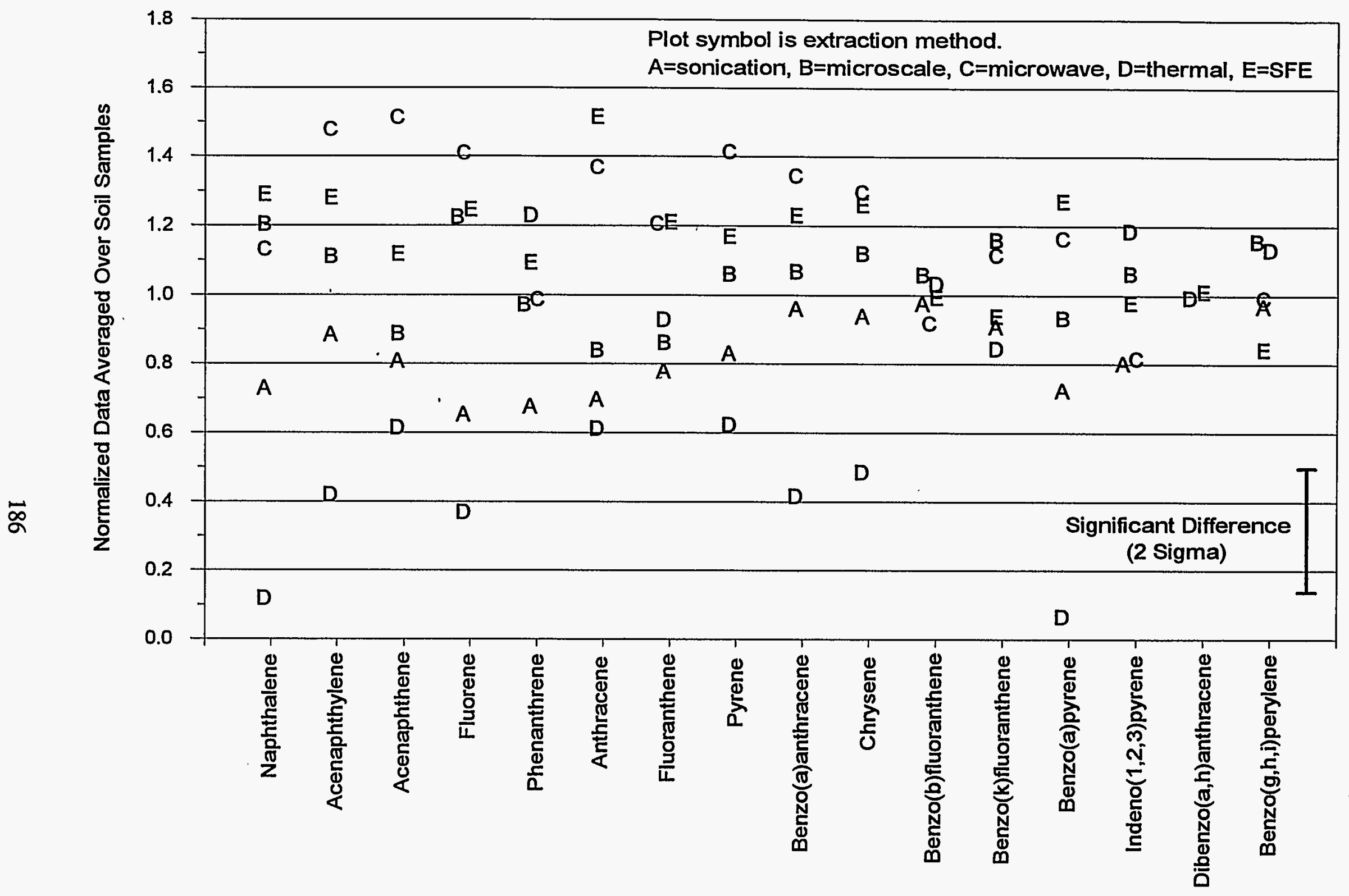

Figure 4.88 Extraction Method Analytical Results - Sandy Soil Sample Means Plotted by Analyte - Data Corrected for Percent Recovery 


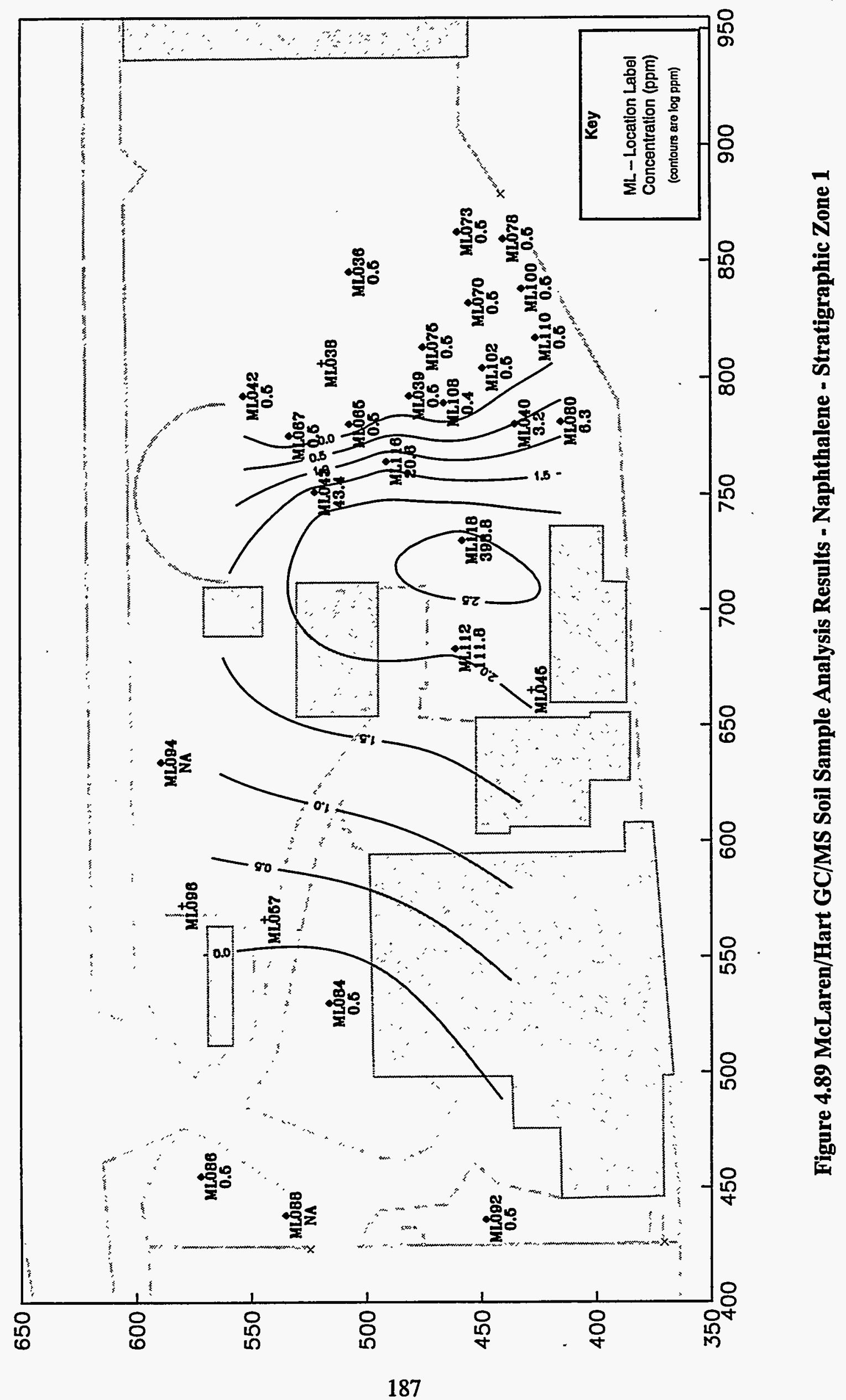




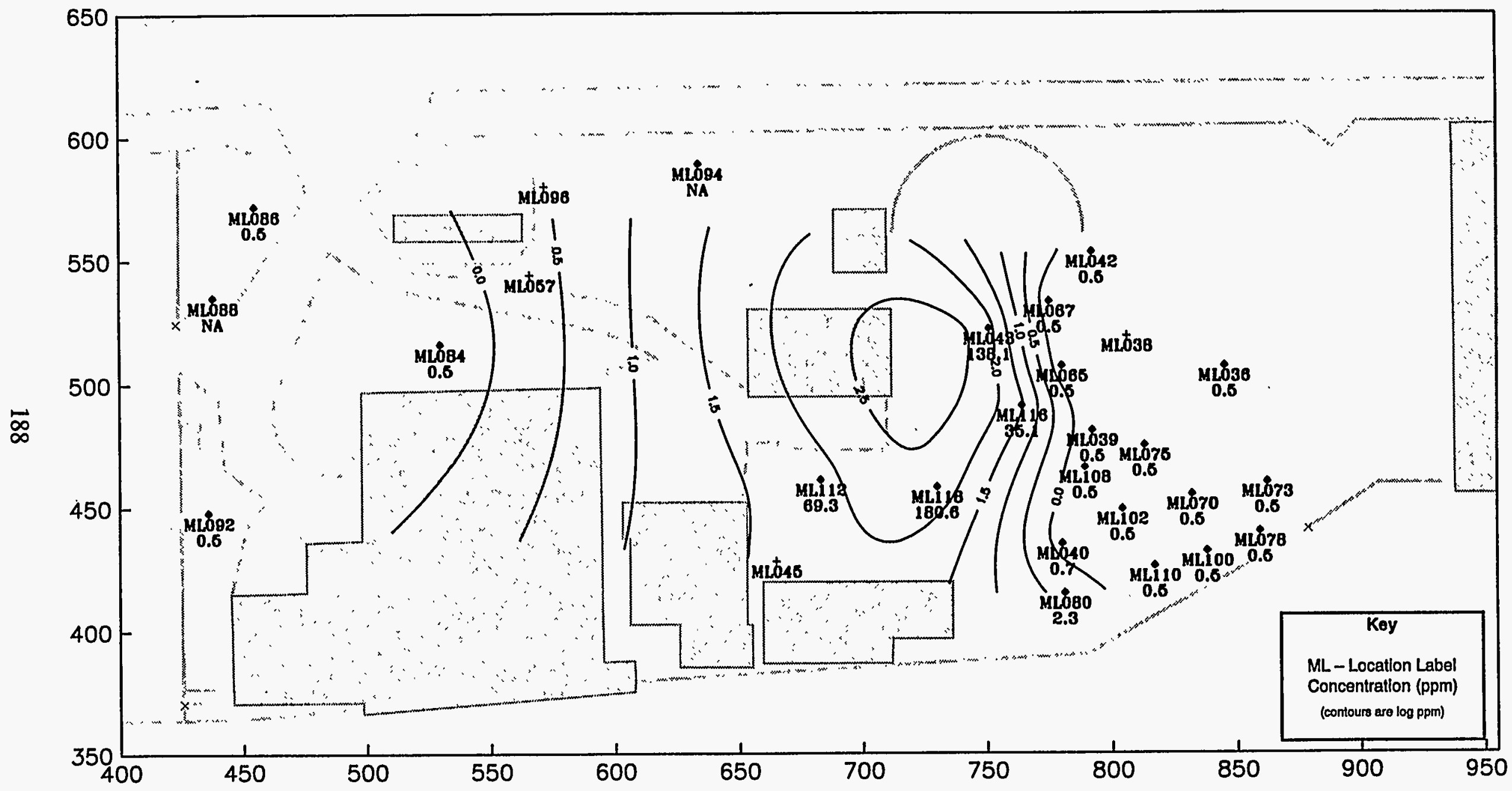

Figure 4.90 McLaren/Hart GC/MS Soil Sample Analysis Results - Phenanthrene - Stratigraphic Zone 1 


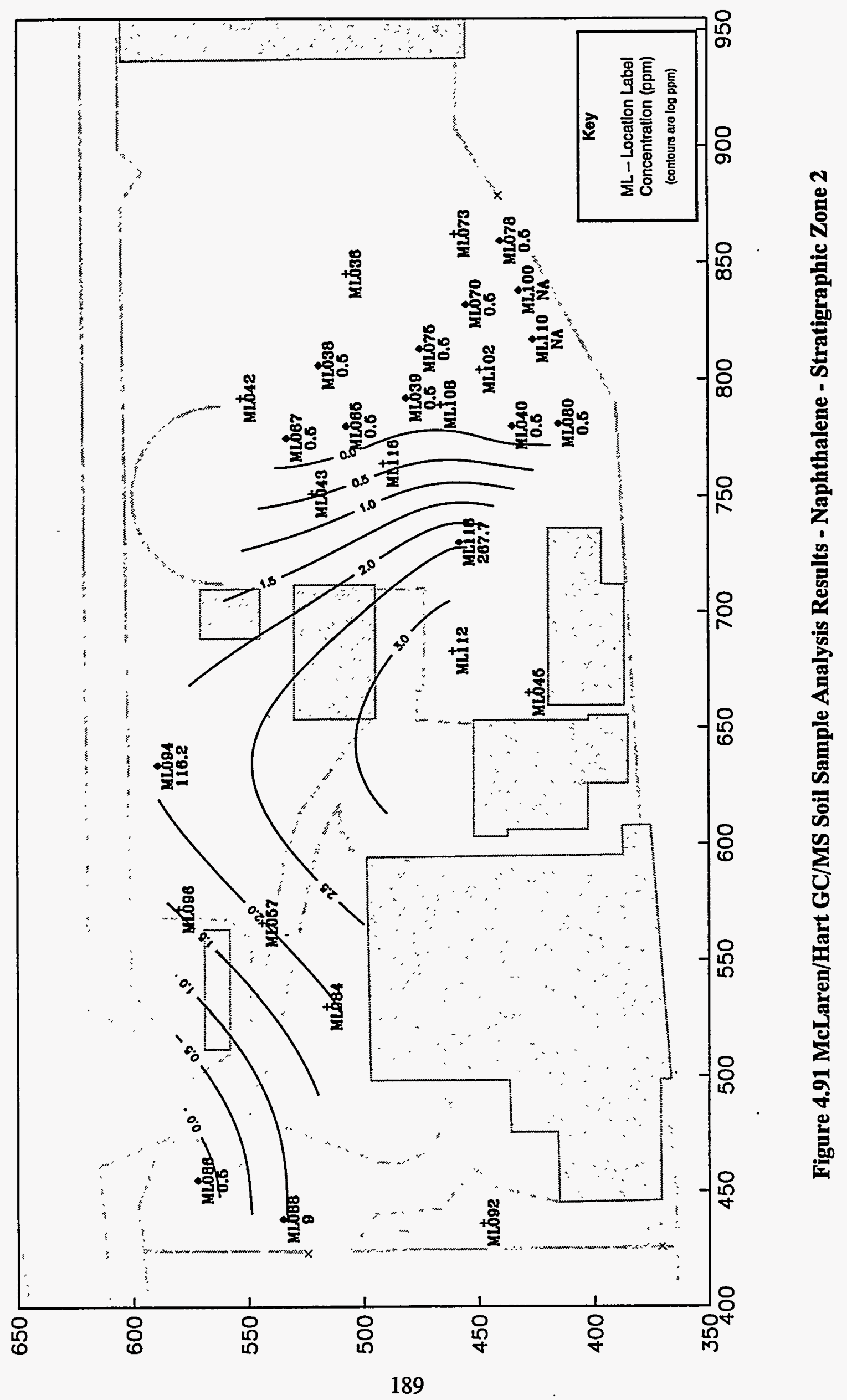




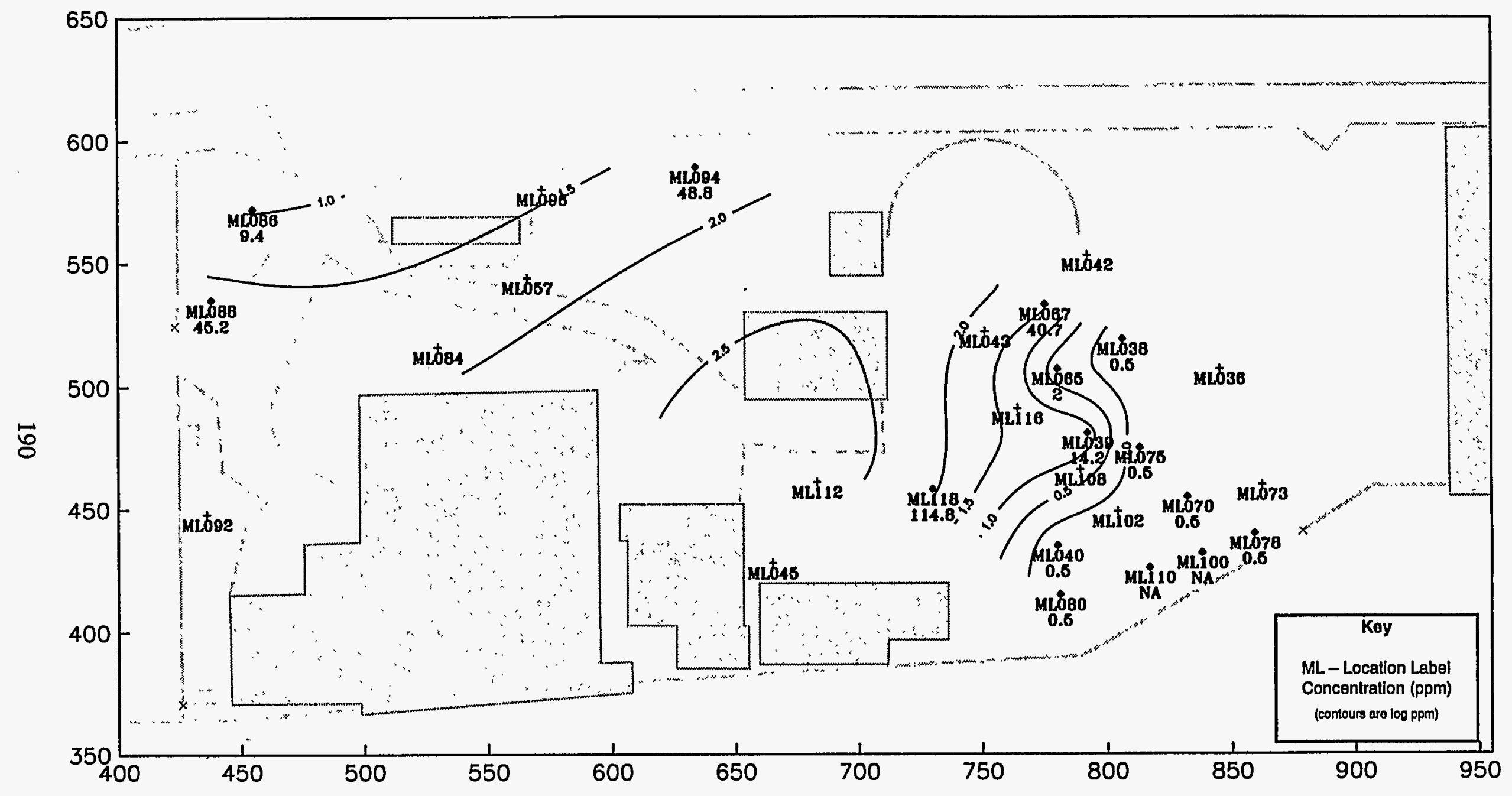

Figure 4.92 McLaren/Hart GC/MS Soil Sample Analysis Results - Phenanthrene - Stratigraphic Zone 2 


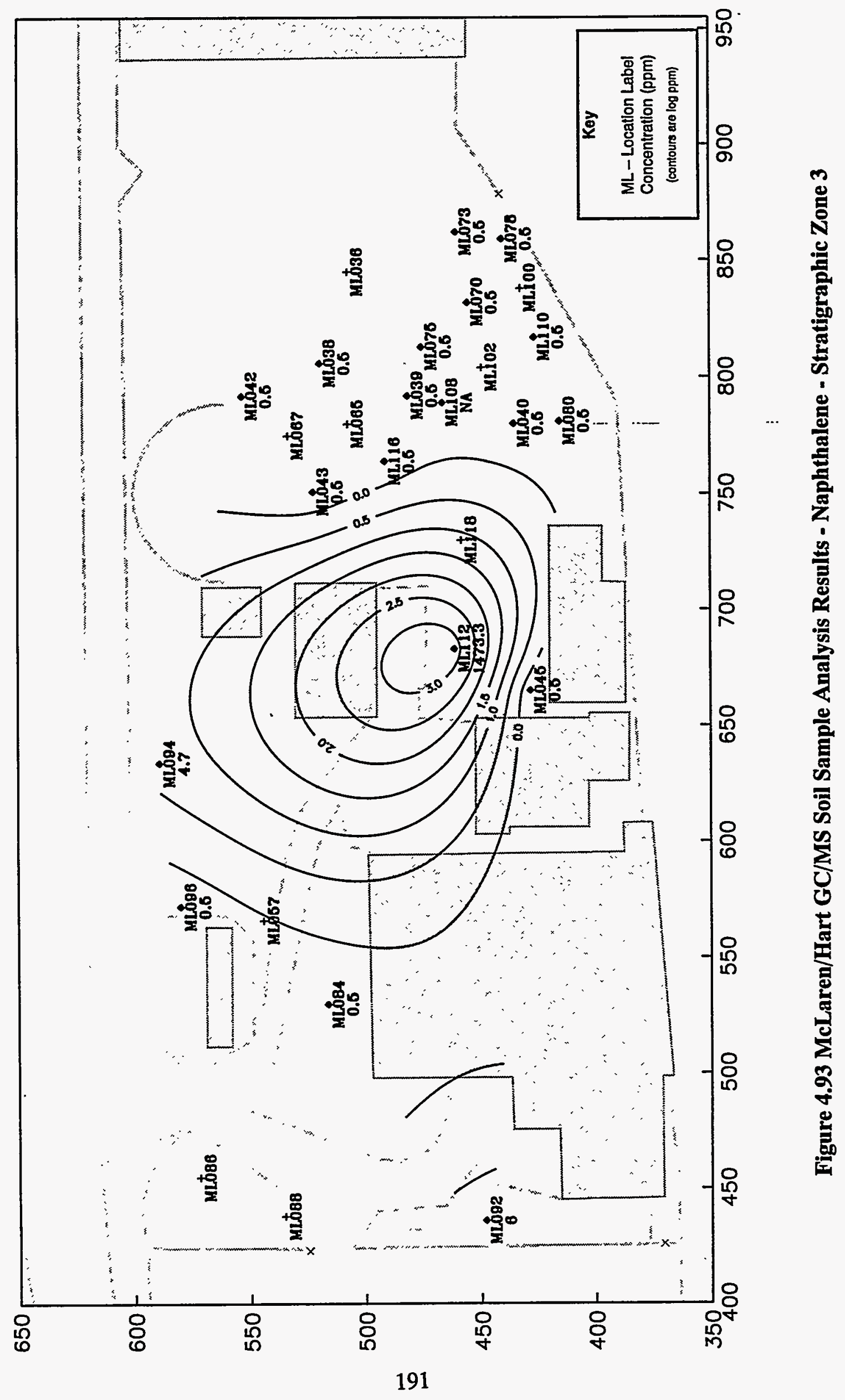




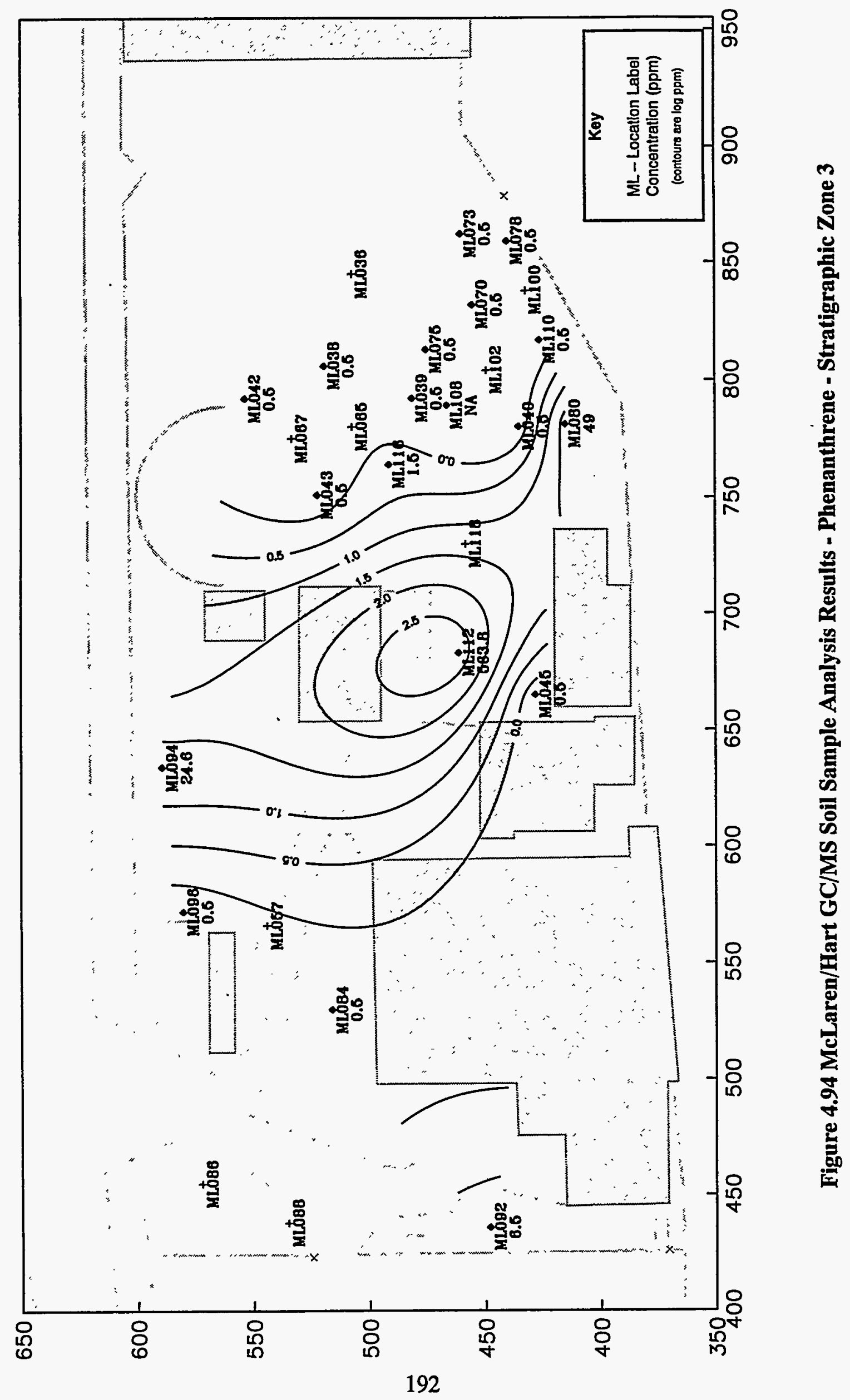




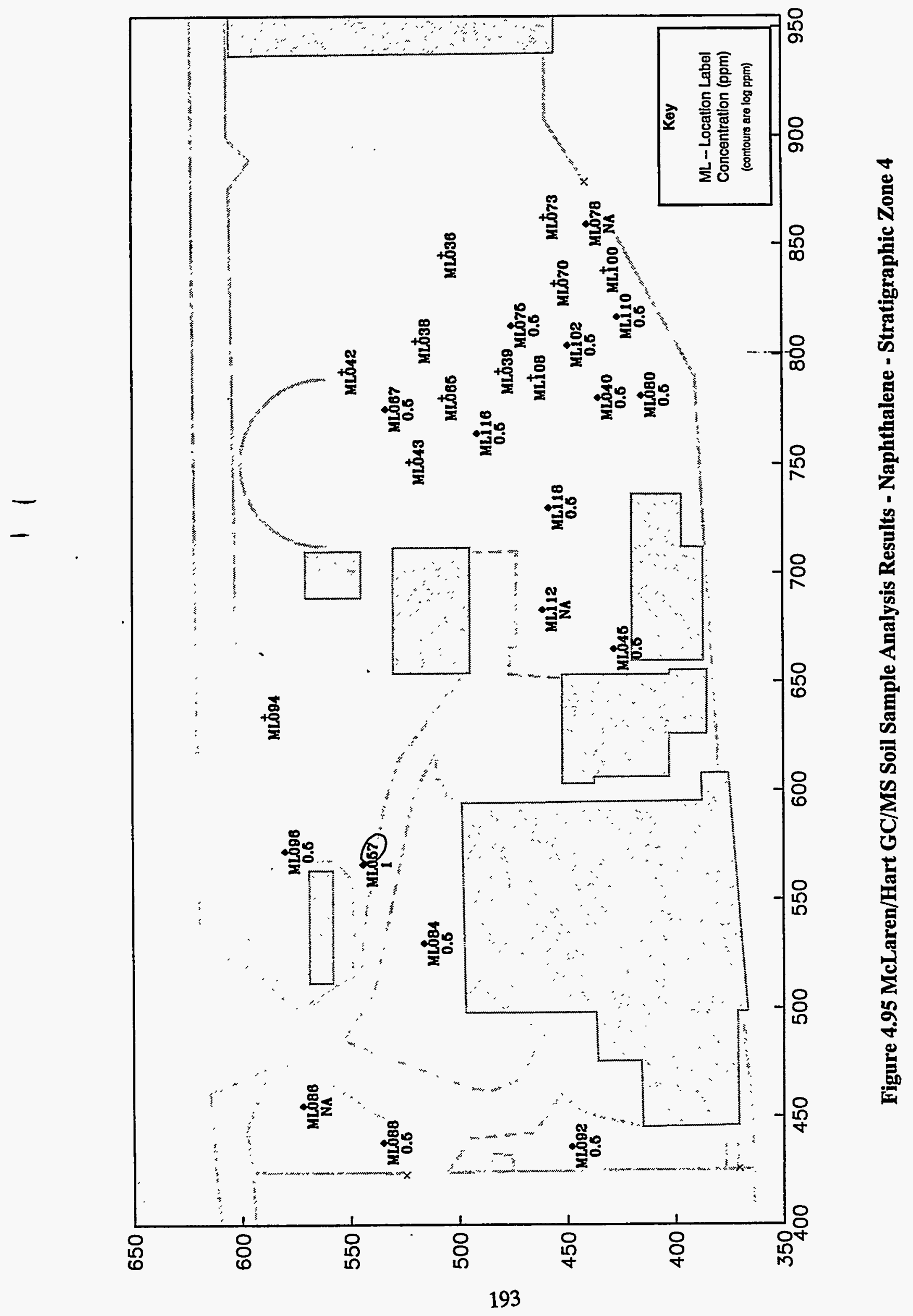




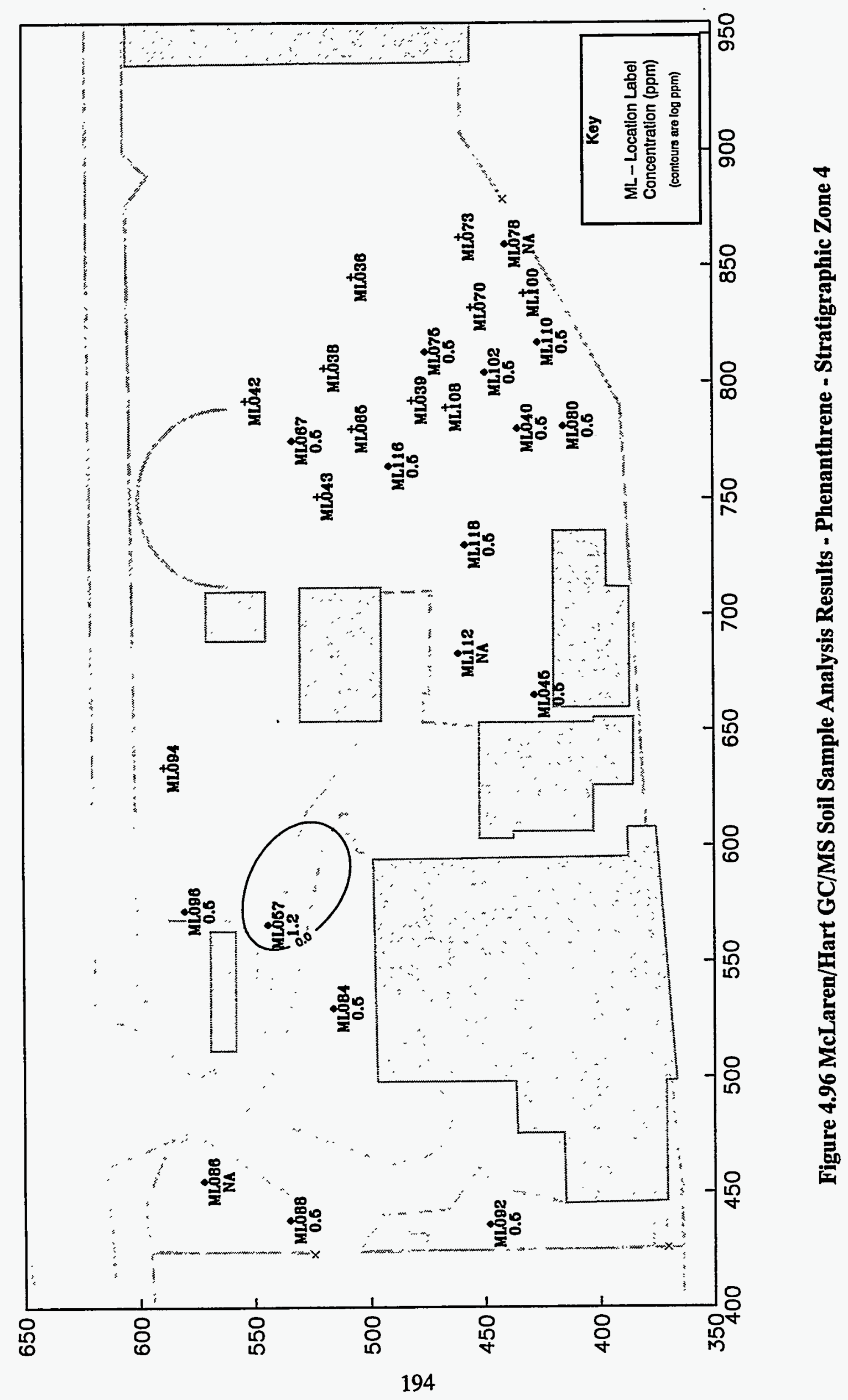




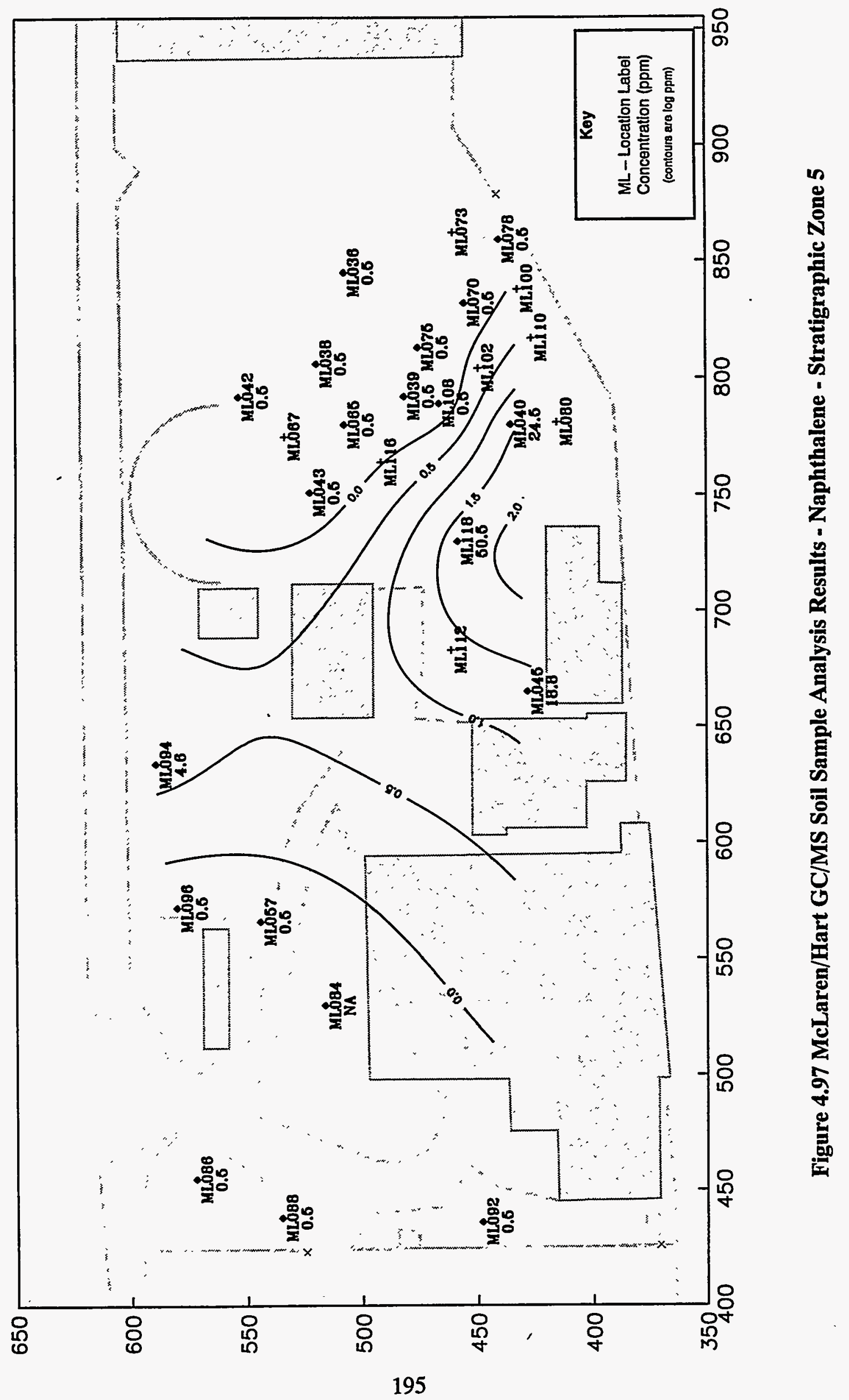




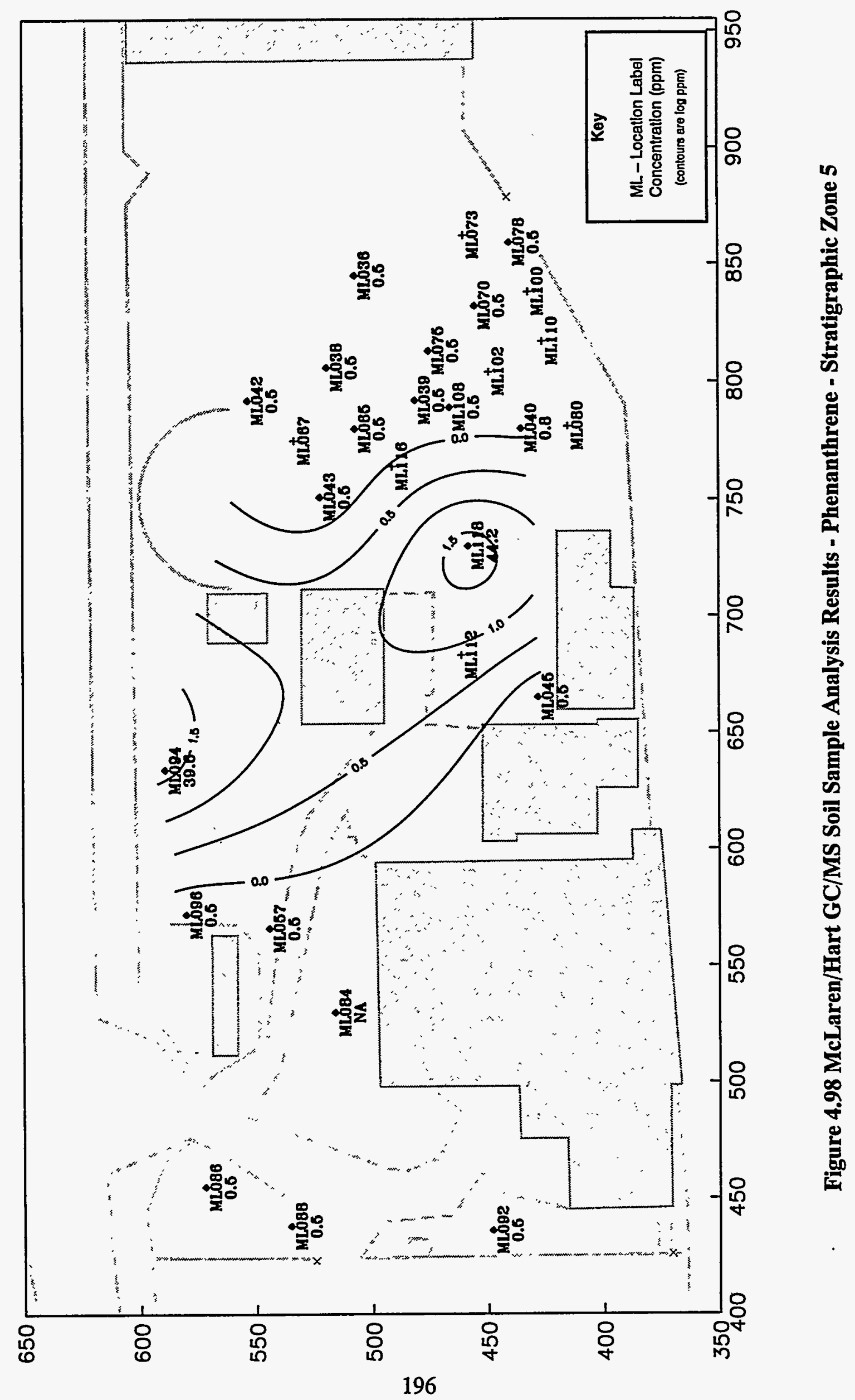




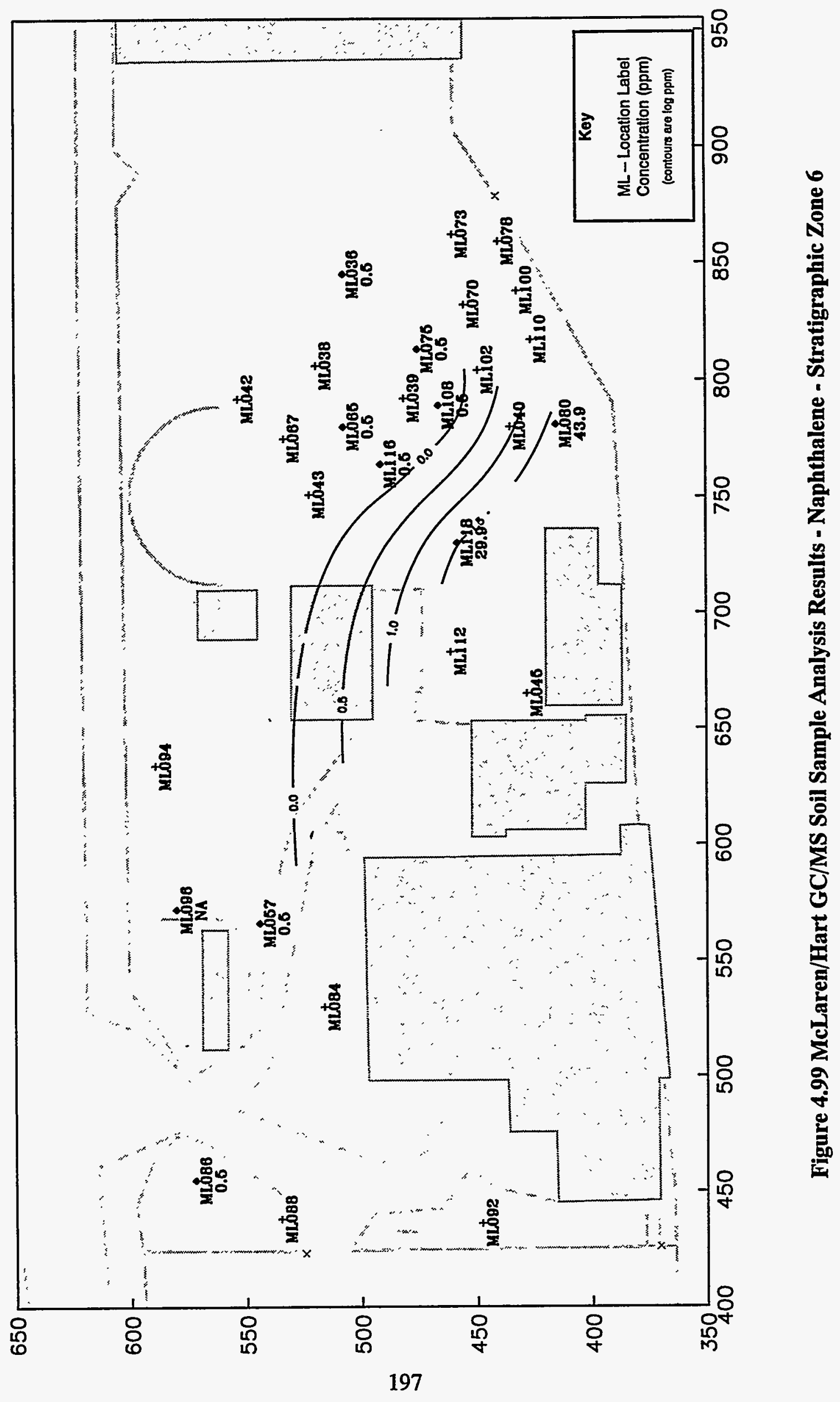




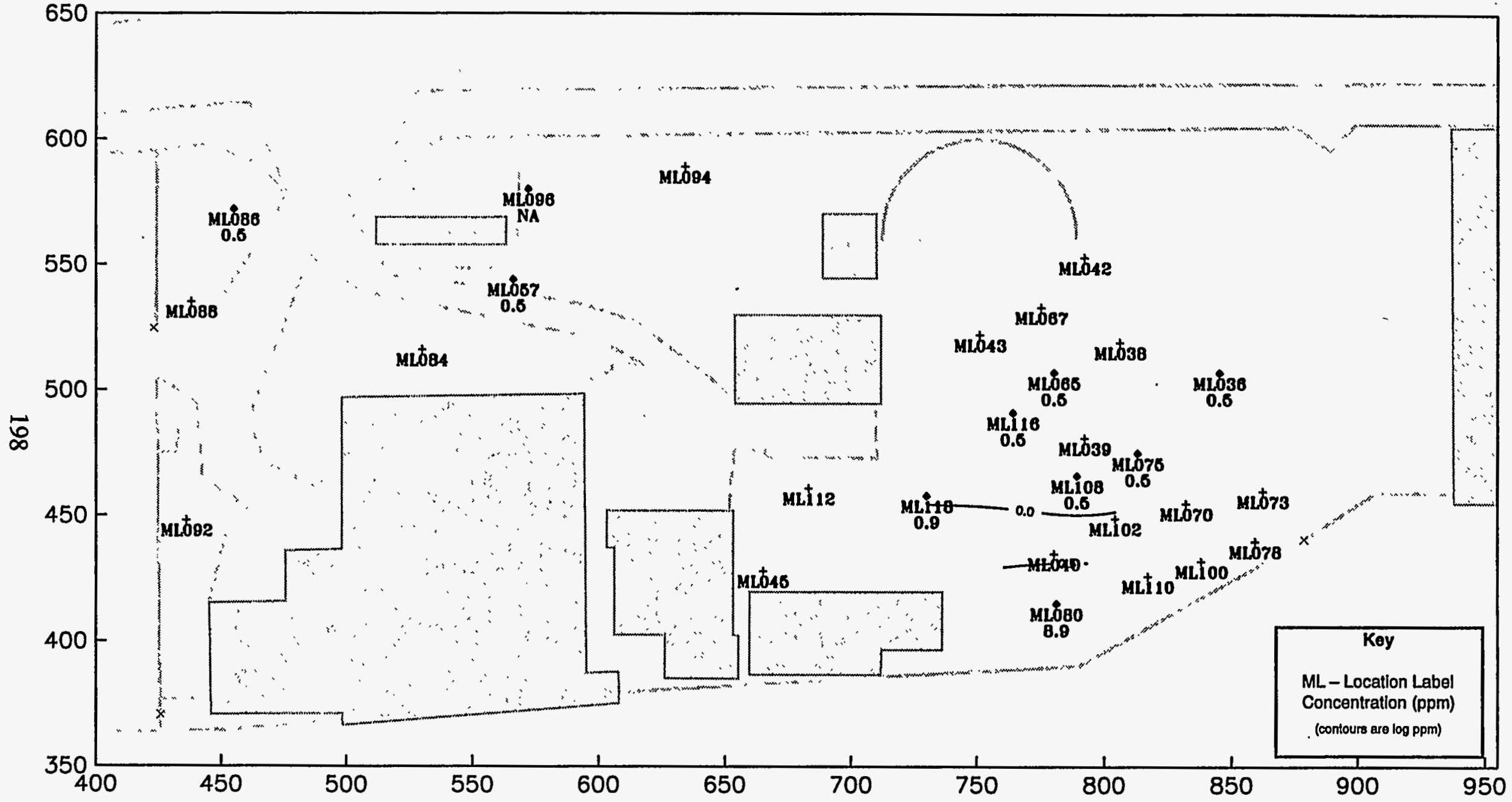

Figure 4.100 McLaren/Hart GC/MS Soil Sample Analysis Results - Phenanthrene - Stratigraphic Zone 6 


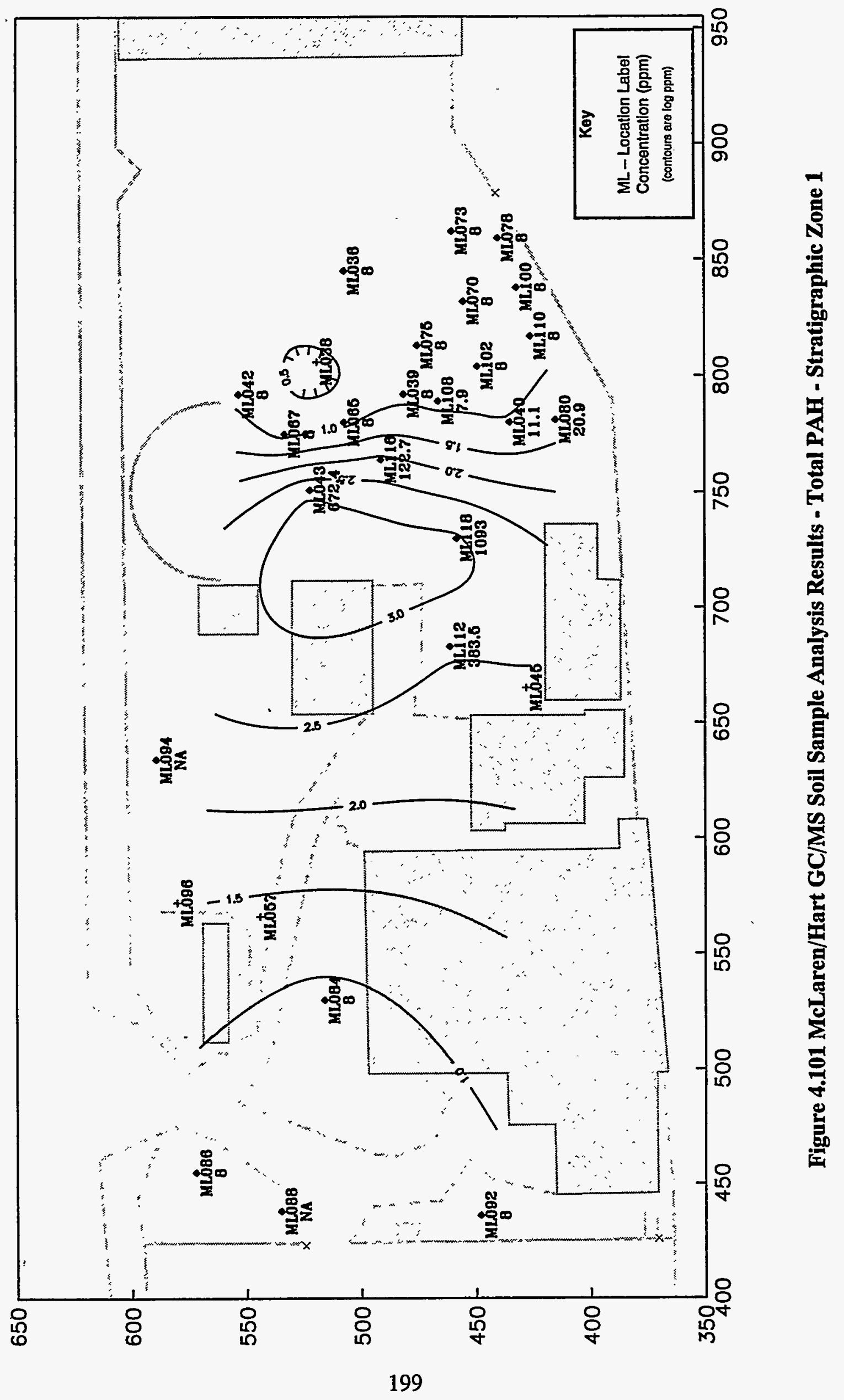




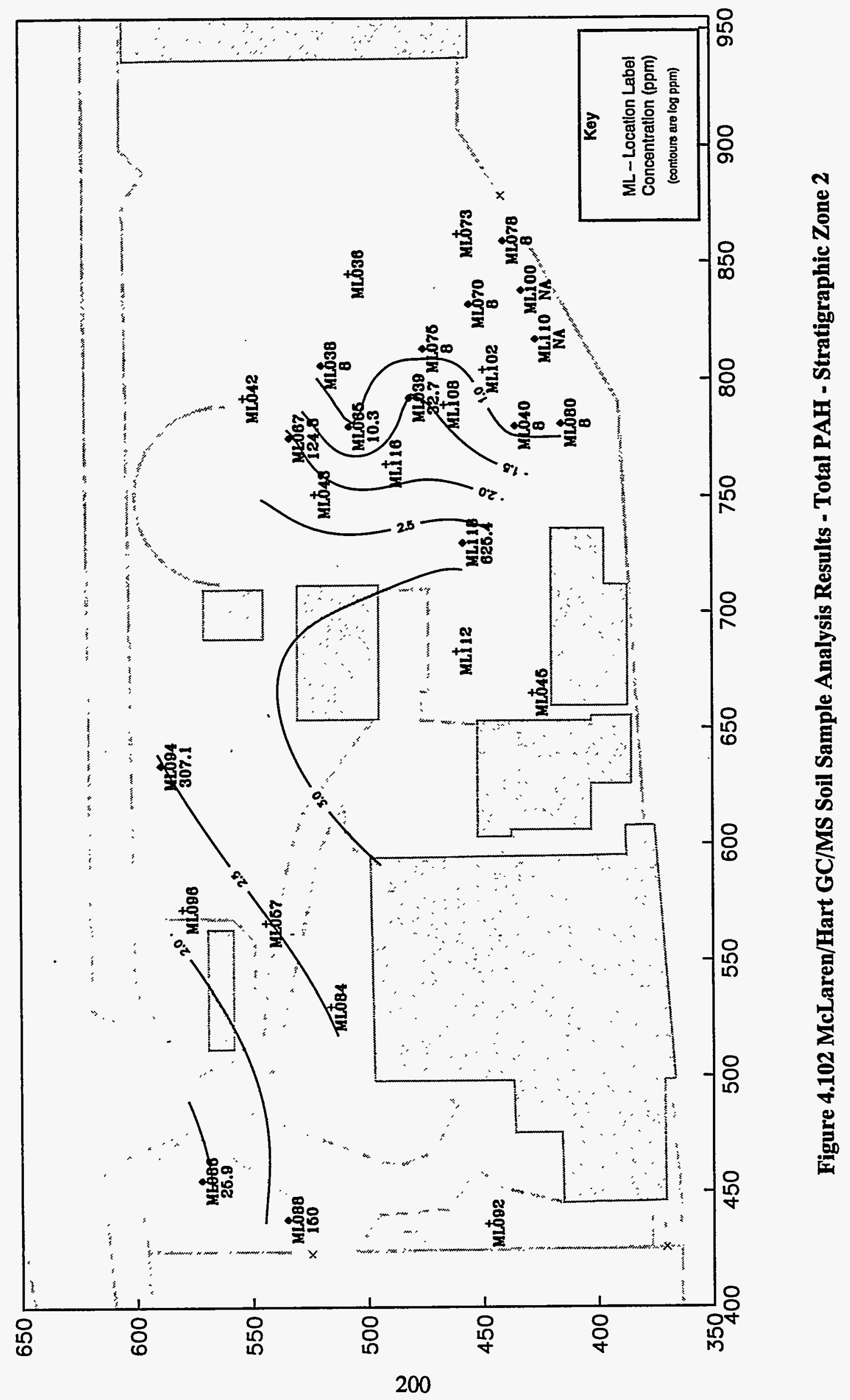




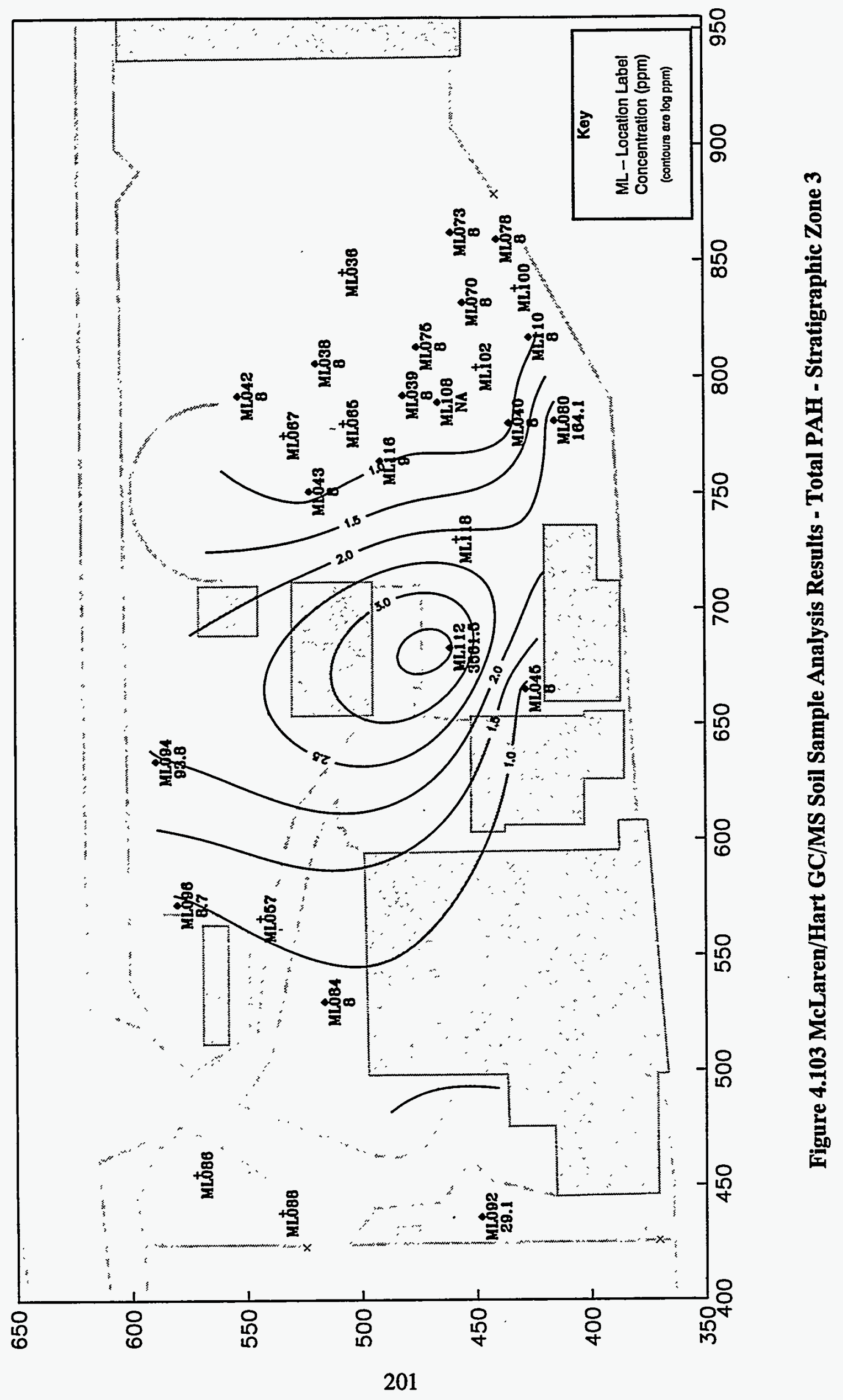




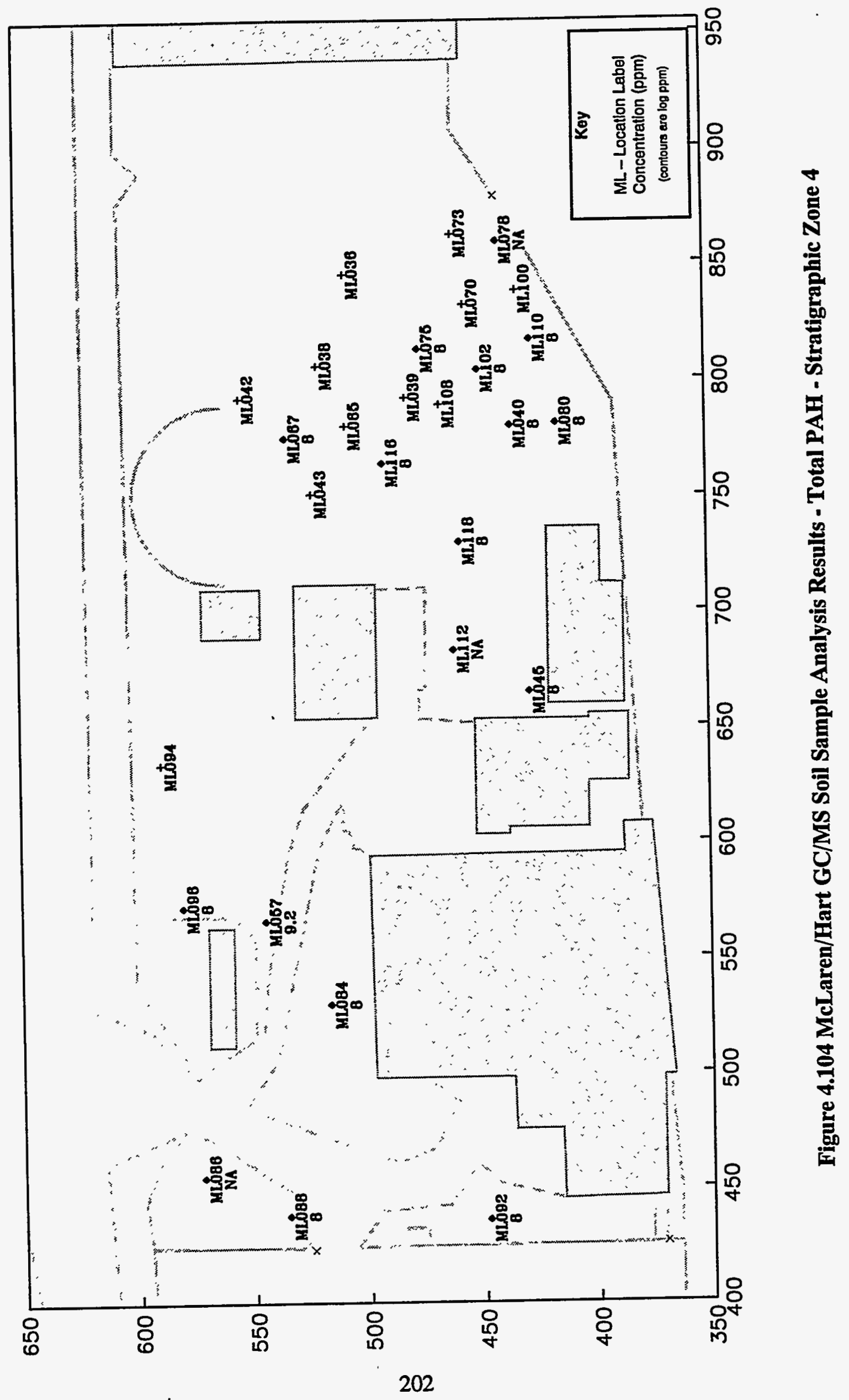




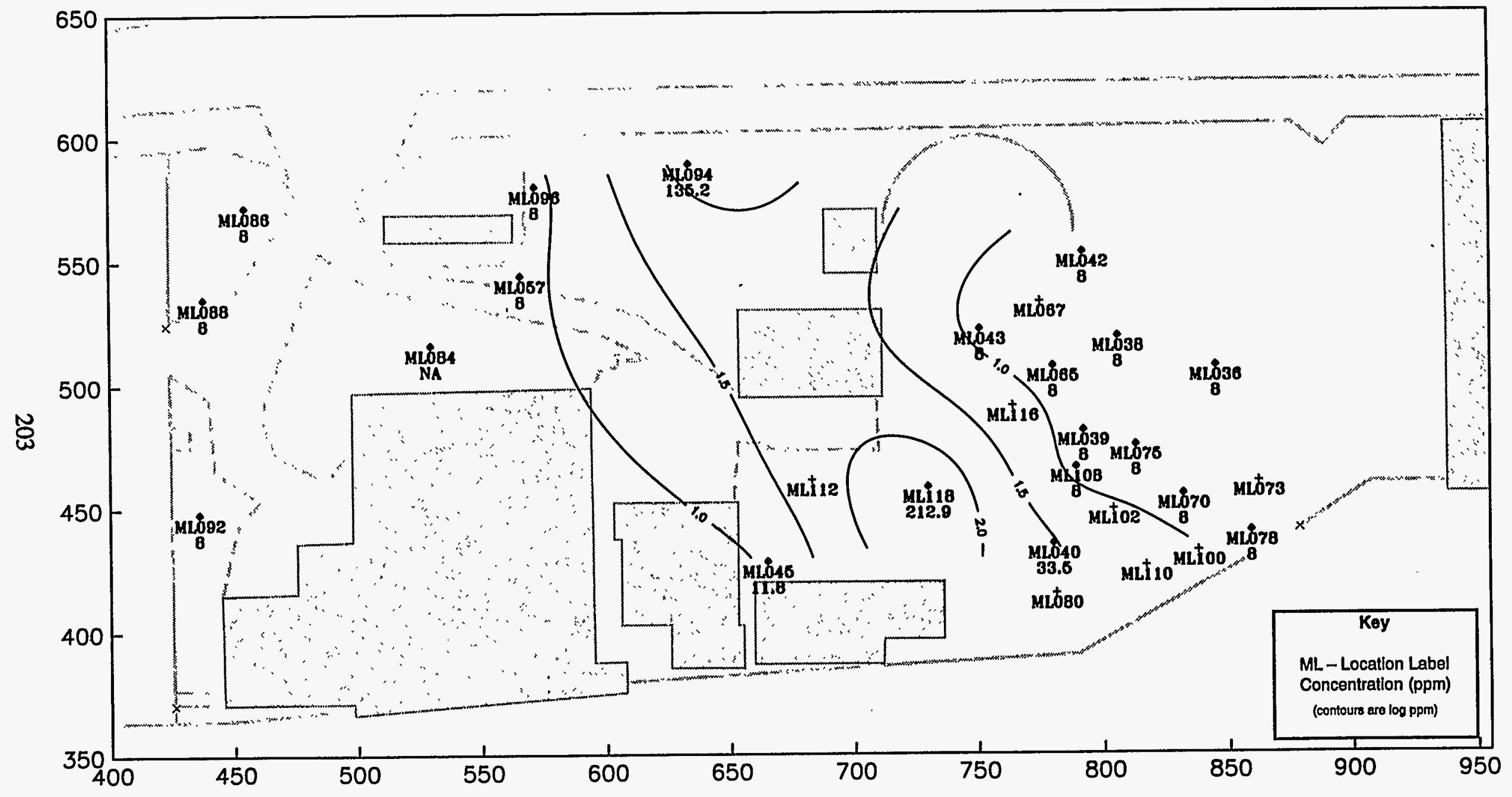

Figure 4.105 McLaren/Hart GC/MS Soil Sample Analysis Results - Total PAH - Stratigraphic Zone 5 


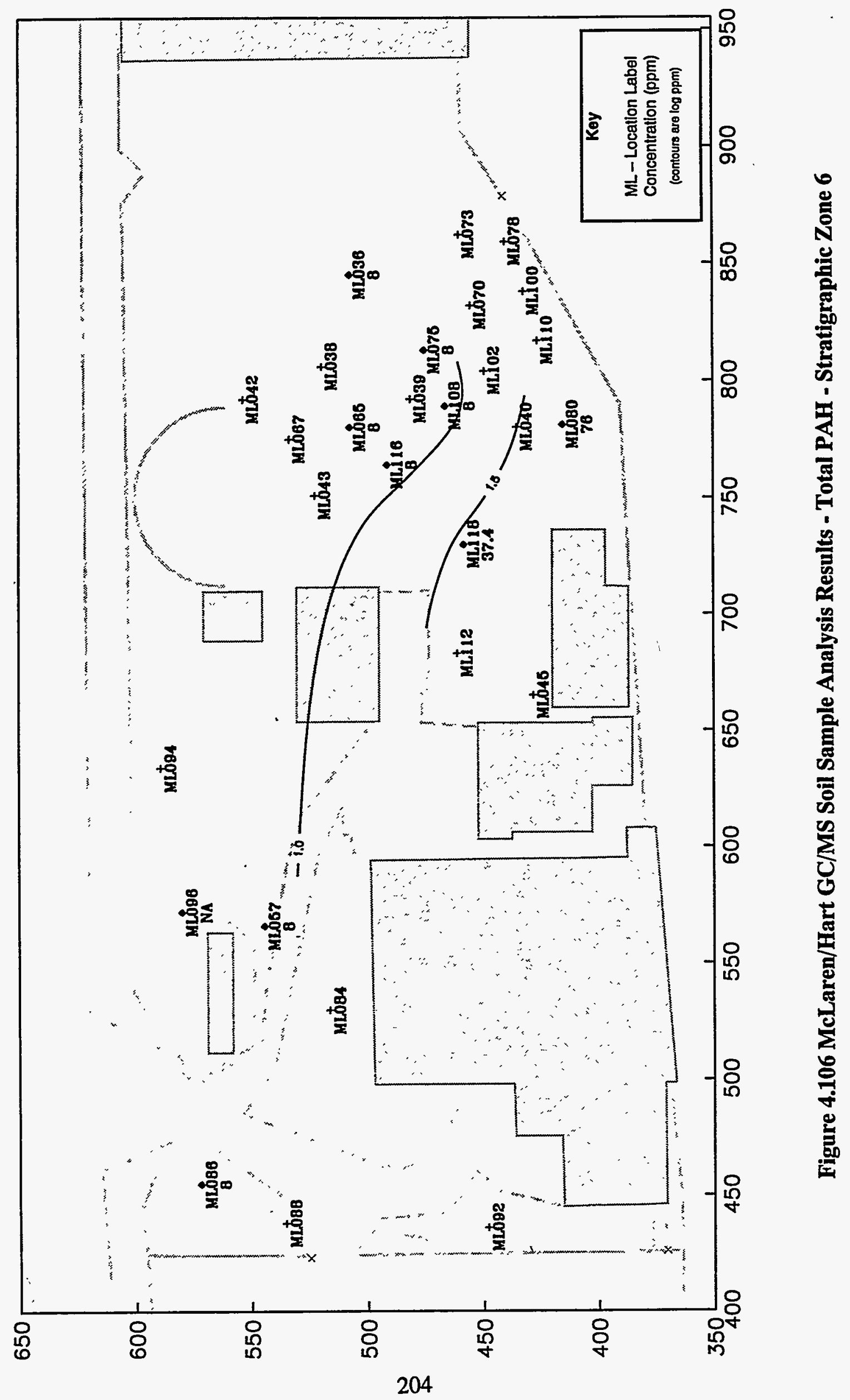




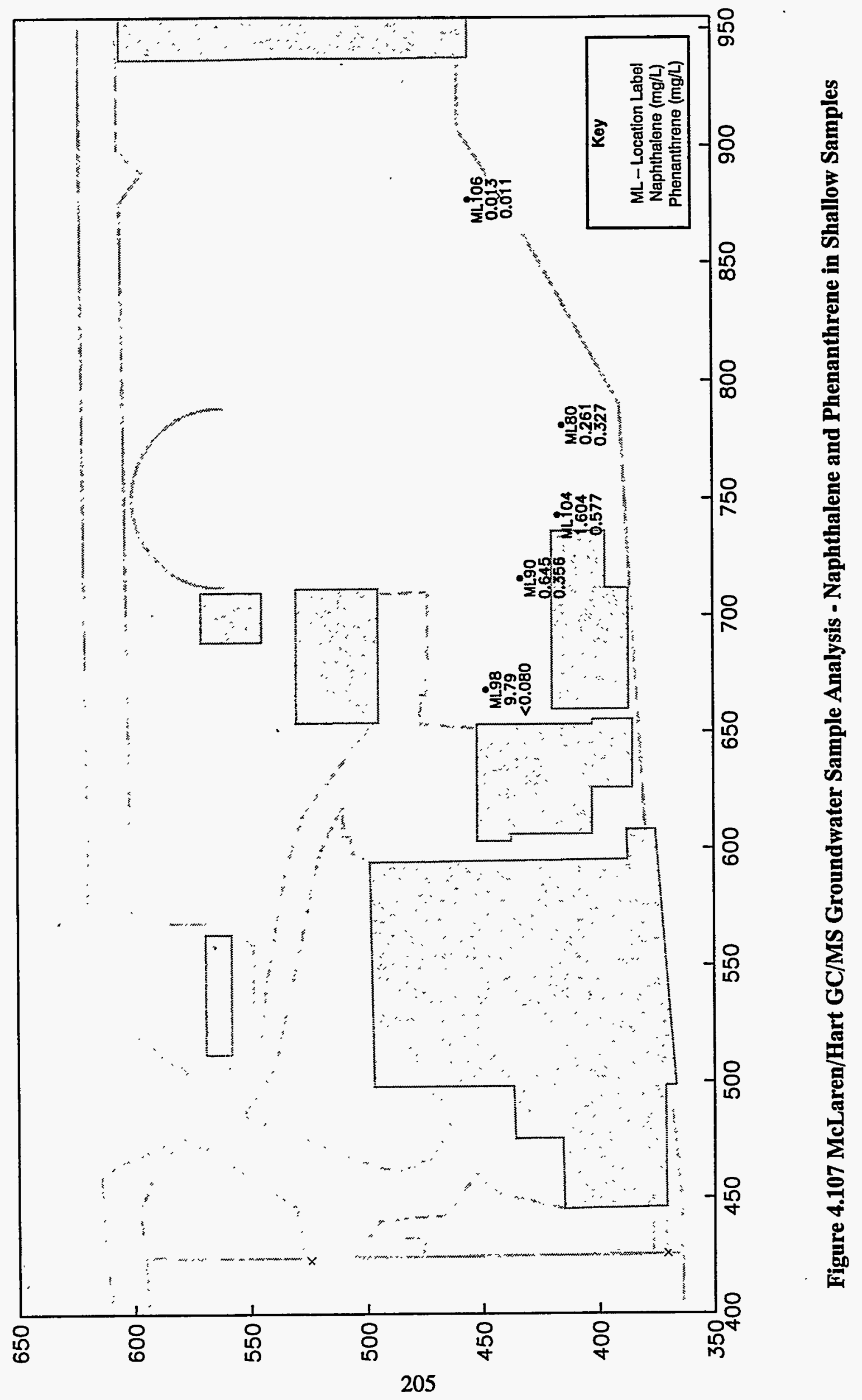




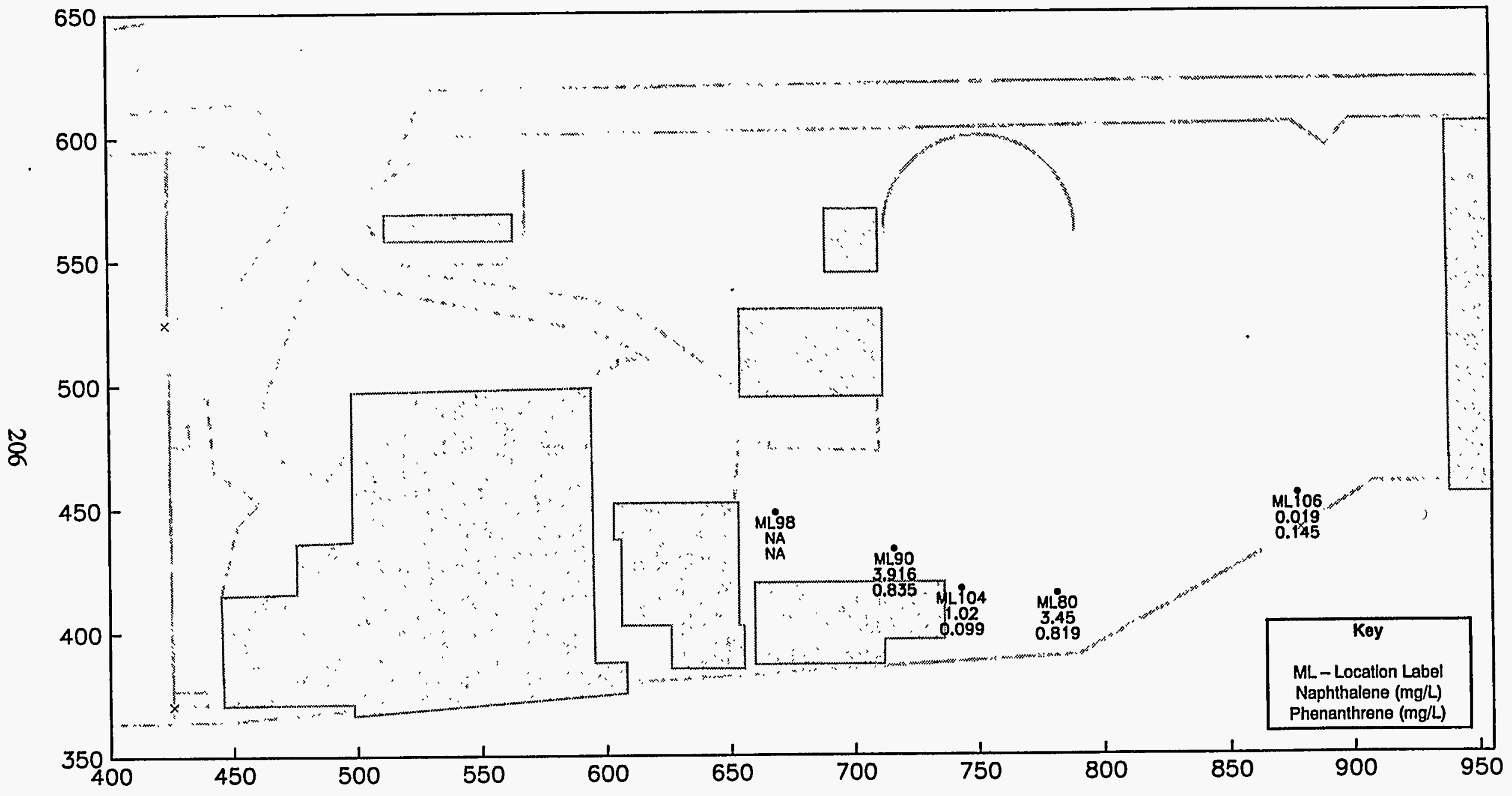

Figure 4.108 McLaren/Hart GC/MS Groundwater Sample Analysis - Naphthalene and Phenanthrene in Deep Samples 
TABLE 4.1*

GPR VELOCITY VS. DEPTH

DERIVED FROM MOVEOUT MEASURED AT

CMP TEST

\begin{tabular}{|c|c|c|c|}
\hline Depth (ft) & Tw0-way travel Time & Interval Velocity & Average Velocity \\
\hline & (ns) & (ns/m two-way travel) & (ns/m two-way travel) \\
\hline $0-6$ & 24.2 & 13.2 & 13.2 \\
\hline $6-21$ & 104.2 & 22.7 & 20.1 \\
\hline $21-30$ & 43.8 & 16 & 18.8 \\
\hline
\end{tabular}

* From D'Appalonia Environmental Services Inc., 1994

TABLE $4.2^{* *}$

APPROXIMATE ELECTRICAL CONDUCTIVITIES, RELATIVE DIELECTRIC CONSTANTS AND TRAVEL TIMES FOR VARIOUS EARTH MATERIALS

\begin{tabular}{|l|c|c|c|}
\hline \multicolumn{1}{|c|}{ Material } & $\begin{array}{c}\text { Approximate } \\
\text { Conductivity } \\
\mathrm{mS} / \mathbf{m}\end{array}$ & $\begin{array}{c}\text { Approximate Relative } \\
\text { Dielectric Constant }\end{array}$ & $\begin{array}{c}\text { Two-way Travel Time } \\
\text { nanoseconds/meter }\end{array}$ \\
\hline Air & 0 & 1 & 6.6 \\
\hline Fresh Water & $10^{-1}-30$ & 81 & 59 \\
\hline Fresh Water Ice & $10^{-1}-10$ & 4 & 13 \\
\hline Permafrost & $10^{-2}-10$ & $4-11$ & $13-15$ \\
\hline Granite & $10^{-6}-1$ & $5.6-8$ & 13.7 \\
\hline Dry Sand & $10^{-4}-1$ & $4-6$ & 36 \\
\hline $\begin{array}{l}\text { Sand, Saturated } \\
\text { (fresh water) }\end{array}$ & $10^{-1}-10^{2}$ & 30 & 21 \\
\hline $\begin{array}{l}\text { Silt, Saturated } \\
\text { fresh water) }\end{array}$ & $10-10^{2}$ & 10 & $18.6-23$ \\
\hline $\begin{array}{l}\text { Clay, Saturated } \\
\text { (fresh water) }\end{array}$ & $10^{2}-10^{4}$ & $8-25$ & $23-30$ \\
\hline Average "Dirt" & $10^{-1}-10^{2}$ & - & \\
\hline
\end{tabular}

** Benson et al. (1983) 
TABLE 4.3 PHASE 1 CONTAMINANT SCREENING-HPLC ANALYTICAL (EPA METHOD 8130) AND IMMUNOASSAY RESULTS

\begin{tabular}{|c|c|c|c|c|c|c|c|c|c|c|c|c|c|c|c|}
\hline \multirow[t]{2}{*}{ Analyte } & \multirow{3}{*}{\begin{tabular}{|c|} 
Detection \\
Limit \\
\end{tabular}} & \multicolumn{14}{|c|}{ Results in $\mathrm{mg} / \mathrm{kg}$} \\
\hline & & MT-1-G001 & MT-1-G002 & MT-1-G003 & MT-1-G004 & MT-1-G005 & MT-1-G006 & MT-1-G007 & MT-1-G008 & MT-1-G009 & MT-1-G010 & MT-1-G011 & MT-1-G012 & MT-1-G013 & MT-1-G014 \\
\hline $\begin{array}{l}\text { Map Location } \\
(\mathrm{S}=\text { shallow }),(\mathrm{D}=\text { deep })\end{array}$ & & ML3.S & ML3-D & ML4-S & ML4-D & MLS-S & MLS-D & MLG-S & ML6-D & ML7-S & ML7-D & ML8-S & MLB-D & ML9-S & MLQ-D \\
\hline Naphthalene & 0.1 & n.a. & n.a. & n.a. & n.a. & n.x. & n.a. & n.a. & n.a. & n.a. & n.a. & n.e. & n... & n.e. & 1.9 \\
\hline Acenaphthylene & 0.1 & n.a. & na. & n.a. & n.a. & n.a. & n.s. & n.e. & n.a. & n.2. & n.a. & n.a. & n.e. & n.a. & 2.1 \\
\hline Fluorene & 0.1 & n.a. & n.a. & n.a. & n. & n.t. & n.a. & n.a. & n.2. & n.a. & n.a. & n.a. & n.a. & n.4. & 0.5 \\
\hline Acenaphthene & 0.1 & n... & n.a. & n.a. & n.a. & n.s. & n.a. & n.a. & n.a. & n.e. & n.2. & n.e. & n.e. & n.8. & 1.9 \\
\hline Phenanthrene & 0.1 & n... & n... & n.a. & n.a. & n.a. & n.2. & n.e. & n.e. & n.2. & $\overline{\text { n.a. }}$ & n.s. & n.a. & n.a. & 1.4 \\
\hline Anthracene & 0.1 & n.a. & n.a. & n.a. & n.a. & n.e. & n.e. & n.a. & n.a. & n.a. & n.a. & n.z. & n.a. & n.e. & 0.3 \\
\hline Fluoranthene & 0.1 & n.a. & n.a. & n.2. & n.a. & n.s. & n.a. & n.a. & n.a. & n.a. & n.a. & n.a. & n.a. & n.e. & 0.7 \\
\hline Pyrene & 0.1 & n.a. & n.s. & n.a. & n.a. & n.e. & n.a. & n.a. & n.a. & n.e. & n.a. & n.a. & n.a. & n.s. & 1 \\
\hline Crysene & 0.1 & n.a. & n.a. & n.a. & n.a. & n.a. & n.a. & n.a. & n.a. & n.e. & n.a. & n.a. & n.2. & n.a. & 1.5 \\
\hline Benzo(b)fluoranthene & 0.1 & n.a. & n.a. & n.a. & n.a. & n.e. & n.s. & n.z. & n.2. & n.a. & n.s. & n.2. & n.a. & n.a. & 0.3 \\
\hline Benzo(k)fluoranthene & 0.1 & n.a. & n.a. & n.a. & n.e. & n.a. & n.a. & n.a. & n.a. & n.e. & n.2. & n.a. & n.a. & n.a. & 0.3 \\
\hline Benzo(a)pyrene & 0.1 & n.a. & n.e. & n.a. & n.a. & n.a. & n.a. & n.a. & n.e. & n.a. & n.a. & n.a. & n.a. & n.a. & 0.6 \\
\hline Dibenzo(a,h,i)perylene & 0.1 & n.a. & n.a. & n.a. & n.a. & n.a. & n., & n.a. & n.a. & n.a. & n.a. & n.2. & n.a. & n.a. & 0.3 \\
\hline Benzo(g,h,i)perylene & 0.1 & n.a. & n.a. & n.a. & n.a. & n.a. & n.a. & n.a. & n.a. & n.a. & n.2. & n.a. & n.a. & n.a. & $<0.1$ \\
\hline Indeno(1,2,3-cd)pyrene & 0.1 & n.s. & n.a. & n.a. & n.a. & n.a. & n.a. & n.a. & n.a. & n.a. & n.s. & n.s. & n.s. & n.a. & 0.3 \\
\hline \multicolumn{2}{|l|}{ TOTAL PAH's (less naphth.) } & n.a. & na. & n.e. & n... & n.a. & n.a. & n.a. & n.a. & n.a. & n.a. & $\overline{\text { n.a. }}$ & n.e. & n.a. & 11.2 \\
\hline TOTAL PAH's (all 15) & & n.a. & n.a. & n.a. & n.a. & n.a. & n.a. & n.a. & n.a. & n..a. & n.a. & n.a. & n.a. & n.a. & 13.1 \\
\hline Percent solids & & 77.2 & 88.8 & 78 & 85 & 78 & 86.7 & 77.3 & 86.8 & 78.1 & 84.4 & n.a. & 75.9 & 77.3 & 76.2 \\
\hline Date collected & & 4-Apr.94 & 4-Apr-94 & 4-Apr-94 & 4-Apr-94 & 4-Apr-94 & 4-Apr-94 & 4-Apr-94 & 4-Apr-94 & 5-Apr-94 & 5.Apr-94 & 5-Apr-94 & 5-Apr-94 & 5.Apr-94 & 5-Apr-94 \\
\hline \multicolumn{16}{|l|}{ IMA screening results } \\
\hline \multicolumn{2}{|l|}{ Results are reported in ppm } & & \multicolumn{6}{|c|}{ (by weight for Ensys and Millipore; by volume for Quantix) } & \multicolumn{3}{|c|}{ *indicates ambiguous results } & & & & \\
\hline Ensys:RISc & & $<1$ & $<1$ & $<1$ & $<1$ & $<1$ & $<1$ & $<1$ & $<1^{*}$ & $<1$ & n.a. & $<1$ & $<1^{*}$ & $<1$ & $>100$ \\
\hline \multicolumn{2}{|l|}{ Millipore/Immunosystems } & $?$ & $?$ & $?$ & $<1.0$ & $<1.0$ & $<1.0$ & $<1.0$ & $<1.0$ & $<1.0$ & n.a. & $<1.0$ & $<1.0$ & $<1.0$ & $>100$ \\
\hline Quantix & & $<0.7$ & $<0.7$ & $<0.7$ & $<0.7$ & $<0.7$ & $<0.7$ & $<0.7$ & $<0.7$ & $<0.7$ & n.s. & $<0.7$ & $<0.7$ & $<0.7$ & $9.0-13.62$ \\
\hline
\end{tabular}


TABLE 4.3 continued

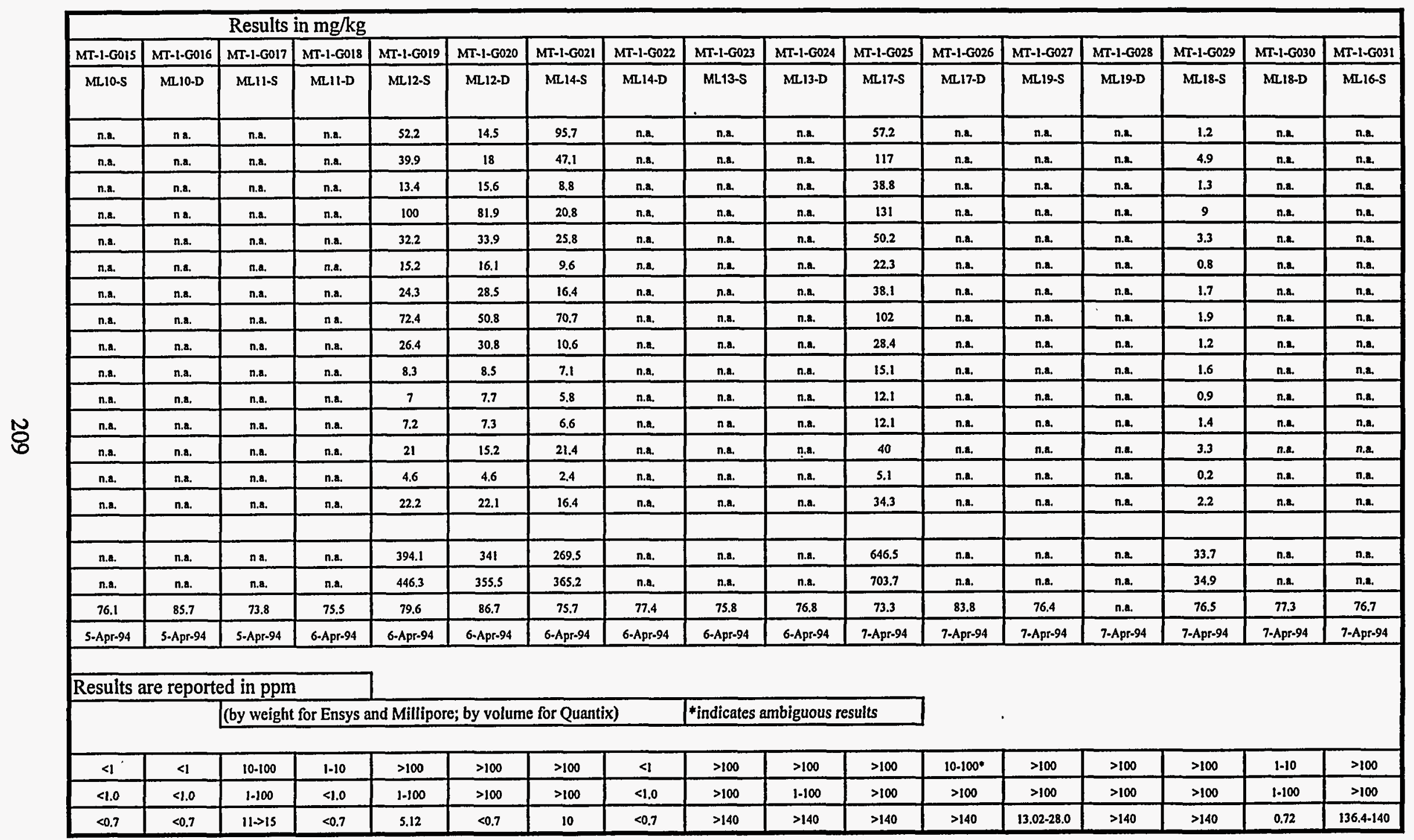


TABLE 4.3 continued

\begin{tabular}{|c|c|c|c|c|c|c|c|c|c|c|c|c|c|c|c|c|}
\hline \multicolumn{17}{|c|}{ Results in $\mathrm{mg} / \mathrm{kg}$} \\
\hline MT-1-G032 & MT-I-G033 & MT-I-G034 & MT-I-G035 & MT-1.G036 & MT-1-G037 & MT-1-G038 & MT-1-0039 & MT-1-G040 & MT-1-G04I & MT-1-G042 & MT-1-G043 & MT-1-G044 & MT-1-G045 & MT-1-G046 & MT-1.G047 & MT-1-G048 \\
\hline ML16-D & MLIS-S & ML15-D & ML27-S & & ML20-S & ML20-D & ML26-S & ML.26-D & ML23-S & ML-23-D & ML22.S & ML22-D & ML24.S & ML24-D & ML25-S & ML25-D \\
\hline n.a. & n...8. & n... & na. & & n...8. & n.a. & n.a. & n.a. & n.a. & n.a. & n.a. & n... & n. 2. & n.a. & n.a. & n.a. \\
\hline n.a. & na. & n.a. & na. & & n.a. & n.a. & n.a. & n.a. & n.a. & n.a. & n.a. & n.a. & n... & n.a. & n.2. & n.a. \\
\hline n.a. & na. & n.a. & n.a. & & n.a. & n.a. & n... & n.a. & n... & n.a. & n.a. & n.a. & n.a. & n.2. & n... & n.a. \\
\hline n.a. & n.a. & n.a. & n.s. & & n.a. & n.a. & n.a. & n.a. & n.a. & n.s. & n.a. & n.a. & n.a. & n.a. & n.a. & n.a. \\
\hline n.a. & n.a. & n.a. & na. & & n.a. & n.a. & n.a. & n... & n... & n... & n.a. & n.a. & n... & n.a. & n.a. & $\mathrm{na.}$ \\
\hline n.a. & n.a. & n.a. & n.a. & & n.a. & n..8. & n.a. & n.a. & n.a. & n.a. & n... & n.a. & n... & n..2. & n.a. & ก.a. \\
\hline $\mathrm{na.}$ & n.a. & n.a. & na. & & n.a. & n.a. & n.a. & n... & n... & n.a. & n.a. & n.a. & n.a. & n.2. & n... & n.a. \\
\hline n.a. & na. & n.a. & n.a. & & n.a. & n.a. & n.a. & n.a. & n.a. & na. & n.a. & n.a. & n.a. & n... & n... & n.a. \\
\hline n.a. & n.a. & n.a. & n.a. & & n.a. & n.a. & n.a. & n.a. & n.a. & n... & n.a. & n... & n.a. & n.e. & n... & n.a. \\
\hline n.a. & n.a. & n.a. & n.a. & & n.a. & n.a. & n.a. & n.a. & n.a. & n.e. & n.a. & n.a. & n.a. & n.a. & n.a. & n.a. \\
\hline n.a. & n.a. & n... & n.a. & & n.s. & n.a. & n.s. & n.a. & n.a. & n.a. & n.a. & n.a. & n.a. & n.a. & n.s. & n.a. \\
\hline n.a. & n.a. & n.a. & n.a. & & n.a. & n... & n.a. & n.a. & n.a. & n.a. & n.a. & n.a. & n.a. & n.a. & n.a. & n.a. \\
\hline n.a. & n.a. & n.a. & n.a. & & n.a. & n.a. & n.a. & n.a. & n.a. & n... & n... & n.a. & n.a. & n.a. & n.a. & n.a. \\
\hline n.a. & n.s. & n.a. & n.a. & & n.a. & n.a. & n..8. & n.a. & n.a. & n.a. & n.a. & n.a. & n.a. & n.a. & n.a. & n... \\
\hline n.a. & n.a. & n.a. & n.a. & & n.a. & n.a. & n.a. & n.a. & n.a. & n.a. & n... & n... & n.a. & n... & n.a. & n.a. \\
\hline & & & & & & & & & & & & & & & & \\
\hline n.a. & n.a. & n.a. & n.a. & n.a. & n.a. & n.a. & n.a. & n.a. & n.a. & n.a. & n.a. & n.a. & n.a. & n.a. & n.a. & n.a. \\
\hline n.a. & n.a. & n.a. & n.a. & n.a. & n.a. & n.a. & n.a. & n.a. & n.a. & n.a. & n.a. & n.a. & n.a. & n.a. & n.a. & n.a. \\
\hline 76.1 & 77.3 & 87 & 75.2 & & 76.9 & 86.7 & 76.3 & 88.5 & 77.7 & 91.3 & 73.8 & 75.7 & 76.2 & 77.3 & 75.1 & 78.2 \\
\hline 7-Apr-94 & 8-Apr-94 & 8-Apr-94 & 8-Apr-94 & & 8-Apr-94 & 8.Apr-94 & 8-Apr-94 & 9-Aprr.94 & 9-Apr-94 & 9-Apr-94 & 9-Apr-94 & 9-Apr-94 & 9-Apr.94 & 9-Apr-94 & 9-Aprr.94 & 9-Aprr-94 \\
\hline \multicolumn{17}{|c|}{ Results are reported in ppm } \\
\hline & & \multicolumn{6}{|c|}{ (by weight for Ensys and Millipore; by volume for Quantix) } & \multicolumn{3}{|c|}{ *indicates ambiguous results } & & & & & & \\
\hline$>100$ & $<1$ & $\leq 1$ & $\leq 1$ & & $>100$ & $>100$ & $10-100$ & $>100^{*}$ & $10^{\circ}-100$ & $10-100$ & $\leq 1$ & 1-10 & $\leq$ & $>100$ & $10 \cdot 100$ & $>100$ \\
\hline$>100$ & $?$ & 2 & 2 & & $>100$ & $>100$ & $>100$ & $>100$ & $>100$ & $1-100$ & $>100$ & $>100$ & $<1.0$ & $1-100$ & $1-100$ & $>100$ \\
\hline 1.44 & $<0.7$ & $<0.7$ & 0.88 & & $>140$ & $>140$ & 0.9 & 107.8 & $<7-10.9$ & $9.8-13.08$ & 48.8 & 115.6 & $<0.7$ & 10.2 & $<0.7$ & 11.2 \\
\hline
\end{tabular}


TABLE 4.4 PHASE 1 CONTAMINANT SCREENING - HPLC ANALYTICAL AND CHEMILUMINESCENCE RESULTS

\begin{tabular}{|c|c|c|c|}
\hline Sample & $\begin{aligned} & \text { Map Location } \\
& \mathrm{S}= \text { Shallow, } \mathbf{D}=\text { Deep } \\
&\end{aligned}$ & $\begin{array}{l}\text { Total PAH's in ppm } \\
\text { Chemiluminescence }\end{array}$ & $\begin{array}{c}\text { HPLC Analytical } \\
\text { (Total PAH's less naphthalene) }\end{array}$ \\
\hline MT-1-G001 & ML3-S & $<10$ & n.a. \\
\hline MT-1-G002 & ML3-D & $<10$ & n.a. \\
\hline MT-1-G003 & ML4-S & $<10$ & n.a. \\
\hline MT-1-G004 & MLA-D & $<10$ & n.a. \\
\hline MT-1-G005 & ML5-S & $<10$ & n.a. \\
\hline MT-1-G006 & ML5-D & $<10$ & n.a. \\
\hline MT-1-G007 & ML6-S & $<10$ & n.a. \\
\hline MT-1-G008 & ML6-D & $<10$ & n.a. \\
\hline MT-1-G009 & ML7-S & $<10$ & n.a. \\
\hline MT-1-G010 & ML7-D & $<10$ & n.a. \\
\hline MT-1-G011 & ML8-S & $<10$ & n.a. \\
\hline MT-1-G012 & ML8-D & $<10$ & n.a. \\
\hline MT-1-G013 & ML9-S & 40 & n.a. \\
\hline MT-1-G014 & ML9-D & 10 & 11.2 \\
\hline MT-1-G015 & ML10-S & $<10$ & n.a. \\
\hline MT-1-G016 & ML10-D & $<10$ & n.a. \\
\hline MT-1-G017 & ML11-S & $<10$ & n.a. \\
\hline MT-1-G018 & ML11-D & $<10$ & n.a. \\
\hline MT-1-G019 & ML12-S & 15 & 394.1 \\
\hline MT-1-G020 & ML12-D & 70 & 341 \\
\hline MT-1-G021 & ML14-S & 160 & 269.5 \\
\hline MT-1-G022 & ML14-D & $7000-8000$ & n.a. \\
\hline $\mathrm{MT}-1-\mathrm{G} 023$ & ML13-S & $1500-2000$ & n.a. \\
\hline MT-1-G024 & ML13-D & $900-1000$ & n.a. \\
\hline MT-1-G025 & ML17-S & $1000-1500$ & 646.5 \\
\hline MT-1-G026 & ML17-D & $2000-2500$ & n.a. \\
\hline MT-1-G027 & ML19-S & $700-800$ & n.a. \\
\hline MT-1-G028 & ML19-D & $>10,000$ & n.a. \\
\hline MT-1-G029 & ML18-S & 140 & 33.7 \\
\hline MT-1-G030 & ML18-D & $<10$ & n.a. \\
\hline MT-1-G031 & ML16-S & $1000-1500$ & n.a. \\
\hline MT-1-G032 & ML16-D & $<10$ & n.a. \\
\hline MT-1-G033 & ML15-S & $<10$ & n.a. \\
\hline MT-1-G034 & ML15-D & $<10$ & n.a. \\
\hline MT-1-G035 & ML27-S & $<10$ & n.a. \\
\hline MT-1-G036 & - & - & n.a. \\
\hline MT-1-G037 & ML20-S & $900-1000$ & n.a. \\
\hline MT-1-G038 & ML20-D & $2500-3000$ & n.a. \\
\hline MT-1-G039 & ML26-S & $500-600$ & n.a. \\
\hline MT-1-G040 & ML26-D & free DNAPL & n.a. \\
\hline MT-1-G041 & ML23-S & 15 & n.a. \\
\hline MT-1-G042 & ML23-D & $<10$ & n.a. \\
\hline MT-1-G043 & ML22-S & 250 & n.a. \\
\hline MT-1-G044 & ML22-D & 250 & n.a. \\
\hline MT-1-G045 & ML24-S & $<10$ & n.a. \\
\hline MT-1-G046 & ML24-D & $<10$ & n.a. \\
\hline MT-1-G047 & ML25-S & 60 & n.a. \\
\hline MT-1-G048 & ML25-D & 25 & n.a. \\
\hline
\end{tabular}


TABLE 4.5 PHASE 1 CONTAMINANT SCREENING - PASSIVE SOIL GAS RESULTS

\begin{tabular}{|c|c|c|c|c|}
\hline \multirow[t]{2}{*}{ Sample } & \multirow{2}{*}{$\begin{array}{c}\text { Map Location } \\
\mathbf{S}=\text { Shallow, } \mathbf{D}=\text { Deep }\end{array}$} & \multicolumn{3}{|c|}{ Analyte (all results in 10E6 counts) } \\
\hline & & $\begin{array}{c}\text { Naphthalene } \\
(2 \mathrm{~m}=128)\end{array}$ & $\begin{array}{c}\text { Anthracene/Phenanthrene } \\
(2 \mathrm{~m}=178)\end{array}$ & $\begin{array}{l}\text { Fluoranthene/Pyrene } \\
(\mathrm{em}=202)\end{array}$ \\
\hline MT-1-G001 & ML3-S & ND & ND & ND \\
\hline MT-1-G002 & ML3-D & ND & ND & ND \\
\hline MT-1-G003 & MLA-S & ND & ND & ND \\
\hline MT-1-G004 & ML4-D & 0.005613 & ND & ND \\
\hline MT-1-G005 & MLS-S & ND & ND & ND \\
\hline MT-1-G006 & ML5-D & ND & ND & ND \\
\hline MT-1-G007 & ML6-S & n.a. & n.a. & n.a. \\
\hline MT-1-G008 & ML6-D & n.a. & na. & n.a. \\
\hline MT-1-G009 & ML7-S & 0.001537 & ND & ND \\
\hline MT-1-G010 & ML7-D & 0.007554 & ND & ND \\
\hline MT-I-G011 & ML8-S & 0.052956 & ND & ND \\
\hline MT-1-G012 & ML8-D & ND & ND & ND \\
\hline MT-1-G013 & ML9-S & 3.65533 & 0.021953 & ND \\
\hline MT-1-G014 & ML9-D & 4.57518 & ND & ND \\
\hline MT-1-G015 & ML10-S & ND & ND & ND \\
\hline MT-1-G016 & ML10-D & ND & $\mathrm{ND}$ & ND. \\
\hline MT-1-G0i7 & ML11-S & 0.359979 & ND & $\mathrm{ND}$ \\
\hline MT-1-G018 & ML11-D & 5.01249 & $\mathrm{ND}$ & ND \\
\hline MT-1-G019 & ML12-S & 3.77898 & 0.122335 & 0.003576 \\
\hline$M T-1-G 020$ & ML12-D & 2.29359 & 0.087985 & 0.003976 \\
\hline MT-1-G021 & ML14-S & ND & ND & $\mathrm{ND}$ \\
\hline $\mathrm{MT}-1-\mathrm{G} 022$ & ML14D & 3.44689 & 0.033333 & $\mathrm{ND}$ \\
\hline MT-1-G023 & ML13-S & 3.37255 & 0.023714 & ND \\
\hline MT-1-G024 & ML13-D & 4.68688 & 0.003604 & ND \\
\hline $\mathrm{MT}-1-\mathrm{G} 025$ & ML17-S & 4.4698 & 0.013798 & $\mathrm{ND}$ \\
\hline MT-1-G026 & ML17-D & 2.60952 & ND & ND \\
\hline MT-1-G027 & ML19-S & 2.62391 & ND & ND \\
\hline MT-1-G028 & ML19-D & 1.82657 & 0.043383 & ND \\
\hline MT-1-G029 & ML18-S & 5.90172 & 0.182617 & 0.017958 \\
\hline MT-1-G030 & ML18-D & 1.86581 & 0.032313 & 0.003747 \\
\hline MT-1-G031 & ML16-S & 4.73797 & 0.024823 & ND \\
\hline$M T-1-G 032$ & ML16-D & 2.25203 & 0.017066 & ND \\
\hline MT-1-G033 & ML15-S & ND & ND & $\mathrm{ND}$ \\
\hline MT-1-G034 & ML15-D & ND & ND & ND \\
\hline MT-1-G035 & ML27-S & 0.000903 & ND & ND \\
\hline MT-1-G036 & - & - & - & - \\
\hline MT-1-G037 & ML20-S & 6.01754 & 0.032288 & ND \\
\hline MT-1-G038 & ML20-D & 3.88412 & 0.042343 & ND \\
\hline MT-1-G039 & ML26-S & 0.710187 & ND & ND \\
\hline MT-1-G040 & ML26-D & 0.34819 & ND & ND \\
\hline MT-1-G041 & ML23-S & 0.050168 & ND & ND \\
\hline MT-1-G042 & ML23-D & 0.520903 & 0.027843 & ND \\
\hline MT-1-G043 & ML22-S & 0.002057 & ND & $\mathrm{ND}$ \\
\hline MT-1-G044 & ML22-D & 0.46519 & ND & ND \\
\hline MT-1-G045 & ML24-S & 0.007131 & ND & ND \\
\hline MT-1-G046 & ML24-D & 0.560711 & 0.020366 & ND \\
\hline MT-1-G047 & ML25-S & 0.005378 & ND & ND \\
\hline MT-1-G048 & ML25-D & 1.69667 & 0.443311 & 0.075436 \\
\hline
\end{tabular}


TABLE 4.6 PHASE 1 CONTAMINANT SCREENING - ACTIVE SOIL GAS RESULTS

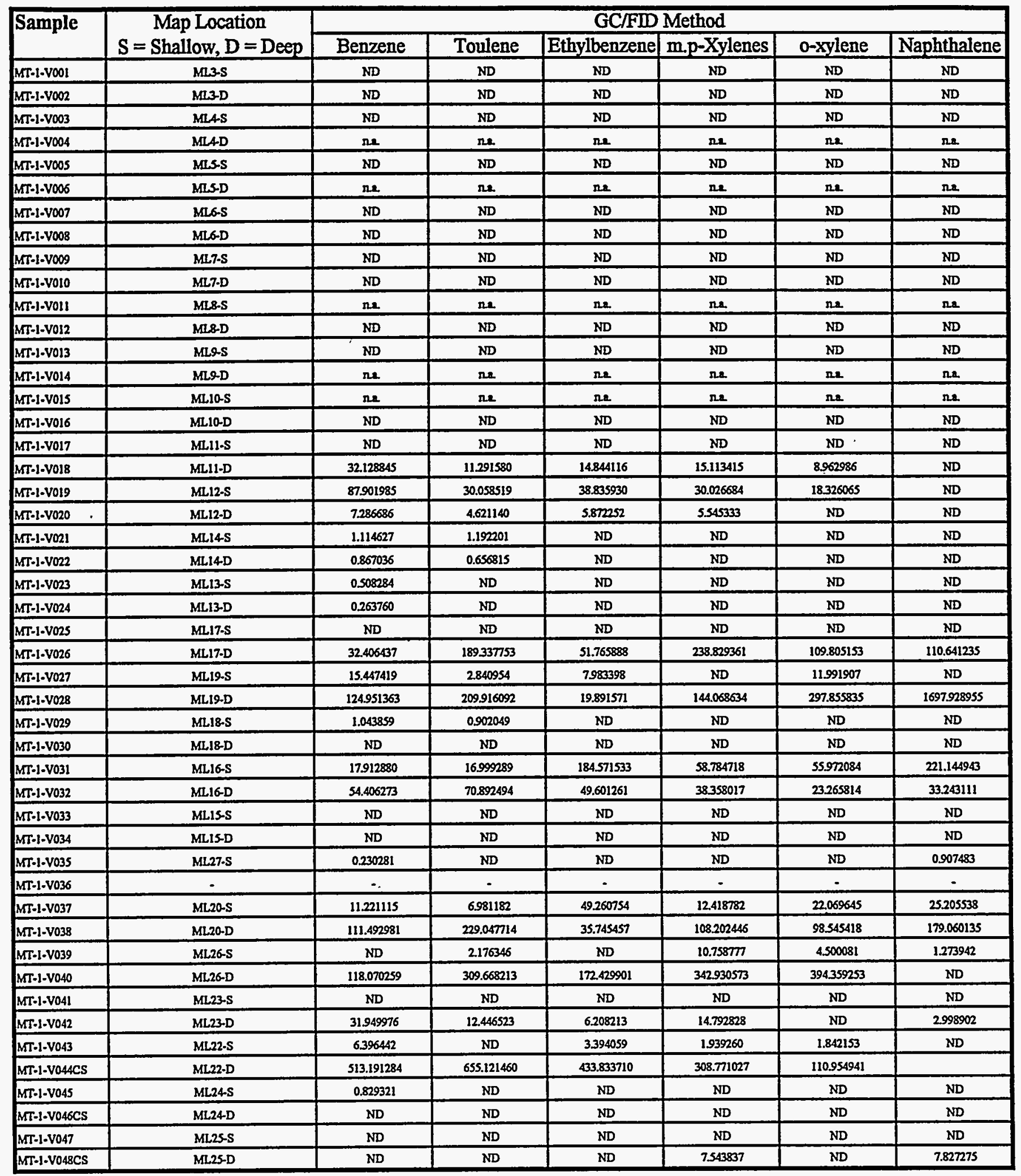


TABLE 4.6 continued

\begin{tabular}{|c|c|c|c|c|c|c|c|}
\hline \multirow[t]{2}{*}{ Sample } & \multirow{2}{*}{$\begin{array}{c}\text { Map Location } \\
\mathrm{S}=\text { Shallow }, \mathrm{D}=\text { Deep }\end{array}$} & \multicolumn{6}{|c|}{ GC/PID Method } \\
\hline & & Benzene & Toulene & Ethylbenzene & m.p-Xylenes & o-xylene & Naphthalene \\
\hline MT-I-V001 & ML3-S & ND & $\mathrm{ND}$ & ND & ND & $\mathrm{ND}$ & $\mathrm{ND}$ \\
\hline MT-1-V0O2 & ML3-D & ND & ND & ND & ND & ND & ND \\
\hline$M T-1-V 003$ & MLAS & $\mathrm{ND}$ & ND & ND & ND & ND & ND \\
\hline MT-1-V004 & MLAD & $\mathrm{nz}$ & $\pi$ & na. & ne. & n. & n. \\
\hline MT-1-Voos & MLS-S & ND & ND & ND & ND & $\mathrm{ND}$ & $\mathrm{ND}$ \\
\hline MT-1.V006 & MLS-D & ne & ne. & ne & ne & ne & ne. \\
\hline MT-1.V007 & MLG-S & ND & $\mathrm{ND}$ & ND & ND & ND & ND \\
\hline MT-1-V008 & MLG-D & ND & ND & ND & $\mathrm{ND}$ & ND & $\mathrm{ND}$ \\
\hline MT-1-V0og & ML7.S & ND & $\mathrm{ND}$ & ND & ND & ND & ND \\
\hline MT-1-V010 & ML7.D & ND & ND & $\mathrm{ND}$ & ND & ND & ND \\
\hline MT-I-V011 & ML8-S & ne. & ne & ne & ne & $\mathrm{ne}$ & $\mathrm{na}$ \\
\hline MT-1-V012 & MLS-D & ND & ND & ND & ND & ND & ND \\
\hline MT-1-V013 & MLe-S & ND & ND & ND & ND & $\mathrm{ND}$ & 3.074724 \\
\hline MTI-1-V014 & ML9-D & ne & ne. & ra & $n_{2}$ & ne & $\mathrm{ra}$ \\
\hline MT-1-VO1S & ML10-S & ne. & ne & $\Omega s$ & na & se. & n... \\
\hline MTI-1-V016 & MLIO-D & ND & ND & ND & ND & ND & ND \\
\hline MT-1.V017 & MLII-S & 0.762419 & 0.388893 & ND & $\mathrm{ND}$ & $\mathrm{ND}$ & $\mathrm{ND}$ \\
\hline$M T-1-V 018$ & MLII-D & 42.081406 & 11.886667 & 16.486229 & 16.071447 & 10.508683 & 0.948226 \\
\hline MTR-1-Volg & ML12-S & 61.539497 & 45350301 & 42.709007 & 32.015926 & 31.759655 & 0.436001 \\
\hline MT-1-V020 & ML12-D & 5.224190 & 4.839434 & 8.070786 & 8349440 & 5.160131 & 0.614379 \\
\hline MTr-1-V021 & MLI4S & 1.720358 & 1.573845 & 1.773427 & 1.661087 & ND & ND \\
\hline MTr-1-V022 & ML14D & 0.944199 & 0.973644 & 1.691951 & 1.553731 & ND & ND \\
\hline MT.1-V023 & MLI3-S & 0.696916 & 0.524565 & 1.185306 & ND & $\mathrm{ND}$ & 0.634707 \\
\hline MT-1-V024 & ML13-D & 0.421216 & 0.412051 & 1.035084 & ND & ND & ND \\
\hline MT-1-V02S & ML17-S & ND & ND & ND & ND & ND & $\mathrm{ND}$ \\
\hline MT-1-V026 & ML17-D & 38.792202 & 202.181534 & 56.042629 & 253.712051 & 120.258537 & 118.893562 \\
\hline MT-1-V027 & MLI9-S & 18.147797 & 3.183892 & 10.937340 & 6.425730 & 14.398224 & 0.476790 \\
\hline MT-1-V028 & ML19-D & 153.833755 & 229.549805 & 22.365625 & 158.336441 & 347.819977 & 1357.087280 \\
\hline MTI-1-V029 & MLI8-S & 1.552771 & 1.174489 & ND & ND & ND & ND \\
\hline MT-1-V030 & ML18-D & 0.166060 & 0.176303 & ND & ND & ND & $\mathrm{ND}$ \\
\hline MT-1.V03! & MLLG-S & 12.794166 & 0.197425 & 175.579727 & ND & 54.705692 & 215.517380 \\
\hline MTR-1-V032 & MLI6-D & 58.704456 & 67.421783 & 47.869938 & 37.766743 & 24987623 & 32.456169 \\
\hline MT-1-V033 & ML15.S & $\mathrm{ND}$ & $N D$ & $\mathrm{ND}$ & $\mathrm{ND}$ & ND & ND \\
\hline MT-1-V034 & MLIS-D • & ND & ND & ND & ND & ND & ND \\
\hline MT-1-V035 & ML27.S & 0.259059 & $\mathrm{ND}$ & $\mathrm{ND}$ & ND & $\mathrm{ND}$ & 1.036475 \\
\hline MT-1-V036 & $\therefore$ & - & - & - & - & - & - \\
\hline MT-1-V037 & ML20-S & 11.654924 & 8.977472 & 68.481377 & 32.538208 & 23.819075 & 27.987640 \\
\hline MT-1.V038 & ML2O-D & 157.798843 & 248.118134 & 40.769600 & 124510078 & 127.324875 & 192.626328 \\
\hline MT-1-V039 & ML26-S & ND & 2018552 & ND & 0.569878 & ND & 2.300092 \\
\hline MTT-1-V040 & ML26-D & 154387711 & 317.272400 & 6.795660 & 326.882874 & 451.848389 & 3.326771 \\
\hline MT-1-V041 & ML23.S & 0.181321 & ND & ND & ND & ND & ND \\
\hline MTI-1-V042 & ML23-D & 10.988695 & 2.228972 & 22.420876 & 10.019212 & 9.833989 & 1.089709 \\
\hline MTI-1-V043 & ML22-S & 9.492801 & ND & 5.466444 & 2.701645 & 4.599745 & 1.019308 \\
\hline MT-1.V044CS & ML22.D & 237.062820 & 588.789856 & 435.934601 & 305.007874 & 117.603355 & 7.800683 \\
\hline MT-1-V045 & ML24-S & 0.936194 & ND & 0.447682 & 0.421743 & ND & 0.191397 \\
\hline MT-1-V046CS & ML24D & ND & 0.420658 & ND & 2.737419 & ND & 0.207056 \\
\hline MT-1-V047 & ML25.S & $\mathrm{ND}$ & ND & ND & ND & ND & ND \\
\hline MT-I-V048CS & ML2S-D & 1.758627 & 0.405296 & 0.217005 & 0.348230 & ND & 0.975272 \\
\hline
\end{tabular}


TABLE 4.7

CPT LIF DATA FOR

SOIL SAMPLES HELD AGAINST THE SAPPHIRE WINDOW VS SPLIT SAMPLE LABORATORY TRPH

\begin{tabular}{|c|c|c|c|c|c|}
\hline $\begin{array}{c}\text { Sample } \\
\text { W }\end{array}$ & $\begin{array}{c}\text { Sample } \\
\text { Depth } \\
\text { (ft.) }\end{array}$ & $\begin{array}{c}\text { EPA 418.1 } \\
\text { Analysis } \\
\text { (mg/kg) }\end{array}$ & $\begin{array}{c}\text { Peak Fluorescence } \\
\text { Intensity } \\
\text { (counts) }\end{array}$ & $\begin{array}{c}\text { Peak } \\
\text { Wavelength } \\
\text { (nm) }\end{array}$ & $\begin{array}{c}\text { Visual Soil } \\
\text { Classification }\end{array}$ \\
\hline $97-1$ & 4.5 & $<25$ & 164 & 400 & clay \\
\hline $101-2$ & 12.5 & $<25$ & 141 & 400 & clay \\
\hline $111-1$ & 12.4 & 232 & 123 & 400 & clay \\
\hline $81-2$ & 8.0 & 1920 & 1072 & 468 & clay \\
\hline $60-1$ & 5.5 & 2334 & 605 & 467 & clay \\
\hline $97-2$ & 10.6 & 5646 & 1052 & 496 & clay \\
\hline $101-1$ & 6.5 & 8178 & 1595 & 475 & clay \\
\hline $60-5$ & 33.5 & 23600 & 530 & 540 & clay \\
\hline $111-2$ & 14.2 & 3542 & 424 & 533 & mix \\
\hline $97-4$ & 25.5 & $<25$ & 154 & 400 & sand \\
\hline $105-2$ & 8.0 & $<25$ & 139 & 400 & sand \\
\hline $60-2$ & 13.0 & 505 & 125 & 400 & sand \\
\hline $97-5$ & 32.1 & 597 & 124 & 400 & sand \\
\hline $105-1$ & 4.2 & 668 & 240 & 400 & sand \\
\hline $101-3$ & 21.5 & 716 & 188 & 400 & sand/gravel \\
\hline $81-1$ & 3.0 & 5849 & 275 & 400 & sand \\
\hline $97-3$ & 14.5 & 7006 & 911 & 506 & sand \\
\hline $111-3$ & 21.0 & 8831 & 322 & 488 & sand \\
\hline $81-3$ & 14.5 & 14122 & 6186 & 425 & sand \\
\hline
\end{tabular}


TABLE 4.8

PANEL PLOT CPT LIF INTENSITY

VS

LABORATORY ANALYSIS OF ADJACENT SOL SAMPLES

\begin{tabular}{|c|c|c|c|c|c|}
\hline $\begin{array}{c}\text { Sample } \\
\mathbf{D}\end{array}$ & $\begin{array}{c}\text { Sample } \\
\text { Depth } \\
\text { (ft.) }\end{array}$ & $\begin{array}{c}\text { EPA 418.1 } \\
\text { Analysis } \\
\text { (mg/kg) }\end{array}$ & $\begin{array}{c}\text { Panel Plot Peak } \\
\text { Fluorescence Intensity } \\
\text { (counts) }\end{array}$ & $\begin{array}{c}\text { Dummy } \\
\text { Pre-push }\end{array}$ & $\begin{array}{c}\text { Visual Soil } \\
\text { Classification }\end{array}$ \\
\hline $97-1$ & 4.5 & $<25$ & 10 & $*$ & clay \\
\hline $101-2$ & 12.5 & $<25$ & 0 & clay \\
\hline $111-1$ & 12.4 & 232 & 400 & clay \\
\hline $81-2$ & 8.0 & 1920 & 700 & clay \\
\hline $60-1$ & 5.5 & 2334 & 400 & clay \\
\hline $97-2$ & 10.6 & 5646 & 300 & clay \\
\hline $101-1$ & 6.5 & 8178 & 650 & clay \\
\hline $60-5$ & 33.5 & 23600 & 380 & clay \\
\hline $111-2$ & 14.2 & 3542 & 550 & mix \\
\hline $97-4$ & 25.5 & $<25$ & 75 & & sand \\
\hline $105-2$ & 8.0 & $<25$ & 0 & & sand \\
\hline $60-2$ & 13.0 & 505 & 200 & & sand \\
\hline $97-5$ & 32.1 & 597 & 150 & & sand \\
\hline $105-1$ & 4.2 & 668 & 100 & & sand \\
\hline $101-3$ & 21.5 & 716 & 250 & & sand/gravel \\
\hline $81-1$ & 3.0 & 5849 & 75 & 1200 & sand \\
\hline $97-3$ & 14.5 & 7006 & 2500 & & sand \\
\hline $111-3$ & 21.0 & 8831 & 6000 & & sand \\
\hline $81-3$ & 14.5 & 14122 & sand \\
\hline
\end{tabular}

* Indicates fluorescence reading was taken in dummy probe pre-push area. 
TABLE 4.9

\section{CPT LIF INTENSITIES WITHIN STRATIGRAPHIC ZONES}

\begin{tabular}{|c|c|c|c|c|c|c|c|c|c|}
\hline Map & $\mathbf{X}$ & $\mathbf{Y}$ & Zone 1 & Zone 2 & Zone 3 & Zone 4 & Zone 5 & Zone 6 & Pre-push* \\
\hline Location & (ft.) & (ft.) & & & & & & & \\
\hline ML28 & 834 & 424 & 10 & 60 & 1 & 100 & 40 & $\mathrm{NA}$ & - \\
\hline ML30 & 908 & 421 & NA & $\mathrm{NA}$ & NA & $\mathrm{NA}$ & NA & $\mathrm{NA}$ & - \\
\hline ML32 & 438 & 408 & 100 & 10 & 10 & 50 & 10 & $\mathrm{NA}$ & - \\
\hline ML33 & 862 & 460 & 0 & 0 & 10 & 75 & 100 & $\mathrm{NA}$ & - \\
\hline ML41 & 450 & 554 & 0 & 0 & 100 & $\mathrm{NA}$ & NA & $\mathrm{NA}$ & - \\
\hline ML53 & 732 & 511 & $\mathrm{NA}$ & NA & NA & 0 & 5 & $\mathrm{NA}$ & 20'LR \\
\hline ML60 & 679 & 436 & 300 & 100 & 200 & 10 & 350 & 10 & 20 'LR \\
\hline ML61 & 668 & 489 & 600 & 250 & 100 & 0 & 300 & 0 & 20'SR \\
\hline ML64 & 787 & 461 & $\mathrm{NA}$ & $\mathrm{NA}$ & NA & 100 & 50 & $\mathrm{NA}$ & $20^{\prime} \mathrm{LR}$ \\
\hline ML72 & 850 & 433 & $\mathrm{NA}$ & NA & $\mathrm{NA}$ & 50 & $\mathrm{NA}$ & NA & 21'SR \\
\hline ML74 & 834 & 440 & NA & $\mathrm{NA}$ & NA & 50 & $\mathrm{NA}$ & $\mathrm{NA}$ & 21 'SR \\
\hline ML76 & 782 & 405 & 0 & 30 & 50 & 30 & 350 & 50 & 20 'SR \\
\hline ML77 & 788 & 467 & NA & NA & NA & 5 & 10 & NA & 20 'SR \\
\hline ML79 & 809 & 440 & NA & $\mathrm{NA}$ & NA & 10 & 50 & 10 & 20'SR \\
\hline ML81 & 581 & 547 & 1000 & 5000 & 800 & 0 & 80 & 5 & $20^{\prime} \mathrm{SR}$ \\
\hline ML83 & 634 & 521 & 200 & 300 & 100 & 10 & 300 & 20 & 20'SR \\
\hline ML85 & 489 & 510 & 30 & 50 & 100 & 80 & 10 & 0 & 20'SR \\
\hline ML87 & 649 & 547 & 100 & 200 & 300 & 100 & 220 & $\mathrm{NA}$ & 20'LR \\
\hline ML89 & 693 & 457 & 200 & 100 & 20 & 100 & 400 & 10 & 20'LR \\
\hline ML91 & 718 & 537 & 0 & 0 & 100 & 0 & 100 & 0 & $20^{\prime} \mathrm{LR}$ \\
\hline ML93 & 587 & 579 & 400 & 2000 & 5000 & 4000 & 20 & 10 & 5'LR \\
\hline ML95 & 771 & 515 & 40 & 0 & 0 & 0 & 80 & 80 & 5'LR \\
\hline ML97 & 744 & 484 & 50 & 450 & 1200 & 70 & 180 & 20 & 5'LR \\
\hline ML99 & 739 & 524 & 80 & 10 & 100 & 60 & 110 & 0 & 7'LR \\
\hline ML101 & 750 & 439 & 700 & 0 & 300 & 0 & 270 & 10 & 5'LR \\
\hline ML105 & 778 & 439 & 60 & 0 & 0 & 0 & 550 & NA & 5'LR \\
\hline ML107 & 767 & 473 & 700 & 5 & 120 & 20 & 260 & NA & 5'LR \\
\hline ML109 & 732 & 453 & 350 & 5 & 300 & 10 & 500 & NA & 5'LR \\
\hline ML111 & 621 & 579 & 220 & 400 & 500 & 2000 & 80 & NA & 5'LR \\
\hline ML113 & 723 & 432 & 1000 & 200 & 200 & 10 & 170 & NA & 5'LR \\
\hline
\end{tabular}

* Depth LR= Large Rod, SR= Small Rod 
TABLE 4.10

COMPARISON OF GEOPROBE CONDUCTIVITY DIPS WITH MCLAREN/HART AND CPT LOGS

\begin{tabular}{|c|c|c|c|c|}
\hline $\begin{array}{l}\text { Geoprobe } \\
\text { Location } \\
\text { ID }\end{array}$ & $\begin{array}{l}\text { Conductivity } \\
\text { Dip Depths } \\
\text { (ft.) }\end{array}$ & Possible Explanation & Source & $\begin{array}{l}\text { Source Distance } \\
\text { from Geoprobe } \\
\text { (ft.) }\end{array}$ \\
\hline & & & & \\
\hline \multirow[t]{2}{*}{$\mathrm{LO28}$} & 21.25 & Gravel at $20 \mathrm{ft}, \mathrm{LIF}$ near $10^{*}$ & \multirow[t]{2}{*}{ CPT L028 } & \multirow[t]{2}{*}{2} \\
\hline & $23-24.5$ & LIF about 100 at 22 to $23 \mathrm{ft}$. & & \\
\hline \multirow[t]{2}{*}{ L033 } & $16 \& 17$ & $\begin{array}{l}\text { Gravel at } 16 \text { to } 17 \mathrm{ft} . \\
(\mathrm{LIF}<25)\end{array}$ & \multirow[t]{2}{*}{ CPT L033 } & \multirow[t]{2}{*}{2} \\
\hline & 21 to 25 & LIF about 50 to 100 at 20 to $24 \mathrm{ft}$. & & \\
\hline L037 & $\begin{array}{l}23.5 \& \\
25 \text { to } 27\end{array}$ & $\begin{array}{l}\text { Gravel plentiful, (no odor) } \\
\text { from } 23 \text { to } 25 \mathrm{ft} \text {. }\end{array}$ & & 2 \\
\hline \multirow[t]{3}{*}{ L040 } & 6 & LIF peaks to 400 at 5 to $6 \mathrm{ft}$. & \multirow[t]{3}{*}{ CPT L105 } & \multirow[t]{3}{*}{5} \\
\hline & 19.5 & $\begin{array}{l}\text { Possible gravel at } 17.5 \mathrm{ft} . \\
(\mathrm{LIF}<10)\end{array}$ & & \\
\hline & 32 to 33 & LIF $>.400$ from 32 to $35.5 \mathrm{ft}$. & & \\
\hline \multirow[t]{4}{*}{ L043 } & 16 & LIF near 100 from 14 to $15 \mathrm{ft}$. & \multirow[t]{4}{*}{ CPT L099 } & \multirow[t]{4}{*}{13} \\
\hline & 18.5 to 19 & $\begin{array}{l}\text { Gravel from } 18 \text { to } 20 \mathrm{ft} \text {. and } \\
\mathrm{LIF}>50 \text { from } 18 \text { to } 23 \mathrm{ft} \text {. }\end{array}$ & & \\
\hline & 20.5 & LIF near 100 from 20 to $22 \mathrm{ft}$. & & \\
\hline & 22 to 23 & LIF > 50 from 22 to $23 \mathrm{ft}$. & & \\
\hline $\mathrm{L} 045$ & 22.5 to 23 & $\begin{array}{l}\text { Gravelly sand with free product from } \\
22 \text { to } 24 \mathrm{ft} \text {. }\end{array}$ & MLH L114 & 2 \\
\hline \multirow[t]{3}{*}{ L052 } & 4.5 to 7 & $\begin{array}{l}\text { Strong odor, black but no sheen } \\
\text { from } 6 \text { to } 8 \mathrm{ft} \text {. }\end{array}$ & \multirow[t]{3}{*}{ MLH L112 } & \multirow{3}{*}{12} \\
\hline & $15.5 \& 18.5$ & $\begin{array}{l}\text { Strong odor, product visible } \\
\text { from } 16 \text { to } 17 \mathrm{ft} \text {. }\end{array}$ & & \\
\hline & 32 to 34.5 & Free phase product from 32 to $34 \mathrm{ft}$. & & \\
\hline \multirow[t]{7}{*}{ L054 } & \multirow[t]{2}{*}{4 to 7} & LIF $>200$ from 5.5 to $8 \mathrm{ft}$. & CPT L109 & 10 \\
\hline & & Strong odor with product from 6 to $7 \mathrm{ft}$. & \multirow[t]{2}{*}{ MLH L118 } & \multirow[t]{2}{*}{8} \\
\hline & 15 & Odor with product from 14 to $16 \mathrm{ft}$. & & \\
\hline & \multirow[t]{2}{*}{$\begin{array}{l}20.5,22.5 \& \\
23.5\end{array}$} & $\begin{array}{l}\text { Gravel from } 20 \text { to } 23 \mathrm{ft} . \\
\text { LIF }>200 \text { from } 16 \text { to } 18 \mathrm{ft} \text {. } \\
\text { LIF about } 300 \text { at } 20 \mathrm{ft} .\end{array}$ & CPT L109 & \\
\hline & & Gravel from 22 to $24 \mathrm{ft}$. & MLH L118 & \\
\hline & \multirow[t]{2}{*}{34.5} & LIF $>200$ from 33 to $35 \mathrm{ft}$. & CPT L109 & \\
\hline & & $\begin{array}{l}\text { Free product from } 32 \text { to } 34 \mathrm{ft} . \\
\text { Strong odor, possible product from } 34 \\
\text { to } 36 \mathrm{ft} \text {. }\end{array}$ & MLH L118 & \\
\hline \multirow[t]{5}{*}{$\mathrm{LO55}$} & 5 & Black soil with strong odor from 5 to $7 \mathrm{ft}$. & \multirow[t]{5}{*}{ MLH 057} & \multirow[t]{5}{*}{19} \\
\hline & 12 to 14 & Free product from 12 to $14 \mathrm{ft}$. & & \\
\hline & 19 & Strong odor, gravel from 18 to $20 \mathrm{ft}$. & & \\
\hline & 25 & Slight odor, no product 24 to $26 \mathrm{ft}$. & & \\
\hline & 36 & Product from 34 to $36 \mathrm{ft}$. & & \\
\hline
\end{tabular}

* LIF intensity units are counts. 
TABLE 4.10 ( continued )

COMPARISON OF GEOPROBE CONDUCTIVTTY DIPS WITH MCLAREN/HART AND CPT LOGS

\begin{tabular}{|c|c|c|c|c|}
\hline $\begin{array}{c}\text { Geoprobe } \\
\text { Location } \\
\text { ID } \\
\end{array}$ & $\begin{array}{c}\text { Conductivity } \\
\text { Dip Depths } \\
\text { (ft) }\end{array}$ & Possible Explanation & Source & $\begin{array}{l}\text { Source Distance } \\
\text { from Geoprobe } \\
\text { (ft.) }\end{array}$ \\
\hline & & & & \\
\hline \multirow[t]{2}{*}{$\overline{L 056}$} & 19 & $\begin{array}{l}\text { Trace gravel, no product mentioned, } \\
\text { from } 18 \text { to } 20 \mathrm{ft} \text {. }\end{array}$ & \multirow[t]{2}{*}{ MLHL065 } & \multirow[t]{2}{*}{2} \\
\hline & 24 to 25 & $\begin{array}{l}\text { Gravel, no product mentioned, } \\
\text { from } 24 \text { to } 26 \mathrm{ft} \text {. }\end{array}$ & & \\
\hline L058 & 10 to 11 & Slight odor at 11 to $13 \mathrm{ft}$. & MLHL067 & $\overline{2}$ \\
\hline \multirow[t]{4}{*}{ L059 } & 5 to 7 & Odor, no visible product & \multirow[t]{2}{*}{ MLHL108 } & \multirow[t]{2}{*}{9} \\
\hline & \multirow[t]{2}{*}{21} & Gravel, no odor from 19 to $21 \mathrm{ft}$. & & \\
\hline & & $\begin{array}{l}\text { Gravel at } 20 \mathrm{ft} . \\
\text { LIF near } 100^{*} \text { from } 20 \text { to } 24 \mathrm{ft} .\end{array}$ & \multirow[t]{2}{*}{ CPT L064 } & \multirow[t]{2}{*}{5} \\
\hline & 23 to 24.5 & $\begin{array}{l}\text { Gravel from } 24 \text { to } 27 \mathrm{ft} . \\
\text { LIF near } 100 \text { from } 20 \text { to } 24 \mathrm{ft} \text {. }\end{array}$ & & \\
\hline \multirow[t]{2}{*}{$\mathrm{L} 062$} & 18 to 20 & Trace gravel, no odor, 18 to $20 \mathrm{ft}$. & \multirow[t]{2}{*}{ MLHL070 } & \multirow[t]{2}{*}{2} \\
\hline & 31 , & Gravel, no odor, 30 to $32 \mathrm{ft}$. & & \\
\hline \multirow[t]{2}{*}{ L063 } & 18 to 19 & Gravel, no odor, 18 to $20 \mathrm{ft}$. & \multirow[t]{2}{*}{ MLHL078 } & \multirow[t]{2}{*}{$\overline{2}$} \\
\hline & 24 & Trace gravel, no odor, 25 to $27 \mathrm{ft}$. & & \\
\hline \multirow[t]{4}{*}{ L066 } & $17 \& 18$ & LIF $>1000$ from 11 to $18 \mathrm{ft}$ & \multirow[t]{4}{*}{ CPT L081 } & \multirow[t]{4}{*}{4} \\
\hline & 20.5 & $\begin{array}{l}\text { LIF about } 800 \text { at } 20.5 \mathrm{ft} \text {. } \\
\text { Gravel at } 20 \text { to } 21.5 \mathrm{ft} \text {. }\end{array}$ & & \\
\hline & $25.5 \& 26$ & Possible gravel from 24 to $25 \mathrm{ft}$. & & \\
\hline & 36 & LIF about 100 from 34 to $36 \mathrm{ft}$. & & \\
\hline \multirow[t]{2}{*}{ L069 } & $18.5 \& 21.5$ & $\begin{array}{l}\text { Free product, some gravel from } 19 \text { to } \\
21 \mathrm{ft} \text {. }\end{array}$ & \multirow[t]{2}{*}{ MLH L94 } & \multirow[t]{2}{*}{14} \\
\hline & $\begin{array}{c}24.5 \& \\
26 \text { to } 27\end{array}$ & $\begin{array}{l}\text { Strong odor, free product, from } 25 \text { to } \\
27 \mathrm{ft} \text {. }\end{array}$ & & \\
\hline
\end{tabular}

* LIF intensity units are counts. 
TABLE 4.11 SOIL CLASSIFICATION COMPARISONS BY SELECTED SAMPLE LOCATION CLUSTERS.

\begin{tabular}{|c|c|c|c|c|}
\hline \multicolumn{5}{|c|}{ Cluster 1} \\
\hline Location & L77 & I64 & L59 & L108 \\
\hline$(\mathrm{X}, \mathrm{Y})(\mathrm{ft})$ & 788,467 & 781,461 & 783,460 & 789,466 \\
\hline Source & $\mathrm{CPT}$ & $\mathrm{CPT}$ & Soil Cond. & McLaren/Hart \\
\hline \multicolumn{5}{|l|}{ Depth (ft.) } \\
\hline 6 to 8 & & & clay & silt with clay \\
\hline 19 to 20 & & & sand & silty sand with gravel \\
\hline 20 to 21 & silt and clay & sand and silt & sand & silty sand with gravel \\
\hline 26 to 28 & sand & sand with gravel & sand/silt? & sand \\
\hline 30 to 31 & silt & sand & sand/silt? & sand \\
\hline 31 to 32 & silt & sand and clayey silt & sand/sut? & clayey silt \\
\hline
\end{tabular}

\begin{tabular}{|l|c|c|c|}
\hline \multicolumn{3}{|c|}{ Cluster 2 } \\
\hline Location & LA7 & L81 & L66 \\
\hline $\mathrm{X}, \mathrm{Y}$ )(ft.) & 583,549 & 581,547 & 584,548 \\
\hline Source & CPT & CPT & Soil Cond. \\
\hline Depth (ft.) & sand with gravel & sand with gravel & sand \\
\hline 20 to 21 & sand & sand & sand \\
\hline 21 to 36 & & silt with clay & clay \\
\hline 36 to 39 & & & \\
\hline
\end{tabular}

\begin{tabular}{|c|c|c|c|}
\hline \multicolumn{4}{|c|}{ Cluster 3} \\
\hline Location & $\mathrm{L} 33$ & L33 & L73 \\
\hline$(\mathrm{X}, \mathrm{Y})(\mathrm{ft})$. & 862,460 & 862,460 & 862,460 \\
\hline Source & CPT LF & CPT Resistivity & McLaren/Hart \\
\hline \multicolumn{4}{|l|}{ Depth (ft.) } \\
\hline 0 to 4 & silt mix & silt mix & \\
\hline 4 to 6 & silt mix & clay & silt with clay \\
\hline 6 to 7 & silt mix & clay & \\
\hline 8 to 10 & clay & clay & clay \\
\hline 10 to 13 & clay & clay & \\
\hline 13 to 14 & clay & clay & silt with sand \\
\hline 14 to 15 & sand mix & sand/silt mix & silt with sand \\
\hline 15 to 17 & sand mix with gravel & sand/silt mix & \\
\hline
\end{tabular}

\begin{tabular}{|c|c|c|c|}
\hline \multicolumn{4}{|c|}{ Cluster 4} \\
\hline Location & L54 & L109 & L118 \\
\hline$(\mathrm{X}, \mathrm{Y})(\mathrm{ft})$ & 737,461 & 732,453 & 730,458 \\
\hline Source & Soil Cond. & CPT & McLaren/Hart \\
\hline \multicolumn{4}{|l|}{ Depth (ft.) } \\
\hline 5 to 7 & silt/sand & silt mix & silty clay \\
\hline 7 to 14 & clay & clay with silt mix & \\
\hline 14 to 15 & clay & clay with silt mix & silty clay \\
\hline 15 to 16 & sand & clay with silt mix & silty clay \\
\hline 16 to 20 & sand & sand with silt mix & \\
\hline 20 to 22 & sand with gravel & sand with gravel & \\
\hline 22 to 24 & sand with gravel & sand with gravel & sand \\
\hline 24 to 26 & sand & sand with gravel & sand \\
\hline 26 to 30 & sand & sand with gravel & sand with silt \\
\hline 30 to 34 & sand & sand with gravel & sand \\
\hline 34 to 36 & silt/clay & silt with clay & silty clay \\
\hline
\end{tabular}


TABLE 4.11 CONTINUED

\begin{tabular}{|c|c|c|c|c|}
\hline \multicolumn{5}{|c|}{ Cluster 5} \\
\hline Location & LAS & $\mathrm{L60}$ & L114 & $\overline{B 8}$ \\
\hline$(\mathrm{X}, \mathrm{Y})$ (ft.) & 665,428 & 679,436 & 667,428 & 675,424 \\
\hline Source & McLaren/Hart & $\mathrm{CPT}$ & McLaren/Hart & BVWST \\
\hline \multicolumn{5}{|l|}{ Depth (ft.) } \\
\hline 6 to 7 & silty clay & & & silty sand \\
\hline 7 to 8 & silty clay & & & silty clay \\
\hline 17 to 19 & silty sand & & & silty clay \\
\hline 20 to 22 & & sand with gravel & & sand \\
\hline 22 to 24 & & sand with gravel & sand with gravel & sand \\
\hline 24 to 26 & sand & sand & & sand \\
\hline 26 to 27 & & sand & & sand \\
\hline 27 to 30 & & sand & & silty sand \\
\hline 30 to 32 & silt and sand & sand & & silty sand \\
\hline 32 to 34 & & sand & & silty sand \\
\hline 34 to 35 & & silt mix with clay & & silty sand \\
\hline 35 to 36 & sand and silt & silt mix with clay & & silty sand \\
\hline 36 to 37 & sand and silt & silt mix with clay & & silt with clay \\
\hline 37 to 39 & & silt mix with clay & & silt with clay \\
\hline
\end{tabular}

\begin{tabular}{|l|c|c|}
\hline \multicolumn{3}{|c|}{ Cluster 6 } \\
\hline Location & L113 & B13 \\
\hline $\mathrm{X}, \mathrm{Y})(\mathrm{ft})$. & 723,432 & 727,429 \\
\hline Source & CPT & BVWST \\
\hline Depth (ft.) & & \\
\hline 6 to 16.5 & clay with silt mix & silty clay \\
\hline 16.5 to 18 & silt mix & silty clay \\
\hline 18 to 19 & sand & silty clay \\
\hline 19 to 25 & sand with gravel & sand \\
\hline 25 to 28 & sand with gravel & silty sand, some gravel \\
\hline 28 to 33 & sand & silty sand, some gravel \\
\hline 33 to 35 & clay & silty sand \\
\hline 36.5 to 40 & & silty clay \\
\hline
\end{tabular}

\begin{tabular}{|l|c|c|}
\hline \multicolumn{3}{|c|}{ Cluster 7 } \\
\hline Location & LA0 & L105 \\
\hline$(\mathrm{X}, \mathrm{Y})(\mathrm{ft})$. & 780,435 & 778,439 \\
\hline Source & McLaren/Hart & $\mathrm{CPT}$ \\
\hline Depth (ft.) & & \\
\hline 6 to 8 & silt over clay & silt over clay \\
\hline 16 to 18 & silty clay & clay, silt and sand \\
\hline 26 to 28 & sand & sand \\
\hline 31 to 33 & sand & sand with gravel \\
\hline 33 to 35 & silt and sand & sand over silt \\
\hline
\end{tabular}

\begin{tabular}{|l|c|c|}
\hline \multicolumn{3}{|c|}{ Cluster 8 } \\
\hline Location & L78 & L63 \\
\hline$(\mathrm{X}, \mathrm{Y})$ (ft.) & 859,440 & 858,439 \\
\hline Source & McLaren/Hart & Soil Cond. \\
\hline \hline Depth (ft.) & & \\
\hline 4 to 6 & silt with clay & silt ? \\
\hline 9 to 11 & clay with silt & clay \\
\hline 13 to 15 & clay with silt & clay over silt \\
\hline 18 to 20 & sand with gravel & sand with gravel \\
\hline 25 to 27 & sand and gravel over clay & sand over silt/clay \\
\hline
\end{tabular}


TABLE 4.11 CONTINUED

\begin{tabular}{|l|c|c|}
\hline \multicolumn{2}{|c|}{ Cluster 9 } \\
\hline Location & L65 & L56 \\
\hline $\mathrm{X}, \mathrm{Y}$ )(ft.) & 780,507 & 780,507 \\
\hline Source & McLaren/Hart & Soil Cond. \\
\hline Depth(ft.) & & \\
\hline 4 to 6 & silt with clay & silt ? \\
\hline 12 to 14 & silty clay & clay \\
\hline 18 to 20 & sand on silt with clay & sand \\
\hline 24 to 26 & silt with gravel on clay & silt/sand with clay? \\
\hline
\end{tabular}

\begin{tabular}{|l|c|c|}
\hline \multicolumn{3}{|c|}{ Cluster 10 } \\
\hline Location & L67 & L58 \\
\hline (X,Y)(ft.) & 775,533 & 775,533 \\
\hline Source & McLaren/Hart & Soil Cond. \\
\hline Depth (ft.) & & \\
\hline 4 to 6 & silt with clay & clay over silt ? \\
\hline 11 to 13 & clay with silt & clay over silt ? \\
\hline 16 to 18 & sand with silt & sand with silt \\
\hline 21 to 23 & silt/sand/gravel on clay & sand over clay \\
\hline
\end{tabular}

\begin{tabular}{|l|c|c|}
\hline \multicolumn{3}{|c|}{ Cluster 11 } \\
\hline Location & L75 & L37 \\
\hline $\mathrm{X}, \mathrm{Y}$ )(ft.) & 813,475 & 812,476 \\
\hline Source & McLaren/Hart & Soil Cond. \\
\hline Depth (ft.) & & \\
\hline 5 to 7 & silt with clay & sand over clay? \\
\hline 10 to 12 & clay with silt & clay \\
\hline 14 to 16 & clay over silt & clay over silt \\
\hline 19 to 21 & silt with sand and clay & sand \\
\hline 23 to 25 & silt & sand and silt \\
\hline
\end{tabular}

\begin{tabular}{|l|c|c|}
\hline \multicolumn{3}{|c|}{ Cluster 12 } \\
\hline Location & LA1 & L68 \\
\hline $\mathrm{X}, \mathrm{Y})(\mathrm{ft})$. & 450,554 & 451,558 \\
\hline Source & $\mathrm{CPT}$ & Soil Cond. \\
\hline Depth (ft.) & & \\
\hline 5 to 12.5 & clay & silt over clay \\
\hline 12.5 to 16.5 & sand & sand with gravel \\
\hline 19 to 21 & sand with gravel & sand with gravel \\
\hline 21 to 36.5 & sand & sand \\
\hline 37 & clay & siltclay? \\
\hline
\end{tabular}


TABLE 4.12 DEPTHS OF STRATIGRAPHIC CONTACTS FROM DIRECT PUSH TECHNOLOGIES

\begin{tabular}{|c|c|c|c|c|c|c|c|c|}
\hline $\begin{array}{l}\text { Map } \\
\text { Location }\end{array}$ & Source & $\begin{array}{c}\mathbf{X} \\
\text { (ft.) }\end{array}$ & $\begin{array}{c}\mathbf{Y} \\
\text { (ft.) }\end{array}$ & Topo. & fill-ucu & ucu-gran & gran-Icu & Icu-bed \\
\hline LI & I & 931.75 & 560.57 & 885.29 & & & & \\
\hline $\mathrm{L3}$ & $I$ & 814.83 & 576.47 & 886.67 & & & & \\
\hline$\overline{\mathrm{L} 4}$ & I & 812.4 & 524.41 & 885.15 & & & & \\
\hline $\mathrm{L} 5$ & I & 785.02 & 476.94 & 883.48 & & & & \\
\hline L7 & I & 840.63 & 501.91 & 884.02 & & & & \\
\hline L9 & $\mathrm{I}$ & 751.16 & 510.02 & 884.56 & & & & \\
\hline$\overline{\mathrm{L} 10}$ & I & 750.6 & 559.96 & 884.86 & & & & \\
\hline$\overline{\mathrm{L} 11}$ & I & 750.39 & 442.33 & 883.99 & & & & \\
\hline$\overline{\mathrm{L} 12}$ & $I$ & 731.66 & 427.6 & 882.55 & & & & \\
\hline$\overline{\mathrm{L} 13}$ & $I$ & 722.9 & 491.92 & 884.65 & & & & \\
\hline$\overline{\mathrm{L} 14}$ & $\bar{I}$ & 721.16 & 537.99 & 884.91 & & & & \\
\hline$\overline{L 15}$ & $I$ & 714.18 & 592.35 & 888.57 & & & & \\
\hline$\overline{\mathrm{L} 16}$ & I & 689.99 & 471.51 & 884.5 & & & & \\
\hline $\mathrm{L} 17$ & $\bar{I}$ & 678.24 & 559.63 & 888.07 & & & & \\
\hline$\overline{\mathrm{L} 18}$ & $I$ & 679.07 & 424.69 & 883.3 & & & & \\
\hline L19 & $I$ & 638.71 & 543.24 & 886.42 & & & & \\
\hline$\overline{\mathrm{L} 20}$ & I & 646.34 & 503.82 & 885.52 & & & & \\
\hline$\overline{\mathrm{L} 22}$ & I & 595.1 & 534.75 & 886.14 & & & & \\
\hline $\mathrm{L} 23$ & I & 590.79 & 575.02 & 886.02 & & & & \\
\hline $\mathrm{L} 24$ & $I$ & 553.31 & 543.28 & 886.45 & & & & \\
\hline $\mathrm{L} 25$ & $\bar{I}$ & 573.97 & 507.37 & 885.79 & & & & \\
\hline$\overline{\mathrm{L} 26}$ & $\bar{I}$ & 634.79 & 574.47 & 886.02 & & & & \\
\hline L28 & $\mathrm{GC}$ & 834 & 424 & 881.5 & 6 & 17 & 30 & 32 \\
\hline $\bar{L} 29$ & $\bar{G}$ & 960 & 418 & 881 & 4 & 17 & 26 & 27 \\
\hline $\mathrm{L} 30$ & $\bar{C}$ & 908 & 421 & 881.2 & ND & 16 & ND & ND \\
\hline $\bar{L} 31$ & $\bar{G}$ & 1036 & 421 & 880.5 & 4 & 16 & 26 & 27 \\
\hline $\mathrm{L} 32$ & $\mathrm{C}$ & 438 & 408 & 884 & 6 & 18 & 34 & 35 \\
\hline L33 & GC & 862 & 460 & 882 & 5 & 14 & 25 & 27 \\
\hline L34 & $G$ & 903 & 515 & 883.5 & 3 & 11 & 19 & 22 \\
\hline$\overline{L 35}$ & $\bar{G}$ & 865 & 550 & 885.1 & 6 & 11 & 13.5 & 15.5 \\
\hline $\bar{L} 36$ & $\overline{\mathrm{GM}}$ & 845 & 507 & 883.7 & 4 & 13 & 22.5 & 45.5 \\
\hline $\mathrm{L} 37$ & $G$ & 812 & 476 & 884 & 3 & 15 & 26 & 29 \\
\hline L38 & GM & 806 & 519 & 884.5 & 2 & 13.5 & 22.5 & 24.5 \\
\hline L39 & $\overline{G M}$ & 792 & 481 & 884.1 & 4 & 15 & 21.5 & 23.5 \\
\hline L40 & $\overline{\mathrm{GM}}$ & 780 & 435 & 883 & 5 & 16 & 33 & 35 \\
\hline $\mathrm{L} 41$ & C & 450 & 554 & 886.5 & $\mathrm{ND}$ & $\mathrm{ND}$ & 37 & 39.5 \\
\hline$\overline{\mathrm{L} 42}$ & $\overline{G M}$ & 792 & 553 & 885.5 & 3 & 13 & 18 & 20 \\
\hline L43 & $\mathrm{GM}$ & 751 & 522 & 884.2 & 4 & 14 & 25.5 & 27.5 \\
\hline$\overline{\mathrm{L} 44}$ & $G$ & 719 & 592 & 887.2 & 3 & 13 & 26 & 29 \\
\hline$\overline{\mathrm{L} 45}$ & $\overline{G M}$ & 665 & 428 & 883 & 5 & 18 & 36 & 38 \\
\hline $\mathrm{L} 46$ & $G$ & 597 & 597 & 889 & 5 & 15 & 34 & 36 \\
\hline$\overline{L 47}$ & $\bar{C}$ & 583 & 549 & 886 & ND & ND & 36.5 & 38 \\
\hline$\overline{L 48}$ & $\bar{G}$ & 655 & 582 & 888 & ND & ND & ND & ND \\
\hline$\overline{\mathrm{L} 49}$ & $\bar{C}$ & 621 & 533 & 886 & ND & ND & 32.5 & 34 \\
\hline
\end{tabular}


TABLE 4.12 continued

\begin{tabular}{|c|c|c|c|c|c|c|c|c|}
\hline $\begin{array}{l}\text { Map } \\
\text { Location }\end{array}$ & Source & $\begin{array}{c}X \\
\text { (ft.) }\end{array}$ & $\begin{array}{c}\mathbf{Y} \\
\text { (ft.) }\end{array}$ & Topo. & fill-ucu & ucu-gran & gran-lcu & Icu-bed \\
\hline L50 & $G$ & 546 & 547 & 886 & ND & ND & ND & ND \\
\hline$\overline{L 51}$ & $\bar{C}$ & 688 & 543 & 885.5 & ND & ND & 32.5 & 34 \\
\hline $\mathrm{L} 52$ & $G$ & 672 & 462 & 883.2 & 3 & 15 & 35 & 38 \\
\hline$\overline{L 53}$ & $\bar{C}$ & 732 & 511 & 884.5 & ND & ND & 31.7 & ND \\
\hline L54 & $\bar{G}$ & 737 & 461 & 884 & 4 & 15 & 34.5 & 37.5 \\
\hline L55 & $G$ & 555 & 529 & 885 & 5 & 14 & 36.5 & 41 \\
\hline$\overline{L 56}$ & $\bar{G}$ & 780 & 507 & 883.9 & 3.5 & 15 & 20 & 22 \\
\hline L57 & $\overline{\mathbf{M}}$ & 566 & 544 & 886 & ND & ND & 35 & 37 \\
\hline$\overline{L 58}$ & $\bar{G}$ & 775 & 533 & 884.2 & 5.5 & 13 & 21.5 & 23.5 \\
\hline L59 & $\bar{G}$ & 783 & 460 & 883.5 & 6.5 & 15.5 & 32.5 & 33 \\
\hline $\mathrm{L} 60$ & C & 679 & 436 & 883 & ND & $\mathrm{ND}$ & 34.5 & 36 \\
\hline L61 & $\bar{C}$ & 668 & 489 & 885 & ND & ND & 36 & 38 \\
\hline $\mathrm{L} 62$ & $G$ & 832 & 455 & 882.5 & 4 & 14.5 & 32 & 34 \\
\hline L63 & $\bar{G}$ & 858 & 439 & 882 & 4 & 14.5 & 26.2 & 28.2 \\
\hline$\overline{L 64}$ & C & 787 & 461 & 883.5 & $\mathrm{ND}$ & ND & 31.5 & 34 \\
\hline L65 & $\bar{M}$ & 780 & 507 & 883.9 & $\mathrm{ND}$ & $\mathrm{ND}$ & 20 & 22 \\
\hline L66 & $\bar{G}$ & 584 & 548 & 886 & 5 & 13 & 36.7 & 38 \\
\hline L68 & G & 451 & 558 & 887.3 & 4 & 12 & 37 & 39 \\
\hline L69 & $\bar{G}$ & 626 & 599 & 889.2 & 5 & 17 & 27 & 29 \\
\hline $\mathrm{L70}$ & $\bar{M}$ & 832 & 455 & 882.5 & ND & $\mathrm{ND}$ & ND & ND \\
\hline L71 & $\mathrm{C}$ & 810 & 452 & 882.5 & ND & ND & ND & $\mathrm{ND}$ \\
\hline$\overline{\mathrm{L} 72}$ & $\mathrm{C}$ & 850 & 452 & 881.5 & ND & ND & ND & ND \\
\hline$\overline{L 73}$ & $\mathbf{M}$ & 862 & 460 & 882 & ND & ND & $\mathrm{ND}$ & $\mathrm{ND}$ \\
\hline L74 & C & 834 & 440 & 882.5 & ND & ND & $\mathrm{ND}$ & ND \\
\hline L75 & $\bar{M}$ & 813 & 475 & 884 & ND & ND & ND & ND \\
\hline$\overline{L 76}$ & $\bar{C}$ & 782 & 405 & 882 & ND & ND & 33.5 & 35.5 \\
\hline L77 & $\bar{C}$ & 788 & 467 & 884 & ND & ND & 31 & 33 \\
\hline L78 & $\overline{\mathbf{M}}$ & 859 & 440 & 882 & $\mathrm{ND}$ & ND & $\mathrm{ND}$ & ND \\
\hline L79 & C & 809 & 440 & 882.5 & ND & ND & 29.5 & 31.5 \\
\hline L80 & $\bar{M}$ & 781 & 415 & 882.4 & $\overline{N A}$ & $\mathrm{NA}$ & 33 & 35 \\
\hline L81 & $\mathrm{C}$ & 581 & 547 & 885.5 & $\mathrm{ND}$ & ND & 37.5 & 39.5 \\
\hline L83 & $\bar{C}$ & 634 & 521 & 885.2 & $\mathrm{ND}$ & ND & 37 & 50 \\
\hline L84 & $\mathbf{M}$ & 530 & 516 & 886 & NA & NA & 37 & 39 \\
\hline L85 & C & 489 & 510 & 886 & ND & ND & 37.5 & 39.5 \\
\hline $\mathrm{L} 86$ & $\bar{M}$ & 455 & 572 & 887.5 & $\mathrm{ND}$ & ND & 36 & 37 \\
\hline L87 & C & 649 & 547 & 886 & ND & ND & 36 & 38 \\
\hline $\mathrm{L} 88$ & $\bar{M}$ & 438 & 535 & 886.5 & ND & ND & 37 & 38 \\
\hline L89 & $\mathrm{C}$ & 693 & 457 & 883.3 & ND & ND & 34 & 37 \\
\hline L90 & $\bar{W}$ & 716 & 433 & 883.2 & ND & ND & ND & ND \\
\hline L91 & $\mathrm{C}$ & 718 & 537 & 884.9 & ND & ND & 24 & 26 \\
\hline L92 & $\overline{\mathrm{M}}$ & 436 & 448 & 885 & ND & ND & ND & ND \\
\hline L93 & $\mathrm{C}$ & 587 & 579 & 886.5 & ND & 13 & 34.5 & 36.5 \\
\hline L94 & $\bar{M}$ & 634 & 589 & 887.5 & ND & ND & 23 & 25 \\
\hline L95 & $\bar{C}$ & 771 & 515 & 884.2 & ND & 15 & 20 & 22 \\
\hline
\end{tabular}


TABLE 4.12 continued

\begin{tabular}{|l|c|c|c|c|c|c|c|c|}
\hline $\begin{array}{l}\text { Map } \\
\text { Location }\end{array}$ & Source & $\begin{array}{c}\mathbf{X} \\
(\mathbf{f t} .)\end{array}$ & $\begin{array}{c}\text { Y } \\
(\mathbf{f t .})\end{array}$ & Topo. & fill-ucu & ucu-gran & gran-lcu & lcu-bed \\
\hline L96 & M & 572 & 580 & 886.7 & ND & ND & 36 & 38 \\
\hline L97 & C & 744 & 484 & 884 & ND & 15 & 34 & 36 \\
\hline L98 & W & 668 & 448 & 883.2 & ND & ND & ND & ND \\
\hline L99 & C & 739 & 524 & 884.2 & ND & 14 & 23 & 25 \\
\hline L100 & M & 838 & 432 & 881.5 & ND & ND & ND & ND \\
\hline L101 & C & 750 & 439 & 883 & ND & 17 & 34 & 36 \\
\hline L102 & M & 804 & 449 & 883 & ND & ND & ND & ND \\
\hline L103 & C & ND & ND & - & - & - & - & - \\
\hline L104 & W & 743 & 417 & 882.5 & ND & ND & ND & ND \\
\hline L105 & C & 778 & 439 & 883.1 & ND & ND & 34.5 & 36.5 \\
\hline L106 & W & 877 & 455 & 881.6 & ND & ND & ND & ND \\
\hline L107 & C & 767 & 473 & 884 & ND & 15 & 33.5 & 35.5 \\
\hline L108 & M & 789 & 466 & 883.8 & ND & ND & 31 & 33 \\
\hline L109 & C & 732 & 453 & 883.5 & ND & 17 & 35.5 & 37.5 \\
\hline L111 & C & 621 & 579 & 886 & ND & 14 & 32.5 & 34.5 \\
\hline L112 & M & 683 & 461 & 883.6 & ND & ND & ND & ND \\
\hline L113 & C & 723 & 432 & 883.2 & ND & 18 & 33 & 35 \\
\hline L114 & M & 667 & 428 & 882.8 & ND & ND & ND & ND \\
\hline L115 & C & 441 & 439 & 884.8 & ND & 15 & 34 & 40 \\
\hline L116 & M & 764 & 491 & 884 & ND & ND & 28 & 32 \\
\hline L117 & ND & ND & ND & - & - & - & - & - \\
\hline L118 & M & 730 & 458 & 883.6 & ND & ND & 33 & 35 \\
\hline
\end{tabular}

Topo $=$ topographic elevation

ucu = upper cohesive unit

Icu $=$ lower cohesive unit

gran $=$ granular soil

bed $=$ bedrock

Sources:

$I=$ Phase 1

$\mathrm{G}=$ Geoprobe soil conductivity

$\mathrm{C}=\mathrm{CPT}$

$\mathrm{M}=\mathrm{McL}$ aren/Hart - soil sample

$\mathrm{W}=$ McLaren/Hart - groundwater sample 
TABLE 4.13

COMPARISON OF KRIGED LCU SURFACE ELEVATIONS WITH LCU SURFACE ELEVATIONS FROM BVWST BOREHOLE LOGS

\begin{tabular}{|l|c|c|c|c|c|c|c|c|}
\hline $\begin{array}{l}\text { BVWST } \\
\text { Boring } \\
\text { (ft.) }\end{array}$ & $\begin{array}{c}\mathbf{X} \\
(\mathbf{f t} .)\end{array}$ & $\begin{array}{c}\mathbf{Y} \\
(\mathbf{f t})\end{array}$ & $\begin{array}{c}\text { LCU Surface } \\
\text { Elevation } \\
\text { (ft. above msl) }\end{array}$ & $\begin{array}{c}\text { Prediction } \\
\text { Std. Dev. } \\
\text { (ft.) }\end{array}$ & $\begin{array}{c}\text { BVWST Surface } \\
\text { Elevation } \\
\text { (ft. a. ms) }\end{array}$ & $\begin{array}{c}\text { Krig-BVWST } \\
\text { Difference } \\
\text { (ft.) }\end{array}$ & $\begin{array}{c}\text { Z-score } \\
*\end{array}$ & Note \\
\hline B1 & 609.10 & 567.10 & 852.20 & 2.67 & 851.30 & 0.90 & 0.34 & - \\
\hline B2 & 655.40 & 557.10 & 853.20 & 2.38 & 846.60 & 6.60 & 2.78 & hole? \\
\hline B3 & 764.40 & 558.30 & 865.00 & 3.02 & 862.50 & 2.40 & 0.81 & - \\
\hline B4 & 624.50 & 522.10 & 848.10 & 1.90 & 847.90 & 0.20 & 0.11 & - \\
\hline B5 & 640.60 & 502.30 & 847.30 & 2.86 & 845.20 & 2.10 & 0.73 & 1 \\
\hline B6 & 705.60 & 460.10 & 849.60 & 2.48 & 848.90 & 0.80 & 0.30 & - \\
\hline B7 & 762.30 & 478.80 & 851.90 & 1.70 & 845.40 & 6.50 & 3.85 & hole? \\
\hline B8 & 675.10 & 424.50 & 847.60 & 2.08 & 846.80 & 0.80 & 0.40 & - \\
\hline B9 & 435.10 & 492.10 & 849.90 & 3.12 & - & - & - & - \\
\hline B10 & 439.30 & 479.10 & 849.80 & 3.10 & 850.60 & -0.80 & -0.26 & - \\
\hline B11 & 431.80 & 509.60 & 849.10 & 3.20 & - & - & - & - \\
\hline B12 & 405.50 & 494.40 & 852.10 & 3.38 & - & - & - & - \\
\hline B13 & 726.80 & 429.10 & 850.00 & 1.66 & 845.80 & 4.20 & 2.53 & hole? \\
\hline B14 & 745.50 & 401.50 & 848.50 & 3.08 & 849.50 & -1.00 & -0.32 & - \\
\hline B15 & 530.10 & 503.10 & 846.70 & 2.51 & 847.30 & -0.60 & -0.24 & - \\
\hline B16 & 853.00 & 485.70 & 858.60 & 2.88 & 858.40 & 0.30 & 0.09 & 2 \\
\hline MW1A & 464.70 & 660.00 & 855.90 & 4.18 & 869.20 & -13.30 & -3.18 & extrap, \\
\hline MW2B & 915.40 & 695.50 & 880.10 & 4.31 & 876.90 & 3.20 & 0.75 & extrap \\
\hline MW3A & 820.90 & 409.60 & 851.00 & 2.85 & 849.00 & 2.00 & 0.71 & - \\
\hline MW4 & 440.00 & 402.10 & 849.40 & 1.89 & 853.30 & -3.90 & -2.06 & - \\
\hline MW5 & 447.90 & 470.50 & 848.70 & 3.06 & - & - & - & - \\
\hline
\end{tabular}

1 depth to LCU not clear from BVWST log, $40,43 \mathrm{ft}$. bgs ( $40 \mathrm{ft}$. used here)

2 depth to bedrock assumed, LCU not encountered on BVWST log

3 depth to LCU not clear on BVWST, 20.5 to $22.5 \mathrm{ft}$. bgs ( $22.5 \mathrm{ft}$. used here)

extrap = extrapolation significantly outside of data bounds

* z-score $=($ Krig-BVWST $) /($ Prediction Std. Dev. $)$

Notes:

Kriging results based on median polish residuals fit to the CPT and Geoprobe Conductivity.

Geoprobe soil sampling data is compared to the BVWST data for LCU surface elevation.

This analysis provides an independent measure of the uncertainty in the model of the LCU

surface elevation and is useful for comparison of the Kriging results.

\section{Conclusions:}

a) The BVWST data suggest lower LCU surface elevations for 12 of the 17 comparisons. Neglecting the extrapolated data, this leaves 4 out of 15 BVWST greater than the predicted value, which is an unusual, but not highly unlikely event.

b) The z-scores should be approximately normally distributed with mean zero and a standard deviation of one if the modeling matches the Kriging prediction theory. With the exception of the extrapolated point MW1 and borings B2, B7, and B13 the z-scores are in good agreement with the prediction theory. This suggests that the prediction standard deviation plot is reasonably accurate. The outlying points B2, B7 and B13 indicate that either the BVWST data happened to hit depressions in the surface, or the predicted standard deviation is too small.

c) The big deviation at MW1A highlights the danger of extrapolation. 
TABLE 4.14 SOIL PAH CONTAMNATION COMPARISONS BY SELECTED SAMPLE LOCATION CLUSTERS.

\begin{tabular}{|c|c|c|c|c|}
\hline \multicolumn{5}{|c|}{ Cluster 1} \\
\hline Localion & L77 & $\mathrm{L64}$ & $\mathbf{L 5 9}$ & L108 \\
\hline$(\mathrm{X}, \mathrm{Y})(\mathrm{n})$. & 788,467 & 781,461 & 783,460 & 789,466 \\
\hline Source & CPT LIF (COUNTS) & $\mathrm{CPT}$ & Soil Cond. & McLaren/Hart \\
\hline \multicolumn{5}{|l|}{ Depth (n.) } \\
\hline 6 to 8 & 50 & 50 & conductivity low 5 to $7 \mathrm{ft}$. & odor, no visible product \\
\hline 19 to 20 & 10 & 50 & conductivity low 18 to $20 \mathrm{ft}$. & no odor \\
\hline 20 to 21 & 10 & 100 & obvious conduc. dip at $21 \mathrm{fl}$. & no odor \\
\hline 231024 & 10 & 100 & obvious conductivity dip & \\
\hline 261028 & 10 & 50 & no PAH signal & no odor \\
\hline 30 to 32 & 10 & 50 & no PAH signal & no odor \\
\hline
\end{tabular}

\begin{tabular}{|l|c|c|}
\hline \multicolumn{2}{|c|}{ Cluster 2 } \\
\hline Location & L81 & L66 \\
\hline$(\mathrm{X}, \mathrm{Y})(\mathrm{ft})$. & 581,547 & 584,548 \\
\hline Source & CPT LIF (counts) & Soil Cond. \\
\hline Depth $(\mathrm{\Omega})$. & & \\
\hline 6 to 21 & $>200$ & conduc. dips 14 to $19 \mathrm{\Omega}$. \\
\hline 7 to 9 & $>400$ & no PAl signal \\
\hline 11 to 18 & $>1000$ & conduc. dips 14 to $19 \mathrm{n}$. \\
\hline 35 to 36 & 100 & slight conduc. dip \\
\hline
\end{tabular}

\begin{tabular}{|l|c|c|}
\hline \multicolumn{3}{|c|}{ Cluster 3 } \\
\hline Location $\cdot$ & L33 & L73 \\
\hline$(\mathrm{X}, \mathrm{Y})(\mathrm{fl})$. & 862,460 & 862,460 \\
\hline Source & CPT LIF (counts) & McLaren/Hart \\
\hline Depth (n.) & & \\
\hline 4 to $(1$ & 10 & no odor incntioned \\
\hline 8 to 10 & 0 & no odor mentioned \\
\hline 13 to 15 & 0 & no odor \\
\hline
\end{tabular}

\begin{tabular}{|c|c|c|c|}
\hline \multicolumn{4}{|c|}{ Cluster 4} \\
\hline Location & L54 & L109 & L118 \\
\hline$(\mathrm{X}, \mathrm{Y})(\mathrm{n})$. & 737,461 & 732,453 & 730,458 \\
\hline Source & Soil Cond. & CPT LIF (counts) & McLaren/Hart \\
\hline \multicolumn{4}{|l|}{ Depth (nt.) } \\
\hline 5107 & conduc. is low & $>200$ & strong odor, product \\
\hline 7 to 14 & conduc. is high & 100 to 200 & \\
\hline 14 10 15 & no clcar signal & 10 & odor \\
\hline 151016 & no clcar signal & 50 & odor and product \\
\hline 16 to 20 & no clcar signal & 200 from 16 to $19 \mathrm{n}$. & \\
\hline 201022 & conduc. dip at $20.5 \Omega$. & 100 & \\
\hline 221024 & conduclivity dips & 10 & no odor \\
\hline 241030 & no PAH signal & 10 & no odor \\
\hline 301034 & no $\mathrm{P} \wedge \mathrm{H}$ signal & 10 & slight odor, product \\
\hline 34 to 36 & conduc. dip at $34.5 \mathrm{ht}$. & 400 & slight odor, product \\
\hline
\end{tabular}


TABLE 4.14 CONTINUED

\begin{tabular}{|c|c|c|c|c|}
\hline \multicolumn{5}{|c|}{ Cluster 5} \\
\hline Location & L45 & $\mathrm{L60}$ & $\mathrm{L114}$ & $\mathrm{B} 8$ \\
\hline$(\mathrm{X}, \mathrm{Y})(\mathrm{ft})$ & 665,428 & 679,436 & 667,428 & 675,424 \\
\hline Source & McLaren/Hart & CPT ILF (counts) & McLaren/Hart & BVWST \\
\hline \multicolumn{5}{|l|}{ Depth (ft.) } \\
\hline 6 to 7 & free product & 300 & & coal and wood fill? \\
\hline 7 to 8 & free product & 300 & & tarry odor \\
\hline 17 to 19 & free product & 100 & & tarry odor, free brown oil \\
\hline 20 to 22 & & 150 & & black free oil at $20 \mathrm{ft}$. \\
\hline 22 to 24 & & 10 & product & \\
\hline 24 to 26 & no contaminant mentioned & 10 & & \\
\hline 30 to 32 & free product & 50 ( 300 from 32 to $34 \mathrm{ft}$.) & & black free oil \\
\hline 35 to 36 & sheen and odor & 50 & & \\
\hline 36 to 37 & sheen and odor & 50 & & \\
\hline
\end{tabular}

\begin{tabular}{|l|c|c|}
\hline \multicolumn{3}{|c|}{ Cluster 6 } \\
\hline Location & L113 & B13 \\
\hline $\mathrm{X}, \mathrm{Y}$ )(ft.) & 723,432 & 727,429 \\
\hline Source & CPT LIF (counts) & BVWST \\
\hline Depth (ft.) & & \\
\hline 2 to 7.5 & 400 at 3 to $4 \mathrm{ft}$, & black tanty free oil \\
& $>400$ at 5 to $8 \mathrm{ft}$. & \\
\hline 7.5 to 31 & irregular trace, $<100$ & no contaminant mentioned \\
\hline 31 to 33 & 100 to 200 & tarry oil, oil sheen \\
\hline
\end{tabular}

\begin{tabular}{|l|c|c|}
\hline \multicolumn{3}{|c|}{ Cluster 7 } \\
\hline Location & LA0 & L105 \\
\hline $\mathrm{X}, \mathrm{Y})(\mathrm{ft})$. & 780,435 & 778,439 \\
\hline Source & McLaren/Hart & CPT LIF (counts) \\
\hline Depth (ft.) & \multicolumn{2}{|c|}{} \\
\hline 6 to 8 & black, strong odor & $\begin{array}{l}\text { >200 at } 5 \text { to } 6 \mathrm{ft} \\
\text { otherwise about } 10\end{array}$ \\
\hline 16 to 18 & black, mild odor & 10 \\
\hline 20 to 22 & no odor & 10 \\
\hline 26 to 28 & slight odor & 10 \\
\hline 31 to 33 & free product & $>400$ at 32 to 34 \\
\hline 33 to 35 & odor, no product & Lif stops at $34 \mathrm{ft}$. \\
\hline
\end{tabular}

\begin{tabular}{|l|c|c|}
\hline \multicolumn{3}{|c|}{ Cluster 8 } \\
\hline Location & L78 & L63 \\
\hline (X,Y)(ft.) & 859,440 & 858,439 \\
\hline Source & McLaren/Hart & Soil Cond. \\
\hline Depth (ft.) & & \\
\hline 4 to 6 & brown to black, no odor & unclear signal \\
\hline 9 to 11 & no odor & no PAH signal \\
\hline 13 to 15 & no odor & no PAH signal \\
\hline 18 to 20 & no odor & slight conduc. dip \\
\hline 25 to 27 & no odor & no PAH signal \\
\hline
\end{tabular}


TABLE 4.14 CONTINUED

\begin{tabular}{|l|c|c|}
\hline \multicolumn{3}{|c|}{ Cluster 9 } \\
\hline Location & L65 & L56 \\
\hline $\mathrm{X}, \mathrm{Y}$ (ft.) & $\mathbf{7 8 0 , 5 0 7}$ & 780,507 \\
\hline Source & McLaren/Hart & Soil Cond. \\
\hline Depth $(\mathrm{ft})$. & & \\
\hline 4 to 6 & no odor & no PAH signal \\
\hline 12 to 14 & $\begin{array}{c}\text { possible product in } \\
\text { isolated zone }\end{array}$ & $\begin{array}{c}\text { no clear signal, possible } \\
\text { conductivity dip }\end{array}$ \\
\hline 18 to 20 & no contaminant mentioned & no clear signal \\
\hline 24 to 26 & no contaminant mentioned & no clear signal \\
\hline
\end{tabular}

\begin{tabular}{|l|c|c|}
\hline \multicolumn{3}{|c|}{ Cluster 10 } \\
\hline Location & L67 & L58 \\
\hline $\mathrm{X}, \mathrm{Y}$ )(ft.) & 775,533 & 775,533 \\
\hline Source & McLaren/Hart & Soil Cond. \\
\hline Depth (ft.) & & \\
\hline 4 to 6 & no odor & no PAH signal \\
\hline 11 to 13 & slight odor? & no PAH signal \\
\hline 16 to 18 & no contaminant mentioned & no PAH signal \\
\hline 21 to 23 & no contaminant mentioned & no PAH signal \\
\hline
\end{tabular}

\begin{tabular}{|c|c|c|}
\hline \multicolumn{3}{|c|}{ Cluster 11} \\
\hline Location & $\overline{L 75}$ & L37 \\
\hline$(\mathrm{X}, \mathrm{Y})(\mathrm{ft})$. & 813,475 & 812,476 \\
\hline Source & McLaren/Hart & Soil Cond. \\
\hline \multicolumn{3}{|l|}{ Depth (ft.) } \\
\hline 5 to 7 & black crumbly soil & no PAH signal \\
\hline 10 to 12 & no contaminant mentioned & no PAH signal \\
\hline 14 to 16 & no odor & no PAH signal \\
\hline 19 to 21 & no odor & no PAH signal \\
\hline 23 to 25 & no odor & no PAH signal \\
\hline
\end{tabular}

\begin{tabular}{|l|c|c|}
\hline \multicolumn{3}{|c|}{ Cluster 12 } \\
\hline Location & LA1 & L68 \\
\hline $\bar{X}, \mathrm{Y}$ )(ft.) & 450,554 & 451,558 \\
\hline Source & CPT LIF (counts ) & Soil Cond. \\
\hline Depth (ft.) & \multicolumn{2}{|c|}{} \\
\hline 12 to 21 & 200 from 12 to 14 & several conductivity dips \\
& zero from 14 to 17 & from 13 to 21 \\
& CPT LIF ends at 17 & - \\
\hline
\end{tabular}


TABLE 4.15 MCLAREN HART SOIL SAMPLES -OBSERVED LEVEL OF PAH CONTAMINATION BY STRATIGRAPHIC ZONE

\begin{tabular}{|l|c|c|c|c|c|c|c|c|}
\hline $\begin{array}{l}\text { Map } \\
\text { Location }\end{array}$ & $\begin{array}{c}\text { X } \\
(\mathbf{f t .})\end{array}$ & $\begin{array}{c}\mathbf{Y} \\
(\mathbf{f t} .)\end{array}$ & Zone 1 & Zone 2 & Zone 3 & Zone 4 & Zone 5 & Zone 6 \\
\hline & & & & & & & & \\
\hline ML36 & 845 & 507 & 0 & 0 & NA & 0 & 0 & NA \\
\hline ML38 & 806 & 519 & NA & 0 & NA & NA & 0 & NA \\
\hline ML39 & 792 & 481 & 1 & 2 & 0 & NA & 0 & 0 \\
\hline ML40 & 780 & 435 & 6 & NA & 1 & 1 & 5 & 2 \\
\hline ML42 & 792 & 553 & 1 & NA & 0 & NA & 0 & 0 \\
\hline ML43 & 751 & 522 & 2 & 2 & 1 & NA & 0 & 0 \\
\hline ML45 & 665 & 428 & 6 & NA & 4 & 5 & 4 & NA \\
\hline ML57 & 566 & 544 & 5 & 6 & 6 & 2 & 6 & 6 \\
\hline ML65 & 780 & 507 & 0 & 2 & NA & NA & 0 & 0 \\
\hline ML67 & 775 & 533 & 0 & 1 & NA & 0 & NA & 0 \\
\hline ML70 & 832 & 455 & 0 & 0 & 0 & 0 & 0 & NA \\
\hline ML75 & 813 & 475 & 0 & 0 & 0 & 0 & 0 & NA \\
\hline ML78 & 859 & 440 & 0 & 0 & 0 & NA & 0 & 0 \\
\hline ML80 & 781 & 415 & 1 & 0 & 6 & 1 & 6 & 6 \\
\hline ML84 & 530 & 516 & 0 & NA & 1 & 0 & 1 & NA \\
\hline ML86 & 455 & 572 & 1 & 6 & NA & 1 & 0 & NA \\
\hline ML88 & 438 & 535 & 2 & 5 & NA & 2 & 0 & NA \\
\hline ML92 & 436 & 448 & 0 & NA & 5 & 0 & 0 & NA \\
\hline ML94 & 634 & 589 & 0 & 2 & 2 & 6 & 6 & NA \\
\hline ML96 & 572 & 580 & 0 & 6 & 6 & 6 & 0 & 0 \\
\hline ML100 & 838 & 432 & 0 & 0 & NA & NA & NA & NA \\
\hline ML102 & 804 & 449 & 0 & NA & NA & 0 & NA & NA \\
\hline ML108 & 789 & 466 & 2 & NA & NA & 0 & 0 & NA \\
\hline ML110 & 817 & 426 & 1 & 1 & 1 & 0 & 0 & NA \\
\hline ML112 & 683 & 461 & 4 & NA & 4 & 0 & 6 & NA \\
\hline ML114 & 667 & 428 & NA & NA & NA & 6 & NA & NA \\
\hline ML116 & 764 & 491 & 1 & NA & 5 & 0 & NA & 0 \\
\hline ML118 & 730 & 458 & 4 & 2 & 5 & 0 & 6 & 5 \\
\hline
\end{tabular}

Note:

Observed Level Of PAH Contamination Designation

Free Product 6

Product Sheen 5

Discoloration 4

Strong Odor 3

Mild Odor 2

Slight Odor 1

No Odor 0 
TABLE 4.16 MCLAREN/HART SOIL SAMPLE ATTRIBUTES

\begin{tabular}{|c|c|c|c|c|c|c|c|}
\hline $\begin{array}{l}\text { Map } \\
\text { Location }\end{array}$ & $\begin{array}{c}\text { Depth } \\
\text { (ft.) }\end{array}$ & $\begin{array}{l}\text { Soil } \\
\text { Type }\end{array}$ & Zone & $\begin{array}{l}\text { Extraction } \\
\text { Methods }\end{array}$ & $\begin{array}{l}\text { Recovery } \\
\text { (feet) }\end{array}$ & Odor & Detects \\
\hline 36 & 5 & c & 1 & $a, b, c, d, e$ & full & $y$ & 0 \\
\hline 36 & 12 & c & 1 & $a, b, c, d, e$ & 1.3 & $\mathrm{y}$ & $\overline{0}$ \\
\hline 36 & 18 & $\bar{c}$ & 6 & $a, b, c, d, e$ & 1.7 & $y$ & 0 \\
\hline 36 & 23 & $\mathrm{c} / \mathrm{s}$ & 7 & $a, b, c, d, e$ & full & $\bar{y}$ & 0 \\
\hline 38 & 12.5 & c & 2 & $a, b, c, d, e$ & full & $\mathrm{y}$ & 0 \\
\hline 38 & 23 & $\mathrm{r} / \mathrm{s}$ & 5 & $a, b ; c, d, e$ & 1.5 & $\bar{y}$ & 0 \\
\hline 39 & 9 & c & 1 & $a, b, c, d, e$ & full & $15 \mathrm{ppm}$ & 1 \\
\hline 39 & 13 & c & 2 & a,b,c,d,e & 1.5 & $\mathrm{y}$ & 2 \\
\hline 39 & 17 & $\mathbf{s}$ & 3 & $a, b, c, d, e$ & 1.75 & $y$ & 0 \\
\hline 39 & 22 & $\mathbf{r}$ & 5 & $a, b, c, d, e$ & 1.75 & $\mathrm{y}$ & 0 \\
\hline 40 & 7.7 & c & 1 & $a, b, c, d, e$ & full & 9ppm & 2 \\
\hline 40 & 17 & c & 2 & $\mathrm{a}, \mathrm{b}, \mathrm{c}, \mathrm{d}, \mathrm{e}$ & 1.5 & $\bar{y}$ & 0 \\
\hline 40 & 21 & $\mathbf{s}$ & 3 & a,b,c,d,e & 1.7 & $y$ & 0 \\
\hline 40 & 27 & $s$ & 4 & $a, b, c, d, e$ & & 30ppm & 1 \\
\hline 40 & 34.5 & $\mathbf{s}$ & 5 & $a, b, c, d, e$ & 1.5 & 200ppm & 5 \\
\hline 43 & 5 & c & 1 & $\mathrm{a}, \mathrm{b}, \mathrm{c}, \mathrm{d}, \mathrm{e}$ & 1.6 & $y$ & 2 \\
\hline 43 & 16 & $s$ & 3 & $\mathrm{a}, \quad \mathrm{d}, \mathrm{e}$ & 1.4 & $\bar{y}$ & 1 \\
\hline 43 & 25 & gravel & 7 & a, $\quad \mathrm{d}, \mathrm{e}$ & 0.9 & $y$ & 0 \\
\hline 42 & 4 & silt & 1 & $a, c, d, e$ & 1.8 & $y$ & 1 \\
\hline 42 & 14 & $\mathbf{s}$ & 3 & $a, \quad c, d, e$ & 1.3 & $y$ & 0 \\
\hline 42 & 19 & $\mathrm{c}, \mathrm{s}$ & 5 & $\mathrm{a}$ & 1.2 & $y$ & 0 \\
\hline 45 & 18.2 & $\mathbf{s}$ & 3 & $a, \quad c, d, e$ & 1.6 & $y$ & 0 \\
\hline 45 & 25 & s & 4 & $a, b, c, d, e$ & 1.6 & $10 \mathrm{ppm}$ & 0 \\
\hline 45 & 35.5 & $\mathrm{~s}$ & 5 & $\mathrm{a}$ & 1.8 & 20ppm & 4 \\
\hline 45 & 36.5 & s & 5 & a, c,d,e & 1.8 & $2 \mathrm{ppm}$ & 4 \\
\hline 57 & 19 & $\mathbf{s}$ & 4 & a, $\quad \mathrm{d}, \mathrm{e}$ & 1.55 & $y$ & 2 \\
\hline 57 & 25 & $s$ & 4 & a, $\quad$ d,e & 1.65 & $y$ & 1 \\
\hline 57 & 34.5 & s & & a, $\quad$ d,e & 1.8 & $y$ & 0 \\
\hline 57 & 35.7 & c & 6 & a, $\quad$ d,e & 1.8 & $y$ & @34.2=60 ppm \\
\hline 65 & 5 & c & 1 & a, d,e & 1.6 & $y$ & 0 \\
\hline 65 & 13 & c & 2 & $a, b, d, e$ & 1 & $3 \mathrm{ppm}$ & 4 \\
\hline 65 & 18.5 & $\mathrm{~s}$ & 5 & $\mathrm{~d}, \mathrm{e}$ & 1.6 & $y$ & 0 \\
\hline 65 & 19.5 & c & 6 & a, d,e & 1.6 & $\mathrm{y}$ & 0 \\
\hline 65 & 25 & si,c & 7 & a, $\quad \mathrm{d}, \mathrm{e}$ & 1.6 & $y$ & 0 \\
\hline 67 & 5 & c & 1 & a, d,e & & $\mathrm{y}$ & 0 \\
\hline 67 & 12 & c & 2 & $a, b, c, d, e$ & 1 & 30ppm & 1 \\
\hline 67 & 17 & $s$ & 4 & $\mathrm{a}, \quad \mathrm{d}, \mathrm{e}$ & 1.6 & $y$ & 0 \\
\hline 67 & 22 & gravel & 7 & a, d,e & 1.5 & $y$ & 0 \\
\hline 70 & 5 & c & 1 & a, $\quad$ d,e & 1.9 & $y$ & 0 \\
\hline 70 & 11 & c & 1 & $\mathrm{a}$ & 1.4 & $y$ & 0 \\
\hline 70 & 14 & c & 2 & $\mathrm{a}$ & 1.95 & $\mathrm{y}$ & 0 \\
\hline 70 & 19 & $\mathbf{s}$ & 3 & $a$ & 1.7 & $\mathrm{y}$ & 0 \\
\hline 70 & 30.5 & $\mathrm{~s}$, gravel & 5 & $a$ & 1.1 & $y$ & 0 \\
\hline
\end{tabular}


TABLE 4.16 continued

\begin{tabular}{|c|c|c|c|c|c|c|c|}
\hline $\begin{array}{l}\text { Map } \\
\text { Location }\end{array}$ & $\begin{array}{c}\text { Depth } \\
\text { (ft.) }\end{array}$ & $\begin{array}{l}\text { Soil } \\
\text { Type }\end{array}$ & Zone & $\begin{array}{l}\text { Extraction } \\
\text { Methods }\end{array}$ & $\begin{array}{c}\text { Recovery } \\
\text { (feet) }\end{array}$ & Odor & Detects \\
\hline 73 & 5 & c & 1 & $\mathrm{a}, \mathrm{c}$ & 1.7 & $\bar{y}$ & $\overline{0}$ \\
\hline 73 & 9 & c & 1 & $\overline{c, d}$ & 1 & $y$ & 0 \\
\hline 73 & 14 & $\mathrm{~s} 1, \mathrm{s3}$ & & $a, b, c, d, e$ & 1.6 & $y$ & 0 \\
\hline 75 & 6 & c & 1 & $c, d$ & 2 & $\mathrm{y}$ & 0 \\
\hline 75 & 11 & c & 1 & $\overline{c, d}$ & 1.3 & $y$ & 0 \\
\hline 75 & 14.5 & c & 2 & $c, d$ & 1.8 & $\mathrm{y}$ & 0 \\
\hline 75 & 15.5 & $\mathbf{s}$ & 3 & $\mathrm{c,d}$ & 1.8 & $y$ & 0 \\
\hline 75 & 20 & $\mathrm{~s} 1, \mathrm{r}$ & 4 & $\overline{c, d}$ & 1.2 & $y$ & 0 \\
\hline 75 & 23.5 & $\mathrm{sl, \textrm {r }}$ & 6 & c, $\mathrm{e}$ & 1.6 & $y$ & 0 \\
\hline 75 & 24.5 & c & 6 & c, e & 1.6 & $y$ & 0 \\
\hline 78 & 5 & c & 1 & $\bar{e}$ & 1.85 & $y$ & 0 \\
\hline 78 & 10 & c & 1 & $\mathrm{e}$ & 1.1 & $y$ & 0 \\
\hline 78 & 14 & c & 2 & $\overline{\mathrm{e}}$ & 1.41 & $y$ & 0 \\
\hline 78 & 19.5 & s & 3 & $a$ & $\overline{1.6}$ & $y$ & 0 \\
\hline 78 & 20 & c & 4 & d & 1.6 & $y$ & 0 \\
\hline 78 & 26 & $\overline{c, s}$ & 5 & e & 1.2 & $y$ & 0 \\
\hline 80 & 6 & c & 1 & a, d,e & 1.85 & $10 \mathrm{ppm}$ & 1 \\
\hline 80 & 9 & c & 1 & $\mathrm{~d}$ & 1.9 & $y$ & 1 \\
\hline 80 & 14 & c & 2 & $a, b, d, e$ & 1.5 & $y$ & 0 \\
\hline 80 & 19 & $\mathbf{s}$ & 3 & $a, b, c, d, e$ & $\overline{1.6}$ & 70ppm & 6 \\
\hline 80 & 29 & $s$ & 4 & e & 1.85 & $\mathrm{y}$ & 1 \\
\hline 80 & 33.5 & c & 6 & $a, b, c, d$ & 1.7 & 70ppm & 2 \\
\hline 80 & 36 & c & $\overline{6}$ & $a, b, c, d, e$ & 2 & $30 \mathrm{ppm}$ & 5 \\
\hline 84 & 6 & c & 1 & $\mathrm{a}$ & 1.9 & $\mathrm{y}$ & 0 \\
\hline 84 & 13.5 & s & 3 & $\mathrm{e}$ & 1.55 & $y$ & 1 \\
\hline 84 & 25 & $\mathrm{~s}$ & 4 & $a$ & 2 & $y$ & 0 \\
\hline 84 & 36 & $\mathrm{~s}$ & 5 & $\mathrm{~d}$ & 1.85 & $y$ & 1 \\
\hline 86 & 5 & $\bar{c}$ & 1 & e & 1.9 & $y$ & 1 \\
\hline 86 & 10.5 & c & 2 & $a, b$ & 1.75 & $y$ & 1 \\
\hline 86 & 20 & $s$ & 4 & $\mathrm{~d}$ & 1.4 & $y$ & 1 \\
\hline 86 & 35.5 & $s$ & 5 & e & 1.4 & $y$ & 0 \\
\hline 86 & 36.5 & $\mathrm{c}$ & 6 & $a$ & 1.4 & $y$ & 0 \\
\hline 88 & 5 & $\mathrm{c}$ & 1 & $\mathrm{~d}$ & 1.9 & $y$ & 2 \\
\hline 88 & 11 & c & 2 & $a, b, c, d, e$ & 1.65 & $10 \mathrm{ppm}$ & 2 \\
\hline 88 & 20 & $s$ & 4 & $\bar{e}$ & 1.65 & $y$ & 2 \\
\hline 88 & 36 & $s$ & 5 & $a$ & 1.3 & $y$ & 0 \\
\hline 92 & 8 & c & 1 & $\mathrm{~d}, \mathrm{e}$ & 1.9 & $y$ & 0 \\
\hline 92 & 17 & $c, s$ & 3 & c, e & 1.6 & $\mathrm{y}$ & 3 \\
\hline 92 & 25 & $s$ & 4 & $\mathrm{a}, \quad \mathrm{d}, \mathrm{e}$ & 2 & $10 \mathrm{ppm}$ & 0 \\
\hline 92 & 33 & s & 5 & $a$ & 1.4 & $y$ & 0 \\
\hline 94 & 6 & c & 1 & $\mathrm{~d}$ & 1.5 & $\mathrm{y}$ & 0 \\
\hline 94 & 13 & $\mathrm{c,s}$ & 2 & $a, b, c, d, e$ & 1.8 & $300 \mathrm{ppm}$ & 3 \\
\hline 94 & 20 & $\mathrm{~s}$ & 3 & $\mathrm{a}, \mathrm{b}, \mathrm{c}, \mathrm{d}, \mathrm{e}$ & 1.7 & $40 \mathrm{ppm}$ & 5 \\
\hline
\end{tabular}


TABLE 4.16 continued

\begin{tabular}{|c|c|c|c|c|c|c|c|}
\hline $\begin{array}{l}\text { Map } \\
\text { Location }\end{array}$ & $\begin{array}{c}\text { Depth } \\
\text { (ft.) }\end{array}$ & $\begin{array}{l}\text { Soil } \\
\text { Type }\end{array}$ & Zone & $\begin{array}{c}\text { Extraction } \\
\text { Methods }\end{array}$ & $\begin{array}{l}\text { Recovery } \\
\text { (feet) }\end{array}$ & Odor & Detects \\
\hline 94 & 26 & $\mathrm{~s}$ & 5 & $a, b, c, d, e$ & 1.4 & 90ppm & 6 \\
\hline 96 & 13.5 & $\mathbf{s}$ & 3 & a, d,e & 1.4 & $5 \mathrm{ppm}$ & 3 \\
\hline 96 & 24.5 & $\mathrm{~s}$ & 4 & e & 1.55 & $y$ & 0 \\
\hline 96 & 30 & $s$ & 4 & $\mathrm{a}$ & 1.5 & $\mathrm{y}$ & 0 \\
\hline 96 & 34.5 & $\mathrm{~s}$ & 5 & $\overline{\mathrm{e}}$ & 1.8 & $y$ & 0 \\
\hline 96 & 35.5 & c & 6 & $\mathrm{~d}$ & 1.8 & $y$ & 0 \\
\hline 100 & 9 & $\bar{c}$ & 1 & $a$ & 1.9 & $\mathrm{y}$ & 0 \\
\hline 100 & 15 & $\bar{c}$ & 2 & $\mathrm{~d}$ & 1.3 & $4 \mathrm{ppm}$ & 0 \\
\hline 102 & 8 & c & 1 & $\bar{e}$ & 1.85 & $y$ & 0 \\
\hline 102 & 16 & $\mathbf{s}$ & 4 & $a$ & 1.7 & $y$ & 0 \\
\hline 108 & 7 & c & 1 & $a, b, d, e$ & 1.45 & $\mathrm{y}$ & 2 \\
\hline 108 & 20 & s & 3 & $\bar{d}$ & 1.2 & $y$ & 0 \\
\hline 108 & 27 & $\mathrm{~s}$ & 5 & $\mathrm{a}, \mathrm{b}, \mathrm{d}, \mathrm{e}$ & 2 & $y$ & 0 \\
\hline 108 & 31 & $\mathrm{s,c}$ & 6 & $\mathrm{e}$ & 1.8 & $y$ & $\overline{0}$ \\
\hline 110 & 9 & c & 1 & $a$ & 1.6 & $y$ & 1 \\
\hline 110 & 16 & $\overline{c, s}$ & 3 & $\bar{d}$ & 1.35 & $y$ & 1 \\
\hline 110 & 19 & $s$ & 3 & e & 1.6 & $y$ & 0 \\
\hline 110 & 23 & $\mathrm{~s}$ & 4 & a & 0.9 & $y$ & 0 \\
\hline 112 & 7 & c & 1 & $\mathrm{a}, \mathrm{b}, \mathrm{d}, \mathrm{e}$ & 1.9 & 140ppm & 3 \\
\hline 112 & 16.5 & $s$ & 3 & $a, b, d, e$ & 1.6 & $1700 \mathrm{ppm}$ & 6 \\
\hline 112 & 17.5 & $s$ & 3 & a, & 1.6 & 70ppm & 2 \\
\hline 112 & 26 & $s$ & 4 & $\mathrm{~d}$ & 2 & 30ppm & 0 \\
\hline 116 & 10.5 & c & 1 & $a, b, d, e$ & 1.8 & $60 \mathrm{ppm}$ & 3 \\
\hline 116 & 11.5 & c & 1 & a, $\quad$ d,e & 1.8 & $10 \mathrm{ppm}$ & 1 \\
\hline 116 & 16 & $s$ & 3 & $\mathrm{a}$, & 1.6 & $2 \mathrm{ppm}$ & 1 \\
\hline 116 & 21 & $s$ & 4 & $\mathrm{~d}$ & 1.65 & $2 \mathrm{ppm}$ & 0 \\
\hline 116 & 25 & s & 4 & $a, b, d, e$ & 1.8 & $2 \mathrm{ppm}$ & 0 \\
\hline 116 & 31 & $\mathrm{c}$ & 6 & $\mathrm{a}, \quad \mathrm{d}, \mathrm{e}$ & 1.2 & 9ppm & 0 \\
\hline 118 & 6.1 & c & 1 & $a, b, d, e$ & 1.6 & 600ppm & 3 \\
\hline 118 & 15 & $\mathbf{s}$ & 2 & $a, b, d$ & 1.4 & 500ppm & 5 \\
\hline 118 & 23 & $\mathrm{~s}$ & 4 & $\mathrm{~d}$ & 1.55 & 3ppm & 0 \\
\hline 118 & 25 & $s$ & 4 & $? ? ?$ & 1.8 & $\mathrm{y}$ & 0 \\
\hline 118 & 27 & $s$ & 4 & $a$ & 1.9 & $y$ & 0 \\
\hline 118 & 29 & $s$ & 4 & $\mathrm{~d}$ & 1.9 & $3 \mathrm{ppm}$ & 0 \\
\hline 118 & 31 & s & 5 & $a, b, d, e$ & 1.2 & 50ppm & 5 \\
\hline 118 & 34.5 & c & 6 & $a, b, d, e$ & 1.7 & $35 \mathrm{ppm}$ & 5 \\
\hline 118 & 35.5 & c & 6 & $a, b, \quad d, e$ & 1.7 & $35 \mathrm{ppm}$ & 4 \\
\hline
\end{tabular}


TABLE 4.17 MCLAREN/HART SAMPLES USED FOR EXTRACTION METHOD COMPARISONS.

\begin{tabular}{|c|c|c|c|c|c|c|}
\hline $\begin{array}{c}\text { Map } \\
\text { Location }\end{array}$ & $\begin{array}{c}\text { McLaren/Hart } \\
\text { Sample II }\end{array}$ & $\begin{array}{c}\mathbf{X} \\
\text { (ft.) }\end{array}$ & $\begin{array}{c}\mathbf{Y} \\
\text { (ft.) }\end{array}$ & $\begin{array}{r}\text { Sample D } \\
\text { from }\end{array}$ & $\begin{array}{c}\left.g s^{* *}\right) \\
\text { to }\end{array}$ & Soil Type \\
\hline ML39 & MT-2-G9 & 792 & 481 & 12 & 14 & clayey \\
\hline MLA0 & MT-2-G17.9 & 780 & 435 & 34 & 35 & sandy \\
\hline ML43 & MT-2-G18 & 751 & 522 & 4 & $6^{\circ}$ & clayey \\
\hline ML67* & MT-2-G40 & 775 & 533 & 11 & 13 & clayey \\
\hline ML80 & MT-2-G65 & 781 & 415 & 18 & 20 & sandy \\
\hline ML88 & MT-2-G78 & 438 & 535 & 10 & 12 & clayey*** \\
\hline ML94 & MT-2-G86 & 634 & 589 & 12 & 14 & clayey*** \\
\hline ML94 & MT-2-G87 & 634 & 589 & 19 & 21 & sandy \\
\hline ML94 & MT-2-G88 & 634 & 589 & 25 & 27 & $\operatorname{sandy} * * *$ \\
\hline ML112 & MT-2-G106 & 683 & 461 & 6 & 8 & clayey \\
\hline ML112 & MT-2-G107.1 & 683 & 461 & 16 & 17.2 & sandy \\
\hline ML116* & MT-2-G111.1 & 764 & 491 & 10 & 11 & clayey \\
\hline ML118 & MT-2-G116.2 & 730 & 458 & 5.5 & 606 & clayey \\
\hline ML118 & MT-2-G117.1 & 730 & 458 & 14 & 15.4 & clayey \\
\hline ML118 & MT-2-G122.1 & 730 & 458 & 30 & 31.2 & sandy \\
\hline
\end{tabular}

* sample not included in the September 1994 report.

** ft. bgs $=$ feet below ground surface.

*** soil type classification changed from the September 1994 report: from "silty clay" (MT-2-G78) and

"clayey sand" (MT-2-G86, predominantly clayey according to the McLaren/Hart logbook) to "clayey" and from

"silty sand" (MT-2-G88) to "sandy." 
TABLE 4.18 MCLAREN/HART SOIL SAMPLE ANALYSIS- AVERAGE PERCENT RECOVERY BY EXTRACTION METHOD AND ANALYTE.

\begin{tabular}{|c|c|c|c|c|c|c|}
\hline \multirow[t]{2}{*}{ Analyte } & \multirow[t]{2}{*}{$\begin{array}{l}\text { Analyte } \\
\text { Code }\end{array}$} & \multicolumn{5}{|c|}{$\begin{array}{l}\text { Average Percent Recovery } \\
\text { by Extraction Method }\end{array}$} \\
\hline & & $\begin{array}{c}\mathbf{A} \\
\text { Sonication }\end{array}$ & $\begin{array}{c}\text { B } \\
\text { Microscale }\end{array}$ & $\begin{array}{c}\text { C } \\
\text { Microwave }\end{array}$ & $\begin{array}{c}\mathbf{D}^{\star} \\
\text { Thermal }\end{array}$ & $\begin{array}{l}\mathbf{E}^{* *} \\
\text { SFE }\end{array}$ \\
\hline Naphthalene & $\mathbf{N}$ & 89 & 120 & 122 & 100 & 85 \\
\hline Acenaphthylene & $\mathrm{AL}$ & 93 & 120 & 120 & 100 & 86 \\
\hline Acenaphthene & $\overline{\mathrm{AT}}$ & 92 & 118 & 120 & 100 & 86 \\
\hline Fluorene & $\bar{F}$ & 99 & 120 & 122 & 100 & 86 \\
\hline Phenanthrene & $\mathrm{PH}$ & 100 & 145 & 124 & 100 & 88 \\
\hline Anthracene & $\bar{A}$ & 97 & 142 & 130 & 100 & 90 \\
\hline Fluoranthene & $\overline{\text { FT }}$ & 102 & 141 & 126 & 100 & 88 \\
\hline Pyrene & PY & 101 & 150 & 110 & 100 & 86 \\
\hline Benzo(a)anthracene & BA & 98 & 131 & 115 & 100 & 86 \\
\hline Chrysene & $\mathrm{C}$ & 97 & 134 & 115 & 100 & 87 \\
\hline Benzo(b)fluoranthene & $\mathrm{BF}$ & 105 & 101 & 121 & 100 & 97 \\
\hline Benzo(k)fluoranthene & $\overline{K F}$ & 105 & 107 & 119 & 100 & 84 \\
\hline Benzo(a)pyrene & $\overline{B P}$ & 103 & 99 & 118 & 100 & 89 \\
\hline Indeno( $(1,2,3, \mathrm{~cd})$ pyrene & IP & 94 & 108 & 129 & 100 & 85 \\
\hline Dibenzo( $(\mathrm{a}, \mathrm{h})$ anthracene & $\overline{\mathrm{AA}}$ & 91 & 96 & 124 & 100 & 89 \\
\hline Benzo(g,h,i)perylene & GP & 81 & 87 & 126 & 100 & 91 \\
\hline
\end{tabular}

* No percent recovery data available; $100 \%$ was assumed, i.e., no data adjustment was made.

** Supercritical fluid extraction, SFE 


\section{TABLE 4.19 SUMMARY STATISTICS OVER SOIL SAMPLES WITHIN ANALYTE/METHOD SUBGROUPS}

\begin{tabular}{|c|c|c|c|c|c|c|c|}
\hline \multirow[t]{2}{*}{ Analyte } & \multirow{2}{*}{$\begin{array}{l}\text { Analyte } \\
\text { Molecular } \\
\text { Weight }\end{array}$} & \multirow[t]{2}{*}{ Statistic ${ }^{\star}$} & \multicolumn{5}{|c|}{ Method } \\
\hline & & & $\begin{array}{c}\text { A } \\
\text { Sonication }\end{array}$ & \begin{tabular}{|c|}
$\mathbf{B}$ \\
Microscale
\end{tabular} & \begin{tabular}{|c|} 
C \\
Microwave
\end{tabular} & $\begin{array}{c}\text { D } \\
\text { Thermal }\end{array}$ & $\begin{array}{c}\mathbf{E} \\
\text { SFE }\end{array}$ \\
\hline \multirow[t]{3}{*}{ Naphthalene } & \multirow[t]{3}{*}{128} & Mean & 0.89 & 1.34 & 1.47 & 0.31 & 1.03 \\
\hline & & s & 0.41 & 0.53 & 0.48 & 0.24 & 0.4 \\
\hline & & n & 10 & 12 & 6 & 9 & 11 \\
\hline \multirow[t]{3}{*}{ Acenaphthylene } & \multirow[t]{3}{*}{152} & Mean & 0.9 & 1.16 & 1.39 & 0.45 & 1.15 \\
\hline & & s & 0.36 & 0.39 & 0.61 & 0.22 & 0.34 \\
\hline & & $\mathbf{n}$ & 9 & 13 & 7 & 10 & 11 \\
\hline \multirow[t]{3}{*}{ Acenaphthene } & \multirow[t]{3}{*}{154} & Mean & 0.82 & 0.99 & 1.47 & 0.68 & 1.05 \\
\hline & & $\mathbf{s}$ & 0.32 & 0.33 & 0.26 & 0.25 & 0.34 \\
\hline & & n & 7 & 12 & 6 & 6 & 11 \\
\hline \multirow[t]{3}{*}{ Fluorene } & \multirow[t]{3}{*}{166} & Mean & 0.77 & 1.33 & 1.28 & 0.48 & 1.04 \\
\hline & & $\mathbf{s}$ & 0.3 & 0.47 & 0.46 & 0.29 & 0.43 \\
\hline & & n & 12 & 14 & 8 & 9 & 12 \\
\hline \multirow[t]{3}{*}{ Phenanthrene } & \multirow[t]{3}{*}{178} & Mean & 0.78 & 1.04 & 1.42 & $\cdot 0.94$ & 0.97 \\
\hline & & $\mathbf{s}$ & 0.27 & 0.51 & 0.74 & 0.54 & 0.37 \\
\hline & & n & 14 & 14 & 9 & 14 & 13 \\
\hline \multirow[t]{3}{*}{ Anthracene } & \multirow[t]{3}{*}{178} & Mean & 0.92 & 0.85 & 1.26 & 0.94 & 1.13 \\
\hline & & s & 0.65 & 0.51 & 0.5 & 0.93 & 0.6 \\
\hline & & $n$ & 10 & 14 & $\cdot 7$ & 8 & 12 \\
\hline \multirow[t]{3}{*}{ Fluoranthene } & \multirow[t]{3}{*}{202} & Mean & 0.77 & 1.16 & 1.27 & 0.75 & 1.03 \\
\hline & & $\mathbf{s}$ & 0.24 & 0.54 & 0.39 & 0.42 & 0.35 \\
\hline & & n & 11 & 14 & 8 & 9 & 13 \\
\hline \multirow[t]{3}{*}{ Pyrene } & \multirow[t]{3}{*}{202} & Mean & 0.83 & 1.15 & 1.39 & 0.62 & 0.98 \\
\hline & & $\mathbf{s}$ & 0.31 & 0.37 & 0.3 & 0.22 & 0.35 \\
\hline & & $\mathrm{n}$ & 14 & 14 & 8 & 7 & 13 \\
\hline Benzo(a)anthracene & 228 & Mean & 0.92 & 1.17 & 1.3 & 0.43 & 1.02 \\
\hline & & $\mathbf{s}$ & 0.41 & 0.39 & 0.26 & 0.2 & 0.41 \\
\hline & & $\mathbf{n}$ & 10 & 14 & 7 & 7 & 12 \\
\hline Chrysene & 228 & Mean & 0.77 & 1.15 & 1.27 & 0.76 & 1 \\
\hline & & $\mathbf{s}$ & 0.27 & 0.44 & 0.23 & 0.87 & 0.45 \\
\hline & & $\mathrm{n}$ & 9 & 14 & 7 & 8 & 12 \\
\hline Benzo(b)fluoranthene & 252 & Mean & 1.03 & 1.22 & 0.96 & 0.91 & 0.86 \\
\hline & & $\mathbf{s}$ & 0.4 & 0.44 & 0.5 & 0.38 & 0.35 \\
\hline & & $\mathrm{n}$ & 5 & 10 & 6 & 7 & 11 \\
\hline Benzo(k)fluoranthene & 252 & Mean & 0.69 & 1.18 & 1.27 & 1.07 & 0.83 \\
\hline & & $\mathbf{s}$ & 0.21 & 0.52 & 0.36 & 1 & 0.38 \\
\hline & & $\mathbf{n}$ & 6 & 11 & 5 & 6 & 11 \\
\hline Benzo(a)pyrene & $\overline{252}$ & Mean & 0.85 & 1.14 & 1.2 & 0.09 & 1.06 \\
\hline & & $\mathbf{s}$ & 0.53 & 0.51 & 0.41 & 0.04 & 0.35 \\
\hline & & $n$ & 7 & 12 & 7 & 3 & 11 \\
\hline Indeno( $(1,2,3$, cd)pyrene & 276 & Mean & 0.99 & 0.9 & 1.01 & 1.15 & 0.99 \\
\hline & & s & 0.26 & 0.4 & 0.3 & 0.41 & 0.2 \\
\hline & & n & 2 & 5 & 4 & 4 & 6 \\
\hline Dibenzo(a,h)anthracene & 278 & Mean & & & & 0.99 & 1.01 \\
\hline & & $\mathbf{s}$ & & & & & \\
\hline & & $\mathbf{n}$ & 0 & 0 & $\mathbf{0}$ & 1 & 1 \\
\hline Benzo(g,h,i)perylene & 276 & Mean & 1.14 & 0.98 & 1.07 & 1.06 & 0.87 \\
\hline & & $\mathbf{s}$ & 0.24 & 0.3 & 0.21 & 0.17 & 0.24 \\
\hline & & n & 2 & 5 & 5 & $\overline{4}$ & 6 \\
\hline
\end{tabular}

${ }^{*} \mathrm{~s}=$ standard deviation, $\mathrm{n}=$ number of observations. 
TABLE 4.20 SANDY VS. CLAYEY SOIL SAMPLE COMPARISONS FOR ALL DATA

\begin{tabular}{|c|c|c|c|c|c|c|c|c|}
\hline \multirow[t]{2}{*}{ Method } & \multicolumn{3}{|c|}{ Clayey } & \multicolumn{3}{|c|}{ Sandy } & \multirow{2}{*}{$\begin{array}{c}\text { Mean Tests } \\
\text { p-value }\end{array}$} & \multirow{2}{*}{$\begin{array}{c}\text { Variance Tests } \\
\text { p-value }\end{array}$} \\
\hline & Mean & $\mathbf{s}$ & n & Mean & $\mathbf{s}$ & $\mathbf{n}$ & & \\
\hline A, Sonication & 0.86 & 0.41 & 84 & 0.81 & 0.22 & 44 & 0.833 & 0.006 \\
\hline B, Microscale & 1.19 & 0.51 & 11 & 1.04 & 0.33 & 67 & 0.032 & 0.003 \\
\hline C, Microwave & 1.34 & 0.53 & 55 & 1.21 & 0.27 & 45 & 0.277 & 0.000 \\
\hline D, Thermal & 0.70 & 0.66 & 55 & 0.72 & 0.42 & 57 & 0.191 & 0.671 \\
\hline $\mathrm{E}, \mathrm{SEF}$ & 0.88 & 0.40 & 92 & 1.17 & 0.29 & 74 & 0.000 & 0.000 \\
\hline
\end{tabular}

$\mathrm{s}=$ standard deviation, $\mathrm{n}=$ number of observations. 
TABLE 4.21 SUMMARY OF METHOD DETECTION LIMIT DATA BY EXTRACTION METHOD.

\begin{tabular}{|l|c|c|c|c|c|c|c|c|c|c|}
\hline \multirow{2}{*}{ Analyte } & \multicolumn{2}{|c|}{$\begin{array}{c}\text { A, } \mathbf{n}=7 \\
\text { Sonication }\end{array}$} & \multicolumn{2}{c|}{$\begin{array}{c}\text { B, } \mathbf{n}=7 \\
\text { Microscale }\end{array}$} & \multicolumn{2}{c|}{$\begin{array}{c}\text { C, } \mathbf{n = 7} \\
\text { Microwave }\end{array}$} & \multicolumn{2}{c|}{$\begin{array}{c}\text { D, } \mathbf{n}=7 \\
\text { Thermal }\end{array}$} & \multicolumn{2}{c|}{$\begin{array}{c}\text { E, } \mathbf{n}=6 \\
\text { SFE }\end{array}$} \\
\cline { 2 - 12 } & $\mathbf{X}$ & $\mathbf{S}$ & $\mathbf{X}$ & $\mathbf{S}$ & $\mathbf{X}$ & $\mathbf{S}$ & $\mathbf{X}$ & $\mathbf{s}$ & $\mathbf{X}$ & $\mathbf{s}$ \\
\hline Naphthalene & 7.95 & 1.2 & 11.54 & 0.56 & 6.93 & 0.25 & 10.40 & 5.6 & 7.40 & 1.9 \\
\hline Acenaphthylene & 8.15 & 1.6 & 12.23 & 0.75 & 7.22 & 0.15 & 24.60 & 9 & 7.76 & 3.2 \\
\hline Acenaphthene & 8.35 & 1.4 & 12.03 & 0.71 & 7.19 & 0.18 & 12.60 & 6.6 & 8.70 & 2.3 \\
\hline Fluorene & 8.91 & 1.6 & 11.95 & 0.73 & 7.21 & 0.1 & 16.90 & 5.2 & 8.74 & 2.3 \\
\hline Phenanthrene & 9.06 & 1.5 & 14.02 & 0.69 & 7.25 & 0.5 & 27.20 & 18.6 & 9.09 & 2.2 \\
\hline Anthracene & 8.82 & 1.7 & 14.87 & 0.75 & 7.15 & 0.38 & 17.40 & 3.2 & 9.20 & 2.5 \\
\hline Fluoranthene & 9.63 & 1.4 & 13.55 & 0.41 & 7.06 & 0.29 & 19.90 & 3 & 9.06 & 2.2 \\
\hline Pyrene & 9.61 & 2 & 16.12 & 2.3 & 7.14 & 0.26 & 19.90 & 3.2 & 8.45 & 2.1 \\
\hline Benzo(a)anthracene & 9.67 & 1.9 & 13.50 & 0.75 & 6.99 & 0.23 & 19.70 & 4.3 & 8.46 & 2.1 \\
\hline Chrysene & 10.06 & 1.7 & 14.21 & 1.2 & 7.11 & 0.29 & 20.60 & 2.6 & 8.65 & 2.2 \\
\hline Benzo(b)fluoranthene & 8.69 & 2.1 & 9.10 & 0.6 & 7.00 & 0.18 & 20.90 & 4.7 & 10.20 & 3 \\
\hline Benzo(k)fluoranthene & 9.61 & 2.3 & 9.73 & 0.48 & 7.43 & 0.25 & 16.50 & 4.8 & 11.08 & 3 \\
\hline Benzo(a)pyrene & 8.94 & 1.8 & 9.18 & 0.6 & 6.73 & 0.2 & 22.30 & 3.1 & 8.53 & 2.7 \\
\hline Indeno(1,2,3,cd)pyrene & 9.59 & 0.9 & 8.50 & 2.1 & 7.16 & 0.2 & 26.20 & 9.1 & 9.70 & 2.7 \\
\hline Dibenzo(a,h)anthracene & 9.14 & 1.2 & 7.74 & 1.1 & 6.64 & 0.27 & 24.30 & 7.6 & 9.69 & 2.6 \\
\hline Benzo(g,h,i)perylene & 9.28 & 1.3 & 8.23 & 1.5 & 6.63 & 0.16 & 27.80 & 9.1 & 9.47 & 2.3 \\
\hline Average & 9.09 & 1.6 & 11.66 & 0.95 & 7.05 & 0.24 & 20.45 & 6.23 & 9.01 & 2.46 \\
\hline Std. error of avg. & 0.41 & & 0.27 & & 0.07 & & 1.80 & & 0.62 & \\
\hline
\end{tabular}

TABLE 4.22 SUMMARY OF METHOD DETECTION LIMITS DEVELOPED ON SITE (mg/kg)

\begin{tabular}{|l|c|c|c|c|c|}
\hline Analyte & $\begin{array}{c}\mathbf{A} \\
\text { Sonication }\end{array}$ & $\begin{array}{c}\text { B } \\
\text { Microscale }\end{array}$ & $\begin{array}{c}\text { C } \\
\text { Microwave }\end{array}$ & $\begin{array}{c}\text { D } \\
\text { Thermal }\end{array}$ & $\begin{array}{c}\text { E } \\
\text { SFE }\end{array}$ \\
\hline Naphthalene & 3.8 & 1.8 & 0.79 & 17.60 & 6.40 \\
\hline Acenaphthylene & 5.0 & 2.4 & 0.47 & 28.30 & 10.80 \\
\hline Acenaphthene & 4.4 & 2.2 & 0.57 & 20.70 & 7.70 \\
\hline Fluorene & 5.0 & 2.3 & 0.31 & 16.30 & 7.70 \\
\hline Phenanthrene & 4.7 & 2.2 & 1.60 & 58.40 & 7.40 \\
\hline Anthracene & 5.3 & 2.4 & 1.20 & 10.00 & 8.40 \\
\hline Fluoranthene & 4.4 & 1.3 & 0.91 & 9.40 & 7.40 \\
\hline Pyrene & 6.3 & 7.2 & 0.82 & 10.00 & 7.10 \\
\hline Benzo(a)anthracene & 6.0 & 2.4 & 0.72 & 13.50 & 7.10 \\
\hline Chrysene & 5.3 & 3.8 & 0.75 & 8.20 & 7.40 \\
\hline Benzo(b)fluoranthene & 6.6 & 1.9 & 0.57 & 14.80 & 10.00 \\
\hline Benzo(k)fluoranthene & 7.2 & 1.5 & 0.79 & 15.10 & 10.00 \\
\hline Benzo(a)pyrene & 5.6 & 1.9 & 0.63 & 9.70 & 9.10 \\
\hline Indeno(1,2,3,cd)pyrene & 2.8 & 6.6 & 0.63 & 28.60 & 9.10 \\
\hline Dibenzo(a,h)anthracene & 3.8 & 3.4 & 0.85 & 23.90 & 8.70 \\
\hline Benzo(g,h,i)perylene & 4.1 & 4.7 & 0.50 & 28.60 & 7.70 \\
\hline
\end{tabular}


TABLE 4.23 RELATIVE ERROR (\%) CALCULATED FROM AVERAGES BY ANALYTE FOR EACH METHOD.

\begin{tabular}{|l|c|c|c|c|}
\hline $\begin{array}{l}\text { Extraction } \\
\text { Method }\end{array}$ & MDL Study & Clayey Soil & Sandy Soil & $\begin{array}{c}\text { Clayey and Sandy } \\
\text { Samples Combined }\end{array}$ \\
\hline A, Sonication & 18 & 50 & 30 & 44 \\
\hline B, Microscale & 8.1 & 44 & 33 & 40 \\
\hline C, Microwave & 3.4 & 42 & 17 & 35 \\
\hline D, Thermal & 30 & 94 & 42 & 73 \\
\hline E, SFE & 27 & 49 & 22 & 39 \\
\hline
\end{tabular}

TABLE 4.24 SUMMARY OF METHOD BIAS AND PRECISION STATISTICAL TESTS.

\begin{tabular}{|c|c|c|}
\hline \multirow[t]{2}{*}{ Comparison/Test } & \multicolumn{2}{|c|}{ Conclusions: Based on } \\
\hline & All available data & $\begin{array}{l}38 \text { subsets with }>\text { ND data } \\
\text { for all five methods }\end{array}$ \\
\hline Method Bias, overall & $\mathrm{D}<\mathrm{A}<\mathrm{E}=\mathrm{B}<\mathrm{C}^{*}$ & $\mathrm{D}<\mathrm{A}=\mathrm{B}<\mathrm{E}=\mathrm{C}$ \\
\hline Method Precision, overall & $\mathrm{D}=\mathrm{B}<\mathrm{A}$, no other differences & $\mathrm{A}=\mathrm{B}=\mathrm{C}=\mathrm{D}=\mathrm{E}$ \\
\hline $\begin{array}{l}\text { Method Bias } \\
\text { Clayey vs Sandy soil means }\end{array}$ & $\begin{array}{l}\text { A: clayey }=\text { sandy } \\
\text { B: clayey }=\text { sandy } \\
\text { C: clayey }=\text { sandy } \\
\text { D: clayey }=\text { sandy } \\
\text { E: clayey }<\text { sandy }\end{array}$ & $\begin{array}{l}\text { A: clayey > sandy } \\
\text { B: clayey }>\text { sandy } \\
\text { C: clayey }=\text { sandy } \\
\text { D: clayey }<\text { sandy } \\
\text { E: clayey }=\text { sandy }\end{array}$ \\
\hline $\begin{array}{l}\text { Method Precision } \\
\text { Clayey vs Sandy soil } \\
\text { standard deviations } \\
\text { Note: low standard deviation } \\
\text { implies high precision }\end{array}$ & $\begin{array}{c}\text { A: } \sigma \text { sandy }<\sigma \text { clayey } \\
\text { B: } \sigma \text { sandy }<\sigma \text { clayey } \\
\text { C: } \sigma \text { sandy }<\sigma \text { clayey } \\
\text { D: } \sigma \text { sandy }=\sigma(<* * *) \text { clayey } \\
\text { E: } \sigma \text { sandy }<\sigma \text { clayey }\end{array}$ & $\begin{array}{l}\text { A: } \sigma \text { sandy }=\sigma \text { clayey } \\
\text { B: } \sigma \text { sandy }=\sigma \text { clayey } \\
\text { C: } \sigma \text { sandy }<\sigma \text { clayey } \\
\text { D: } \sigma \text { sandy }=\sigma \text { clayey } \\
\text { E: } \sigma \text { sandy }<\sigma \text { clayey }\end{array}$ \\
\hline $\begin{array}{l}\text { Analyte Means vs. MW, }{ }^{* *} \\
\text { combined samples }\end{array}$ & $\begin{array}{l}\text { D increases with increasing MW } \\
C \text { decreases with increasing MW }\end{array}$ & \multirow{3}{*}{ No analyses performed } \\
\hline $\begin{array}{l}\text { Analyte Means vs. MW, ** } \\
\text { clayey samples }\end{array}$ & No changes with increasing MW & \\
\hline $\begin{array}{l}\text { Analyte Means vs. MW, ** } \\
\text { sandy samples }\end{array}$ & $\begin{array}{l}\text { D increases with increasing MW } \\
\text { E and C decrease with increasing } \\
M W\end{array}$ & \\
\hline
\end{tabular}

* $\mathrm{A}=$ Sonication, $\mathrm{B}=$ Microscale, $\mathrm{C}=$ Microwave, $\mathrm{D}=$ Thermal and $\mathrm{E}=\mathrm{SFE}$

** Based on a linear regression of analyte mean on molecular weight (MW)

*** The F-Test indicates "<" but the nonparametric test indicates "=". 
TABLE 4.25 MCLAREN/HART GROUNDWATER SAMPLE RESULTS

\begin{tabular}{|c|c|c|c|c|c|c|c|c|c|c|c|}
\hline & & Compound & Naphthalene & Acenaphthylene & Acenaphthene & Fluorene & Phenanthrene & Anthracene & Fluoranthene & Pyrene & Benzo(a)anthracene \\
\hline & & Matrix & WATER & WATER & WATER & WATER & WATER & WATER & WATER & WATER & WATER \\
\hline & & Conc:mg/ & & & & & & & & & \\
\hline ML & Depth & $\begin{array}{l}\text { Sample } \\
\text { Name }\end{array}$ & & & & & & & & & \\
\hline 80 & $19^{\prime}-21^{\prime}$ & GW001-DL & 0.261 & 0.151 & $0.030 \mathrm{~J}$ & $0.079 \mathrm{~J}$ & 0.327 & 0.123 & 0.174 & 0.152 & 0.086 \\
\hline 80 & $30^{\prime}-32^{\prime}$ & GW002-DL & 3.450 & $0.464 \mathrm{~J}$ & $<0.080$ & $0.317 \mathrm{~J}$ & 0.819 & $0.376 \mathrm{~J}$ & $0.448 \mathrm{~J}$ & $0.340 \mathrm{~J}$ & $0.190 \mathrm{~J}$ \\
\hline 90 & 23' & GW003-DL & 0.645 & 0.090 & $0.032 \mathrm{~J}$ & 0.094 & 0.356 & $0.098 \mathrm{~J}$ & $0.154 \mathrm{~J}$ & $0.148 \mathrm{~J}$ & $0.067 \mathrm{~J}$ \\
\hline 90 & $33^{\prime}$ & GW004DL & 3.916 & $0.710 \mathrm{~J}$ & $0.088 \mathrm{~J}$ & $0.305 \mathrm{~J}$ & 0.835 & $0.244 \mathrm{~J}$ & $0.251 \mathrm{~J}$ & $0.300 \mathrm{~J}$ & $0.134 \mathrm{~J}$ \\
\hline 98 & $24^{\prime}-26^{\prime}$ & GWOOS-UNFILT-DL & 9.790 & $0.624 \mathrm{~J}$ & $<0.080$ & $<0.080$ & $<0.080$ & $<0.080$ & $<0.080$ & $<0.080$ & $<0.080$ \\
\hline 98 & $24^{\prime}-26^{\prime}$ & GW005-FILT.DL & 6.112 & $0.348 \mathrm{~J}$ & ND & ND & $\mathrm{ND}$ & ND & ND & ND & ND \\
\hline 104 & $21^{\prime}-23^{\prime}$ & GW007-DL & 1.604 & $0.321 \mathrm{~J}$ & $<0.080$ & $0.200 \mathrm{~J}$ & $0.577 \mathrm{~J}$ & $0.115 \mathrm{~J}$ & $0.179 \mathrm{~J}$ & $0.252 \mathrm{~J}$ & $0.102 \mathrm{~J}$ \\
\hline 104 & $30^{\prime}-32^{\prime}$ & GW008-DL & 1.020 & $0.108 \mathrm{~J}$ & $<0.020$ & $0.031 \mathrm{~J}$ & $0.099 \mathrm{~J}$ & $0.035 \mathrm{~J}$ & $0.057 \mathrm{~J}$ & $0.053 \mathrm{~J}$ & $0.026 \mathrm{~J}$ \\
\hline 106 & $22^{\prime}-24^{\prime}$ & GW009 & $0.019 \mathrm{~J}$ & $0.004 \mathrm{~J}$ & ND & $0.005 \mathrm{~J}$ & 0.145 & $0.005 \mathrm{~J}$ & $0.011 \mathrm{~J}$ & $0.010 \mathrm{~J}$ & $0.005 \mathrm{~J}$ \\
\hline 106 & $16^{\prime}-18^{\prime}$ & GW010 & $0.013 \mathrm{~J}$ & ND & ND & ND & $0.011 \mathrm{~J}$ & $0.020 \mathrm{~J}$ & $0.010 \mathrm{~J}$ & $0.007 \mathrm{~J}$ & $<0.004$ \\
\hline & & Solubility $(m g /)^{*}$ & 30 & 3.93 & 3.47 & 1.69 & 1 & 0.045 & 0.24 & 0.135 & 0.012 \\
\hline
\end{tabular}

Qualifiers:

$\mathrm{ND}=$ No detection of this target analyte at or above the MDL.

$\mathrm{MDL}=4.0 \mathrm{ug} /$

$\mathrm{DL}=$ Dilution required to bring target analyte concentration within linear and or qualifier range.

$\mathrm{J}=$ Estimated value outside the instrument's linear range.

* at 25 degrees centigrade 
TABLE 4.25 MCLAREN/HART GROUNDWATER SAMPLE RESULTS (continued)

\begin{tabular}{|c|c|c|c|c|c|c|c|c|c|}
\hline & & Compound & Chrysene & Benzo(b)fluoranthene & Benzo(k)fluoranthene & Benzo(a)pyrene & Indeno $(1,2,3-\infty d)$ pyrene & Dibenzo(a,h)anthracene & Benzo(g,h,i)perylene \\
\hline & & Matrix & WATER & WATER & WATER & WATER & WATER & WATER & WATER \\
\hline & & \multicolumn{8}{|l|}{ Conc:mg/ } \\
\hline ML & Depth & \begin{tabular}{|l} 
Sample \\
Name
\end{tabular} & & & & & & & \\
\hline 80 & $19.21^{\prime}$ & GW001-DL & $0.077 \mathrm{~J}$ & $0.049 \mathrm{~J}$ & $0.066 \mathrm{~J}$ & $0.065 \mathrm{~J}$ & $0.033 \mathrm{~J}$ & $0.012 \mathrm{~J}$ & $0.027 \mathrm{~J}$ \\
\hline 80 & $30^{\prime}-32^{\prime}$ & GWO02-DL & $0.180 \mathrm{~J}$ & $<0.080$ & $<0.080$ & $0.126 \mathrm{~J}$ & $<0.080$ & $<0.080$ & $<0.080$ \\
\hline 90 & $33^{\prime}$ & GW004-DL & $0.122 \mathrm{~J}$ & $<0.080$ & $<0.080$ & $0.083 \mathrm{~J}$ & $<0.080$ & $<0.080$ & $<0.080$ \\
\hline 98 & $24^{\prime}-26^{\prime}$ & GWOOS-UNFILT-DL & $<0.080$ & $<0.080$ & $<0.080$ & $<0.080$ & $<0.080$ & $<0.080$ & $<0.080$ \\
\hline 98 & $24^{\prime}-26^{\prime}$ & GW00S-FILT-DL & ND & ND & ND & ND & ND & ND & ND \\
\hline 104 & $21^{\prime}-23^{\prime}$ & GW007.DL & $<0.080$ & $<0.080$ & $<0.080$ & $<0.080$ & $<0.080$ & $<0.080$ & $<0.080$ \\
\hline 104 & $30^{\prime}-322^{\prime}$ & GW008-DL & $0.025 \mathrm{~J}$ & $<0.020$ & $<0.020$ & $<0.020$ & $<0.020$ & $<0.020$ & $<0.020$ \\
\hline 106 & $22^{\prime}-24^{\prime}$ & ow0os & $0.005 \mathrm{~J}$ & ND & ND & ND & ND & ND & ND \\
\hline 106 & $16-18^{\prime}$ & GW010 & ND & ND & ND & ND & ND & ND & ND \\
\hline
\end{tabular}

Qualifiers:

$\mathrm{ND}=$ No detection of this target analyte at or above the MDL.

$\mathrm{MDL}=4.0 \mathrm{ug} / \mathrm{l}$

$\mathrm{DL}=$ Dilution required to bring target analyte concentration within linear and or qualifier range.

$\mathrm{J}=$ Estimated value outside the instrument's linear range.

* at 25 degrees centigrade 
Ames Expedited Site Characterization - Marshalltown FMGP Site

FINAL Site Report

March 25, 1996

\subsection{SUMMARY AND CONCLUSIONS}

\subsection{Introduction}

Ames Laboratory of the U.S. DOE undertook a project sponsored by its Office of Technology Development (OTD) in the Environmental Management (EM) program to promote the adoption of ESC and its associated technologies. The goals of the project are to seek out and develop better, safer, faster, cheaper technologies and approaches to solving DOE environmental problems, to improve the basis for analyzing site characterization data, to compare the various analytical technologies, and to encourage stakeholder participation in the environmental arena. The ESC methodology incorporates on-site decision making that facilitates the use of dynamic work plans, allowing site characterizations to be completed in a single continuous effort rather than in a protracted, iterative manner. It has been demonstrated that characterizations can be streamlined and made more efficient without compromising data quality. Two fundamental precepts of the ESC method are, first, the use of both on-site quantitative analytical and multiple geological characterization technologies to minimize the need to perform massive intrusive sampling and time consuming off-site laboratory analysis and, second, the use of on-site decision making to significantly reduce the probability of returning to the site to fill data gaps. As a result, the traditional multi-phase sequence of sampling-analysis-planning-sampling-analysisetc., becomes compressed into a single integrated effort.

In many situations, ESC may be executed in two steps or phases. The first can be described as a focusing phase, in which a direction for the execution, or at least initiation, of the detailed quantitative investigation (Phase II) is planned. Phase I may involve paring down an extensive list of possible contaminants to a short list of target analytes, and/or it may involve identification of the area(s) in which to focus or begin a detailed characterization. In many situations, Phase I has been previously accomplished through work conducted in preliminary site assessments, scoping investigations, or review of historical records.

Several categories of effort can be applied to Phase I, depending on site-specific factors, regulatory considerations, and the existing data set. These categories include:

- Breliminary sampling and quantitative analysis to identify contaminants of concern. This is applicable at sites where multiple wastes or products may have been released and a target list has not been developed.

- Qualitative chemical analysis to identify gross dimensions of the contaminated areas, identify contaminant media, and to qualitatively identify analytes for Phase II. 
Ames Expedited Site Characterization - Marshalltown FMGP Site, FINAL Site Report

March 25, 1996

- Geologic characterization from principally non-intrusive methods (e.g., geophysical surveys) to build a conceptual model of the subsurface from which, with knowledge of contaminant properties, likely contaminant migration pathways can be assessed.

ESC Phase II involves the process of confirming and further developing the site geological model with the application of more intrusive technologies than Phase I and gathering detailed quantitative data regarding the nature and extent of contaminants in the relevant media, which might be called the chemical site model. The data collection plan is designed and continually updated to maximize efficiency by effectively eliminating unneeded sampling. Samples are usually collected by rapid methods which produce minimal disturbance and analyzed in on-site, mobile laboratories instrumented to strictly comply with regulator-approved methods (e.g., U.S.EPA SW-846) traditionally performed in off-site laboratories. Data generated by the mobile laboratories is verified on site and integrated into the conceptual site model on a daily basis, allowing for real-time interpretation.

ESC was first developed and executed by a group at Argonne National Laboratory on a landfill site in New Mexico. Since then the Argonne group has conducted ESC projects at sites in Texas and on several sites in the central U.S. During its first year, the Ames ESC Project mission was to evaluate and promote both IT and SOPT environmental characterization technologies within the ESC framework of real-time data evaluation and a dynamic work plan. Work at the first site, described in this report, was performed as a demonstration outside of the regulatory compliance domain. With the assistance of the Iowa Department of Natural Resources, a FMGP site in Marshalltown, Iowa, was identified as a suitable subject site for the Ames ESC demonstration.

The summary and conclusions of the Marshalltown FMGP ESC investigation focus on an evaluation of the applicability, effectiveness and reliability of both the SOPT and IT fielded at the site in the broad categories of geological/stratigraphic characterization, detection of the nature, distribution and extent of contamination and on-site data integration. In addition, the lessons learned from the Marshalltown ESC demonstration, including significant contributions from this investigation to the advancement of the application of the ESC process in general, are elucidated.

\subsection{Geological/Stratigraphic Characterization}

The site is situated within a glacial drift terrain on the edge of the floodplain of a minor stream. The ground surface is flat to gently sloping, with approximately 10 feet of relief across the site. The BVWST RI report provided a significant amount of stratigraphic information with which to plan the Ames ESC demonstration. The report indicated that the depth to limestone bedrock at the site varies from about 25 feet in the northern part of the site to about 45 feet in the southwestern part of the site. Overlying the bedrock is a sequence of alluvial and 
glacial/lacustrine soils generally characterized by the sequence cohesive over granular over cohesive. The soils were in turn overlain by a heterogeneous dense/hard fill.

Geophysical survey techniques including GPR, seismic reflection and refraction, EOL and borehole logging were fielded at the site primarily to define the surface of the bedrock. Secondary objectives were to provide soil stratigraphic information and information regarding the distribution of PAH contamination. Geophysical survey techniques are considered to be the primary component in the early phase of the ESC process. In this case, the geophysical survey techniques were required to operate under very difficult site conditions. Cultural interferences such as noise from the railroad tracks and from activities within the site, overhead power lines, buildings and surface metallic objects and site stratigraphic conditions such as the heterogeneous upper dense/hard fill unit and the uneven, weathered bedrock surface all contributed to the difficulties. In addition to the geophysical survey techniques, two minimally intrusive geophysical/geotechnical techniques, CPT and soil conductivity logging, were fielded primarily to define the soil stratigraphy and, in particular, the surface topography of the LCU as the DNAPL coal tar residue could tend to pool on and flow along this stratigraphic contact. The CPT unit was equipped with a LIF sensor designed to detect petroleum, oil and lubricant contaminants in the subsurface; the CPT thus played a dual characterization role.

Results of the geophysical surveying were compared with both the BVWST data and with the minimally intrusive data collected as a part of this investigation. After these comparisons were made, it appears that only the SOPT borehole logging provided useful, reliable information. The $300 \mathrm{MHz}$ GPR survey data provided some information on the locations of shallow foundations and services/utilities, but this was not an important aspect of this project. Significant above ground reflectors are apparent in the traces. The processing of the data in a three-dimensional format and the production of time slices through the model, which is an IT associated with this work, shows promise. A significant portion of the data set, however, was not used in generating the three-dimensional model because of software limitations. More appropriate software and computing capabilities should be considered for future endeavors.

The dense/hard upper fill layer and the highly electrically conductive upper cohesive unit are believed to have restricted the depth of penetration of the $100 \mathrm{MHz}$ surveying equipment. The contractor provided profiles in terms of nanoseconds only on the vertical scale; it was left up to ESC personnel to try and interpret depths in feet. Potential variations in velocities could create errors in the depths to contacts on the order of 5 to 10 feet. Moreover, the bedrock surface inferred from the $100 \mathrm{MHz}$ profile did not compare well qualitatively with the general topography of the bedrock surface interpreted from intrusive technologies.

The seismic refraction data was acquired using good techniques and was presented in a clear and concise manner in the contractor's report. Comparisons were generally quite good with the existing BVWST data (which they had at the time of their data interpretation) but comparisons 
Ames Expedited Site Characterization - Marshalltown FMGP Site

FINAL Site Report

March 25, 1996

with the minimally intrusive data indicated that their interpreted bedrock depth contours could be in error by as much as 13 feet; 5 to 10 foot disagreements were frequent. The seismic reflection contractor, as with the GPR contractor, provided uninterpreted seismic profiles with milliseconds on the vertical scale. Their velocity interpretations were unclear, including the application of a "static correction" between two lines, thus making the data virtually impossible to interpret in a quantitative sense by Ames ESC personnel. Qualitative comparisons with minimally intrusive data indicate that their interpretation of the depth to bedrock is in error on the order of 10 feet in places. Moreover, seismic reflection trials carried out by the seismic refraction contractor with a similar sledge hammer source indicated that no clear reflections were apparent. Trials with an IT vibratory source also did not produce any clear reflecting events.

The IT EOL results indicated a large high resistivity anomaly was present in the central part of the site where contamination was believed to be the highest. When the resistivity values determined for this anomaly were compared with representative resistivity values calculated from the borehole geophysical logging and the soil conductivity probe results, it was noted that the EOL resistivities were significantly higher than those detected by either of the other technologies - the borehole geophysical conductivities and soil conductivity probe values are similar. The EOL resistivity anomaly remains without a rational explanation or correlation with other technologies. The fact that the transmitter loop was deployed within a few feet of vehicles, chain link fences, stacks of steel piping and even within a steel shed and the fact that only one receiver well was used probably detracted from the accuracy of the results.

As noted above, the SOPT borehole geophysics may be considered the only reliable information generated from the suite of geophysical surveying technologies fielded. These data proved useful in defining the transition from weathered to unweathered limestone and also provided useful information for correlation with other geophysical data, such as the soil conductivity probe and the EOL surveying data. This information also could have been useful in assessing the depth of penetration of the GPR, but was not used for this purpose at this site. (The GPR contractor is the same contractor who carried out the borehole geophysics.) On the down side, however, the borehole geophysical logging required previously drilled boreholes with wells installed and thus did not provide much additional stratigraphic information, beyond that mentioned above. Moreover, the conductivity responses from the borehole conductivity logging in particular did not produce either as sharp a stratigraphic contact or the ranges of conductivity values produced by the soil conductivity probe. This is probably due to the fact that the soil conductivity probe is in direct contact with the soil and is measuring the bulk conductivity of a relatively small volume of soil whereas the borehole conductivity measurements, in order to avoid measuring the conductivity of the well materials, need to be made farther out into the stratigraphic units and thus measure the bulk conductivity of a much greater volume of material.

It is clear that at the Marshalltown FMGP site the interpretation of the bedrock surface from the geophysical survey techniques is tenuous and can be in error by as much as 10 to 15 feet when 


\section{Ames Expedited Site Characterization - Marshallown FMGP Site}

FINAL Site Report

March 25, 1996

compared with data from intrusive technologies. The negative impact of the cultural interferences and relatively difficult stratigraphic conditions, coupled with uninterpreted data being submitted by some contractors, make evaluation of the techniques very difficult. All the techniques, however, point to the fact that the bedrock surface is weathered, uneven and highly variable.

The SCAPS CPT unit and the Geoprobe soil conductivity probe provided very useful and reliable stratigraphic data. Side-by-side comparisons of the direct push technology logs with BVWST borehole logs indicated stratigraphic correspondence of the unit contacts generally within about one to two feet. The CPT is basically a stand-alone stratigraphic logging tool, requiring limited on-site calibration while stratigraphic interpretation from the soil conductivity probe requires a higher level of calibration. The soil conductivity probe could be regarded as having a role of filling in stratigraphic information between two locations with known stratigraphic profiles. The major unit stratigraphic contacts were generally easily picked off of both the CPT and soil conductivity logs and used to generate a database for an EarthVision three-dimensional site stratigraphic model. When sections from this site stratigraphic model were compared with nearby BVWST borehole logs, correlation of stratigraphic units was generally within about one to two feet. It should be noted, however, that the BVWST data tended to produce a slightly deeper granular unit/LCU contact than the direct push data. This could be the result of differing interpretations of the often transitional lower contact or also could be due to inherent stratigraphic variability over relatively small distances. It should be noted that both the CPT and soil conductivity probe produce continuous logs and significantly more stratigraphic detail than can be obtained through auger borehole logging. This level of detail can be important for DNAPL fate and transport considerations.

Several difficulties experienced with the direct push technologies are as follows: the heterogeneous upper fill layer created problems for penetration of both the CPT and the soil conductivity probes; neither technology could reliably indicate the surface of the bedrock; and, probe breakage was a problem if large gravel or cobble sizes or the bedrock surface was unexpectantly encountered. The Geoprobe soil conductivity unit had the advantages of better mobility and more rapid production than the CPT; however, the CPT had the advantages of being more stand alone in terms of stratigraphic logging, provided more direct contaminant screening data and also had more push capacity than the Geoprobe. Hollow stem augering or a similar technology is probably the only means by which the surface of the bedrock may be reliably determined at this site.

\subsection{Contaminant Detection}

Based on the previous BVWST site characterization work, the lateral and vertical distribution of the dissolved PAHs and residual non-aqueous phase liquid contamination was estimated. 


\section{Ames Expedited Site Characterization - Marshalltown FMGP Site}

FINAL Site Report

March 25, 1996

Assessment of the nature and distribution of the PAH contaminants was carried out using three types of technologies: Phase I screening technologies involving three commercially available IT immunoassay (IMA) kits, an IT CL system and SOPT active and IT passive soil gas measurements; Phase II screening involving IT CPT LIF and IT Geoprobe soil conductivity measurements; and, Phase II quantitative chemical analysis of soil samples using five different on-site extraction methods coupled with GC/MS instruments. For the purpose of developing and assessing the site contamination model, the soil stratigraphy was subdivided into six zones based on the site soil stratigraphy. The six zones defined are as follows:

- middle and bottom of upper cohesive unit (termed zones 1 and 2 respectively),

- top, middle and bottom of granular unit (termed zones 3,4 and 5 respectively), and

- top of LCU (termed zone 6).

The Phase I suite of contaminant screening technologies were applied in an effort to evaluate their ability to identify the approximate boundaries of the organically contaminated area. A triangular 24-point, 50 foot grid was designed to overlap suspected contaminated and uncontaminated areas. At each grid node, Geoprobe equipment was used to collect one shallow ( 4 to 5 feet deep) and one deep (10 to 15 feet deep) soil sample - these depths correspond to zones 1 and 2, and in places zone 3 , as described above. Duplicates from soil core samples were analyzed by all three IMA techniques; in addition, the pore fluids were extracted from an additional duplicate sample and analyzed using the CL system. Since this step in the characterization process was conducted to simulate a screening-level interpretation of the distribution of contaminants, soil collected in the sample tubes was not homogenized. In addition, a soil vapor sample was drawn at each sampling level and analyzed using a field gas chromatograph housed in a Geoprobe vehicle. Finally, a soil gas collector containing two or three adsorption elements in a resealable glass container was placed in a pre-cleaned copper tube in each probe hole at the approximate soil sampling depths to passively adsorb organic compounds in soil vapors over a period of a week.

The data from the three IMA analyses indicated that the presence or absence of detectable PAHs for each vendor's data correlates fairly well with either of the other two. Furthermore, each of the three shallow and deep data sets agree very well with each other on the location and shape of the PAH contamination distribution. The deep data sets places the centroid of the plume further west than the shallow data, consistent with the depth dependent results from other analytical techniques used. When compared to the limited off-site analysis set, the relative degree of agreement within each of the three IMA results are good. Better agreement and fewer false negatives were produced by IMA techniques using gravimetric measurement of soil rather than the volumetric measurements. 
Ames Expedited Site Characterization - Marshalltown FMGP Site

FINAL Site Report

March 25, 1996

The CL analysis involved a proprietary organic liquid phase extraction from the soil sample and addition of chemicals to bring about luminescence of PAH compounds in the extract. This method required independent laboratory analysis of samples from the same media to develop a calibration curve. Results were reported as total PAH and did not correlate well with other methods used. Moreover, the method requires significantly more calibration, sample preparation and handling than the other screening methods.

Passive soil vapor samplers were analyzed off site by thermal desorption and direct mass spectrometer (MS) analysis. Results are thus reported in ion counts of three different molecular masses. The passive collectors provided the advantage of detecting some of the less volatile PAHs not detectable with active soil gas techniques. The shallow data for all three molecular masses agree well with each other. Little of the heavier PAHs compounds were found in the deeper data set, and as expected, lighter molecular weight appears to correspond to higher volatility and concentration in soil gas.

Active soil gas measurements for aromatic hydrocarbons and naphthalene were made by drawing a vacuum on the probe hole, capturing the effluent vapor stream, and injecting it into the GC. Detection of naphthalene was facilitated by heating the vapor sample prior to injection. Results from both photoionization and flame ionization detectors were recorded for aromatic hydrocarbons and naphthalene. Good agreement between the two detectors was found. Concentration differences between the two depths were small and consistent with a more extensive eastern component of the shallow plume.

Overall, the results of the Phase I contaminant screening technologies generally compare well with the BVWST results for stratigraphic zones 1,2 and 3. The plumes are generally centered around the former purifier building and extend down toward the storage shed at the southern end of the site. Analytical results for BVWST boreholes B-02, B-04, B-05 and B-06 generally indicated the highest levels of PAH contamination. Analytical results for boreholes B-08, B-13 and B-14 indicated that the top two stratigraphic zones in the southcentral part of the site also contained PAH contaminants. The gravimetric IMA results and the passive gas results for the deep screening zone indicated significant PAH contamination in this region; the other screening techniques have partially detected it (CL) or completely missed it (active gas). A significant finding of the Phase I contaminant screening study was that PAH contamination existed further to the west than it would appear from the BVWST data. In particular, the BVWST analytical data for B-15 from zone 2, corresponding to the deep screening tests, indicate no PAH contamination (the analytical results for samples from this depth range gave non detects). The deep zone IMA results and passive gas results, in particular, exhibit significant levels of PAH contamination at ML-24 and ML-25, indicating that the plume could in fact extend farther to the west. 
Ames Expedited Site Characterization - Marshalltown FMGP Site

FINAL Site Report

March 25, 1996

Phase II contaminant screening was performed using the SCAPS LIF sensor system and interpreting dips in the Geoprobe soil conductivity profiles. Chemical analysis of soil samples collected adjacent to LIF "hits" indicated that while the LIF sensor data could not be considered as quantitative, the LIF sensor could reliably detect regions of low, medium or high contamination in a qualitative sense. In addition, comparisons of LIF data with olfactory/visual contamination descriptions from nearby BVWST boreholes and MLH sampling locations provided further support for the efficacy of the LIF data.

Average LIF intensities for each of the six stratigraphic zones were interpreted from the LIF panel plots. Contour plots for each zone gave an excellent indication of the distribution of contaminants at the site. In the upper four zones the most significant feature is the heavily contaminated zone in the northwest portion of the site, just to the east of the substation. The general trend of the contaminated region is still, however, from the northwest to the southeast through the central part of the site. Relatively high LIF readings were also noted in the region of ML-97 (off the SE corner of the former purifier building) in the upper four zones. The main plume for zone 5 (base of the granular unit) extends from just west of the former purifier building to the southern portion of the site; elevated LIF readings near the southern site boundary indicate that contamination has most likely moved off the site in this zone. Significant hits were only observed at two locations in zone 6 (top of the LCU).

Many of the panel plots showed elevated fluorescence intensity values within the upper cohesive unit and within the upper and lower portions of the granular unit. Examination of the LIF panel plots revealed that elevated fluorescence intensity levels commonly occurred in the two to four feet of sandy soil immediately overlying the top of the LCU indicating a pooling of DNAPL on the LCU. When LIF measurements were taken in the top of the LCU, the fluorescence intensity reading typically dropped to near zero. This indicates that the LCU is tending to retard the downward migration of the DNAPL contamination at these locations.

Although the BVWST borehole B-1 indicates some fairly significant contamination in zone 3 in the northwest part of the site, the CPT LIF data suggest that this area of the site is more heavily contaminated than is indicated by the BVWST data alone. Moreover, the CPT LIF results in this region tend to confirm the hits in the deep zone by the IMA and the passive gas naphthalene tests - the deep zone for these tests approximately corresponds to zone 2. The CPT LIF responses in the southern part of the site agree both with the BVWST data and the IMA and passive soil gas screening data for the deep zone. In addition, the lobe centered at ML-97 in the LIF data is captured very nicely by the deep IMA screening data, which has this lobe centered on ML-9 some 4 feet to the south of ML-97. No evidence of this lobe appears in the shallow IMA data. The passive soil gas data for the shallow and deep zones, especially for naphthalene, shows this feature as well. Only shallow GC/PID naphthalene shows this feature while no evidence for it appears in any of the CL data. The relatively low but persistent LIF hits at ML-33 on the eastern 
Ames Expedited Site Characterization - Marshalltown FMGP Site

FINAL Site Report

March 25, 1996

side of the site are not confirmed by any other measurements of the PAH concentration in this region.

When correlated with olfactory/visual or LIF data, the Geoprobe soil conductivity logs could be used to interpret zones of PAH contamination. Zones of heavy coal tar residue contamination showed up as distinctive dips on the conductivity trace. Thus, while the soil conductivity probe data can not be considered stand-alone, it can be used to enhance the detail of the site contamination model between more definitive data locations. Another source of data which should not be overlooked is the olfactory/visual comments from the sample logs. A logarithmictype scale ranging from zero (essentially no contamination) to six (essentially free product) has been developed which can quite clearly distinguish heavily contaminated zones from lightly or non-contaminated zones. These type of plots have been developed for both the BVWST and MLH descriptions and correlate very well with and provide support for the more quantitative data.

In terms of contaminant screening, the CPT LIF may be considered the most direct qualitative methodology for indicating regions of PAH contamination. The LIF responses have been corroborated by side-by-side sampling with chemical analyses, by correspondence with nearby BVWST logs and indirectly by the soil conductivity logs. The CPT LIF contaminant screening program has indicated the extent of the heavily contaminated zone in the northwestern part of the site near the substation and also highlighted the extent of coal tar residue contamination which is sitting on the LCU.

Phase II quantitative plume delineation efforts were planned and implemented based on the results of the BVWST RI report, contaminant screening data collected in Phase I and Phase II, and the updated site geologic model. The primary technology evaluation function of this part of Phase II was the comparison and assessment of five on-site extraction methods (both SOPT and IT) for PAHs in soil. In terms of the site characterization goal of this project, samples would ordinarily be selected outside the expected plume area followed by moving toward the suspected plume with the intent of using the low quantitation limits of the GC/MSs to map the action limits of the plume. This approach, however, would lead to a large number of ND samples which could not be used for the technology evaluation task. In the end, as a result of lower than expected sample analysis throughput and the realization that very few samples would be available for the extraction method comparison task, modifications were made to the sampling and analysis plan so that only samples judged to be contaminated were sent to all five extraction methods for quantitative analysis. Consequently, only the eastern edge of the plume was well defined by the quantitative methodologies.

Soil core samples for this effort were collected with minimal subsurface disturbance using a Geoprobe system. A total of 127 samples were run through one or more of the five extraction methods and analyzed by one of three identical GC/MS systems. The extraction methods used 
included three organic solvent-based methods (sonication, microscale, microwave-enhanced extraction) plus thermal desorption and SFE. Sonication extraction is a SOPT while all the others are ITs. To acquire matrix-specific MDLs, a soil sample was spiked with $10 \mathrm{mg} / \mathrm{kg}$ of each of the sixteen targeted PAHs, homogenized and split into 35 subsamples. Analyses for each of the 16 analytes were run seven times (six for SFE) using each of the five extraction methods. In addition, a series of percent recovery tests were carried out to examine the relative extraction efficiency of the different methods. These data were generated by testing five or six samples in duplicate (two for microwave) spiked with $50 \mathrm{mg} / \mathrm{kg}$ of each of the PAH compounds.

The evaluation of the on-site extraction methods was complicated by the fact that the data set being used was unbalanced (i.e., unequal representation in the data set between soil types, analytes or extraction methods), there were still a significant number of ND results in the data set and two basic soil type matrices (sandy and clayey soils) were present. Given these limitations, the method bias comparisons clearly indicate that the thermal extraction efficiency is less than (lower mean, negative bias) the sonication extraction efficiency which is less than the microwave extraction efficiency (higher mean, positive bias). The microscale and SFE extraction methods generally give intermediate results. There is no definitive indication that any extraction method is more precise overall, although there is a tendency for the method precision for the thermal and microscale extraction methods to be significantly less than the precision for the sonication method on the basis of all the available data. On the basis of all the available data, the clayey soil analytical results show greater variation than the sandy soil results indicating greater precision with sandy samples for all but the thermal extraction method. The 38 subsets of this data, whereby all methods gave a greater than ND determination, indicate that only the microwave and SFE methods show greater variation with clayey than with sandy soils, while the other methods show no variation differences between soil types.

The MDL study performed on site indicates that the MDL is greater than $1.0 \mathrm{mg} / \mathrm{kg}$ for all extraction methods except the microscale method. The relative errors based on the field data generally indicate poorer analytical precision than is indicated by the MDL data. The MDL study data sets are small and were collected over a relatively short time frame during which the on-site procedures were being established. The MDL data indicate that the microwave method shows a negative bias while the thermal method shows a large positive bias; these results conflict with biases indicated by the field data for both the thermal and microwave methods.

In summary, within the inherent limitations of these analyses, we found low thermal extraction results with high relative error and high microwave extraction results with low relative error, while the absolute value of the bias for both of these methods appears to decrease with increasing analyte molecular weight. A significant finding of the study was the potential for inconsistencies in procedures and results to arise, even within the strict adherence to SW-846 methods. 
Due to the areal distribution of the sampling locations, as noted above, and the fact that heavily contaminated samples were systematically excluded from quantitative PAH analysis, the quantitative data generated on site adds little to further the development of the site contamination model. This data does, however, confirm the eastern edge of the contaminated area as defined by the Phase I and Phase II screening technologies and also correlates well, in places, with the quantitative BVWST data. A key issue is that while the screening tools have been able to define the areas of contamination much more clearly, they do so only in a qualitative sense.

Quantitative data like the MLH data presented in this report are required for regulatory decision making.

In terms of analysis of the limited number of groundwater samples collected, it may be noted that PAH compounds were detected above the IDNR standards in every one of the groundwater samples analyzed. Due to the distance of the sampling locations from BVWST monitoring wells and the different groundwater sampling collection procedures, direct comparison of these results with the BVWST results is not possible. It is apparent, however, that the concentrations given in the central part of the plume are significantly higher than any concentrations recorded by BVWST.

\subsection{Data Integration}

EarthVision was used for integration and visualization of the data on a daily basis and it was extremely useful in assessing the status of the site geological and contamination models and in planning the next day's sampling locations. Data handling, analysis and visualization could be improved with a more flexible database arrangement and also with the inclusion of geostatistical capabilities such as kriging and quantitative uncertainty analysis.

\subsection{Lessons Learned}

In making the conclusions noted above and detailing the lessons learned from this investigation, it must be kept in mind that a primary goal of the Ames ESC project at Marshalltown was to compare and evaluate SOPT and ITs; the goal was not to carry out a full ESC style characterization of the site. In this context, in terms of geological characterization technologies, a lesson learned from this investigation is that while geophysical survey techniques are an integral part of the ESC process, all techniques may not be appropriate for all sites. In this case, the difficult stratigraphic conditions and significant cultural interferences, along with, in some cases poor data collection and analysis/reporting procedures, led to significant error and uncertainty in the geophysical survey results and severely limited their useful application. The potential limitations of the methods being proposed need to be carefully considered on a site 


\section{Ames Expedited Site Characterization - Marshalltown FMGP Site FINAL Site Report}

March 25, 1996

specific basis. Future endeavors also need to insist on receiving fully interpreted data from the contractors in terms of stratigraphy and depths to major geologic contacts.

Information regarding the location of the phreatic surface and hydraulic heads and gradients was lacking in this investigation and tends to, in general, play somewhat of a secondary role in the ESC process as currently defined. An understanding of the site groundwater conditions is essential to the development of a realistic conceptual model of contaminant transport and thus to the preliminary evaluation of potential remedial schemes. Part of the difficulty is that traditional regulatory-accepted groundwater sampling requires monitoring wells while the selection of monitoring well locations is one of the end products of an ESC investigation. Progress in this regard could be made with the further development and regulatory acceptance of groundwater data from samples collected using direct push and probing tools. There is still the difficulty, however, of assessing the temporal variations of groundwater conditions. An ESC investigation, by design, is meant to be initiated and completed in the shortest possible time; this approach is not conducive to assessing the temporal variation of groundwater conditions. The use of temporary, direct-push-technology-installed wells for continued collection of data during the analysis/reporting stage is one potential alternative which could be built into ESC procedures.

The application, versatility and the high quality of data from direct push technologies was demonstrated and proven at this site. The SCAPS CPT demonstrated the reliability and efficiency of both the stratigraphic logging capabilities and LIF sensing capabilities in developing the site stratigraphic model and delineating areas of PAH contamination. The capabilities of the Geoprobe soil conductivity system were also proven. It had more maneuverability than the CPT and was also more operationally efficient. With proper calibration, it can be reliably used to fill in stratigraphic data between two locations with known stratigraphic profiles. In addition, again with proper calibration and verification, it can be used to enhance the site contamination model. Both direct push technologies have the capability to provide much more detailed stratigraphic information than conventional auger boring, which is important when considering contaminant fate and transport. On the down side, both direct push technologies had difficulties detecting weathered bedrock, and unexpected bedrock or large gravel/cobbles resulted in broken probes. More rugged equipment for getting through upper heterogeneous fill layers, which could be present on many developed sites, could be considered.

There was a great deal of sampling in the east and northeast areas of the site. Once it was established that contamination in this area was low or nonexistent, this sampling activity should have been shifted west to where higher levels of contamination were detected. The eastern boundary of the contaminant plume was defined with relatively high accuracy, however, there is a large uncertainty regarding the location of the western boundary of the plume. This is due in large part to several large buildings in the southwest corner of the site; however, it may have been possible to obtain several more samples at selected locations adjacent to these buildings. Probably the most useful additional data could be obtained from sampling just south of the site 
adjacent to the railroad tracks, if this were possible. As one of the primary objectives of the Ames Laboratory ESC project was to collect enough data to allow direct comparison of SOPT and ITs, this does not impact on the success of project. It would have been extremely useful, however, to have quantitatively assessed the level of uncertainty in both the stratigraphic and contaminant distribution models on a daily basis with the use of geostatistical tools.

Lastly, this study indicates the potential for significant variation of chemical analysis results for PAHs in soils, even when following regulatory approved SW-846 procedures. The uncertainty and potential variability associated with soil matrix effects, sample selection, preparation and extraction procedures far outweigh inaccuracies in the chemical analysis methodologies themselves. The Phase I and Phase II screening results, including the olfactory/visual data, give a far better picture of the distribution and extent of contamination than the quantitative analysis results. Technologies such as CPT LIF give a continuous profile of contamination at a specific location and the results are largely operator independent. The promotion of these technologies to regulatory acceptance would fit the goals of this project in developing better, safer, faster and cheaper site characterization technologies. The quantitative analysis technologies could perhaps be limited to confirmation of the ranges of contamination measured by screening type analyses. The ranges could tie into both action levels and the evaluation/selection of potential remedial schemes. 


\subsection{ACKNOWLEDGMENTS}

The value of this demonstration has been enhanced by the efforts of many people. As principal investigator (PI) I take full responsibility for any of its failures and declare that its successes are owed exclusively to the following people.

First are the members of the Ames Laboratory ESC Team: Dave Wonder, hydrogeologist, whose experience and judgment was simply invaluable, Connie Bailey, communications specialist, whose insights on how to meet stakeholder needs are unique to the DOE complex, Joel Prail, computer engineer, whose willingness to explore new ways to use data management tolls was vital and finally Beth Weiser, information \& logistics coordinator, whose creativity, insight and energy were so effective and infectious. Jo Holland and Kathy Petersen, project secretaries, performed beautifully under conditions often characterized by uncertainty and urgency.

Support from Assistant Professor Bruce Kjartanson of the Civil Engineering Department at Iowa State University ranged from being the major author of this report to field and off-site analysis of the direct push technologies. His insights were greatly valued as were those of his $\mathrm{PhD}$ student Greg Stenback, whose statistical expertise was crucial for this report. Greg Junk served as analytical advisor to the PI and his expertise was very helpful in planning for the analytical program of this demonstration. A debt of gratitude goes to Bill Haas for several reasons but most importantly for identifying McLaren/Hart as a company having experience with many aspects of ESC in the private sector.

Steve Gelb, national director of field analytical services for McLaren/Hart, offered critical guidance and generous support on project strategies as well as the implementation of the field sampling and analysis program. Another major contributor was Johanshir Golchin, environmental engineer of the Iowa Department of Natural Resources, who took an active, personal interest in the implementation of this demonstration. Without his generous assistance our schedule would not have been met. Dean Hargens of IES Industries was a model of a site manager willing to try something new that could result in significant savings in the characterization the FMGP sites currently owned by IES. He and his staff provided effective and timely support to for the myriad needs of an ESC demonstration with its new ways of doing business.

Tom Noble and Jim Corones provided an opportunity for this PI to lead the project on very little evidence of his ability to do so. Such faith and courage is often absent in a program and nearly always goes unrecognized. I trust that this acknowledgment helps in a small way to repay their extraordinary support. 
Ames Expedited Site Characterization - Marshalltown FMGP Site

FINAL Site Report

March 25, 1996

Finally, none of this work could have occurred without the vision and unwavering support of Caroline Purdy, DOE headquarters program manager. Her management style serves as a model for all to emulate and her considerable efforts are cheerfully and gratefully acknowledged.

Ames Laboratory is operated for the U.S.-DOE by Iowa State University under Contract No. W7405-ENG-82 and is a member of ISU's Institute for Physical Research and Technology. This report was prepared as a deliverable on the project "Ames Laboratory Expedited Site Characterization Demonstrations," Technical Task Plan CH1-3-10-05, as part of the Characterization, Monitoring, and Technology Crosscutting Program in the OTD within the DOE's EM program. 
Ames Expedited Site Characterization - Marshalltown FMGP Site

FINAL Site Report

March 25, 1996

\subsection{BIBLIOGRAPHY}

Conover, W. J., 1980, Practical Nenparametric Statistics, 2 ed. John Wiley \& Sons, NY, 493 pgs.

Burton, J. C., Walker, J. L., Meyer, T., Rose, C., Aggarwal, P., Aver,y M. and Jennings, T., "A Rapid, Cost-Effective, Multidisciplinary, Minimal Drilling Process for Site Characterization," 1993 Environmental Restoration Conference, washington D.C.

BVWST, 1992, Remedial Investigation Report: Marshalltown, Iowa, Former Manufactured Gas Plant Site. October 1992.

Chiang, C. Y., Loos, K. R. and Klopp, R. A., 1992, Field Determination of Geological/Chemical Properties of an Aquifer by Cone Penetrometry and Headspace Analysis, Ground Water, Vol. 30, No. 3 pp. 428-436.

Cressie, N. A. C., 1991, Statistics for Spatial Data, John Wiley \& Sons, Inc., NY, 900 pgs.

Christy, C. D. , Christy, T. M. and Volker, W. "A Percussion Probing Tool for the Direct Sensing of Soil Conductivity," Geoprobe Systems, Technical Paper No. 94-100, March 1994, pp. 1-14.

DOE, 1994, "A New Approach to Environmental Research and Technology Development at the U.S. Department of Energy - Action Plan," prepared by the U.S. Department of Energy.

Douglas, B. J. and Olsen, R. S. 1981, Soil Classification Using Electric Cone Penetrometer, Symposium on Cone Penetration Testing and Experience, Geotechnical Engineering Division, ASCE, St. Louis, pp. 207-227.

Grunig,J. and Hunt, T., 1984, Managing Public Relations, Holt, Rinehart and Winston.

Hansen, R. E., 1985, Bedrock Topography of Central Iowa, Map I-1609. 1985.

Lankston, R. W. 1989. The Hidden Layer Problem in Refraction Seismic Surveying Dies Hard. Proceedings SAGEEP, Golden, CO.

Palmer, D. 1980. The Generalized Reciprocal Method of Seismic Refraction Interpretation. SEG Publ.

McDonald, D. B., 1986; D. B. McDonald Research Associates, Preliminary Investigation of the Manufactured Gas Plant at Marshalltown, Iowa, September 1986. 
Ames Expedited Site Characterization - Marshallown FMGP Site

FINAL Site Report

March 25, 1996

McLaren/Hart, 1994 Results of Chemical Analyses; Contractor's Report.

Olson, R. S. 1988, Using the CPT for Dynamic Site Response Characterization, Earthquake Engineering and Soil Dynamics II - Recent Advances in Ground-Motion Evaluation, ASCE, J. L. Von Thun, editor, June 27-30, 1988, pp. 375-388.

Olson, R. S. and Farr, J. V., 1986, Site Characterization Using the Cone Penetrometer Test, Proceedings of the ASCE Conference on Use of In-Situ Testing in Geotechnical Engineering, ASCE, New York, pp. 854-868.

Stenback, G.A., 1995, personal communication.

Stenback, G. A. and Kjartanson, B. H., 1994, Summary of Data Analyses: Ames Laboratory ESC Demonstration at FMGP Site in Marshalltown, Iowa, Prepared for Ames Laboratory/TIP, September 1994.

Stenback, G.A., B. H. Kjartanson, K. Older and Drinkwine, A., 1994, Site Characterization and Analysis Penetrometer System: Investigation of a Former Manufactured Gas Plant Site in Marshalltown, Iowa. June 1994.

Stenback, G.A. and Kjartanson, B.H., 1995, McLaren/Hart Extraction Method Analyses Comparisons from Marshalltown ESC Demonstration Data, May, 1995.

Technos, Inc., 1994, Summary of Geophysics; Contractor's Report.

Tuthill, 1988, Tuthill, Inc. Preliminary Report Marshalltown, Iowa Marshalltown Gas Light Company, Iowa Railway and Light Corporation, FMGP Site No 48.1 August 1, 1988.

Tuthill, 1989, Tuthill, Inc., Cumulative Report of Public Water Quality Assurance and Past Investigations at Marshalltown, Iowa, February 27, 1980. 
Ames Expedited Site Characterization - Marshalltown FMGP Site FINAL Site Report

March 25, 1996

APPENDIX A: $\quad$ STRATIGRAPHIC LOGS FOR BOREHOLES HSA-1 AND HSA-2 


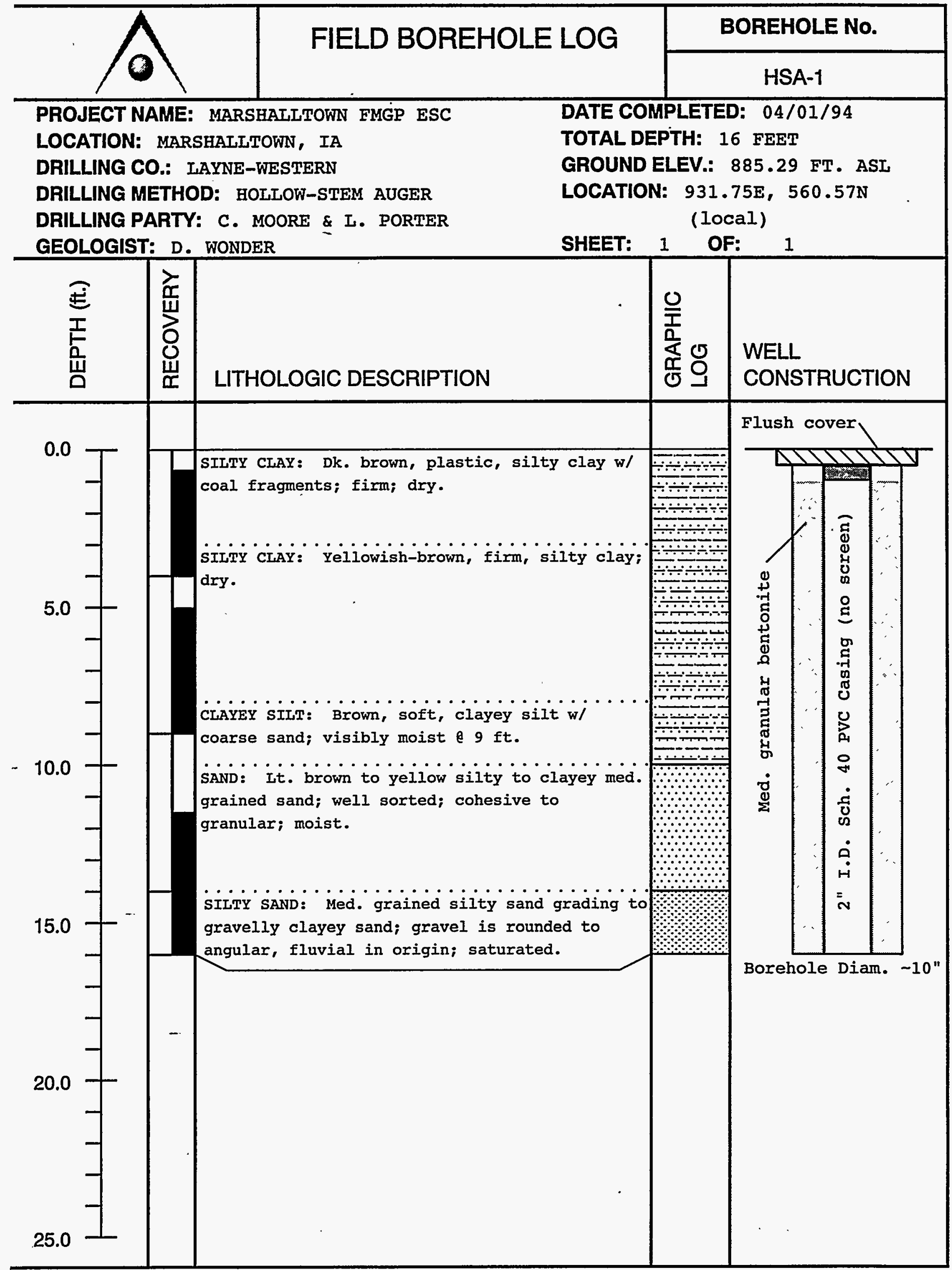




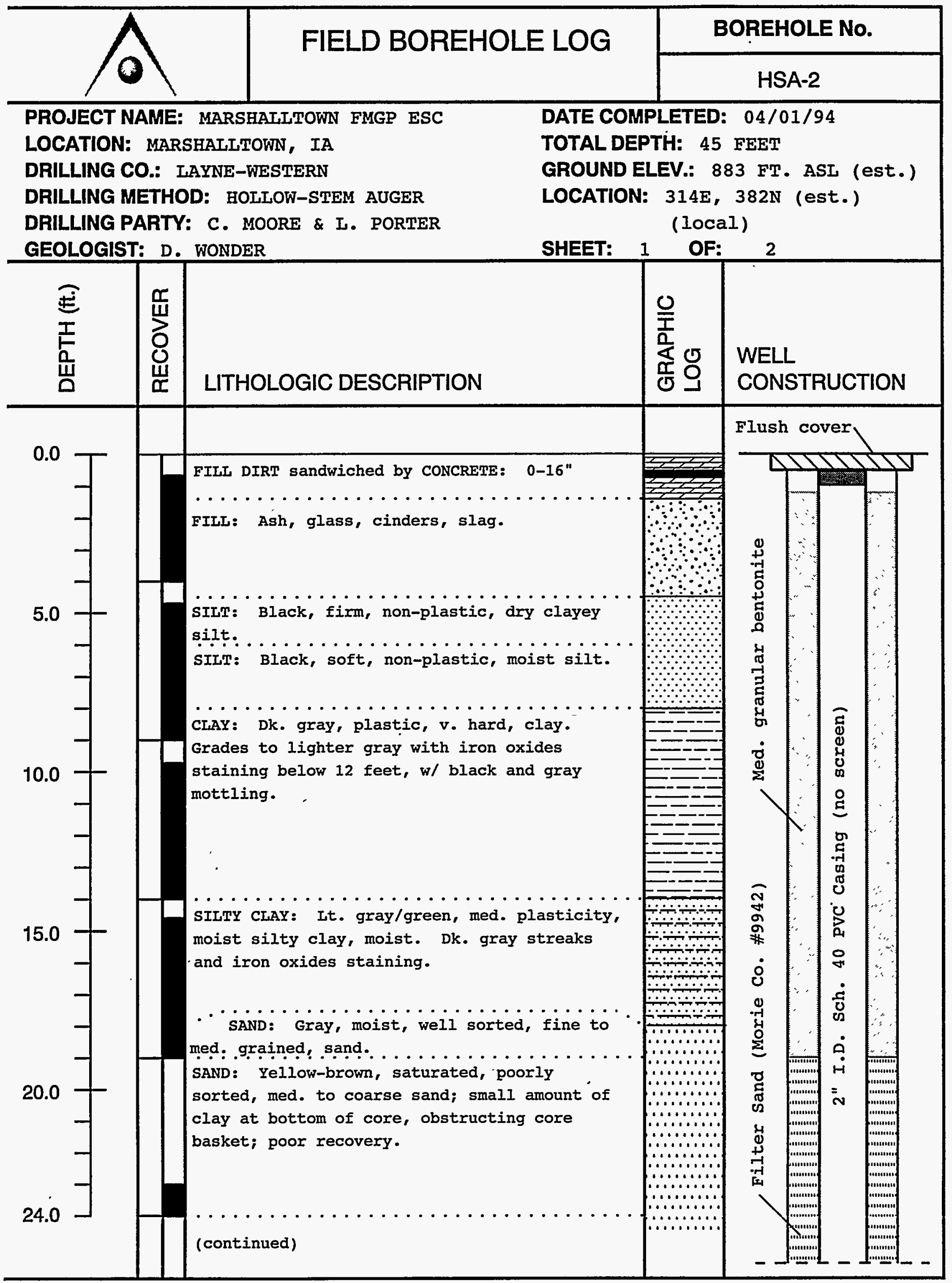




\section{AIELD BOREHOLE LOG}

BOREHOLE NO.

HSA-2

PROJECT NAME: MARSHALLTOWN FMGP ESC

LOCATION: MARSHALLTOWN, IA

DRILLING CO.: LAYNE-WESTERN

DRILLING METHOD: HOLLOW-STEM AUGER

DRILLING PARTY: C. MOORE \& I. PORTER

GEOLOGIST: D. WONDER
DATE COMPLETED: $04 / 01 / 94$

TOTAL DEPTH: 45 FEET

GROUND ELEV.: 883 FT. ASL (est.)

LOCATION: 314E, 382N (est.)

(local)

SHEET: 2 OF: 2

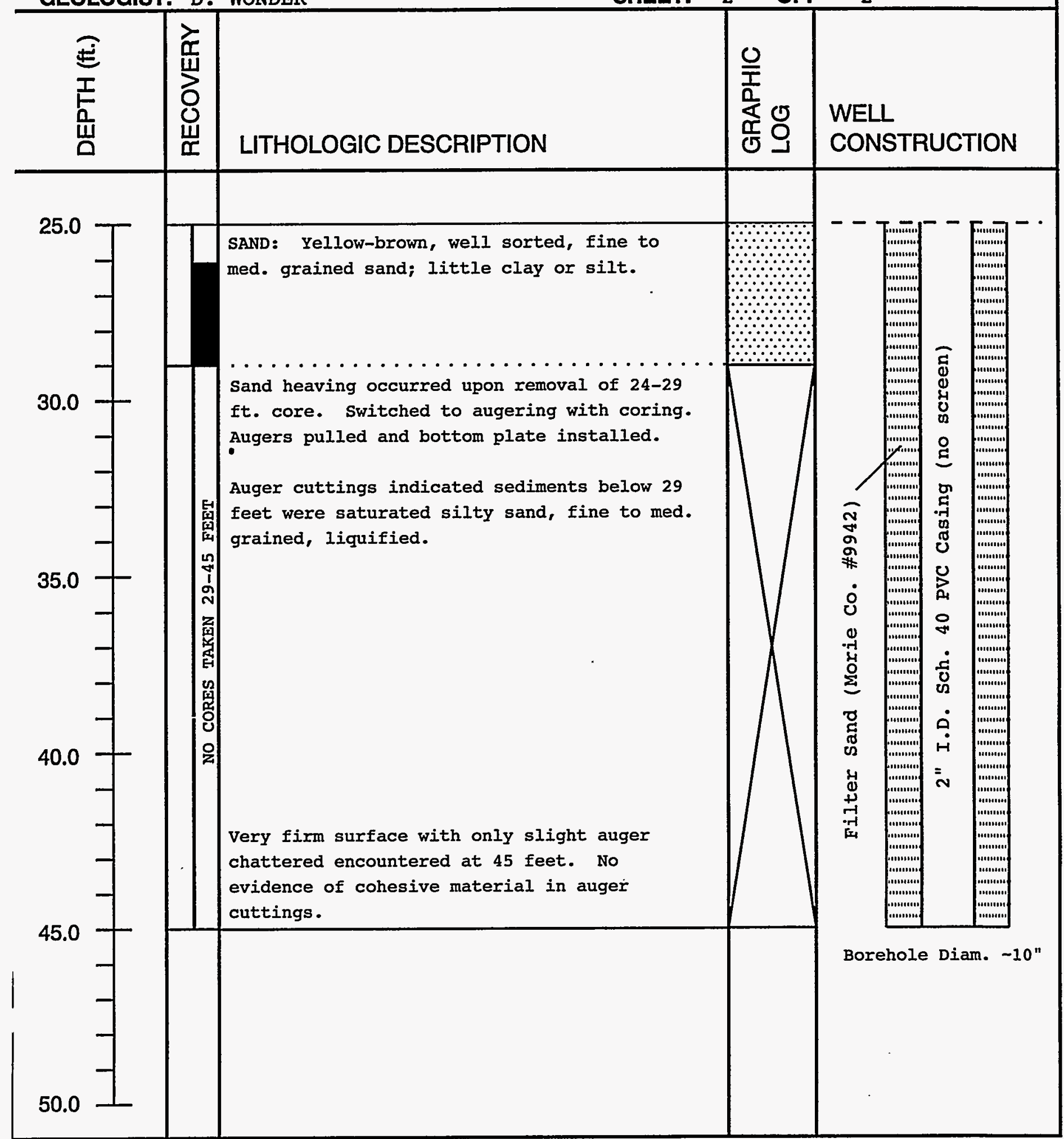


Ames Expedited Site Characterization - Marshalltown FMGP Site DRAFT Site Report

October 19, 1995

APPENDIX B: $\quad$ PANEL PLOTS FOR CPT PUSHES 
(7аวł) 47da0
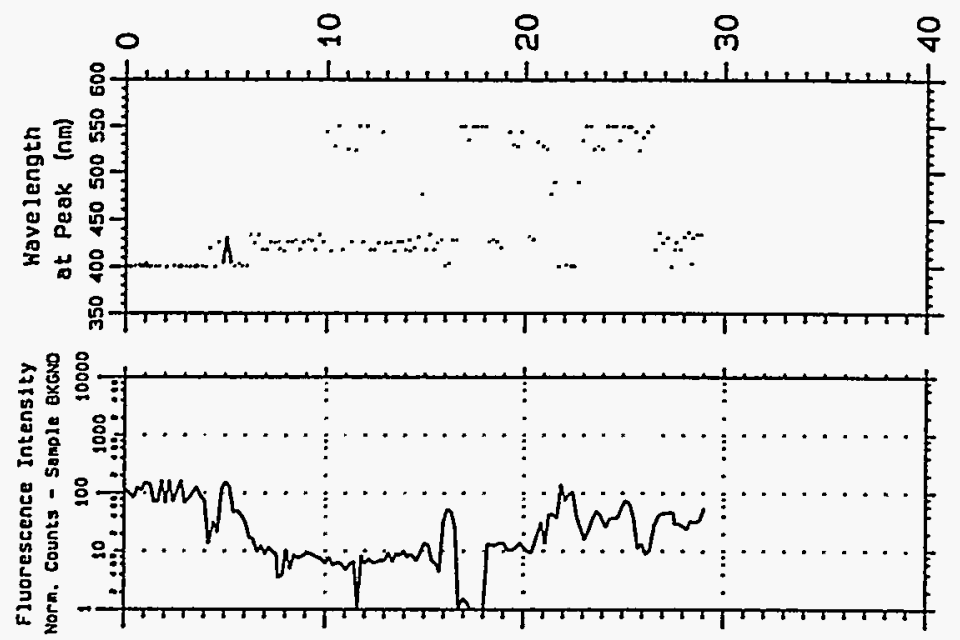

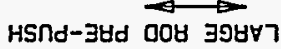
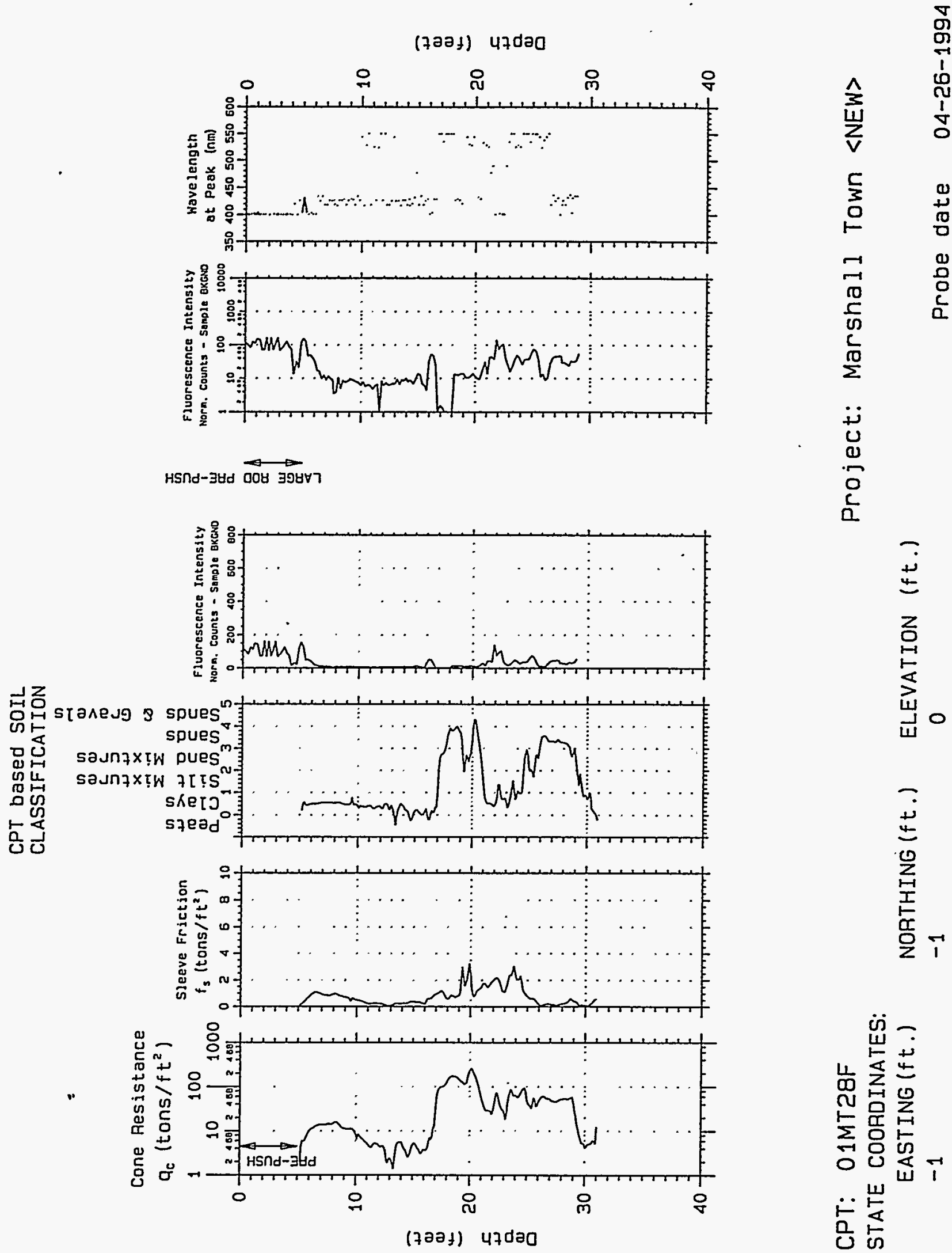

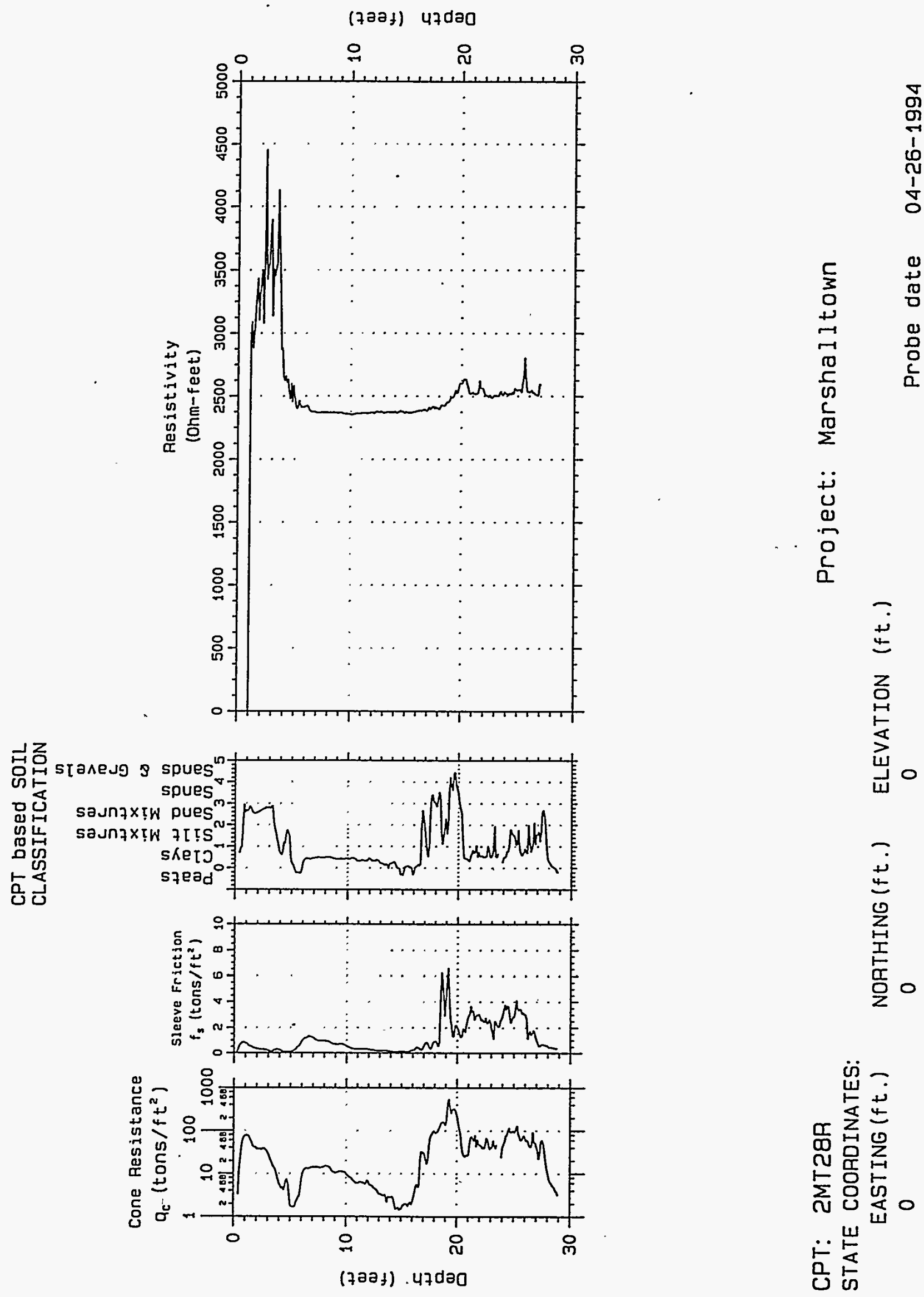

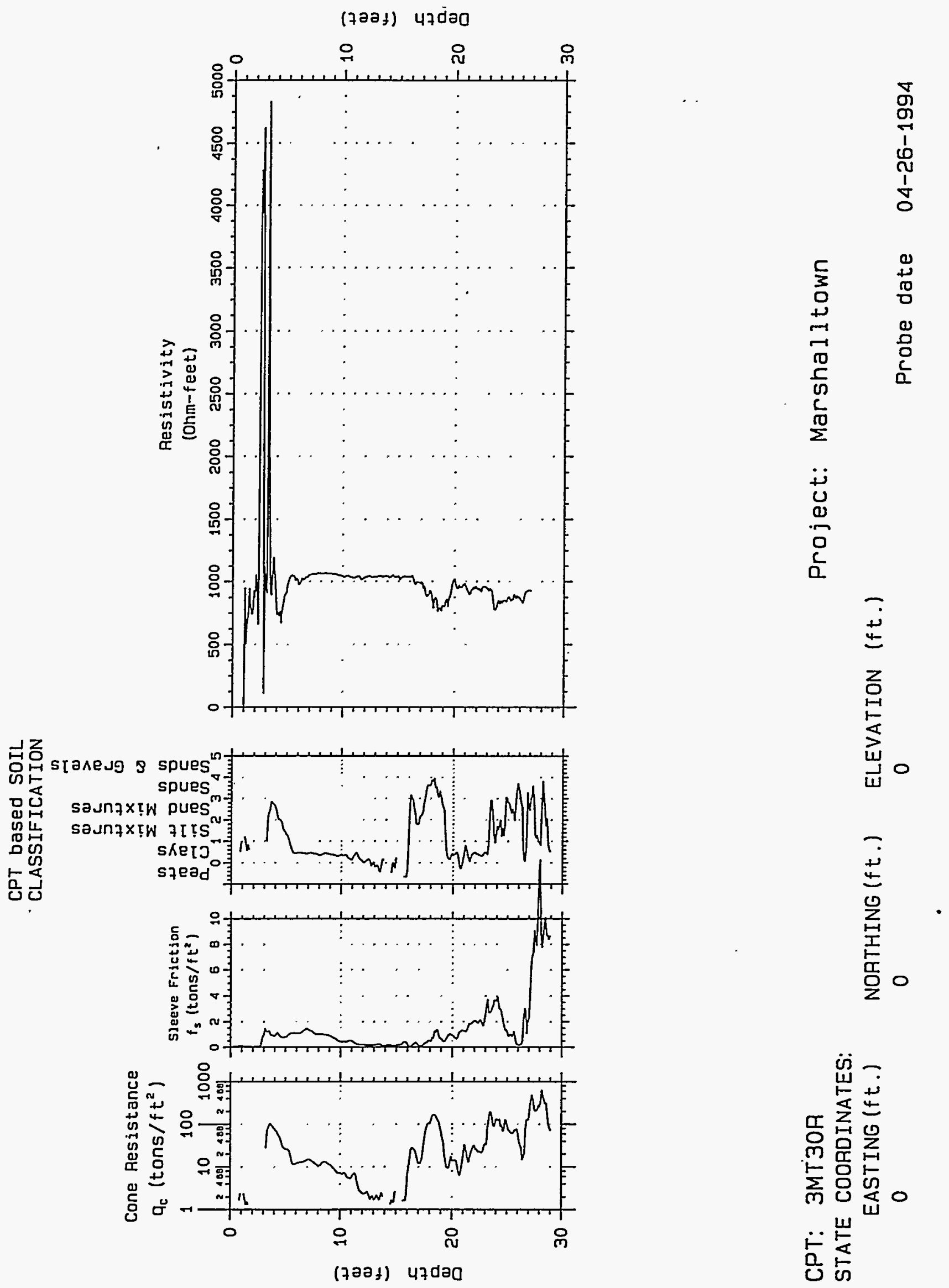

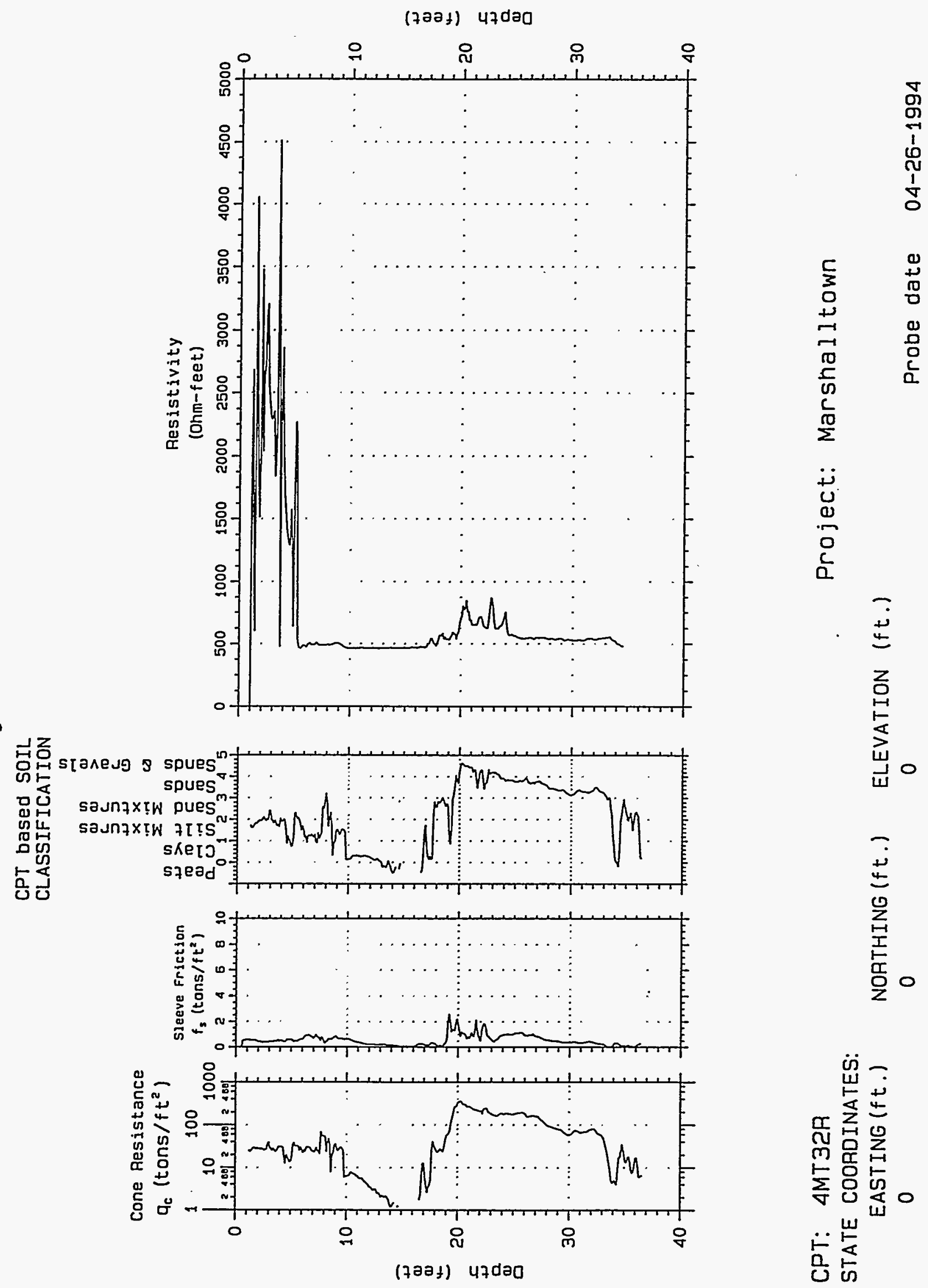

نํ.
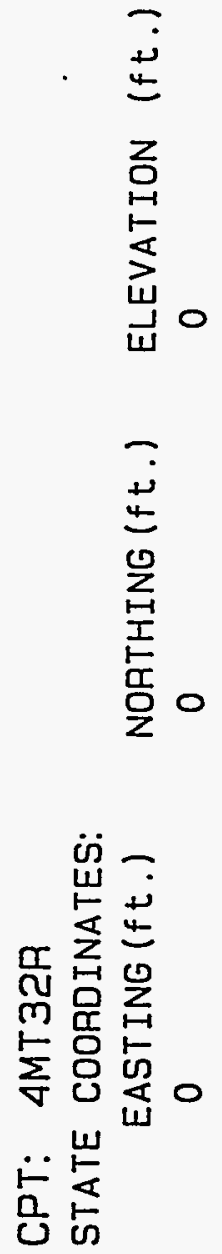


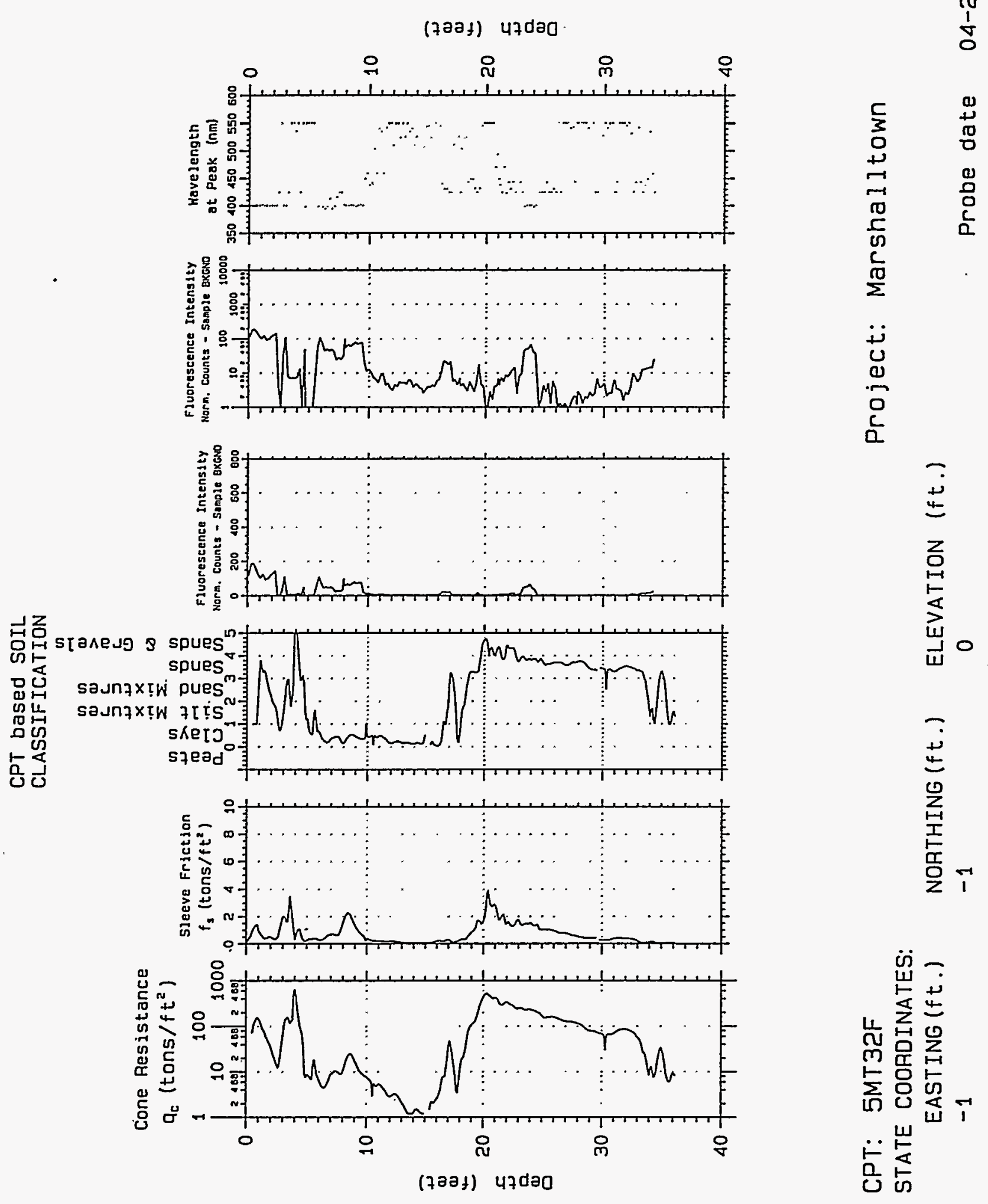



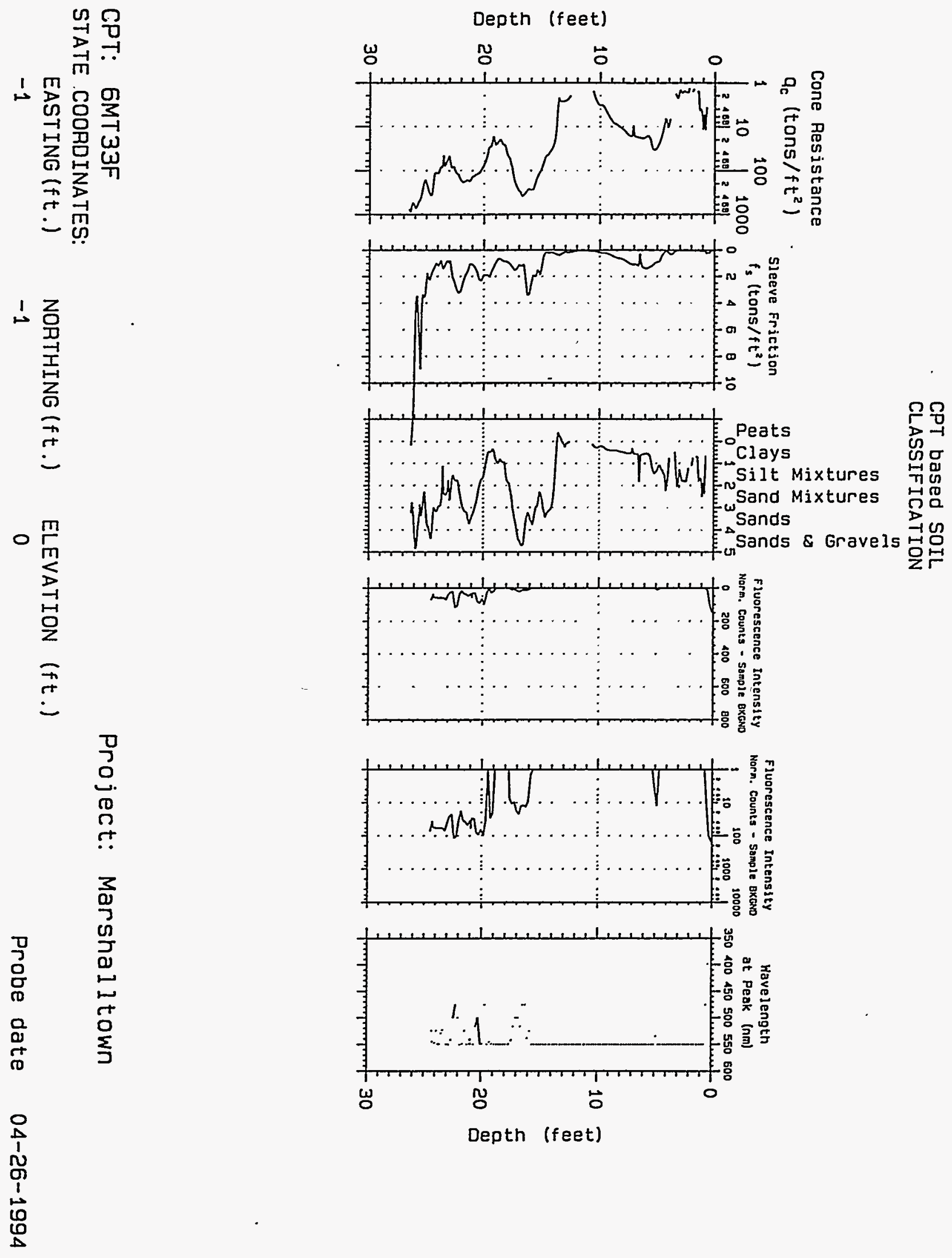

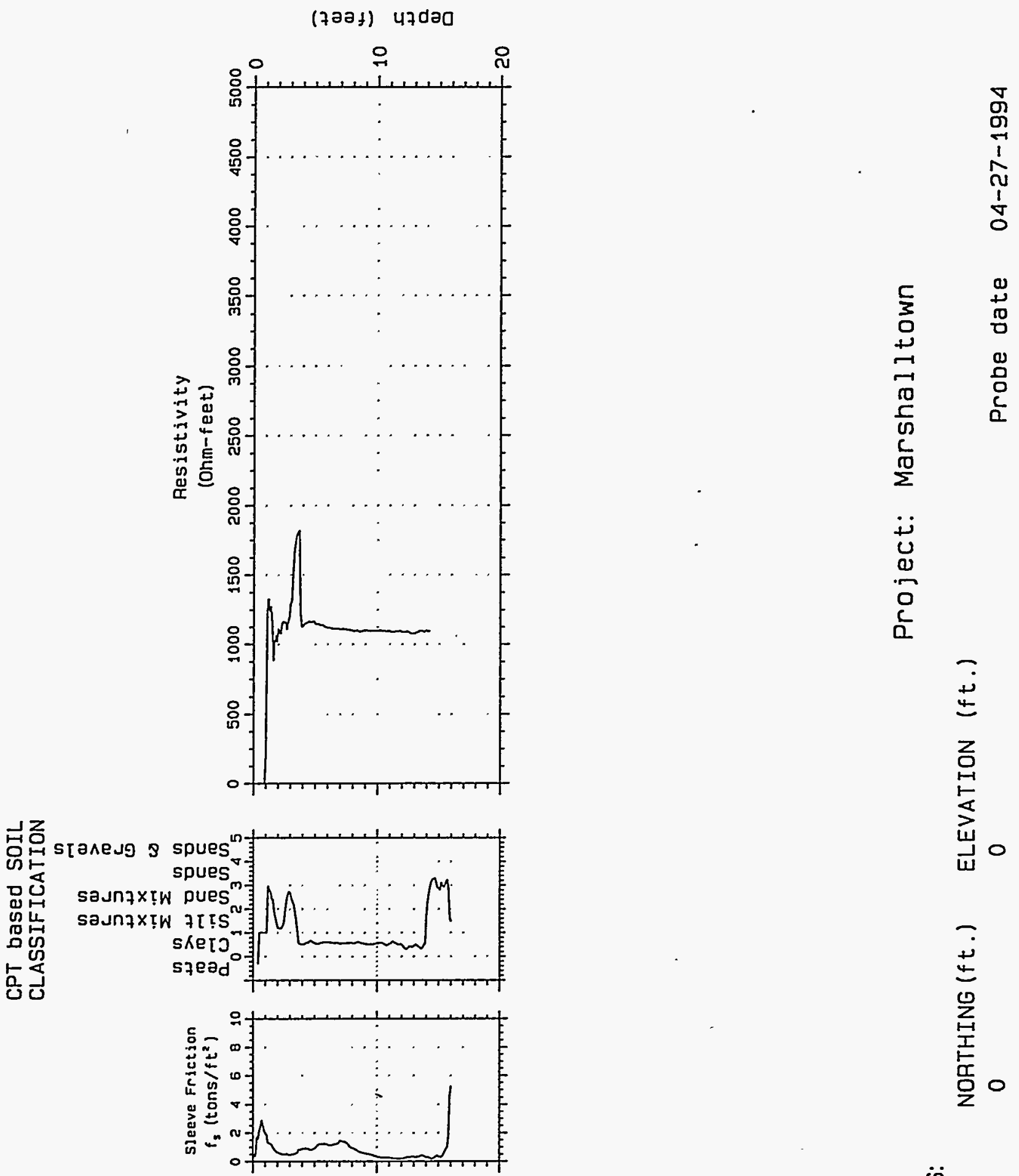

ii

4

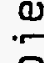

듬

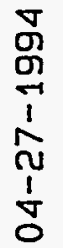

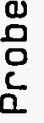

ن

古

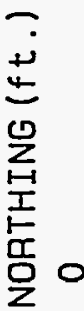

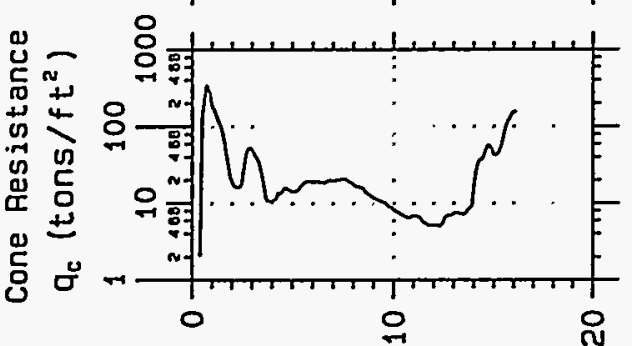

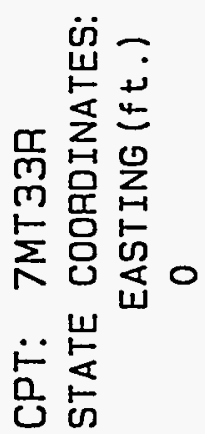

(7аa1) 470a0 


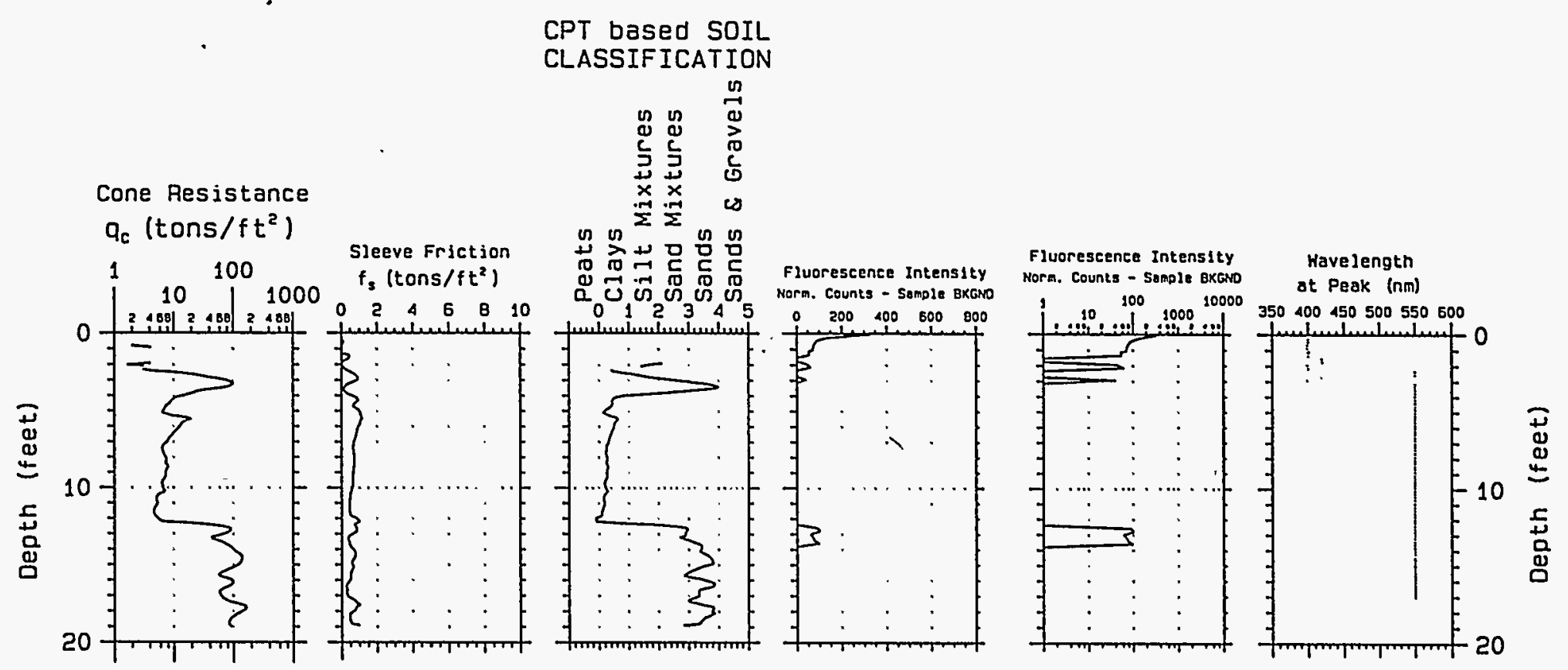

СРT: $8 M T 41 F$

Project: Marshalltown

STATE COORDINATES:

EASTING ( $f t$.)

NORTHING $(f t$.$) ELEVATION (ft.)$

$-1$

$-1$

0

Probe date

$04-27-1994$ 
0

HSกd-3甘d 008 39847

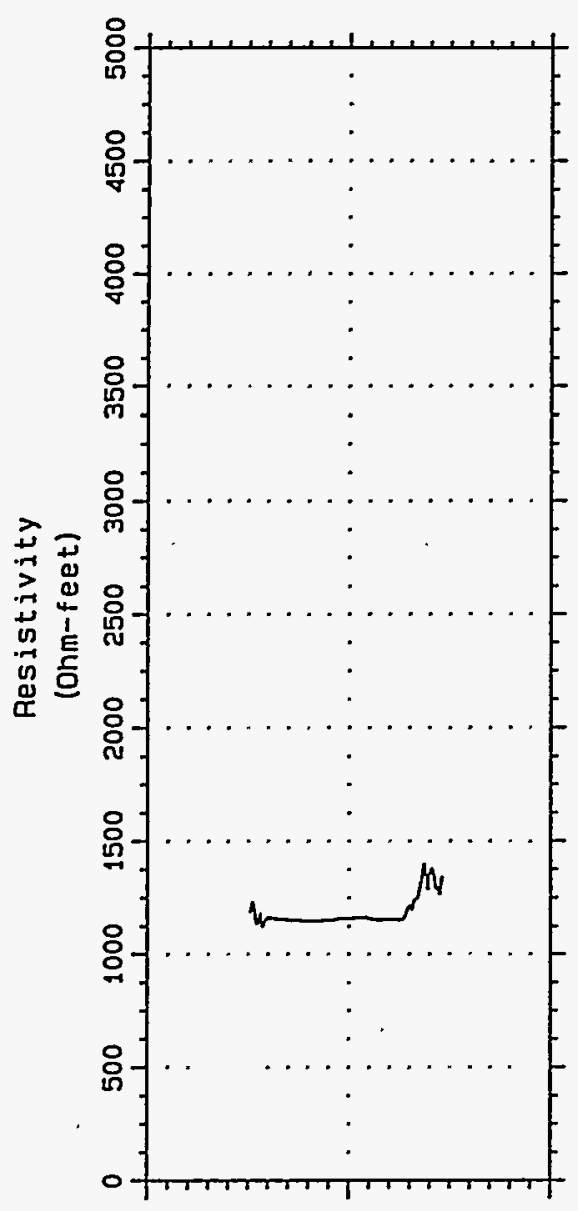

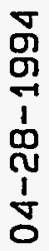

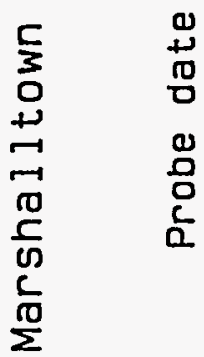

iن

U

음
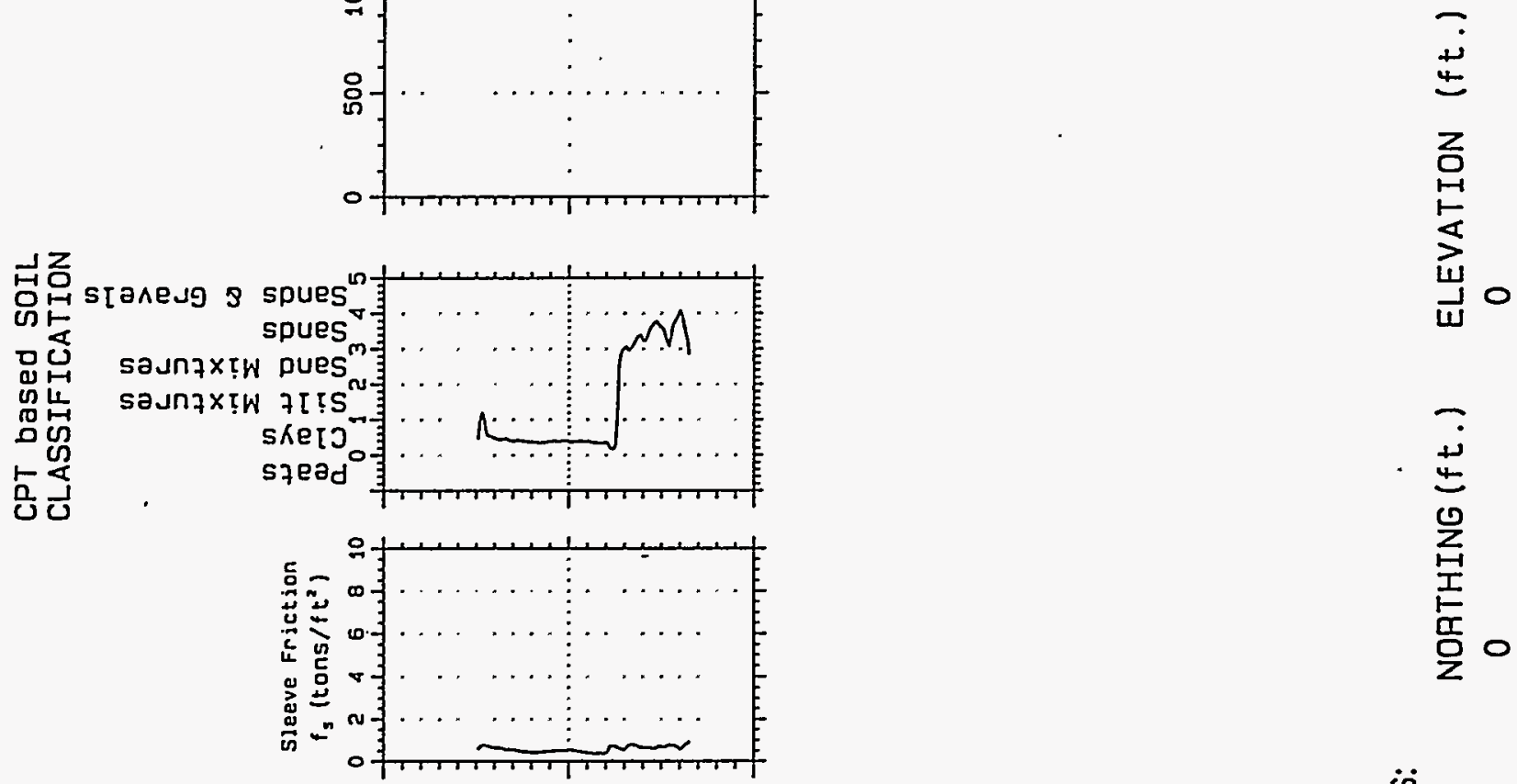

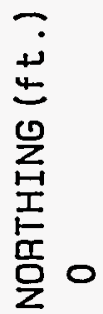

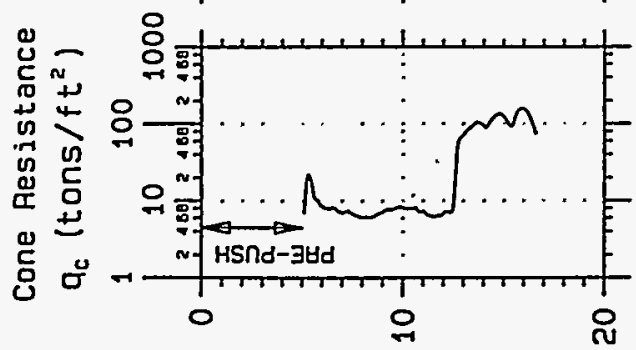

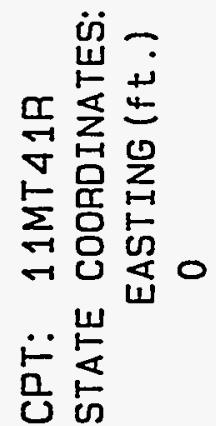



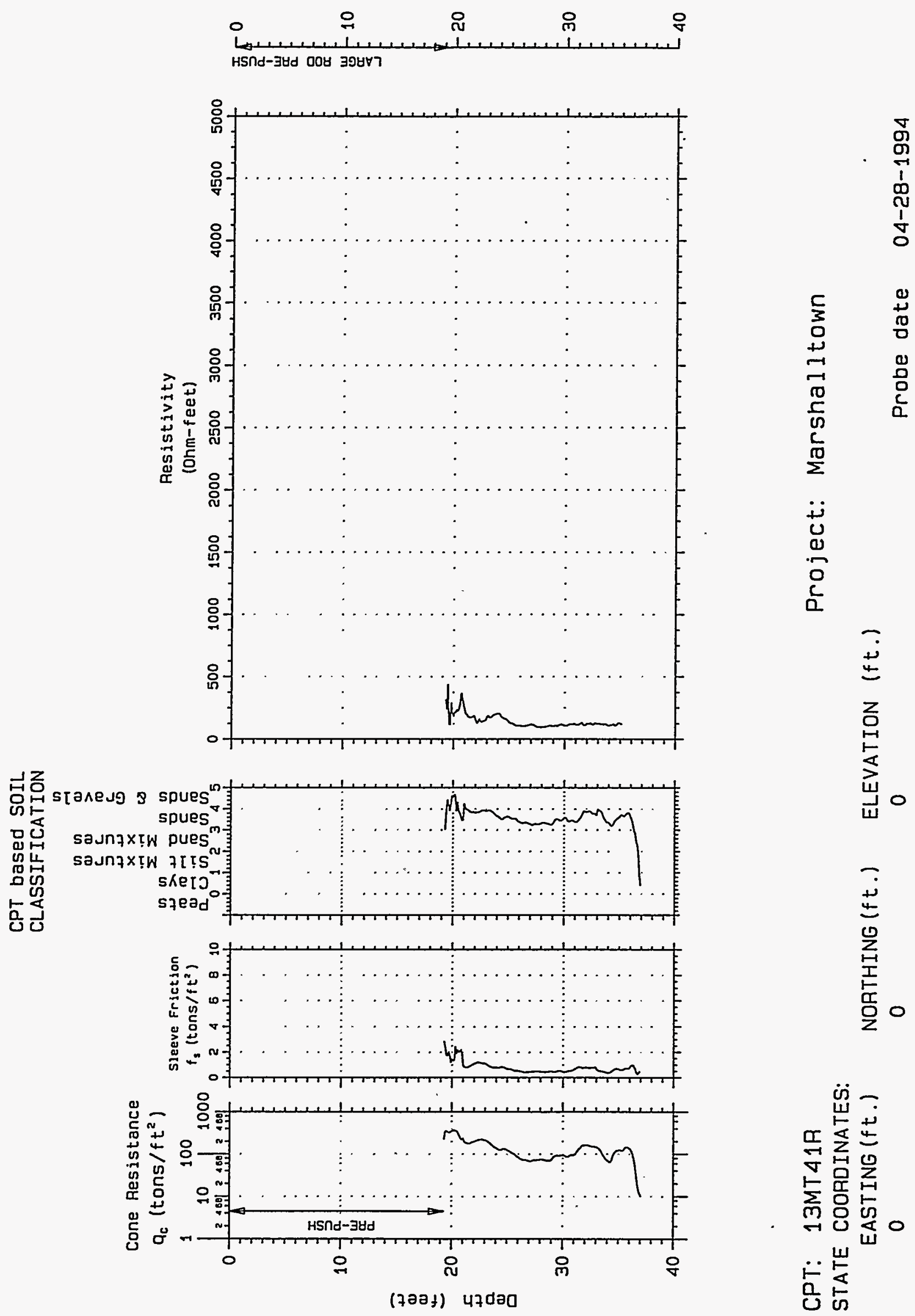

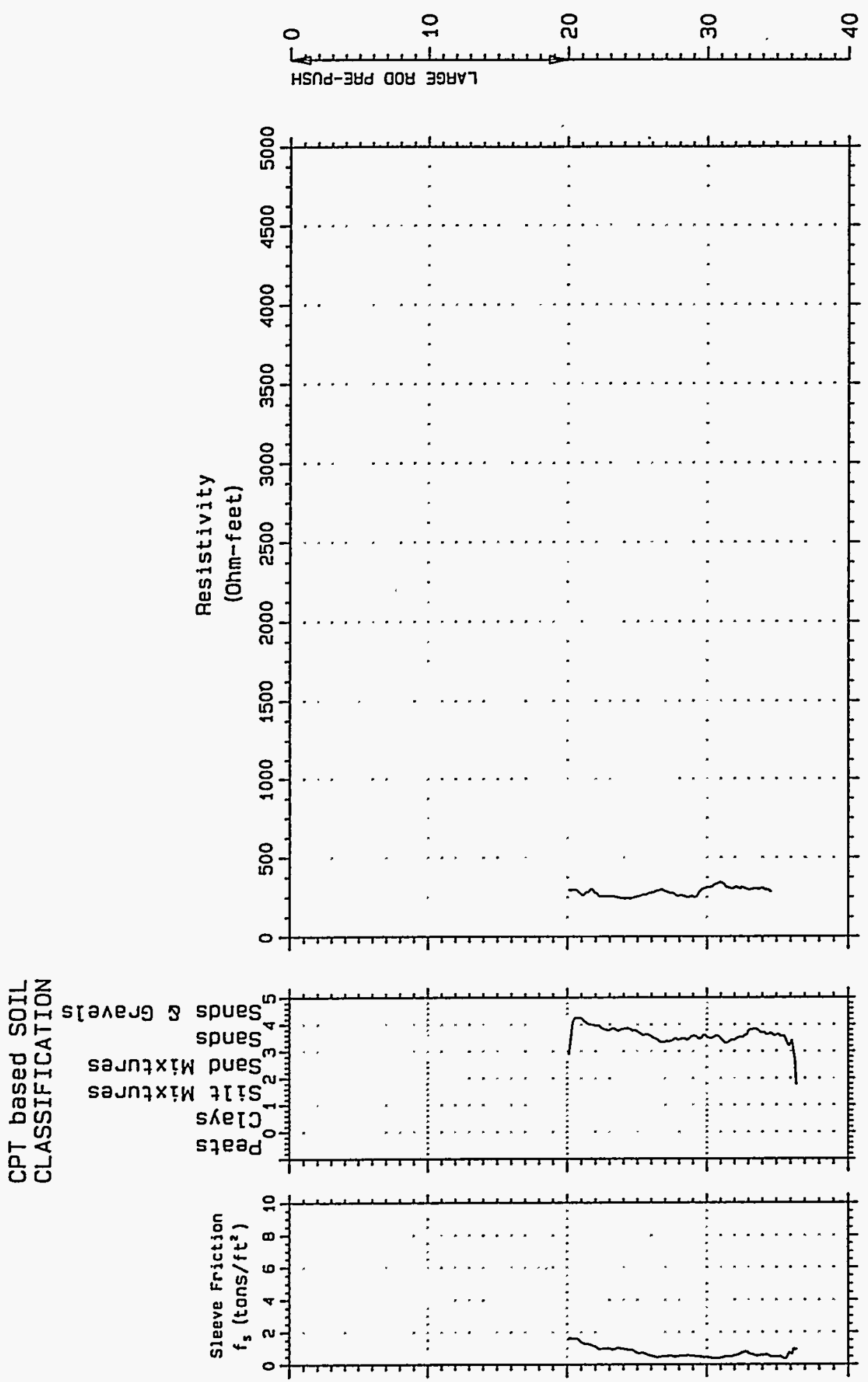

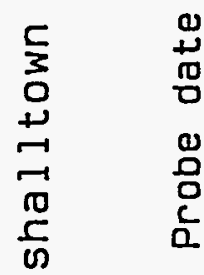

w

¿
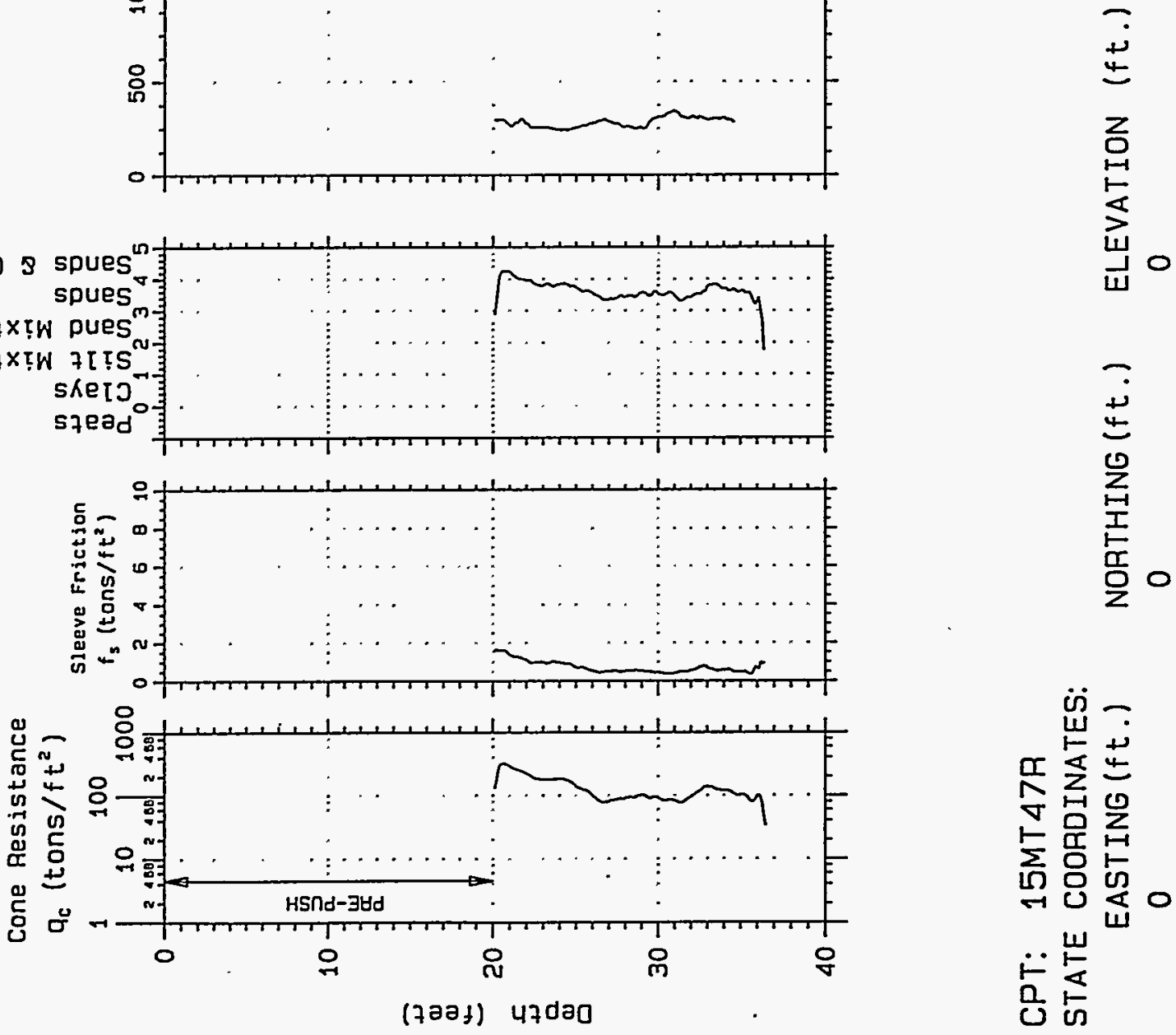

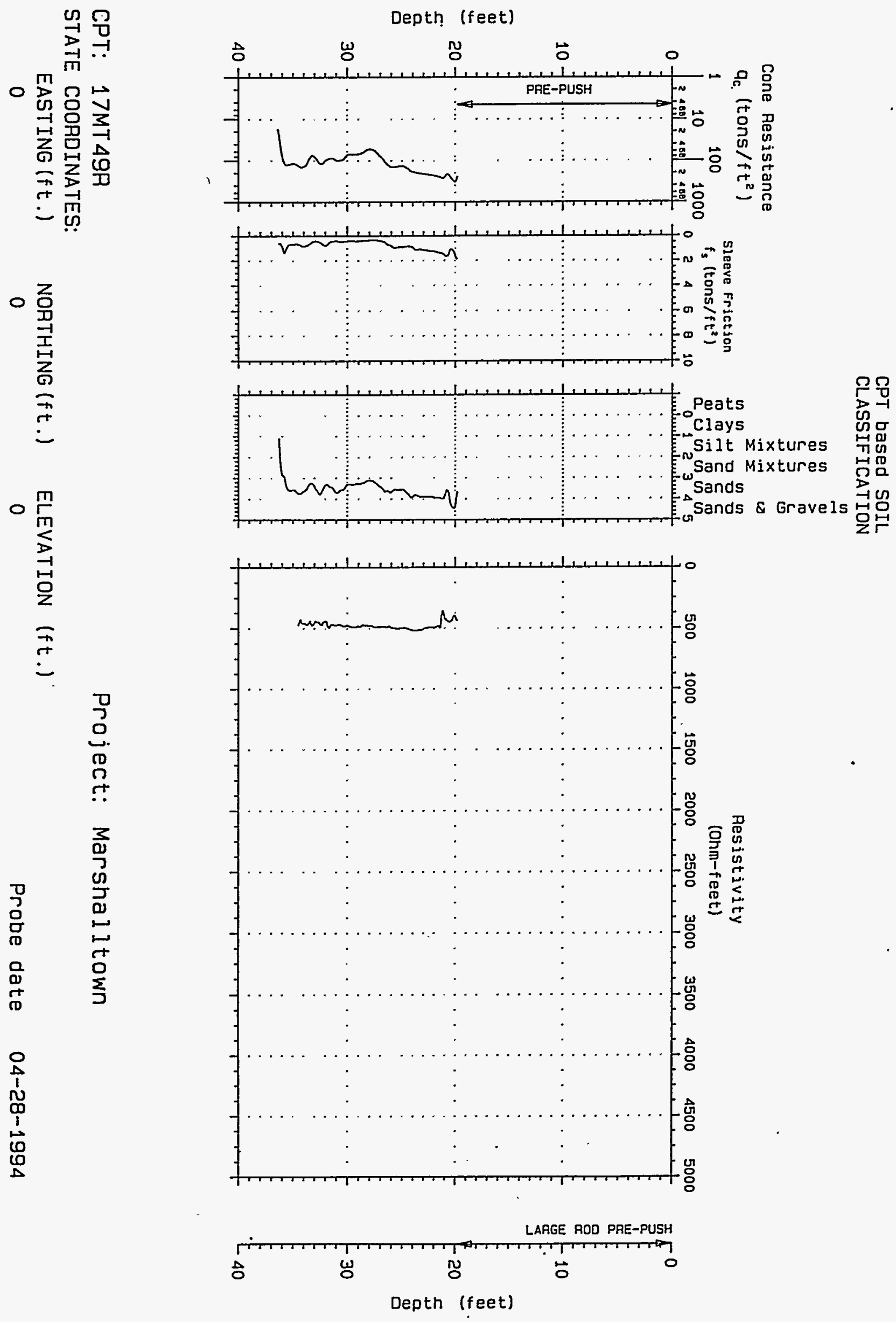
(72025) 47d20
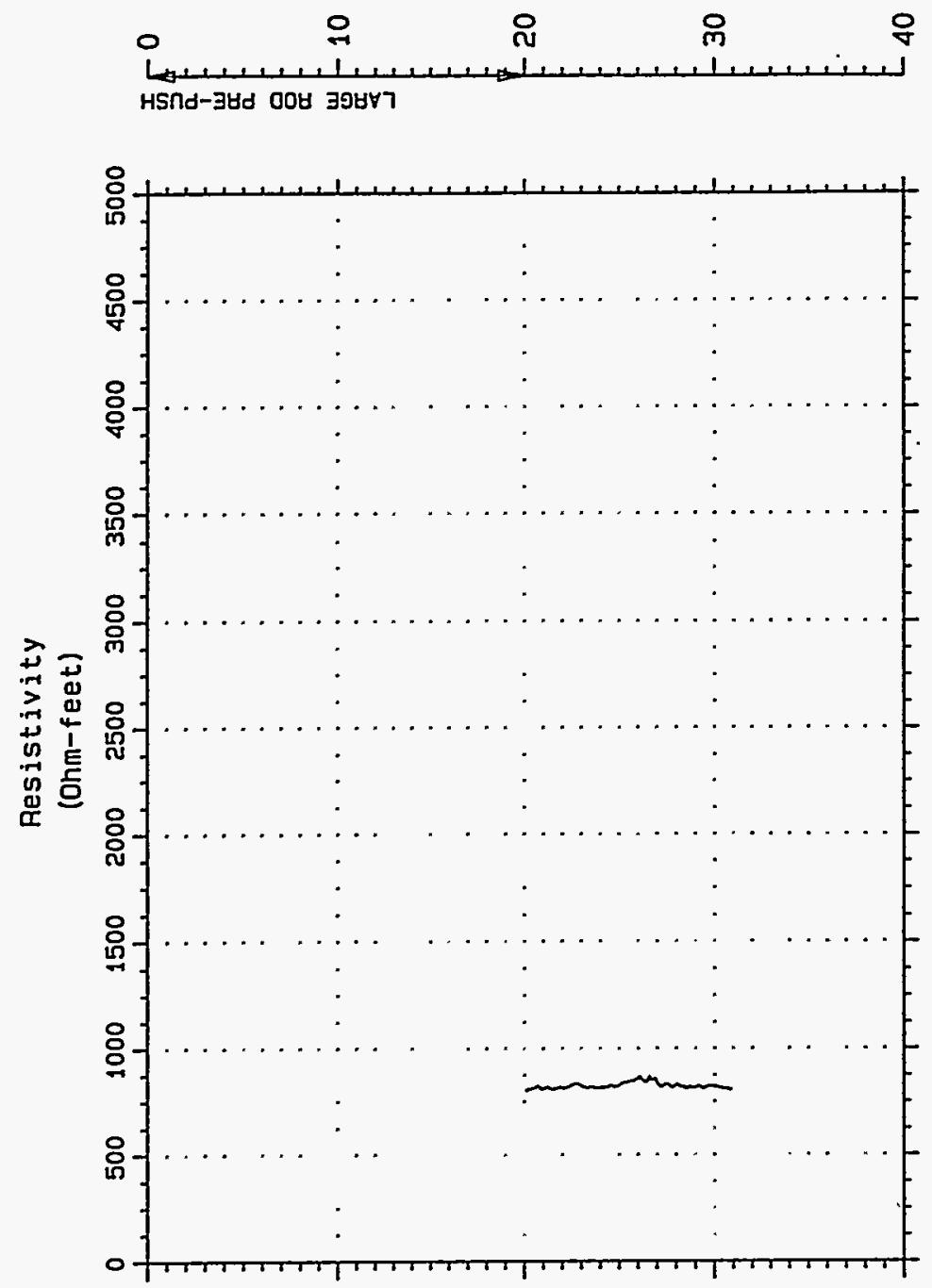

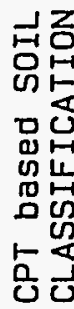
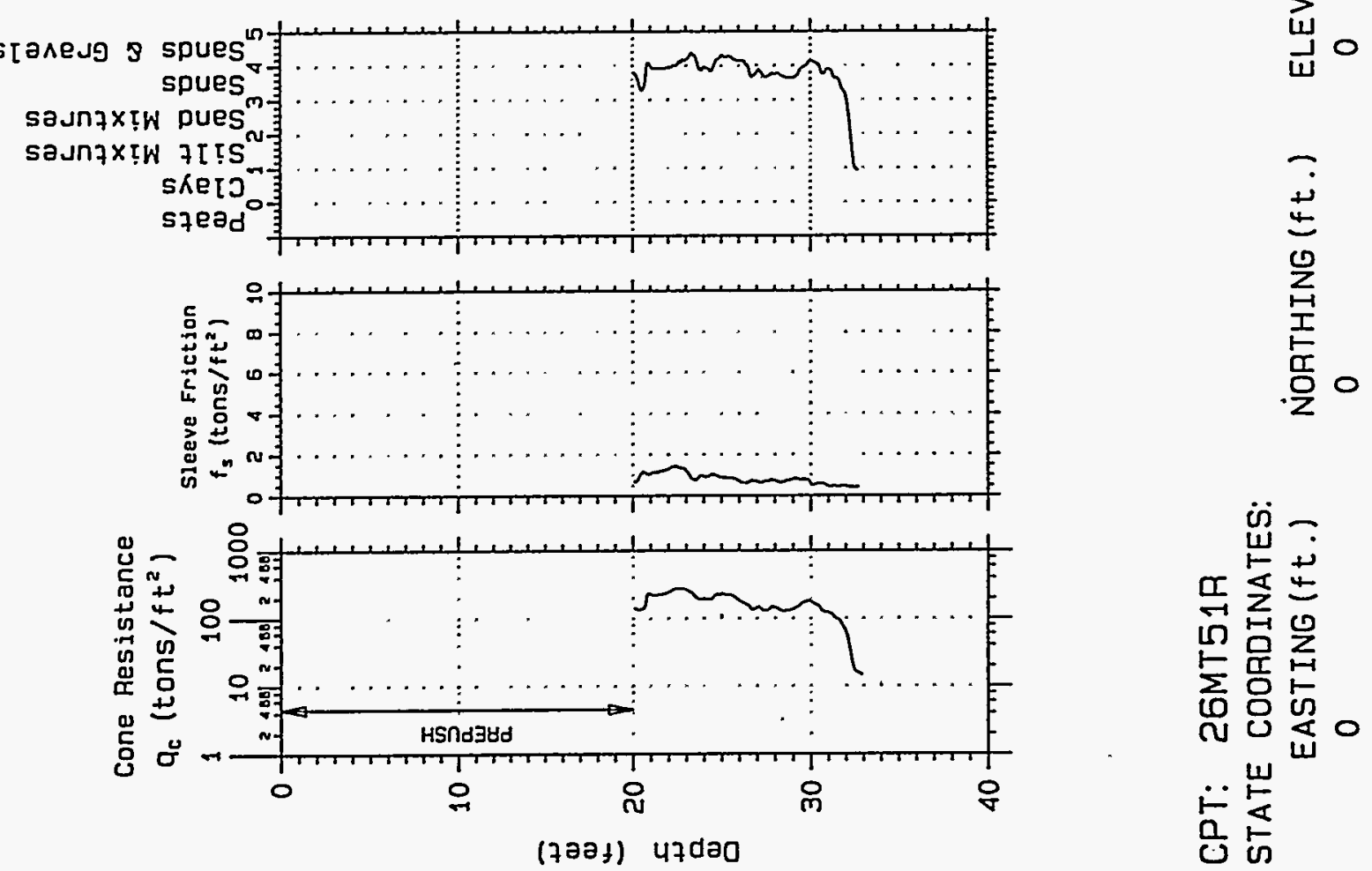

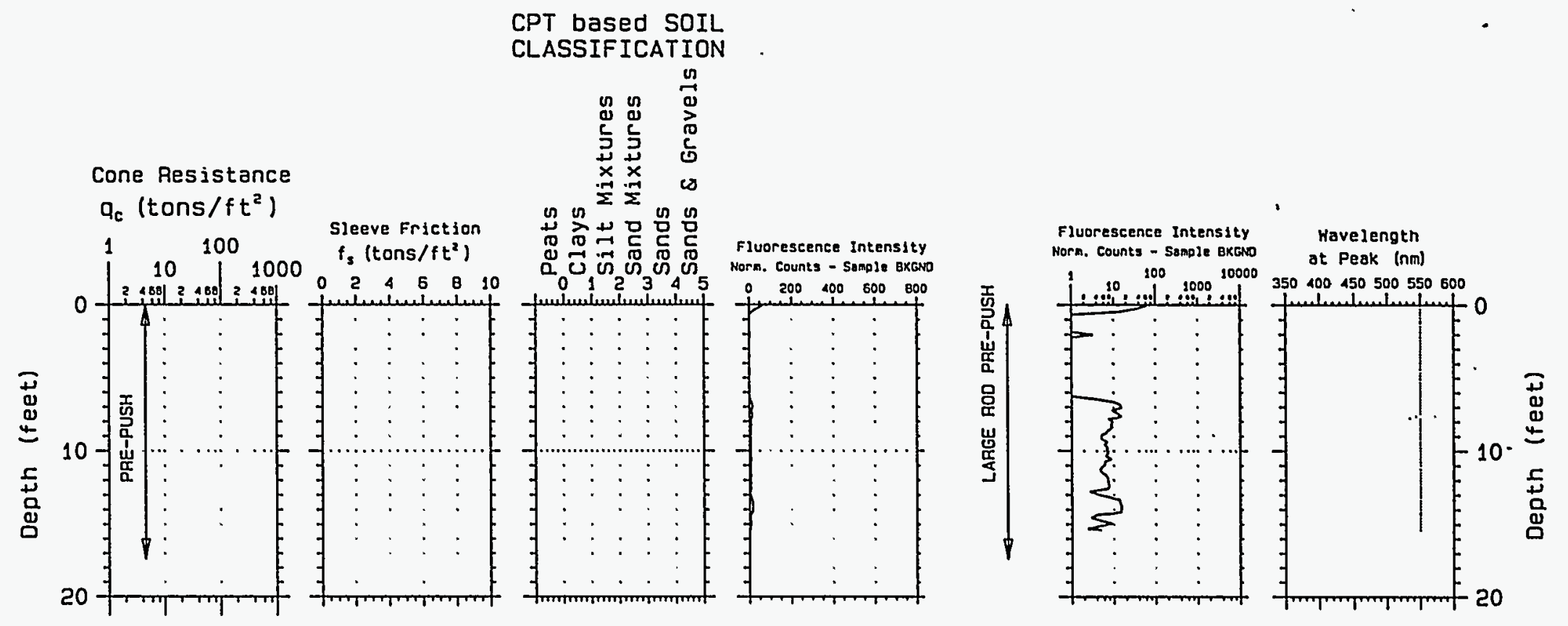

CPT: 28MT53F

Project: Marshalltown

STATE COORDINATES:

EASTING (ft.) NORTHING (ft.) ELEVATION $(f t$.

$-1$ 


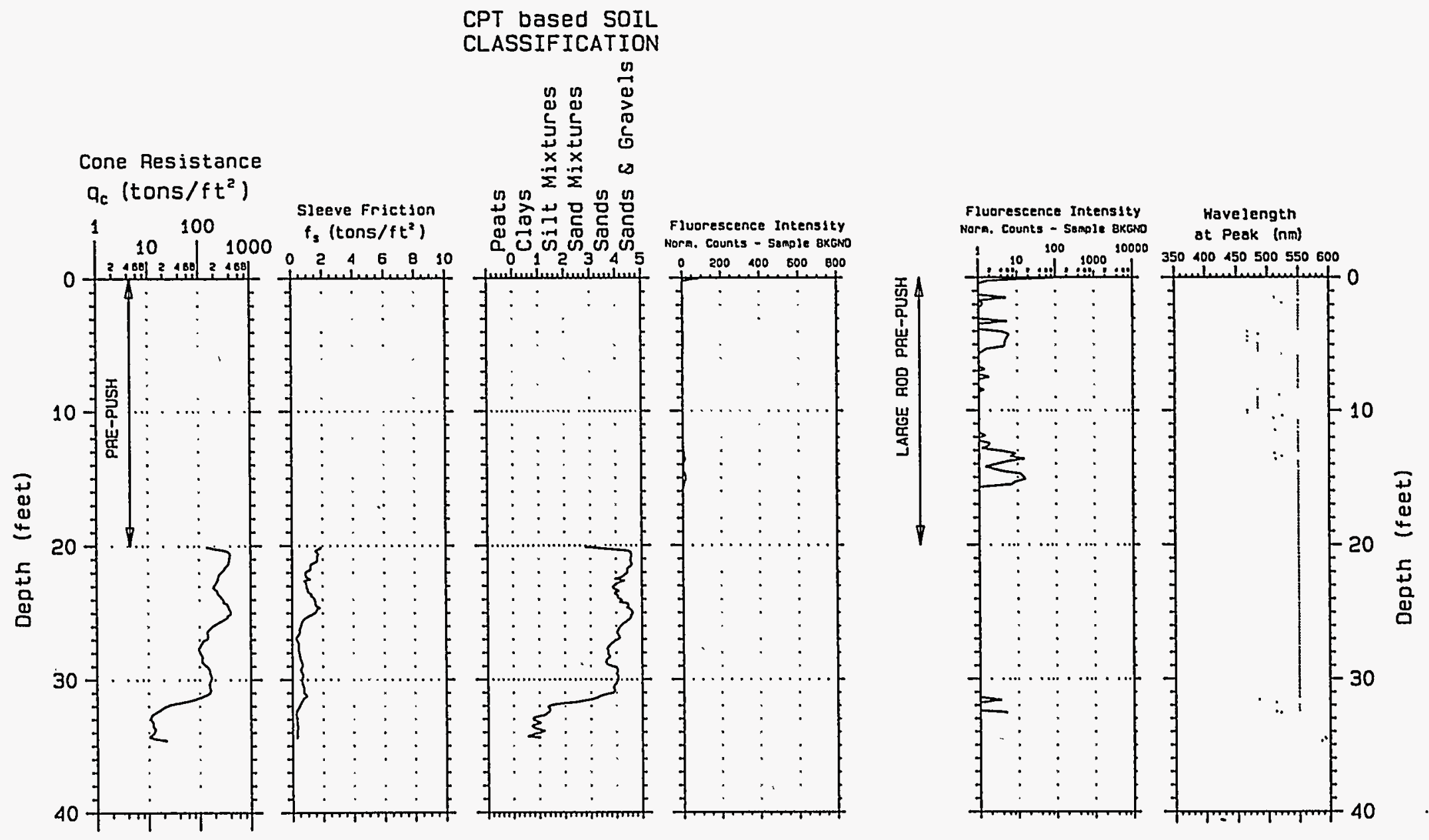

CPT: 30MT53F

Project: Marshalltown

STATE COORDINATES:

EASTING (ft.) NORTHING (ft.) ELEVATION (ft.)

$-1$

$-1$

0

Probe date

$04-29-1994$ 

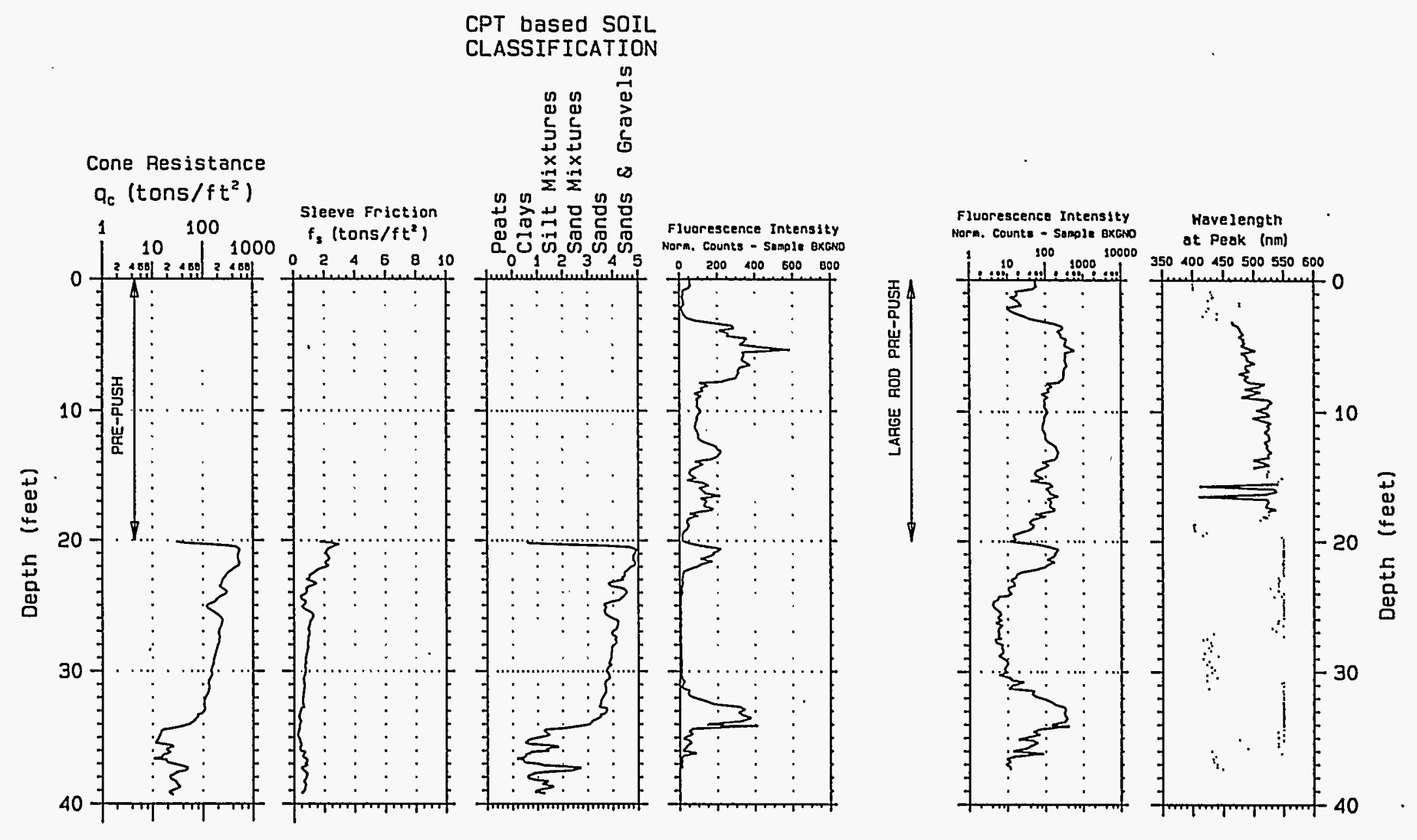

\section{CPT: 33MT60F \\ STATE COORDINATES: \\ EASTING ( $f t$.)}

$-1$
NORTHING ( $f t$.)

$-1$
Project: Marshalltown

ELEVATION (ft.) 0
Probe date 04-29-1994 


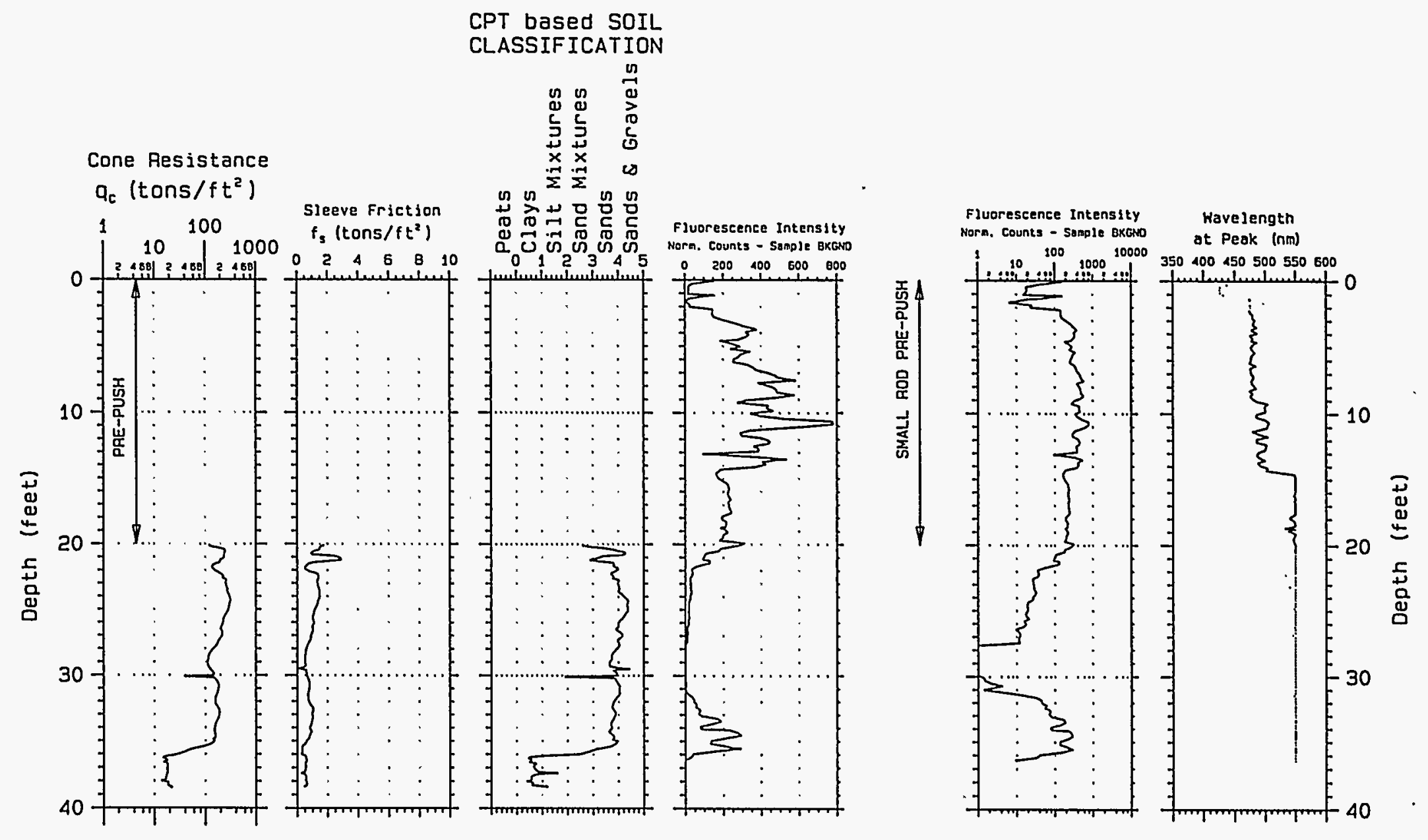

CPT: 35MT61F

Project: Marshalltown

STATE COORDINATES:

EASTING ( $f t$.)

NORTHING (ft.) ELEVATION (ft.)

$-1$

$-1$

0

Probe date

$04-29-1994$ 


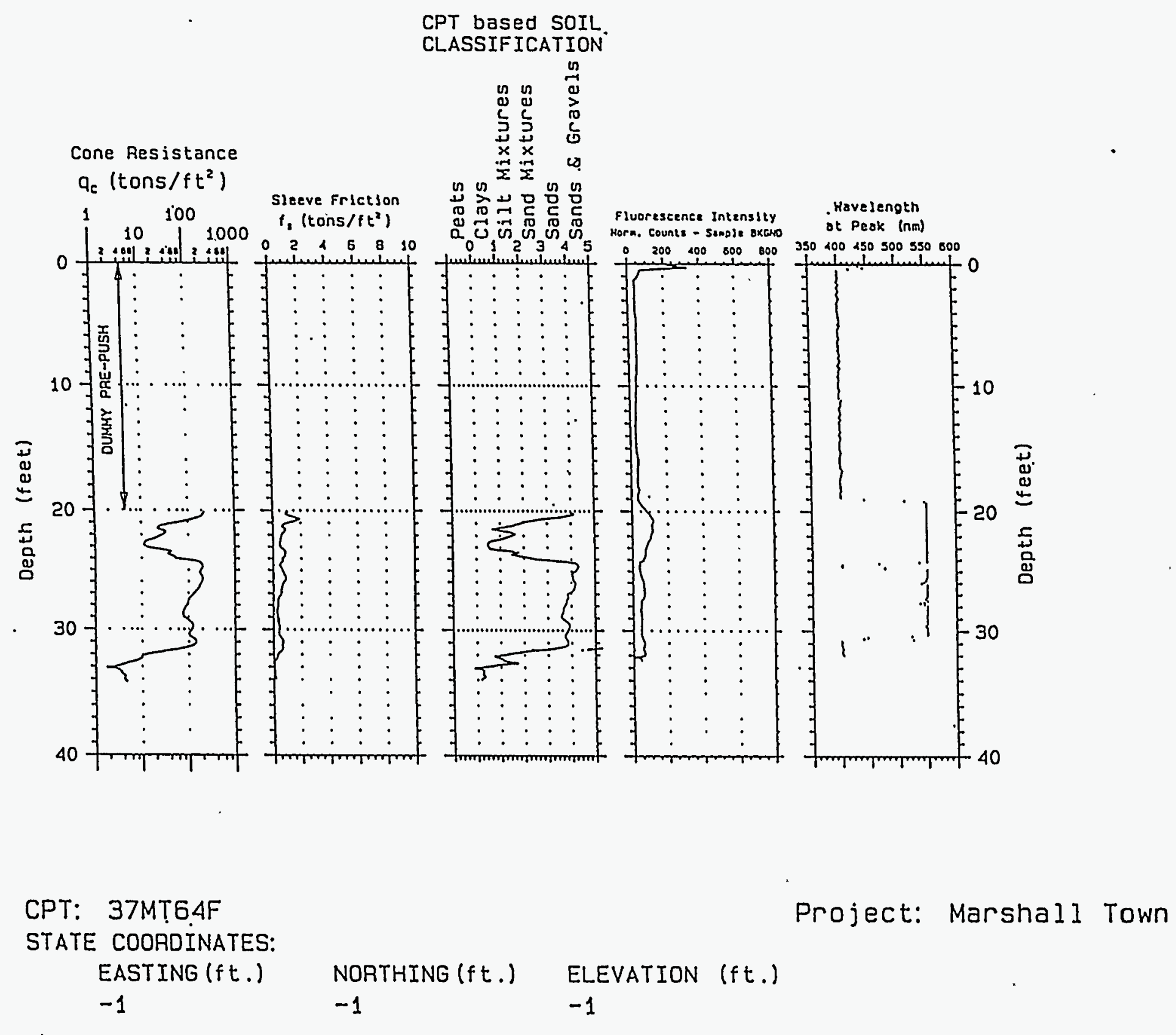



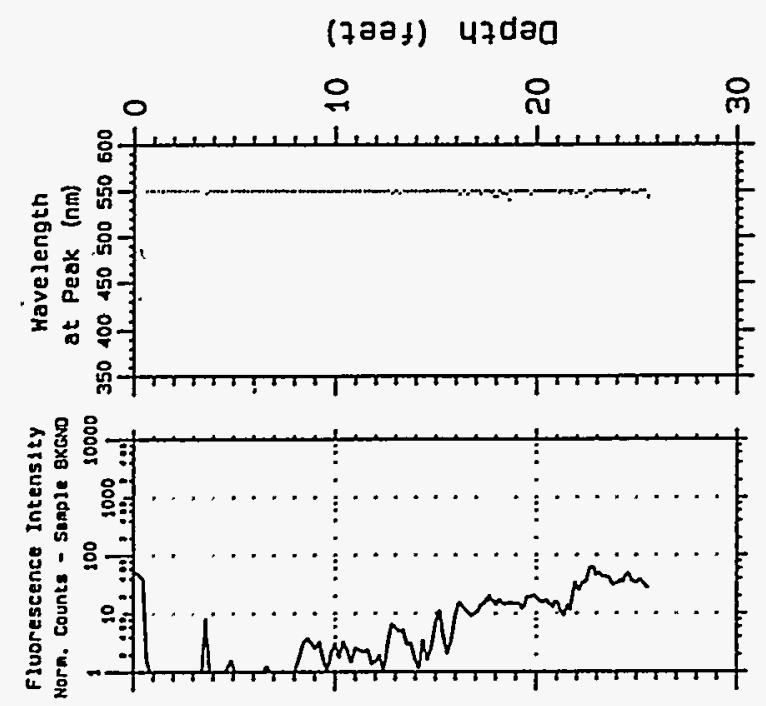

\begin{tabular}{l} 
J \\
g \\
\hdashline \\
요 \\
1 \\
j
\end{tabular}

HSกd-38d 008 าาษพS
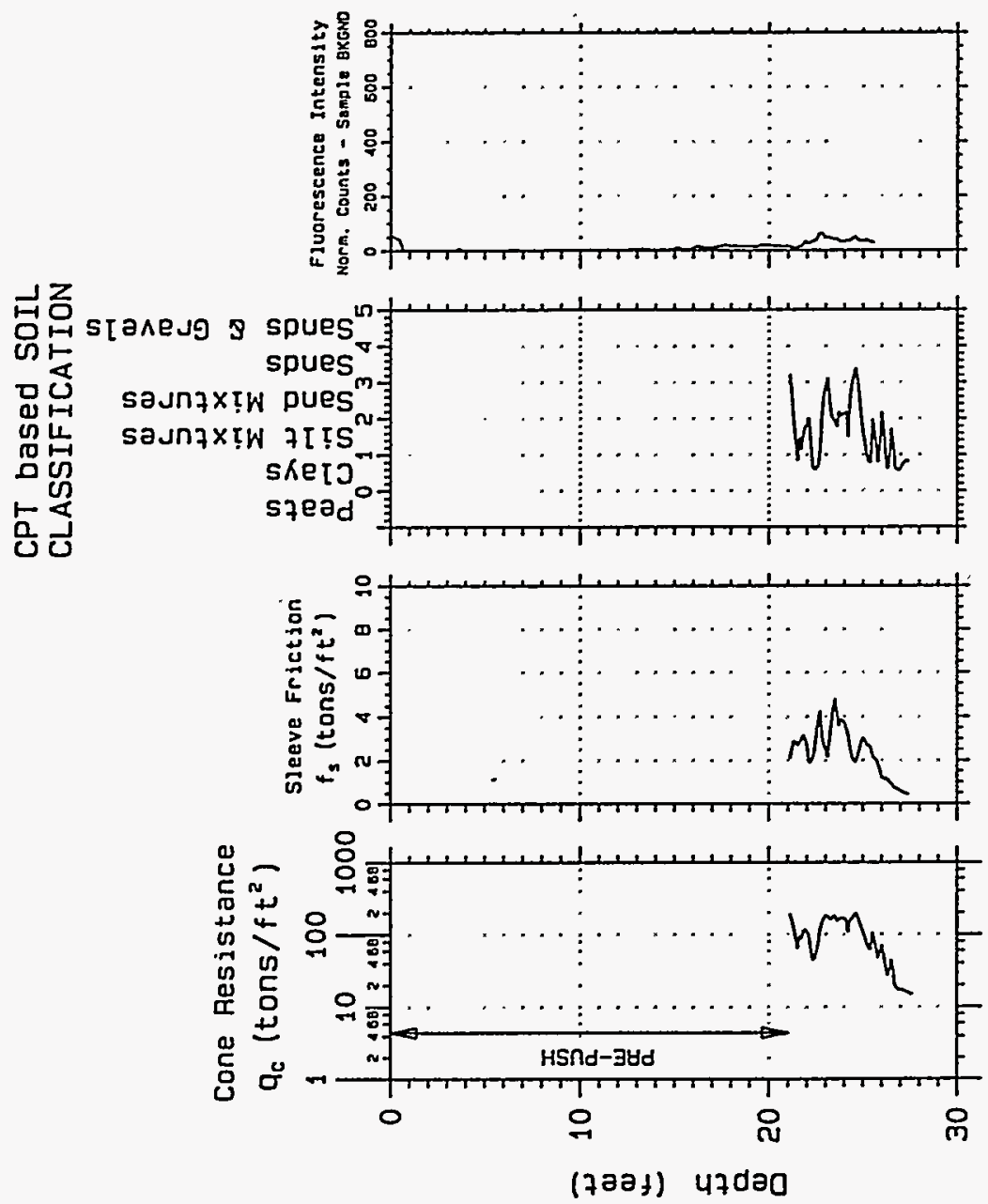

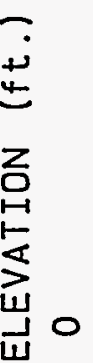
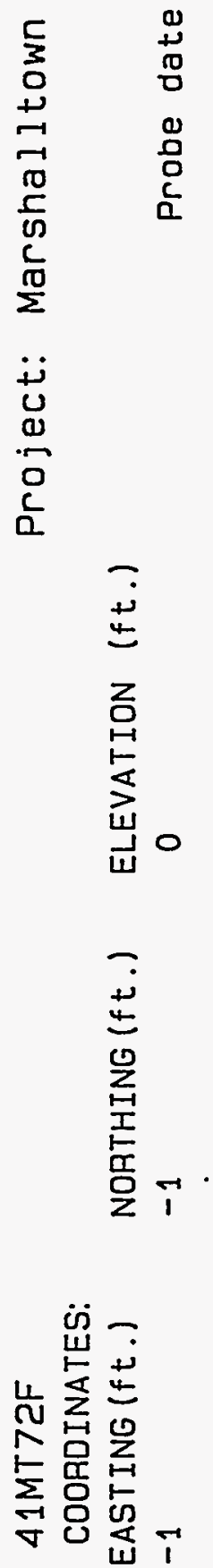

它皆 


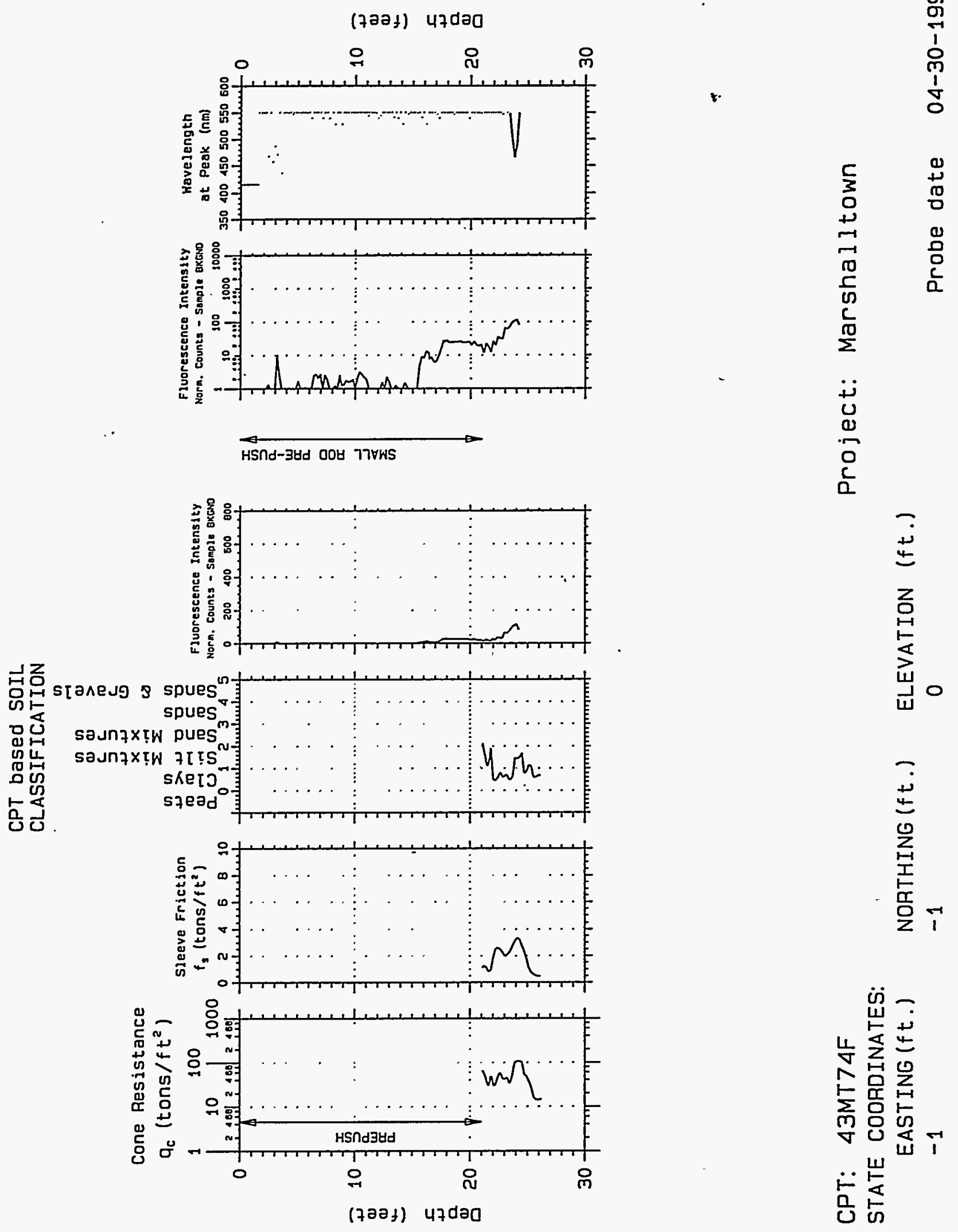



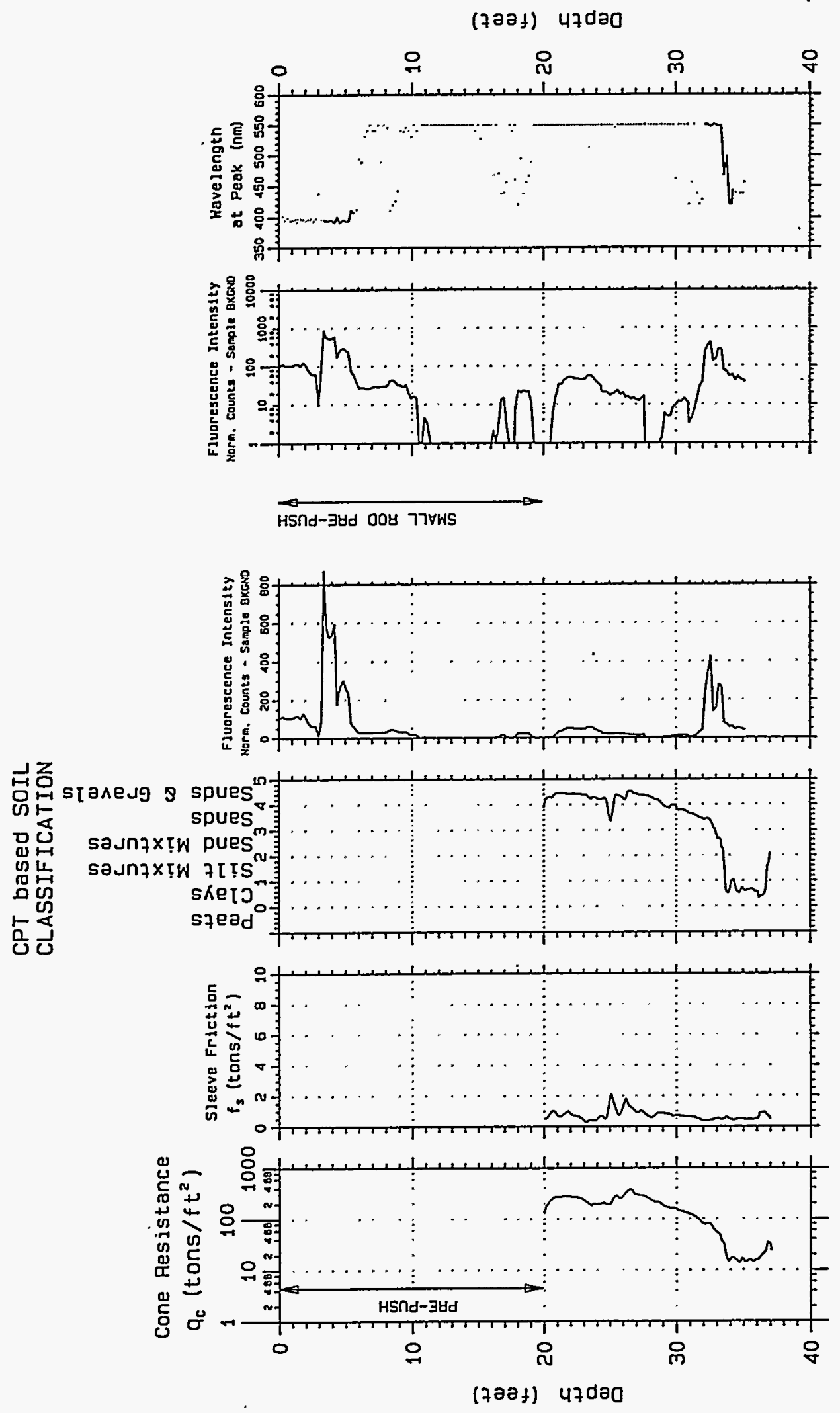

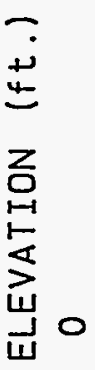
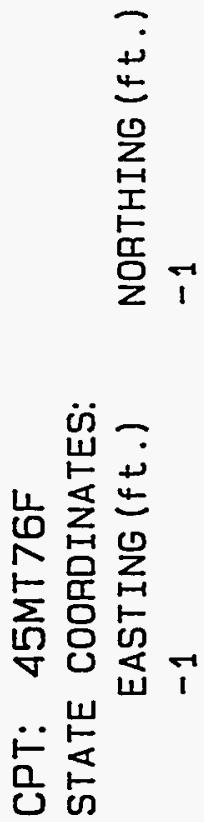


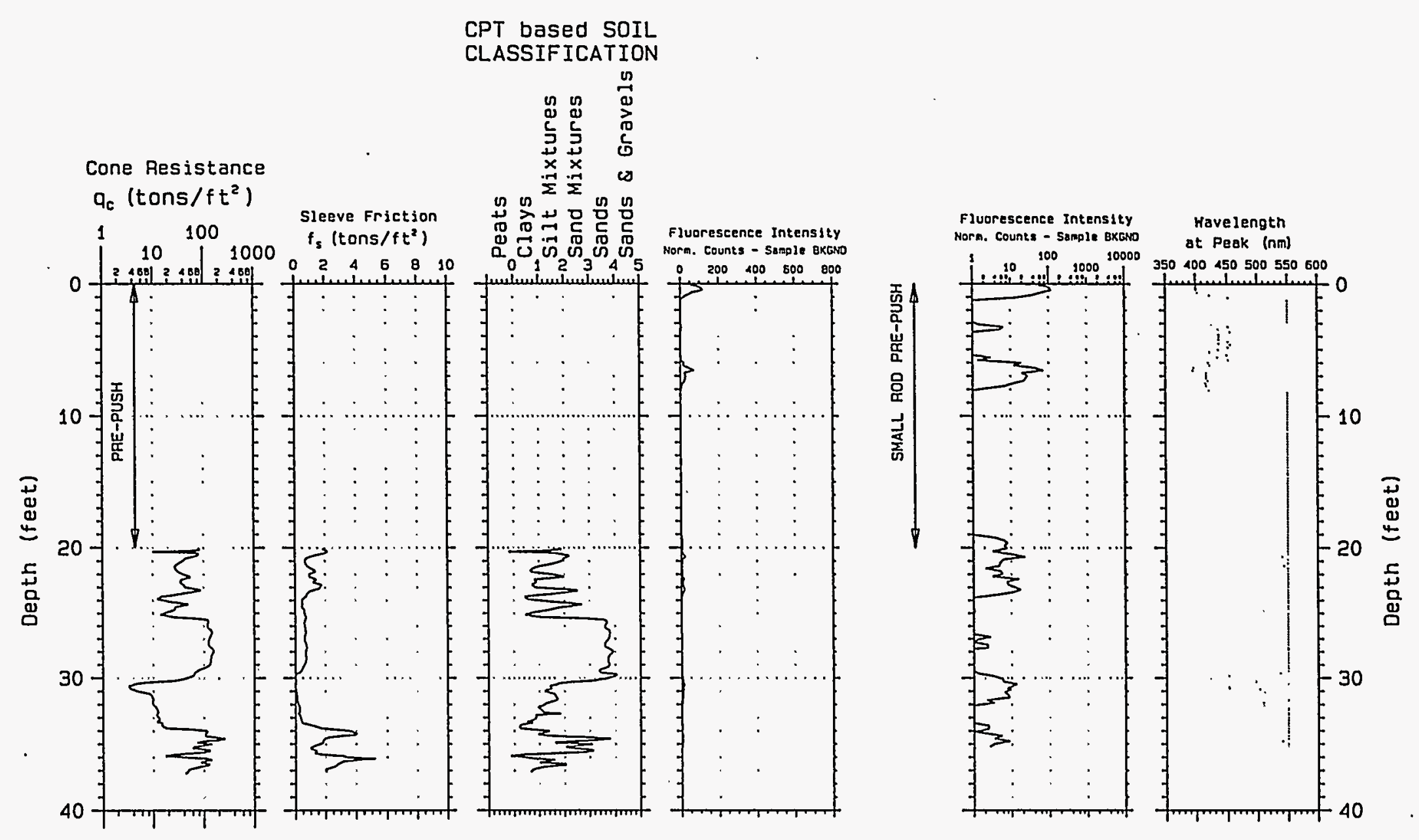

CPT: 47MT77F

STATE COORDINATES:

EASTING (ft.)

$-1$
NORTHING ( $f t$.$) ELEVATION (f t$.

$-1$
Project: Marshalltown

Probe date 04-30-1994 


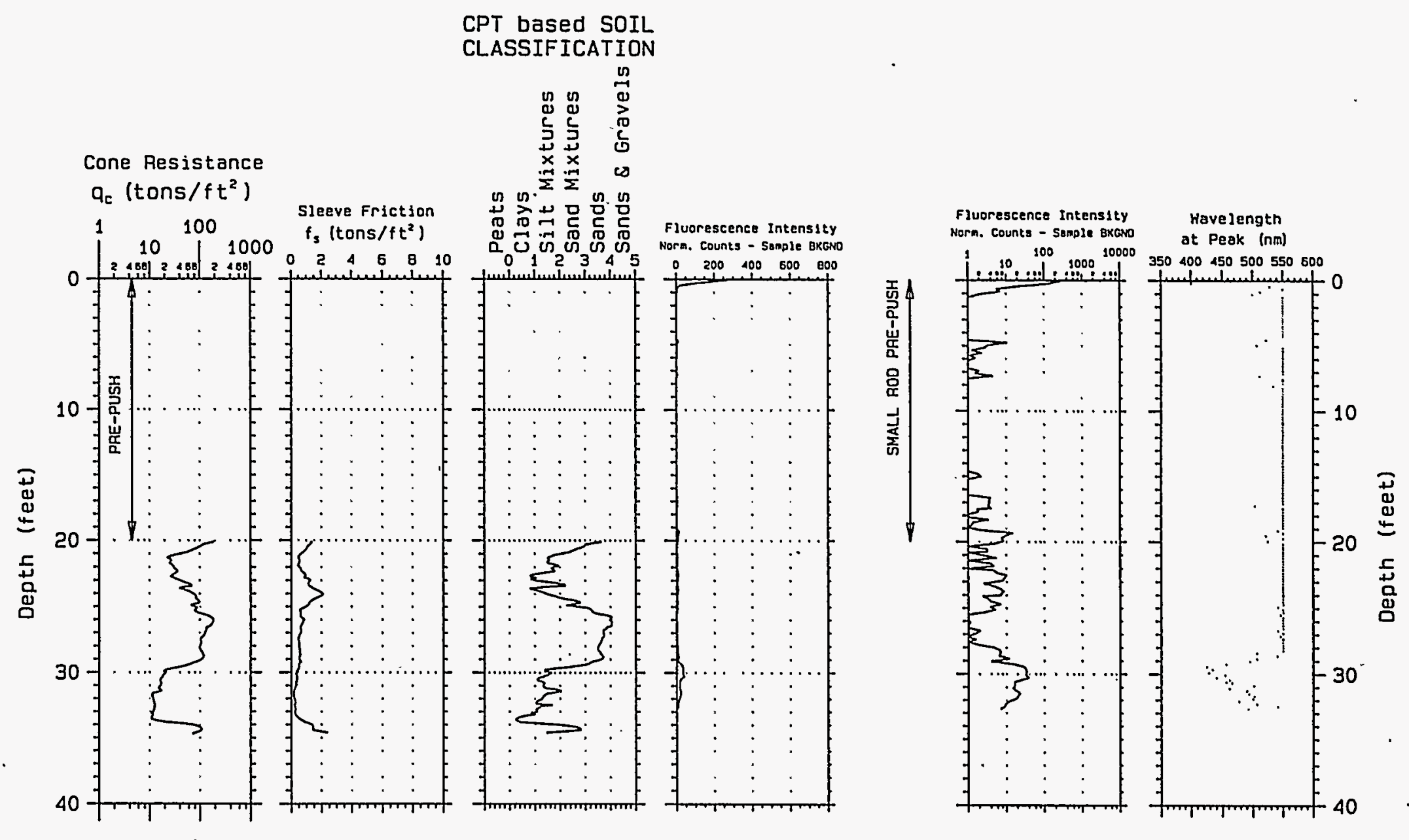

CPT: 49MT79F

Project: Marshalltown

STATE COORDINATES: EASTING $(f t$.$) NORTHING ( f t$.$) ELEVATION ( f t$.

$-1$

$-1$

0

Probe date 05-01-1994 


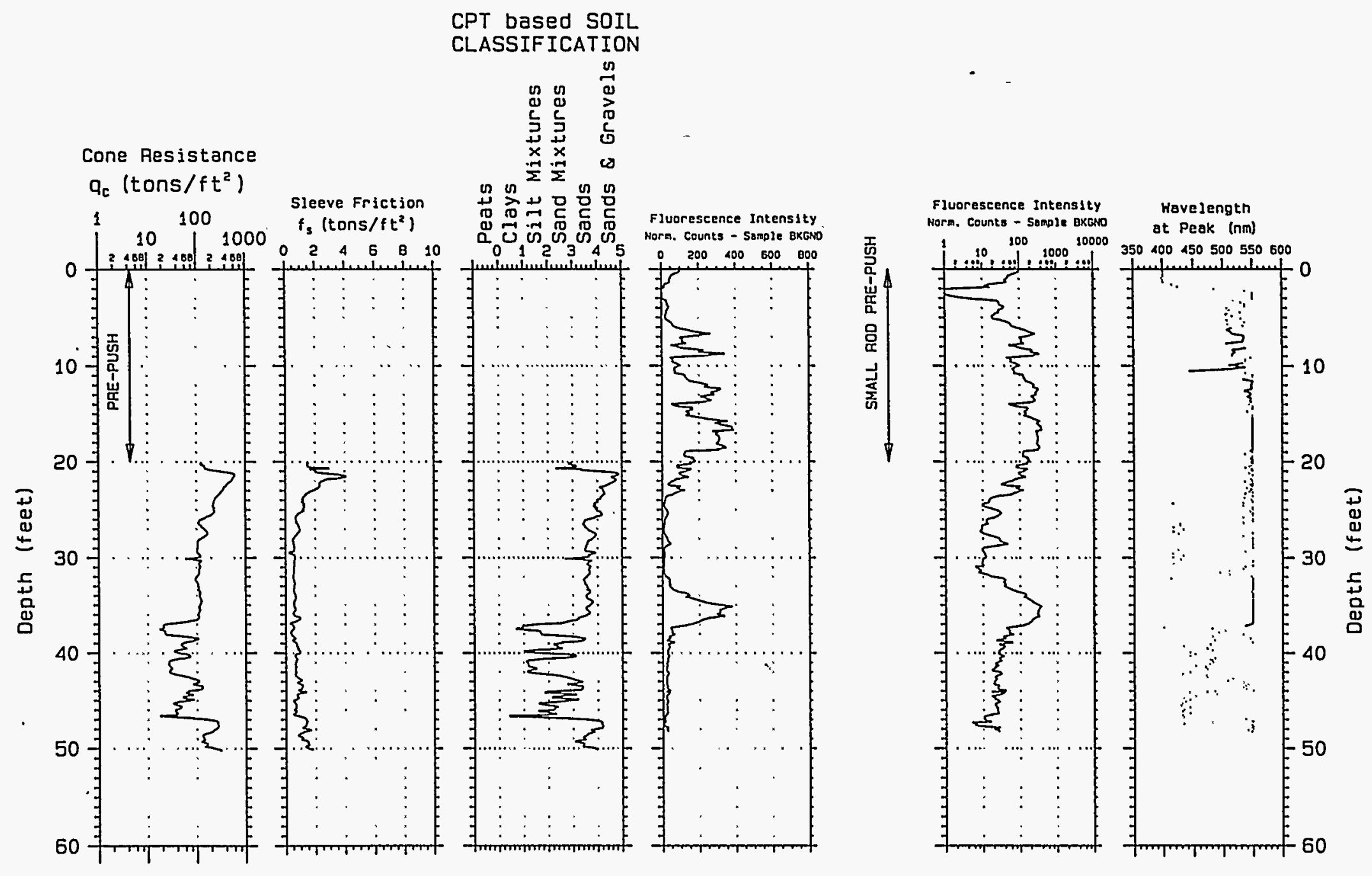

CPT: 54MT83F

Project: Marshalltown

STATE COORDINATES:

EASTING (ft.) NORTHING (ft.) ELEVATION (ft.)

$-1$

$-1$

0

Probe date 05-01-1994 


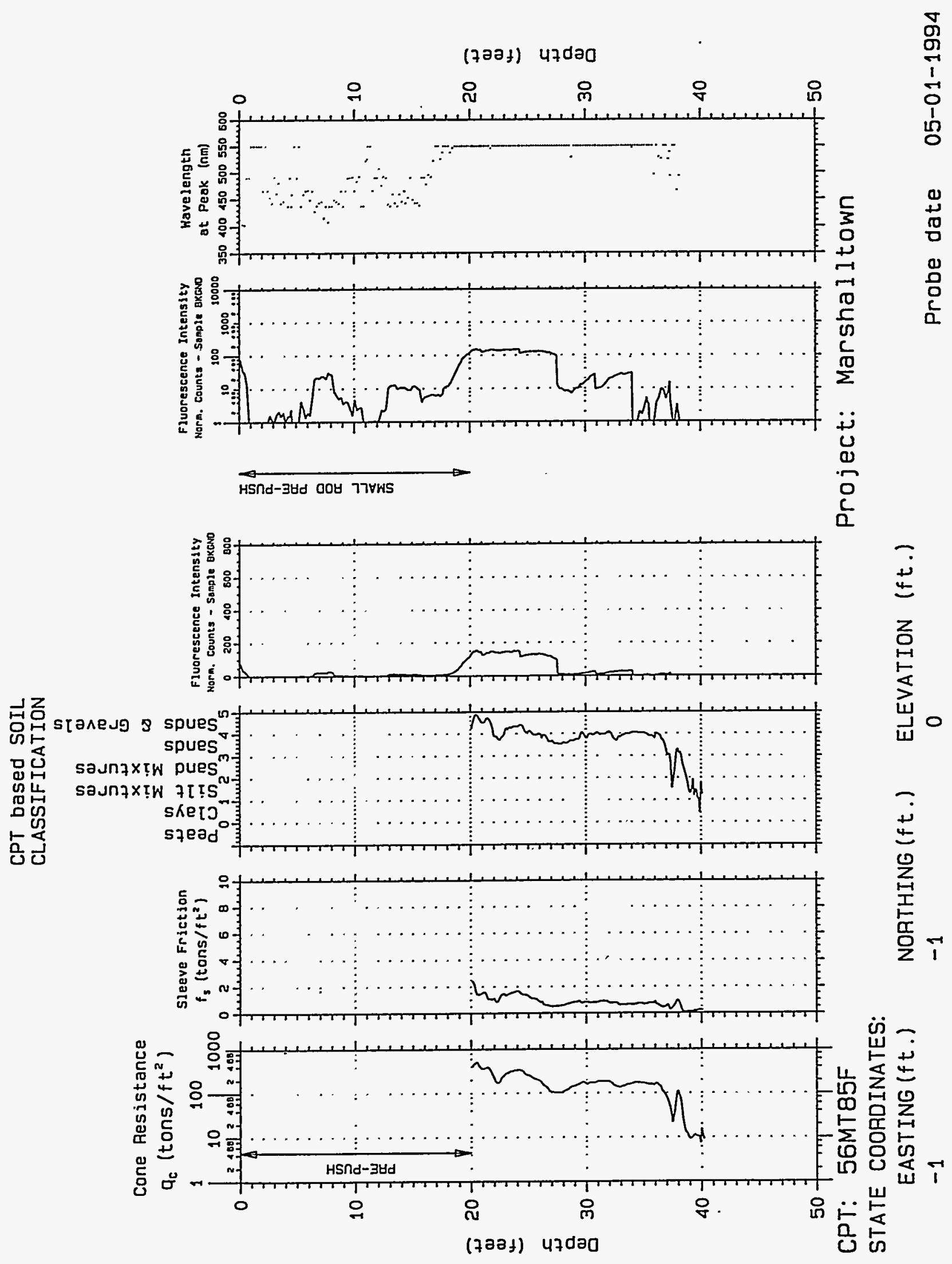


(7aa\$) 470อ0
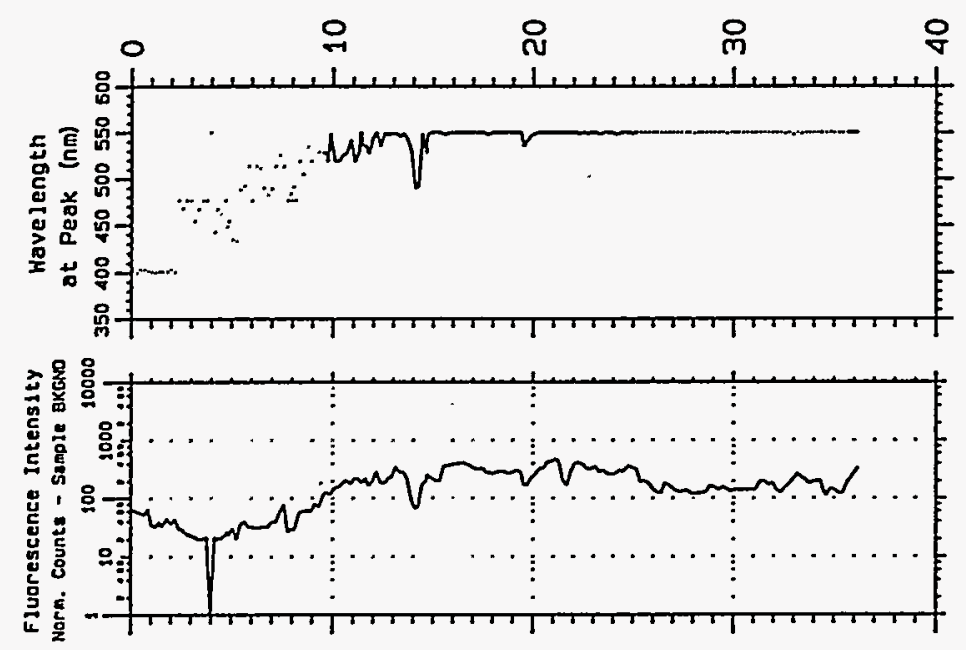

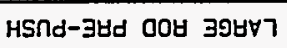
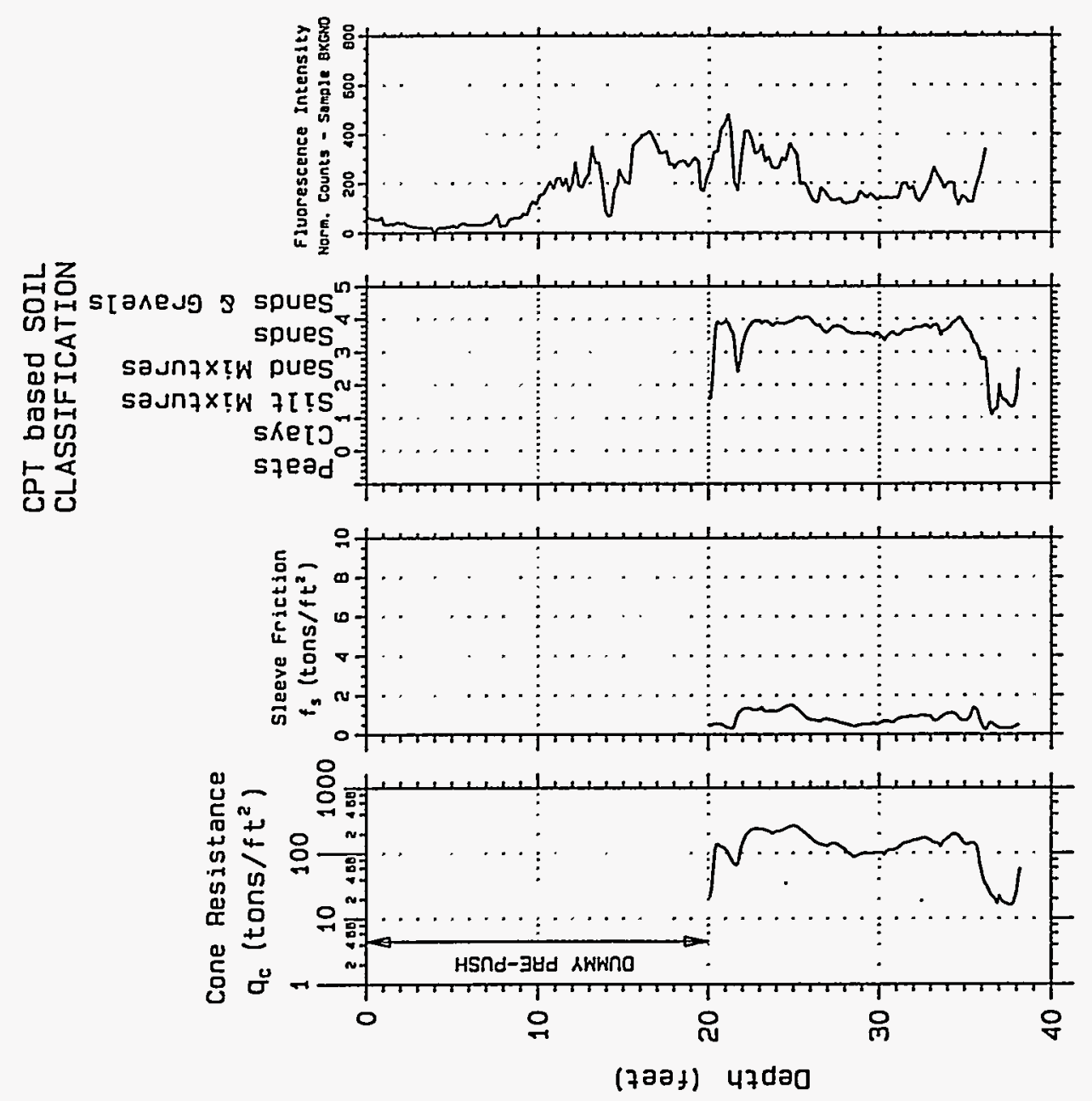

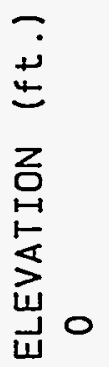

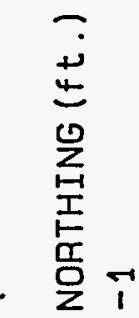

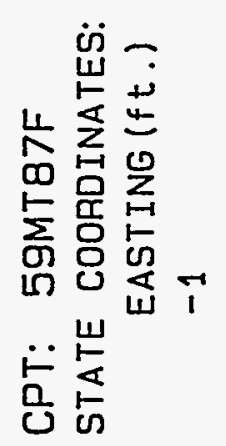


(7aaf) 47da0
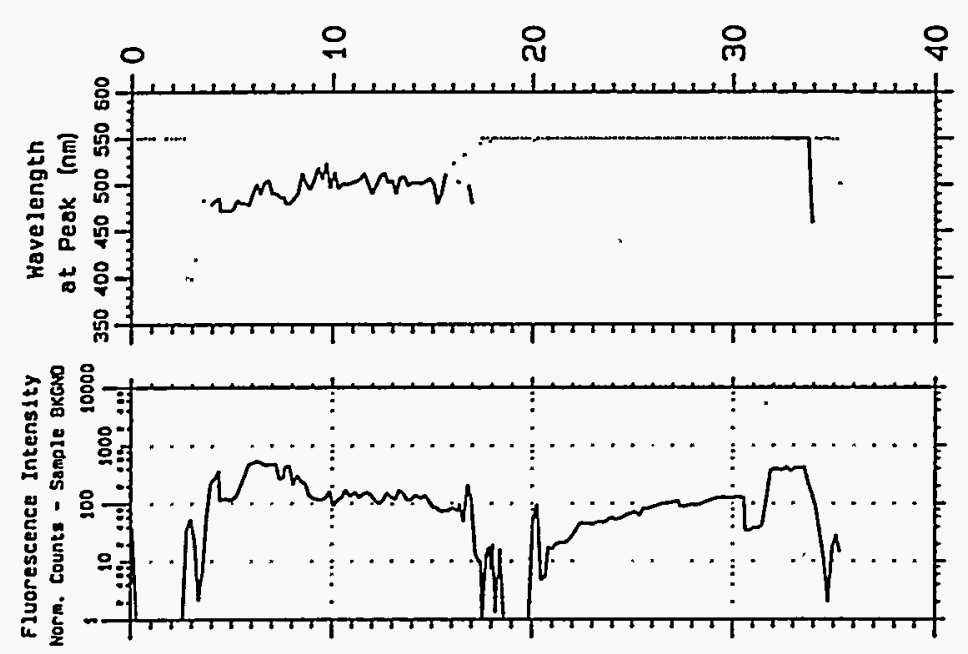

Hsกd-38d 008 394ชา
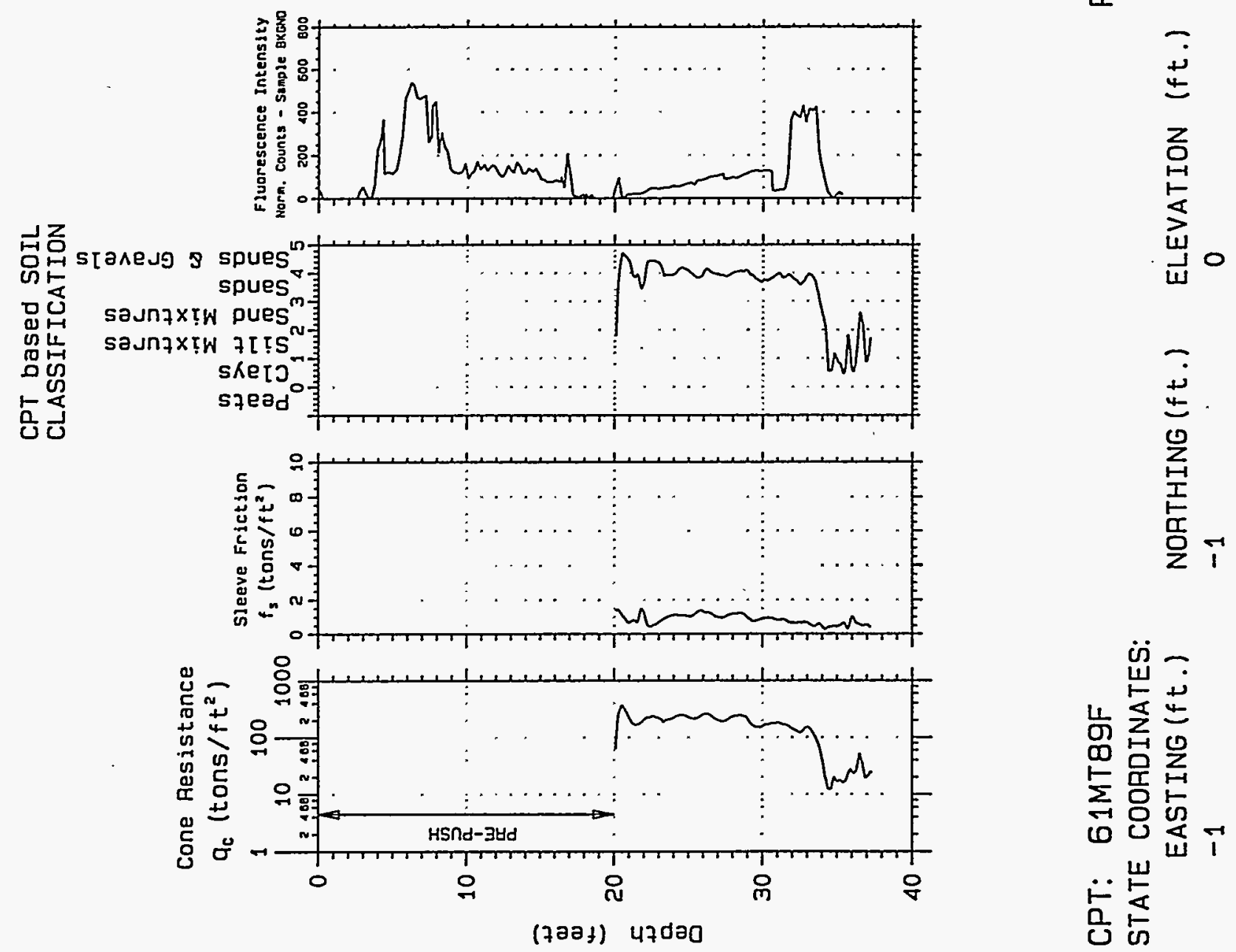


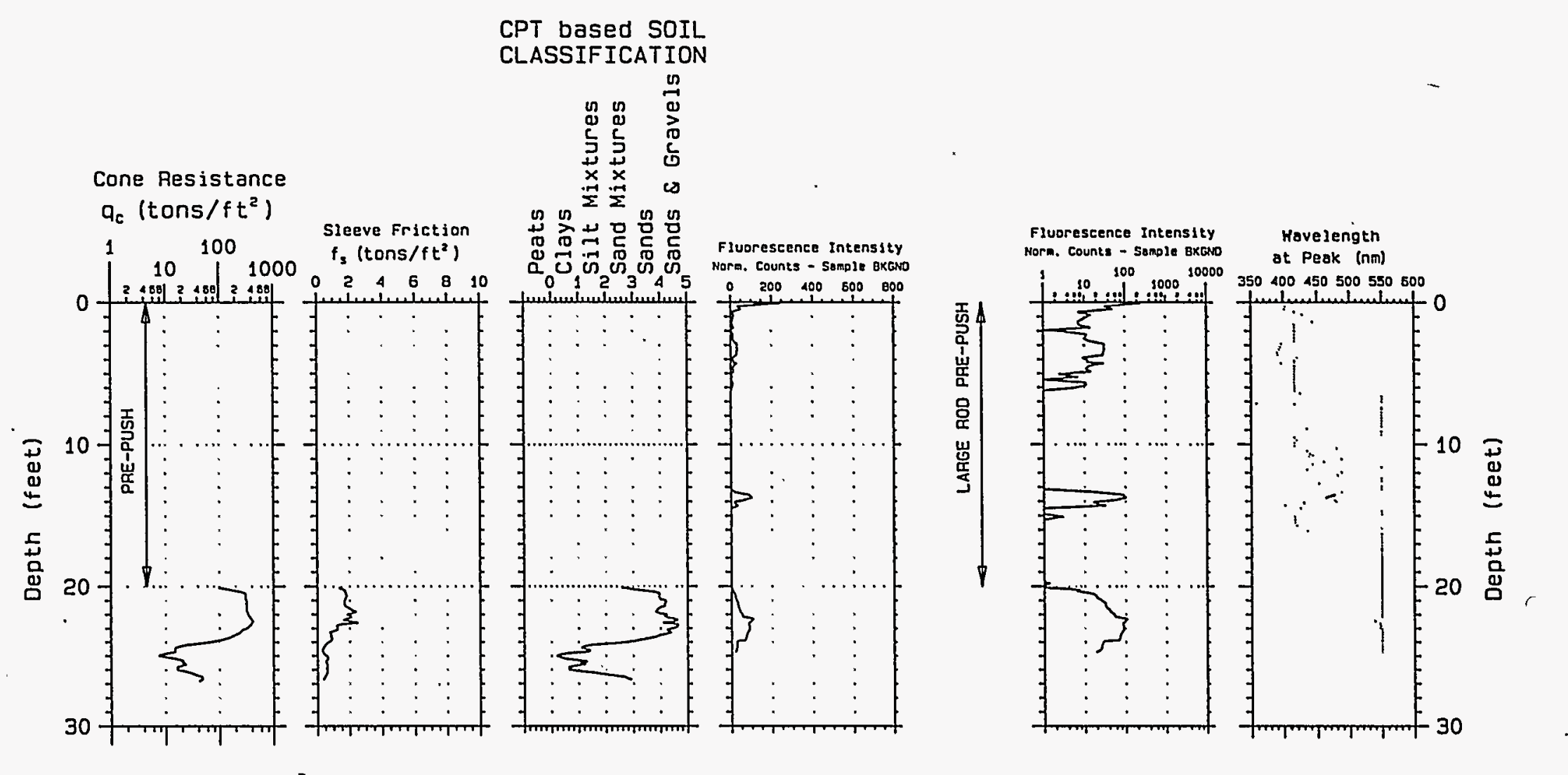

CPT: 63MT91F

Project: Marshalltown

STATE COORDINATES:

EASTING ( $f t$.$) NORTHING (f t$.$) ELEVATION (f t$.

$-1$

$-1$

0

Probe date 05-02-1994 


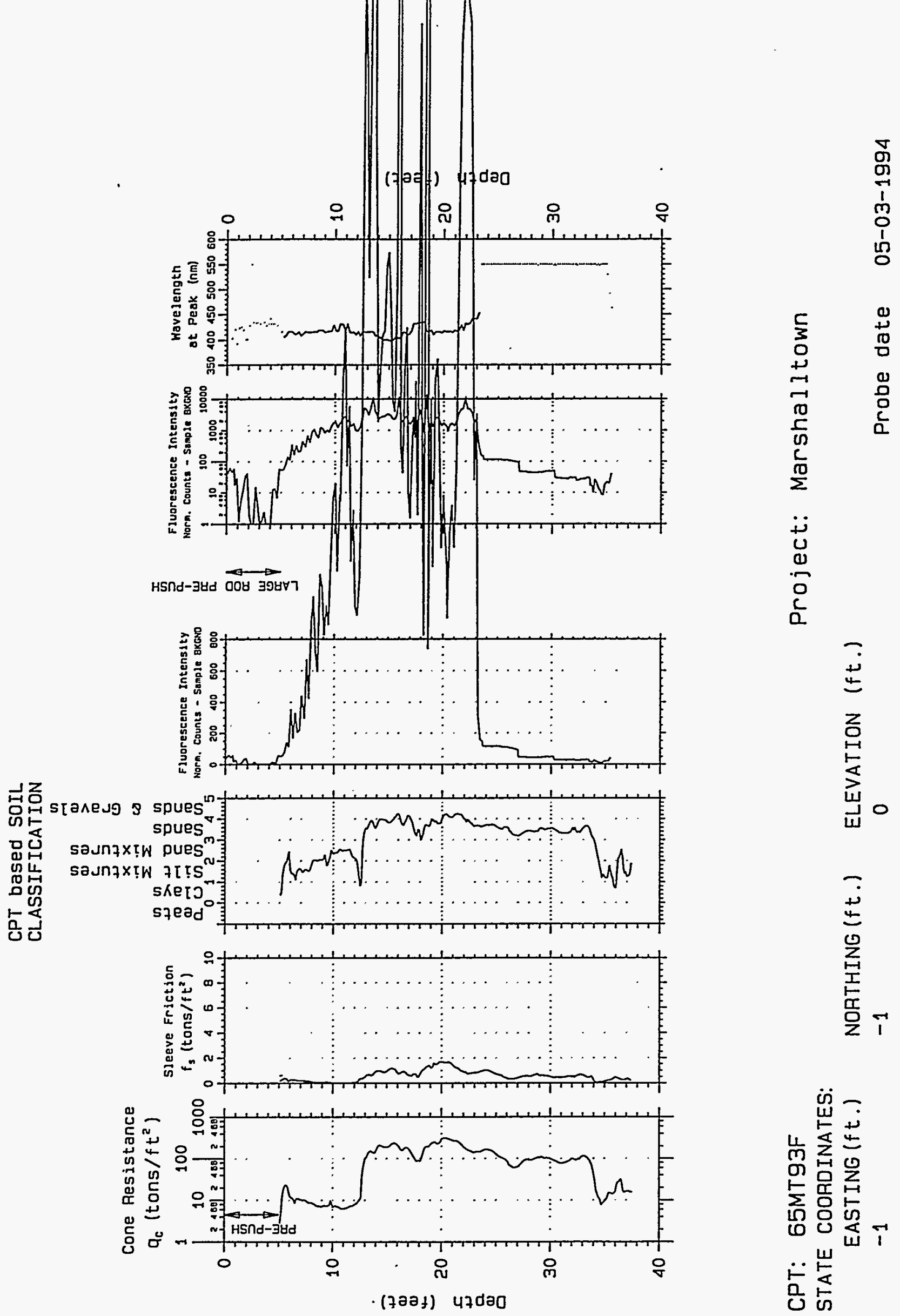



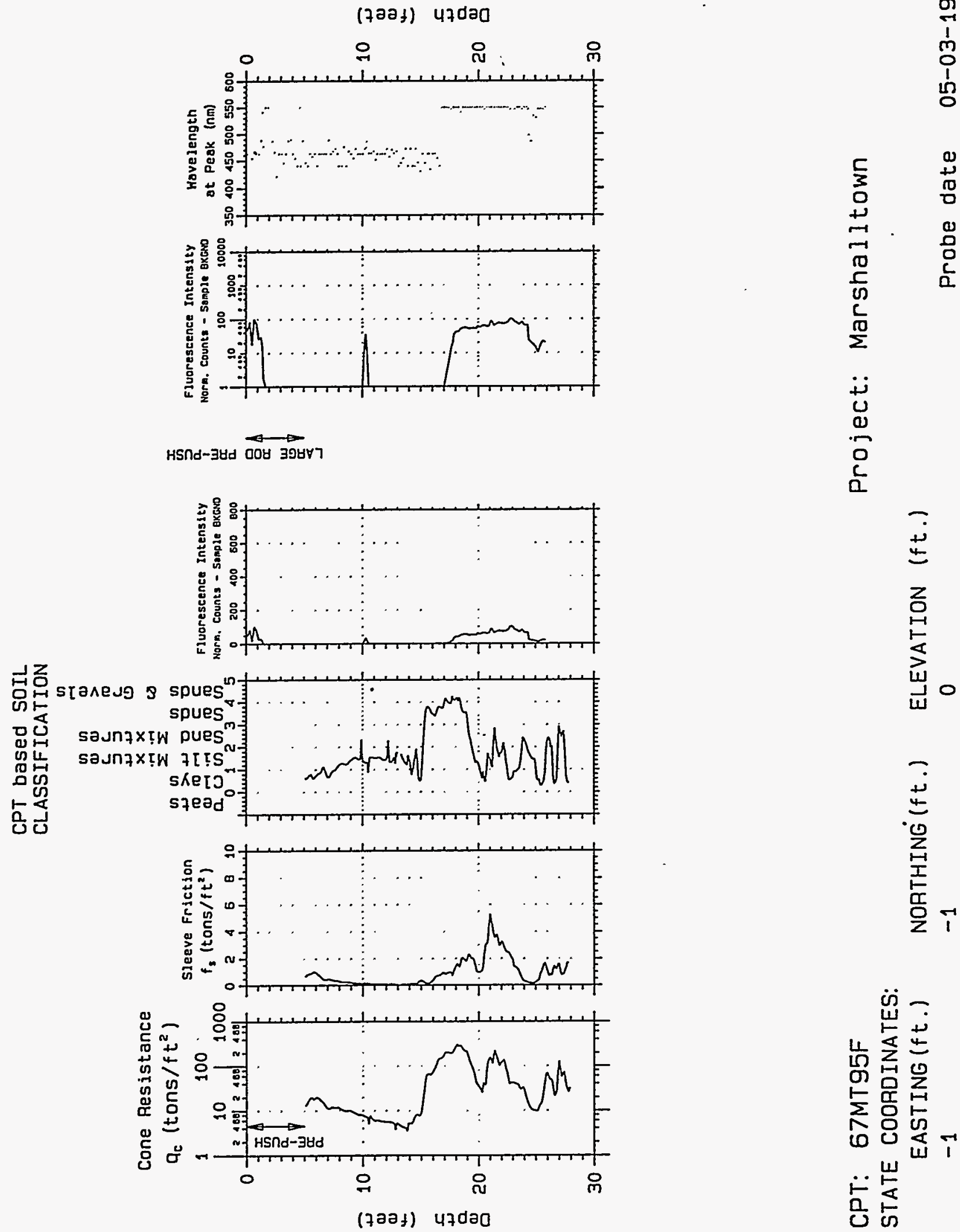


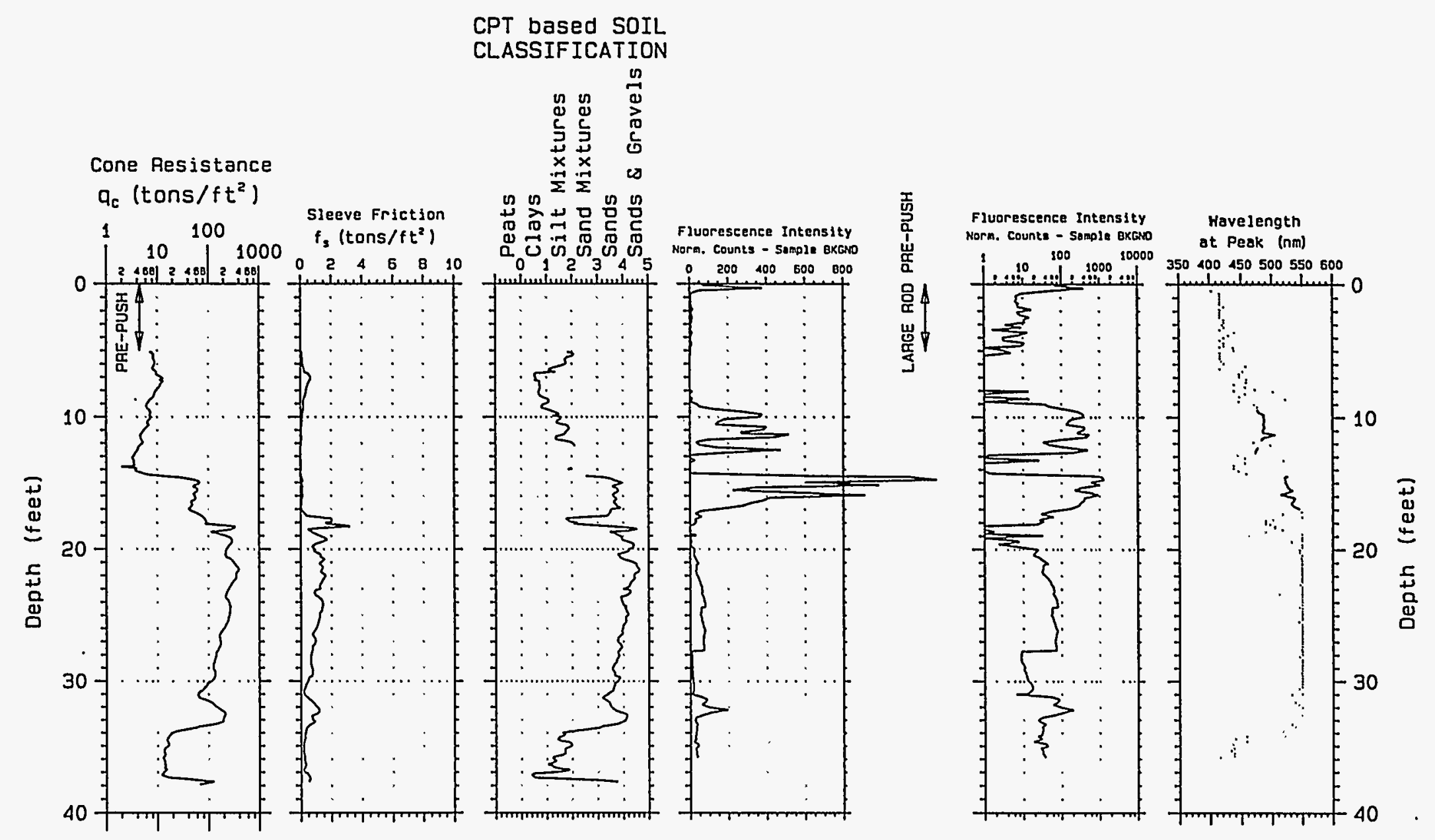

CPT: 73MT97F

STATE COORDINATES:

EASTING ( $\mathrm{ft}$. )

$-1$
NORTHING ( $f t$.)

$-1$
Project: Marshalltown <NEW>

ELEVATION ( $\mathrm{ft}$.

0
Probe date

05-03-1994 


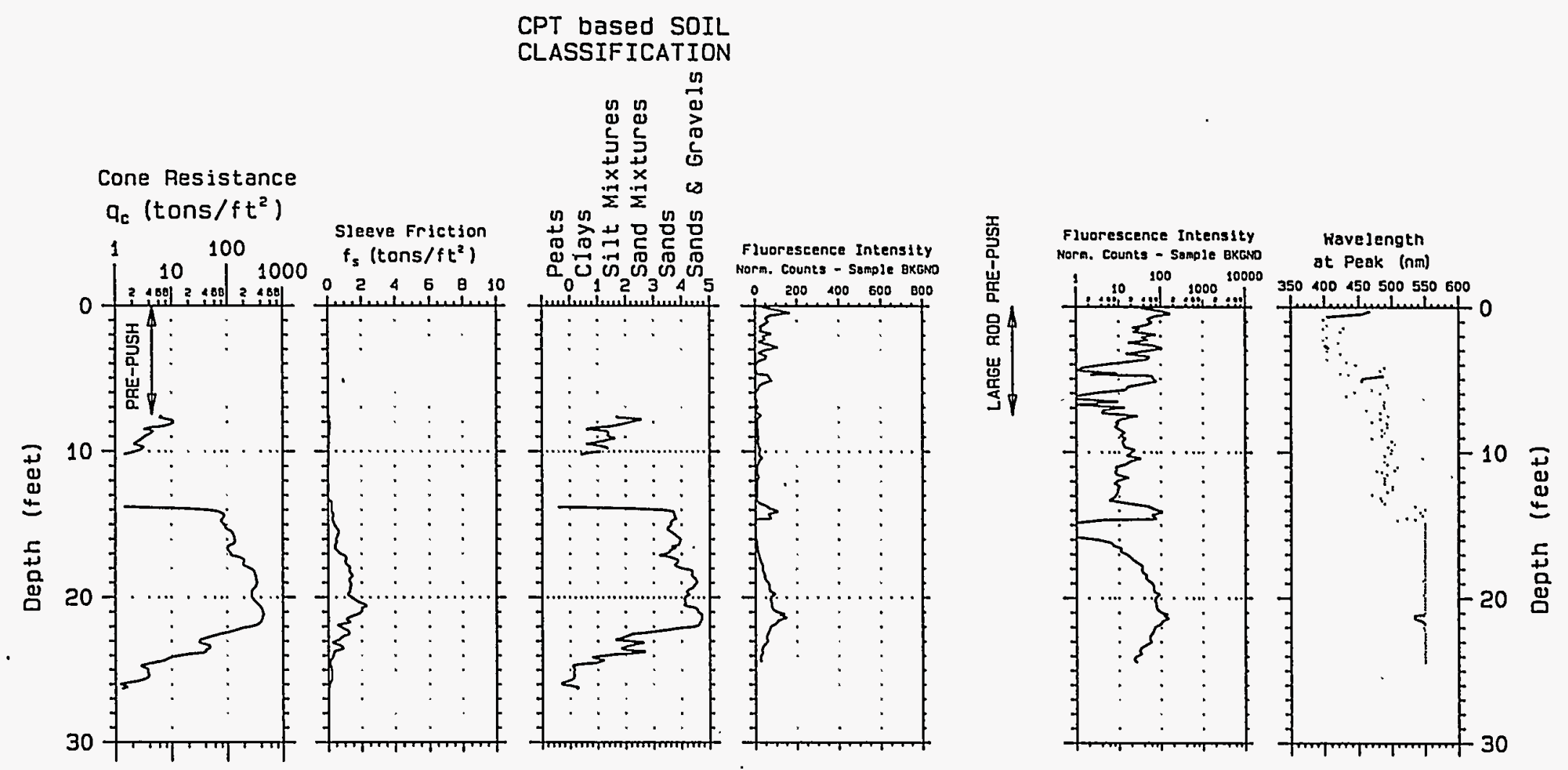

CPT: 81MT99F

STATE COORDINATES:

EASTING ( $f t$.

NORTHING (ft.) ELEVATION $(\mathrm{ft}$.

$-1$

$-1$

0

Probe date 05-04-1994 


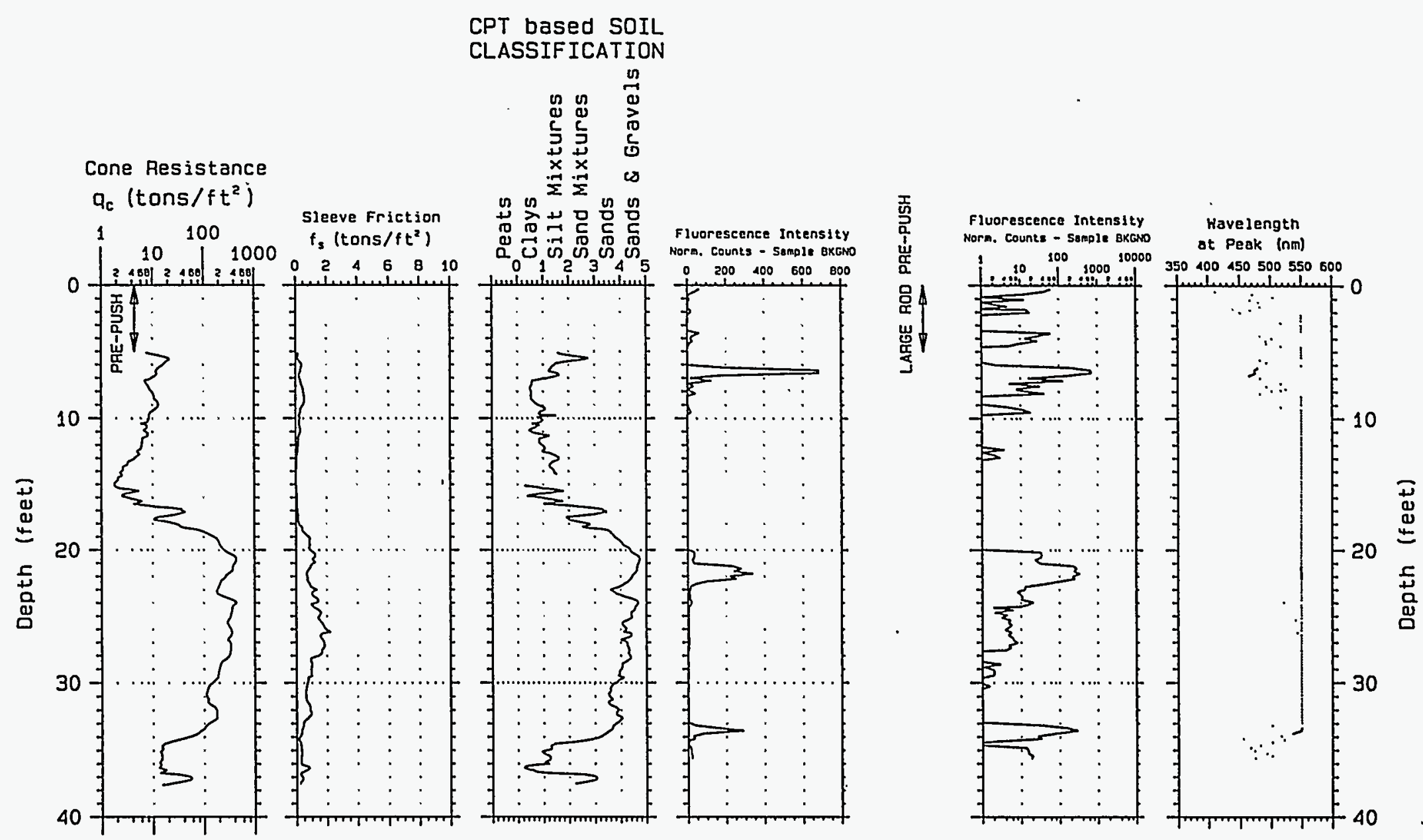

CPT: 83MT101F

STATE COORDINATES:

EASTING ( $\mathrm{ft}$.)

$-1$
NORTHING ( $\mathrm{ft}$. )

$-1$
Project: Marshalltown

ELEVATION ( $\mathrm{ft}$.

Probe date 05-04-1994 
CPT based SOIL

CLASSIFICATION

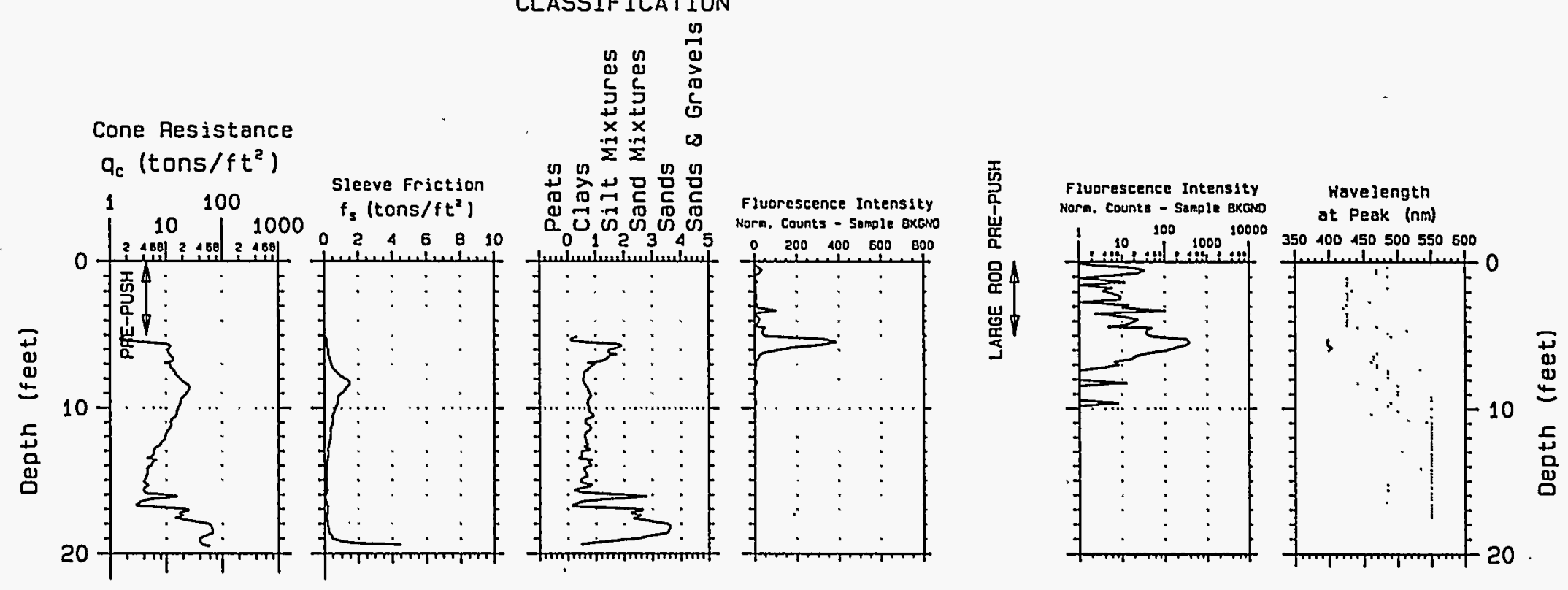

CPT: 90MT105F

STATE COORDINATES:

EASTING ( $f t$.)

$-1$
NORTHING $(f t$.

$-1$
Project: Marshalltown

ELEVATION (ft.)
Probe date 05-04-1994 

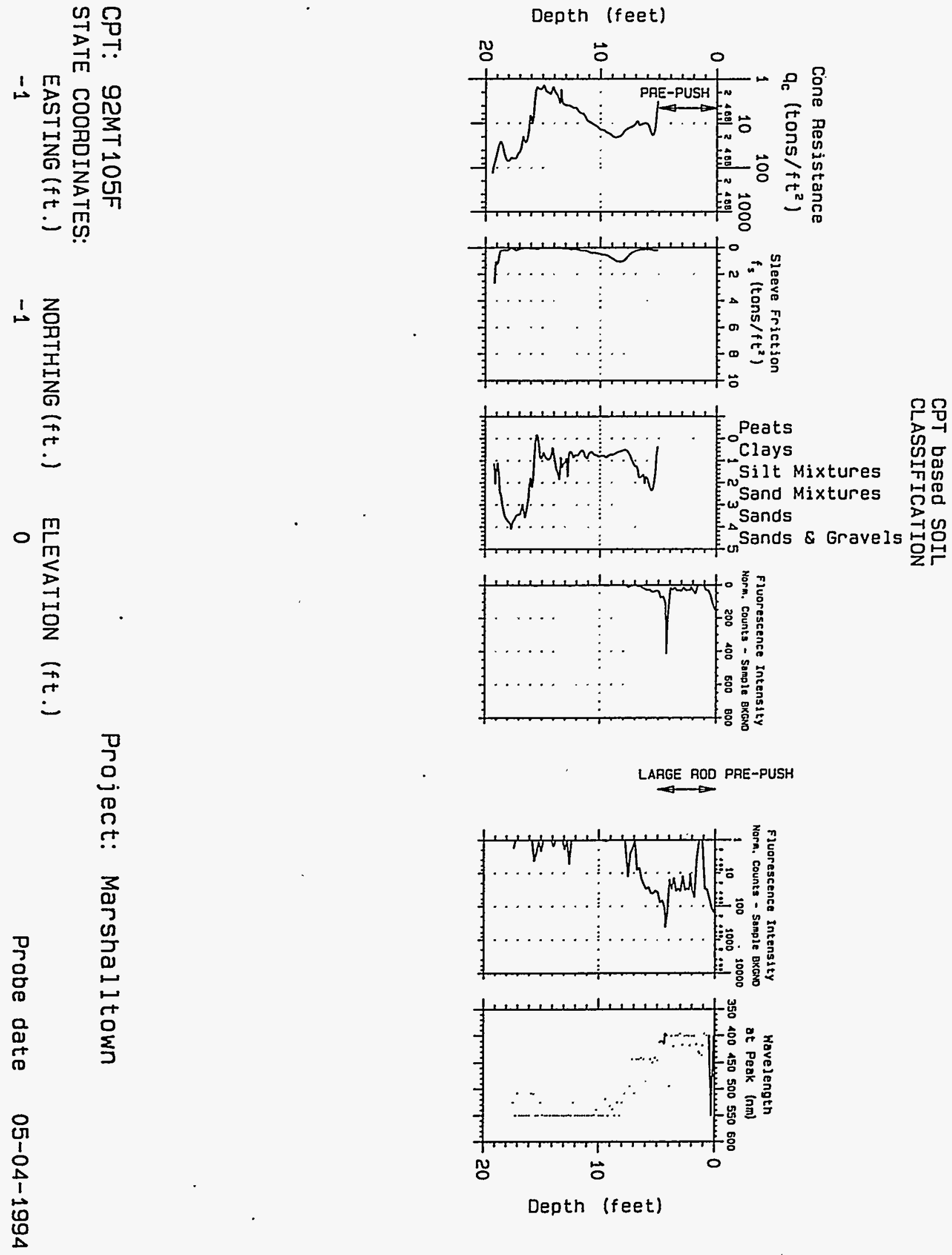


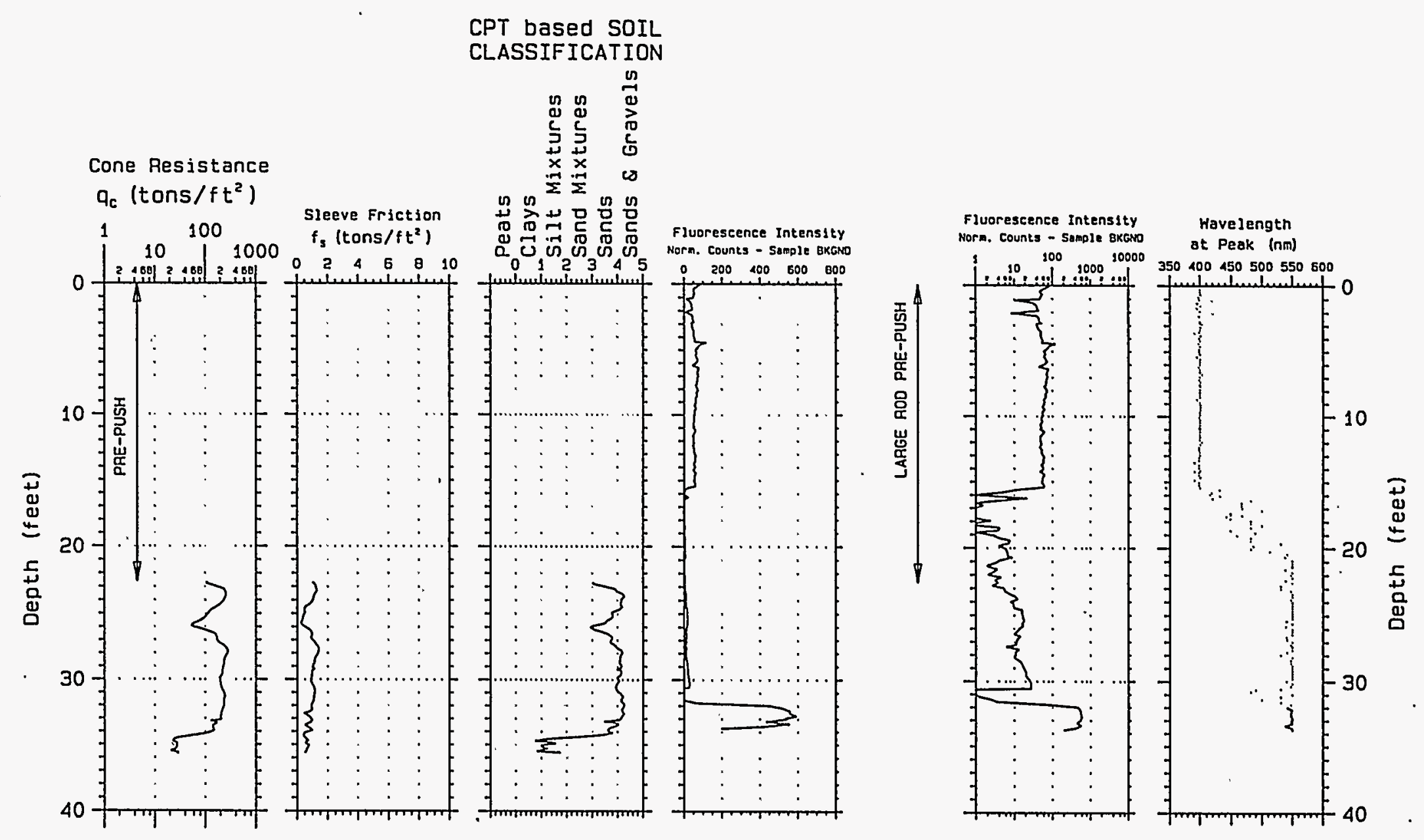

CPT: 96MT105F

STATE COORDINATES: EASTING ( $f t$.) $-1$
NORTHING $(f t$.$) ELEVATION (f t$.
Project: Marshall town

Probe date 05-05-1994 

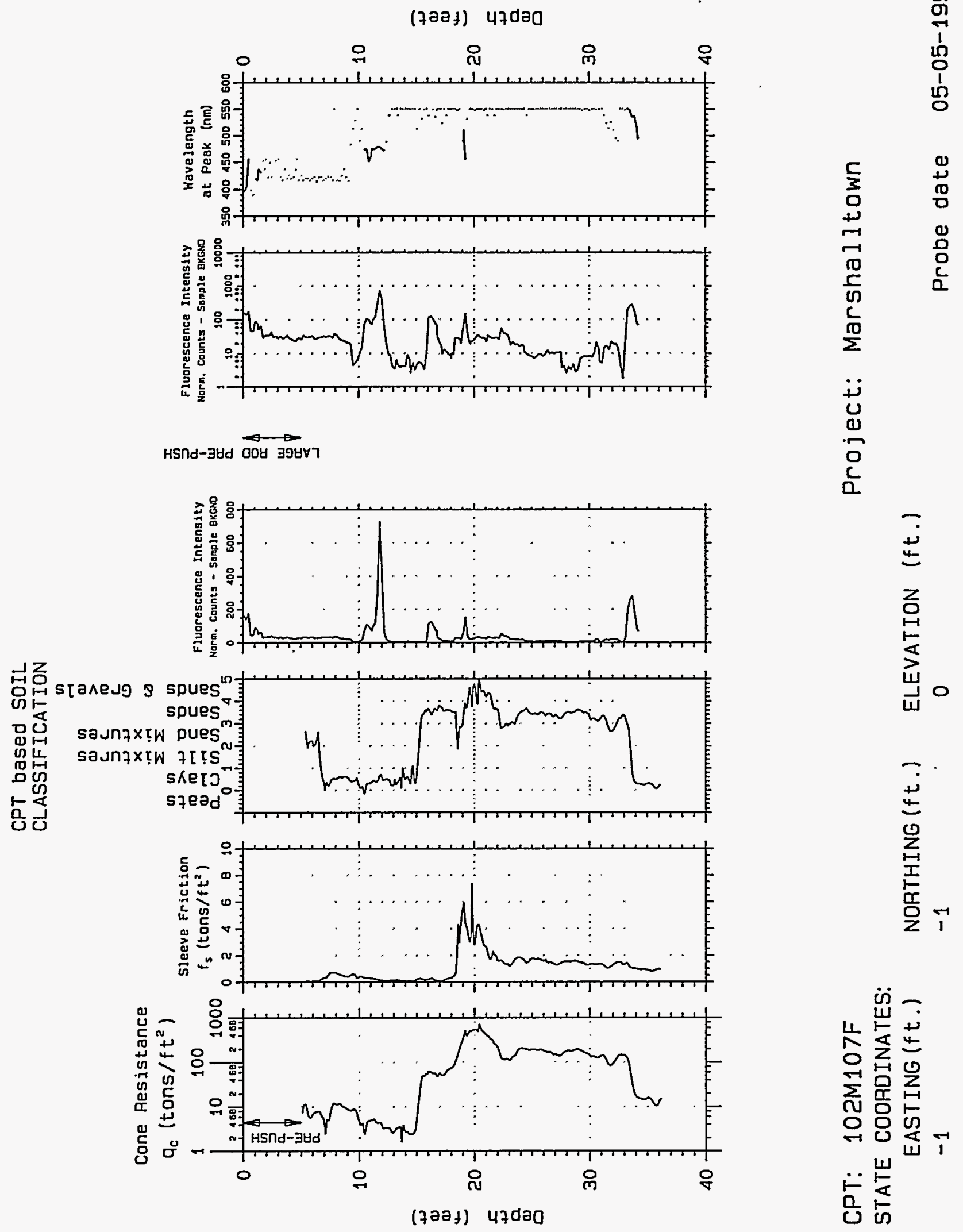
(7aaf) 47da0
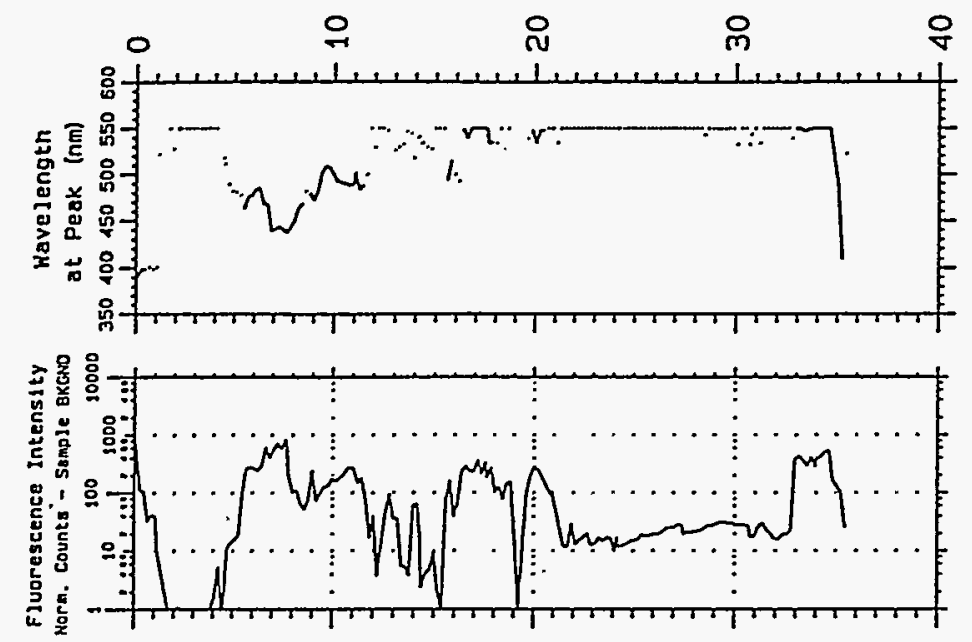

HSกd-3ษd $\overrightarrow{00 \mathrm{~B} 3987 า}$
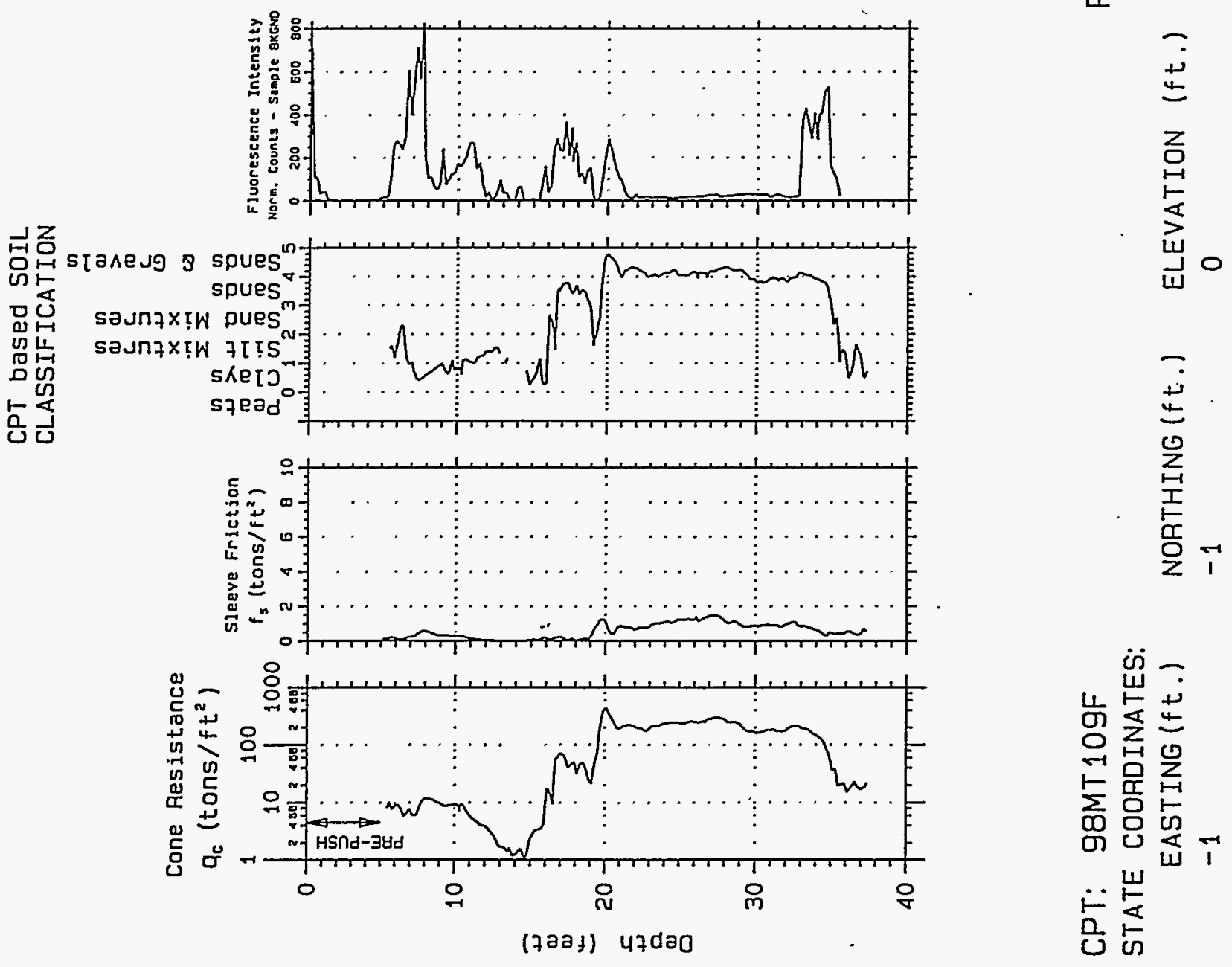

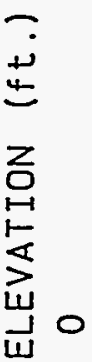

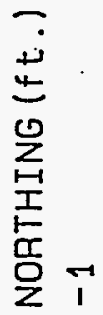


HSnd-34d 008 $\exists 98 \forall 7$
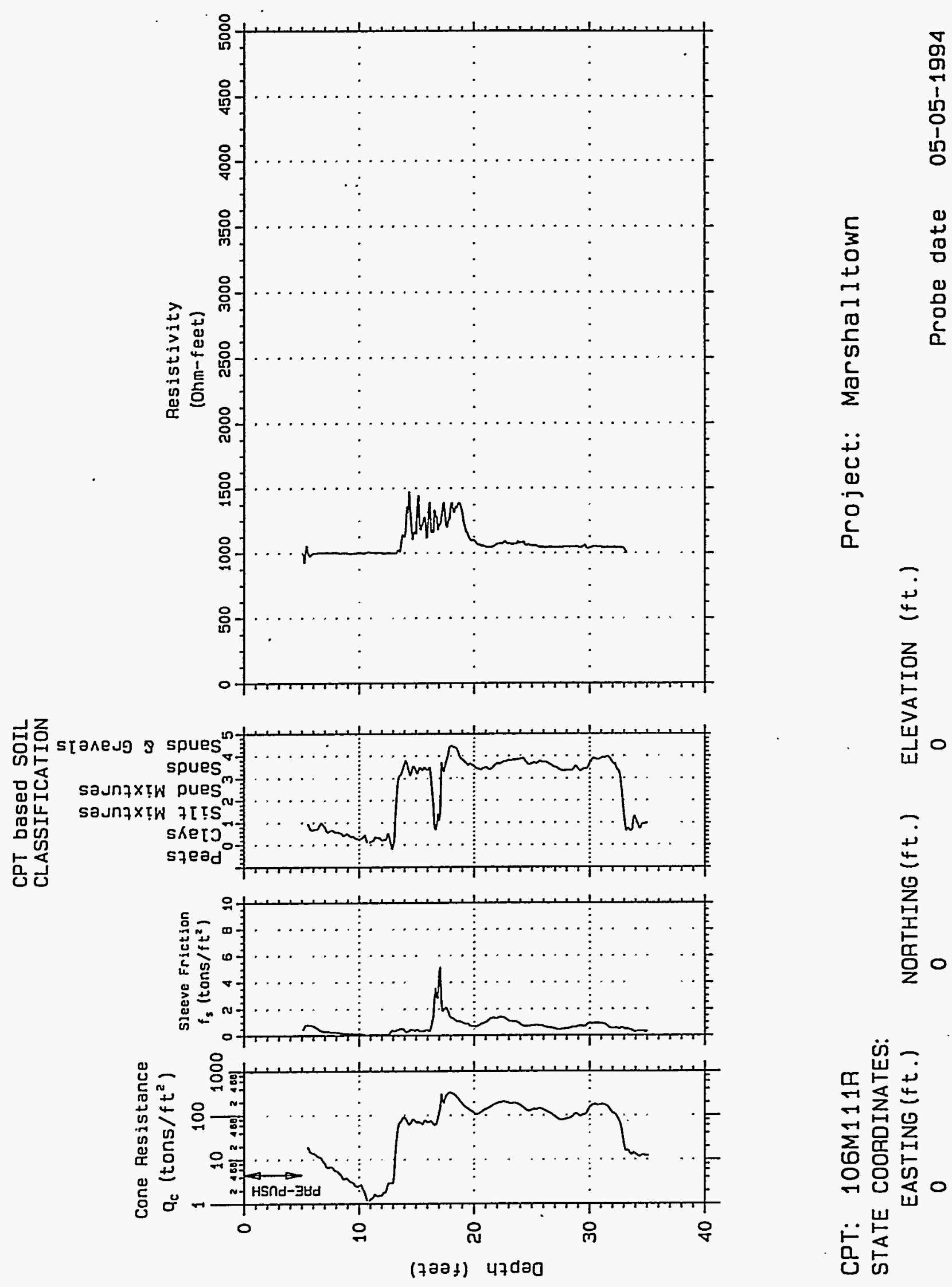

它点点

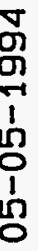
0
0
0
0
0
0
0 


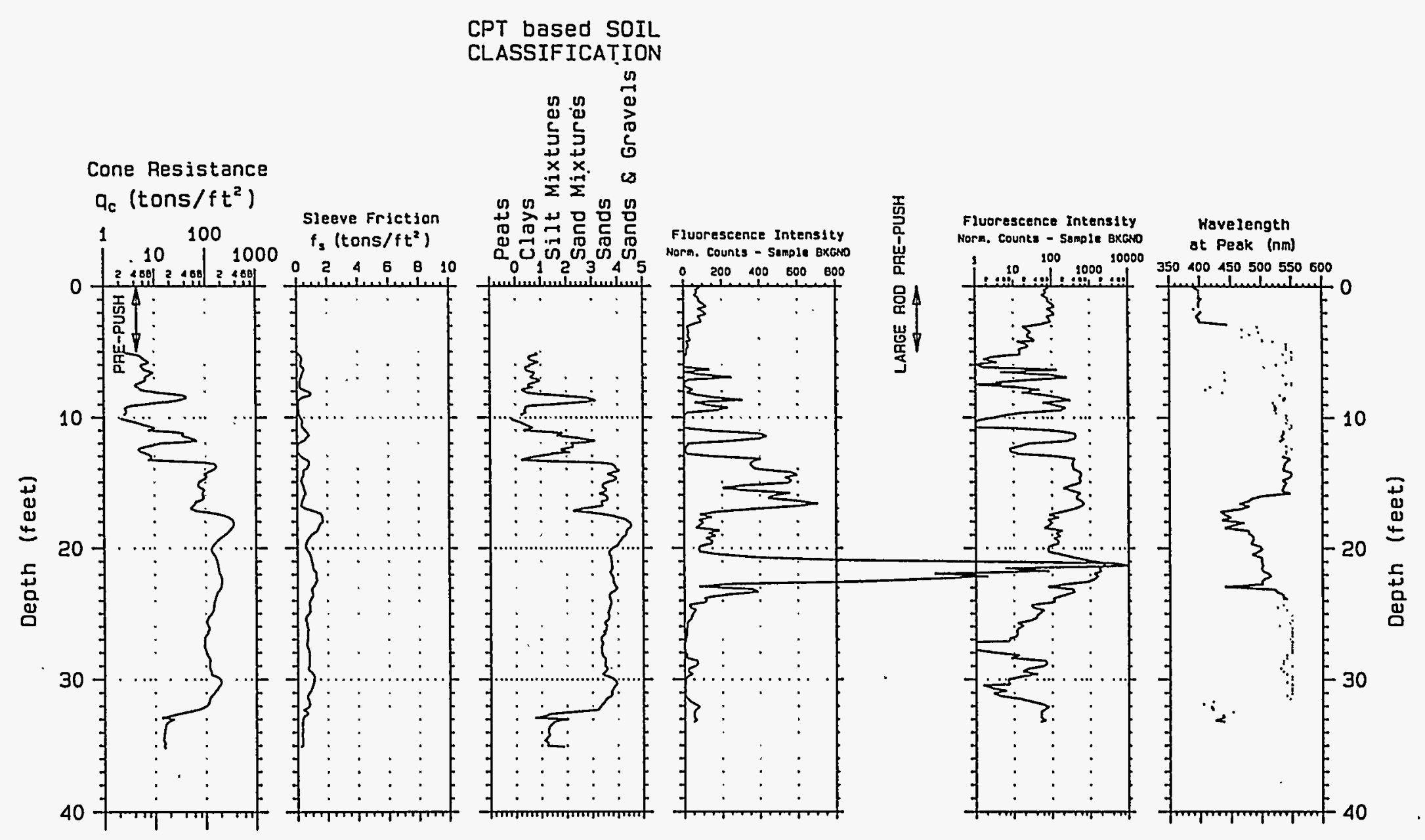

CPT: 104M111F

STATE COORDINATES:

EASTING ( $f t$.)
$-1$
NORTHING ( $\mathrm{ft}$.)

$-1$
Project: Marshalltown

ELEVATION $(\mathrm{ft}$.

0
Probe date

05-05-1994 
(7аat) 47020
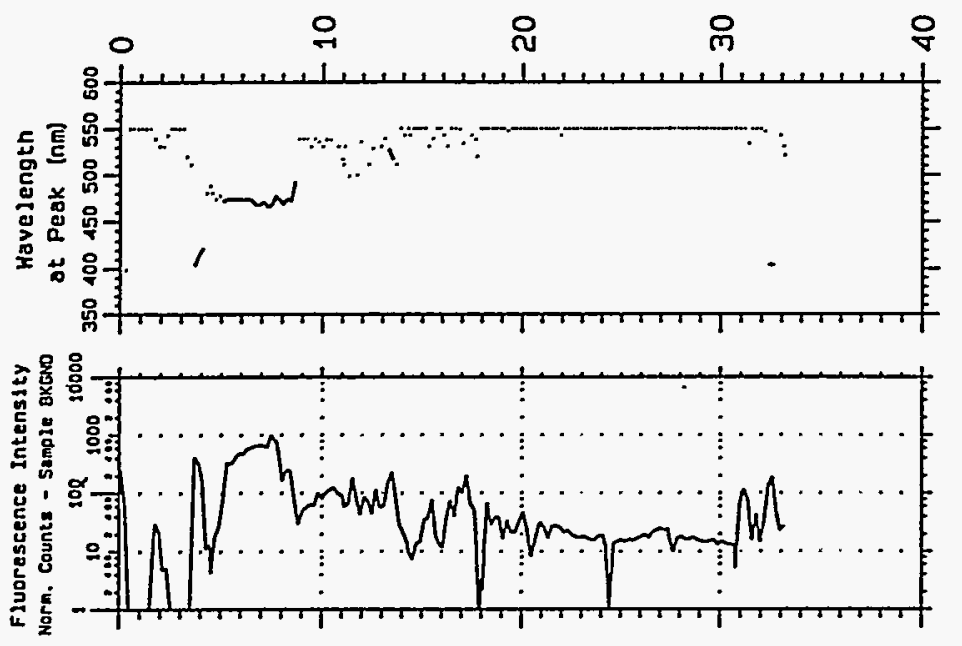

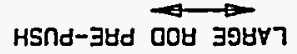

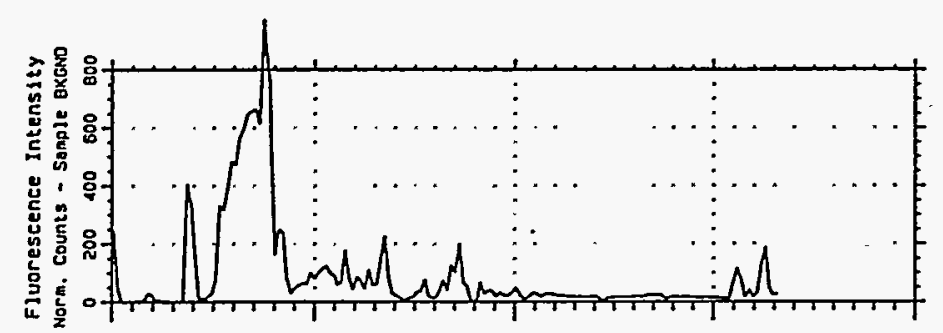

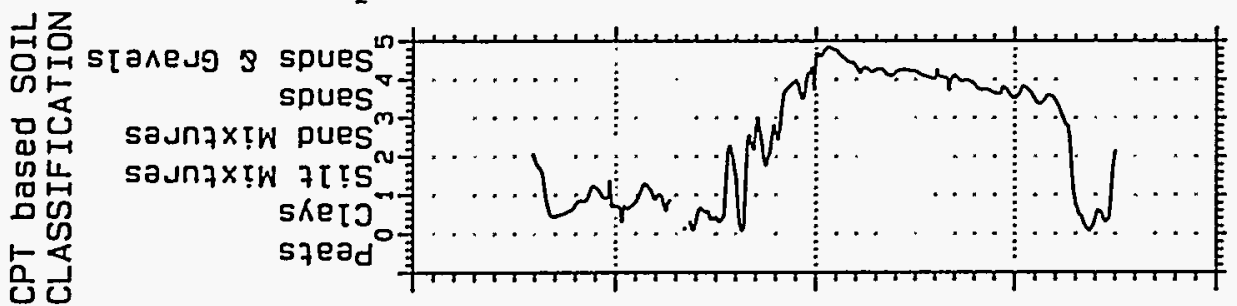
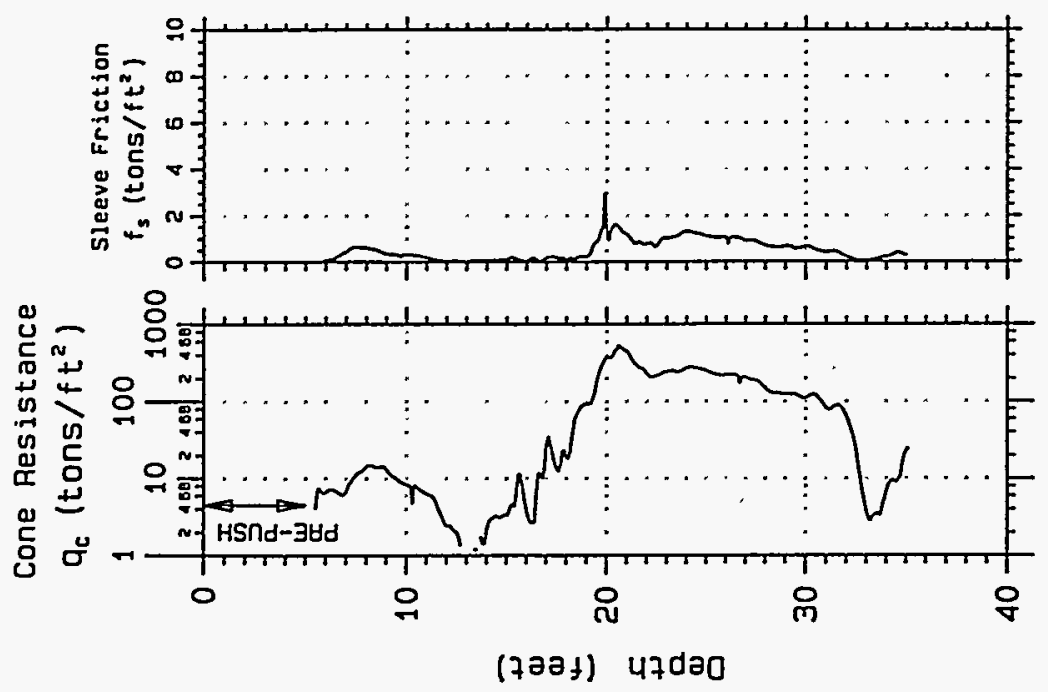

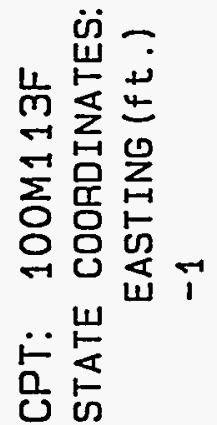


HSกd-3ษd 008 $39 \forall \forall 7$
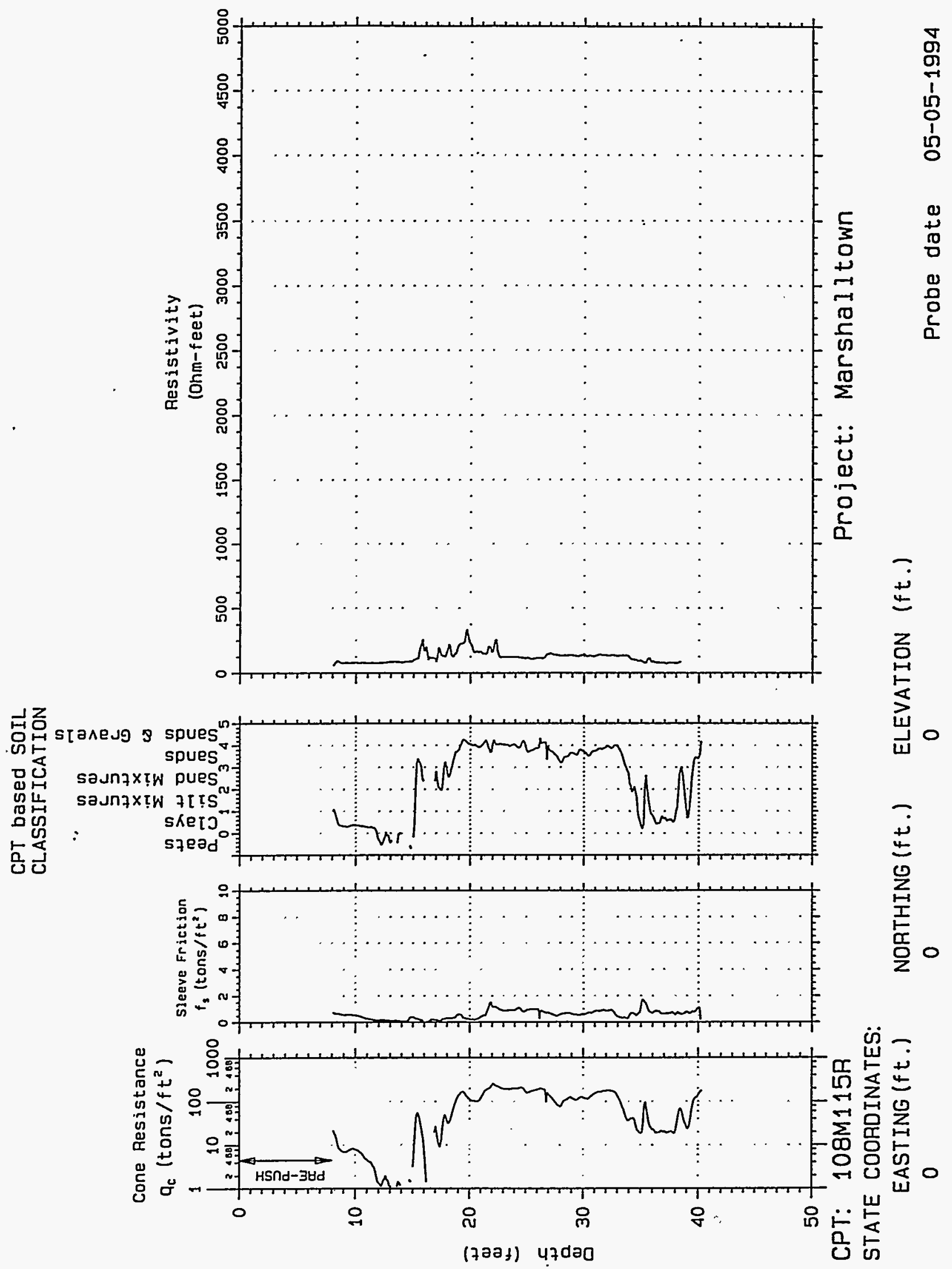
Ames Expedited Site Characterization - Marshalltown FMGP Site DRAFT Site Report

October 19, 1995

APPENDIX C:

CONDUCTIVITY PROFULS -- CONDUCTIVITY VERSUS DEPTH LOGS 


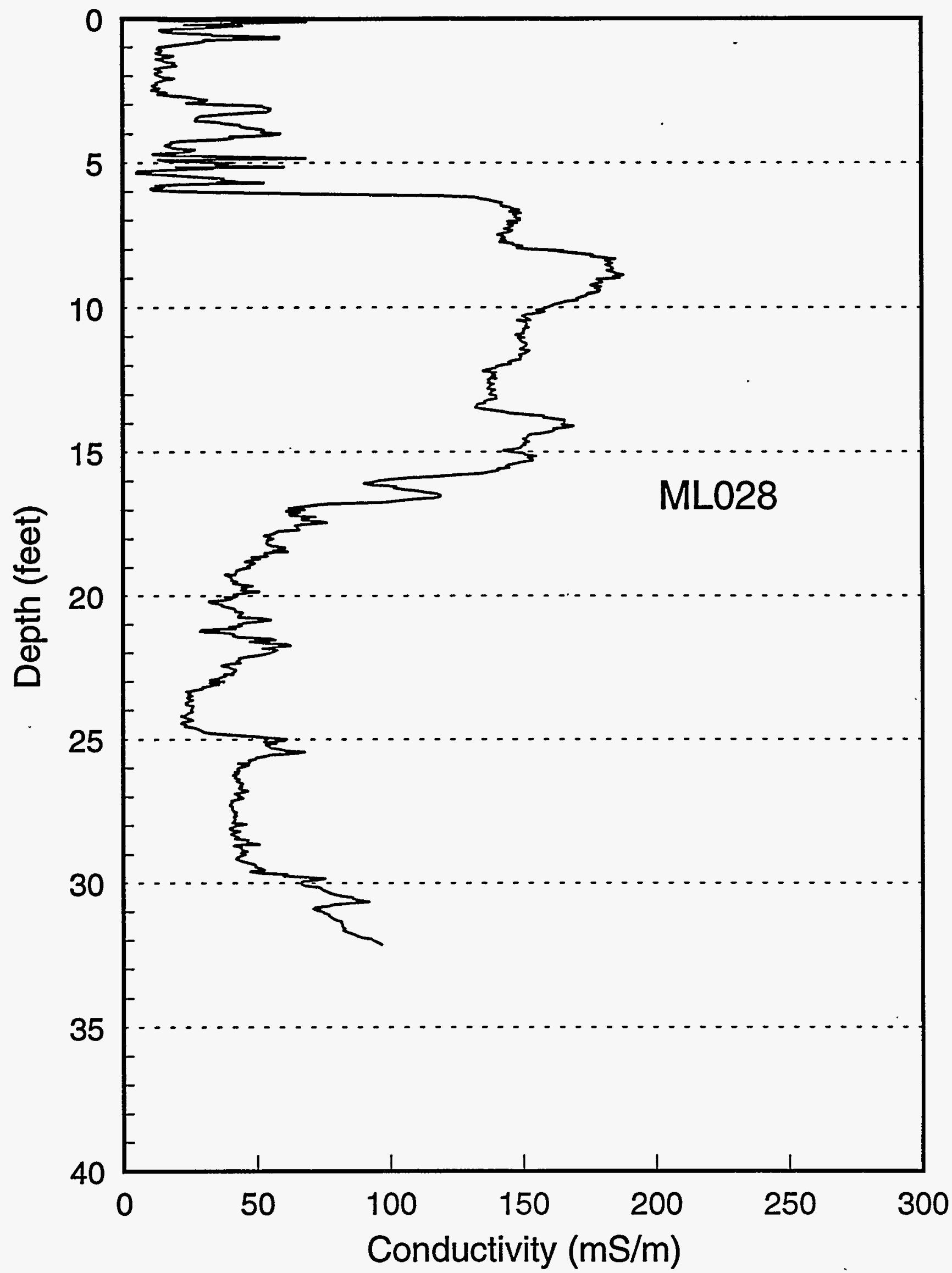




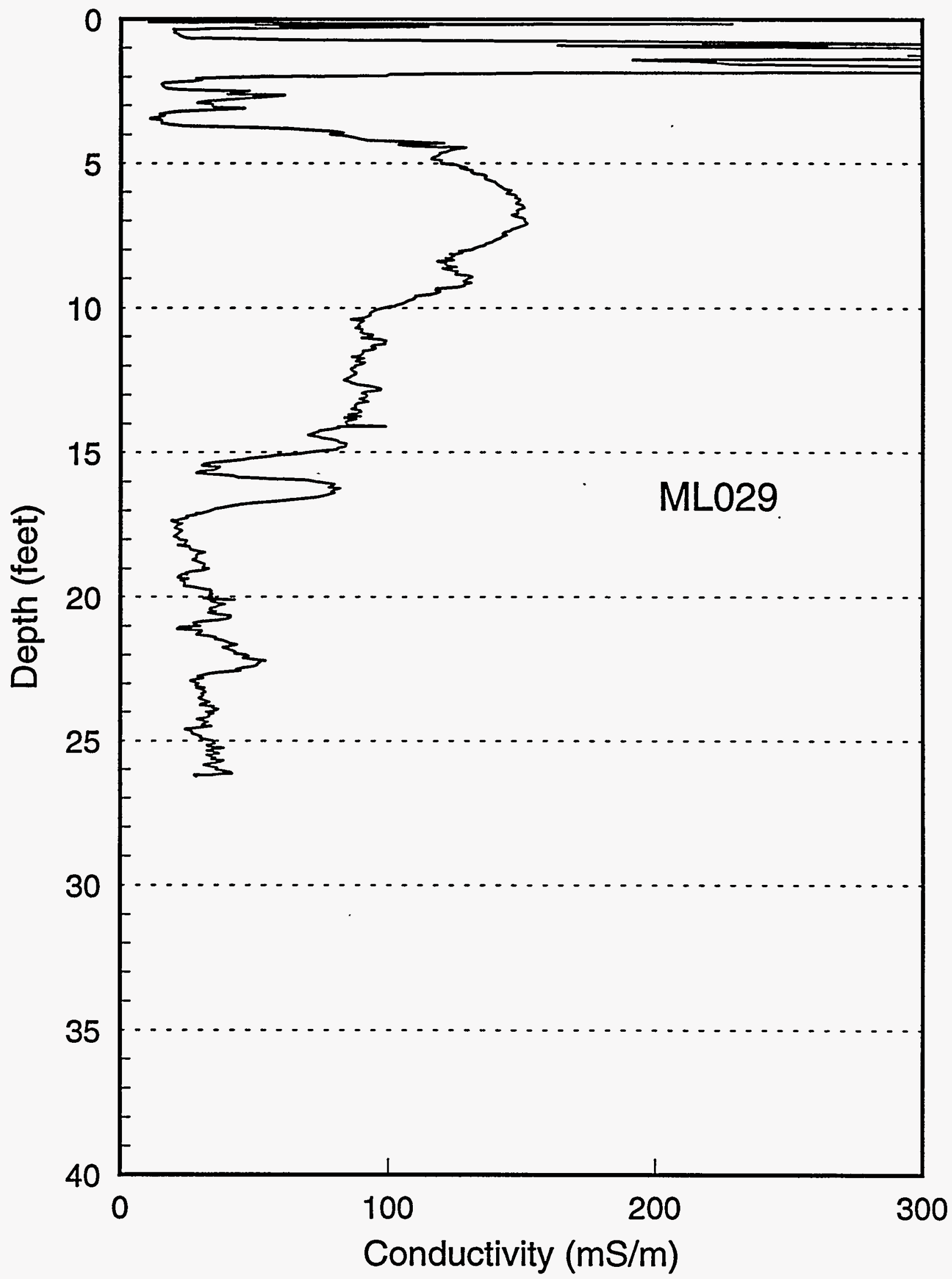




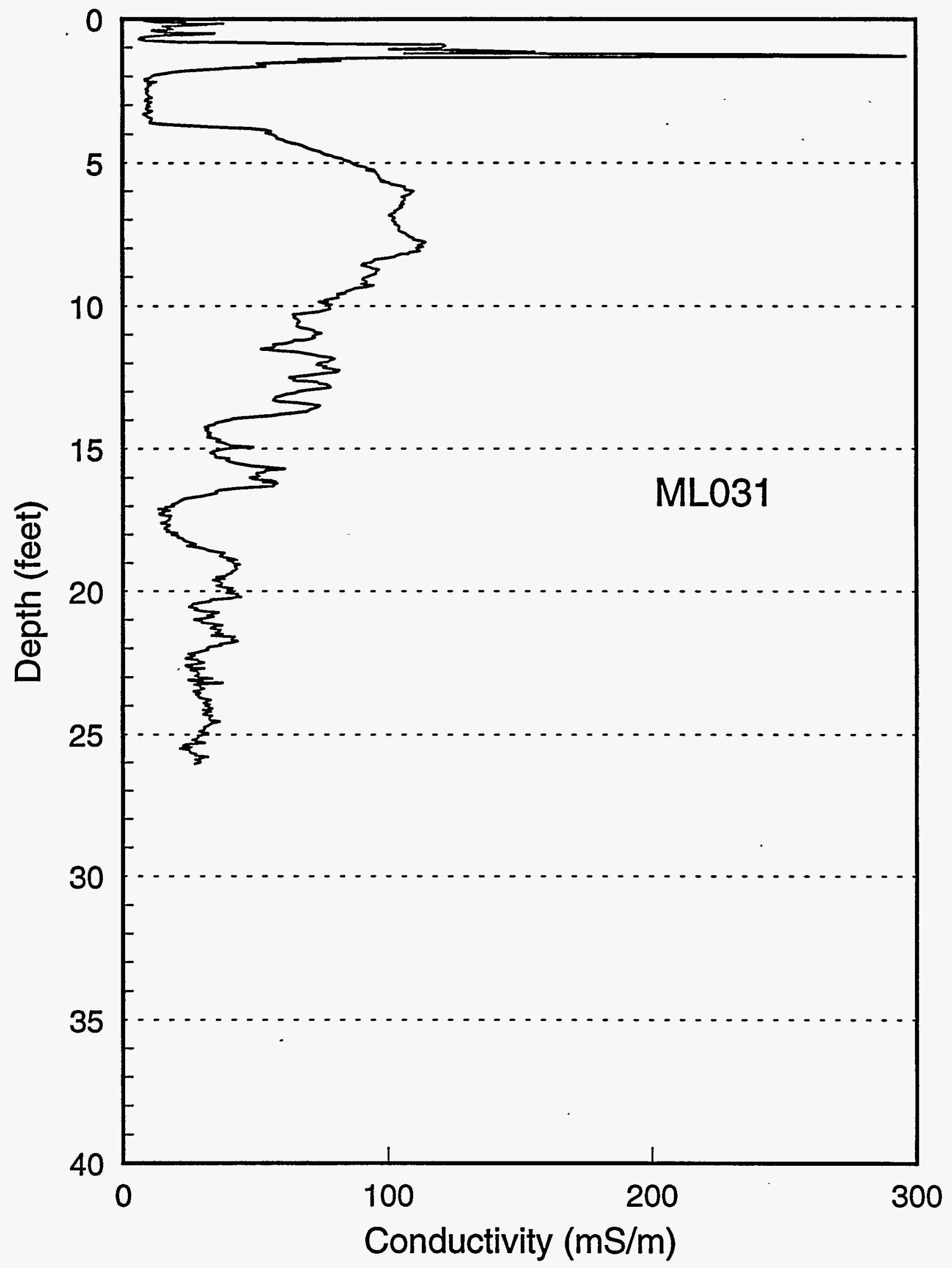




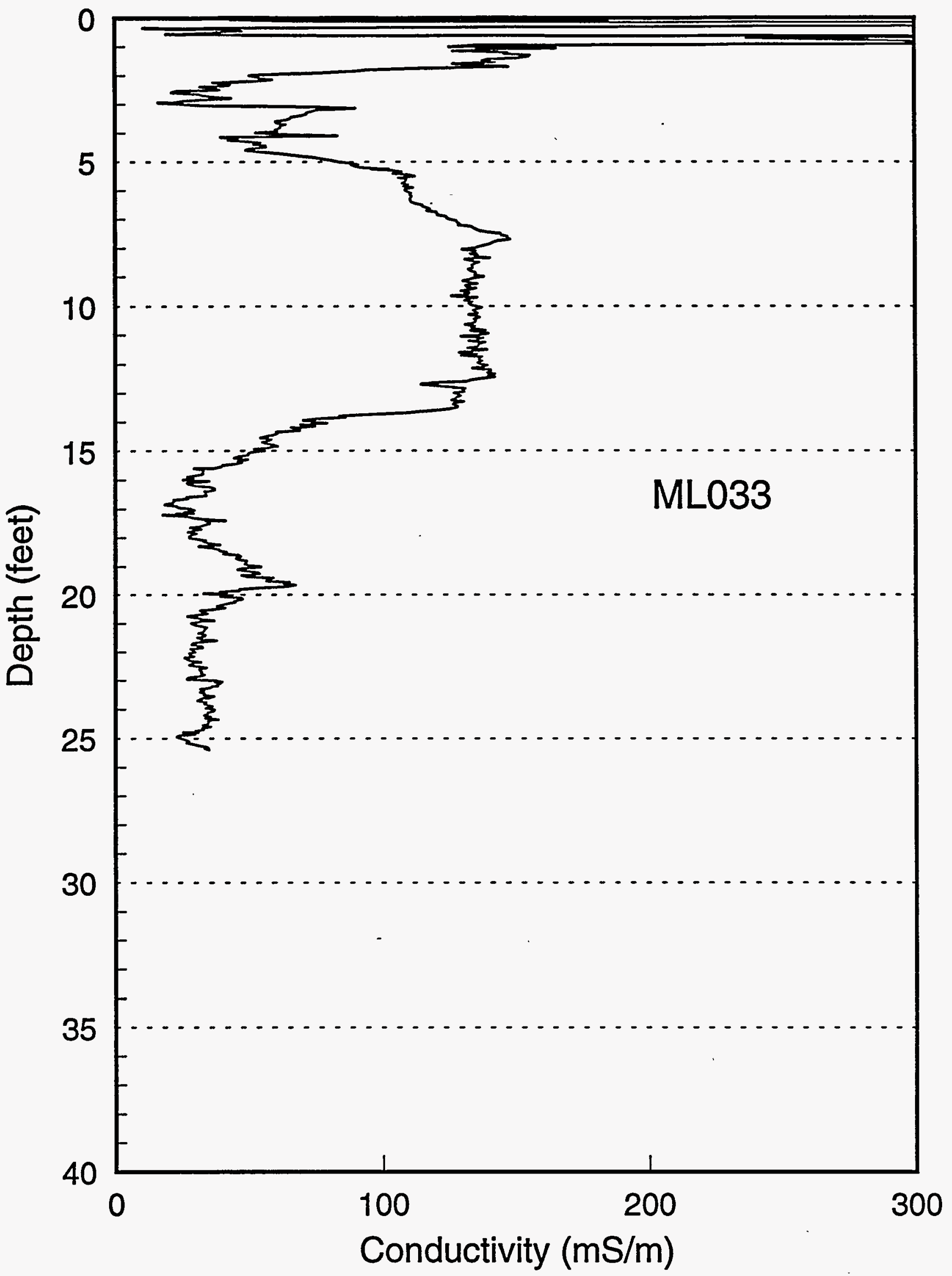




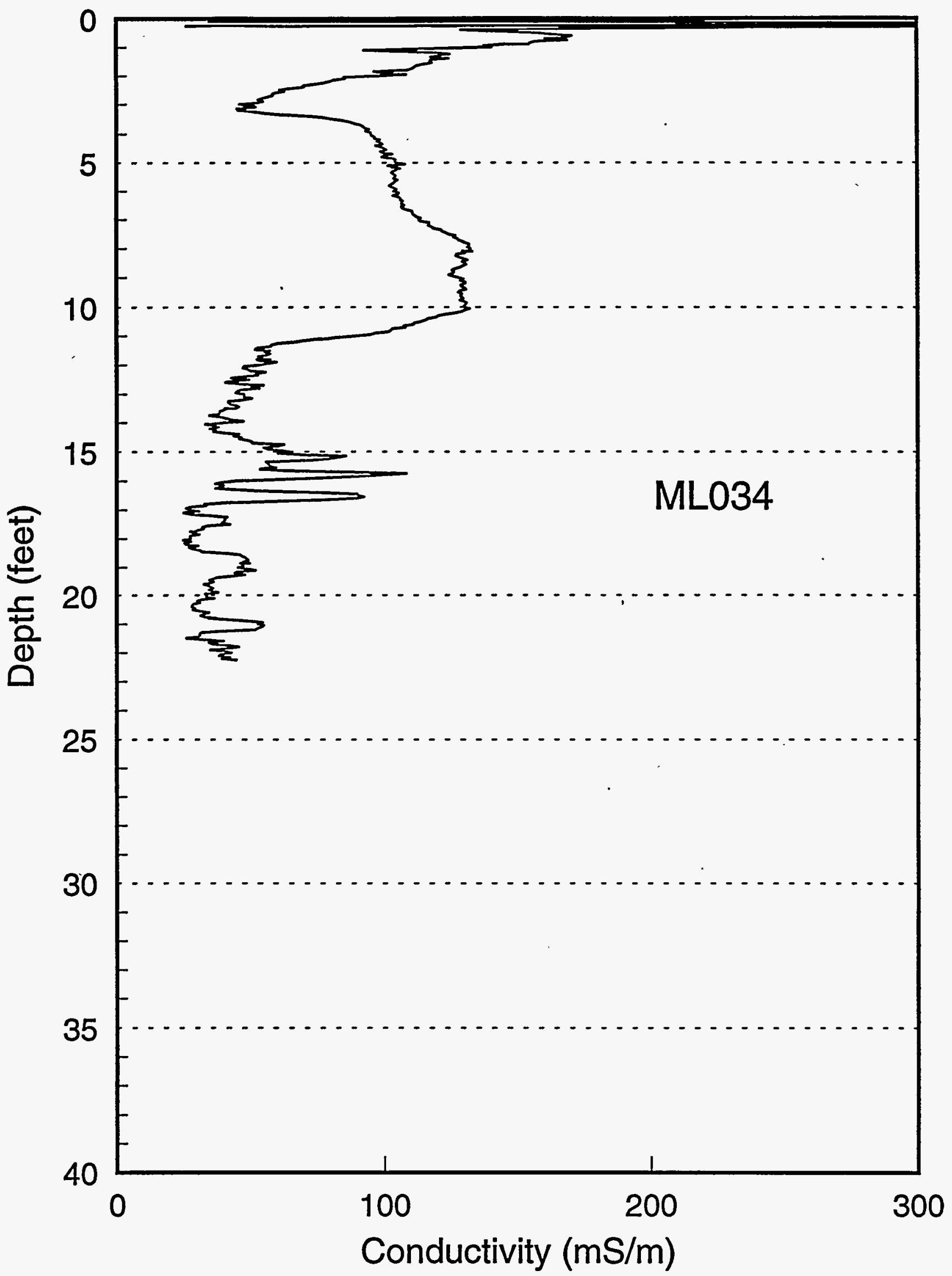




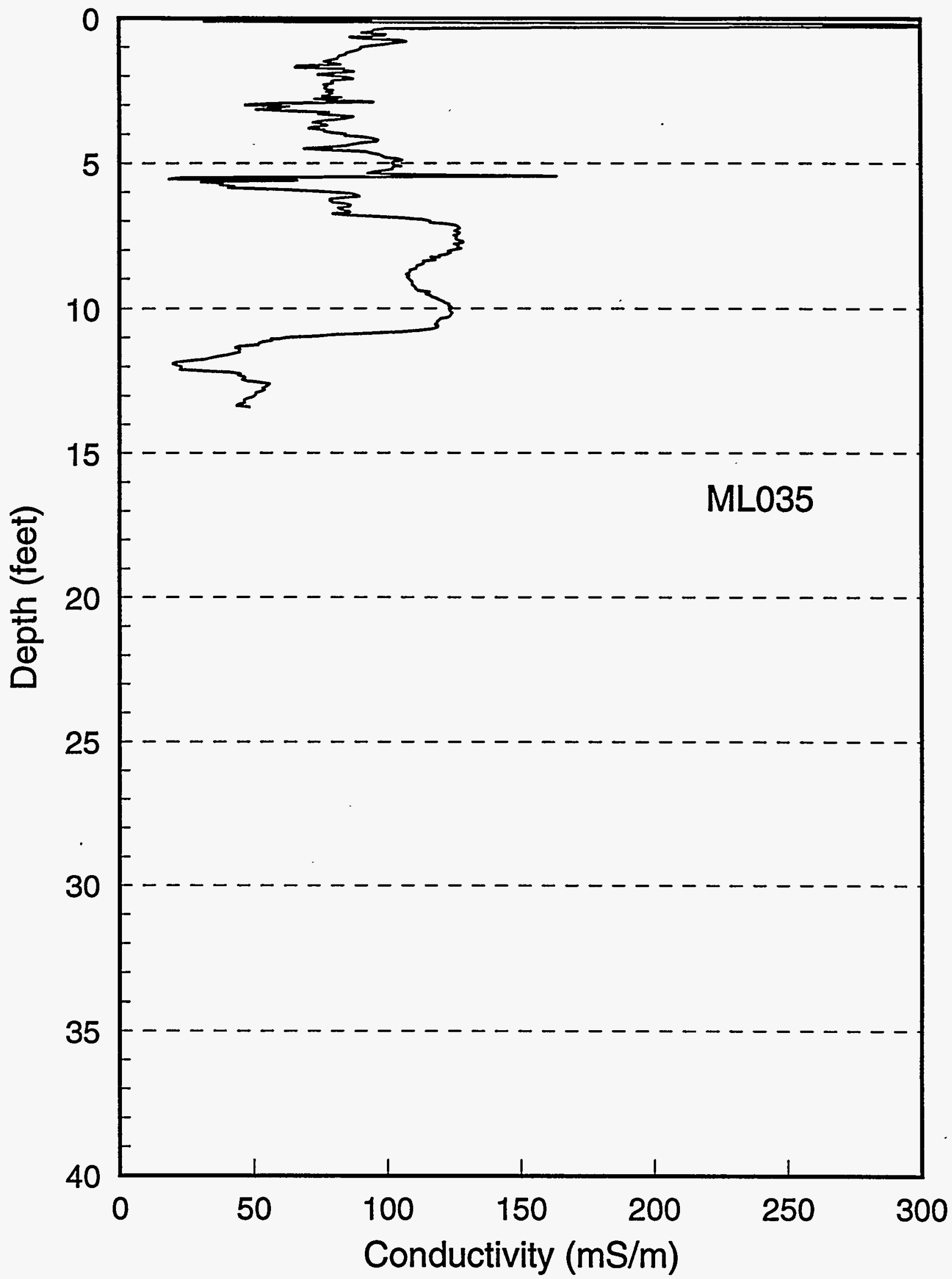




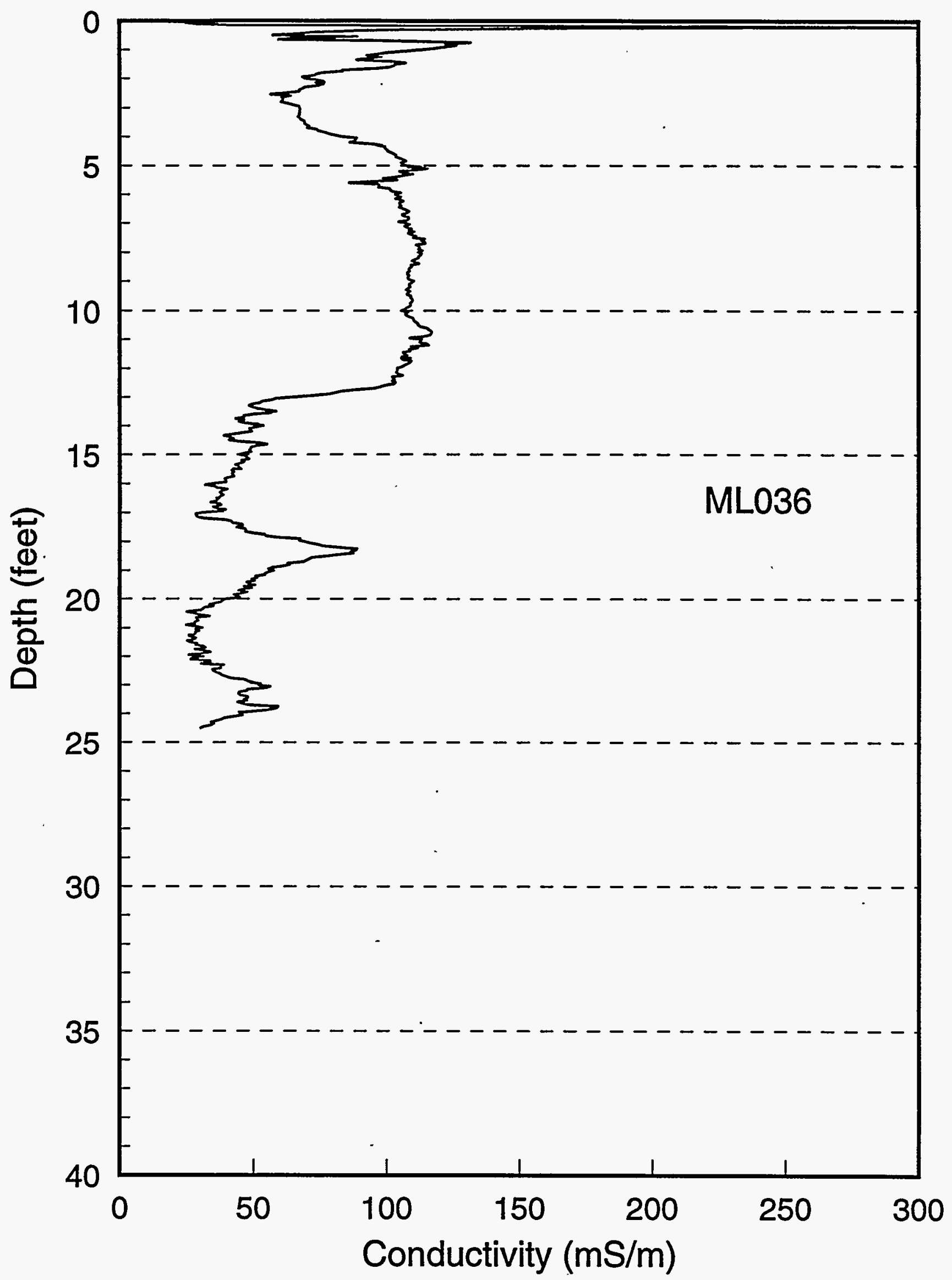




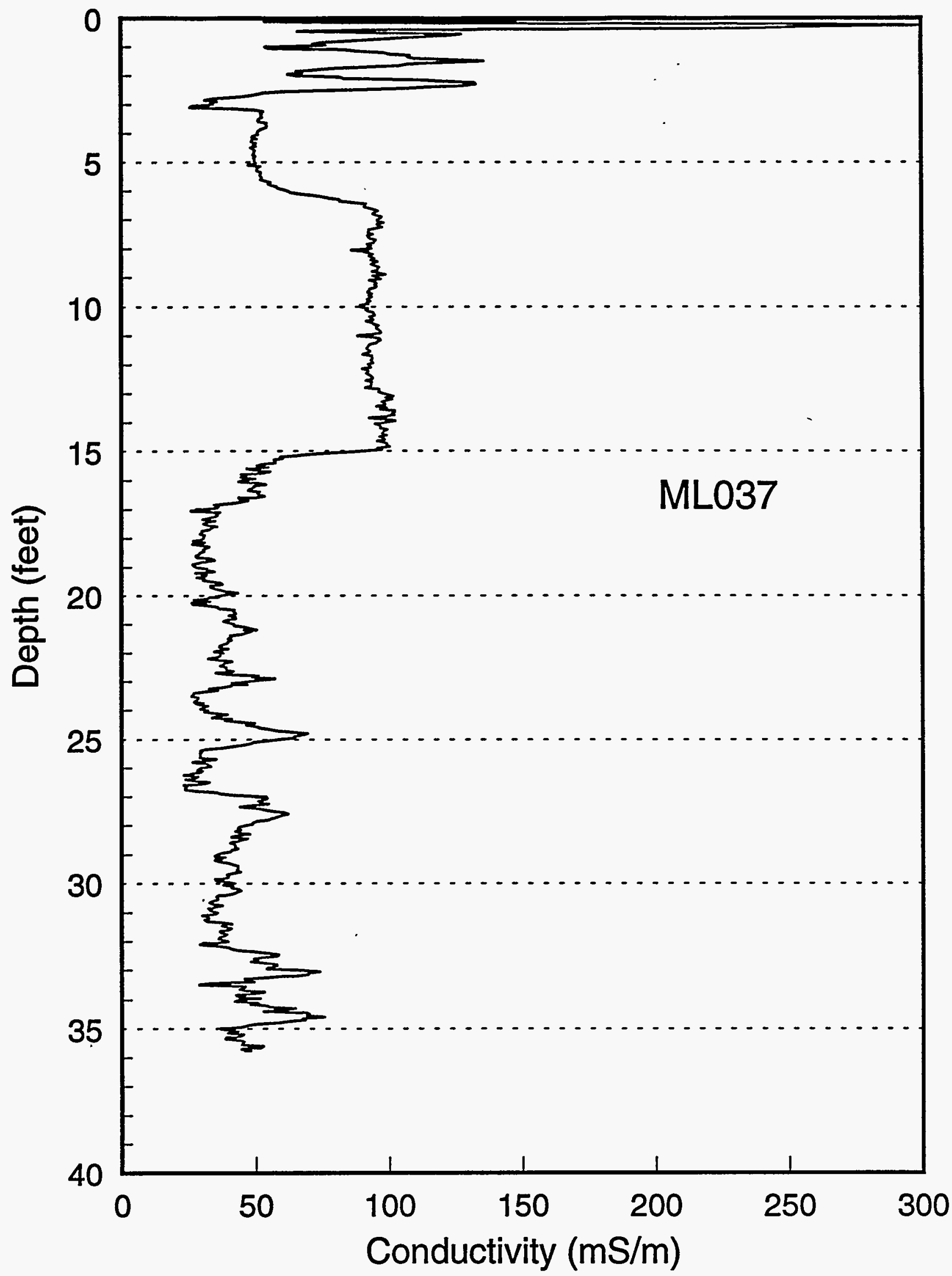




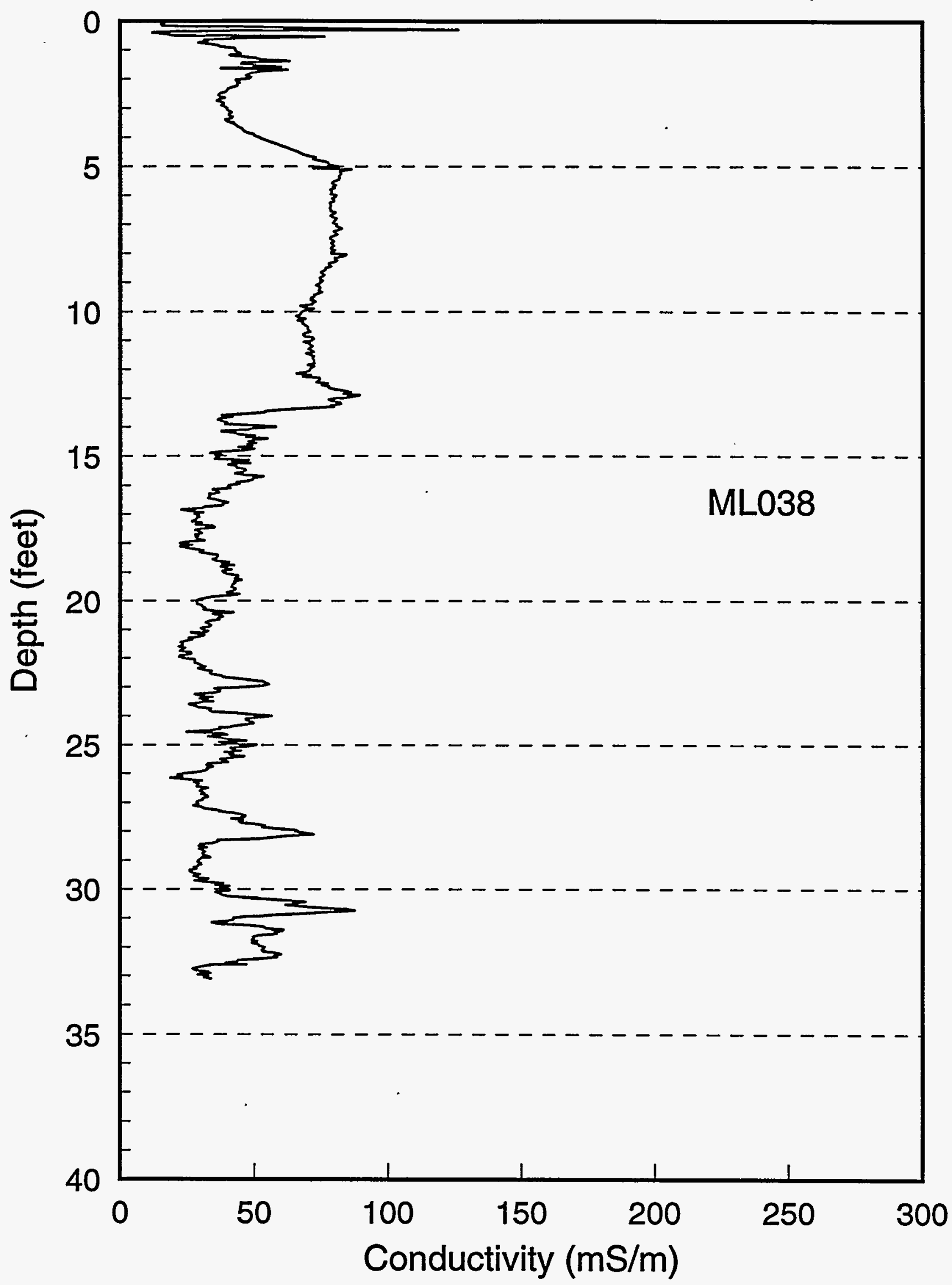




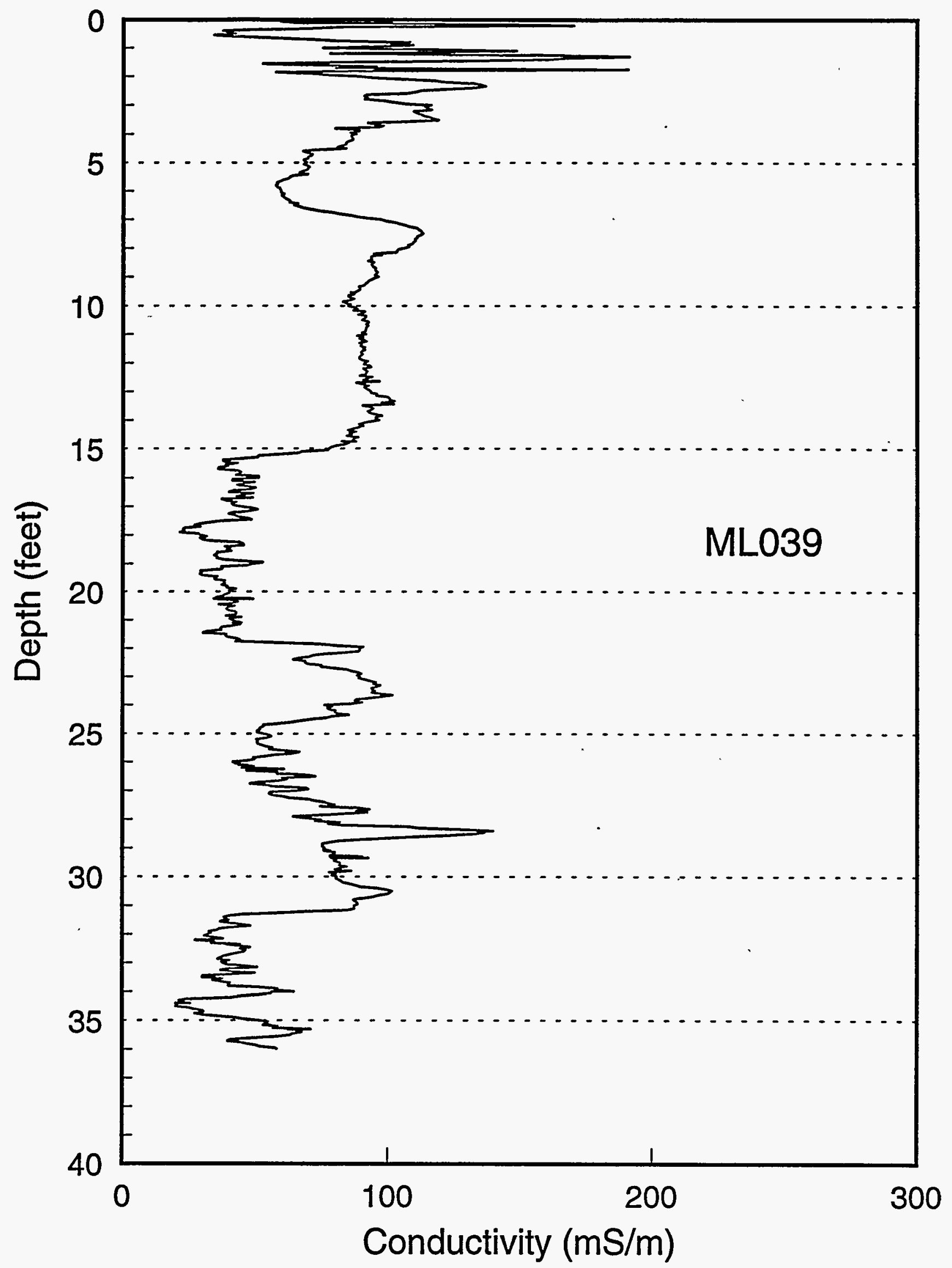




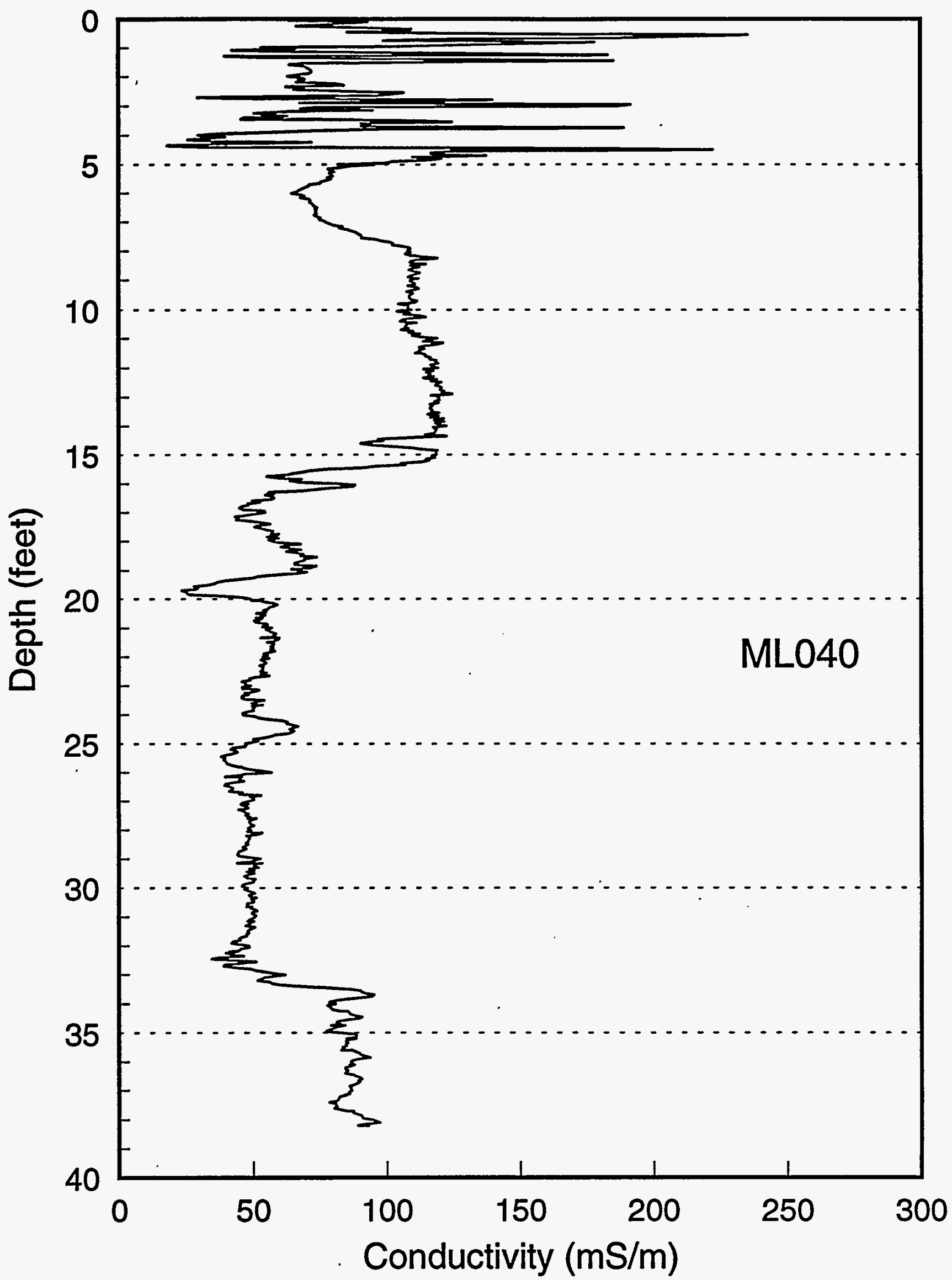




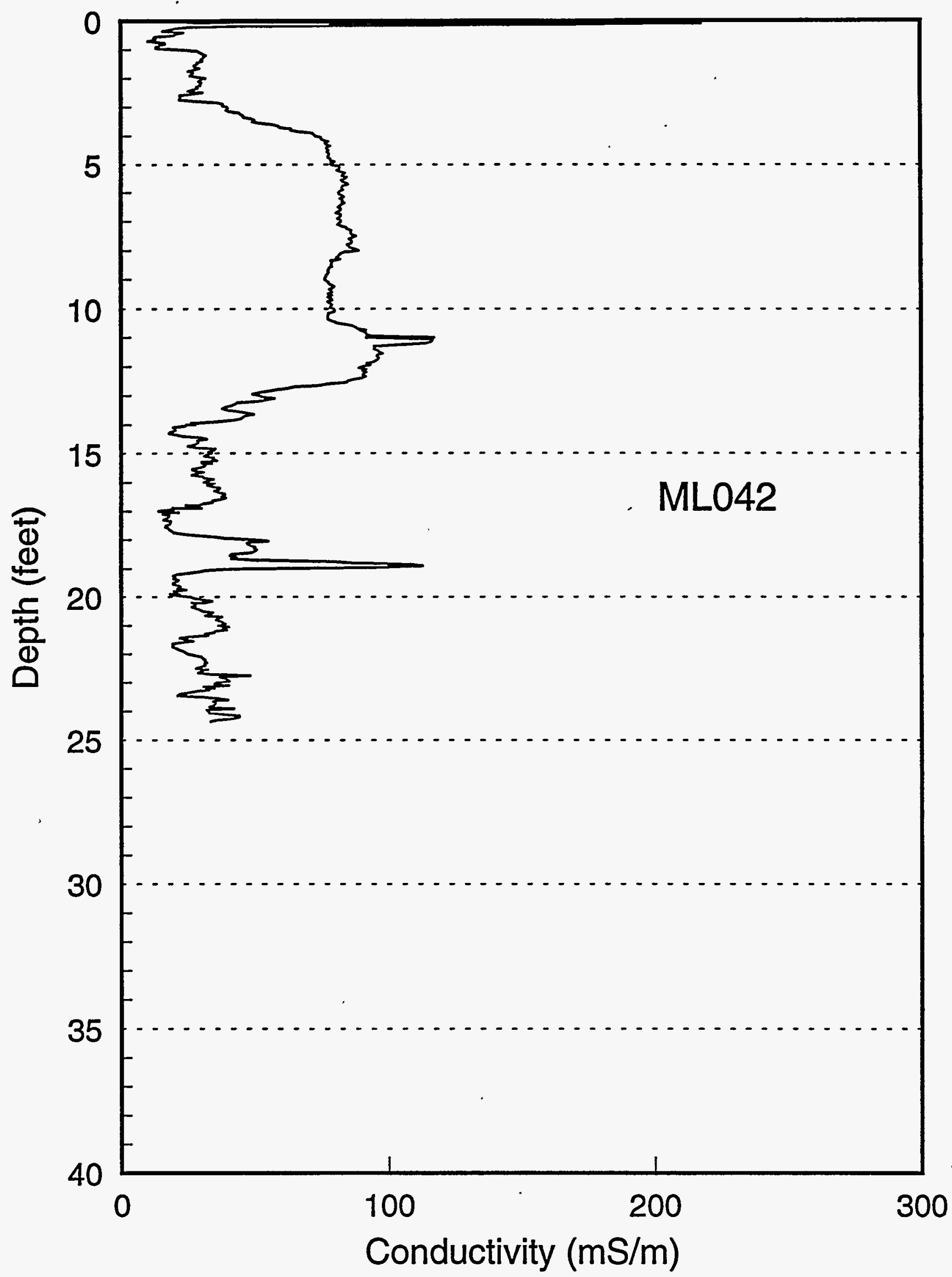




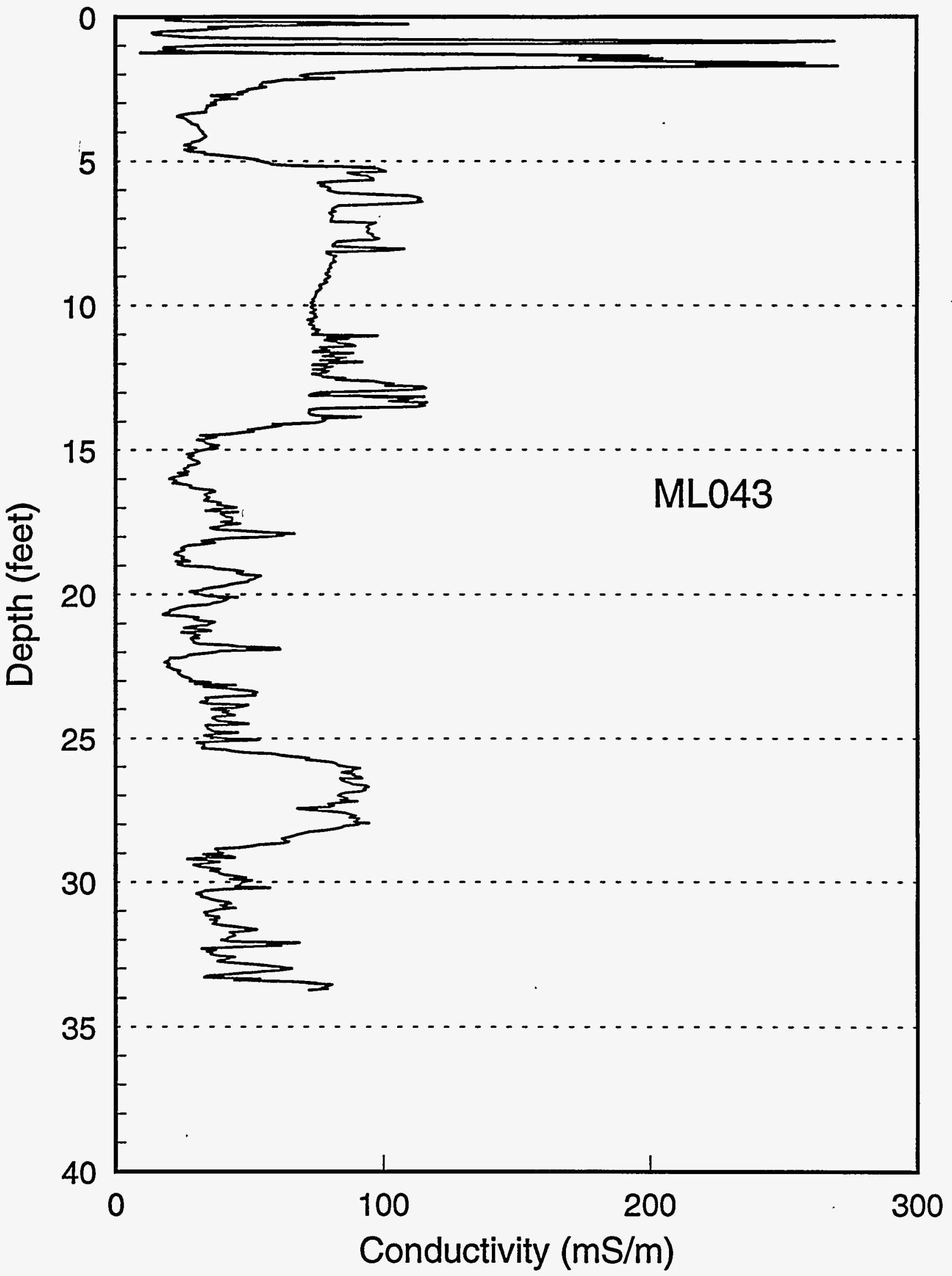




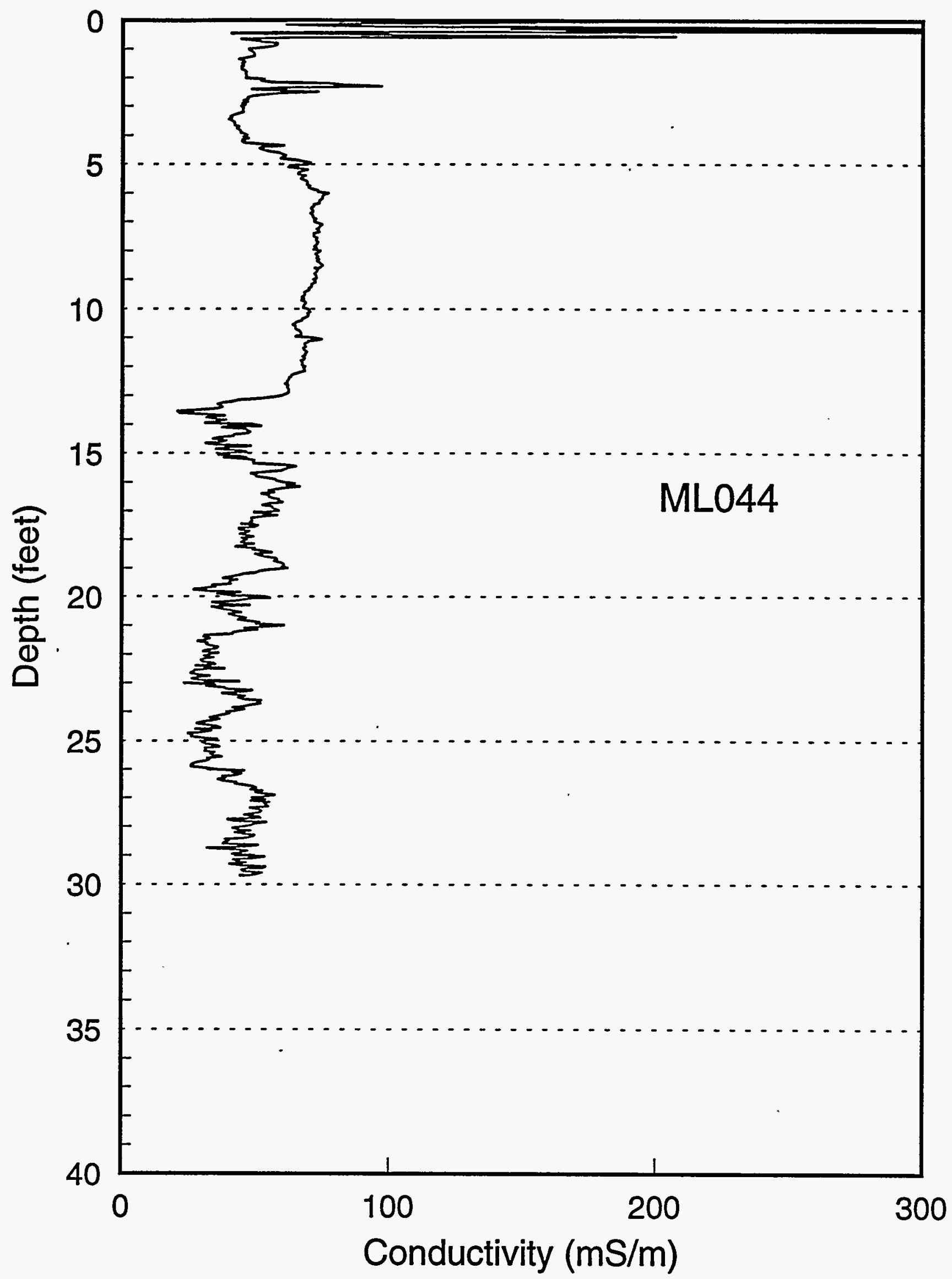




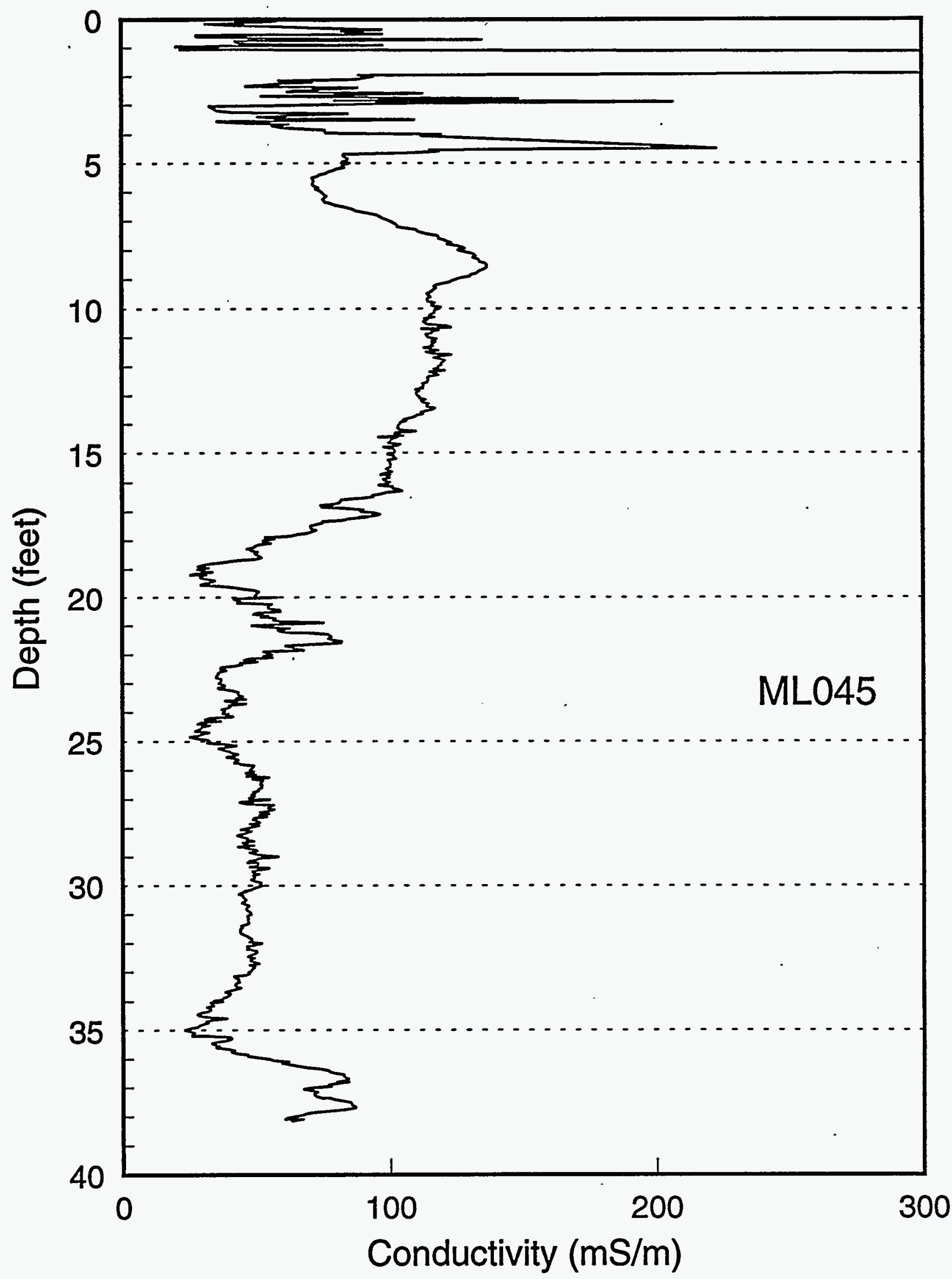




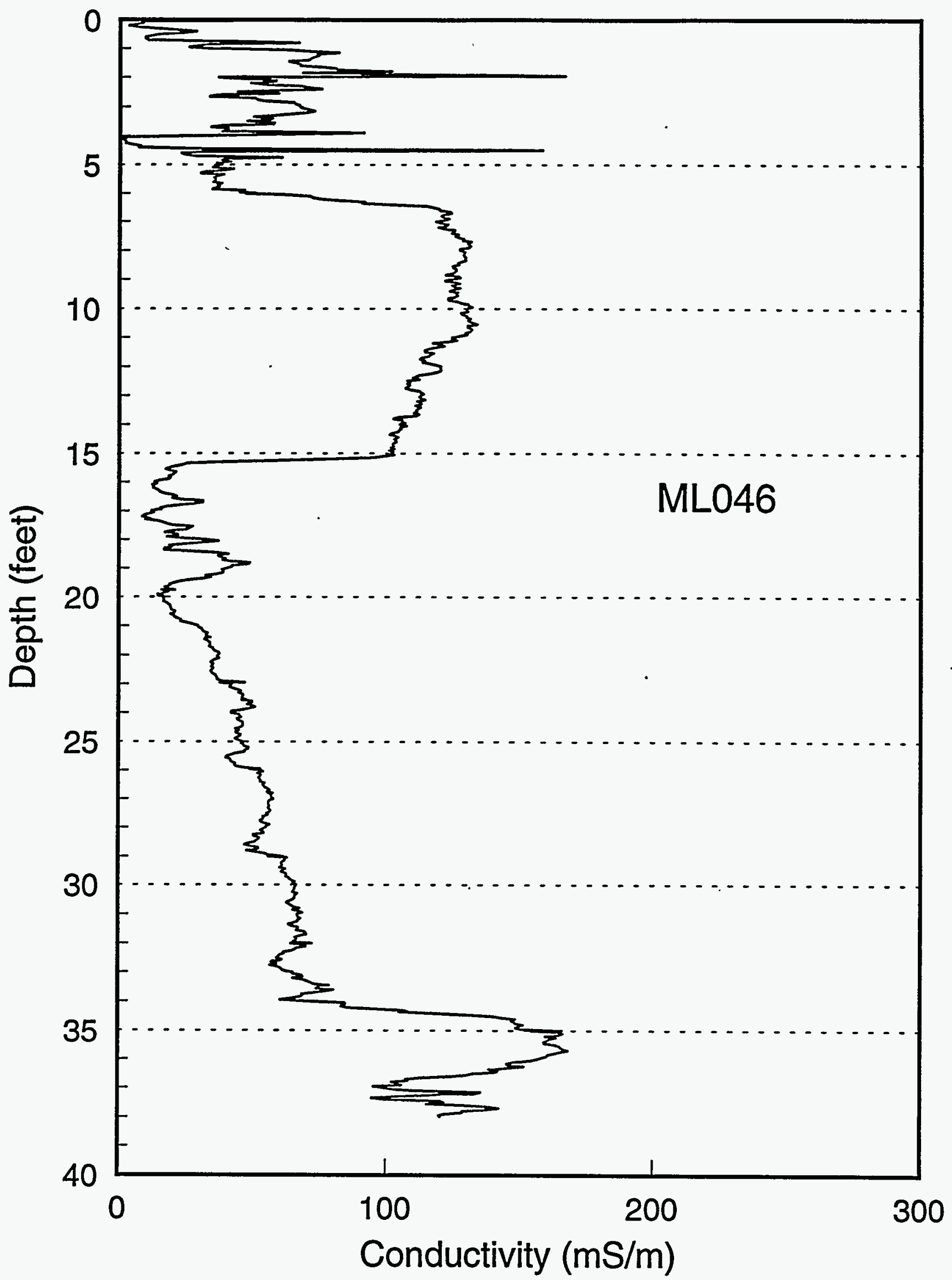




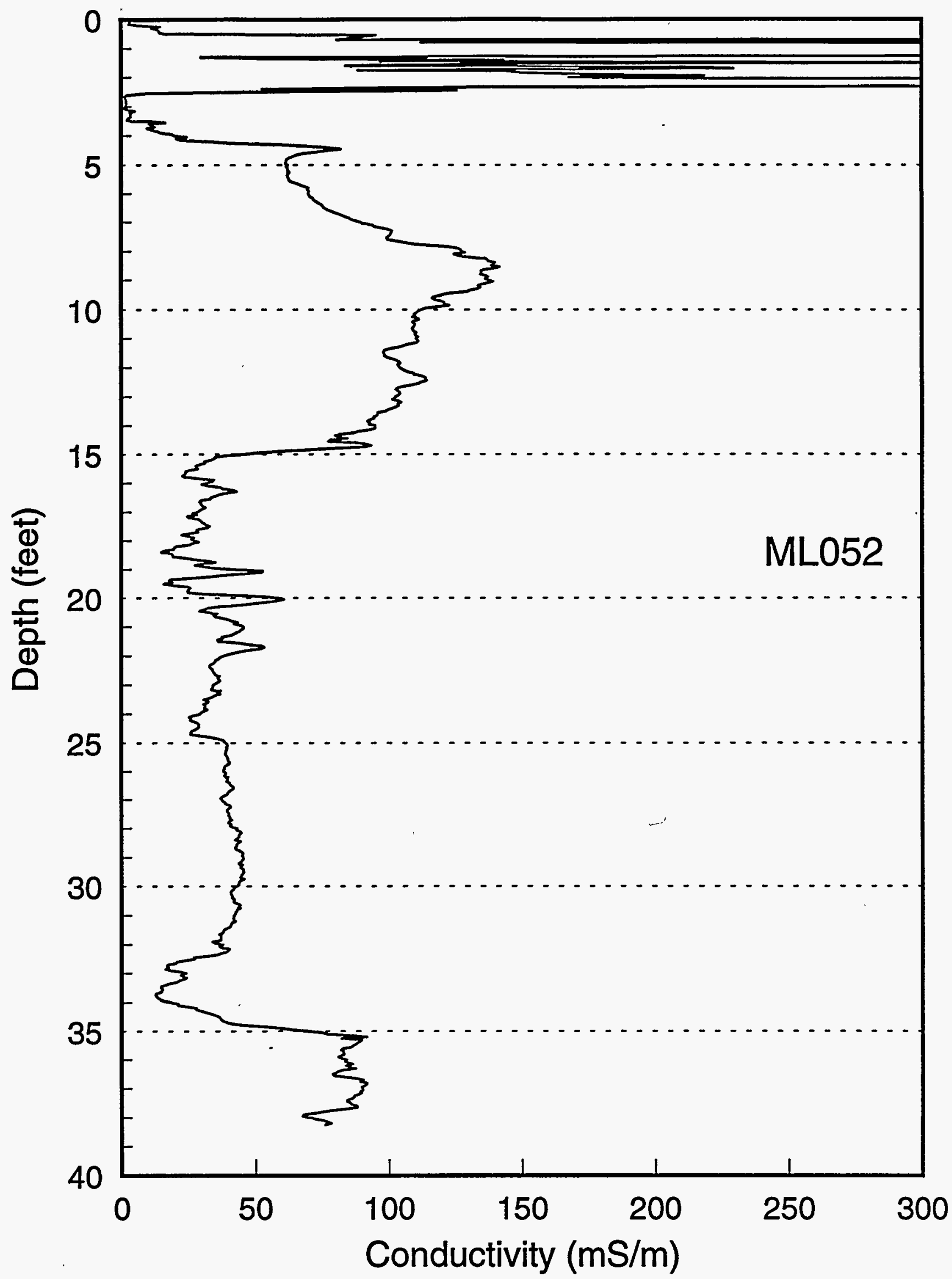




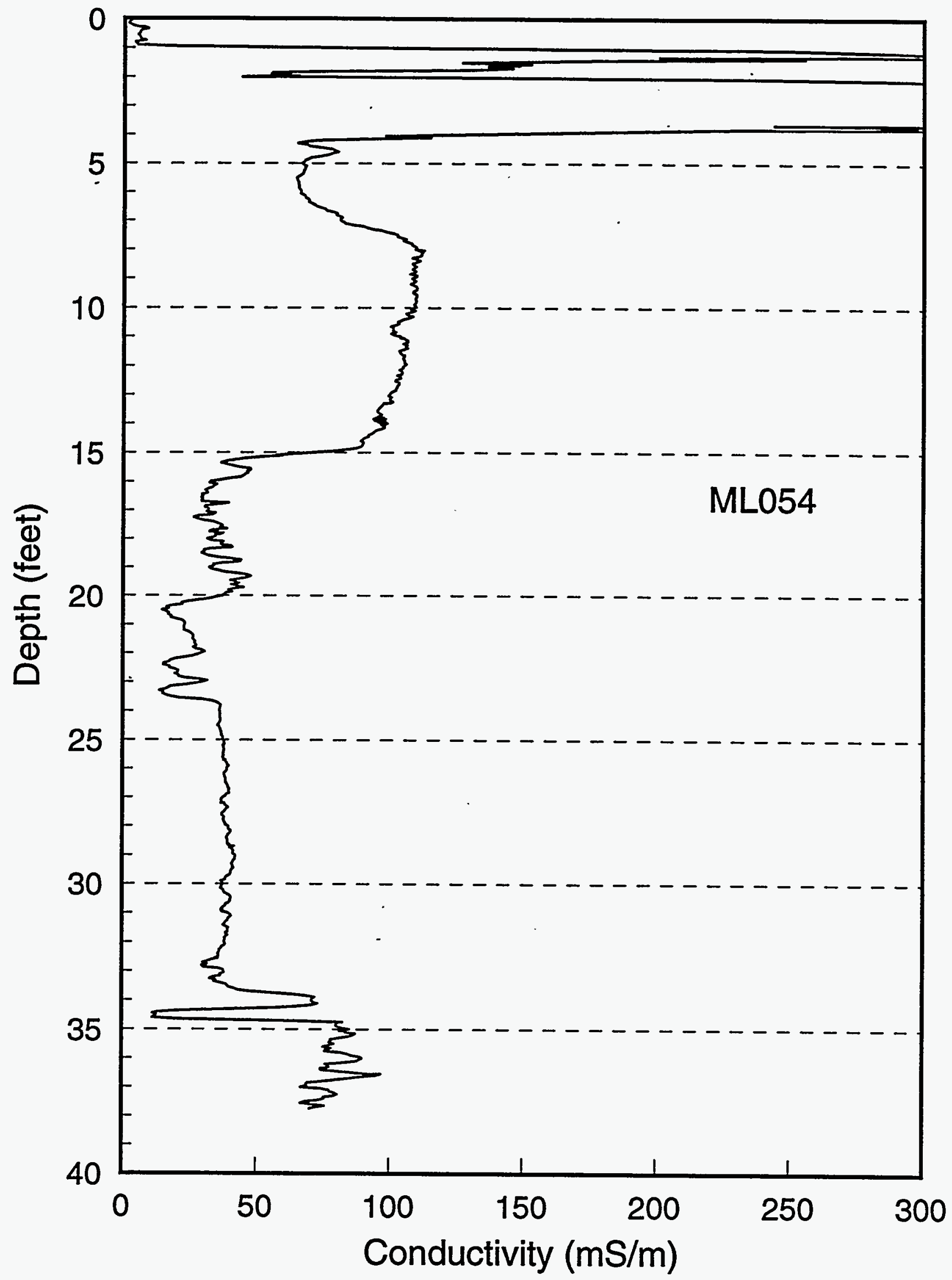




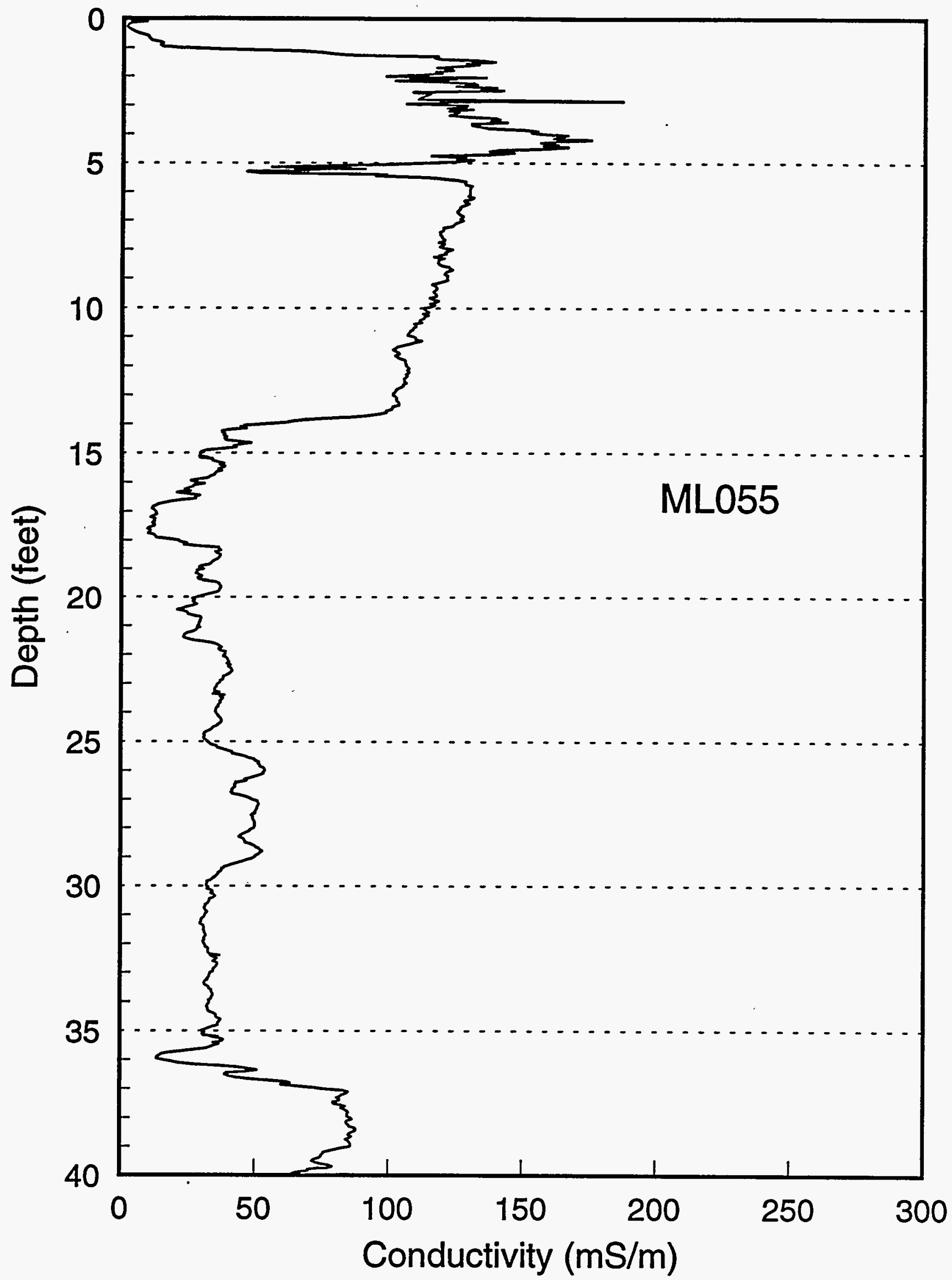




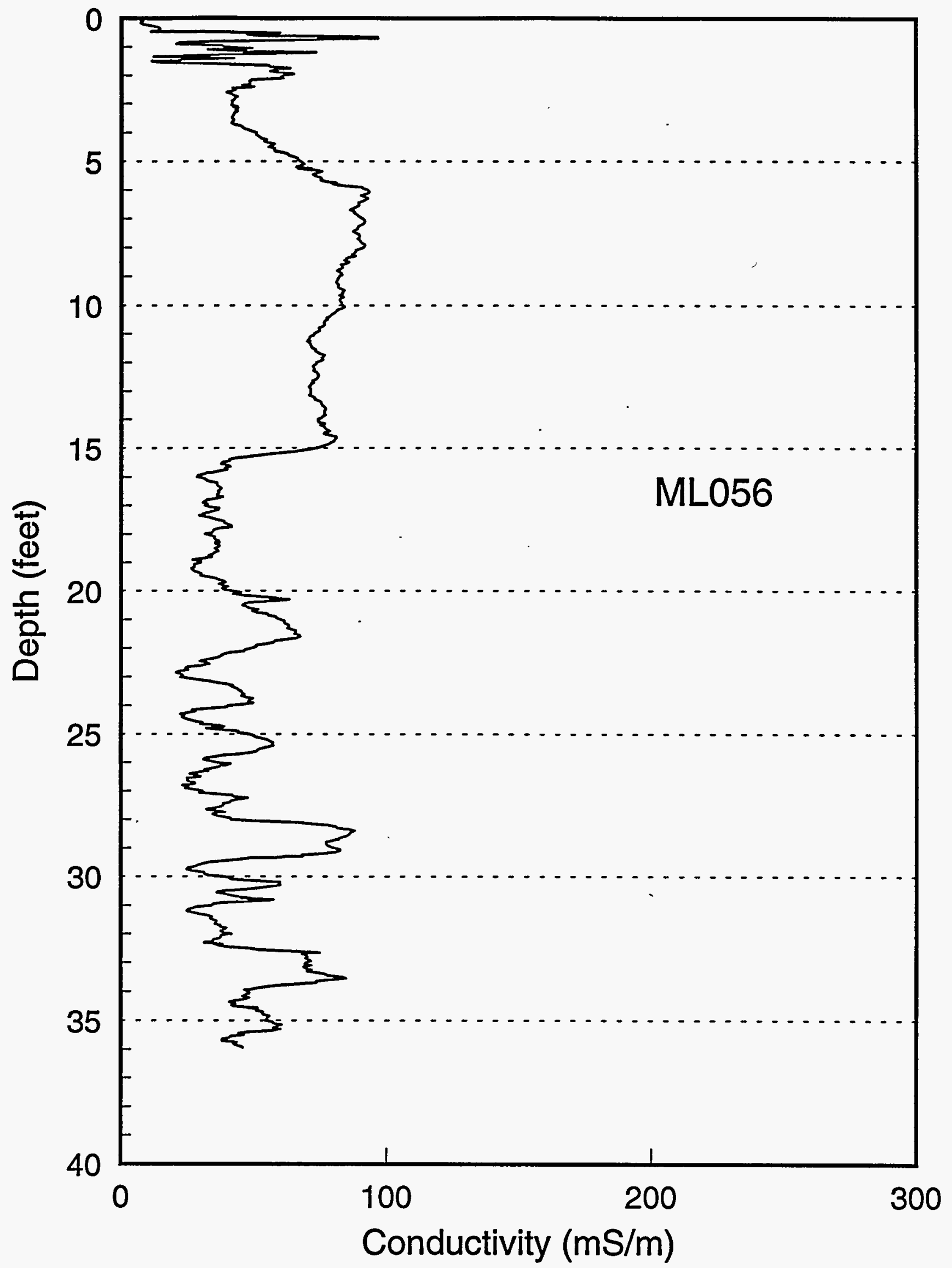




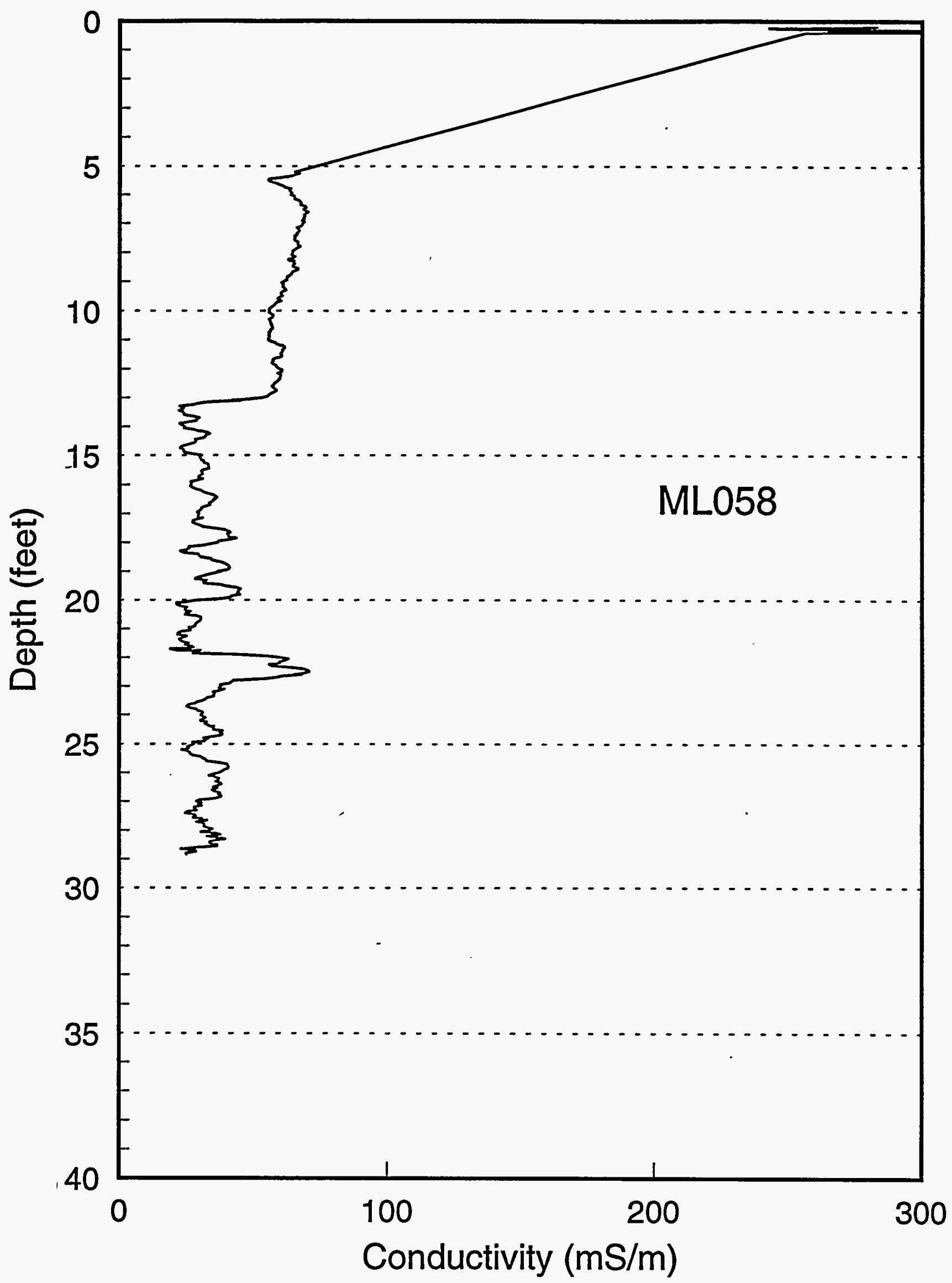




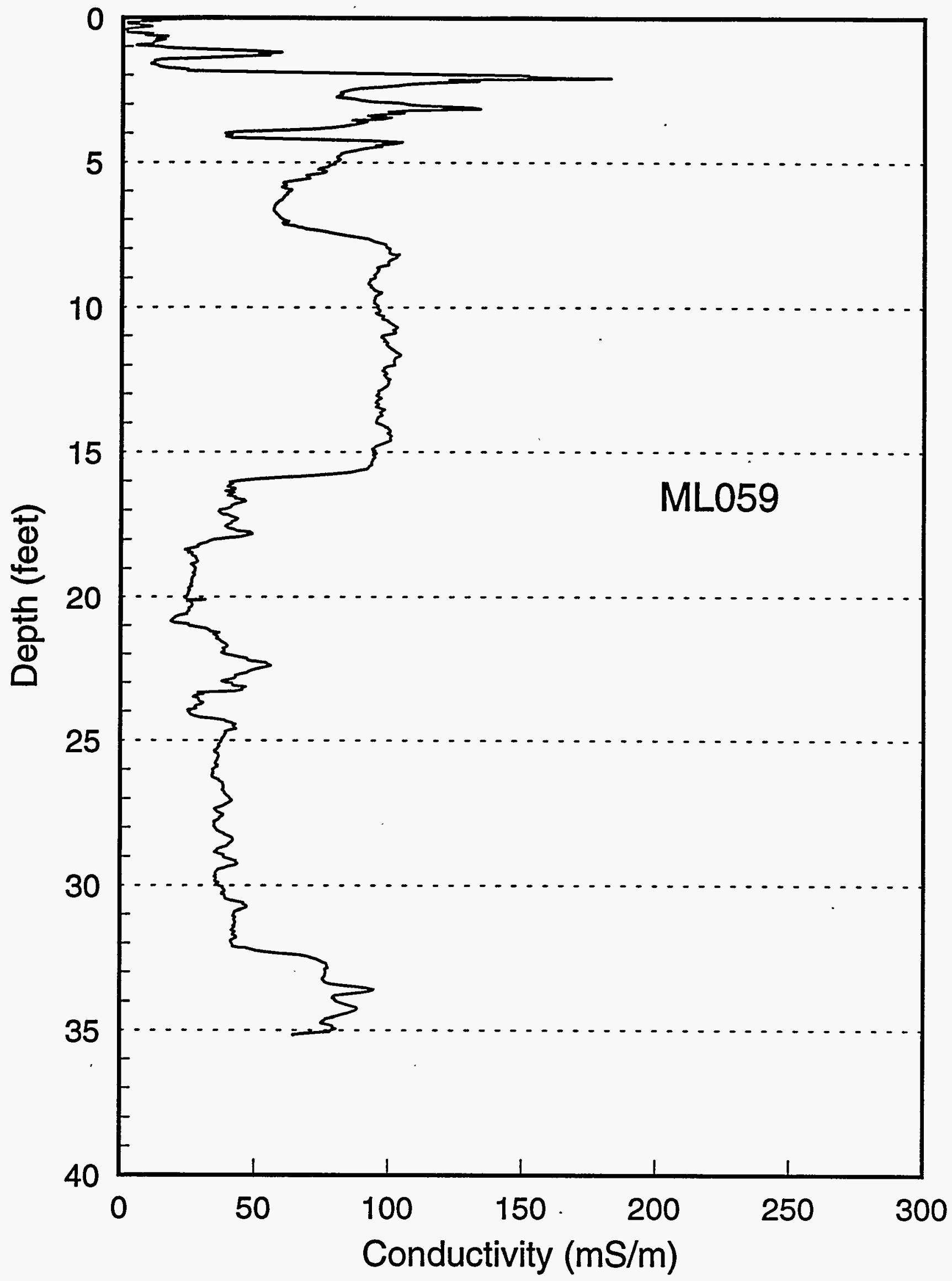




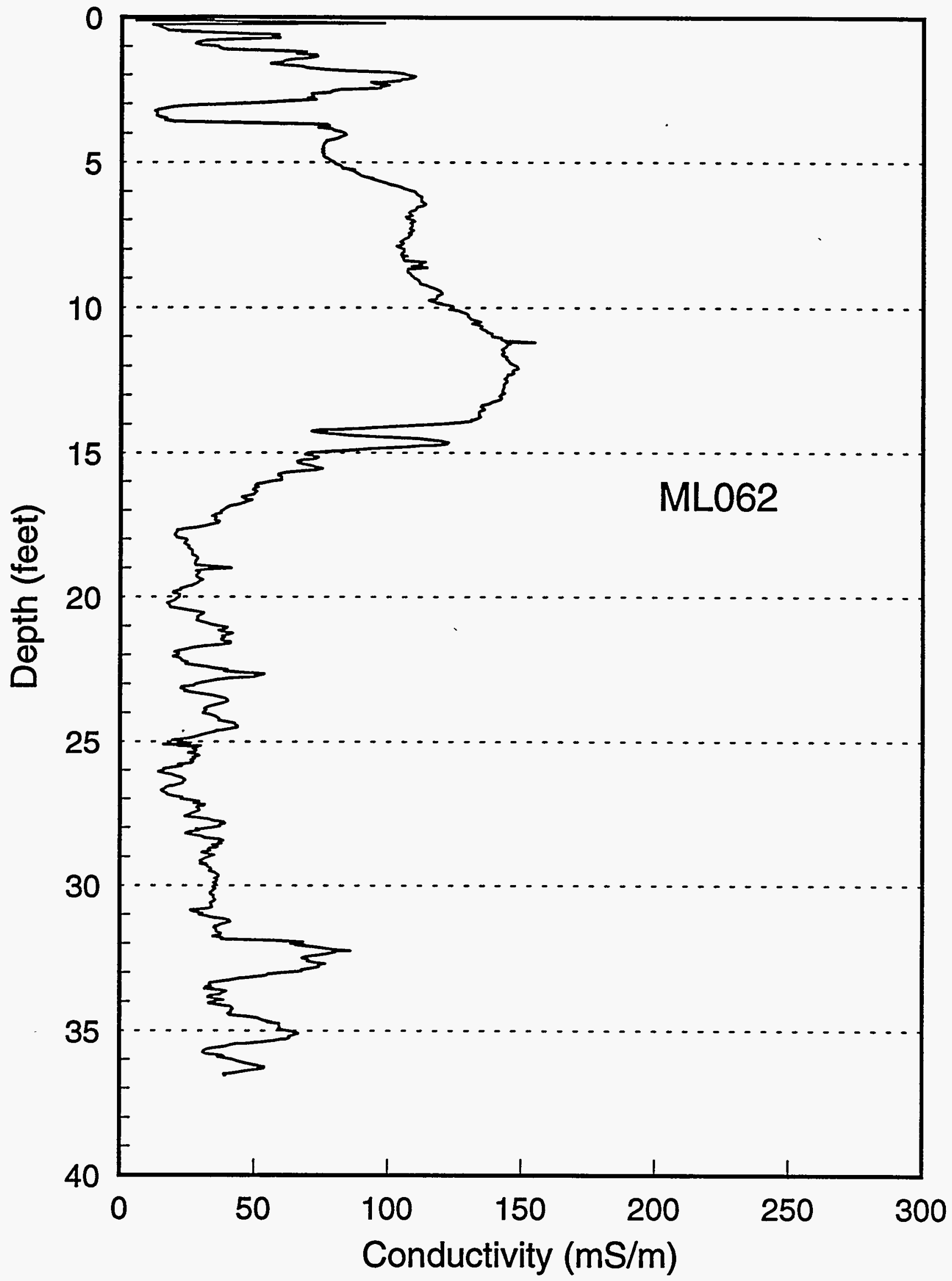




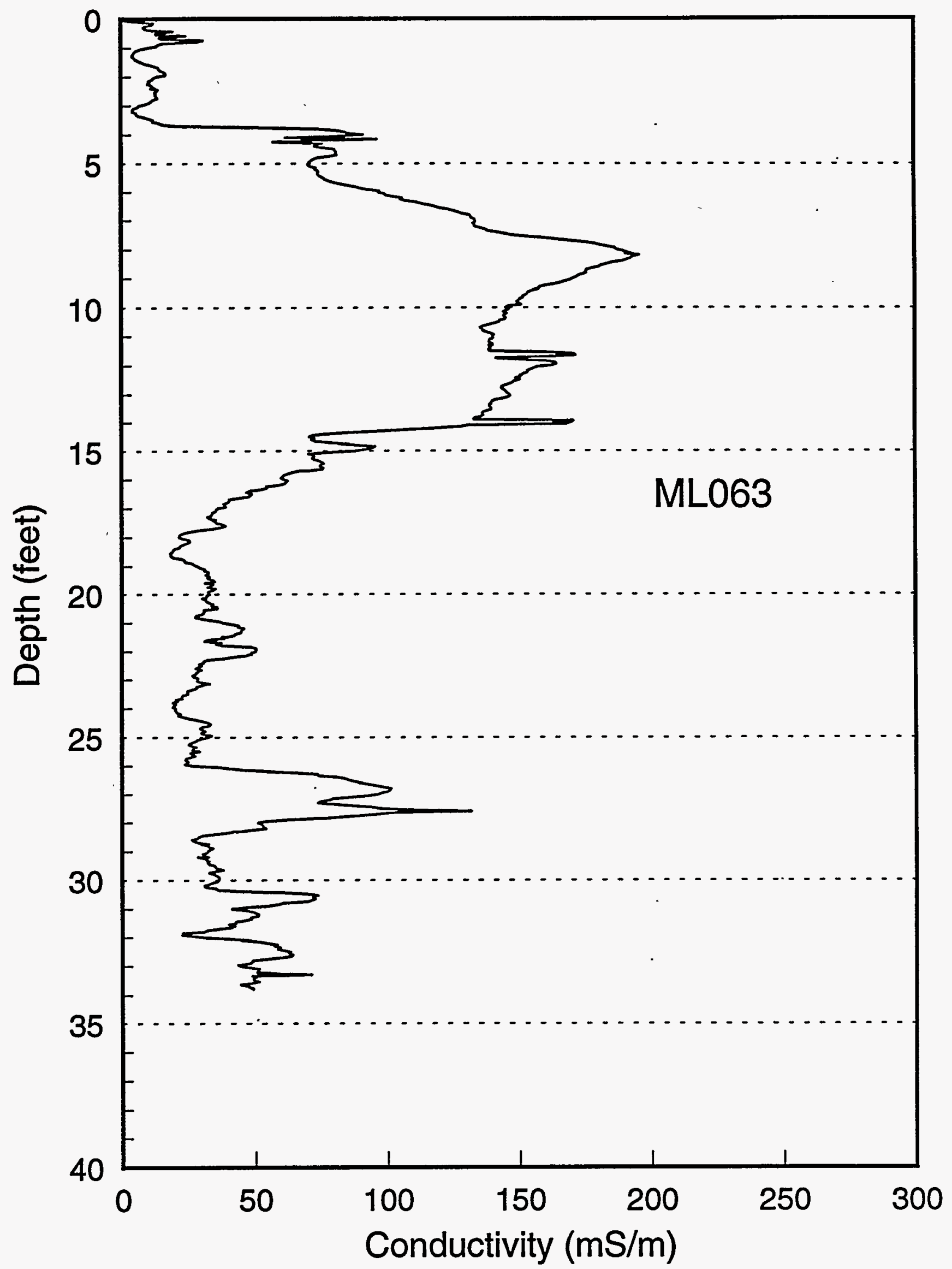




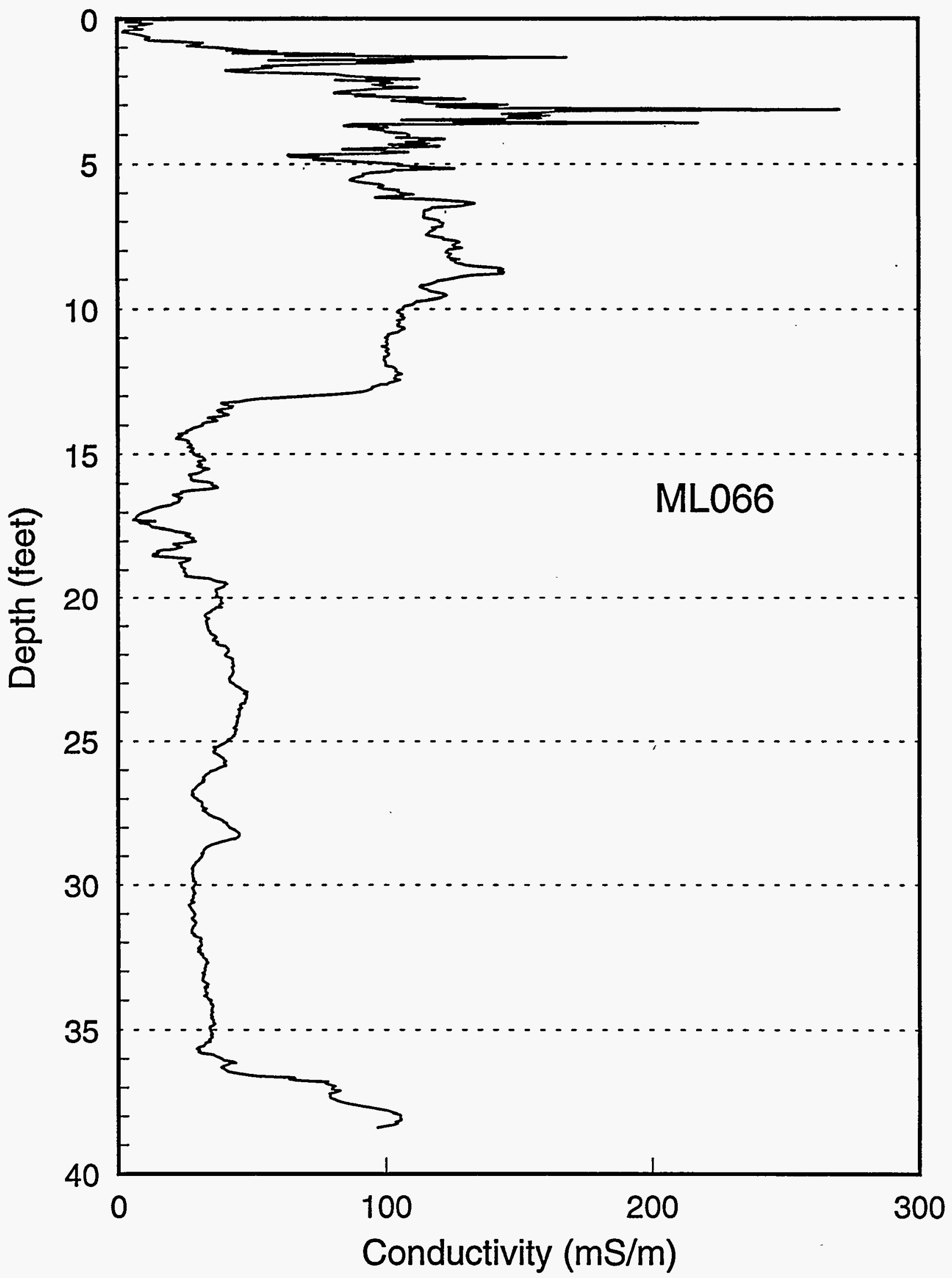




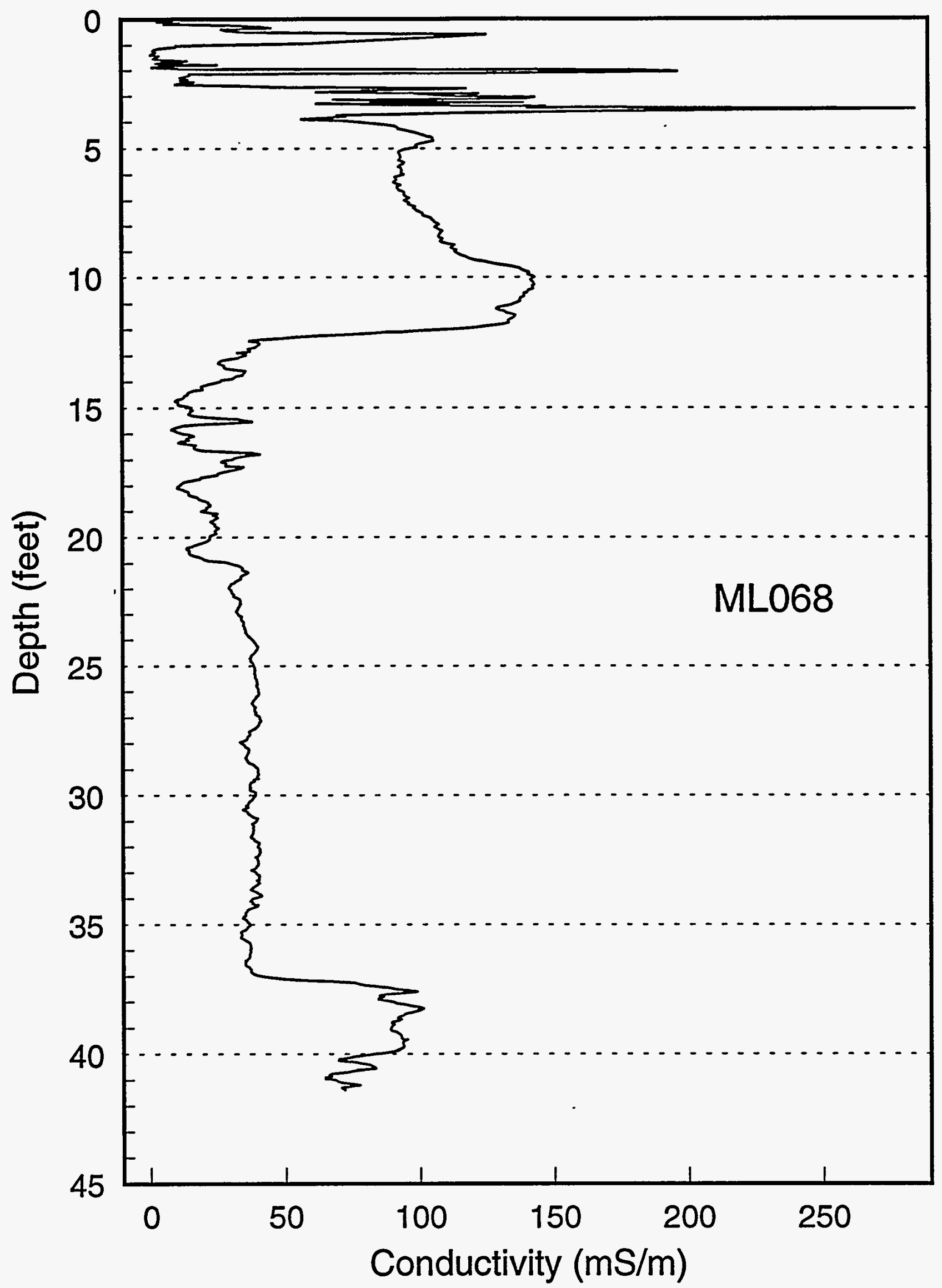




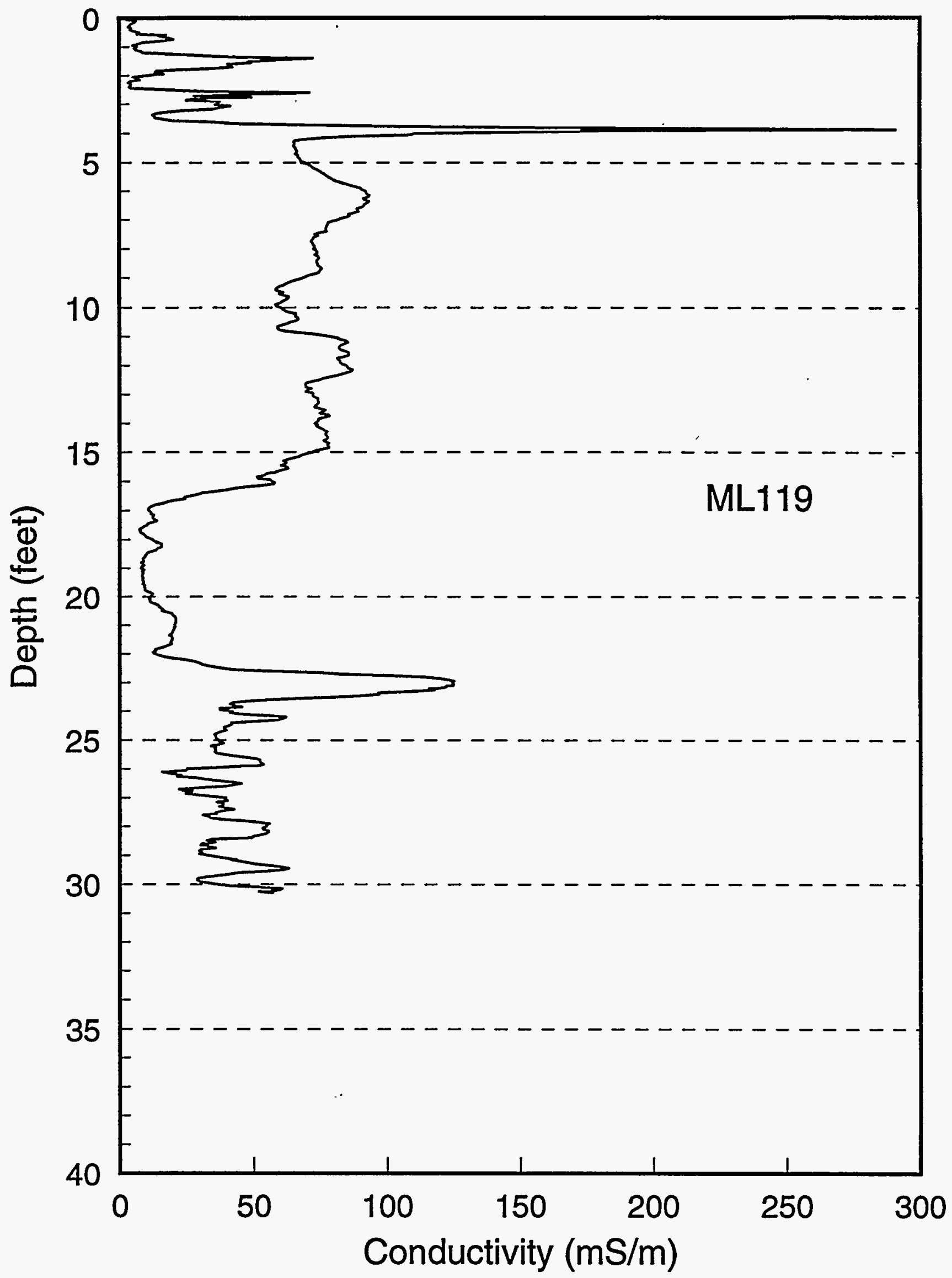


Depth (feet)

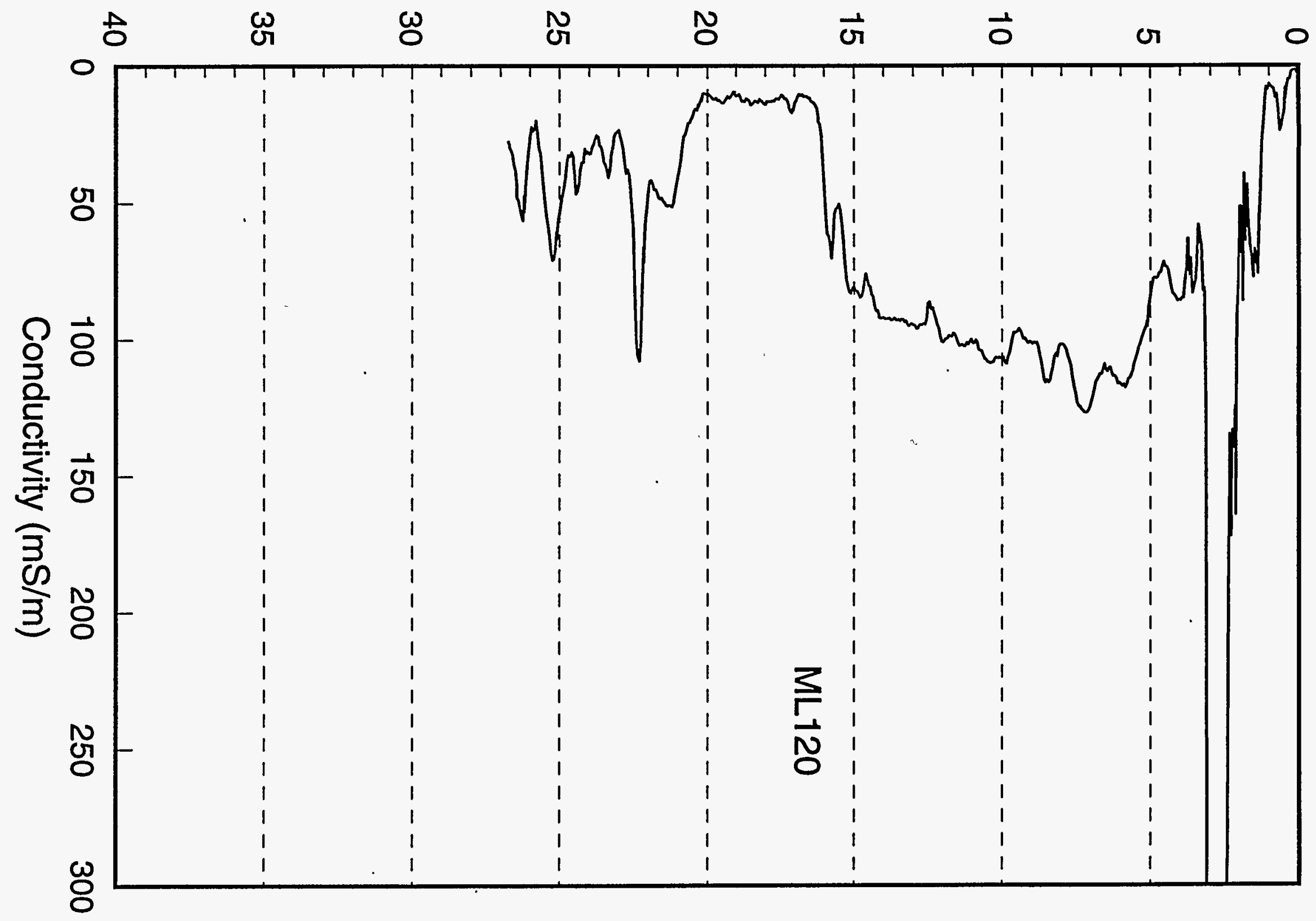




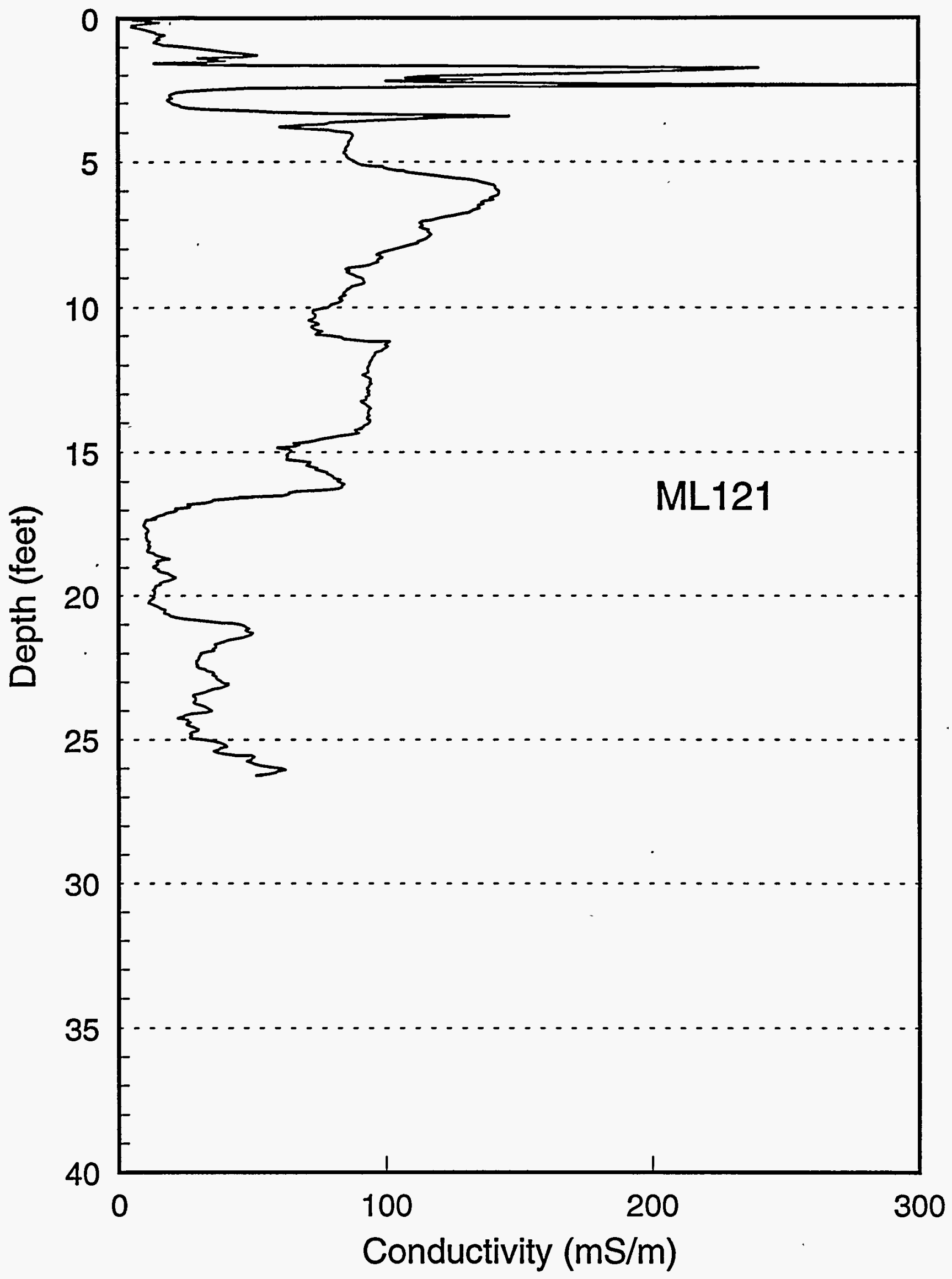


Ames Expedited Site Characterization - Marshalltown FMGP Site DRAFT Site Report

October 19, 1995

APPENDIX D:

ANALYTICAL RESULTS OF EACH SAMPLE FOR THE 16 PAFS 


\section{GCMS Data}

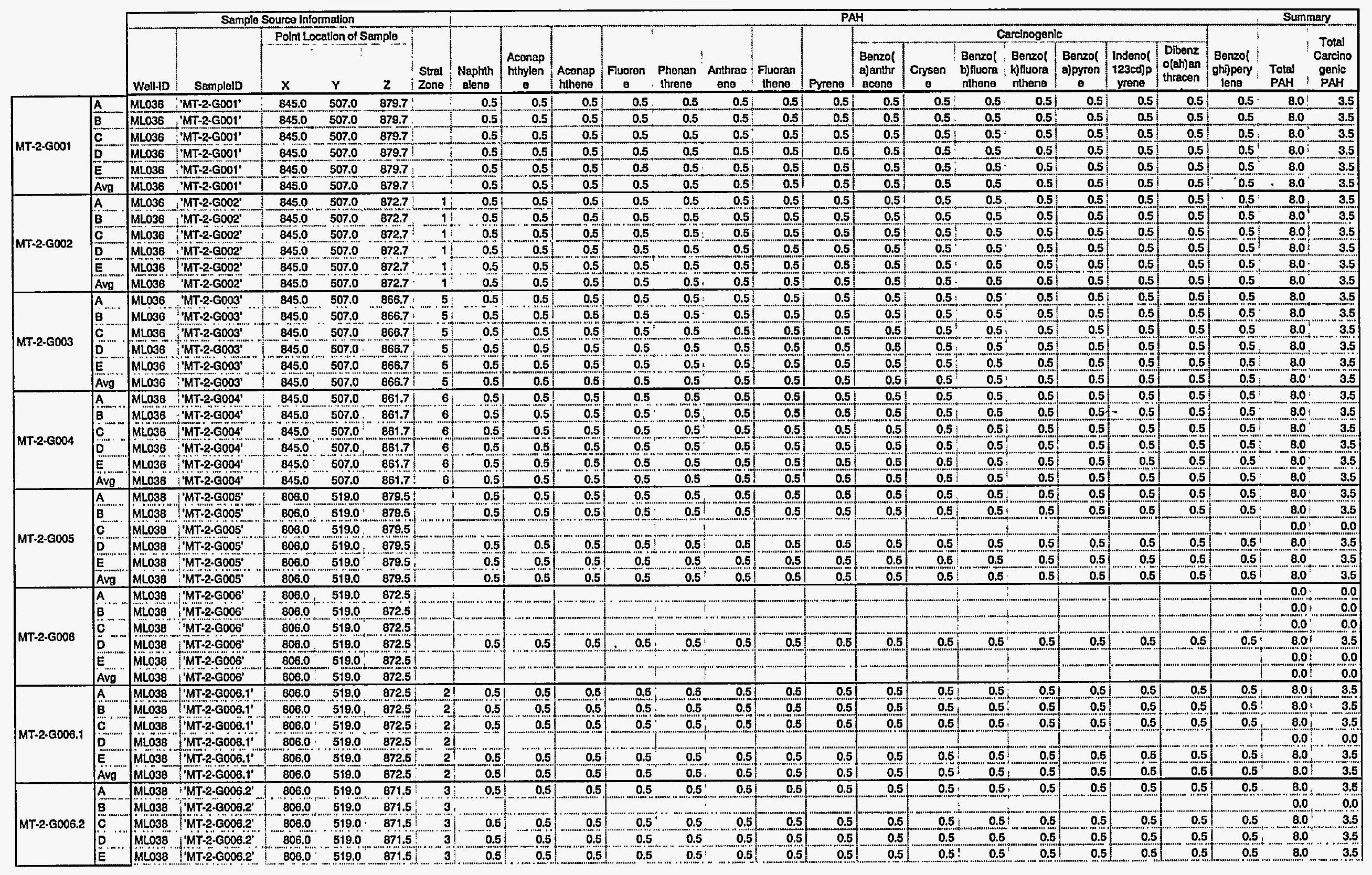

1 of 17

Data from Method D not used in calculations of averages 


\section{GCMS Data}

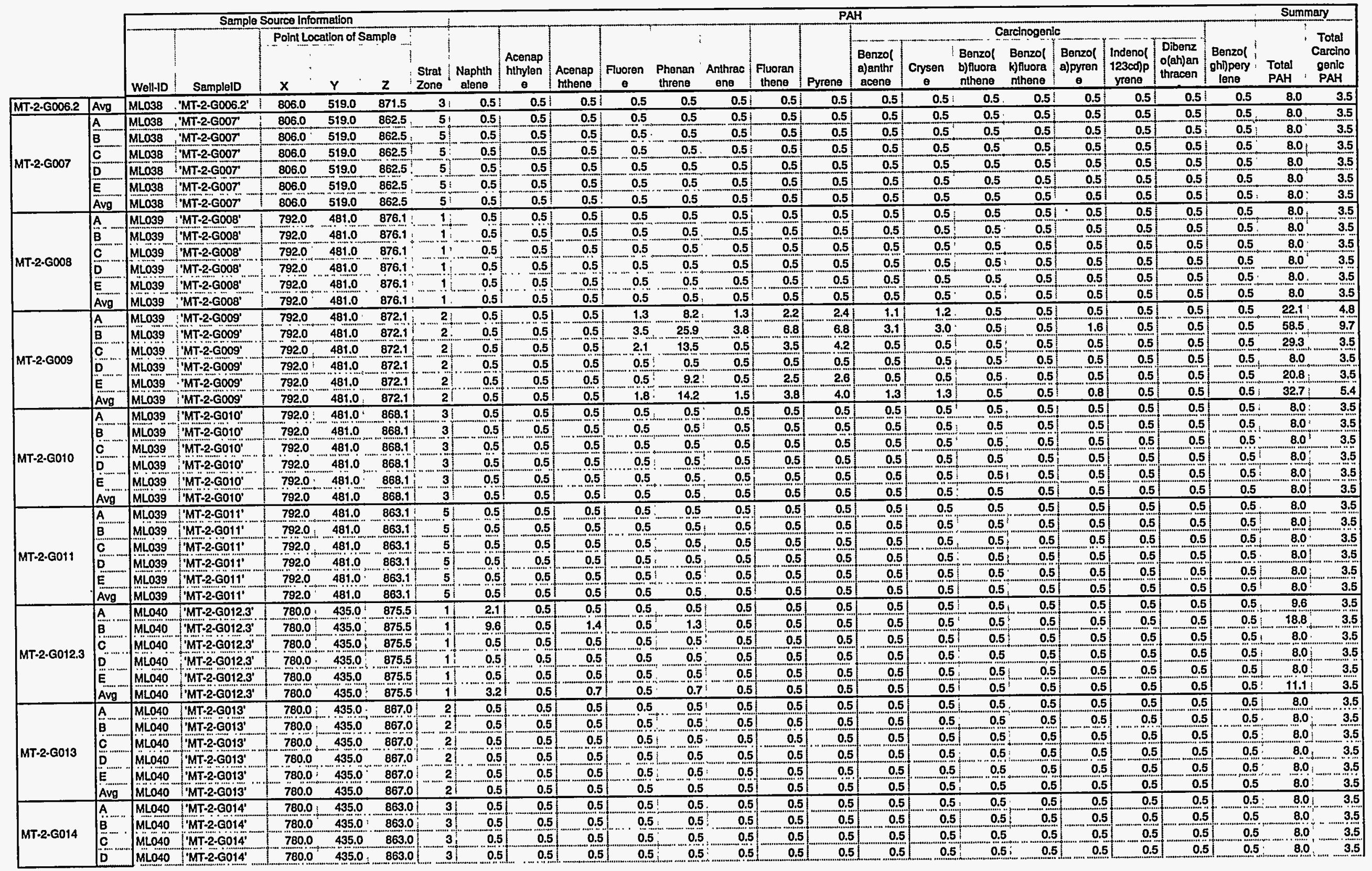

\section{2 of 17}

Data from Method D not used in calculations of averages 


\section{GCMS Data}

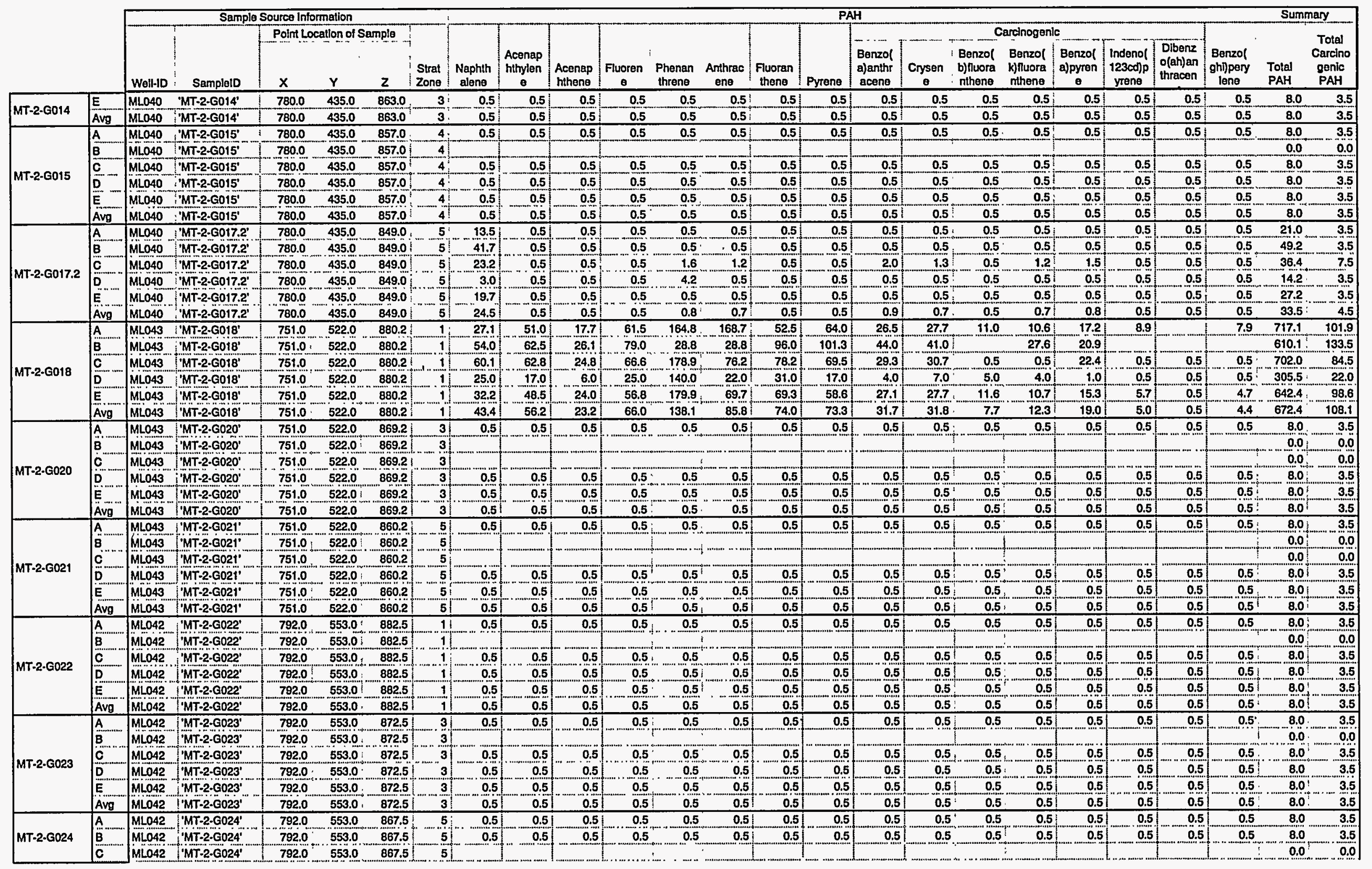

3 of 17

Data from Method D not used in calculations of averages 


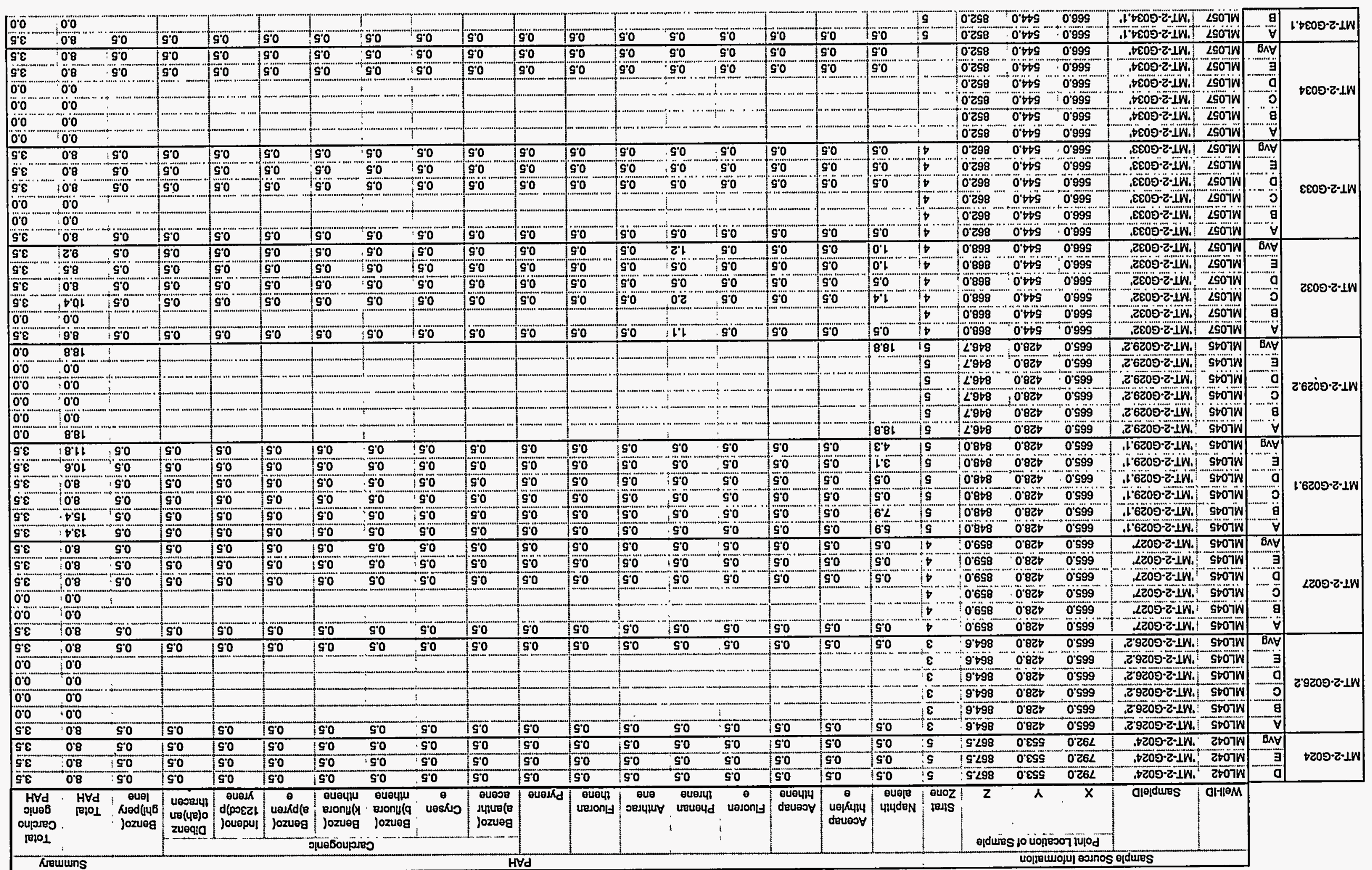

\section{efea SWDO}


GCMS Data

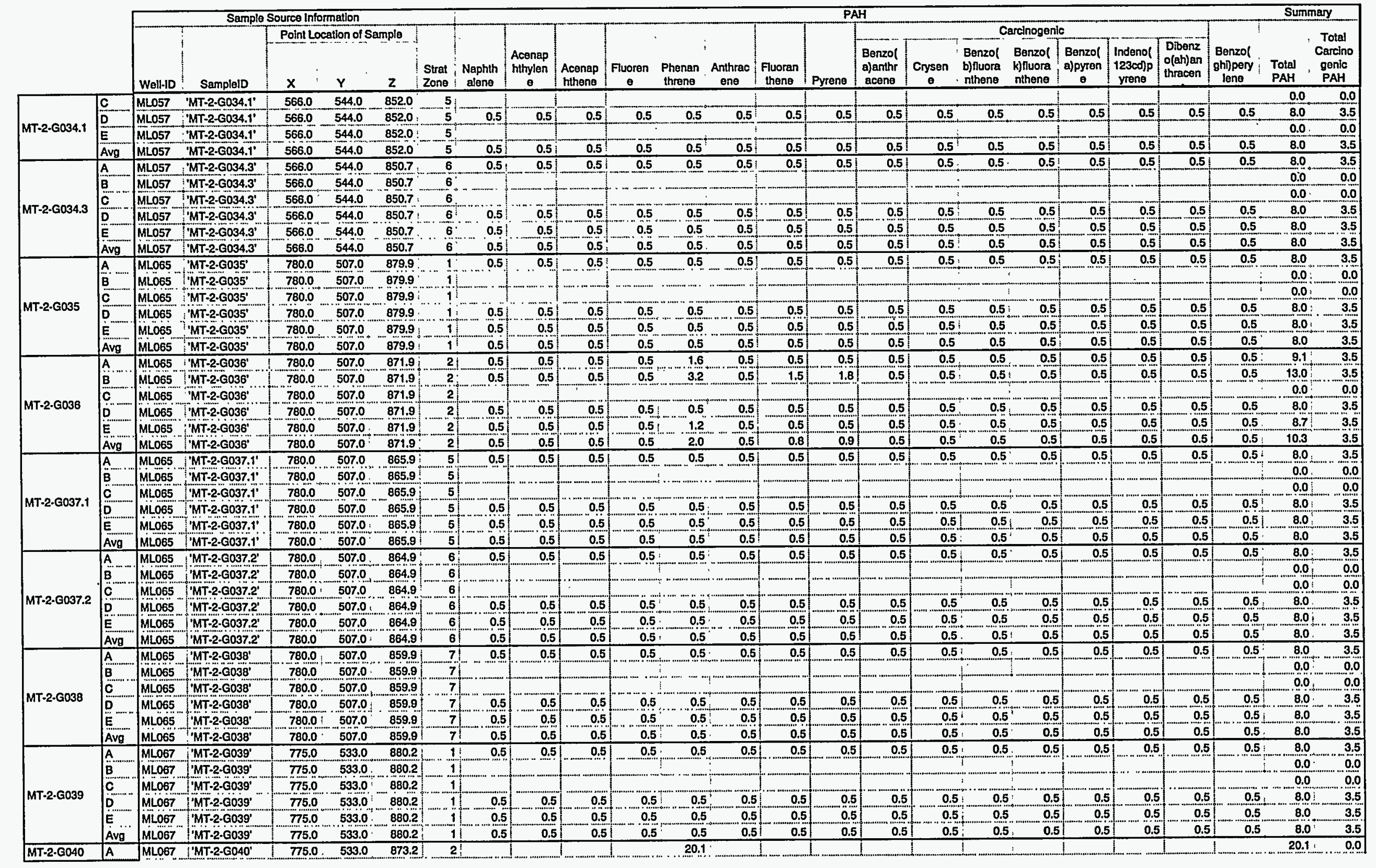

\section{5 of 17}

Data from Method D not used in calculations of averages 


\section{GCMS Data}

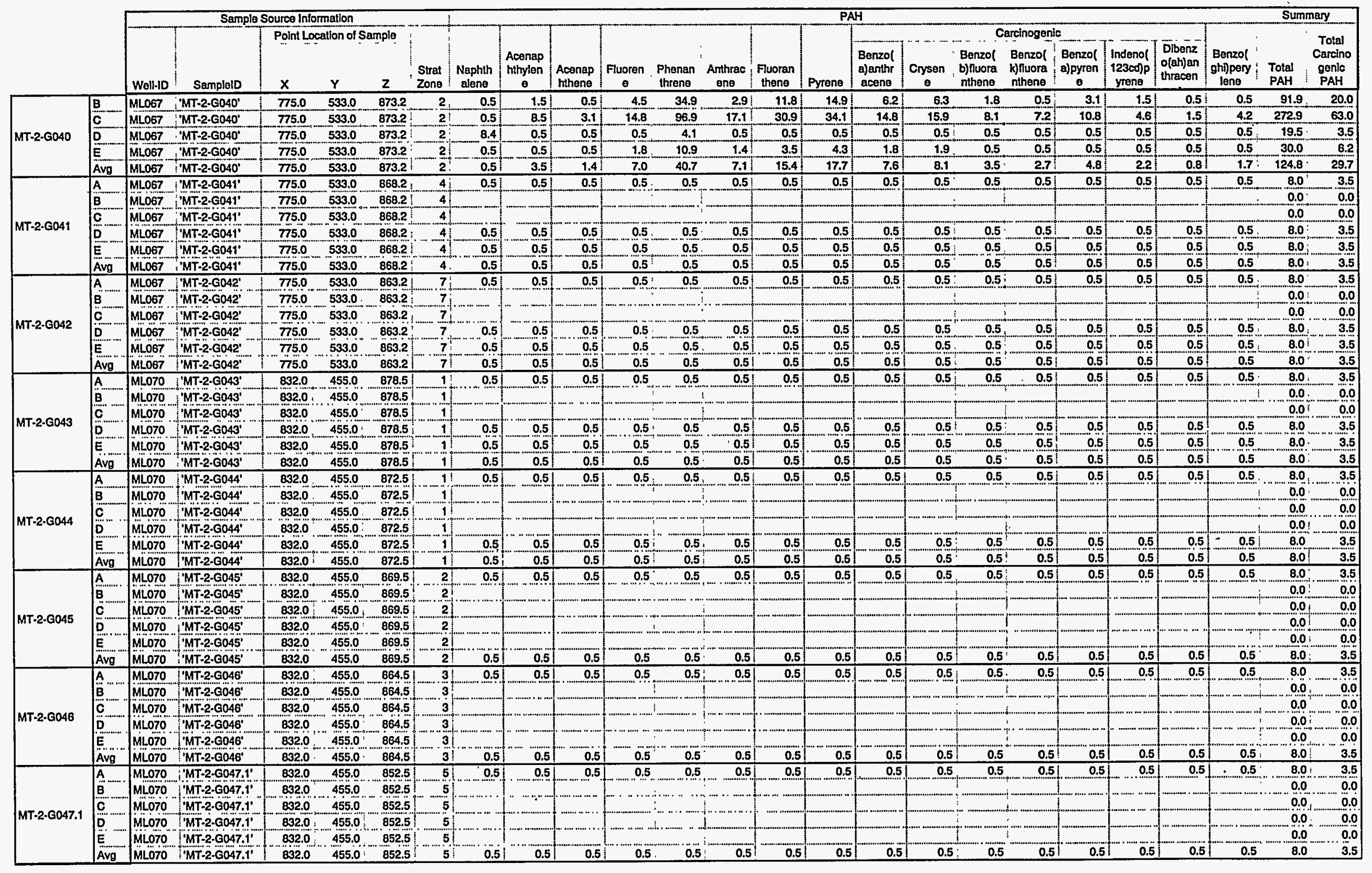

6 of 17

Data from Method D not used in calculations of averages 
GCMS Data

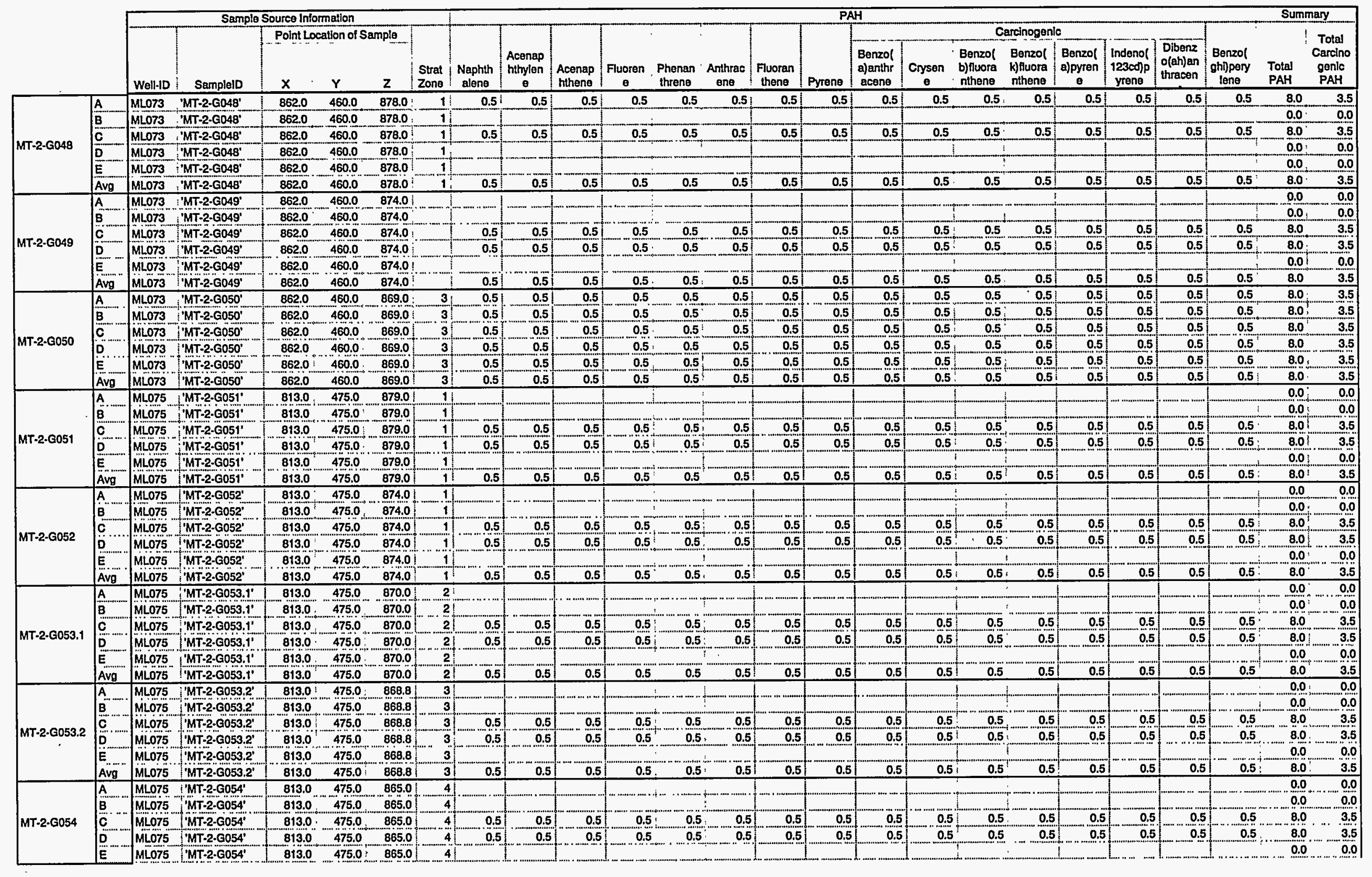

7 of 17

Data from Method D not used in calculations of averages 


\section{GCMS Data}

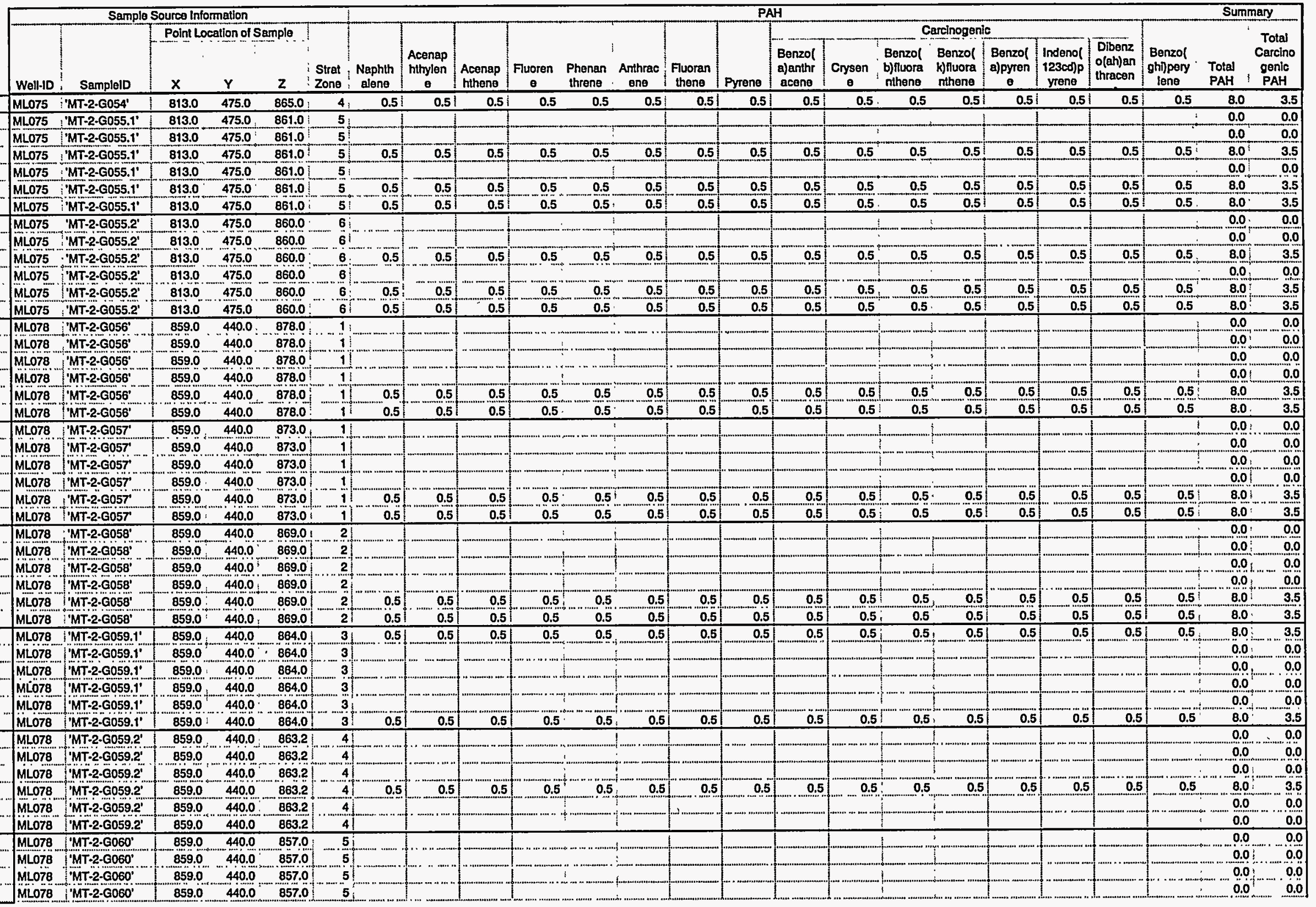

8 of 17

Data from Method D not used in calculations of averages 


\section{GCMS Data}

\begin{tabular}{|c|c|c|c|c|c|c|c|c|c|c|c|c|c|c|c|c|c|c|c|c|c|c|c|c|c|}
\hline & & \multicolumn{16}{|c|}{ PAH } & \multicolumn{3}{|c|}{ Summary } \\
\hline & & \multirow[b]{2}{*}{ Welltid } & \multirow[b]{2}{*}{ Sampleld } & \multicolumn{3}{|c|}{ Point Location of Sample } & \multirow{2}{*}{\multicolumn{2}{|c|}{\begin{tabular}{|c}
$i$ \\
$\mid \begin{array}{c}\text { Naphth } \\
\text { alene }\end{array}$ \\
\end{tabular}}} & \multirow[b]{2}{*}{$\begin{array}{c}\text { Acenap } \\
\text { hithylen } \\
\vdots\end{array}$} & \multirow[b]{2}{*}{$\begin{array}{l}\text { Acenap } \\
\text { hthene }\end{array}$} & \multirow[b]{2}{*}{$\begin{array}{c}\text { Fluoren ? } \\
8\end{array}$} & \multirow[b]{2}{*}{$\begin{array}{l}\text { Phenan } \\
\text { threne }\end{array}$} & & & & & & $C_{B}$ & archnogenic & & & & & & \\
\hline & & & & $x$ & $Y$ & $z$ & & & & & & & $\begin{array}{c}\text { Anthrac } \\
\text { ens }\end{array}$ & \begin{tabular}{|l|}
$\begin{array}{l}\text { Fuoran } \\
\text { thena }\end{array}$ \\
\end{tabular} & Pyrene & $\begin{array}{l}\text { Benzol } \\
\text { a)anthr } \\
\text { acena }\end{array}$ & $\begin{array}{c}\text { Cysen } \\
\theta\end{array}$ & $\begin{array}{l}\text { Benzzol } \\
\text { byfluora } \\
\text { nthene }\end{array}$ & $\begin{array}{l}\text { Benzol } \\
\text { kifluora } \\
\text { nthene }\end{array}$ & $\begin{array}{l}\text { Benzol } \\
\text { a)pyren } \\
e\end{array}$ & 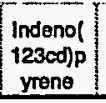 & 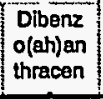 & $\begin{array}{l}\text { Benzol } \\
\text { ghippery } \\
\text { leng }\end{array}$ & $\begin{array}{l}\text { Total } \\
\text { PAH } \\
\end{array}$ & $\begin{array}{l}\text { loral } \\
\text { carcino } \\
\text { genle } \\
\text { PAH }\end{array}$ \\
\hline & E & MLOT8 & MT-2.6060 & 859.0 & 440.0 & $857.0 !$ & 5 & 0.5 & 0.5 & 0.5 & 0.5 & 0.5 & 0.5 & 0.5 & 0.5 & 0.5 & 0.5 . & 0.5 & 0.5 & 0.5 & 0.5 & 0.5 & 0.5 & 8.0 & 3.5 \\
\hline 30 & Avg & ML078 & MT-2-6060 & 859.0 & 440.0 & 857.0 & 5 & 0.5 & 0.5 & 0.5 & 0.5 & 0.5 & 0.5 & 0.5 & 0.5 & 0.5 & 0.5 & 0.5 & 0.5 & 0.5 & 0.5 & 0.5 & 0.5 & 8.0 & 3.5 \\
\hline & $A$ & MLOBO & MTT-2.6062' & 11.0 & 415.0 & 877.4 & 1 & 6.5 & 0.5 & 0.5 & 1.3 & 4.0 & 4.1 & $2.2 !$ & 2.4 & 1.1 & 1.1 & 1.2 & 1.4 & 0.5 & 0.5 & 0.5 & 0.5 & 28.2 & 6.3 \\
\hline & $\mathrm{B}$ & MLOBO & 'MT-2-G062' & 781.0 & 415.0 & 877.4 & 1 & & & & & & & & & & & & & & & & & 0.0 & 0.0 \\
\hline & $c$ & MLOBO & MT-2.6062 & 781.0 & 415.0 & 877.4 & 11 & & & & & & & & & & & & & & & & & 0.0 & 0.0 \\
\hline TT-2-G062 & D & & 'MT-2.6062' & 781.0 & 415.0 & 877.4 & 1 & 0.5 & 0.5 & 0.5 & 0.5 & 0.5 & 0.5 & 0.5 & 0.5 & 0.5 & 0.5 & 0.5 & $0.5 !$ & 0.5 & 0.5 & 0.5 & 0.5 & 8.0 & 3.5 \\
\hline & E & & $3062^{\prime}$ & 781.0 & 415.0 & 877.4 & 1 & 6.1 & 0.5 & 0.5 & 0.5 & 0.5 & 0.5 & 0.5 & 0.5 & 0.5 & 0.5 & 0.5 & 0.5 & 0.5 & 0.5 & 0.5 & $0.5 \mathrm{i}$ & 13.6 & 3.5 \\
\hline & Avg & MLOBO & MT-2 & 781.0 & 415 & 877.4 & 1 & 6.3 & 0.5 & 0.5 & 0.9 & 2.3 & 2.31 & 1.4 & 1.5 & 0.81 & 0.8 & 0.9 & 1.0 & 0.5 & 0.5 & 0.5 & $0.5:$ & 20.9 & 4.9 \\
\hline & A & MLOBO & MT-2-6063". & 781.0 & 415.0 & 874.4 & 1 & & & & & & 1 & & & 1 & & & & & & & & 0.0 & 0.0 \\
\hline & $B$ & MLOBO & $M M-2-6063$ & 1.0 & 415.0 & 874.4 & 1 & & & & 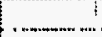 & & & & & & & & & & & & & 0.0 & \\
\hline & c & 80 & MT-2-G063' & 1.0 & 415.0 & 874.4 & 1 & & & & & & & & & & & & & & & & & 0.0 & 0.0 \\
\hline MT-2-G063 & 0 & & $M T-2 . G 063^{\prime}$ & $781.0^{\circ}$ & $415.0^{\circ}$ & 874.4 & 1 & 0.5 & 0.5 & 0.5 & 0.5 & 0.5 & $0.5 !$ & 0.5 & 0.5 & 0.5 & 0.5 & 0.5 & 0.5 & 0.5 & 0.5 & 0.5 & 0.5 & 8.0 & 3.5 \\
\hline & & & & & -415 & 874.4 & 1 & & & & & & & & & & & & & & & & & 0.0 & 0.0 \\
\hline & Avg & ML & & 78 & 41 & 874.4 & i & & & & & & & & & & & & & & & & & 0.0 & 0.0 \\
\hline & A & MI & MT-2:G064 & $781: 0$ & 415.0 & 869.4 & 2 & 0.5 & 0.5 & 0.5 & 0.5 & 0.5 & $0.5 \mathrm{j}$ & 0.5 & 0.5 & 0.51 & 0.5 & 0.5 & 0.5 & 0.5 & 0.5 & 0.5 & 0.5 & 8.0 & 3.5 \\
\hline & $B$ & 80 & $M T-20064$ & 7810 & 415.0 & 869.4 & 2 & 0.5 & 0.5 & 0.5 & 0.5 & 0.5 & 0.5 & 0.5 & $0.5 !$ & 0.5 & 0.5 & 0.5 & 0.5 & 0.5 & 0.5 & 0.5 & 0.5 & 8.0 & \\
\hline & $c$ & MLO80 & $M T-2.0064^{\prime}$ & 781.0 & 415.0 & 869.4 & 2 & & & & & & & & & & & & & & & & & 0.0 & 0.0 \\
\hline MT-2-G064 & D & & MT-2-G064 & .0 & $415.0^{\circ}$ & 869.41 & 2 & 0.5 & 0.5 & 0.5 & 0.5 & 1.6 & 0.5 & 0.5 & 0.5 & 0.5 & 0.5 & 0.5 & 0.51 & 0.5 & 0.5 & 0.5 & 0.5 & 9.1 & 3.5 \\
\hline & E & & 064 & & 415.0 & 869.4 & 2 & 0.5 & 0.5 & 0.5 & 0.5 & .0 .5 & 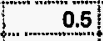 & 5 & 0.5 & 0.5 & 5 & 0.5 & 0.5 & 0.5 & 0.5 & 0.5 & 0.5 & 8.0 & 3.5 \\
\hline & Aिg & & & 78 & 41 & 869.4 & & 0.5 & 0.5 & 0.5 & 0.5 & 0.5 & 0.5 & 0.5 & 0.5 & 0.5 & 0.5 & 0.5 & 0.5 & 0.5 & 0.5 & 0.5 & 0.5 & 8.0 & 3.5 \\
\hline & A & MLO080 & MT-2.G065' & 781.0 & 415.0 & 864.4 & $3 !$ & & 9.6 & & 9.1 & 38.8 & & 12.5 & 18.8 & 6.4 & $5.9 \mathrm{i}$ & & & & & & & 112.4 & 12.4 \\
\hline & $\mathrm{B}$ & MLOOBO & & $7810^{\circ}$ & 415.0 & 864.4 & 3 & 0.5 & 32.4 & 2.1 & 9.8. & 52.8 & 15.0 & 17 & & 7 & 7.3. & 0.5 & & 4.5 & 2.3 & 0.5 & 2.4 & 154.4 & 23.1 \\
\hline & $\mathrm{C}$ & MLO080 & MT-2-6065' & 781.0 & 415. & 864.4 & $3 !$ & 0.5 & 19.8 & 4.1 & 17.1. & 64.5 & 24.1 & 21.2 & 25 & 9.9 & 9.3 & 4.6 & 5.3 & 7.9 & 0.5 & & 3.7 & 217.8 & 37.4 \\
\hline |MT-2-G065 & $D$ & MLOBo & MT-2-6065' & 781.0 & 415.0 & 864.4 & 3 & 0.5 & 3.9 & 1.4 & 5.3 & 71.8 & 10.8 & 16.6 & 13.6 & - & 3.3 & 5.7. & 4.9 & 0.5 & 5.8 & 5 & 3.7 & 52.0 & 24.4 \\
\hline & $\mathrm{E}$ & & $M T-2.6065^{\prime}$ & 781.0 & 415.0 & 864.4 & & 0.5 & 13.3 & 2.6 & 10.5 & $\begin{array}{r}39.8 \\
\end{array}$ & 14.0 & 15.6 & 19.5 & 800 & 7.8 & 3.4 & 4.4 & 2 & 23 & 0.5 & 2.3 & 150.5 & i \\
\hline & Avg & & MT-2-c065 & 781.0 & 415 & 864.4 & & 0.5 & 13.8 & 3.0 & 11.6 & 49.0 & 16.1 & 16.6 & 20.7 & 8.07 & 7.6 & 2.8 & 3.4 & 2 & 1.7 & 0.5 & 2.8 & 164.1 & 30.1 \\
\hline & $\frac{T}{A}$ & & & 781 & .415 .0 & $854.4 !$ & & & & & & & & & & & & & & & & & & 0.0 & 0.0 \\
\hline & B. & & & 7810 & 415.01 & 854.4 & 4 & & & & .............. & . . ..... & & & & & & & & & & & & 0.0 & \\
\hline & c'”' & Milogo & MT-2.0066 & 781 & 415 & 854.4 & 4. & & & & $\ldots . . . .$. & $\cdots$ & & & & & ....... & 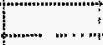 & & ...... & ?.:- & & & 0.0 & 0.0 \\
\hline & D. & MiLOOBO & $M T-2-0066$ & 781 & 415 & 854.4 & & & & & & & & & & & & & & & & & & 0.0 & \\
\hline & $E$ & & MT 2.6 & 781 & 415 & 854.4 & & 0.5 & 0.5 & o... & 0.5 & 0.5 & 0.5 & 0.5 & 0.5 & 0.5 & 0.5 & 0.5 & 0.5 & 0.5 & 0.5 & 0.5 & 0.51 & 8.0 & 3.5 \\
\hline & Ávg & & MT & 781 & 415.0 & 854.4 & 4 & 0.5 & 0.5 & - 0.5 & 0.5 & 0.5 & 0.5 & 0.5 & 0.5 & 0.5 & 0.5 & 0.5 & 0.01 & 5 & 0.5 & 0.5 & .5. & .0 & \\
\hline & A & & & 78 & 41 & 84 & (6) & 42. & & & & 9.1 & & & & & & & & & & & & 51.4 & 0.0 \\
\hline & B & $\mid M$ & & 781.0 & 415. & & 8 & 19. & 1.7 & 0.5 & 1.0 & 3.3 & 0.5 & 0.5 & 1.0 & 0.5 & $\pi$ & 0.5 & $0.5 !$ & 0. & 0.5 & 0.5 & 0.5 & 31.8 & 3.5 \\
\hline & c & & MT-2 & 781. & 415 & & 8 & 70.1 & 7.9 & 1.6 & 5.6 & 14.2 & 5.1 & 4.0 & 4.7 & 2.0 & 28 & 0.5 & 1.1 .1 & 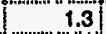 & 0.5 & 0 & 0.5 & 121.6 & 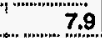 \\
\hline MT-2-G067.2 & D & MLOBO & MT-2-G067.2 & 781.0 & 415.0 & 84 & 6 & 9.3 & 0.5 & 0.5 & 7.7 & 7.7 & 0.5 & 1.1 & 0.5 & 0.5 & 0.5 & 0.5 & 0.5 & 0.5 & 0.5 & 0.5 & 0.5 & 25.8 & 3.5 \\
\hline & $E$ & MLOBO & MT-2.0087.2 & 781 & 415.0 & & 6 & & & & & & & & & & & & & & & & & & \\
\hline & Avg & & M & & $41:$ & 84 & 6 & 43.9 & 4.8 & 11 & 3,3 & 8.9 & 2.8 & 3 & 2.8 & 1.2 & 1.2 & 0.5 & 0.8 ! & 0 & 0.5 & & 0.5 & & \\
\hline & $\mid$ & & & 781 & 415 & 847 & 6 & & 0.5 & 0.5 & 0.5 & 0.5 & 0.5 & 0.5 & 0.5 & 0.5 & 0.5 & 0.5 & 0.5 & I & 0.5 & & & 0 & 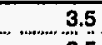 \\
\hline & $B$ & iii & & 781 & 415 & 84 & 6 & 9.9 & 0.5 & 0.5 & 0.5 & 0.5 & 0.5 & 0.5 & 0.5 & 0.5 & 0.5 & 0.5 & 0.5 & 0 & 0.5 & & $1: 6$. & & \\
\hline & & & & $78 !$ & 41 & 84 & & 28.4 & 0.2 & 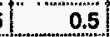 & 0.5 & 0.5 & 0.5 & 0.5 & 0.5 & 0.5 & 0.5 & 0.5 & 0.5 & 0 & 0.5 & & 0.5 & 35.9 & 3.5 \\
\hline MT & D & Mi & MT & 781 & 415 & 847.4 & 6 & 4.1 & 0.5 & 0. & 0.5 & 3.8 & 0.5 & 0 & 0.5 & 0.5 & & a & & 0 & & & $0=$ & 14 & \\
\hline & $E$ & & & 78 & 41 & 84 & 6 & 9.4 & 0.5 & i) & 0.5 & 0.5 & 0 & & & & 0.9 & 0.5 & & & & & & & \\
\hline & Avg & | MLOово & $M T-2 . G$ & 781.0 & $415.0^{\circ}$ & 847.4 & 6 & 14.8 & 0.5 & 9 & 0.5 & 0.5 & 0.5 & 0.5 & 0 & $\underline{-}$ & 0.5 & 0.5 & 1 & & ic & & 0.5 & & \\
\hline & $A_{.}$ & MLOOS4 & MT-2-G & 530.0 & 516.0 & 881.0 & 1 & 0.5 & 0.5 & 0.5 & 0.5 & 0.5 & 0.5 & 0.5 & 0.5 & 0.5 & 0.5 & 0.5 & 0.5 & 0.5 & 0.5 & 0.5 & 0.5 & 10 & 3.5 \\
\hline MT-2-G069 & B & Mi & MT- & 530 & $5 j 6$ & 881.0 & 1 & & & & & & & & & & & & & & & & & 0 & 0.0 \\
\hline & $7=$ & ML084 & MT-2.6069' & 530. & 516 & Sot & 1 & & & & & & & & & & & & & & & & & 0.0 & 0 \\
\hline
\end{tabular}

9 of 17

Data from Method $D$ not used in calculations of averages 
GCMS Data

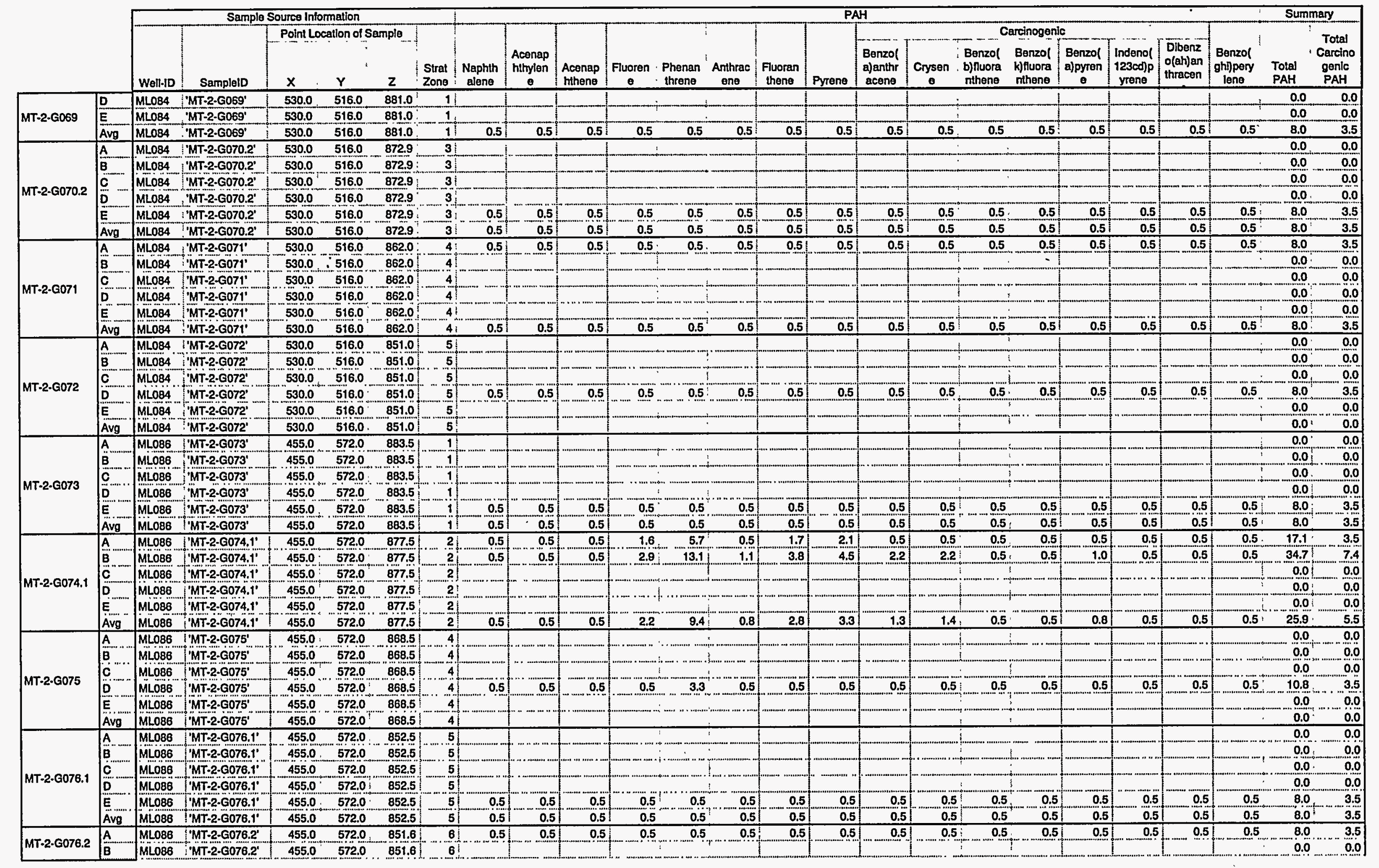

\section{0 of 17}

Data from Method $D$ not used in calculations of averages 


\section{GCMS Data}

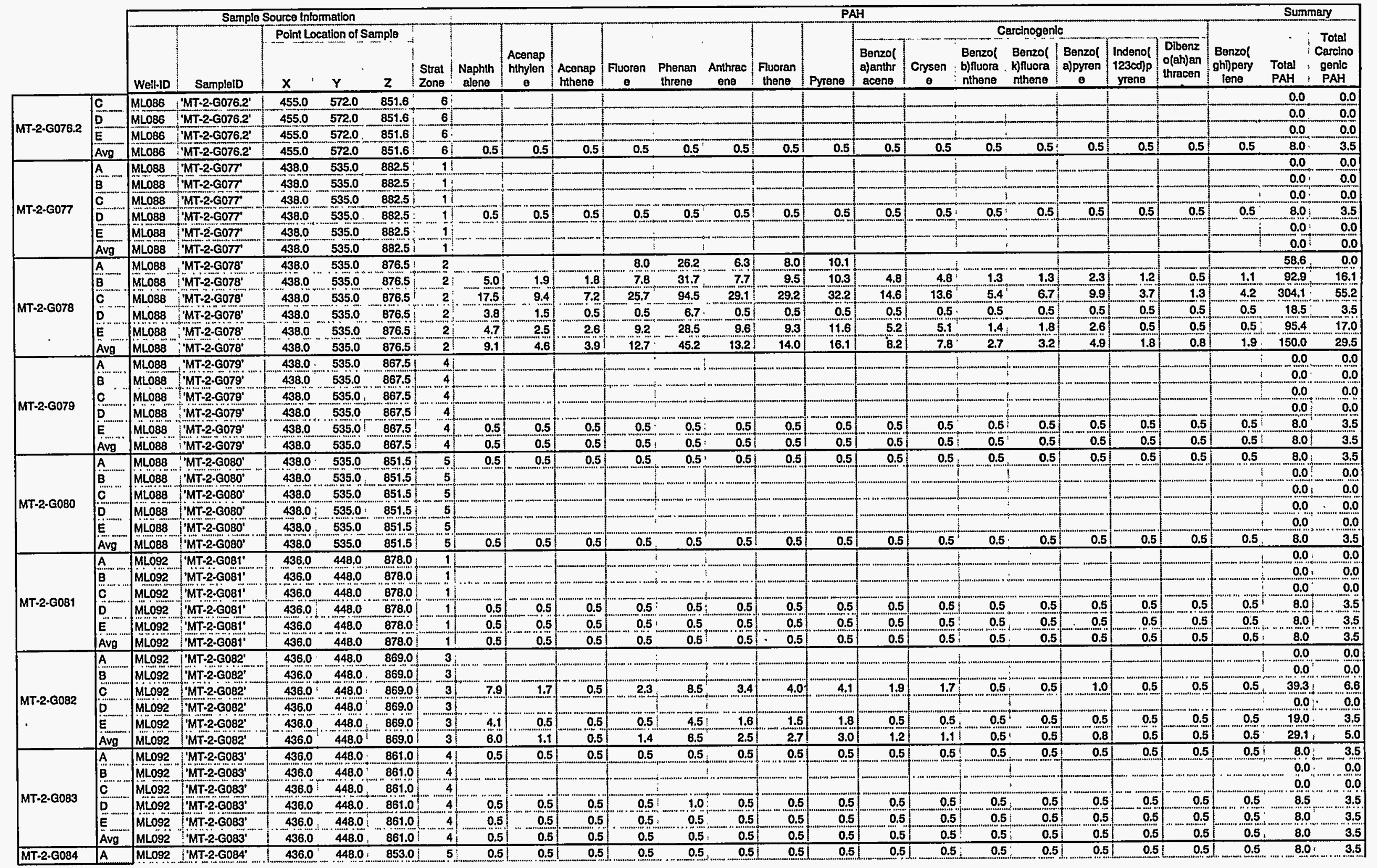

\section{1 of 17}

Data from Method $D$ not used in calculations of averages 
GCMs Data

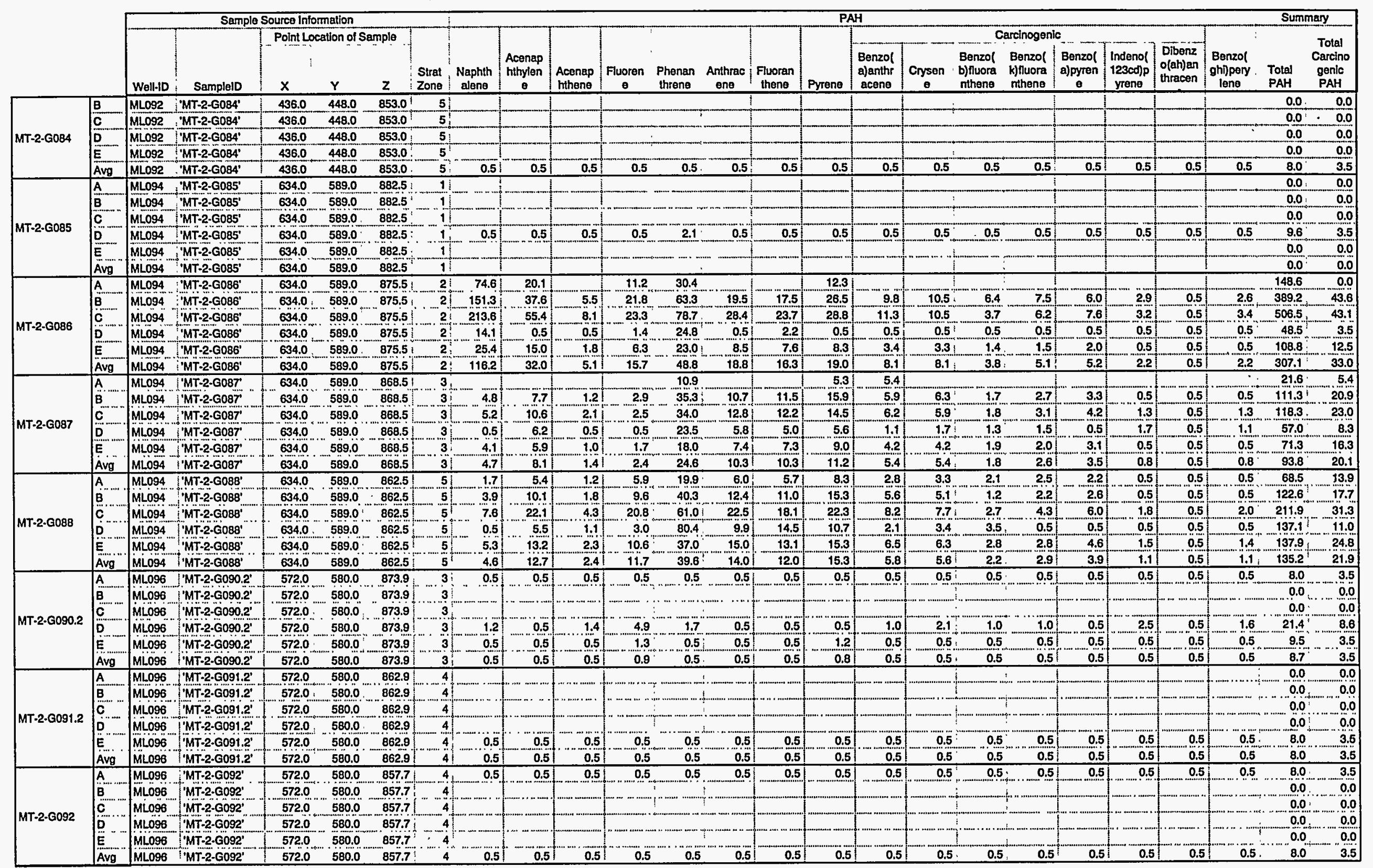

12 of 17

Data from Method $D$ not used in calculations of averages 
GCMS Data

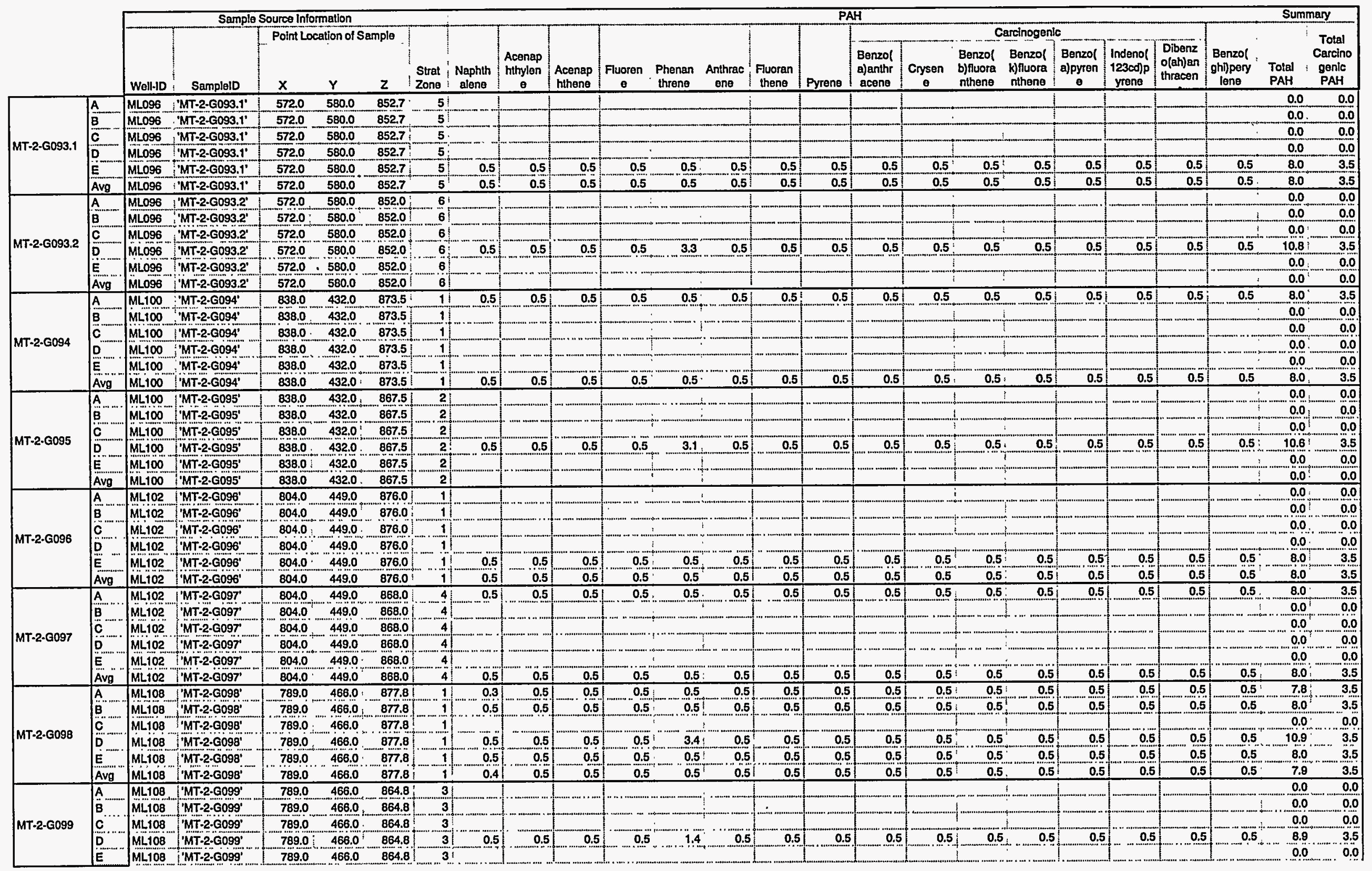

13 of 17

Data from Method D not used in calculations of averages 
GCMS Data

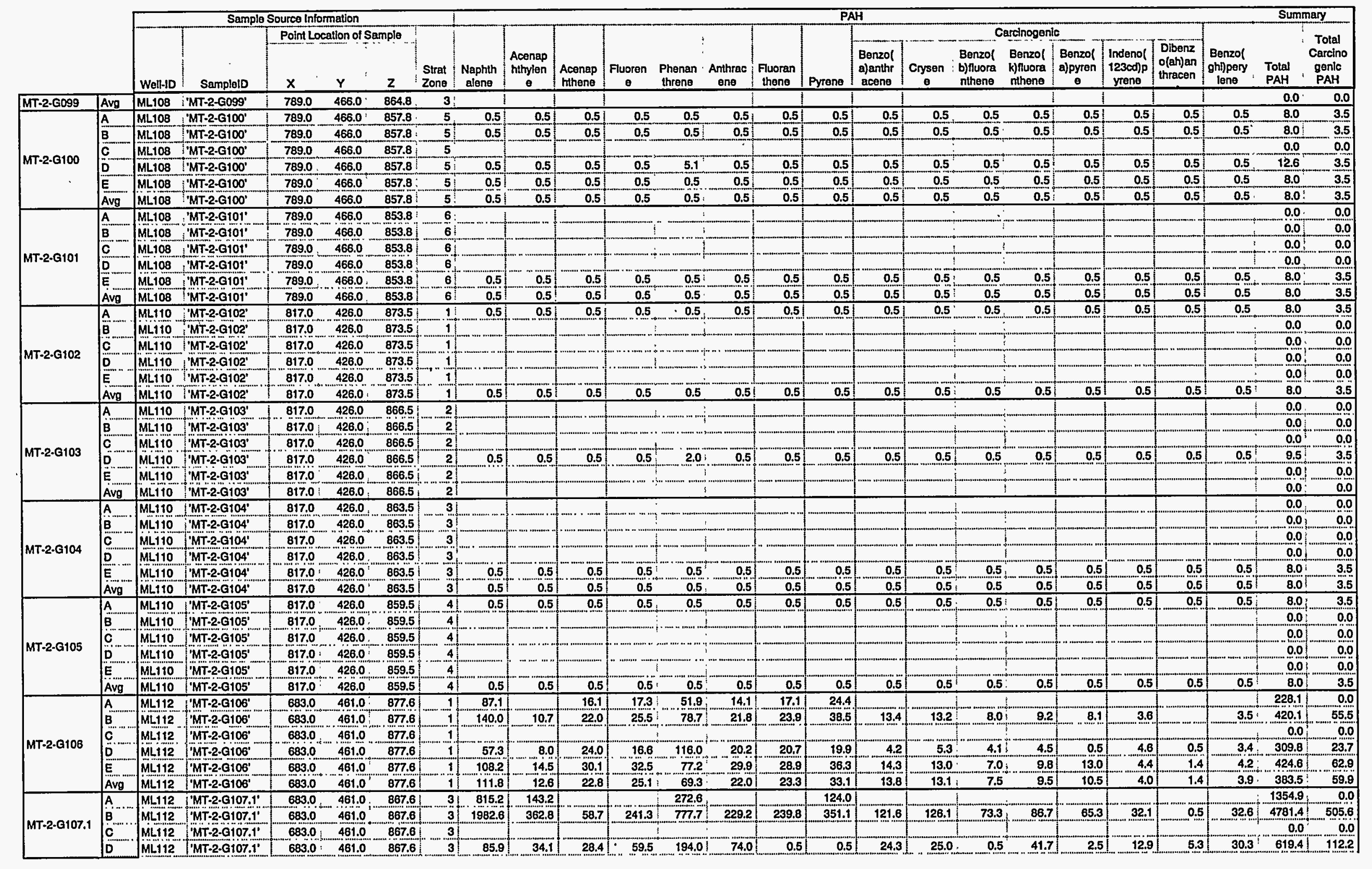

14 of 17

Data from Method D not used in calculations of averages 


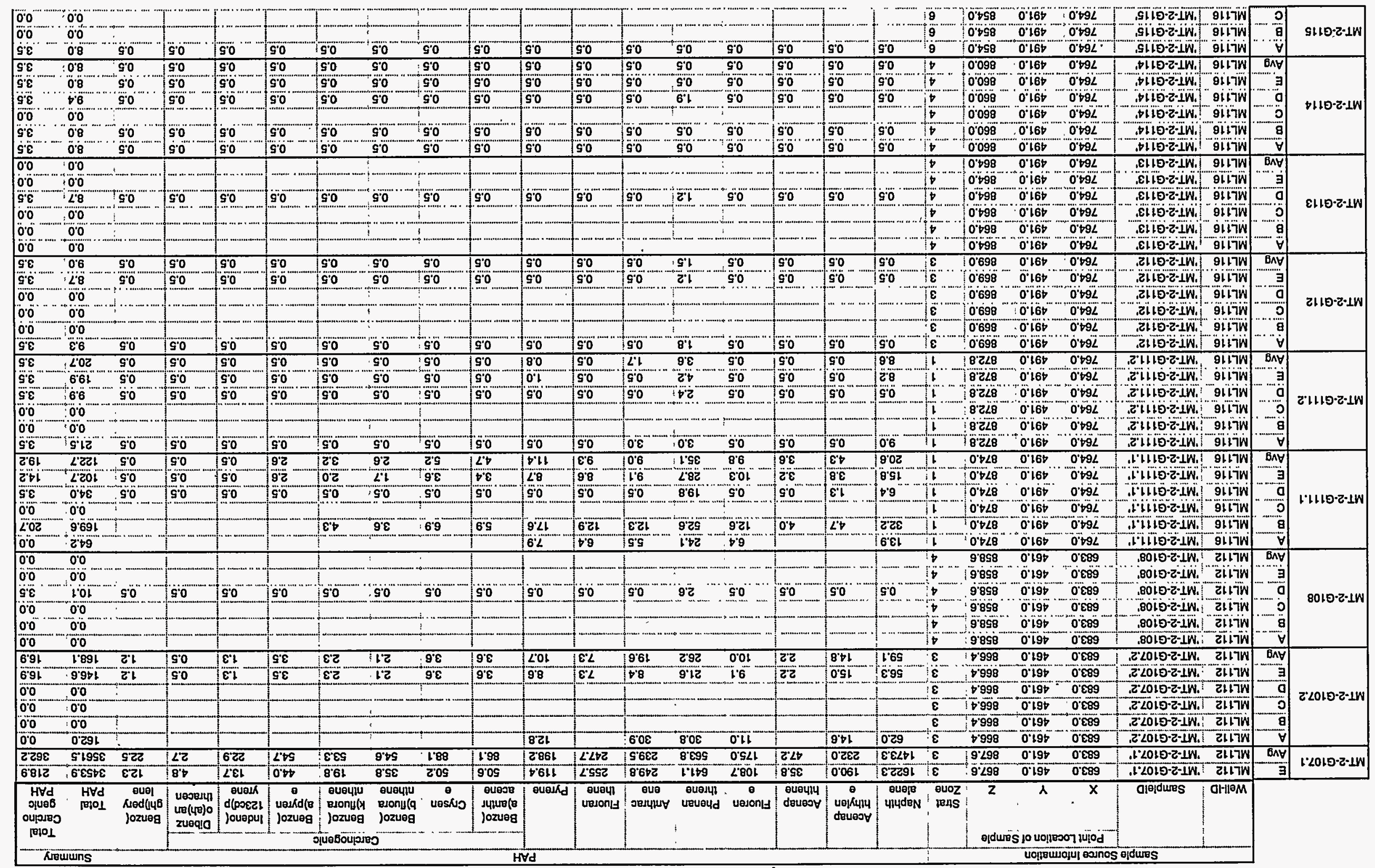

\section{efea SWDอ}


GCMS Data

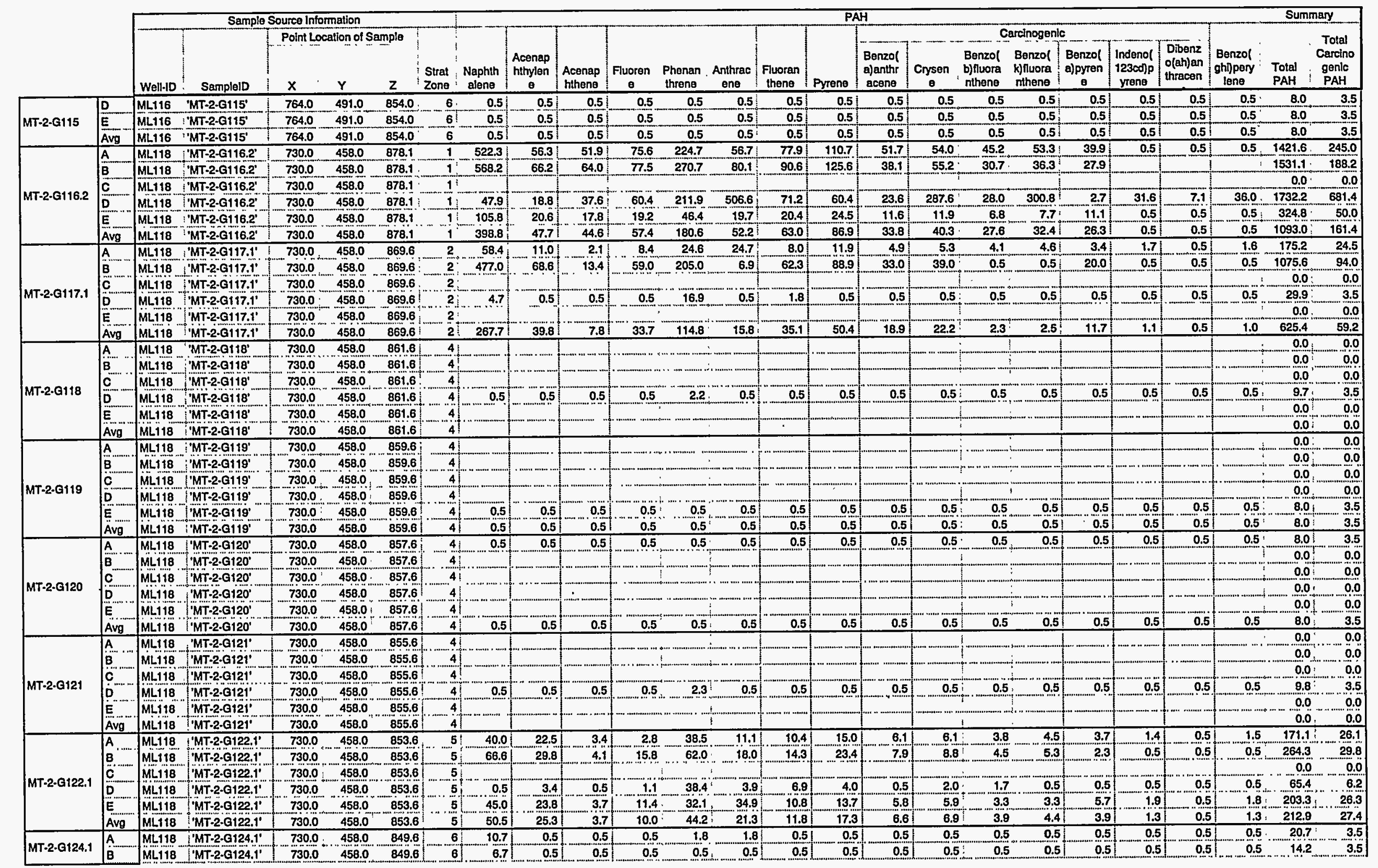

16 of 17

Data from Method D not used in calculations of averages 
GCMS Data

\begin{tabular}{|c|c|c|c|c|c|c|c|c|c|c|c|c|c|c|c|c|c|c|c|c|c|c|c|c|c|}
\hline & & \multicolumn{6}{|c|}{ Sample Source Information } & \multicolumn{15}{|c|}{ PAH } & \multicolumn{3}{|c|}{ Summany } \\
\hline & & \multirow[b]{2}{*}{ Well-1D } & \multirow[b]{2}{*}{ Sampleld } & \multicolumn{3}{|c|}{ Point Location of Semple } & \multirow[b]{2}{*}{$\begin{array}{l}\text { Strat } \\
\text { Zone }\end{array}$} & \multirow[b]{2}{*}{$\begin{array}{c}\text { Naphth } \\
\text { slens }\end{array}$} & \multirow[b]{2}{*}{$\begin{array}{c}\text { Acenep } \\
\text { htthylen } \\
8\end{array}$} & \multirow[b]{2}{*}{$\begin{array}{l}\text { Acenap } \\
\text { hthene }\end{array}$} & \multirow[b]{2}{*}{$\begin{array}{c}\text { Fluoren } \\
e\end{array}$} & \multirow[b]{2}{*}{$\begin{array}{c}\text { Phenan } \\
\text { threns }\end{array}$} & \multirow[b]{2}{*}{$\begin{array}{c}\text { Anthrac } \\
\text { ene }\end{array}$} & \multirow[b]{2}{*}{$\begin{array}{c}\begin{array}{c}\text { Fuoran } \\
\text { thene }\end{array} \\
\end{array}$} & \multirow[b]{2}{*}{ Pyreng } & \multicolumn{7}{|c|}{ Carcinogenic } & \multirow[b]{2}{*}{$\begin{array}{c}\text { Benzol } \\
\text { ghi)pery } \\
\text { lene }\end{array}$} & \multirow[b]{2}{*}{$\begin{array}{l}\text { Total } \\
\text { PAH }\end{array}$} & \multirow[b]{2}{*}{$\begin{array}{c}\text { Total } \\
\text { Carclno } \\
\text { genic } \\
\text { PAH }\end{array}$} \\
\hline - & & & & $\mathrm{x}$ & $Y$ & $z$ & & & & & & & & & & $\begin{array}{c}\text { Benzol } \\
\text { a)anthr } \\
\text { acone }\end{array}$ & $\begin{array}{c}\text { Crysen } \\
\quad\end{array}$ & $\begin{array}{l}\text { Benzol } \\
\text { b)fluora } \\
\text { nthene }\end{array}$ & $\begin{array}{l}\text { Benzol } \\
\text { k)fluora } \\
\text { nthene }\end{array}$ & $\begin{array}{c}\text { Benzol } \\
\text { a)pyren } \\
0\end{array}$ & $\begin{array}{c}\text { Indeno( } \\
123 \cos ) \mathrm{p} \\
\text { yrene } \\
\end{array}$ & $\begin{array}{l}\text { Dibenz } \\
\text { o(ah)an } \\
\text { thracen }\end{array}$ & & & \\
\hline \multirow{4}{*}{ MT-2-G124.1 } & C & ML118 & 'MT-2-G124.I' & 730.0 & 458.0 & 849.6 & 6 & & & & & & & & & & & & & & & & & 0.0 & 0.0 \\
\hline & $D$ & ML11B & 'MT-2-G124.1' & 730.0 & 458.0 & 849.6 & 6 & 1.3 & 0.5 & 0.5 & 0.5 & 3.9 & 0.5 & 0.5 & 0.5 & 0.5 & 0.5 & 0.5 & 0.5 & 0.5 & 0.5 & 0.5 & 0.5 & 12.2 & 3.5 \\
\hline & $E$ & ML118 & IMT-2-G124.1 & 730.0 & 458.0 & 849.6 & 6 & 5.0 & 0.5 & 0.5 & 0.5 & 0.5 & 0.5 & 0.5 & 0.5 & 0.5 & 0.5 & 0.5 & 0.5 & 0.5 & 0.5 & 0.5 & 0.5 & 12.5 & 3.5 \\
\hline & Avg & ML118 & IIT-2-G124.1' & 730.0 & 458.0 & 849.6 & 6 & 7.5 & 0.5 & 0.5 & 0.5 : & 0.9 & 0.9 & 0.5 & 0.5 & 0.5 & 0.5 & 0.5 & 0.5 & 0.5 & 0.5 & 0.5 & 0.5 & 15.8 & 3.5 \\
\hline \multirow{6}{*}{ MT-2-Gt24.2 } & $A$ & ML118 & 'MT-2-G124.2' & 730.0 & 458.0 & 848.9 & 6 & 31.3 & 0.5 & 0.5 & 0.5 & 0.5 & 0.5 & 0.5 & 0.5 & 0.5 & 0.5 & 0.5 & 0.5 & 0.5 & 0.5 & 0.5 & 0.5 & 38.8 & 3.5 \\
\hline & $B$ & ML118 & MT-2-G124.2 & 730.0 & 458.0 & 848.9 & 6 & 29.3 & 0.5 & 0.5 & 0.5 & 0.5 & 0.5 & 0.5 & 0.5 & 0.5 & 0.5 & 0.5 & 0.5 & 0.5 & 0.5 & 0.5 & 0.5 & 36.8 & 3.5 \\
\hline & c & $M L 118$ & MT-2-G124.2 & 730.0 & 458.0 & 848.9 & 6 & & & & & & & & & & & & & & & & & 0.0 & 0.0 \\
\hline & D & MLIIB & MT-2-G124.2 & 730.0 & 458.0 & 848.9 & 6 & 4.2 & 0.5 & 0.5 & 0.5 & 4.5 & 0.5 & 0.5 & 0.5 & 0.5 & 0.5 & 0.5 & 0.5 & 0.5 & 0.5 & 0.5 & 0.5 & 15.7 & 3.5 \\
\hline & $E$ & ML.118 & MT-2-G124.2 & 730.0 & 458.0 & 848.9 & 6 & 29.2 & 0.5 & 0.5 & 0.5 & 0.5 & 0.5 & 0.5 & 0.5 & 0.5 & 0.5 & 0.5 & 0.5 & 0.5 & 0.5 & 0.5 & 0.5 & $36.7 !$ & 3.5 \\
\hline & Avg & ML118 & 'MT-2-G124.2' & 730.0 & 458.0 & 848.9 & 6 & 29.9 & 0.5 & 0.5 & 0.5 & 0.5 . & $0.5 i$ & 0.5 & 0.5 & 0.5 & 0.5 & 0.5 & 0.5 & 0.5 & 0.5 & 0.5 & 0.5 & 37.41 & 3.5 \\
\hline
\end{tabular}

17 of 17

Data from Method D not used in calculations of averages 
Ames Expedited Site Characterization - Marshalltown FMGP Site

FINAL Site Report

March 25, 1996

\section{APPENDIX E: $\quad$ CONTRACTORS AND CONSULTANTS}

Mobile Analytical Services and

Equipment:

McLaren/Hart Environmental

25 Independence Boulevard

Warren, NJ 07059

$908 / 627-8162$

PACE, Inc.

1710 Douglas Drive North

Minneapolis, MN 55422

612/544-5543

CEM Corporation

3100 Smith Farm Road

P. O. Box 200

Matthews, NC 28106-200

913/492-2006

Field screening technologies:

ENSYS

P. O. Box 14063

Research Triangle Park, NY 27709

919/941-5509 Ext129

Millipore Corporation

80 Ashby Road

Bedford, MA 01730

800/722-5996 Ext 8654

Quantix Systems

2611 Branch Pike

Cinnaminson, NJ 08077

609/829-0110
Northeast Research Institute (NERI)

390 Farmington Avenue, Suite A100

Farmington, CT 06032

203/677-9666

Global Environmental

5311 Spring Canyon Trail

Rapid City, SD 57 702-9176

605/342-5311

Golder Federal

4104 148th Avenue North

Redmond, WA 98052

206/883-0777

MTI Analytical Institute

41762 Christy Street

Fremont, CA 94538

510/490-0901 Ext 242

U.S. Army Corps of Engineers

Kansas City District

700 Federal Building

601 E. 12th

Kansas City, MO 64106-2896

816/426-3553

Geophysical surveys:

Resolution Resources

9501 Ferris Road

Springport, MI 49284

517/857-3939 
Ames Expedited Site Characterization - Marshalltown FMGP Site

FINAL Site Report

March 25, 1996

Coleman Research Corporation

Blackhawk Geosciences Division

301 Commercial Road,

Suite B

Golden, CO 80401

301/621-8600

GEHM Corporation

1417 Bingham Road

P. O. Box 65

Boonville, MO 65233

816/882-3485

D'Appolonia Environmental Services

One Monroeville Center

Suite 500

Monroeville, PA 15146

303/248-6424

Drilling services:

Layne-Western

25450 Highway 275

Valley, NE 68064

402/359-2042

\section{Health and safety oversight:}

Layne Safety \& Environmental Health Sciences

1900 Shawnee Mission Parkway

Shawnee Mission, KS 66201

913/677-6852
Stratigraphic characterization services:

Geoprobe Systems, Inc.

605 Barney

Salina, KS 67401

913/825-1842

U.S. Army Corps of Engineers

Kansas City District

700 Federal Building

601 E. 12th

Kansas City, MO 64106-2896

816/426-3553

2-D and 3-D data visualization:

Dynamic Graphics, Inc.

1015 Atlantic Avenue

Alameda, CA 94501

510/522-0700

Off-site laboratory analysis:

Keystone Laboratories

501 West 3rd Street North

Newton, IA 50208

800/858-5227

Field management support:

Knightly Environmental, Inc.

P. O. Box 15104

Lenexa, KS 66285

913/888-1985 
Ames Expedited Site Characterization -Marshalltown FMGP Site FINAL Site Report

March 25, 1996

Geophysics consultant:

Technos, Inc.

3333 Northwest 21st Street

Miami, FL 33142

305/634-4507 
Ames Expedited Site Characterization - Marshalltown FMGP Site

FINAL Site Report

March 25, 1996

\section{APPENDIX F: $\quad$ STAKEHOLDER LIST}

New and State-of-the-Practice Technology Providers

DOE national labs, technology transfer officers and environmental groups/programs)

Army Corps of Engineers

Companies responding to the ESC Request for Proposals

Personal contacts made by ESC project managers

University technology development centers

DOE EM-50 managers

Technology Users - Customers of ESC

DOE EM-30 points of contacts

DOE EM-40 managers

Iowa Utility Association (includes utilities who are privately owned but whose stock is public)

Iowa Association of Municipal Utilities (government owned-e.g., Ames Municipal

Utilities, Cedar Falls, Muscatine)

EPRI (Electric Power Research Institute)

Gas Research Institute (GRD members

EEI (Edison Electric Institute)

IGT (Institute of Gas Technology)

Environmental managers of utilities in the U. S. (from EEI database)

Iowa Department of Transportation

Chicago Northwestern Railroad (borders the site)

City Govemment

Ames Chamber of Commerce

Marshalltown Mayor and Chamber of Commerce

Iowa League of Municipalities members

National League of Municipalities members

Regulators

Iowa State Association of Counties (includes County Board of Supervisors who make decisions on FMGPs)

Division directors of EPA staff nationwide

Neighboring (regional) state environmental protection departments

Iowa Department of Natural Resources

Iowa Department of Health \& Human Services

Iowa Utility Board

Iowa Office of Consumer Advocate 
Ames Expedited Site Characterization - Marshalltown FMGP Site

FINAL Site Report

March 25, 1996

Media and Trade Press

Hazmat World

Environmental Protection Magazine

Environmental Science and Technology

KDHO, 2 radio stations in Marshalltown

Marshalltown Times Republican newspaper

Des Moines Register

The Daily Tribune (Ames)

WHO TV

WOI TV

Omaha World Herald

Newsletters that reach utilities -- (Utility Environment Report, Electric Utility Week, Electric Power Daily)

Users of the Washington State Energy Office's electronic bulletin board

[3000 users (United States and Intemational) includes consultants working with utilities]

DOE publications/newsletters

Associations and Societies

Iowa Petroleum Council

Soil and Water Conservation Society

Iowa Engineering Society (includes Consulting Engineers Council of Iowa)

Iowa Geologic Society

Iowa Geologic Survey Bureau

Petroleum Marketers of Iowa

National Association of Engineering Geologists

Kansas City Region of AEG

National Groundwater Association

Iowa Groundwater Association

Iowa Association of Business and Industry

Environmental Professionals of Iowa

Air and Waste Management Association

American Society for Testing of Materials

Association of engineering firms practicing in the geosciences

Hazardous Waste Action Coalition

National Registry of Environmental Professionals

\section{Educators}

Iowa High School Science Educators

AEA (Area Education Agency)

College Placement Council Newsletter

Iowa Community Colleges (e.g., Kirkwood)

Department heads of the science departments at Iowa State University, University of Iowa, University of Northem Iowa 
Ames Expedited Site Characterization - Marshalltown FMGP Site

FINAL Site Report

March 25, 1996

Iowa point of contacts for DOE EM's PETE program

Iowa Legislators

Ralph Rosenberg and others on environmental committee

Other govermments

Sac and Fox Tribe

\section{Public Interest Groups and other citizens}

Iowa Citizens for Community Improvement

Iowa Citizen Action Network

Businesses located adjacent to the site 
Ames Expedited Site Characterization - Marshalltown FMGP Site FINAL Site Report

March 25, 1996

APPENDIX G:

ATTENDEES AT THE VISITORS' DAYS 


\section{ESC Attendees List for May 2}

Shiv Ambardar

Seneca Environmental Services

4509 NE 14 Street

Des Moines, IA 50313

(515)292-3464

Kevin Armstrong

Montgomery Watson

11107 Aurora Avenue

Des Moines, IA 50322-7938

(515)253-0830

Doug Betts

Kiowa Corporation

P.O. Box 657-T

610 S. 12th Avenue

Marshallown, IA 50158

(515)753-5566

Christopher Cooper

Advanced Environmental Services, Inc.

Corporated Centre 200

PO Box 160

Marion, IA 52302

(319)377-6357

Lou Corones

2600 Timberland Road

Ames, IA 50010

(515)292-9654

Steve Crane

Montgomery Watson

11107 Aurora Avenue

Des Moines, IA 50322-7938

(515)253-0830

James Dzubay

Matrix Technologies, Inc.

2978 12th Street, NW

St. Paul, MN 55112

(612)424-4803
Marty Fleuette

Allwaste/Northcentral

305 21st Street

Camanche, IA 52730

(319)243-7951

Regie Goodale

lowa Utilities Board

5 th Floor, Lucas Bldg.

Des Moines, IA 50309

(515)281-5979

Jerry Hoffman

Burns \& McDonnell Waste

10881 Lowell

Suite 200

Overland Park, KS 66210

(816)822-3076

John Hom

KCPL

PO Box 418679

4400 East Front St.

Kansas City, MO 64141-9679

(816)245-3821

Randy Kroneman

Montgomery Watson

11107 Aurora Avenue

Des Moines, IA 50322-7938

(515)253-0830

Marie Leat

lowa DNR

Wallace State Office Bldg.

Des Moines, IA 50319

(515)281-8045

Mick Leat

Environmental Science \& Engr.

10520 Hickman Rd, Suite F

Des Moines, IA 50325

(515)252-0088
Shirley A. Liebman

The SECON Group, Inc.

91 Pinnacle Road West

Holtwood, PA 17532

(717)284-5225

Perry Morris

Kirkwood Community College

6301 Kirkwood Blvd

Cedar Rapids, IA 52341

(319)398-5678

Joani Nicoll

lowa Utilities Board

5 th Floor, Lucas Bldg.

Des Moines, IA 50309

(515)281-5979

Cary Pieterick

LBG Consultants

4009 W. 49th St.

Stuite 204

Sioux Falls, SD 57106

(605)361-6000

George Robataille

Army Environmental Center

SFIM-AEC-TSD

Bldg E4435

Aberdeen Proving Ground, MD 210

(410)671-1576

Bill Robinson

Advanced Environmental Services,

Corporated Centre 200

PO Box 160

Marion, IA 52302

(319)377-6357

Dennis Rosauer

lowa Utilities Board

5 th Floor, Lucas Bldg.

Des Moines, IA 50309

(515)281-5979 


\section{ESC Attendees List for May 2}

\author{
Bill Smith \\ lowa Utilities Board \\ 5th Floor, Lucas Bldg. \\ Des Moines, IA 50309 \\ (515)281-5469
}

Jim Starrett

Coleman Energy and Enviro. Systems

301 Commercial Road, Ste B

Golden, CO 80401

\author{
Doug Wirth \\ Int'I Ag Prog./bringing 15 Bolivians \\ 6 Curtiss Hall \\ lowa State University \\ Ames, IA 50011 \\ 4-2845 \\ Vicki Wood \\ Kirkwood Community College \\ 6301 Kirkwood Blvd \\ Cedar Rapids, IA 52341 \\ (319)398-5678
}

Clay Swanson
lowa DNR
Box 1443
Mason City, IA 50402
$(515) 424-4073$
T. R. Thompson
Mayor of Marshalltown
1406 S. 12th St.
Marshalltown, IA 50158

Al Tompkins

lowa DNR

Box 1443

Mason City, IA 50402

(515)424-4073

Bill Weiss

Burns \& McDonnel

10881 Lowell

Overland Park, KS 66210

(816)333-8787

Patrick Wheat

Univ. of Northem lowa

Rm-9, ITC

Cedar Falls, IA 50614-0178

(319)273-2561 


\section{ESC Attendees List for May 3}

\author{
Glenn Carper \\ lowa DNR \\ Box 1443 \\ Mason City, IA 50402 \\ (515) $424-4072$
}

Jay Emerson

University of lowa

316 Jessup Hall

lowa City, IA 52242

(319)339-1969

Fred Fitter

Groundwater Service \& Supply

2701 SE Convenience Blvd.

Ankeny, IA 50021

(515)964-0304

Richard Freeman

Fox Engineering

1531 Airport Rd.

Ames, IA 50010

(515)233-0000

Brian Gedlinske

lowa Waste Reduction Center

Univ. of Northern lowa

Field Office 5, Wallace Bldg

Des Moines, IA 50319

(515)281-6484

Bill Gress

Dept. of Natural Resources

Field Office \#5, Wallace Bldg.

Des Moines, IA 50319

(515)281-6484

Bill Haas

lowa Office of Consumer Advocate

Office of Utilities

Lucas State Office BIdg.

Des Moines, IA 50319

(515)281-5984

Argie Hall

RR \#2, Box 342

Maxwell, IA 50161
Julianna Johnston

lowa Citizens Action Network

124 1/2 E. Washington St.

lowa City, IA 52240

(319)354-8116

Greg Junk

Ames Laboratory

108 Spedding

lowa State University

Ames, IA 50011

4-9488

\section{Curt Krieger}

lowa DNR

Box 1443

Mason City, IA 50402

(515)424-4072

Peter Law

Tighe \& Bond

Westfield Executive Park

53 Southampton Road

Westfield, MA 01085-5308

(413)572-3209

Chi Li

lowa Office of Consumer Advocate

Office of Utilities

Lucas State Office Bldg.

Des Moines, IA 50319

(515)281-5984

Fred Lindeman

Interstate Power Co.

PO Box 769

Dubuque, IA 52004-0769

(319)582-5421

Jim Marek

Groundwater Technology, Inc.

3000 Justin Drive, Suile $K$

Urbandale, IA 50322

(515)252-0234

(319)339-4830 


\section{ESC Attendees List for May 3}

Ken McFadden

Environmental Science \& Engineering 10520 Hickman Rd, Suite F

Des Moines, IA 50325

(515)252-0088

Jim McKay

Environmental Science \& Engineering

10520 Hickman Rd, Suite $F$

Des Moines, IA 50325

(515)252-0088

Deanna Neldeberg

Marshalltown Co. Emergency Mgmt.

24 N. Center Street

Marshalltown, IA 50158

(515)754-6385

Brock Nelson

Chicago NW

165 N. Canal Street

Chicago, IL 60606

(312)559-6585

\section{Gerald Palau}

Bechtel

151 Lafayette Drive

PO Box 350

Oak Ridge, TN 37831-0350

(615)220-2000

Sheila Parker

lowa Office of Consumer Advocate

Office of Utilities

Lucas State Office Bldg.

Des Moines, IA 50319

(515)281-5984

Ron Polle

lowa Office of Consumer Advocate

Office of Utilities

Lucas State Office Bldg.

Des Moines, IA 50319

(515)281-5984
Mary Porter

RR \#2, Box 342

Maxwell, IA 50161

William Porter

RR \#2, Box 342

Maxwell, IA 50161

Keith Potts

Walsh Environmental

9802 Nicholas St.

Suite 105

Omaha, NE 68114

(402)393-1010

Ruth A. Rosdail

lowa DNR

900 East Grand

Des Moines, IA 50319

Charlie Ross

Carolina Power \& Light

PO Box 1551

CPB - 342

Ralëigh, NC 27602

Thomas Sheber

Brown Engineering

1051 Office Park Road

West Des Moines, IA 50265

(515)225-6900

Larry Shi

lowa Office of Consumer Advocate

Office of Utilities

Lucas State Office BIdg.

Des Moines, IA 50319

(515)281-5984
Edward J. Staples

Amerasia Technology, Inc.

2248 Townsgate

Westlake Village, CA 91361

(805) $495-9388$

John Vedder

lowa DNR

900 East Grand

Des Moines, IA 50319

Greg Vitale

lowa Office of Consumer Advocate

Office of Utilities

Lucas State Office Bldg.

Des Moines, IA 50319

(515)281-5984

Paul Wang

Ames Laboratory

106 Spedding Hall

Ames, IA 50011-3020

4-6773

Gary Watson

Amerasia Technology, Inc.

2248 Townsgate

Westlake Village, CA 91361

(805)495-9388

John Wheeler

Environmental Science \& Engineeri 10520 Hickman Rd, Suite F

Des Moines, IA 50325

(515)252-0088

Todd D. Whipple

Fox Engineering Associates, Inc.

1531 Airport Rd.

Ames, IA 50010

(515)233-0000 


\title{
ESC Attendees List for May 3
}

\author{
Alesia Whitney-Knight \\ lowa DNR \\ 900 East Grand \\ Des Moines, IA 50319 \\ (515)281-8925 \\ Darrel Wolff \\ Burlington Environmental \\ PO Box 230 \\ Columbia, IL 62236 \\ (618)281-7173 \\ Ying Yan \\ lowa Office of Consumer Advocate \\ Office of Utilities \\ Lucas State Office Bldg. \\ Des Moines, IA 50319 \\ (515)281-5984
}




\section{ESC Attendees List for May 4}

Mary-Linda Adams

Resolution Resources, Inc.

1740 W. Rebel Road

Palmdale, CA 93551

(805)273-8592

Tom Albertson

lowa-Illinois Gas and Electric Company

206 E. 2nd Street

Davenport, IA 52801

(319)326-7384

Rich Blohm

Coleman Energy and Enviro. Systems

301 Commercial Road, Ste B

Golden, CO 80401

\section{Curt Clowe}

Woodward-Clyde Consultants

101 S. 108th Avenue

Omaha, NE 68154

(402)334-8181

Kevin Cornwell

Geotechnical Services

5730 S. 86th Circle

Omaha, NE 68127

(402)339-6104

Jack Cummings

Ames Laboratory

211 TASF

Ames, IA 50011

4-4582

Janet Estep

PACE, Incorporated

i 1710 Douglas Drive North

Minneapolis, MN 55422

(612)525-3307
John Haas

lowa Dept. of Transportation

800 Lincolnway

Bureau of Management

Ames, IA 50010

(515)239-1040

Brian Herridge

Resolution Resources, Inc.

310 West 52nd Street

Minneapolis, MN 55419

(612)824-3234

Bart Hoekstra

Coleman Energy and Enviro. Systems

301 Commercial Road, Ste B

Golden, CO 80401

(303)278-8700

Gary K. Hubbard

Unisys Environmental Systems

PO Box 64525

MS U1F 15

St. Paul, MN 55164-0525

(612)456-3721

Dan Huffaker

Terra Clean, Inc.

PO Box 65248

West Des Moines, IA 50265

(515)221-9823

Robin Husman

Seneca Environmental Services

5113 Tremont

Davenport, IA 52807

(319)386-2522

Gary Johnson

Midwest Gas

401 Douglas, PO Box 778

Sioux Cily, IA 51102

(712)277-7586
Vemon Jordan

lowa Utilities Board

5th Floor, Lucas Bldg.

Des Moines, la 50309

(515)281-5979

Bob Libra

lowa DNR

109 Trowbridge Hall

lowa City, IA 52240

(319)335-1585

Jim Majure

lowa State University

218 Durham Center

Ames, IA 50011

Cal Mather

Terra Clean, Inc.

PO Box 65248

West Des Moines, IA 50265

(515)221-9823

Steve Mayberry

IADOT

800 Lincolnway

Bureau of Management

Ames, IA 50010

(515)239-1040

Teresa McConnell

lowa Utilities Board

5th Floor, Lucas Bldg.

Des Moines, IA 50309

(515)281-5979

Tomiann McDaniel

US Army Corps of Engineers

20 Mass. Ave., NW

Washington, DC 20314-1000

(202)504-4363 


\section{ESC Attendees List for May 4}

B. Keith McMahan EG \& G Mound Applied Tech.

PO Box 3000

Miamisburg, $\mathrm{OH} 45342$

(513)865-3462

Jill Meran

IES Industries, Inc.

200 First Street S.E.

PO Box 351

Cedar Rapids, IA 52402

(319)398.4394

Les Merritt

Ames Laboratory

133 Spedding

Ames, IA 50011

Jiane Munns

lowa Utilities Board

5th Floor, Lucas Bldg.

Des Moines, IA 50309

(515)281-5979

Sam Nelson

Midwest Gas

401 Douglas, PO Box 778

Sioux City, IA 51102

(712)277-7930

Dennis Ostwinkle

lowa DNR

Box 1443

Mason City, IA 50402

(515)424-4073

Barb Oswalt

lowa Utilities Board

5th Floor, Lucas Bldg.

Des Moines, IA 50309

(515)281-5979
Rick Petersen

Midwest Gas

401 Douglas, PO Box 778

Sioux City, IA 51102

(712)277-7953

Vicki Place

lowa Utilities Board

5th Floor, Lucas Bldg.

Des Moines, IA 50309

(515)281-5979

David W. Porter

Coleman Research Corp.

9891 Broken Land Parkway

Suite 200

Columbia, MD 21045

(410)720-5155

Jim Ralston

lowa \& Illinois Gas \& Elec Co.

206 E. 2nd St.

Davenport, IA 52801

(319)326-7384

Bill Rice

Midwest Gas

401 Douglas, PO Box 778

Sioux City, IA 51102

(712)277-7572

Dennis Sensenbrenner

Seneca Environmental

4509 NE 14th Street

Des Moines, IA 50313

(515)262-3500

Charlie Shannon

Hogentogler \& Co., Inc.

9515 Gerwig Lane

PO Drawer 2219

Columbia, MD 21045

(410)381-2390
Bill Simpkins

lowa State University

Geology Dept.

55 Science I

Ames, IA 50011

4-7814

Dawn Vance

lowa Utilities Board

5th Floor, Lucas Bidg.

Des Moines, IA 50309

(515)281-5979

Jeff Vansteenburg

DNR

P.O. Box 1443

2300 - 15th St., SW

Mason City, IA 50402

(515)424-4073

Mitch White

Seneca Environmental Services

5113 Tremont

Davenport, IA 52807

(319)386-2522

Tally Wright-Wells

Barr Enginnering Co

8300 Norman Center Dr

Minneapolis, MN 55437

(612)832-2739 


\section{APPENDIX H: $\quad$ ABBREVIATIONS AND ACRONYMS}

$\mathrm{A} / \mathrm{P}$

ASL

BVWST

CDP

CL

CLP

CMP

CPT

DNAPL

DOE

E\&E

EM

EOL

ESC

F/P

FMGP

GC

GC/FID

GC/MS

GC/PID

GIS

GPR

GRM

GSSI

HPLC

$\mathrm{Hz}$

IDNR

IES

IMA

IT

LCU

LIF

MDL

$\mathrm{MHz}$

ML

MLH

MS

$\mathrm{ms}$

MW
Anthracene/phenanthrene

Above sea level

Black and Veatch Waste Science and Technology

Common depth point

Chemiluminescence

Contract laboratory program

Common midpoint

Cone penetration testing

Denser-than-water non-aqueous phase liquid

U. S. Department of Energy

Ecology and Environmental

Environmental Management

Electromagnetic offset logging

Expedited Site Characterization

Fluoranthene/pyrene

Former manufactured gas plant

Gas chromatography

Gas chromatography/flame ionization detector

Gas chromatography/Mass Spectrometry

Gas chromatography/photoionization detector

Geographical Information System

Ground penetrating radar

Generalized Reciprocal Method

Geophysical Survey Systems, Inc.

High pressure liquid chromatography

Hertz

Iowa Department of Natural Resources

Iowa Electric Service

Immunoassay

Innovative technologies

Lower cohesive unit

Laser induced fluorescence

Method detection limit

Megahertz

Map locations

McLaren/Hart

Mass spectrometry

Milliseconds

Molecular Weight 
Ames Expedited Site Characterization - Marshalltown FMGP Site

FINAL Site Report

March 25, 1996

NAPL

ND

NERI

ns

OTD

PAHs

POL

PPM

QA/QC

RI

SCAPS

SFE

SOPT

SVOCs

TD-GC/MS

TD-MS

TRPH

UCU

U.S.-EPA

UST

WES

VOA

VOCs
Non-aqueous phase liquid

Non-detect

Northeast Research Institute

Nanoseconds

Office of Technology Development

Polynuclear aromatic hydrocarbons

Petroleum, oil, and lubricant

Parts per million

Quality assurance and quality control

Remedial Investigation

Site Characterization and Analysis Penetrometer System

Supercritical fluid extraction

State-of-the-practice technologies

Semivolatile organic compounds

Thermal Desorption-Gas Chromatography/Mass Spectrometry

Thermal Desorption/Mass Spectrometry

Total recoverable petroleum hydrocarbon

Upper cohesive unit

United States Environmental Protection Agency

Underground storage tank

Waterways Experiment Station

Volatile organic analysis

Volatile organic compounds 
Ames Expedited Site Characterization - Marshalltown FMGP Site

FINAL Site Report

March 25, 1996

\subsection{DISTRIBUTION LIST}

Ames Laboratory (2)

Office of Public Affairs and Information

111 TASF Building

Iowa State University

Ames, Iowa 50011

Office of Scientific and Technical

Information (OSTI) (2)

P. O. Box 75

Oak Ridge, TN 37831

Patent Counsel (1)

Chicago Operations

Argonne National Laboratory

9800 S. Cass

Argonne, IL 60439

Dr. Al Bevolo (25)

Ames Laboratory

Iowa State University

125 S. 3rd Street

Ames, IA 50010

Dr. Martin Edelson (1)

Ames Laboratory

Iowa State University

130 Spedding Hall

Ames, IA 50011

Dr. James Corones (1)

Ames Laboratory

Iowa State University

329 Wilhelm Hall

Ames, IA 50011
Dr. Paul Wang (1)

Ames Laboratory

Iowa State University

106 Spedding Hall

Ames, IA 50011

Dr. Caroline Purdy (10)

U. S. DOE/EM-54

Trevion II, Suite 400

12800 Middlebrook Road

Germantown, MD 20874

Eric Lightner (1)

U. S. DOE/EM-541

Trevion II, Suite 400

12800 Middlebrook Road

Germantown, MD 20874

Mr. Dean Hargens (2)

IES Industries, Inc.

200 First Street S. E.

P. O. Box 351

Cedar Rapids, IA 52402

Mr. George Robataille (10)

Army Environmental Center

SFIM-AEC-TSD

Building E4430

Aberdeen Proving Ground, MD 21010

Mr. David Wonder (5)

Ames Laboratory

Iowa State University

125 S. 3rd Street

Ames, IA 50010 
Ames Expedited Site Characterization - Marshalltown FMGP Site

FINAL Site Report

March 25, 1996

Ms. Connie Bailey (5)

Ames Laboratory

Iowa State University

125 S. 3rd Street

Ames, IA 50010

Ms. Beth Weiser (1)

Ames Laboratory

Iowa State University

125 S. 3rd Street

Ames, IA 50010

Dr. Bruce Kjartanson (1)

Iowa State University

394 Town Engineering

Ames, IA 50011

Mr. Johanshir Golchin (2)

Iowa Dept. of Natural Resources

900 E. Grand Avenue, Wallace Building

Des Moines, IA 50319

Mr. Steve Gelb (1)

McLaren/Hart Environmental

25 Independence Boulevard

Warren, NJ 07059

Mr. Dick Benson (1)

Technos

3333 Northwest 21st Street

Miami, FL 33142

Mr. George Burns (1)

PACE, Inc.

1710 Douglas Drive North

Minneapolis, MN 55422
David Howard (1)

CEM Corporation

3100 Smith Farm Road

P. O. Box 200

Matthews, NC 28106-200

Mr. Will Elcoate (1)

ENSYS

P. O. Box 14063

Research Triangle Park, NY 27709

Mr. Vincent Donovan (1)

Millipore Corporation

80 Ashby Road

Bedford, MA 01730

Mr. Christopher Heywood (1)

Quantix Systems

2611 Branch Pike

Cinnaminson, NJ 08077

Ms. Rosalie Mandaro (1)

Northeast Research Institute (NERI)

390 Farmington Avenue, Suite A100

Farmington, CT 06032

Mr. Adel Heriba (1)

Global Environmental

5311 Spring Canyon Trail

Rapid City, SD 57702-9176

Mr. Charles Voss (1)

Golder Federal

4104 148th Avenue North

Redmond, WA 98052

Ms. Deborah Gonzalez (1)

MTI Analytical Institute

41762 Christy Street

Fremont, CA 94538 
Ames Expedited Site Characterization - Marshalltown FMGP Site

FINAL Site Report

March 25, 1996

Brian Herridge (1)

Resolution Resources

9501 Ferris Road

Springport, MI 49284

Mr. Bart Hoekstra (1)

Coleman Research Corporation

Blackhawk Geosciences Division

301 Commercial Road,

Suite B

Golden, CO 80401

Mr. Tom Maxwell (1)

GEHM Corporation

1417 Bingham Road

P. O. Box 65

Boonville, MO 65233

Mr. William Johnson (1)

D'Appolonia Environmental Services

One Monroeville Center

Suite 500

Monroeville, PA 15146

Mr. Colin Christy (1)

Geoprobe Systems, Inc.

605 Barney

Salina, KS 67401

Ms. Kathy Older (1)

U.S. Army Corps of Engineers

Kansas City District

700 Federal Building

601 E. 12th

Kansas City, MO 64106-2896
Mr. James Shinn (1)

Applied Research Associates

Waterman Road, P. O. Box 120A

South Royalton, VT 05068

Dr. Ewa Marchwinska (1)

Institute for Ecology of Industrial Areas

6 Kossutha Street

40-833 Katowice, Poland

Mr. Gerald Hill (1)

Southern States Energy Board

3091 Governor Lake Drive

Suite 400

Norcross, GA 30071

Mr. Jeffrey Brown (1)

Land Tech Remedial

569 Main Street

Monroe, CT 06468

Robert Cleary (1)

Princeton Groundwater

P. O. Box 263033

Tampa, FL 33685

Ms. Kelly Bitner (1)

Mactec

2309 Renard Place SE, Suite 103

Albuquerque, NM 87106

Terry Corneil (1)

GETF

7010 Little River Turnpike

Suite 300

Annandale, VA 22003 
1 Ames Expedited Site Characterization - Marshalltown FMGP Site FINAL Site Report

March 25, 1996

Mr. Michael Barcelona

University of Michigan

Suite 181

EWRE Bldg

1351 Beal Avenue

Ann Arbor, MI 48109 